Seleção de atributos para aprendizado multirrótulo Newton Spolaôr 

SERVIÇO DE PÓS-GRADUAÇÃO DO ICMC-USP

Data de Depósito:

Assinatura:

\section{Seleção de atributos para aprendizado multirrótulo}

\section{Newton Spolaôr}

Orientadora: Profa. Dra. Maria Carolina Monard

Coorientadora: Profa. Dra. Huei Diana Lee

Tese apresentada ao Instituto de Ciências Matemáticas e de Computação - ICMC-USP, como parte dos requisitos para obtenção do título de Doutor em Ciências - Ciências de Computação e Matemática Computacional. VERSÃO REVISADA 
Ficha catalográfica elaborada pela Biblioteca Prof. Achille Bassi e Seção Técnica de Informática, ICMC/USP, com os dados fornecidos pelo(a) autor(a)

S762s Spolaôr, Newton

Seleção de atributos para aprendizado multirrótulo / Newton Spolaôr; orientadora Maria Carolina Monard; co-orientadora Huei Diana Lee. -- São Carlos, 2014. $178 \mathrm{p}$.

Tese (Doutorado - Programa de Pós-Graduação em Ciências de Computação e Matemática Computacional) -Instituto de Ciências Matemáticas e de Computação, Universidade de São Paulo, 2014.

1. seleção de atributos multirrótulo. 2. revisão sistemática. 3. construção de rótulos. 4. Relieff. 5. ganho de informação. I. Monard, Maria Carolina,

orient. II. Lee, Huei Diana, co-orient. III. Título. 
Este documento foi preparado com o formatador de textos $\mathrm{ET}_{\mathrm{E}} \mathrm{X}$. O sistema de citações de referências bibliográficas utiliza o padrão Apalike do sistema $\mathrm{BibT} \mathrm{E}$. 
"Meu único desejo é um pouco mais de respeito pelo mundo, que começou sem o homem e vai terminar sem ele - isso é algo que sempre deveríamos ter presente" Levi-Strauss 


\section{Agradecimentos}

A Deus, pela companhia e proteção que tem proporcionado.

Aos meus pais Maria da P. N. S. Spolaôr e Donizeti Spolaôr, pela base familiar sólida, pelo amor incondicional e pelo apoio que tem me proporcionado. Palavras são pouco para expressar o quanto desejo lhes agradecer. Sou grato porque nos mantemos unidos mesmo morando em diferentes cidades. À minha irmã e amiga Thays Spolaôr, que desde o berço já assumia o papel de minha maior companheira. A nostalgia dos momentos alegres da infância consolam a distância. Ao meu avô Leandro Spolaôr, pelos ensinamentos e doutrinas que se mantém mesmo após sua partida. Sua fé, altruísmo e amor pela música são ideais nobres que tive a honra de compartilhar. Ao meu tio Eliziário e aos meus demais familiares.

À minha orientadora e amiga, Maria C. Monard, pela oportunidade de compartilhar conhecimentos científicos e não-científicos nestes anos, bem como pela paciência e sabedoria com que me orientou. Lembro de quando ela teve um leve acidente, nada sério, e lhe estendi a mão. Aquilo não foi nada comparado a todas as vezes em que ela me estendeu sua mão. Sua companhia durante as primeiras viagens ao exterior e o estágio sanduíche foi exemplar. A oportunidade de trabalhar com alunos de graduação sob orientação dela também deve ser destacada. A parceria tem sido boa e ela complementa várias das minhas fraquezas. À minha co-orientadora e amiga, Huei D. Lee, pela companhia desde a graduação, pelos ensinamentos que tem compartilhado e pelos caminhos exemplares que construiu e tem mantido com ética e trabalho sério.

Aos educadores e amigos Wu F. Chung, Renato B. Machado e Richardson F. Voltolini, por todos os aprendizados que tem compartilhado, pela confiança e pela formação de várias pessoas. Aos demais amigos que integraram o LABI, como Antonio R. S. Parmezan, Bianca Espindola, Caio A. A. Araujo, Felipe C. Fernandes, Jefferson T. Oliva, Jorge A. Júnior, Leandro B. dos Santos, Narco A. R. Maciejewski, Neimar Neitzel, Paulo César, Rafaella A. L. Neitzel, 
Ricardo G. B. Nabo, Vanize Meneghetti, Willian Zalewski e Wilson Jung por compartilhar conhecimentos e entretenimentos.

Ao professor Grigorios Tsoumakas, pelo trabalho em colaboração na Aristotle University of Thessaloniki (AUTh) e pelo apoio na resolução de questões burocráticas. Aos colegas da universidade grega Anestis Fachantidis, Eleftherios Spyromitros-Xioufis, Emmanouil Rigas, Thanos S. Stavropoulos e Yannis Kavakiotis pelo acolhimento.

Aos amigos e colegas do LABIC, com destaque para Jorge C. ValverdeRebaza, Pedro Shiguihara, Nils M. Llerena, Ricardo M. Marcacini, Thiago de P. Faleiros, Fabiano F. dos Santos, Celso A. R. de Sousa, Lilian Berton, Ígor A. Braga, Merley da S. Conrado, Rafael Giusti, Diego F. Silva, Vinícius M. A. de Souza, Jean Metz, Jimena T. Tomás e Victor A. M. Carvalho. A Everton A. Cherman pela colaboração em diversos momentos do desenvolvimento deste trabalho e pela contribuição para a realização do estágio sanduíche. A Rafael G. Rossi pelo apoio na resolução de questões técnicas. Agradeço também aos professores do Labic, Gustavo E. A. P. A. Batista e Solange O. Rezende.

Aos amigos e colegas da USP Paulo H. Pisani, Marina Osio, Gabriel Humpire, Laura E. Florian, Fernando A. A. Nóbrega, Roni A. Aceituno, Didier A. Vega-Oliveros, Eduardo Pezutti, Fabiano B. de Sousa, Roberto A. Gueleri, Murillo G. Carneiro, Erick R. Fonseca, Renata C. B. Madeo, Christian C. Bones e Guilherme F. de Arruda. Aos bons professores e servidores que tive contato nessa universidade. A Ana C. Lorena pela oportunidade de desenvolver trabalhos em colaboração e de divulgar o método da revisão sistemática.

Aos amigos de outras nacionalidades Ezgi Kahraman (Turquia), Nevila Zirai (Albânia), Nikos Tay (Grécia), Olena Loburenko (Ucrânia) e Sama Safavi (Irã), que tornaram a estadia no estrangeiro mais proveitosa. Deixo a eles um obrigado nas respectivas línguas:

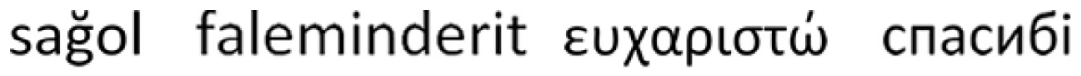

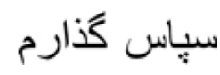

A Thiago C. da Rosa, Gustavo H. B. Ramón, Dijiane R. de Paula, Charles Zimmerman, Luís Brito, Daniele da S. Rogério e Janice Costa pela longa amizade.

A todas as pessoas que diretamente ou indiretamente me ajudaram e apoiaram para a realização deste trabalho.

Ao Conselho Nacional de Desenvolvimento Científico e Tecnológico (CNPq) e à Fundação de Amparo à Pesquisa do Estado de São Paulo (FAPESP), pelo apoio financeiro ao longo da realização deste trabalho. À FAPESP em particular por financiar o estágio sanduíche e pela atuação exemplar em vários momentos. À USP e à AUTh pela infra-estrutura oferecida. 


\section{Resumo}

A presença de atributos não importantes, i.e., atributos irrelevantes ou redundantes nos dados, pode prejudicar o desempenho de classificadores gerados a partir desses dados por algoritmos de aprendizado de máquina. $\mathrm{O}$ objetivo de algoritmos de seleção de atributos consiste em identificar esses atributos não importantes para removê-los dos dados antes da construção de classificadores. A seleção de atributos em dados monorrótulo, nos quais cada exemplo do conjunto de treinamento é associado com somente um rótulo, tem sido amplamente estudada na literatura. Entretanto, esse não é o caso para dados multirrótulo, nos quais cada exemplo é associado com um conjunto de rótulos (multirrótulo). Além disso, como esse tipo de dados usualmente apresenta relações entre os rótulos do multirrótulo, algoritmos de aprendizado de máquina deveriam considerar essas relações. De modo similar, a dependência de rótulos deveria também ser explorada por algoritmos de seleção de atributos multirrótulo. A abordagem filtro é uma das mais utilizadas por algoritmos de seleção de atributos, pois ela apresenta um custo computacional potencialmente menor que outras abordagens e utiliza características gerais dos dados para calcular as medidas de importância de atributos, tais como correlação atributo-classe, entre outras. A hipótese deste trabalho é que algoritmos de seleção de atributos em dados multirrótulo que consideram a dependência de rótulos terão um melhor desempenho que aqueles que ignoram essa informação. Para tanto, é proposto como objetivo deste trabalho o projeto e a implementação de algoritmos filtro de seleção de atributos multirrótulo que consideram relações entre rótulos. Em particular, foram propostos dois métodos que levam em conta essas relações por meio da construção de rótulos e da adaptação inovadora do algoritmo de seleção de atributos monorrótulo ReliefF. Esses métodos foram avaliados experimentalmente e apresentaram bom desempenho em termos de redução no número de atributos e qualidade dos classificadores construídos usando os atributos selecionados. 


\section{Abstract}

Irrelevant and/or redundant features in data can deteriorate the performance of the classifiers built from this data by machine learning algorithms. The aim of feature selection algorithms consists in identifying these features and removing them from the data before constructing classifiers. Feature selection in single-label data, in which each instance in the training set is associated with only one label, has been widely studied in the literature. However, this is not the case for multi-label data, in which each instance is associated with a set of labels. Moreover, as multi-label data usually exhibit relationships among the labels in the set of labels, machine learning algorithms should take this relationship into account. Therefore, label dependence should also be explored by multi-label feature selection algorithms. The filter approach is one of the most usual approaches considered by feature selection algorithms, as it has a potentially lower computational cost than other approaches and uses general properties from data to calculate feature importance measures, such as the feature-class correlation. The hypothesis of this work is that feature selection algorithms which consider label dependence will perform better than the ones that disregard label dependence. To this end, this work proposes and develops filter approach multi-label feature selection algorithms which take into account relations among labels. In particular, we proposed two methods that take into account these relations by performing label construction and adapting the single-label feature selection algorithm ReliefF. These methods

were experimentally evaluated, showing good performance in terms of feature reduction and predictability of the classifiers built using the selected features. 


\section{Sumário}

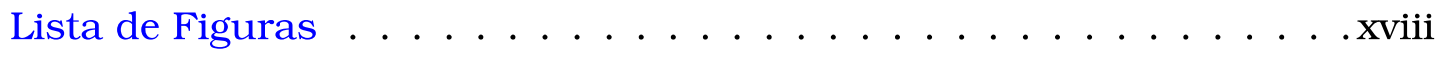

Lista de Tabelas . . . . . . . . . . . . . . . . .xxiii

Lista de Abreviaturas . . . . . . . . . . . . . . . xxvii

Lista de Algoritmos . . . . . . . . . . . . . . . xxix

Lista de Nomenclaturas . . . . . . . . . . . . . . . . . xxxxiii

1 Introdução 1

1.1 Objetivo e Hipótese . . . . . . . . . . . . . . . . . . . 4

1.2 Principais Contribuições $\ldots \ldots \ldots \ldots \ldots \ldots$

1.3 Organização do Trabalho . . . . . . . . . . . . . 8

2 Aprendizado Multirrótulo $\quad 11$

2.1 Definições . . . . . . . . . . . . . . . . 11

2.2 Métodos de Aprendizado Multirrótulo . . . . . . . . . . . . 14

2.2.1 Transformação do Problema . . . . . . . . . . . . 15

2.2.2 Adaptação do Algoritmo . . . . . . . . . . . . . . 17

2.3 Avaliação de Classificadores Multirrótulo . . . . . . . . . . . 20

2.3.1 Medidas de Avaliação baseadas em Exemplo . . . . . . . . . 21

2.3.2 Medidas de Avaliação baseadas em Rótulo . . . . . . . . . 24

2.3.3 Classificadores Baseline . . . . . . . . . . . . . . . 26

2.4 Dependência de Rótulos . . . . . . . . . . . . . . . . . . . . . 27

2.5 Caracterização do Conjunto de Dados . . . . . . . . . . . . 30

2.6 Aplicações de Aprendizado Multirrótulo . . . . . . . . . . 34

2.7 Considerações Finais . . . . . . . . . . . . . . . 35

3 Seleção de Atributos para Aprendizado Multirrótulo 37

3.1 Dimensões da Seleção de Atributos Multirrótulo . . . . . . . . . 38

3.1 .1 Interação com Classificador . . . . . . . . . . . . . 39

3.1 .2 Escopo do Multirrótulo . . . . . . . . . . . . 41

3.1 .3 Dependência de Rótulos . . . . . . . . . . . . . . . 41

3.2 Importância de Atributos . . . . . . . . . . . . . . . . . . 42

3.3 Trabalhos Relacionados . . . . . . . . . . . . . . . . . . 44

3.3.1 Extensões do Algoritmo ReliefF . . . . . . . . . . . . 45

3.3.2 Outros Métodos para Seleção de Atributos Multirrótulo Propostos na Literatura . . . . . . . . . . . . . 47 
3.4 Considerações Finais $\ldots \ldots \ldots \ldots$. . . . . . . . 50

4 Seleção de Atributos baseada em Transformação do Problema 51

4.1 Seleção de Atributos via Abordagens Tradicionais de Transformação do Problema . . . . . . . . . . . . . . . . . 52

4.1.1 Abordagem Binary Relevance . . . . . . . . . . . . . . . . 52

4.1.2 Abordagem Label Powerset . . . . . . . . . . . . . . . . . 56

4.2 Método Label Construction for Feature Selection . . . . . . . . . . . 56

4.2.1 Etapa 1: Seleção ... . . . . . . . . . . . 57

4.2 .2 Etapa 2: Geração . . . . . . . . . . . . . . 59

4.3 Medidas de Importância de Atributos Utilizadas . . . . . . . . . . . 60

4.4 Configuração Experimental . . . . . . . . . . . . . . . . 62

4.4.1 Configurações Específicas das Abordagens Tradicionais . . 63

4.4.2 Configurações Específicas do Label Construction for Feature Selection . . . . . . . . . . . . . . 64

4.5 Resultados e Discussão . . . . . . . . . . . . . . . 65

4.5.1 Abordagens Tradicionais de Transformação do Problema . 65

4.5.2 Label Construction for Feature Selection . . . . . . . . . . . . 70

4.6 Considerações Finais . . . . . . . . . . . . . 75

5 Adaptação do Algoritmo Relieff para Seleção de Atributos Multir$\begin{array}{ll}\text { rótulo } & \mathbf{7 7}\end{array}$

5.1 Família de Algoritmos Relief . . . . . . . . . . . . . 78

5.1 .1 Relief .................. 78

5.1 .2 Relieff ................... . . 79

5.1 .3 RReliefF . . . . . . . . . . . . . . 80

5.2 Extensões Propostas para Seleção de Atributos Multirrótulo via

ReliefF .................... 83

5.2 .1 ReliefF Multirrótulo ................ 83

5.2 .2 ReliefF Mútuo Multirrótulo . . . . . . . . . . . . . 85

5.3 Configuração Experimental . . . . . . . . . . . . . . 87

5.3.1 Conjuntos de Dados Artificiais . . . . . . . . . . . . 89

5.3.2 Medidas de Avaliação Específicas para Seleção de Atributos 90

5.4 Resultados e Discussão . . . . . . . . . . . . . . . . . . 92

5.4.1 Comparação entre Configurações dos Algoritmos RF-ML e RFM-ML . . . . . . . . . . . . . . . 92

5.4 .2 Algoritmos baseados em ReliefF e IG-LP . . . . . . . . . . 95

5.5 Considerações Finais . . . . . . . . . . . . . . . . 102

6 Conclusões 105

6.1 Revisão Sistemática sobre Seleção de Atributos Multirrótulo . . . 105

6.2 Seleção de Atributos de Segunda Ordem via Construção de Rótulosi 06 
6.3 Extensão do Algoritmo ReliefF para Seleção de Atributos Multir-

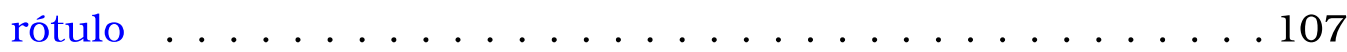

A Apêndice

111

A. 1 Resultados Complementares Relacionados à Revisão Sistemática 111

A.2 Resultados Experimentais Complementares ao Capítulo 4 . . . 112 A.2.1 Abordagens Tradicionais de Transformação do Problema . 113 A.2.2 Label Construction for Feature Selection . . . . . . . . . . . 120

A.3 Resultados Experimentais Complementares ao Capítulo 5 . . . . 135 A.3.1 Configurações dos Algoritmos RF-ML e RFM-ML . . . . . . 135

A.3.2 Algoritmos baseados em ReliefF e IG-LP . . . . . . . . . . . 142 
xvi 


\section{Lista de Figuras}

1.1 Uma possivel taxonomia para organizar trabalhos em seleção de atributos multirrótulo . . . . . . . . . . . . 3

2.1 Taxonomia de medidas de avaliação do aprendizado multirrótulo

2.2 Ilustração do desempenho de quatro algoritmos de aprendizado multirrótulo em termos de duas medidas de avaliação . . . . . . . 24

2.3 Resumo do procedimento de seleção utilizado para encontrar 64 publicações relevantes de um total de 1543 trabalhos . . . . . . 27

2.4 Guantidade de valores de medidas coletados das 64 publicações para cada medida de avaliação que são piores/iguais ou melhores que os valores calculados pelo classificador baseline $\mathrm{Geral}_{B} \ldots$. . 28

2.5 Guantidade de publicações usando cada medida de avaliação . 28

2.6 Ilustração de boxplot para analisar a distribuição da frequência

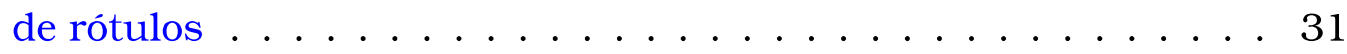

2.7 Boxplots representando a distribuição da frequência dos rótulos de cada conjunto de dados benchmark utilizado neste trabalho . .

3.1 Dimensões da seleção de atributos: interação com classificador, escopo do multirrótulo, dependência de rótulos e medida de importância ........................ 38

3.2 Visão geral das abordagens para seleção de atributos (a) filtro, (b) wrapper e (c) embutida . . . . . . . . . . . . . . . . . 39

3.3 Abordagem filtro . . . . . . . . . . . . . . . . . . 39

3.4 Abordagem wrapper . . . . . . . . . . . . . . . . . 40

3.5 Abordagem embutida . . . . . . . . . . . . . 40

3.6 Taxonomia de tipos de medidas de importância de atributos . . . 43

3.7 Número de trabalhos relacionados por ano de publicação, de acordo com a última atualização da revisão sistemática (junho de 2014) . . . . . . . . . . . . . . . . . . . 45

4.1 Abordagem Binary Relevance para seleção de atributos . . . . . . 53

4.2 Ilustração da aplicação das duas etapas de LCFS para construir

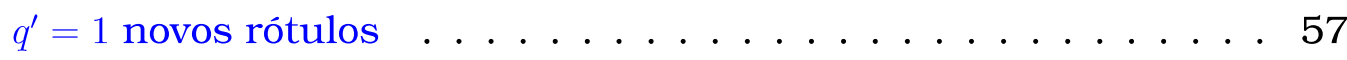

4.3 Desempenho de algoritmos de $\mathrm{SA}$ em termos dos valores de $F$ Measure obtidos pelo algoritmo de classificação $B R k N N-b$. . . . 67 
4.4 Desempenho de algoritmos de SA em termos dos valores das quatro medidas de avaliação obtidos pelo algoritmo de classificação $B R k N N-b$ no conjunto de dados 8-Magtag5k . . . . . . . . . . 68

4.5 Desempenho de configurações do LCFS em termos dos valores de F-Measure obtidos pelo algoritmo de classificação BRkNN-b . . . . 71

5.1 Conjunto de dados no qual o exemplo $E_{4}$ possui $k=3$ vizinhos mais próximos, mas nenhum vizinho mútuo $\left(k^{\prime}=0\right) \quad \ldots$. . . . 87

5.2 Ilustração da configuração experimental . . . . . . . . . . . . 88

5.3 Ilustração da medida Area Under Curve for Feature Ranking eva-

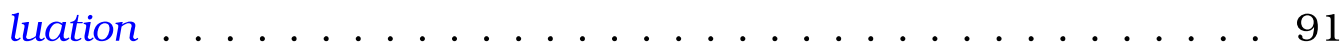

5.4 Diagrama do pós-teste de Nemenyi para algoritmos baseados em ReliefF e IG-LP nos conjuntos de dados artificiais com $\mu=10 \%$. . 96

5.5 Diagrama do pós-teste de Nemenyi para algoritmos baseados em ReliefF e IG-LP nos conjuntos de dados artificiais com $\mu=40 \%$. . 96

5.6 Evolução da quantidade de multirrótulos distintos obtidos utilizando a abordagem $L P$ em função do nível de ruído dos 45 conjuntos de dados artificiais . . . . . . . . . . . . . . 98

A. 1 Desempenho de algoritmos de SA em termos dos valores de Hamming Loss obtidos pelo algoritmo de classificação BRkNN-b . . . 128

A.2 Desempenho de algoritmos de SA em termos dos valores de Accuracy obtidos pelo algoritmo de classificação $B R k N N-b$. . . . . . 129

A.3 Desempenho de algoritmos de SA em termos dos valores de $F_{b}$ obtidos pelo algoritmo de classificação $B R k N N-b$. . . . . . . . . 130

A.4 Agrupamento hierárquico dos exemplos do conjunto de dados 6Genbase* por meio do método single link . . . . . . . . . . . . . . 131

A.5 Desempenho de configurações do LCFS em termos dos valores de Hamming Loss obtidos pelo algoritmo de classificação BRkNN-b . 132

A.6 Desempenho de configurações do LCFS em termos dos valores de Accuracy obtidos pelo algoritmo de classificação BRkNN-b . . . . . 133

A.7 Desempenho de configurações do LCFS em termos dos valores de $F_{b}$ obtidos pelo algoritmo de classificação $B R k N N-b$. . . . . . . . . 134 


\section{Lista de Tabelas}

2.1 Exemplos rotulados no formato atributo-valor . . . . . . . . . . 12

2.2 Conjunto de exames médicos com um único diagnóstico (rótulo) por exame . . . . . . . . . . . . . . . 13

2.3 Conjunto de exames médicos com um ou mais diagnósticos (rótulos) por exame . . . . . . . . . . . . . 13

2.4 Ilustração de um conjunto de dados multirrótulo . . . . . . . . . 15

2.5 Conjuntos de dados monorrótulo resultantes da aplicação da abordagem $B R$ no conjunto multirrótulo da Tabela $2.4 \ldots \ldots$

2.6 Conjuntos de dados monorrótulo como um problema multiclasse resultante da aplicação da abordagem $L P$ no conjunto multirrótulo da Tabela $2.4 \ldots \ldots \ldots$. . . . . . . . . . . . 17

2.7 Ilustração das medidas de avaliação multirrótulo . . . . . . . . . 23

2.8 Matrizes de confusão para um conjunto $D_{t e} \operatorname{com} L=\{a, b, c, d\} \ldots 26$

2.9 Valores das medidas macro-averaged e micro-averaged para as quatro medidas binárias consideradas . . . . . . . . . . . . 26

2.10 Descrição dos conjuntos de dados benchmark utilizados neste

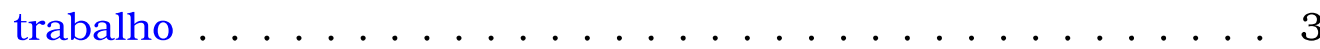

3.1 Guantidade de trabalhos instanciando cada dimensão da SA . . . 44

4.1 Combinação de resultados da avaliação individual de atributos realizada por um algoritmo de SA monorrótulo para estimar os valores de importância final relacionados a um conjunto de dados multirrótulo . . . . . . . . . . . . . . . . 54

4.2 Conjunto de dados ilustrativo para o método LCFS . . . . . . . . 58

4.3 Conjunto de dados ilustrativo para a Etapa 2 do método LCFS . . 60

4.4 Configurações do método Label Construction for Feature Selection avaliadas neste trabalho . . . . . . . . . . . . . . . . . 64

4.5 Categorização dos métodos de seleção de atributos multirrótulo baseados em transformação do problema avaliados neste trabalho de acordo com a taxonomia proposta neste trabalho . . . . . 65

4.6 Melhores rankings médios atingidos pelo algoritmo de classificação $B R k N N-b$ para cada quantidade de atributos $\left|X^{\prime}\right|$ selecionada pelos algoritmos de SA e para cada medida de avaliação . . . . . . . 
4.7 Melhores rankings médios atingidos pelo algoritmo de classificação BRkNN-b para cada quantidade de atributos $\left|X^{\prime}\right|$ selecionada pelas configurações do método LCFS e para cada medida de avaliação . . . . . . . . . . . . . . . . . . . . 72

4.8 Valores dos rankings médios atingidos por classificadores BRkNN$b$ gerados a partir de $I G-L P$ e IG-BR para cada quantidade de atributos selecionados $\left|X^{\prime}\right| \ldots \ldots \ldots \ldots$. . . . . . . . 74

4.9 Valores dos rankings médios atingidos por classificadores BRkNN$b$ gerados a partir de $I G-L P$ e $S R-X$ para cada quantidade de atributos selecionados $\left|X^{\prime}\right| \ldots \ldots \ldots \ldots \ldots$. . . . . . . 74

5.1 Categorização dos algoritmos de seleção de atributos multirrótulo baseados em ReliefF e de $I G-L P$ avaliados neste trabalho de acordo com a taxonomia proposta neste trabalho . . . . . . . .

5.2 Nove configurações para conjuntos de dados artificiais com o mesmo nível de ruído . . . . . . . . . . . . . . . . . 90

5.3 Configuração RF-ML significativamente melhor com nível de significância $\alpha=0,05 \ldots \ldots \ldots$. . . . . . . . . . 93

5.4 Melhores rankings médios atingidos pelo algoritmo de classificação $B R k N N-b$ para cada quantidade de atributos $\left|X^{\prime}\right|$ selecionada pelo algoritmo $R F-M L$ com medidas de dissimilaridade Jaccard e Hamming e para cada medida de avaliação . . . . . . . . . 93

5.5 Porcentagem de empates nos valores de cada medida de avaliação obtidos pelos classificadores $B R k N N$ - $b$ gerados a partir do algoritmo RF-ML com medidas de dissimilaridade Jaccard e Hamming 94

5.6 Melhores rankings médios atingidos pelo algoritmo de classificação $B R k N N-b$ para cada quantidade de atributos $\left|X^{\prime}\right|$ selecionada pelos algoritmos $R F-M L$ e $R F M-M L$ e para cada medida de avaliação 95

5.7 Resumo dos resultados experimentais nos conjuntos de dados artificiais de acordo com FR-AUC: algoritmos de SA baseados em ReliefF e IG-LP . . . . . . . . . . . . . . . . . . . . 96

5.8 Número de casos nos quais FR-AUC não atingiu seu valor máximo 97

5.9 Melhores rankings médios atingidos pelo algoritmo de classificação $B R k N N-b$ para cada quantidade de atributos $\left|X^{\prime}\right|$ selecionada pelos algoritmos baseados em ReliefF e IG- $L P$ e para cada medida de avaliação . . . . . . . . . . . . . . . . . . . . 999

5.10 Porcentagem de empates nos valores de cada medida de avaliação obtidos por classificadores BRkNN-b gerados a partir de algoritmos de SA baseados em ReliefF e IG-LP . . . . . . . . . . 100 
5.11 Similaridade média dos subconjuntos de atributos de tamanho $\left|X^{\prime}\right|=10 \% M$ encontrados pelos algoritmos de SA baseados em ReliefF e IG-LP . . . . . . . . . . . . . . . . . . . . . . . 102

A.1 Informações das 74 publicações identificadas pela revisão sistemática . . . . . . . . . . . . . . . . . . . . . 111

A.2 Resultados do classificador baseline Geral $_{B}$ nos 10 conjuntos de dados considerados . . . . . . . . . . . . . . . . . 112

A.3 Desempenho dos classificadores $B R k N N-b$ construídos usando todos os atributos dos 10 conjuntos de dados considerados . . . . 113

A.4 Resultados experimentais de acordo com $F$-measure baseada em exemplos: cinco métodos de SA baseados em abordagens tradicionais de transformação do problema . . . . . . . . . . . . . . 113

A.5 Resultados experimentais de acordo com Hamming Loss: cinco métodos de SA baseados em abordagens tradicionais de transformação do problema . . . . . . . . . . . . . . . . . . . 115

A.6 Resultados experimentais de acordo com Accuracy: cinco métodos de SA baseados em abordagens tradicionais de transformação do problema . . . . . . . . . . . . . . . . . . 117

A.7 Resultados experimentais de acordo com $F_{b}$ : cinco métodos de SA baseados em abordagens tradicionais de transformação do

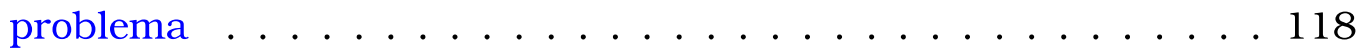

A.8 Resultados experimentais de acordo com $F$-measure baseada em exemplos: quatro configurações do LCFS e algoritmo IG-BR . . . 120

A.9 Resultados experimentais de acordo com Hamming Loss: quatro configurações do LCFS e algoritmo IG-BR . . . . . . . . . . . .

A.10 Resultados experimentais de acordo com Accuracy: quatro configurações do LCFS e algoritmo IG-BR . . . . . . . . . . . . .

A.11 Resultados experimentais de acordo com $F_{b}$ : quatro configurações do LCFS e algoritmo IG-BR . . . . . . . . . . . . . . . 126

A.12 Resultados experimentais de acordo com $F$-measure baseada em exemplos: algoritmo de SA RF-ML com medidas de dissimilaridade Jaccard e Hamming . . . . . . . . . . . . . . . . . . . . . 135

A.13 Resultados experimentais de acordo com Hamming Loss: algoritmo de SA RF-ML com medidas de dissimilaridade Jaccard e Hamming . . . . . . . . . . . . . . .

A.14 Resultados experimentais de acordo com Accuracy: algoritmo de SA RF-ML com medidas de dissimilaridade Jaccard e Hamming . 138

A. 15 Resultados experimentais de acordo com $F_{b}$ : algoritmo de SA $R F$ ML com medidas de dissimilaridade Jaccard e Hamming . . . . 140 
A.16 Rankings de atributos e valores de importância estimados por $R F-M L$ nos conjuntos de dados artificiais $\operatorname{com} \mu=0 \% \ldots \ldots . . .142$

A.17 Rankings de atributos e valores de importância estimados por $R F-M L$ nos conjuntos de dados artificiais $\operatorname{com} \mu=5 \% \quad \ldots \ldots$.

A.18 Rankings de atributos e valores de importância estimados por $R F-M L$ nos conjuntos de dados artificiais com $\mu=10 \% \ldots \ldots . .143$

A.19 Rankings de atributos e valores de importância estimados por $R F-M L$ nos conjuntos de dados artificiais com $\mu=20 \% \ldots \ldots . .143$

A.20 Rankings de atributos e valores de importância estimados por $R F-M L$ nos conjuntos de dados artificiais $\operatorname{com} \mu=40 \% \ldots \ldots . .144$

A.21 Rankings de atributos e valores de importância estimados por $R F-L P$ nos conjuntos de dados artificiais $\operatorname{com} \mu=0 \% \ldots \ldots . . .144$

A.22 Rankings de atributos e valores de importância estimados por $R F-L P$ nos conjuntos de dados artificiais com $\mu=5 \%$. . . . . 145

A.23 Rankings de atributos e valores de importância estimados por $R F-L P$ nos conjuntos de dados artificiais $\operatorname{com} \mu=10 \% \ldots \ldots . .145$

A.24 Rankings de atributos e valores de importância estimados por $R F-L P$ nos conjuntos de dados artificiais $\operatorname{com} \mu=20 \% \ldots \ldots . .145$

A.25 Rankings de atributos e valores de importância estimados por $R F-L P$ nos conjuntos de dados artificiais $\operatorname{com} \mu=40 \% \ldots \ldots . .146$

A.26 Rankings de atributos e valores de importância estimados por $R F-B R$ nos conjuntos de dados artificiais $\operatorname{com} \mu=0 \% \ldots \ldots \ldots 146$

A.27 Rankings de atributos e valores de importância estimados por $R F-B R$ nos conjuntos de dados artificiais com $\mu=5 \% \ldots \ldots . .147$

A.28 Rankings de atributos e valores de importância estimados por $R F-B R$ nos conjuntos de dados artificiais com $\mu=10 \% \ldots \ldots . .147$

A.29 Rankings de atributos e valores de importância estimados por $R F-B R$ nos conjuntos de dados artificiais $\operatorname{com} \mu=20 \% \ldots \ldots . .148$

A.30 Rankings de atributos e valores de importância estimados por $R F-B R$ nos conjuntos de dados artificiais $\operatorname{com} \mu=40 \% \ldots \ldots . .148$

A.31 Rankings de atributos e valores de importância estimados por IG-LP nos conjuntos de dados artificiais com $\mu=0 \% \ldots \ldots$

A.32 Rankings de atributos e valores de importância estimados por IG-LP nos conjuntos de dados artificiais com $\mu=5 \% \ldots \ldots . .149$

A.33 Rankings de atributos e valores de importância estimados por IG-LP nos conjuntos de dados artificiais com $\mu=10 \% \ldots \ldots$

A.34 Rankings de atributos e valores de importância estimados por IG-LP nos conjuntos de dados artificiais com $\mu=20 \% \ldots \ldots$

A.35 Rankings de atributos e valores de importância estimados por IG-LP nos conjuntos de dados artificiais com $\mu=40 \% \ldots \ldots 151$ 
A.36 Resultados experimentais nos conjuntos de dados artificiais de acordo com FR-AUC: algoritmos de SA baseados em ReliefF e IG-LP151

A.37 Resultados experimentais de acordo com $F$-measure baseada em exemplos: algoritmos de SA baseados em ReliefF e IG-LP . . . . . 152

A.38 Resultados experimentais de acordo com Hamming Loss: algoritmos de SA baseados em Relieff e IG-LP . . . . . . . . . . . . . . . 154

A.39 Resultados experimentais de acordo com Accuracy: algoritmos de SA baseados em ReliefF e IG-LP . . . . . . . . . . . . . . . 155

A.40 Resultados experimentais de acordo com $F_{b}$ : algoritmos de SA baseados em Relieff e IG-LP . . . . . . . . . . . . . . . . 157

A.41 Similaridade média dos subconjuntos de atributos de tamanho $\left|X^{\prime}\right|=20 \% M$ encontrados por algoritmos de SA baseados em ReliefF e $I G-L P$. . . . . . . . . . . . . . . . . . . . . . . . . . . . . 159

A.42 Similaridade média dos subconjuntos de atributos de tamanho $\left|X^{\prime}\right|=30 \% M$ encontrados por algoritmos de SA baseados em ReliefF e $I G-L P \ldots \ldots$. . . . . . . . . . . . . . . . . . 159

A.43 Similaridade média dos subconjuntos de atributos de tamanho $\left|X^{\prime}\right|=40 \% M$ encontrados por algoritmos de SA baseados em ReliefF e $I G-L P \ldots \ldots$. . . . . . . . . . . . . . . . . . . 160

A.44 Similaridade média dos subconjuntos de atributos de tamanho $\left|X^{\prime}\right|=50 \% M$ encontrados por algoritmos de SA baseados em ReliefF e $I G-L P \ldots \ldots$. . . . . . . . . . . . . . . . 160

A.45 Similaridade média dos subconjuntos de atributos de tamanho $\left|X^{\prime}\right|=60 \% M$ encontrados por algoritmos de SA baseados em ReliefF e $I G-L P \ldots \ldots$. . . . . . . . . . . . . . . . 160

A.46 Similaridade média dos subconjuntos de atributos de tamanho $\left|X^{\prime}\right|=70 \% M$ encontrados por algoritmos de SA baseados em ReliefF e $I G-L P \ldots \ldots$. . . . . . . . . . . . . . . . . 160

A.47 Similaridade média dos subconjuntos de atributos de tamanho $\left|X^{\prime}\right|=80 \% M$ encontrados por algoritmos de SA baseados em ReliefF e $I G-L P \ldots \ldots$. . . . . . . . . . . . . . . 160

A.48 Similaridade média dos subconjuntos de atributos de tamanho $\left|X^{\prime}\right|=90 \% M$ encontrados por algoritmos de SA baseados em ReliefF e $I G-L P \ldots \ldots$. . . . . . . . . . . . . . . . . . . . . 160 
Xxiv 


\section{Lista de Abreviaturas}

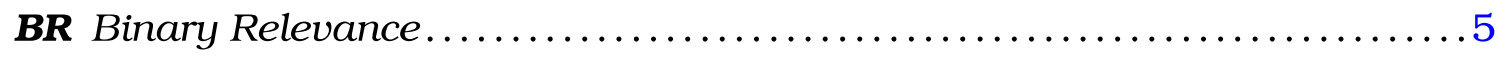

BRkNN Binary Relevance $k$ Nearest Neighbor $\ldots \ldots \ldots \ldots \ldots \ldots \ldots \ldots \ldots$

BRkNN-b Binary Relevance $k$ Nearest Neighbor extensão B ............. 19

BRLL Binary Relevance and Linear Support Vector Machines ........... 16

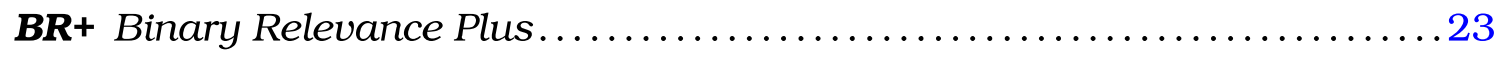

C45M C4.5 Multirrótulo ...................................... 18

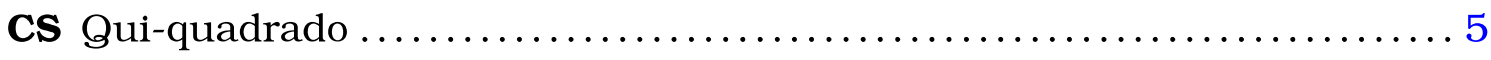

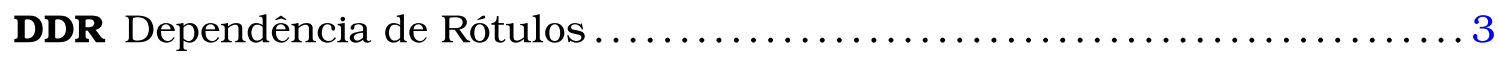

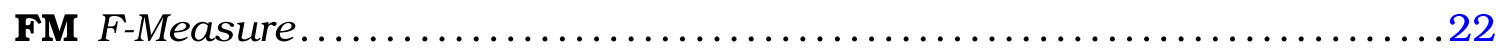

FR-AUC Area Under Curve for Feature Ranking evaluation ...............90

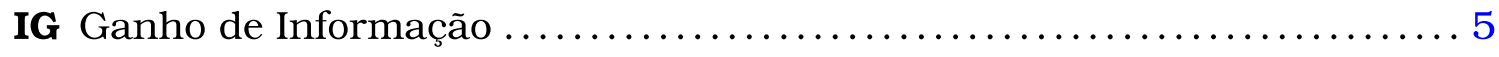

IG-BR Ganho de informação baseado na abordagem $B R \ldots \ldots \ldots \ldots \ldots 64$ 
IG-LP Ganho de informação baseado na abordagem $L P$. .64

kNN $k$ Nearest Neighbor. 6

LCFS Label Construction for Feature Selection. .5

LP Label Powerset 6

MLMUT Multilabel Mutual k Nearest Neighbor 18

MLNB Multilabel Naive Bayes 18

$\boldsymbol{R F}$-BR ReliefF baseado na abordagem $B R$ 64

$\boldsymbol{R F}$-LP ReliefF baseado na abordagem $L P$ 64

RF-ML ReliefF Multirrótulo .6

RFM-ML ReliefF Mútuo Multirrótulo .6

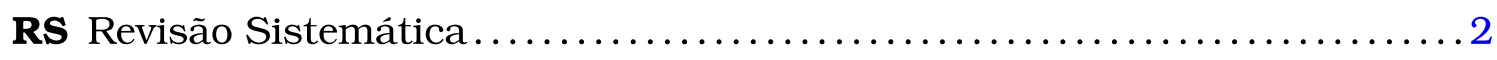

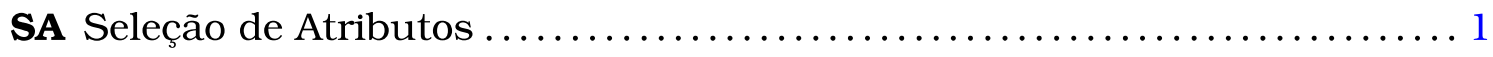

SAR Seleção de Atributos Randômica ........................... 64

SC Seleção baseada em Coocorrência .......................... 57

SC-A Seleção baseada em Coocorrência e operador AND . . . . . . . . . . 64

SL Seleção baseada em rótulos relacionados $\ldots \ldots \ldots \ldots \ldots \ldots \ldots \ldots \ldots 7$ 
SL-X Seleção baseada em rótulos relacionados e operadores XNOR e XOR.64

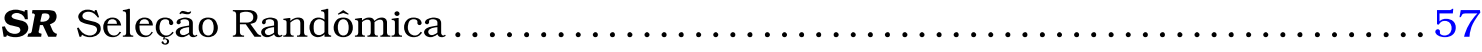

SR-A Seleção Randômica e operador AND ......................... 64

SR-X Seleção Randômica e operadores XOR e XNOR .................. 64

SVM Support Vector Machines............................... 16 
Xxviii 


\section{Lista de Algoritmos}

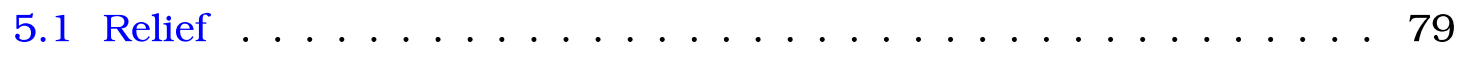

5.2 ReliefF ...................... 81

5.3 RReliefF . . . . . . . . . . . . . . . . . . 82

5.4 ReliefF Multirrótulo . . . . . . . . . . . . . . . . . . . 84

5.5 ReliefF Mútuo Multirrótulo . . . . . . . . . . . . . 86 
$\mathrm{XXX}$ 


\section{Lista de Nomenclaturas}

$\mu \quad$ Nível de ruído nos multirrótulos de um conjunto de dados artificial, página 89

$\mathbf{x}_{i} \quad i$-ésimo vetor com valores categóricos ou numéricos, página 11

$B_{k}(E)$ Conjunto de $k$ vizinhos mais próximos do exemplo $E$, página 19

$B_{m u t}(E)$ Conjunto de $k$ vizinhos mútuos mais próximos do exemplo $E$, página 19

c Número de iterações de algoritmos baseados em Relief, página 84

$c_{c}\left(y_{1}, y_{2}\right)$ Número de exemplos nos quais os rótulos $y_{1}$ e $y_{2}$ são coocorrentes, página 58

$c_{d}\left(y_{1}, y_{2}\right)$ Número de exemplos nos quais os rótulos $y_{1}$ e $y_{2}$ discordam, página 58

$c_{e}\left(y_{1}, y_{2}\right)$ Número de exemplos nos quais os rótulos $y_{1}$ e $y_{2}$ concordam, página 58

$C R(D)$ Cardinalidade de rótulo de um conjunto de dados $D$, página 30

D $\quad$ Conjunto de dados, página 12

$D_{d} R(D)$ Diversidade de rótulo de um conjunto de dados $D$, página 30

$D_{t e} \quad$ Conjunto de teste, página 13

$D_{t r} \quad$ Conjunto de treinamento, página 13

$D_{v a} \quad$ Conjunto de validação, página 13

$D R(D)$ Densidade de rótulo de um conjunto de dados $D$, página 30

$E_{i} \quad i$-ésimo exemplo, página 6 
$E K_{z} \quad z$-ésimo vizinho mais próximo de um exemplo $E_{i}$, página 84

$F_{b} \quad$ Medida de avaliação micro-averaged F-measure, página 63

$f_{b} \quad$ Complexidade do algoritmo base utilizado no $B R$, página 16

$f_{m} \quad$ Complexidade do algoritmo base utilizado no $L P$, página 17

$k \quad$ Número de vizinhos mais próximos, página 18

$k^{\prime} \quad$ Número de vizinhos mútuos mais próximos, página 85

L Conjunto de rótulos simples de um conjunto de dados, página 12

$M \quad$ Número de atributos de um conjunto de dados, página 11

$M_{i r r} \quad$ Número de atributos irrelevantes de um conjunto de dados, página 89

$M_{r e l} \quad$ Número de atributos relevantes de um conjunto de dados, página 89

$N \quad$ Número de exemplos de um conjunto de dados $D$, página 11

$N_{t e} \quad$ Número de exemplos do conjunto de teste $D_{t e}$, página 13

$N_{t r} \quad$ Número de exemplos do conjunto de treinamento $D_{t r}$, página 13

NI Número de exemplos ignorados no algoritmo $R F M-M L$, página 87

$p \quad$ Número de multirrótulos distintos, página 56

$P\left(y_{k}\right)$ Frequência relativa do rótulo simples $y_{k}$, página 18

$q \quad$ Número de rótulos simples de um conjunto de dados, página 12

$q^{\prime} \quad$ Número de novos rótulos simples gerados por LCFS, página 57

$r \quad$ Cardinalidade da intersecção de dois subconjuntos de atributos $S_{1}$ e $S_{2}$, página 91

$R F \quad$ Medida de importância inerente ao algoritmo Relief, página 2

$s \quad$ Tamanho do multirrótulo predito por $B R k N N-b$ para um exemplo, página 19

$X^{\prime} \quad$ Subconjunto de atributos, página 52

$X_{j} \quad j$-ésimo atributo, página 11

$x_{i j} \quad$ Valor do atributo $X_{j}$ para o exemplo $E_{i}$, página 11

$Y \quad$ Classe, página 11 
$Y_{i} \quad$ Multirrótulo verdadeiro para o exemplo $E_{i}$, página 12

$y_{i} \quad i$-ésimo rótulo simples, página 11

$y_{i j} \quad$ Novo rótulo simples obtido via combinação dos rótulos $y_{i}$ e $y_{j}$, página 57

$Z \quad$ Multirrótulo predito para o exemplo $E$, página 19 
Xxxiv 


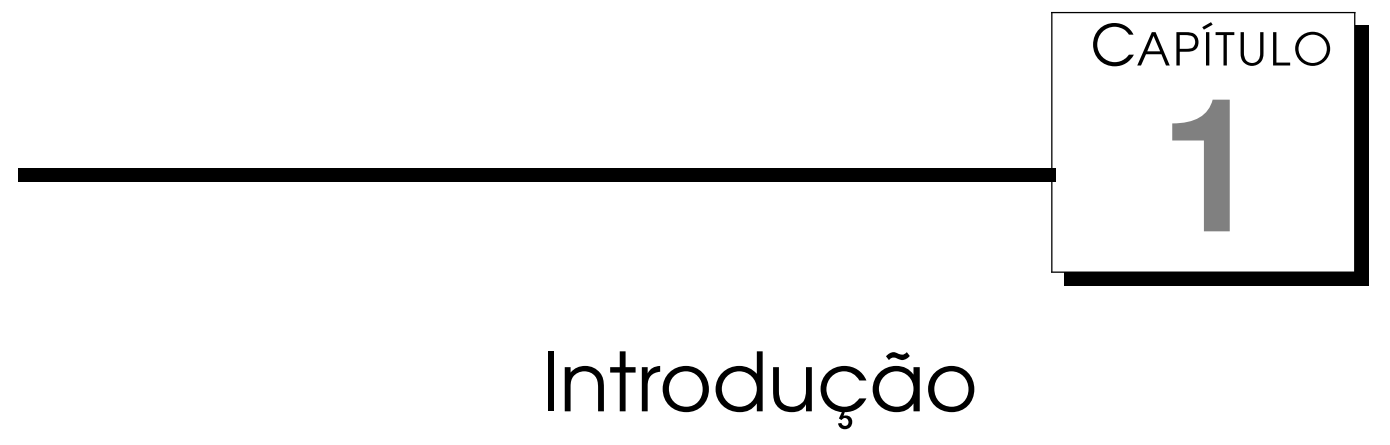

O avanço tecnológico possibilita a geração e o armazenamento de dados representativos em distintos domínios em uma intensidade crescente. A complexidade na análise desses dados motiva o desenvolvimento de processos inteligentes de extração de conhecimento, como o de mineração de dados (Han e Kamber, 2011). Esse processo consiste de três etapas principais: préprocessamento, extração de padrões e pós-processamento. O resultado desse processo são modelos (hipóteses) construídos utilizando algoritmos de aprendizado. Esses modelos representam conhecimento (padrões) sobre um determinado domínio.

A etapa de pré-processamento é de interesse, dentre outros motivos, pela influência que apresenta no processo de aprendizado, podendo consumir boa parte do tempo despendido na mineração de dados (Pyle, 1999). Uma das tarefas que pode ser realizada como parte do pré-processamento é a Seleção de Atributos (SA), a qual é usualmente aplicada em um conjunto de dados descrito por uma tabela atributo-valor. Nessa tabela, cada linha representa um exemplo desse conjunto de dados e cada coluna os valores de um dos atributos que descreve os exemplos.

A SA pode ser definida como um processo de busca por um subconjunto de atributos importantes em termos de uma medida ou critério de importância que reflete relevância e/ou não redundância de atributos ${ }^{2}$, e remoção dos atributos restantes. Esse processo auxilia na posterior extração de padrões inerentes aos dados, apresentando uma potencial redução de efeitos

\footnotetext{
${ }^{2}$ Um atributo pode ser definido como relevante se, ao ser removido do conjunto de dados, implicar em perda de desempenho de um modelo. Dois atributos são considerados não redundantes quando seus valores não são significativamente correlacionados.
} 
da "maldição da dimensionalidade" (Liu e Motoda, 2007) que prejudicam o aprendizado, bem como economia de tempo/dinheiro caso atributos custosos do domínio sejam removidos por não serem necessários para o aprendizado do conceito a ser modelado.

Muitas das medidas de importância propostas na literatura são aplicáveis a conjuntos de dados monorrótulo, em que cada exemplo está associado a um único rótulo (conceito meta) (Mitchell, 1997). No entanto, é intuitivo para o ser humano associar suas observações (exemplos) a mais de um conceito, caracterizando dados multirrótulo. O interesse na extração de conhecimento desses dados tem aumentado na última década, sendo possivel observar aplicações variadas, como análise de emoções (Bhowmick et al., 2009), anotação de imagens e vídeos (Boutell et al., 2004), categorização de textos (Esuli et al., 2008) e predição da resistência de vírus a medicamentos (Heider et al., 2013). De fato, na revisão bibliográfica deste trabalho (Spolaôr et al., 2012b), realizada de acordo com o método de Revisão Sistemática (RS) e atualizada recentemente, foram encontrados 74 trabalhos que consideram o uso de medidas de importância em dados multirrótulo.

Dado o interesse crescente no assunto, é possivel levantar a seguinte questão:

\section{Como organizar trabalhos em seleção de atributos multirrótulo?}

Uma alternativa para responder a questão é obtida por meio da determinação de dimensões para categorizar os trabalhos. Uma das dimensões, já considerada na literatura de aprendizado monorrótulo (Liu e Motoda, 2007), bem como em surveys de aprendizado multirrótulo (Zhang e Zhou, 2013; Tsoumakas et al., 2010), consiste na interação do processo de SA com o algoritmo de aprendizado. Nesse sentido, três abordagens podem ser identificadas: embutida, filtro e wrapper. A abordagem filtro é a única que independe do algoritmo de aprendizado. Em outras palavras, métodos filtro procuram estimar a importância dos atributos considerando somente informações extraídas dos dados. Algumas medidas que ilustram a aplicação dessa abordagem na seleção de atributos multirrótulo incluem a informação mútua (Doquire e Verleysen, 2013), a medida $R F$ inerente ao algoritmo Relief (Spolaôr et al., 2013b, 2011a), o ganho de informação (Karabulut, 2013), a incerteza simétrica (Lastra et al., 2011) e o qui-quadrado (Trohidis et al., 2008).

Além da interação com o algoritmo de aprendizado, outra dimensão que pode ser considerada para organizar métodos de SA multirrótulo, adaptada da classificação hierárquica ${ }^{3}$ (Esuli et al., 2006), é o escopo do multirrótulo considerado pelo método. A seleção de atributos com escopo multirrótulo está

\footnotetext{
${ }^{3} \mathrm{Na}$ classificação hierárquica, uma ou mais classes podem ser divididas em subclasses ou agrupadas em superclasses.
} 
associada com métodos que identificam um subconjunto de atributos para o conjunto de dados. Por outro lado, a seleção de atributos com escopo monorrótulo consiste de métodos que escolhem um subconjunto de atributos diferente para cada rótulo simples no multirrótulo e que, opcionalmente, aplicam em seguida uma estratégia para combinar os resultados monorrótulo. Adicionalmente, o escopo híbrido abrange métodos que apresentam simultaneamente características de escopo multirrótulo e monorrótulo.

Uma dimensão adicional para organização dos métodos de SA, adaptada do aprendizado multirrótulo (Zhang e Zhou, 2013), corresponde à intensidade da exploração da Dependência de Rótulos (DDR), a qual é um tema que tem sido destacado pela comunidade (Dembczyński et al., 2012). Nessa dimensão é possivel classificar estratégias de primeira ordem, segunda ordem e alta ordem. Estratégias de primeira ordem consideram rótulos individualmente, ignorando a DDR. Na segunda abordagem são consideradas, por exemplo, relações entre pares de rótulos, enquanto que na última são tratadas relações entre uma quantidade maior de rótulos. As três dimensões de SA multirrótulo estão representadas na Figura 1.1.

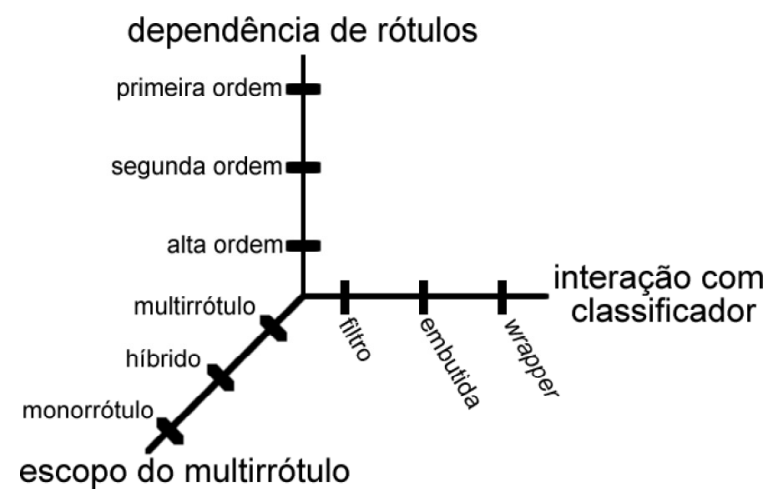

Figura 1.1: Uma possível taxonomia para organizar trabalhos em seleção de atributos multirrótulo

De modo geral, a dependência de rótulos pode ser vista como alguma relação entre rótulos de um conjunto de dados multirrótulo. Uma relação simples que exemplifica esse aspecto consiste na coocorrência de rótulos. Nesse sentido, quanto maior a coocorrência entre dois rótulos, mais dependentes eles seriam.

A comunidade tem defendido que a exploração da dependência de rótulos deve contribuir para melhorar o desempenho da classificação multirrótulo. A revisão bibliográfica realizada neste trabalho corrobora com isto e permite observar que a consideração da dependência de rótulos na seleção de atributos multirrótulo tem levado a bons resultados em trabalhos relacionados. A partir desses indícios, acredita-se que a consideração da dependência de rótulos durante a seleção de atributos deve contribuir para se obter um melhor 
desempenho no aprendizado.

\subsection{Objetivo e Hipótese}

Neste trabalho são investigados algoritmos de seleção de atributos para dados multirrótulo, considerando as seguintes assertivas:

1. O conjunto de rótulos que participam dos multirrótulos é conhecido a priori, tem relevância para o domínio do problema e é interpretável por humano;

2. O número de possíveis rótulos é limitado, tipicamente não maior que o número de atributos que descrevem os exemplos;

3. Cada exemplo está associado a um multirrótulo não vazio, i.e., o multirrótulo deve conter pelo menos um rótulo;

4. A seleção de atributos importantes auxilia na simplificação da linguagem de descrição de exemplos permitindo, entre outros, a redução da dimensionalidade dos dados sem ônus significativo de desempenho ${ }^{4}$.

A partir dessas assertivas, é levantada a hipótese de que algoritmos de seleção de atributos em dados multirrótulo que consideram a dependência de rótulos terão um melhor desempenho que aqueles que ignoram essa informação. Para verificar essa hipótese, é proposto como objetivo do trabalho o projeto e a implementação de algoritmos filtro de seleção de atributos multirrótulo que consideram relações entre rótulos. Esses algoritmos são avaliados, por exemplo, de acordo com o desempenho dos modelos gerados usando os atributos selecionados por cada algoritmo.

\subsection{Principais Contribuições}

O presente trabalho apresenta várias contribuições, as quais são descritas sucintamente a seguir.

\section{Revisão Sistemática sobre Seleção de Atributos Multirrótulo}

O método da revisão sistemática possibilita uma exploração ampla, rigorosa e reprodutivel da literatura (Kitchenham e Charters, 2007). Desse modo, é obtida uma leitura diferenciada dos trabalhos relacionados que é útil, por

\footnotetext{
${ }^{4} \mathrm{O}$ ônus significativo ou não de desempenho do modelo construído após a SA pode ser medido de diferentes maneiras e depende dos objetivos pretendidos no processo de extração de conhecimento.
} 
exemplo, para posicionar o presente trabalho no estado da arte correspondente.

Além disso, há uma falta de revisões amplas sobre publicações descrevendo o uso de seleção de atributos em dados multirrótulo, motivando a aplicação da RS nesse contexto. A revisão realizada neste trabalho foi atualizada desde a versão inicial (Spolaôr et al., 2012b), na qual foram encontradas 49 publicações. Na versão atual, esse número incrementou para 74 publicações, as quais foram sintetizadas e organizadas de acordo com as três dimensões mencionadas anteriormente - Figura 1.1. Observando a dimensão de dependência de rótulos, foi possivel obter evidências de que a consideração da dependência de rótulos na seleção de atributos é incipiente.

Seleção de Atributos de Segunda Ordem via Construção de Rótulos

Como mencionado, estratégias de segunda ordem consideram relações entre pares de rótulos, o que viabiliza alguma exploração da dependência de rótulos. Uma maneira de obter métodos de seleção de atributos de segunda ordem consiste em adaptar medidas de importância monorrótulo tradicionais, como Qui-quadrado (CS) e Ganho de Informação (IG), para permitir a análise dessas relações sem transformação do problema. Contudo, dependendo da medida, isso poderia ser uma tarefa difícil. Além disso, de modo geral, cada medida teria que ser adaptada individualmente.

Neste trabalho é proposto o método Label Construction for Feature Selection (LCFS) que pode facilitar a consideração de relações de segunda ordem por meio da construção de rótulos antes de realizar a seleção de atributos propriamente dita, independentemente da medida de importância utilizada. Dado um conjunto de dados, para a construção de cada rótulo são realizadas duas etapas: (1) seleção do par de rótulos originais que são combinados para a (2) geração do novo rótulo. Após gerar todos os novos rótulos, o conjunto de dados é expandido com esses rótulos. Em seguida, é possível usar nesses dados a abordagem tradicional de transformação do problema multirrótulo para monorrótulo, denominada Binary Relevance $(B R)$, o que viabiliza o uso de qualquer medida de SA monorrótulo. É necessário ressaltar que, mesmo com a transformação do problema via $B R$, é esperado que os novos rótulos ainda representem informações obtidas de pares de rótulos (segunda ordem).

A avaliação experimental realizada com 10 conjuntos de dados benchmark Capítulo 4 - indica que a melhor configuração do LCFS, aplicada junto com a medida IG, leva a alguns modelos significativamente melhores que os modelos gerados pela combinação da usual abordagem BR com IG (Spolaôr et al., 2014b). Além disso, como apresentado no Capítulo 4, há alguns resultados positivos dessa configuração em relação à tradicional abordagem de transfor- 
mação do problema Label Powerset $(L P)$, a qual também considera relações entre rótulos. Convém ressaltar que o método proposto foi implementado de acordo com o framework Mulan ${ }^{5}$ (Tsoumakas et al., 2011) e se encontra disponivel para a comunidade de aprendizado multirrótulo.

\section{Extensão do Algoritmo Relieff para Seleção de Atributos Multirrótulo}

A revisão sistemática indica que métodos usuais para seleção de atributos multirrótulo incluem variações das medidas de importância monorrótulo IG e CS. Apesar da popularidade, muitas dessas variações são estritamente univariadas, ou seja, não consideram interações entre atributos durante o processo de seleção de atributos. Além disso, dependendo da medida e do conjunto de dados sob investigação, a discretização dos dados é necessária para viabilizar a avaliação dos atributos. Uma desvantagem adicional de vários métodos usuais é que o problema de aprendizado multirrótulo é transformado para monorrótulo antes da SA, o que pode prejudicar a exploração da dependência de rótulos.

Neste trabalho são propostas duas extensões do algoritmo ReliefF (Kononenko, 1994) e sua variação para regressão RReliefF (Robnik-Sikonja e Kononenko, 2003), as quais evitam as três desvantagens mencionadas anteriormente. Primeiro, as extensões mantém a propriedade do algoritmo original de considerar interações entre atributos ${ }^{6}$. Segundo, as extensões também mantém a aplicabilidade do ReliefF a dados numéricos e categóricos. Terceiro, as extensões propostas tratam dados multirrótulo diretamente, sem a necessidade de transformação para dados monorrótulo, o que preserva relações entre rótulos.

A principal modificação realizada por ambas extensões consiste no uso da dissimilaridade entre conjuntos de rótulos (multirrótulos), ao invés da dissimilaridade entre valores de classe monorrótulo inerente a RReliefF, para modelar a probabilidade de que dois exemplos tenham rotulação diferente. Em particular, a extensão ReliefF Multirrótulo ( $R F-M L)$ trabalha com a vizinhança tradicionalmente utilizada no algoritmo $k$ Nearest Neighbor $(k N N)$. Por outro lado, a extensão ReliefF Mútuo Multirrótulo (RFM-ML) estabelece uma vizinhança entre os exemplos $E_{i}$ e $E_{j}$ se e somente se $E_{j}$ é vizinho mais próximo de $E_{i}$ e vice-versa (Spolaôr et al., 2013c).

A avaliação experimental com 45 conjuntos de dados artificiais (Spolaôr et al., 2013c) e 10 conjuntos benchmark - Capítulo 5 - sugere que a extensão proposta $R F-M L$ leva, em determinados casos, a resultados melhores que

\footnotetext{
${ }^{5}$ Pacote de aprendizado multirrótulo baseado no Weka e disponível em http://mulan. sourceforge. net.

${ }^{6}$ Interações entre atributos são abordadas quando a dissimilaridade entre vizinhos (exemplos) mais próximos, inerentes a Relieff e RRelieff, é calculada a partir de todos os atributos.
} 
métodos que combinam as abordagens $B R$ e $L P$ com o ReliefF original (Spolaôr et al., 2013b). Além disso, os subconjuntos de atributos selecionados por essa extensão apresentam em geral similaridade baixa com os demais algoritmos avaliados, sugerindo uma boa opção para combinação com outros algoritmos de seleção de atributos por meio de métodos como ensemble (Cannas et al., 2013). Assim como LCFS, as extensões propostas foram implementadas no framework Mulan e se encontram disponiveis para a comunidade.

Convém ressaltar a relativa novidade das propostas deste trabalho. Diferentemente do método de Kong et al. (2012), nenhum exemplo multirrótulo é ignorado na extensão $R F-M L$. Tanto $R F-M L$ quanto $R F M-M L$ reúnem, de modo único, características de extensões contemporâneas da literatura (Pupo et al., 2013; Slavkov et al., 2013) para SA filtro no suporte ao aprendizado multirrótulo não hierárquico. Além disso, as extensões apresentam menor complexidade que a medida informação mútua multivariada, a qual integra o estado da arte e também considera interações entre atributos (Lee e Kim, 2013) ${ }^{7}$.

\section{Outras Contribuições}

Este trabalho também apresenta várias contribuições relacionadas ao aprendizado multirrótulo e monorrótulo, listadas a seguir.

- Seleção de atributos monorrótulo por meio de algoritmos genéticos multiobjetivo (Spolaôr et al., 2011b);

- Análise de índices de complexidade para problemas de classificação monorrótulo (Lorena et al., 2012);

- Aprendizado multirrótulo baseado na vizinhança mútua (Cherman et al., 2014, 2013);

- Aprendizado multirrótulo baseado no paradigma lazy (Reis et al., 2012);

- Framework para geração de conjuntos de dados artificiais multirrótulo (Tomás et al., 2014, 2013);

- Framework para análise exploratória de dados multirrótulo (Carvalho et al., 2014);

- Revisão sistemática sobre algoritmos de aprendizado multirrótulo que reportam resultados experimentais (Metz et al., 2014; Spolaôr et al., 2013a);

- Revisão sobre a família de algoritmos Relief e suas extensões para seleção de atributos monorrótulo (Spolaôr et al., 2014a).

\footnotetext{
${ }^{7} \mathrm{~A}$ complexidade da informação mútua multivariada é exponencial (quarta ordem).
} 


\subsection{Organização do Trabalho}

O restante da tese é organizado da seguinte maneira.

\section{Capítulo 2: Aprendizado Multirrótulo}

Neste capítulo são descritos conceitos de aprendizado multirrótulo, incluindo métodos de aprendizado e medidas de avaliação de desempenho. Além disso, temas como a dependência de rótulos, os conjuntos de dados benchmark utilizados neste trabalho e aplicações de aprendizado multirrótulo são apresentados.

\section{Capítulo 3: Seleção de Atributos para Aprendizado Multirrótulo}

Neste capítulo são tratados aspectos como dimensões para organização de métodos de seleção de atributos multirrótulo e algumas medidas de importância de atributos. Os principais resultados da aplicação do processo de revisão sistemática, úteis para retratar a literatura relacionada ao presente trabalho, também são abordados neste capítulo.

\section{Capítulo 4: Seleção de Atributos baseada em Transformação do Problema}

Neste capítulo são avaliados dois grupos de métodos para seleção de atributos em dados multirrótulo. O primeiro grupo consiste de métodos que combinam uma abordagem tradicional de transformação do problema com um algoritmo de SA monorrótulo tradicional. O segundo grupo é baseado na combinação de um desses algoritmos com diferentes configurações do método LCFS, proposto neste trabalho. LCFS constroi rótulos antes da transformação do problema com o intuito de facilitar a investigação de relações de segunda ordem durante a seleção de atributos. Experimentos comparativos em cada grupo e entre os melhores representantes de cada grupo em conjuntos de dados benchmark são apresentados e discutidos.

Capítulo 5: Adaptação do Algoritmo Relieff para Seleção de Atributos Multirrótulo

Neste capítulo são apresentadas as extensões $R F-M L$ e $R F M-M L$ do algoritmo ReliefF propostas neste trabalho. Na avaliação experimental são realizados dois grupos de experimentos. Enquanto o primeiro consiste de comparações envolvendo configurações de $R F-M L$ e $R F M-M L$, o segundo avalia a melhor extensão proposta em relação a métodos de transformação do problema baseados em ReliefF e ganho de informação. Além dos conjuntos de dados 
benchmark já utilizados no Capítulo 4, a avaliação experimental inclui conjuntos de dados artificiais gerados por meio do framework web desenvolvido em colaboração.

Capítulo 6: Conclusões

Neste capítulo, as considerações finais desta tese, bem como limitações e trabalhos futuros, são apresentados.

Apêndice $A$

O Apêndice contém resultados complementares à revisão sistemática da literatura e às avaliações experimentais descritas nos Capítulos 4 e 5 . 


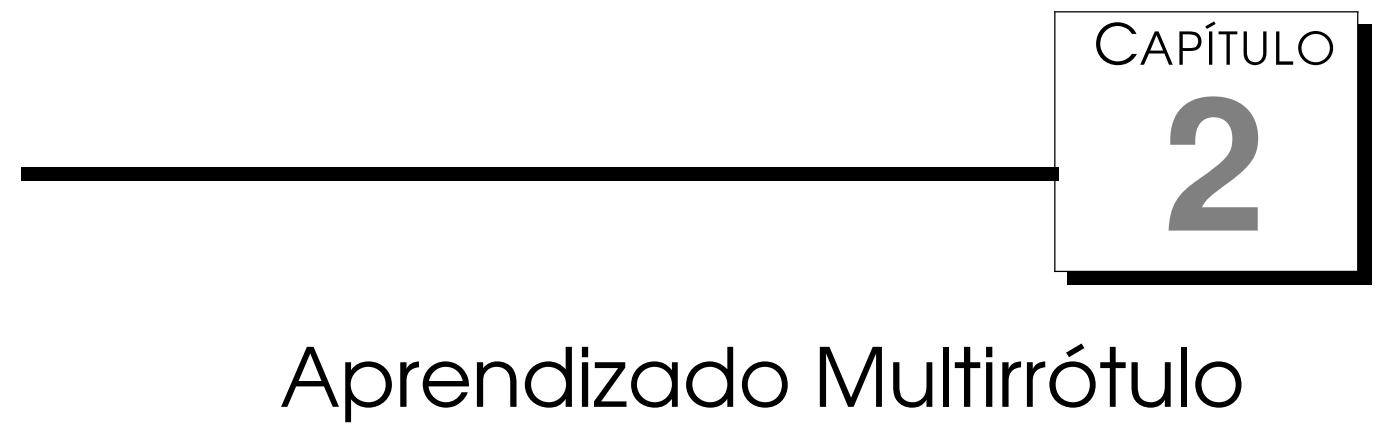

Uma maneira de avaliar o desempenho de métodos de seleção de atributos é por meio da comparação da qualidade dos modelos gerados utilizando todos os atributos do conjunto de dados e utilizando somente os atributos selecionados. Neste capítulo são abordados conceitos e definições de aprendizado multirrótulo, bem como são descritos os principais métodos de aprendizado e medidas de avaliação utilizados no desenvolvimento deste trabalho. Também são tratados conceitos relacionados à dependência de rótulos, a qual é importante no desenvolvimento de algoritmos que atuam em dados multirrótulo, bem como a caracterização dos conjuntos de dados multirrótulo utilizados neste trabalho.

\subsection{Definições}

Para a tarefa de aprendizado monorrótulo, a entrada do algoritmo consiste de um conjunto de $N$ exemplos $E_{i}$, com $i=1, \ldots, N$, escolhidos de um domínio com uma distribuição $\mathcal{D}$ fixa, desconhecida e arbitrária, da forma $\left\{\left(\mathbf{x}_{1}, y_{1}\right), \ldots,\left(\mathbf{x}_{N}, y_{N}\right)\right\}$ para alguma função desconhecida $y=f(\mathbf{x})$. Os $\mathbf{x}_{i}$ são tipicamente vetores da forma $\left(x_{i 1}, x_{i 2}, \ldots, x_{i M}\right)$ com valores categóricos ou numéri$\cos$, tal que $x_{i j}$ refere-se ao valor do atributo $j$, denominado $X_{j}$, para o exemplo $E_{i}$, como apresentado na Tabela 2.1(a). Os valores (rótulos) $y_{i}$ referem-se ao valor que o atributo $Y$, frequentemente denominado classe, pode assumir.

Dado um conjunto $D$ de exemplos a um algoritmo de classificação, um classificador ou modelo $H$ que aproxima $f$, i.e., $H(\mathbf{x}) \approx f(\mathbf{x})$, será induzido. O classificador consiste na hipótese feita sobre a verdadeira (mas desconhecida) função $f$. Dado um novo exemplo $\mathbf{x}$, o classificador, ou hipótese $H$, prediz o 
valor correspondente de $y$.

Assim, na classificação monorrótulo, cada exemplo $E_{i}$ está associado a um único valor da classe, o qual é o rótulo $y_{i}$ contido no conjunto de rótulos $L$, i.e., $y_{i} \in L$, com $|L|>1$. Guando existem mais de dois valores de classe possíveis $(|L|>2)$, o problema é chamado de classificação multiclasse. Caso o valor de classe seja sim ou não, o problema é chamado de classificação binária.

Por outro lado, na classificação multirrótulo, um exemplo pode estar associado a mais de um rótulo simultaneamente, i.e., um exemplo $E_{i}$ está associado a um conjunto de rótulos $Y_{i}$. O exemplo $E_{i}$ é representado da forma $\left(\mathbf{x}_{i}, Y_{i}\right)$, onde $Y_{i} \subseteq L, Y_{i} \neq \emptyset$ e $L=\left\{y_{1}, y_{2}, y_{3}, \ldots, y_{q}\right\}$ é o conjunto dos $q=|L|$ rótulos simples que participam dos multirrótulos $Y_{i}, i=1 . . N$, como representado na Tabela $2.1(b)^{8}$. Neste caso, dado um novo exemplo $\mathbf{x}$, o classificador deve predizer o multirrótulo $Y$ correspondente, i.e., $H(\mathbf{x})=Y$.

Tabela 2.1: Exemplos rotulados no formato atributo-valor

(a) Multiclasse

\begin{tabular}{c|cccc|c}
\cline { 2 - 6 } & $X_{1}$ & $X_{2}$ & $\ldots$ & $X_{M}$ & $Y$ \\
\hline \hline$E_{1}$ & $x_{11}$ & $x_{12}$ & $\ldots$ & $x_{1 M}$ & $y_{1}$ \\
$E_{2}$ & $x_{21}$ & $x_{22}$ & $\ldots$ & $x_{2 M}$ & $y_{2}$ \\
$\vdots$ & $\vdots$ & $\vdots$ & $\ddots$ & $\vdots$ & $\vdots$ \\
$E_{N}$ & $x_{N 1}$ & $x_{N 2}$ & $\ldots$ & $x_{N M}$ & $y_{N}$ \\
\hline \hline
\end{tabular}

(b) Multirrótulo

\begin{tabular}{c|cccc|c}
\cline { 2 - 6 } \multicolumn{1}{c|}{} & $X_{1}$ & $X_{2}$ & $\ldots$ & $X_{M}$ & $Y$ \\
\hline \hline$E_{1}$ & $x_{11}$ & $x_{12}$ & $\ldots$ & $x_{1 M}$ & $Y_{1}$ \\
$E_{2}$ & $x_{21}$ & $x_{22}$ & $\ldots$ & $x_{2 M}$ & $Y_{2}$ \\
$\vdots$ & $\vdots$ & $\vdots$ & $\ddots$ & $\vdots$ & $\vdots$ \\
$E_{N}$ & $x_{N 1}$ & $x_{N 2}$ & $\ldots$ & $x_{N M}$ & $Y_{N}$ \\
\hline \hline
\end{tabular}

A principal diferença entre aprendizado multirrótulo e aprendizado monorrótulo é que os rótulos no conjunto de rótulos no aprendizado multirrótulo estão frequentemente correlacionados, enquanto que os possiveis valores da classe (rótulos) no aprendizado monorrótulo são mutuamente exclusivos.

Para ilustrar essa diferença, é possível considerar os conjuntos de dados monorrótulo e multirrótulo referentes a exames médicos com diagnóstico apresentados, respectivamente, nas Tabelas 2.2 e 2.3. Nesses conjuntos de dados, cada exemplo corresponde ao exame médico de um paciente, enquanto cada atributo descreve uma informação presente nos exames. No conjunto de dados monorrótulo - Tabela 2.2 - cada exame possui um único diagnóstico (rótulo) registrado. Por outro lado, no conjunto de dados multirrótulo - Tabela 2.3 - cada exame registra um ou mais diagnósticos ao mesmo tempo.

É interessante notar que no conjunto de dados multirrótulo da Tabela 2.3 pode haver uma dependência entre os rótulos gastrite e duodenite, relacionados a doenças que acometem órgãos adjacentes. A consideração da dependência desses rótulos durante o aprendizado multirrótulo pode levar à construção

\footnotetext{
${ }^{8}$ Existe também a classificação hierárquica multirrótulo, que combina classificação hierárquica, na qual uma ou mais classes podem ser divididas em subclasses ou agrupadas em superclasses, com classificação multirrótulo (Cerri et al., 2012; Metz, 2011).
} 
Tabela 2.2: Conjunto de exames médicos com um único diagnóstico (rótulo) por exame

\begin{tabular}{c|cccc|c}
\cline { 2 - 5 } & Dor & Febre & $\ldots$ & Peso & $Y$ \\
\hline \hline Exame $_{1}$ & sim & sim & $\ldots$ & 101,5 & gastrite \\
Exame $_{2}$ & nao & sim & $\ldots$ & 61,2 & esofagite \\
$\vdots$ & $\vdots$ & $\vdots$ & $\ddots$ & $\vdots$ & $\vdots$ \\
Exame $_{N}$ & nao & nao & $\ldots$ & 79,8 & duodenite \\
\hline \hline
\end{tabular}

Tabela 2.3: Conjunto de exames médicos com um ou mais diagnósticos (rótulos) por exame

\begin{tabular}{c|cccc|c}
\cline { 2 - 5 } & Dor & Febre & $\ldots$ & Peso & $Y$ \\
\hline \hline Exame $_{1}$ & sim & nao & $\ldots$ & 98,5 & $\{$ gastrite,duodenite $\}$ \\
Exame $_{2}$ & nao & sim & $\ldots$ & 61,2 & $\{$ esofagite $\}$ \\
$\vdots$ & $\vdots$ & $\vdots$ & $\ddots$ & $\vdots$ & $\vdots$ \\
Exame $_{N}$ & nao & sim & $\ldots$ & 84,8 & \{esofagite,gastrite,duodenite $\}$ \\
\hline \hline
\end{tabular}

de melhores classificadores do que os que seriam obtidos se essa informação adicional fosse ignorada.

Dado um conjunto de exemplos $D$ é importante distinguir os três conjuntos utilizados no processo de aprendizado descritos a seguir:

Conjunto de treinamento $D_{t r}$. Esse conjunto de $N_{t r}$ exemplos é a principal entrada dos algoritmos de aprendizado. É a partir dele que são construídos os classificadores e, portanto, ele deve ser representativo da distribuição da população dos dados do domínio. Ou seja, os $N_{t r}$ exemplos desse conjunto são "vistos" pelo algoritmo de aprendizado durante a construção do modelo.

Conjunto de teste $D_{t e}$. Esse conjunto de $N_{t e}$ exemplos é utilizado para avaliar o classificador construído. O conjunto de teste não deve ser apresentado ao algoritmo de aprendizado durante a construção do classificador. Idealmente, $D_{t e}$ não deveria ter exemplos em comum com o conjunto de treinamento.

Conjunto de validação $D_{v a}$. Em alguns casos, como otimização de parâmetros de um algoritmo, pode ser necessário utilizar exemplos para realizar ajustes no classificador em construção pelo algoritmo de aprendizado. Esses exemplos não são utilizados diretamente na construção do classificador, mas são considerados para o seu ajuste. Dessa maneira, esses exemplos são indiretamente "vistos" durante o processo de aprendizado, o que obriga que os exemplos de validação sejam distintos dos exemplos de teste. 
Os exemplos que compõem esses conjuntos são descritos por um conjunto de atributos. Cada atributo pode apresentar propriedades como as destacadas a seguir (Kononenko e Kukar, 2007):

Atributo ruidoso. É um atributo no qual alguns valores podem estar incorretos devido a erros tipográficos ou de medição;

Atributo incompleto. É um atributo no qual alguns valores são faltantes.

Além dessas propriedades individuais, dependências entre atributos e entre atributos e a classe também podem ser consideradas:

Atributo randômico. É um atributo não relacionado à classe e que deveria ser ignorado para evitar influência negativa no conceito alvo (classe);

Atributo redundante. É um atributo que contém informação totalmente contida em outro atributo;

Atributo correlacionado. É um atributo que contém informação parcialmente contida em outro atributo ou subconjunto de atributos. Quanto mais correlacionados, mais interdependentes os atributos são;

Atributo fortemente interdependente com relação à classe. Quando há interdependência forte entre atributos e a classe, o conceito alvo é difícil de determinar, pois a importância de um desses atributos é detectável somente no contexto de outro(s) atributo(s). Em problemas de classificação monorrótulo, um exemplo de forte interdependência com relação à classe é a função paridade. A forma mais simples é a função binária XOR; sua negação (equivalência) é igualmente difícil. Em ambos casos, cada atributo isoladamente não apresenta nenhuma informação sobre a classe, o que pode dificultar o aprendizado.

\subsection{Métodos de Aprendizado Multirrótulo}

Diversos métodos de aprendizado multirrótulo têm sido propostos e podem ser categorizados de diversas maneiras (Tsoumakas et al., 2010; Carvalho e Freitas, 2009). Neste trabalho é adotada uma categorização simples (Zhang e Zhou, 2013), a qual organiza métodos em dois grupos ${ }^{9}$ :

Transformação do Problema. Transformam o conjunto de dados multirrótulo em um ou mais conjuntos de dados monorrótulo. Após a transformação, algoritmos tradicionais de aprendizado são utilizados para a re-

\footnotetext{
${ }^{9}$ Outras categorizações incluem ainda um terceiro grupo constituído por métodos de classificação a partir de ranking (Tsoumakas et al., 2010).
} 
solução do(s) problema(s) monorrótulo separadamente. Ao final do processo, resultados monorrótulo podem ser combinados para viabilizar o aprendizado multirrótulo.

Adaptação do Algoritmo. Realizam aprendizado em conjuntos de dados multirrótulo diretamente, i.e., sem transformar o conjunto de dados. Em muitos casos, esses algoritmos consistem em adaptações de métodos propostos anteriormente para aprendizado monorrótulo.

A ideia principal dos métodos de transformação do problema é ajustar os dados ao algoritmo, enquanto que os métodos de adaptação do algoritmo ajustam o algoritmo aos dados (Zhang e Zhou, 2013).

\subsubsection{Transformação do Problema}

Dentre os vários métodos de transformação do problema propostos na literatura, dois métodos simples e usualmente relacionados ao processo de seleção de atributos são:

1. Binary Relevance (BR); e

2. Label Powerset (LP).

Na descrição desses métodos é utilizado o conjunto de dados da Tabela 2.4. Esse conjunto possui $N=5$ exemplos multirrótulo com $L=\left\{y_{1}, y_{2}, y_{3}, y_{4}\right\}$. O espaço de atributos descritores é omitido, pois permanece inalterado pelos métodos da abordagem de transformação do problema.

Tabela 2.4: Ilustração de um conjunto de dados multirrótulo

\begin{tabular}{c|l}
\cline { 2 - 2 } & \multicolumn{1}{c}{$Y$} \\
\hline \hline$E_{1}$ & $Y_{1}=\left\{y_{2}, y_{3}\right\}$ \\
$E_{2}$ & $Y_{2}=\left\{y_{1}, y_{3}, y_{4}\right\}$ \\
$E_{3}$ & $Y_{3}=\left\{y_{4}\right\}$ \\
$E_{4}$ & $Y_{4}=\left\{y_{2}, y_{3}\right\}$ \\
$E_{5}$ & $Y_{5}=\left\{y_{3}, y_{4}\right\}$ \\
\hline \hline
\end{tabular}

Binary Relevance (Tsoumakas et al., 2010) consiste em uma abordagem simples que decompõe um problema multirrótulo em $|L|$ problemas de classificação binária, um para cada rótulo simples contido em $L$. Na Tabela 2.5, essa decomposição é exemplificada para o conjunto de dados multirrótulo com $|L|=4$ representado na Tabela 2.4 .

Após a transformação, cada um dos $|L|$ conjuntos de dados monorrótulo resultantes é submetido a um algoritmo de aprendizado monorrótulo (algoritmo base) para construção de um classificador binário. A predição de um 
Tabela 2.5: Conjuntos de dados monorrótulo resultantes da aplicação da abordagem $B R$ no conjunto multirrótulo da Tabela 2.4

\begin{tabular}{c|c}
\cline { 2 - 2 } & $Y$ \\
\hline \hline$E_{1}$ & $\neg y_{1}$ \\
$E_{2}$ & $y_{1}$ \\
$E_{3}$ & $\neg y_{1}$ \\
$E_{4}$ & $\neg y_{1}$ \\
$E_{5}$ & $\neg y_{1}$ \\
\hline \hline
\end{tabular}

\begin{tabular}{c|c}
\cline { 2 - 2 } & $Y$ \\
\hline \hline$E_{1}$ & $y_{2}$ \\
$E_{2}$ & $\neg y_{2}$ \\
$E_{3}$ & $\neg y_{2}$ \\
$E_{4}$ & $y_{2}$ \\
$E_{5}$ & $\neg y_{2}$ \\
\hline \hline
\end{tabular}

\begin{tabular}{c|c}
\cline { 2 - 2 } & $Y$ \\
\hline \hline$E_{1}$ & $y_{3}$ \\
$E_{2}$ & $y_{3}$ \\
$E_{3}$ & $\neg y_{3}$ \\
$E_{4}$ & $y_{3}$ \\
$E_{5}$ & $y_{3}$ \\
\hline \hline
\end{tabular}

\begin{tabular}{c|c}
\cline { 2 - 2 } & $Y$ \\
\hline \hline$E_{1}$ & $\neg y_{4}$ \\
$E_{2}$ & $y_{4}$ \\
$E_{3}$ & $y_{4}$ \\
$E_{4}$ & $\neg y_{4}$ \\
$E_{5}$ & $y_{4}$ \\
\hline \hline
\end{tabular}

novo exemplo $E$ é obtida pela união dos rótulos positivos preditos pelos $|L|$ classificadores monorrótulo para $E$.

A complexidade computacional da abordagem $B R$ é $O\left(q \times f_{b}\left(M, N_{t r}\right)\right)$, onde $q=|L|$ e $f_{b}\left(M, N_{t r}\right)$ é a complexidade do algoritmo base que recebe como entrada um conjunto de treinamento de $N_{t r}$ exemplos monorrótulo descritos por $M$ atributos. Como os $q=|L|$ problemas monorrótulo são independentes, a implementação da abordagem pode ser facilmente paralelizada.

Apesar da simplicidade, a abordagem $B R$ possui como uma desvantagem a desconsideração da dependência de rótulos na construção do modelo de classificação multirrótulo, já que os classificadores monorrótulo são construídos independentemente. Essa desvantagem pode ser minimizada, por exemplo, por meio de métodos que permitem uma interação entre esses classificadores para descobrir possíveis dependências de rótulos (Cherman et al., 2012; Read et al., 2009).

Além do aprendizado multirrótulo, a abordagem $B R$ tem sido relacionada com o processo de seleção de atributos, como descrito em (Karabulut, 2013; Rios e Kavuluru, 2013; Spolaôr et al., 2013b; Spolaôr e Tsoumakas, 2013; Ye et al., 2012; Trohidis et al., 2008; Tsoumakas e Vlahavas, 2007; Cong e Tong, 2008; Olsson e Oard, 2006; Yang e Pedersen, 1997). Em particular, o método Binary Relevance and Linear Support Vector Machines (BRLL) usa Support Vector Machines (SVM) linear como algoritmo base para BR, o que é considerado eficiente para classificar grandes conjuntos de dados esparsos (Fan et al., 2008). O método BRLL foi adotado em um estudo que integra este trabalho, no qual foram realizados vários experimentos com seleção de atributos multirrótulo em conjuntos de dados textuais (Spolaôr e Tsoumakas, 2013).

Label Powerset (Tsoumakas et al., 2010) consiste em uma abordagem simples que transforma o problema multirrótulo em um problema multiclasse, i.e., monorrótulo. Essa transformação considera os multirrótulos distintos no conjunto de dados como os valores possiveis do atributo classe no conjunto multiclasse correspondente. Desse modo, cada multirrótulo distinto é mapeado para um rótulo nos dados multiclasse. Na Tabela 2.6, essa transformação é ilustrada utilizando o conjunto de dados multirrótulo representado na Ta- 
bela 2.4. Note, por exemplo, que o rótulo associado a $E_{1}$ no conjunto de dados multiclasse - Tabela 2.6 - é denotado por $y_{2,3}$ e corresponde ao multirrótulo $Y_{1}=\left\{y_{2}, y_{3}\right\}$ do conjunto de dados multirrótulo - Tabela 2.4.

Tabela 2.6: Conjuntos de dados monorrótulo como um problema multiclasse resultante da aplicação da abordagem $L P$ no conjunto multirrótulo da Tabela 2.4

\begin{tabular}{c|c}
\cline { 2 - 2 } \multicolumn{1}{c|}{} & $Y$ \\
\hline \hline$E_{1}$ & $y_{2,3}$ \\
$E_{2}$ & $y_{1,3,4}$ \\
$E_{3}$ & $y_{4}$ \\
$E_{4}$ & $y_{2,3}$ \\
$E_{5}$ & $y_{3,4}$ \\
\hline \hline
\end{tabular}

Após a transformação, um algoritmo de aprendizado multiclasse (algoritmo base) é utilizado no conjunto de dados resultante. A predição de um novo exemplo é obtida diretamente por esse classificador.

A complexidade computacional da abordagem $L P$ é $O\left(f_{m}\left(M, N_{t r}, \min \left(2^{q}, N_{t r}\right)\right)\right.$ ), em que $f_{m}\left(M, N_{t r}, \min \left(2^{q}, N_{t r}\right)\right)$ é a complexidade do algoritmo base que recebe como entrada um conjunto de treinamento de $N_{t r}$ exemplos descritos por $M$ atributos e com até $\min \left(2^{q}, N_{t r}\right)$ multirrótulos distintos possíveis. Diferentemente do $B R$, uma eventual paralelização da implementação da abordagem $L P$ é dependente do algoritmo base utilizado.

A abordagem $L P$ é capaz de tratar a dependência de rótulos em cada exemplo de uma maneira simples, o que pode influenciar positivamente no desempenho do aprendizado. Por outro lado, uma quantidade elevada de rótulos possiveis $\left(\min \left(2^{q}, N\right)\right)$ usualmente implica no desbalanceamento dos dados multiclasse (maioria dos rótulos associada a poucos exemplos), o que por sua vez pode prejudicar o desempenho. Outra desvantagem de $L P$ é a sua incapacidade de predizer multirrótulos que não participam do conjunto de treinamento.

Assim como observado para $B R$, a abordagem $L P$ tem sido aplicada conjuntamente com a seleção de atributos (Spolaôr et al., 2013b; Trohidis et al., 2008). Ambas abordagens são adotadas neste trabalho para comparação com extensões de SA propostas neste trabalho - Capítulos 4 e 5 .

\subsubsection{Adaptação do Algoritmo}

Vários métodos representantes dessa abordagem têm sido propostos (Zhang e Zhou, 2013). Esses métodos são capazes de tratar diretamente o problema multirrótulo, dispensando a transformação do conjunto de dados. Quatro desses métodos, os quais têm sido relacionados de alguma maneira ao processo de seleção de atributos, são: 


\section{C4.5 Multirrótulo (C45M);}

\section{Binary Relevance $k$ Nearest Neighbor $(B R k N N)$;}

3. Multilabel Mutual k Nearest Neighbor (MLMUT); e

4. Multilabel Naive Bayes (MLNB).

O algoritmo de classificação monorrótulo $\mathrm{C} 4.5$ possibilita construir um modelo na forma de uma árvore de decisão para dados monorrótulo (Quinlan, 1993). Os testes realizados em cada nó são definidos com auxílio da medida convencional de entropia ${ }^{10}$ e cada nó folha está associado a uma determinada classe. A partir desse cenário, foi proposto o algoritmo C45M (Clare e King, 2001), o qual realiza duas adaptações importantes no C4.5 para classificar dados multirrótulo. Uma dessas adaptações consiste na utilização de nós folhas que representam um conjunto de rótulos, ao invés de rótulos simples. A outra adaptação corresponde à modificação da fórmula da entropia, originalmente concebida para dados monorrótulo, de modo a considerar as probabilidades dos rótulos que aparecem nos multirrótulos - Equação 2.1. Nessa equação, $P\left(y_{k}\right)$ é a frequência relativa do rótulo simples $y_{k}$ e $\bar{P}\left(y_{k}\right)=1-P\left(y_{k}\right)$.

$$
\text { Entropia }=-\sum_{k=1}^{|L|}\left(\left(P\left(y_{k}\right) \log P\left(y_{k}\right)\right)+\left(\bar{P}\left(y_{k}\right) \log \bar{P}\left(y_{k}\right)\right)\right) \text {. }
$$

Em particular, a última adaptação foi isolada e aplicada em (Spolaôr et al., 2012a) para realizar seleção de atributos multirrótulo conforme a abordagem filtro, i.e., independentemente do algoritmo C45M. Assim como ocorre na classificação multirrótulo, o problema multirrótulo foi tratado diretamente para selecionar atributos, dispensando a transformação dos dados. Read et al. (2012) também isolam essa adaptação para a realização de SA embutida em dados stream.

$\mathrm{O}$ algoritmo de classificação $k$ Nearest Neighbor $(k N N)$ baseia-se na busca pelos $k$ exemplos mais próximos, de acordo com uma medida de similaridade, do exemplo a ser predito. O novo rótulo pode ser obtido, por exemplo, pela moda dos rótulos dos $k$ vizinhos. A popularidade desse algoritmo para aprendizado monorrótulo também tem sido observada no aprendizado multirrótulo e várias adaptações têm sido propostas (Younes et al., 2011; Spyromitros et al., 2008; Brinker e Hüllermeier, 2007; Luo e Zincir-Heywood, 2005; Zhang e Zhou, 2005). Duas dessas adaptações, BRkNN e MLMUT, descritas a seguir, são utilizadas no desenvolvimento deste trabalho.

\footnotetext{
${ }^{10}$ Incerteza inerente aos dados.
} 
O algoritmo $B R k N N$ é $|L|$ vezes mais rápido que uma aplicação da abordagem $B R$ usando $k N N$ como algoritmo base, pois realiza somente uma busca pelos $k$ vizinhos mais próximos. Adaptações para tratar o problema multirrótulo diretamente, com relativo destaque comparado a outro algoritmo baseado em kNN (Zhang e Zhou, 2005), são realizadas nas extensões Binary Relevance $k$ Nearest Neighbor extensão A (BRkNN-a) e Binary Relevance k Nearest Neighbor extensão B (BRkNN-b) (Spyromitros et al., 2008). Ambas extensões são baseadas em um valor de confiança de rótulo, o qual é estimado, para cada rótulo, a partir da porcentagem dos $k$ vizinhos que contêm esse rótulo. A extensão $B R k N N$-a classifica um novo exemplo $E$ usando os rótulos com confiança superior a 0,5 , i.e., rótulos incluídos em pelo menos metade dos $k$ vizinhos mais próximos de $E$. Se nenhum rótulo atende essa condição, o multirrótulo predito $Z=H(E)$ é constituído somente pelo rótulo de maior confiança, de modo a evitar a predição de multirrótulos vazios. Por outro lado, a extensão $B R k N N-b$ classifica $E$ com os $[s]$ rótulos $^{11}$ que possuem a maior confiança, em que $s$ é o tamanho médio dos multirrótulos dos $k$ vizinhos mais próximos de $E$.

O BRkNN também foi estendido em um trabalho realizado em colaboração (Reis et al., 2012). Em particular, três modificações foram propostas, as quais são relacionadas à contribuição dada por cada um dos $k$ vizinhos no momento de definição do multirrótulo a ser predito $Z$, ao uso da informação de dissimilaridade entre exemplos para definição do número de rótulos $s$ e à substituição da medida de dissimilaridade entre exemplos pela medida $\mathrm{He}$ terogeneous Value Difference Metric (Wilson e Martinez, 2000). Experimentos ilustrativos realizados em quatro conjuntos de dados benchmark sugerem que essas extensões melhoram o desempenho do BRkNN.

Convém destacar que algoritmos lazy são suscetiveis a atributos irrelevantes. Assim, eles são úteis para a avaliação de métodos de seleção de atributos. Considerando as características mencionadas, neste trabalho a extensão $B R k N N-b$ foi utilizada para avaliar métodos de SA multirrótulo - Capítulos 4 e 5 .

O algoritmo MLMUT também foi desenvolvido neste trabalho em colaboração (Cherman et al., 2013). Assim como BRkNN-b, MLMUT usa a estratégia $k$ vizinhos mais próximos para identificar o conjunto de exemplos $B_{k}$ que será considerado para predizer o multirrótulo de um novo exemplo $E$, i.e., $\left|B_{k}\right|=k$. Seja $B_{k}(E)$ o conjunto de $k$ vizinhos mais próximos de um novo exemplo $E$. O conjunto $B_{m u t}(E)$ consiste nos exemplos $E_{i}$ que atendem a condição expressa na Equação 2.2.

$$
E_{i} \in B_{\text {mut }}(E) \text { se } E_{i} \in B_{k}(E) \text { e } E \in B_{k}\left(E_{i}\right)
$$

\footnotetext{
${ }^{11}$ A função $f(x)=[x]$ retorna o número inteiro mais próximo de $x$.
} 
Como essa estratégia exige que exemplos em $B_{m u t}(E)$ não devem apenas estar entre os $k$ vizinhos mais próximos de $E$, mas também exige que o próprio exemplo $E$ esteja entre os $k$ vizinhos mais próximos dos exemplos em $B_{\text {mut }}(E)$, $M L M U T$ restringe a quantidade de exemplos em $B_{m u t}(E)$. Em outras palavras, $\left|B_{\text {mut }}(E)\right| \leq\left|B_{k}(E)\right|$.

No Capítulo 5 são propostas duas extensões para um algoritmo de seleção de atributos: $R F-M L$ e $R F M-M L$. O procedimento de busca por vizinhos próximos, inerente ao algoritmo $k N N$, é adotado por ambas extensões como parte do processo de avaliação de atributos, embora $R F M-M L$ considere a ideia da vizinhança mútua adotada pelo MLMUT.

Outro algoritmo de aprendizado monorrótulo tradicional estendido para tratar dados multirrótulo diretamente é o Naive Bayes. Em particular, a adaptação do Naive Bayes denominada MLNB modifica a maneira como o princípio Maximum a Posteriori é implementado para cada rótulo simples do conjunto de dados multirrótulo (Zhang et al., 2009). Um método de seleção de atributos que avalia atributos considerando a dependência de rótulos é aplicado internamente ao MLNB conforme a abordagem wrapper. Esse método utiliza algoritmos genéticos multiobjetivo para otimizar simultaneamente duas medidas de avaliação de classificação multirrótulo. O uso do algoritmo MLNB para a abordagem wrapper de seleção de atributos é também avaliado por Zhang et al. (2009), sugerindo competitividade em relação a outros métodos da literatura.

\subsection{Avaliação de Classificadores Multirrótulo}

Medidas convencionais de avaliação do desempenho do aprendizado monorrótulo, como Acurácia, F-Measure e Área sob a curva ROC (Flach, 2012), consideram que a predição de um exemplo pode ser correta ou incorreta. Entretanto, a avaliação do aprendizado multirrótulo deve também levar em conta predições parcialmente corretas, i.e., predições nas quais apenas uma parte dos rótulos verdadeiros está contida no conjunto de rótulos (multirrótulo) predito, exigindo medidas específicas para esse problema (Tsoumakas et al., 2010; Tsoumakas e Katakis, 2007; Godbole e Sarawagi, 2004). Além disso, medidas de avaliação multirrótulo estimam o desempenho do aprendizado conforme distintos aspectos teóricos (Zhang e Zhou, 2013), os quais podem ser explicita ou implicitamente otimizados pelos algoritmos de aprendizado. Assim, a avaliação de classificadores multirrótulo deveria considerar múltiplas medidas ao invés de focar apenas na(s) medida(s) otimizada(s) pelo algoritmo de aprendizado.

Medidas de avaliação multirrótulo podem ser organizadas conforme a ta- 
xonomia ilustrada na Figura 2.1 (Zhang e Zhou, 2013). Neste trabalho são utilizadas medidas baseadas em exemplo e baseadas em rótulo para avaliar a qualidade da classificação. As medidas baseadas em exemplo avaliam a performance do classificador em cada exemplo do conjunto de teste $D_{t e}$ separadamente e retornam o valor médio desses valores no conjunto de teste. Por outro lado, as medidas baseadas em rótulo avaliam a performance do classificador em cada rótulo separadamente e retornam o macro/micro averaged em todos os rótulos do conjunto de teste. Ambos tipos de medidas são descritos a seguir.

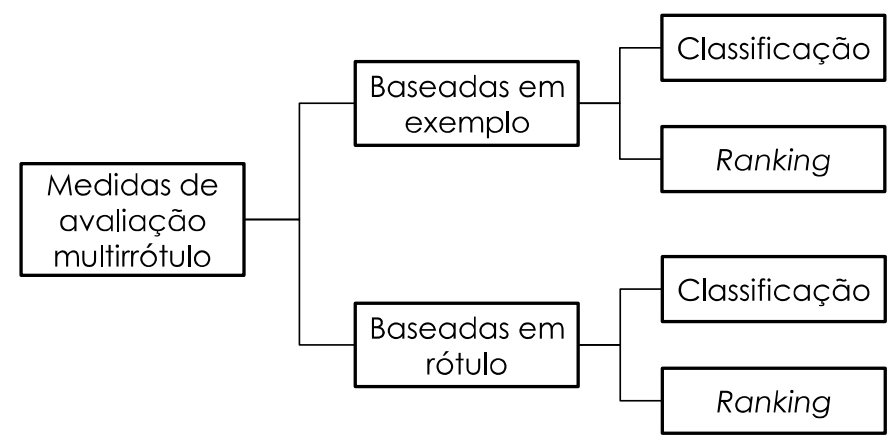

Figura 2.1: Taxonomia de medidas de avaliação do aprendizado multirrótulo (Zhang e Zhou, 2013)

\subsubsection{Medidas de Avaliação baseadas em Exemplo}

Seja $D$ um conjunto de dados constituído por $N$ exemplos $\left(\mathbf{x}_{i}, Y_{i}\right)$, com $i=$ $1 \ldots N, Y_{i} \subseteq L$ e $Y_{i} \neq \emptyset$ o conjunto de rótulos verdadeiros (multirrótulo) do $i$ ésimo exemplo. $H$ é um classificador multirrótulo gerado utilizando o conjunto de treinamento $D_{t r}$ que prediz o multirrótulo $Z_{i}=H\left(\mathbf{x}_{i}\right)$ para um dado $\mathbf{x}_{i}$ do conjunto de teste $D_{t e}$.

As seguintes medidas de avaliação consideram a diferença entre o multirrótulo verdadeiro $\left(Y_{i}\right)$ e o predito $\left(Z_{i}\right)$ associados a cada um dos $N_{t e}$ exemplos do conjunto de teste $D_{t e}$. O valor das medidas varia no intervalo $[0,1]$.

Hamming Loss (HL). A medida Hamming Loss, definida pela Equação 2.3, baseia-se na distância de Hamming entre os conjuntos $Y_{i}$ e $Z_{i}$, denotada por $\Delta$. Em outras palavras, $\Delta$ corresponde ao operador lógico XOR (diferença simétrica) entre $Y_{i}$ e $Z_{i}$. Assim, Hamming Loss considera tanto o caso da não predição de um rótulo que deveria ser predito, quanto a predição de um rótulo que não deveria ser predito. Ao contrário das demais medidas baseadas em exemplo, quanto menor for o valor de Hamming Loss, melhor é a classificação. O valor ótimo da medida 0 (zero) ocorre quando a distância de Hamming é 0, i.e., quando $Y_{i}=Z_{i} \forall i$. 


$$
\operatorname{Hamming} \operatorname{Loss}\left(H, D_{t e}\right)=\frac{1}{N_{t e}} \sum_{i=1}^{N_{t e}} \frac{\left|Y_{i} \Delta Z_{i}\right|}{|L|}
$$

Subset Accuracy (SAC). A medida Subset Accuracy é definida pela Equação 2.4, onde $I$ (verdadeiro $)=1$ e $I($ falso $)=0$. Assim, Subset Accuracy somente considera predições totalmente corretas, semelhante à medida Acurácia para avaliação monorrótulo.

$$
\operatorname{Subset} \operatorname{Accuracy}\left(H, D_{t e}\right)=\frac{1}{N_{t e}} \sum_{i=1}^{N_{t e}} I\left(Z_{i}=Y_{i}\right) .
$$

Accuracy (AC). A medida Accuracy, definida pela Equação 2.5, consiste na adaptação da acurácia usada na avaliação do aprendizado monorrótulo. A Accuracy considera os acertos ao predizer rótulos presentes no multirrótulo $Y_{i}$, bem como o erro que ocorre ao não predizer rótulos em $Y_{i}$.

$$
\operatorname{Accuracy}\left(H, D_{t e}\right)=\frac{1}{N_{t e}} \sum_{i=1}^{N_{t e}} \frac{\left|Y_{i} \cap Z_{i}\right|}{\left|Y_{i} \cup Z_{i}\right|} .
$$

Precision (PC). A medida Precision, definida pela Equação 2.6, considera quantos rótulos preditos também pertencem ao multirrótulo verdadeiro $Y_{i}$, i.e., verifica se os rótulos preditos estão no multirrótulo $Y_{i}$. Se todos os rótulos preditos atendem essa condição $\left(Z_{i} \subseteq Y_{i}\right)$, o valor da medida é máximo (igual a 1).

$$
\operatorname{Precision}\left(H, D_{t e}\right)=\frac{1}{N_{t e}} \sum_{i=1}^{N_{t e}} \frac{\left|Y_{i} \cap Z_{i}\right|}{\left|Z_{i}\right|}
$$

Recall (RL). A medida Recall, definida pela Equação 2.7, leva em conta a quantidade de rótulos verdadeiros que foram preditos em $Z_{i}$. Se todos os rótulos verdadeiros estão contidos no conjunto de rótulos preditos $\left(Y_{i} \subseteq Z_{i}\right)$, o valor da medida é máximo (igual a 1 ).

$$
\operatorname{Recall}\left(H, D_{t e}\right)=\frac{1}{N_{t e}} \sum_{i=1}^{N_{t e}} \frac{\left|Y_{i} \cap Z_{i}\right|}{\left|Y_{i}\right|} .
$$

F-Measure (FM). Assim como a Acurácia, a F-Measure foi inspirada em uma medida utilizada na avaliação de classificadores monorrótulo. Essa me- 
dida, definida pela Equação 2.8, calcula a média harmônica entre as medidas de avaliação Precision e Recall em busca de equilíbrio.

$$
F \text {-Measure }\left(H, D_{t e}\right)=\frac{1}{N_{t e}} \sum_{i=1}^{N_{t e}} \frac{2\left|Y_{i} \cap Z_{i}\right|}{\left|Z_{i}\right|+\left|Y_{i}\right|} .
$$

Para ilustrar essas medidas, na Tabela 2.7 é apresentada a classificação de $N_{t e}=7$ exemplos em um conjunto de teste $D_{t e}$ multirrótulo com $L=\{a, b, c, d\}$, onde $Y_{i}$ e $Z_{i}$ denotam respectivamente o multirrótulo verdadeiro e o multirrótulo predito para o exemplo $E_{i}$. Nessa tabela são mostrados os valores das medidas de avaliação para cada exemplo e, na última linha, a média desses valores.

Tabela 2.7: Ilustração das medidas de avaliação multirrótulo

\begin{tabular}{c|c|c|c|c|c|c|c|c|c|c}
\cline { 2 - 10 } & $Y_{i}$ & $Z_{i}$ & $Y_{i} \cup Z_{i}$ & $Y_{i} \cap Z_{i}$ & HL & SAC & AC & $P C$ & $R L$ & $F M$ \\
\hline \hline$E_{1}$ & $\{c\}$ & $\{c\}$ & $\{c\}$ & $\{c\}$ & 0 & 1 & 1 & 1 & 1 & 1 \\
$E_{2}$ & $\{a, b\}$ & $\{a, b\}$ & $\{a, b\}$ & $\{a, b\}$ & 0 & 1 & 1 & 1 & 1 & 1 \\
$E_{3}$ & $\{a, b\}$ & $\{c, d\}$ & $\{a, b, c, d\}$ & $\{\emptyset\}$ & 1 & 0 & 0 & 0 & 0 & 0 \\
$E_{4}$ & $\{a, c, d\}$ & $\{a, c\}$ & $\{a, c, d\}$ & $\{a, c\}$ & 0,25 & 0 & 0,67 & 1 & 0,67 & 0,8 \\
$E_{5}$ & $\{a, c\}$ & $\{a, c, d\}$ & $\{a, c, d\}$ & $\{a, c\}$ & 0,25 & 0 & 0,67 & 0,67 & 1 & 0,8 \\
$E_{6}$ & $\{a, b\}$ & $\{b, c\}$ & $\{a, b, c\}$ & $\{b\}$ & 0,5 & 0 & 0,33 & 0,5 & 0,5 & 0,5 \\
$E_{7}$ & $\{b, d\}$ & $\{a, b, c\}$ & $\{a, b, c, d\}$ & $\{b\}$ & 0,75 & 0 & 0,25 & 0,33 & 0,5 & 0,4 \\
\hline \multicolumn{7}{c}{} & \multicolumn{7}{c}{ Média } & 0,39 & 0,29 & 0,56 & 0,64 & 0,67 & 0,64 \\
\hline
\end{tabular}

Note que somente os exemplos $E_{1}$ e $E_{2}$ obtiveram classificação totalmente correta, enquanto que o exemplo $E_{3}$ mostra um dos piores casos de classificação. Nos demais exemplos, a predição parcialmente correta é refletida diferentemente por cada medida de avaliação.

Pode ser observado que a otimização - maximização ou minimização, dependendo do caso - de uma medida de avaliação não necessariamente implica na otimização de outra medida. Na Figura 2.2 é possível notar outro exemplo relacionado, pois nenhum dos quatro métodos de aprendizado multirrótulo considerados - BRkNN-b, Binary Relevance Plus (BR+) e BR com algoritmo base Naive Bayes (NB) (Spolaôr e Monard, 2014) e MLMUT - consegue atingir um valor muito bom para ambas as medidas Hamming Loss e F-measure, o qual está localizado no canto superior esquerdo do espaço bidimensional considerado. Assim, como mencionado anteriormente, a avaliação de classificadores multirrótulo deveria sempre considerar múltiplas medidas de avaliação, pois elas avaliam diferentes aspectos da classificação. Esse requisito faz com que a avaliação de classificadores multirrótulo seja uma tarefa difícil. 


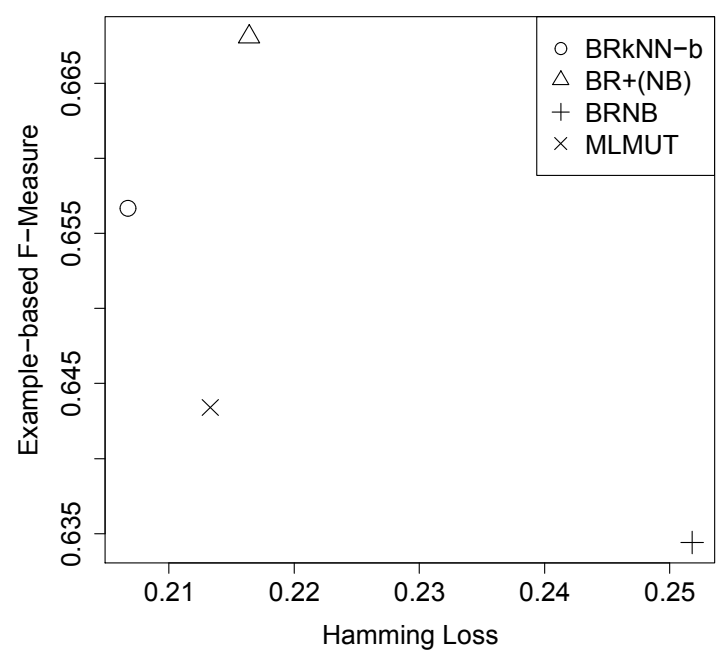

Figura 2.2: Ilustração do desempenho de quatro algoritmos de aprendizado multirrótulo em termos de duas medidas de avaliação

\subsubsection{Medidas de Avaliação baseadas em Rótulo}

Essas medidas dissecam o processo de avaliação dos algoritmos em medidas separadas para cada rótulo, e então combinam esse valor dentre todos os rótulos.

Os valores da classe em um problema de classificação binária monorrótulo são, geralmente, denominados sim (positiva) e não (negativa). Uma maneira natural de avaliar esses classificadores é por meio dos valores de $V_{P}$, $F_{P}, F_{N}$ e $V_{N}$, os quais correspondem, respectivamente, à quantidade de verdadeiros/falsos positivos/negativos ${ }^{12}$ de um total de $N_{t e}=V_{P}+F_{P}+F_{N}+V_{N}$ exemplos do conjunto de teste $D_{t e}$.

Em função desses valores, as medidas de avaliação de classificação binária Precision (PC), Recall (RL), F-Measure (FM) e Accuracy (AC) são definidas, respectivamente, pelas Equações 2.9 a 2.12 .

$$
\begin{array}{ccc}
P C\left(H, D_{t e}\right)=\frac{V_{P}}{V_{P}+F_{P}} & \text { (2.9) } & R L\left(H, D_{t e}\right)=\frac{V_{P}}{V_{P}+F_{N}} \\
F M\left(H, D_{t e}\right)=\frac{2 V_{P}}{2 V_{P}+F_{P}+F_{N}} . & \text { (2.11) } & A C\left(H, D_{t e}\right)=\frac{V_{P}+V_{N}}{N}
\end{array}
$$

A partir dessas medidas, é possível definir as duas medidas de avaliação baseadas em rótulo descritas a seguir (Tsoumakas et al., 2010; Lewis et al.,

\footnotetext{
${ }^{12}$ Se um exemplo cuja classe verdadeira é positiva é classificado como positivo, ele é denominado verdadeiro positivo $\left(V_{P}\right)$. Caso a classe verdadeira seja negativa e ele for classificado como positivo, ele é denominado falso positivo $\left(F_{P}\right)$. Notação similar é utilizada no caso dos exemplos negativos.
} 
2004). Essas medidas consideram os $L=\left\{y_{1}, y_{2}, \ldots, y_{q}\right\}$ rótulos que participam dos multirrótulos e uma medida de avaliação da classificação binária, denotada por $B\left(V_{P}, V_{N}, F_{P}, F_{N}\right)$ e calculada em função de uma matriz de confusão. Seja $V_{P_{y_{i}}}, F_{P_{y_{i}}}, V_{N_{y_{i}}}$ e $F_{N_{y_{i}}}$ o número de verdadeiros/falsos positivos/negativos do classificador binário para o rótulo $y_{i}$ do conjunto de rótulos $L$. Esses números participam das medidas macro e micro-averaged definidas a seguir.

Macro-averaged ( $B_{\text {macro }}$ ). A medida Macro-averaged, definida pela Equação 2.13, calcula uma medida de avaliação binária para cada rótulo separadamente e então retorna a média dentre todos os rótulos. $B_{\text {macro }}$ é influenciada por todos os rótulos da mesma maneira, de modo que o desempenho em rótulos pouco frequentes ganha relevância.

$$
B_{\text {macro }}\left(H, D_{t e}\right)=\frac{1}{q} \sum_{i=1}^{q} B\left(V_{P_{y_{i}}}, F_{P_{y_{i}}}, V_{N_{y_{i}}}, F_{N_{y_{i}}}\right) .
$$

Micro-averaged ( $B_{\text {micro }}$ ). A medida Micro-averaged, definida pela Equação 2.14, soma os valores das $q$ matrizes de confusão antes de aplicar a medida de avaliação binária. $B_{\text {micro }}$ é influenciada pela distribuição de frequência de rótulos e, assim, é dominada pelo desempenho obtido em rótulos mais frequentes.

$$
B_{\text {micro }}\left(H, D_{\text {te }}\right)=B\left(\sum_{i=1}^{q} V_{P_{y_{i}}}, \sum_{i=1}^{q} F_{P_{y_{i}}}, \sum_{i=1}^{q} V_{N_{y_{i}}}, \sum_{i=1}^{q} F_{N_{y_{i}}}\right) .
$$

Conceitualmente, as medidas macro-averaged assumem igual peso para os rótulos e as micro-averaged assumem igual peso para os exemplos. Além disso, elas verificam as Equações 2.15 e 2.16.

$$
\begin{array}{r}
A C_{\text {macro }}\left(H, D_{t e}\right)=A C_{\text {micro }}\left(H, D_{t e}\right) \\
A C_{\text {micro }}\left(H, D_{t e}\right)+H a m m i n g \operatorname{Loss}\left(H, D_{t e}\right)=1 .
\end{array}
$$

Para exemplificar, considere um classificador multirrótulo $H$ e um conjunto de 40 exemplos de teste, $\operatorname{com} L=\{a, b, c, d\}$. Para cada um dos rótulos simples em $L$ é gerada uma das matrizes de confusão apresentadas na Tabela 2.8.

Na Tabela 2.9 são apresentados os valores de $B_{\text {macro }}$ e $B_{\text {micro }}$, os quais foram obtidos a partir do cálculo das medidas de classificação binária Precision, Recall, F-Measure e Accuracy sobre as matrizes de confusão da Tabela 2.8. 
Tabela 2.8: Matrizes de confusão para um conjunto $D_{t e} \operatorname{com} L=\{a, b, c, d\}$

(a) Rótulo
\begin{tabular}{|c|c|}
\hline 20 & 0 \\
$V_{P}$ & $F_{N}$ \\
\hline 0 & 20 \\
\hline$F_{P}$ & $V_{N}$ \\
\hline
\end{tabular}

(b) Rótulo $b$
\begin{tabular}{|c|c|}
\hline 20 & 10 \\
$V_{P}$ & ${ }_{F_{N}}$ \\
\hline 0 & 10 \\
$F_{P}$ & $V_{N}$ \\
\hline
\end{tabular}

(c) Rótulo $c$
\begin{tabular}{|c|c|}
\hline 10 & 0 \\
$V_{P}$ & $\underset{F_{N}}{ }$ \\
\hline 20 & 10 \\
\hline$F_{P}$ & $\underset{V_{N}}{ }$ \\
\hline
\end{tabular}

(d) Rótulo $d$

\begin{tabular}{|l|l|}
\hline 10 & 10 \\
$V_{P}$ & $F_{N}$ \\
\hline 10 & 10 \\
$F_{P}$ & $V_{N}$ \\
\hline
\end{tabular}

Tabela 2.9: Valores das medidas macro-averaged e micro-averaged para as quatro medidas binárias consideradas

\begin{tabular}{c|c|c|c|c}
\cline { 2 - 5 } & Precision & Recall & F-Measure & Accuracy \\
\hline \hline macro-averaged & 0,75 & 0,83 & 0,70 & 0,69 \\
micro-averaged & 0,67 & 0,75 & 0,71 & 0,69 \\
\hline \hline
\end{tabular}

\subsubsection{Classificadores Baseline}

No aprendizado monorrótulo, a partir de uma ou mais medidas de avaliação, é possível comparar o desempenho de classificador(es) em relação ao desempenho de classificadores considerados baselines. Um exemplo reconhecido desses baselines é baseado no erro majoritário do conjunto de dados monorrótulo, no qual a predição de exemplos ocorre sem considerar o conjunto de atributos. Ao comparar o erro majoritário com outros classificadores, é possível verificar, por exemplo, se os últimos são melhores que o baseline.

No entanto, o uso de baselines baseados em erro majoritário de conjuntos de dados multirrótulo é praticamente inexplorado, o que motivou o desenvolvimento de trabalhos em colaboração (Metz et al., 2014; Spolaôr et al., 2013a). Inicialmente, classificadores baselines específicos para determinadas medidas de avaliação foram elaborados. Posteriormente, foi proposto $\mathrm{Geral}_{B}$, o qual tem como intuito servir como baseline geral para mais de uma medida. A idéia do Geral $_{B}$ é construir um ranking dos rótulos simples contidos em $L$ de acordo com a frequência deles no conjunto de dados $D$. Os $[t]$ rótulos ${ }^{11}$ mais frequentes constituem o multirrótulo $Z$ a ser predito, onde $t$ é o tamanho médio dos multirrótulos (cardinalidade, ou número médio de rótulos por exemplo) do conjunto de dados.

Esses baselines, implementados de acordo com o framework $\mathrm{Mu}-$ lan e disponíveis em http://www.labic.icmc.usp.br/pub/mcmonard/ Implementations/Multilabel/mlbaseline-code.zip, foram avaliados experimentalmente em (Metz et al., 2012). O $\mathrm{Geral}_{B}$ foi considerado uma alternativa simples para destacar classificadores que atingem desempenho pior que esse baseline em termos de várias medidas de avaliação. De fato, Geral $_{B}$ dispensa o uso de diferentes classificadores baselines, um por medida de avaliação, e pode ser adequado como referência para algoritmos de aprendizado multirrótulo que otimizam mais de uma medida de avaliação (multiobjetivo). 
Dado o destaque obtido, $\mathrm{Geral}_{B}$ foi selecionado com o intuito de analisar se resultados experimentais publicados na literatura de aprendizado multirrótulo, identificados por meio do método de revisão sistemática (Spolaôr et al., 2013a), eram superiores a esse baseline. O uso de critérios de seleção nos 1543 trabalhos inicialmente coletados permitiu reduzir a quantidade para 64 publicações, a partir das quais foram extraídos 5342 valores de medidas de avaliação, que por sua vez foram comparadas com os valores obtidos por Geral $_{B}{ }^{13}$ (Metz et al., 2014). Essa redução no número de trabalhos é resumida na Figura 2.3.
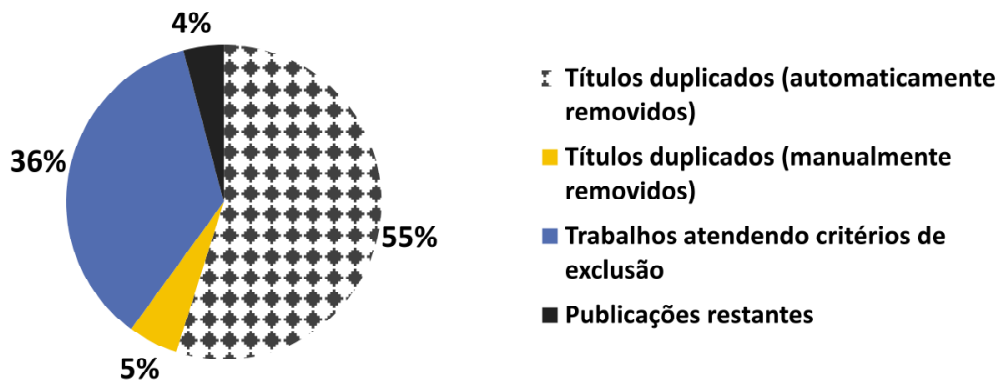

Figura 2.3: Resumo do procedimento de seleção para encontrar 64 publicações relevantes de um total de 1543 trabalhos (Metz et al., 2014)

A partir dos 5342 valores coletados, foi observado também quantas vezes cada medida de avaliação foi utilizada nos trabalhos selecionados. Nas Figuras 2.4 e 2.5 são apresentadas as frequências das oito medidas de avaliação mais frequentes nos 64 trabalhos em termos da quantidade de valores registrados e da quantidade de publicações usando cada medida, respectivamente.

Foi verificado que $12,8 \%$ dos resultados experimentais são piores ou iguais aos resultados obtidos por Geral $_{B}$. Em particular, $43 \%$ dos resultados publicados para o conjunto de dados 2-Corel5k não superam o baseline. Além disso, foi observado que a medida Hamming Loss tem sido a mais utilizada na literatura.

\subsection{Dependência de Rótulos}

A dependência de rótulos é um tema que vem se destacando na comunidade de aprendizado multirrótulo, pois acredita-se que a consideração desse aspecto pelos algoritmos de aprendizado contribui para melhorar o desempenho dos classificadores multirrótulo gerados. De fato, uma quantidade crescente de trabalhos que buscam explorar a dependência de rótulos na cons-

\footnotetext{
${ }^{13}$ Todos os resultados considerados encontram-se disponíveis em http://www.labic.icmc.usp.br/pub/mcmonard/ExperimentalResults/ Metz-GeneralB-SupplementaryMaterial.pdf.
} 


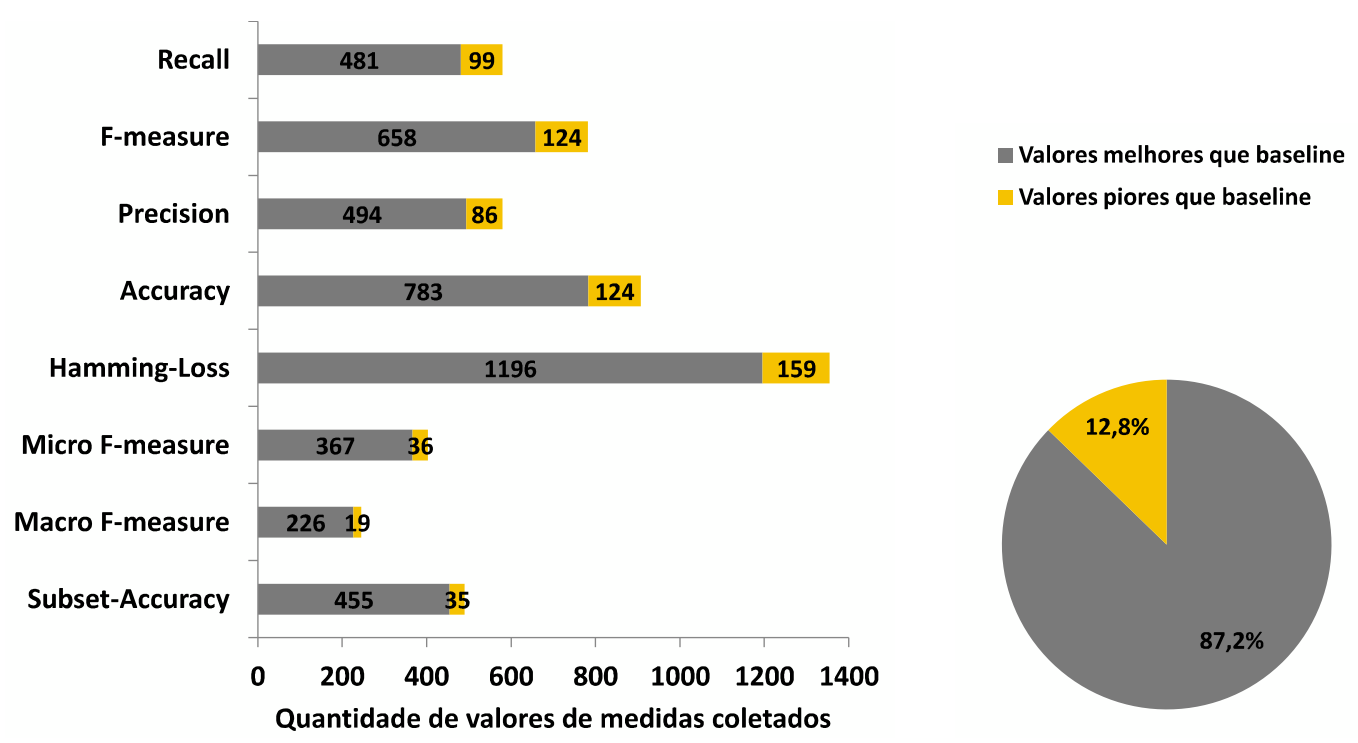

Figura 2.4: Quantidade de valores de medidas coletados das 64 publicações para cada medida de avaliação que são piores/iguais ou melhores que os valores calculados pelo classificador baseline Geral $_{B}$ (Metz et al., 2014)

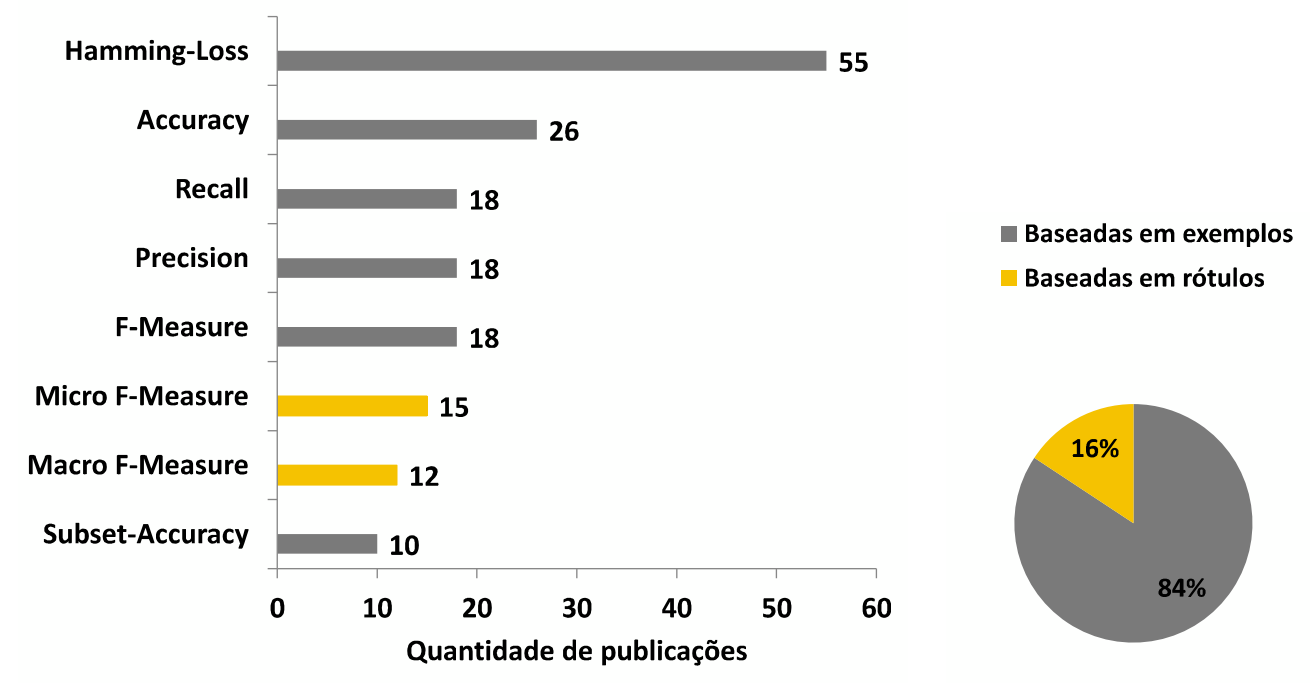

Figura 2.5: Quantidade de publicações usando cada medida de avaliação (Metz et al., 2014) 
trução de classificadores tem sido publicada (Chekina et al., 2013; Cherman et al., 2012; Younes et al., 2011; Read et al., 2009; Tsoumakas et al., 2009; Tsoumakas e Vlahavas, 2007; Godbole e Sarawagi, 2004). Além disso, a dependência de rótulos é um fator essencial que diferencia o aprendizado multirrótulo do aprendizado monorrótulo.

O interesse da comunidade na exploração da dependência de rótulos é relativamente recente. Dembczyński et al. (2010) comentaram que a maioria dos trabalhos de classificação multirrótulo da época apresentam uma análise insuficiente da influência da dependência de rótulo no processo de aprendizado. Uma das razões consiste na falta de clareza com que melhorias de desempenho obtidas por um classificador são associadas exclusivamente à dependência de rótulos. Os mesmos autores também propõem distinguir dois tipos diferentes de dependência de rótulos:

1. Dependência condicional: dependência de rótulos condicionada a um exemplo $\mathbf{x}$ descrito por $M$ atributos;

2. Dependência incondicional: dependência de rótulos global, i.e., independente de exemplos específicos.

Para exemplificar, a dependência condicional pode ser estimada ao atribuir ao algoritmo de aprendizado a responsabilidade de aprender essa dependência (Chekina et al., 2013; Cherman et al., 2012). Por outro lado, a dependência incondicional pode ser estimada por meio da correlação entre rótulos.

Com relação à maneira como a dependência de rótulos é explorada, os algoritmos de aprendizado multirrótulo existentes podem ser organizados em três grupos (Zhang e Zhou, 2013):

Estratégias de primeira ordem. Inclui métodos que ignoram relações entre rótulos ao considerar o aprendizado de cada rótulo separadamente. $\mathrm{O}$ método Binary Relevance é uma estratégia clássica desse grupo, pois transforma o problema multirrótulo em $|L|$ problemas monorrótulo binários e os resolve um a um. Apesar da simplicidade e custo computacional relativamente reduzido (a dependência de rótulos não é estimada), essas estratégias podem ter baixa qualidade preditiva ao supor a independência entre os rótulos no aprendizado multirrótulo.

Estratégias de segunda ordem. Inclui métodos que consideram relações entre pares de rótulos. Por explorar a dependência de rótulos com intensidade intermediária, essas estratégias podem atingir qualidade preditiva e custo computacional razoáveis. Contudo, em algumas aplicações, as relações entre uma quantidade maior de rótulos são mais representativas e outras estratégias são necessárias. 
Estratégias de alta ordem. Inclui métodos que analisam relações entre três ou mais rótulos. Apesar de explorar com maior intensidade a dependência de rótulos, as estratégias de alta ordem podem exigir um maior custo computacional. Dependendo da aplicação, esse custo computacional pode ser proibitivo, motivando o uso de estratégias de menor ordem.

Apesar do potencial benefício que a exploração da dependência de rótulos apresenta, uma revisão ampla e reprodutível da literatura realizada neste trabalho (Spolaôr et al., 2012b) e atualizada recentemente, encontrou poucos trabalhos de seleção de atributos para dados multirrótulo que consideram explicitamente a dependência de rótulos. Esse cenário, descrito com maior detalhe na Seção 3.3, página 44, foi uma das motivações no desenvolvimento deste trabalho.

\subsection{Caracterização do Conjunto de Dados}

Características como a quantidade de rótulos de cada exemplo influenciam o desempenho dos métodos de classificação (Tsoumakas e Katakis, 2007). Nesse cenário, a Cardinalidade de Rótulo $(C R)$, definida pela Equação 2.17, representa o número médio de rótulos simples atribuídos a cada exemplo de um conjunto de dados multirrótulo $D$.

$$
C R(D)=\frac{1}{|D|} \sum_{i=1}^{|D|}\left|Y_{i}\right| .
$$

Por outro lado, a Densidade de Rótulo $(D R)$ normaliza o número médio de rótulos simples atribuídos a cada exemplo pela quantidade de rótulos simples que constituem os multirrótulos - Equação 2.18. Note que ambas as medidas estão relacionadas por $C R(D)=|L| D R(D)$.

$$
D R(D)=\frac{1}{|D|} \sum_{i=1}^{|D|} \frac{\left|Y_{i}\right|}{|L|} .
$$

Outra medida utilizada para caracterizar dados multirrótulo é a Diversidade de Rótulo $\left(D_{d} R\right)$, definida pela Equação 2.19. Em outras palavras, a medida calcula o número de multirrótulos diferentes no conjunto de dados $D$.

$$
D_{d} R(D)=|\{Y \mid \exists \mathbf{x}:(\mathbf{x}, Y) \in D\}|
$$

A frequência de cada rótulo $y_{i} \in L=\left\{y_{1}, y_{2}, \ldots, y_{q}\right\}$, i.e., o número de exemplos rotulados com $y_{i}$, é também uma característica importante para, por 
exemplo, identificar o desbalanceamento dos rótulos. Como o número de rótulos $|L|=q$ pode ser elevado, Tsoumakas (2013) sugere o uso de estatística descritiva baseada em quartil ${ }^{14}$ para caracterizar a distribuição da frequência de rótulos. Em particular, essa caracterização ordena as $q$ frequências de rótulos simples em um conjunto de dados multirrótulo $D$ para encontrar as cinco medidas descritas a seguir:

Min: Frequência do rótulo associado à menor quantidade de exemplos em $D$, i.e., do rótulo menos frequente;

$1 Q$ : Frequência do rótulo até o qual se encontram $25 \%$ dos rótulos menos frequentes em $D\left(1^{\circ}\right.$ Quartil);

$M e d$ : Frequência do rótulo até o qual se encontram a metade (50\%) dos rótulos menos frequentes em $D\left(2^{\circ}\right.$ Quartil ou Mediana);

$3 Q$ : Frequência do rótulo até o qual se encontram $75 \%$ dos rótulos menos frequentes em $D$ ( $3^{\circ}$ Quartil);

Max: Frequência do rótulo associado à maior quantidade de exemplos em $D$, i.e., do rótulo mais frequente.

Para ilustrar, considere um conjunto de dados multirrótulo que contém $N=100$ exemplos $\operatorname{com} q=9$ rótulos e um conjunto ordenado de frequências de rótulo dado por $[5,8,10,12,15,30,35,45,55]$. A partir desse conjunto, os valores das cinco medidas são: $\operatorname{Min}=5,1 Q=10$, Med $=15,3 Q=35$ e Max $=55$. Esses valores são representados graficamente por meio de um boxplot - Figura 2.6.

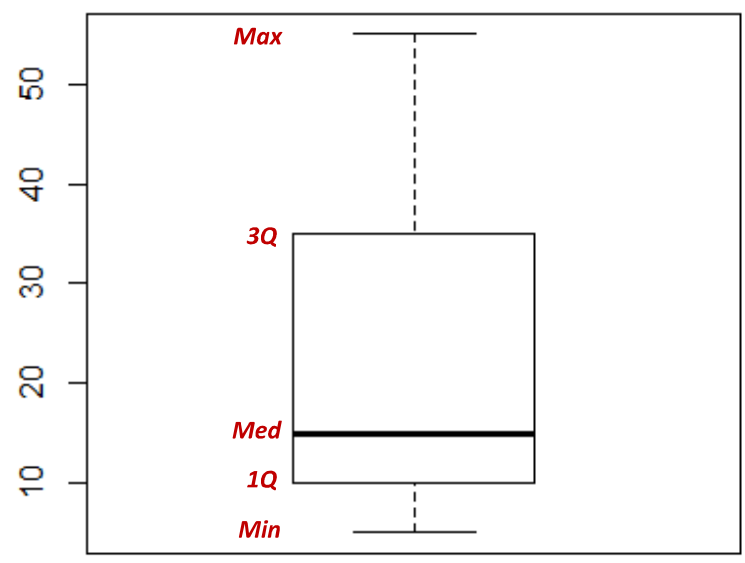

Figura 2.6: Ilustração de boxplot para analisar a distribuição da frequência de rótulos

\footnotetext{
${ }^{14}$ Um dos três valores que divide um conjunto ordenado de dados em quatro partes iguais, cada uma com $25 \%$ dos dados.
} 
Vários conjuntos de dados benchmark relacionados a aplicações multirrótulo estão disponíveis para a comunidade em repositórios como o das ferramentas Mulan ${ }^{15}$, Meka ${ }^{16}$ e Keel $^{17}$. Na Tabela 2.10 são descritas as características dos 10 conjuntos de dados multirrótulo utilizados nos experimentos dos Capítulos 4 e 5. A primeira coluna, Nome, identifica o conjunto de dados. A coluna Domínio é relacionada ao tipo de dados utilizado; nas colunas $N$ e $M$ são indicados respectivamente o número de exemplos e de atributos no conjunto de dados; na coluna $|L|$ é apresentada a quantidade de rótulos presentes no problema; as colunas $C R(D), D R(D)$ e $D_{d} R(D)$ se referem respectivamente à cardinalidade de rótulo, à densidade de rótulo e à diversidade de rótulo de um conjunto de dados $D$. As últimas cinco colunas apresentam as informações que caracterizam a distribuição de frequência de rótulos.

Tabela 2.10: Descrição dos conjuntos de dados benchmark utilizados neste trabalho

\begin{tabular}{llrrrrrrr||rrrrr}
\hline & \multicolumn{4}{c}{ Informações gerais } & \multicolumn{4}{c}{ Distribuição da frequência de rótulos } \\
Nome & Domínio & $N$ & $M$ & $|L|$ & $C R(D)$ & $D R(D)$ & $D_{d} R(D)$ & Min & $1 Q$ & Med & $3 Q$ & $M a x$ \\
\hline 1-Cal500 & música & 502 & 68 & 174 & 26,04 & 0,15 & 502 & 5 & 15,00 & 39,50 & 109,00 & 444 \\
2-Corel5k & imagem & 5000 & 499 & 374 & 3,52 & 0,01 & 3175 & 1 & 6,00 & 15,00 & 38,75 & 1120 \\
3-Corel16k001 & imagem & 13766 & 500 & 153 & 2,86 & 0,02 & 4803 & 25 & 67,00 & 115,00 & 264,00 & 3170 \\
4-Emotions & música & 593 & 72 & 6 & 1,87 & 0,31 & 27 & 148 & 166,50 & 170,50 & 185,00 & 264 \\
5-Fapesp & texto & 332 & 8669 & 66 & 1,77 & 0,03 & 206 & 1 & 4,00 & 6,00 & 11,00 & 37 \\
6-Genbase* & biologia & 662 & 1186 & 27 & 1,25 & 0,05 & 32 & 1 & 3,50 & 17,00 & 45,00 & 171 \\
7-Llog-f* & texto & 1253 & 1004 & 75 & 1,38 & 0,02 & 303 & 1 & 4,00 & 11,00 & 22,50 & 171 \\
8-Magtag5k & música & 5260 & 68 & 136 & 4,84 & 0,04 & 4163 & 9 & 28,75 & 86,50 & 258,50 & 1591 \\
9-Scene & imagem & 2407 & 294 & 6 & 1,07 & 0,18 & 15 & 364 & 404,50 & 429,00 & 432,50 & 533 \\
10-Yeast & biologia & 2417 & 103 & 14 & 4,24 & 0,30 & 198 & 34 & 323,75 & 659,50 & 952,75 & 1816 \\
\hline
\end{tabular}

O conjunto de dados multirrótulo 5-Fapesp foi construído por membros de nosso laboratório de pesquisa, $\mathrm{LABIC}^{18}$, a partir da extração de descritores (atributos) bag-of-related-words de reportagens (exemplos) de uma revista on$\operatorname{line}^{19}$, as quais são anotadas pela equipe da revista de acordo com a(s) área(s) científica(s) reportada(s) (rótulos) (Rossi e Rezende, 2011). O conjunto de dados musicais 8-Magtag $5 k^{20}$ encontra-se descrito em (Marques et al., 2011).

Os demais conjuntos de dados foram obtidos por meio dos repositórios Mulan $^{15}$ e Meka ${ }^{16}$. Os conjuntos 6-Genbase* e 7-Llog-f* foram por nós préprocessados, respectivamente, por meio da remoção de um atributo de identificação e de exemplos não rotulados.

Na Figura 2.7 são apresentados gráficos do tipo boxplot que representam a distribuição da frequência dos rótulos de cada conjunto de dados da Tabela 2.10. Esses gráficos destacam a grande variação na frequência de rótulos

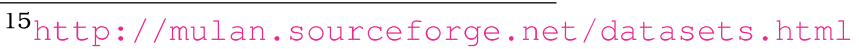

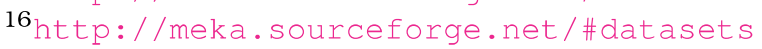

17 http: / / sci2s.ugr.es/keel/multilabel.php

${ }^{18} \mathrm{O}$ conjunto de dados pode ser obtido com os autores.

19 www.revistapesquisa. fapesp.br

20 http://tl.di.fc.ul.pt/t/magtag5k.zip
} 
dentre os 10 conjuntos de dados. Para exemplificar, os conjuntos de dados 3-Corel16k001 (Figura 2.7\#3) e 5-Fapesp (Figura 2.7\#5) apresentam, respectivamente, a maior e a menor diferença absoluta entre as frequências de rótulo Min e Max.
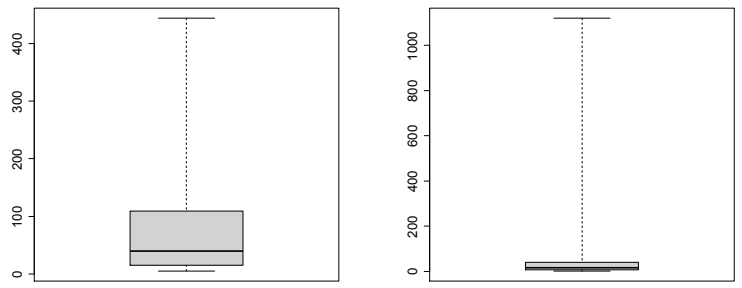

\#1 cal500

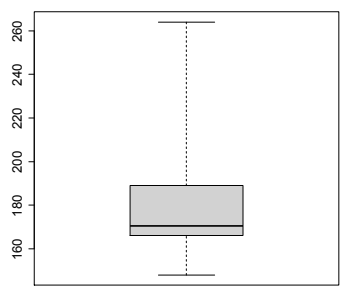

\#4 emotions

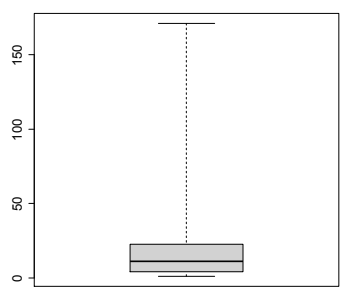

\#7 llog

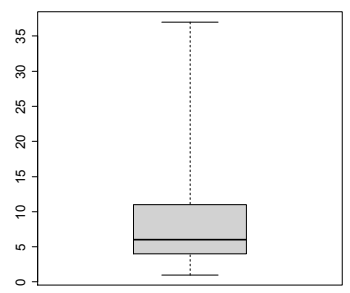

\#5 fapesp

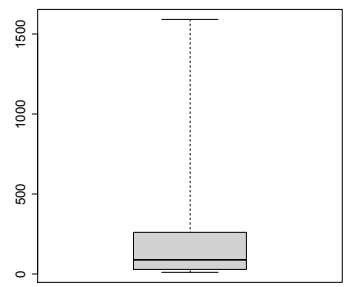

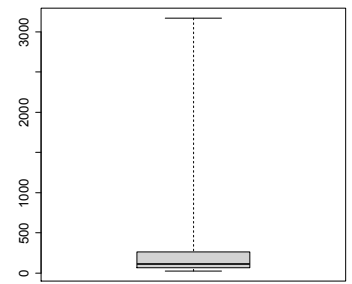

\#3 corel16k

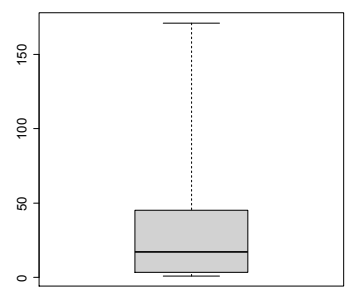

\#6 genbase

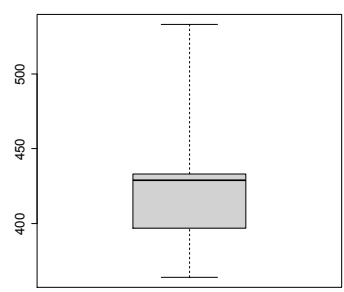

\#9 scene

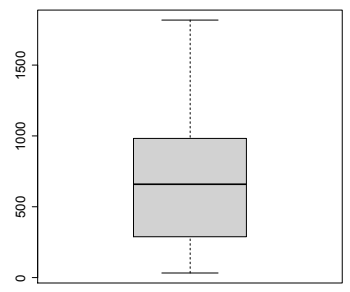

\#10 yeast

Figura 2.7: Boxplots representando a distribuição da frequência dos rótulos de cada conjunto de dados benchmark utilizado neste trabalho 


\subsection{Aplicações de Aprendizado Multirrótulo}

Como mencionado, a classificação multirrótulo vem se mostrando útil no apoio a distintos tipos de aplicação, como anotação de imagem e vídeo, classificação de dados textuais e identificação de funções em proteínas. A seguir são abordadas algumas dessas aplicações, em sua maioria ilustradas por meio de conjuntos de dados benchmark utilizados no desenvolvimento deste trabalho.

Análise de emoções. Uma aplicação potencial de aprendizado multirrótulo consiste na análise de emoções provocadas após a leitura de notícias ou outro tipo de texto, em que cada emoção é mapeada em um rótulo (Bhowmick et al., 2009). A natureza multirrótulo fica evidente na leitura da notícia "ataque de terroristas mata 30 pessoas na Nigéria", a qual pode despertar emoções de tristeza e medo.

Anotação de imagem e vídeo. Imagens e vídeos podem apresentar vários objetos ou contextos simultaneamente. No trabalho de Boutell et al. (2004) é realizada uma classificação de cenas, em que cada imagem apresenta conjuntamente diferentes objetos, como montanha, céu e mar. Cada um desses contextos se torna um rótulo durante a modelagem dos dados para o aprendizado multirrótulo. O estudo realizado em (Worring et al., 2007) representa um tipo de aplicação relacionada, na qual quadros de vídeos são capturados e categorizados em distintos rótulos correspondentes aos conceitos presentes nos quadros.

Categorização de documentos textuais. Um documento textual pode estar associado a mais de um conceito ou categoria. Cada um desses conceitos é modelado como um rótulo, como exemplificado em distintos trabalhos de classificação multirrótulo (Dendamrongvit et al., 2011; Wang e Jiang, 2009; Esuli et al., 2008; Chen et al., 2007). Por exemplo, documentos relacionados a notícias podem ser classificados como política e economia simultaneamente. Há ainda projetos que exemplificam a categorização de dados textuais como emails ${ }^{21}$ e páginas web ${ }^{22}$. De fato, o aprendizado multirrótulo para categorização de documentos textuais é uma das aplicações mais ativas, o que se reflete por exemplo na quantidade de conjuntos de dados textuais benchmark disponiveis para a comunidade.

Identificação de funções em proteínas. Cada proteína pode ser associada a um conjunto de funções (rótulos) (Elisseef e Weston, 2002), caracterizando uma aplicação de aprendizado multirrótulo potencialmente útil

\footnotetext{
${ }^{21}$ http://bailando.sims.berkeley.edu/enron_email.html

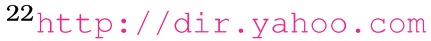


para apoiar estudos em áreas como a biologia. Adicionalmente, uma organização hierárquica pode ser utilizada, de modo que cada nível da hierarquia apresente várias funções (Cerri et al., 2012).

Predição de resistência viral a múltiplos fármacos. A identificação da resistência de vírus a fármacos é de interesse na área de bioinformática, tendo sido abordada inclusive por métodos de aprendizado de máquina. Contudo, apenas recentemente a resistência simultânea de vírus para múltiplos medicamentos tem sido analisada por métodos de aprendizado multirrótulo (Heider et al., 2013). Para tanto, cada sequência do genótipo viral consiste em um exemplo, o qual pode ser resistente a um ou mais fármacos (rótulos). É necessário ressaltar que essa aplicação ilustra a relevância da exploração da dependência de rótulos para determinados domínios.

\subsection{Considerações Finais}

Neste capítulo foram apresentados os principais conceitos, definições e aplicações relacionados ao aprendizado multirrótulo. Em particular, dois grupos de métodos de aprendizado e de medidas de avaliação multirrótulo foram abordados, bem como classificadores baseline que fornecem uma referência para o desempenho de aprendizado de um classificador multirrótulo. A dependência de rótulos, também considerada no capítulo, é um tema que deve ser melhor explorado, pois acreditamos que pode contribuir significativamente para o foco deste trabalho: o projeto e desenvolvimento de algoritmos de seleção de atributos de dados multirrótulo.

No próximo capítulo é apresentada a seleção de atributos para aprendizado multirrótulo, bem como uma síntese dos trabalhos relacionados encontrados por meio do método de revisão sistemática da literatura. 


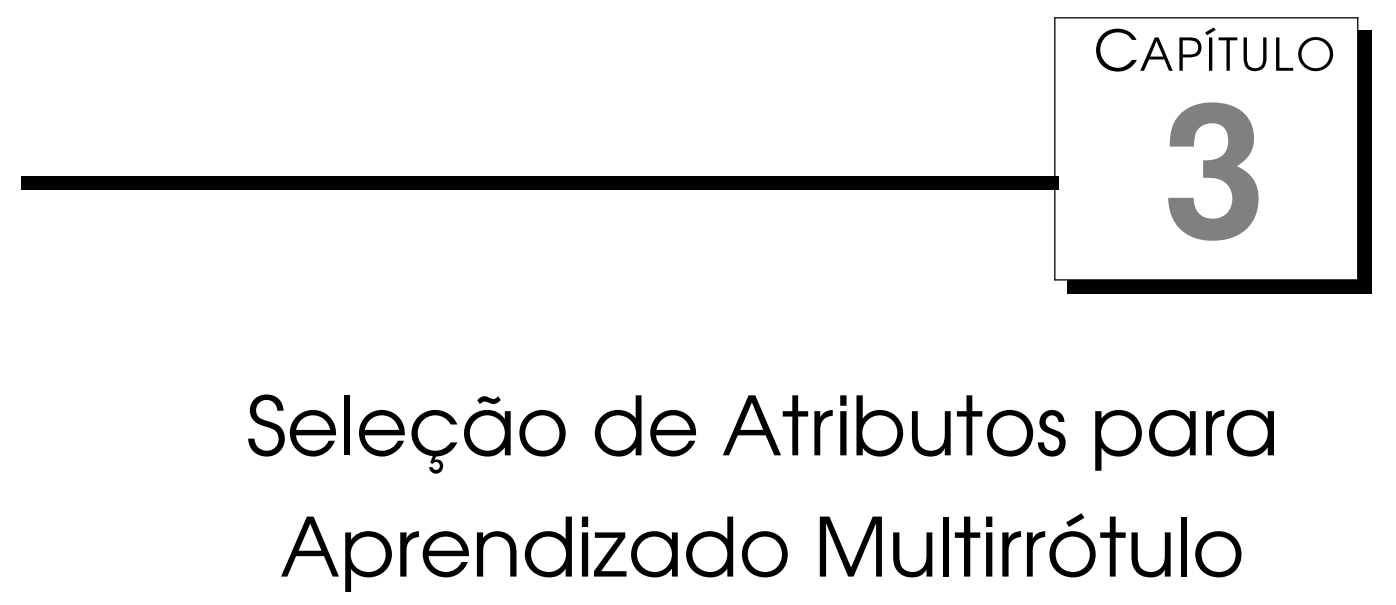

A seleção de atributos em aprendizado supervisionado tem como objetivo a identificação de atributos significativos. Alguns dos benefícios potenciais obtidos com a redução de efeitos da "maldição da dimensionalidade" incluem a geração de um classificador com desempenho melhor ou semelhante ao obtido pelo classificador construído a partir de todos os atributos, i.e., sem seleção de atributos (Hastie et al., 2001). Além disso, atributos de extração custosa em um determinado domínio poderiam ser descartados se eles não forem considerados significativos durante a seleção de atributos.

Como observado no aprendizado monorrótulo, o uso de tarefas auxiliares como a seleção de atributos constitui uma alternativa para obter melhores classificadores multirrótulo. De fato, uma revisão ampla, reprodutivel e rigorosa da literatura, realizada neste trabalho por meio do método de revisão sistemática, permitiu encontrar dezenas de publicações relacionadas à seleção de atributos para dados multirrótulo.

Neste capítulo são descritas características (dimensões) para organizar métodos de seleção de atributos multirrótulo e são abordados tipos de medidas para estimar a importância de atributos. Adicionalmente, são considerados os 74 trabalhos identificados por meio da revisão sistemática, o que constitui uma das contribuições deste trabalho. 


\section{1 Dimensões da Seleção de Atributos Multirrótulo}

Métodos de seleção de atributos para dados monorrótulo podem ser organizados em dimensões relacionadas, por exemplo, ao tipo de medida de importância envolvida (Liu e Motoda, 1998) ou à interação com o algoritmo de aprendizado (Kohavi e John, 1997). Em particular, a interação entre a medida de importância e o classificador já tem sido considerada em surveys de aprendizado multirrótulo (Zhang e Zhou, 2013; Tsoumakas et al., 2010) para organizar alguns métodos de SA. Neste trabalho, é proposta a adição de duas dimensões específicas para seleção de atributos multirrótulo:

1. Escopo do multirrótulo considerado durante a avaliação dos atributos;

2. Intensidade da exploração da dependência de rótulos.

As dimensões consideradas são ilustradas na Figura 3.1 e descritas a seguir. Essa taxonomia é flexível e permite tanto expandir as dimensões atuais, usando por exemplo grupos de medidas de importância de atributos definidas no contexto monorrótulo - Seção 3.2 - , quanto adicionar novas dimensões.

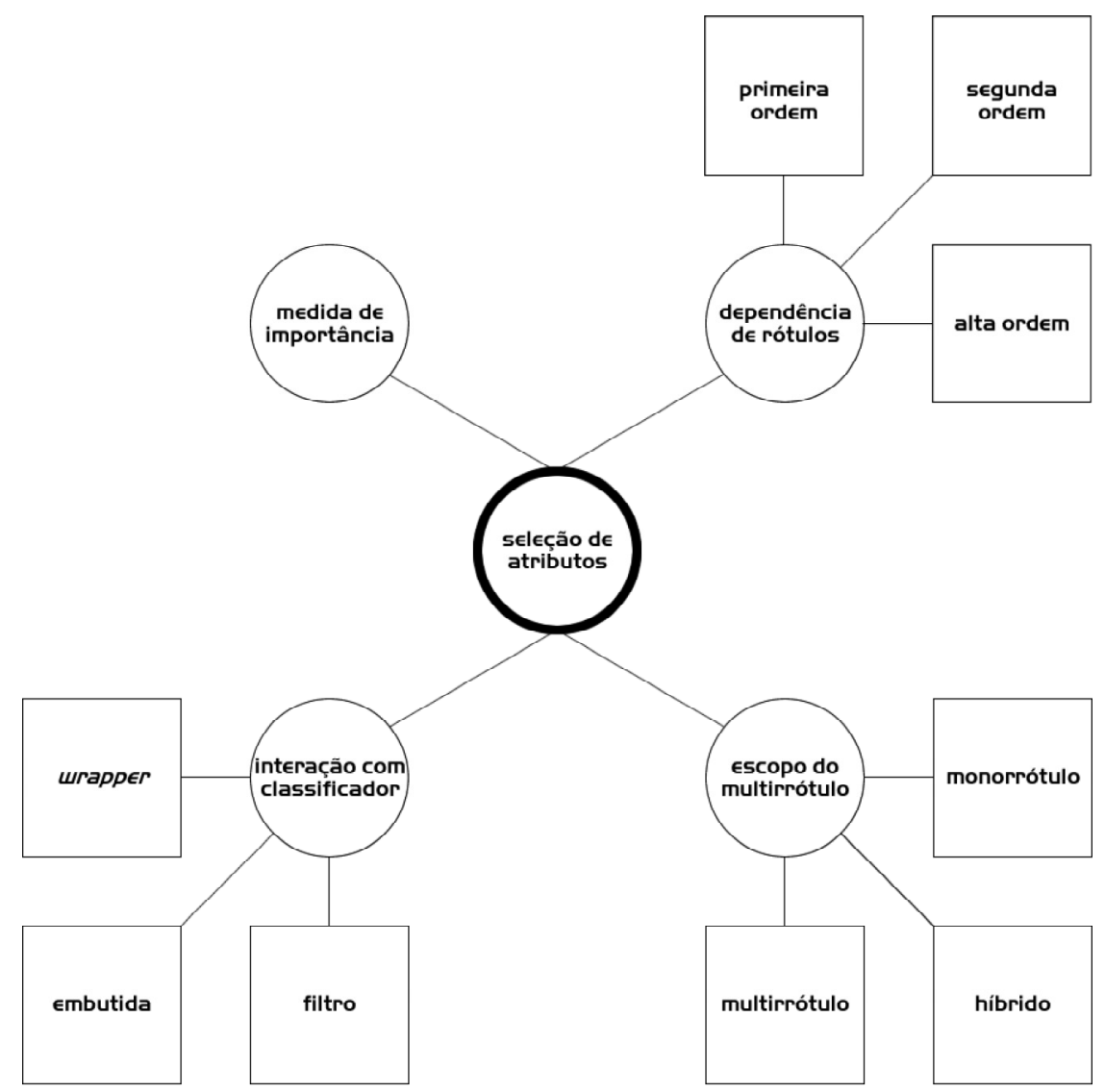

Figura 3.1: Dimensões da seleção de atributos: interação com classificador, escopo do multirrótulo, dependência de rótulos e medida de importância 


\subsubsection{Interação com Classificador}

Uma característica inerente a métodos de seleção de atributos consiste na interação que as medidas de importância associadas a esses métodos apresentam com o algoritmo de aprendizado. Três abordagens que tratam dessa característica consistem nas abordagens filtro, wrapper e embutida (Liu e Motoda, 2007), as quais são ilustradas na Figura 3.2.

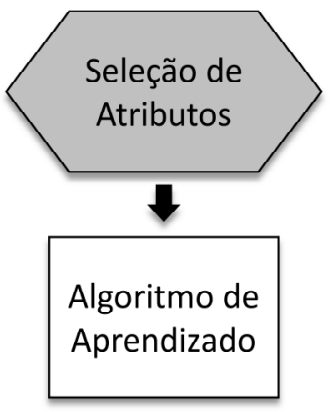

(a)

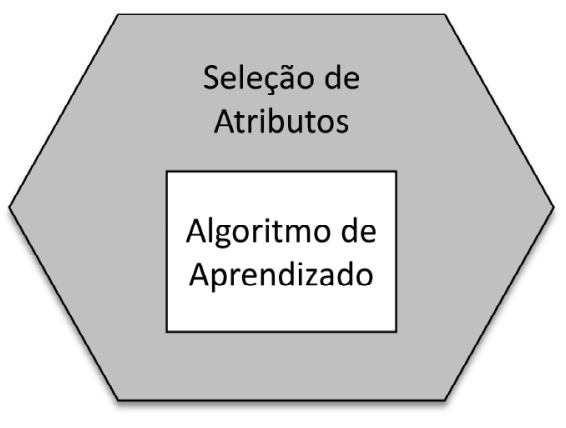

(b)

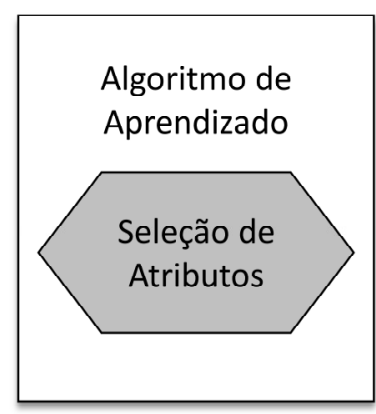

(c)

Figura 3.2: Visão geral das abordagens para seleção de atributos (a) filtro, (b) wrapper e (c) embutida (Covões, 2010)

Pode ser observado nessa figura que a abordagem filtro é a única que não exige interação com o algoritmo de aprendizado. A ideia é filtrar atributos irrelevantes e/ou redundantes, conforme alguma medida de importância, antes do aprendizado ocorrer (John et al., 1994). Portanto, essa medida se fundamenta em propriedades intrínsecas dos dados e independentes do classificador. Na Figura 3.3 é ilustrada a abordagem filtro para selecionar atributos.

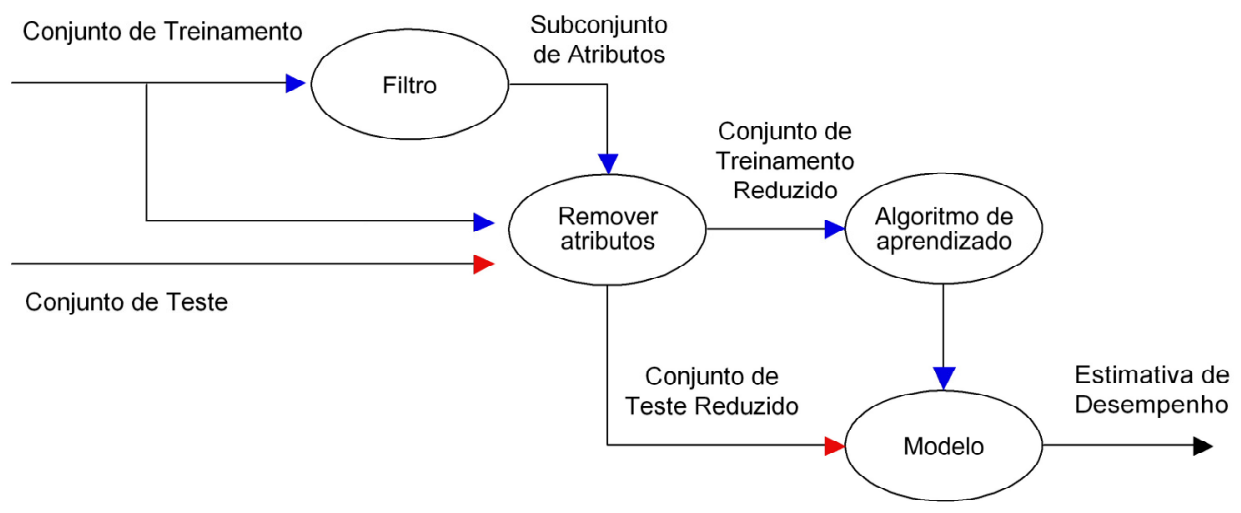

Figura 3.3: Abordagem filtro (Baranauskas, 2001)

$\mathrm{Na}$ abordagem wrapper, o algoritmo de aprendizado é utilizado como "caixa preta" para estimar a importância de atributos, como ilustrado na Figura 3.4. Como o mesmo algoritmo de aprendizado é usado para a seleção de atributos e para o aprendizado, espera-se obter um classificador com bom desempenho 
para esse algoritmo específico. Entretanto, além da dependência do algoritmo de aprendizado, essa abordagem é custosa devido à necessidade de gerar um classificador para cada subconjunto de atributos candidato no processo de busca da SA.

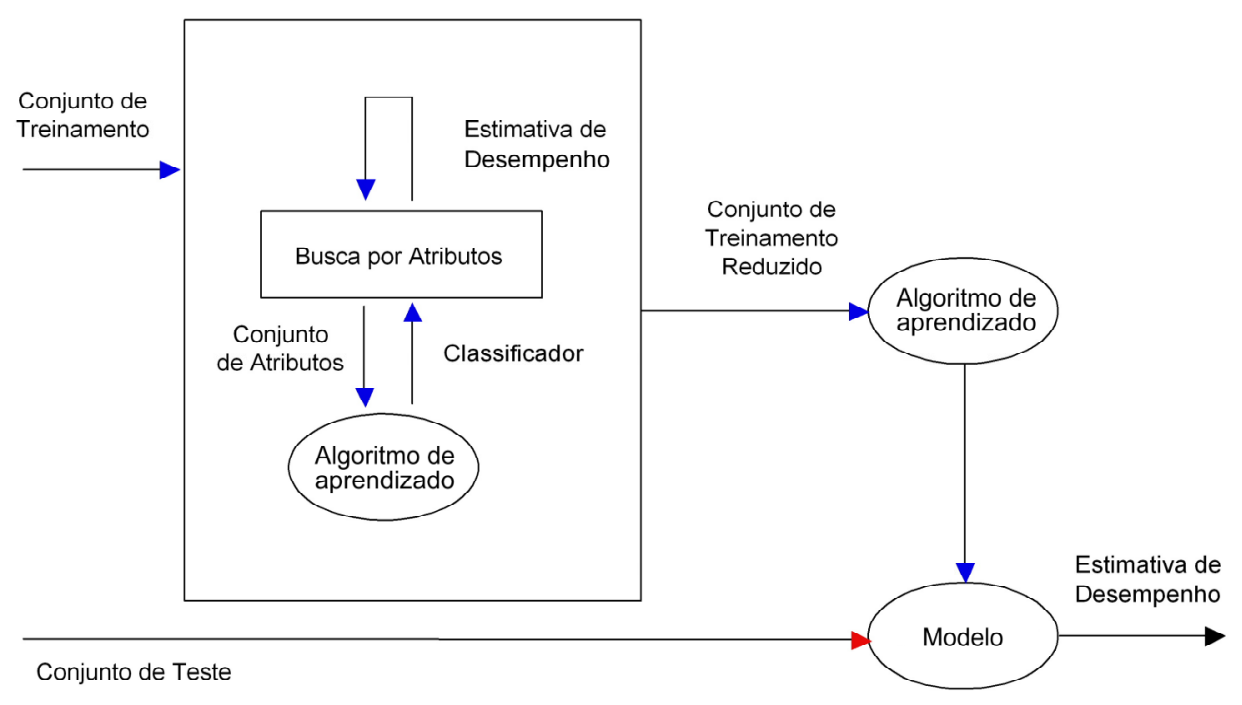

Figura 3.4: Abordagem wrapper (Lee, 2005)

A abordagem embutida se distingue das demais porque o próprio algoritmo de aprendizado realiza a seleção de atributos durante o processo de construção do classificador, como ilustrado na Figura 3.5.

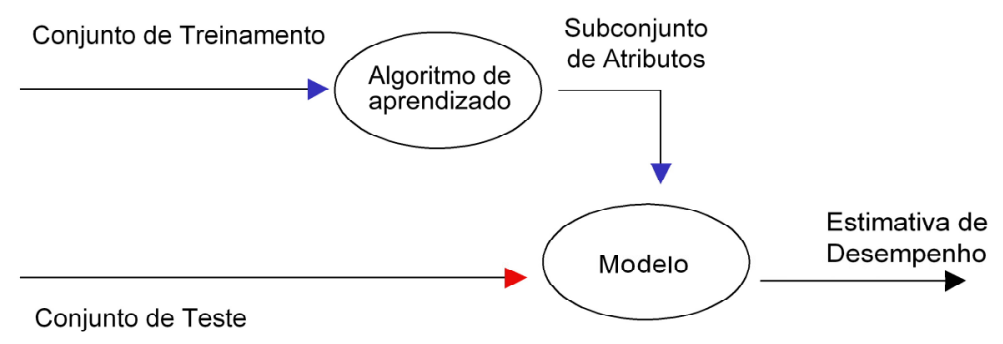

Figura 3.5: Abordagem embutida (Lee, 2005)

Como destacado na Seção 3.3, a maioria dos métodos de seleção de atributos para aprendizado multirrótulo utiliza a abordagem filtro. O uso da abordagem filtro em dados multirrótulo pode ser justificado, entre outros fatores, pelo fato dessa abordagem se concentrar em propriedades intrínsecas dos dados. Assim, se dispensa a repetição da construção de classificadores para avaliar a importância de atributos em conjuntos de dados com alta dimensionalidade, tanto de atributos quanto de rótulos, os quais são comuns em aplicações multirrótulo como a categorização de documentos textuais. 


\subsubsection{Escopo do Multirrótulo}

Outra dimensão que incluímos neste trabalho para organizar métodos de SA consiste no escopo do multirrótulo considerado durante a seleção de atributos - Figura 3.1. As três categorias dessa dimensão, as quais foram adaptadas da literatura de classificação hierárquica (Esuli et al., 2006), são descritas a seguir.

A seleção de atributos com escopo multirrótulo envolve a seleção de um mesmo subconjunto de atributos baseado nos multirrótulos do conjunto de dados. Em outras palavras, os dados multirrótulo são tratados sob uma perspectiva global, sem o uso de uma abordagem de transformação do problema.

Por outro lado, a seleção de atributos com escopo monorrótulo identifica atributos importantes para cada rótulo simples em $L$, i.e., sob uma perspectiva local. Após, é possível optar pela agregação dos subconjuntos de atributos obtidos, resultando em um único subconjunto, ou mantê-los separados para o restante do processo de aprendizado. Alguns métodos que utilizam essa abordagem inclusive permitem a aplicação de algoritmos tradicionais de SA monorrótulo.

Adicionalmente, o escopo híbrido abrange métodos que apresentam simultaneamente características dos escopos multirrótulo e monorrótulo. A hibridização é obtida, por exemplo, ao considerar em cada iteração do método de SA dois ou mais rótulos simples conjuntamente.

A SA com escopo monorrótulo tem sido a mais usual na literatura - Seção 3.3. Contudo, o escopo monorrótulo apresenta a desvantagem de realizar a avaliação da importância dos atributos $q$ vezes, uma por rótulo do conjunto de rótulos $L$. Adicionalmente, se a seleção de atributos for realizada independentemente em cada um dos $q$ conjuntos de dados monorrótulo, i.e., não houver agregação para determinar um único subconjunto de atributos, há uma chance de selecionar todos os atributos e, portanto, não reduzir a dimensionalidade, se a união desses subconjuntos for o conjunto original com $M$ atributos.

\subsubsection{Dependência de Rótulos}

A outra dimensão adicionada neste trabalho para organização dos métodos de SA consiste na intensidade de exploração da dependência de rótulos durante a avaliação da importância de atributos - Figura 3.1. As três categorias dessa dimensão, adaptadas do aprendizado multirrótulo (Zhang e Zhou, 2013; Zhang e Zhang, 2010), são descritas a seguir.

As estratégias de primeira ordem realizam a seleção de atributos rótulo a rótulo, ignorando a coexistência de outros rótulos. Porém, a relativa eficiência 
computacional devido à análise independente de rótulos pode não ser suficiente para compensar a potencial perda de qualidade obtida ao ignorar relações entre dois ou mais rótulos.

As estratégias de segunda ordem avaliam atributos enquanto consideram algum tipo de relação entre dois rótulos do conjunto $L$. Embora essas estratégias exijam, em geral, um custo computacional maior que as de primeira ordem, elas são capazes de explorar a dependência de rótulos com maior intensidade.

As estratégias de alta ordem incluem métodos de SA que abordam relações entre três ou mais rótulos. Em relação às demais estratégias, é esperado um maior custo computacional, o qual pode ser compensado em aplicações nas quais relações entre múltiplos rótulos são relevantes.

As estratégias de primeira ordem têm predominado na literatura. No entanto, como mencionado na Seção 3.3, já é possível encontrar algumas publicações que utilizam as outras abordagens e que, consequentemente, tratam algum tipo de relação entre rótulos.

Na seleção de atributos monorrótulo, cinco grupos de medidas de importância são definidas (Liu e Motoda, 1998). Contudo, a exploração de todos esses grupos, descritos na próxima seção, ainda é incipiente em SA multirrótulo.

\subsection{Importância de Atributos}

Para selecionar um bom atributo, é necessário definir sua importância. De modo geral, a importância de um atributo pode ser definida como:

Definição 3.2.1 Importância de um Atributo (Liu e Motoda, 1998): Um atributo é dito importante se, quando removido, a medida de importância considerada em relação aos atributos restantes é deteriorada.

A partir dessa definição, medidas de importância de atributos podem ser utilizadas para determinar em relação a quê um atributo é importante. Para organizar essas medidas, Liu e Motoda (1998) propõem a taxonomia ilustrada na Figura 3.6 para a seleção de atributos monorrótulo. Na sequência, são descritos os grupos de medidas de consistência, dependência, distância, informação e precisão.

Medidas de Consistência. A importância de atributos baseada em consistência busca encontrar atributos que reduzam a ocorrência de exemplos com valores similares em cada atributo e rótulos distintos. Assim, um subconjunto de atributos é considerado importante se o conjunto de treinamento descrito por esses atributos apresenta uma consistência satisfa- 


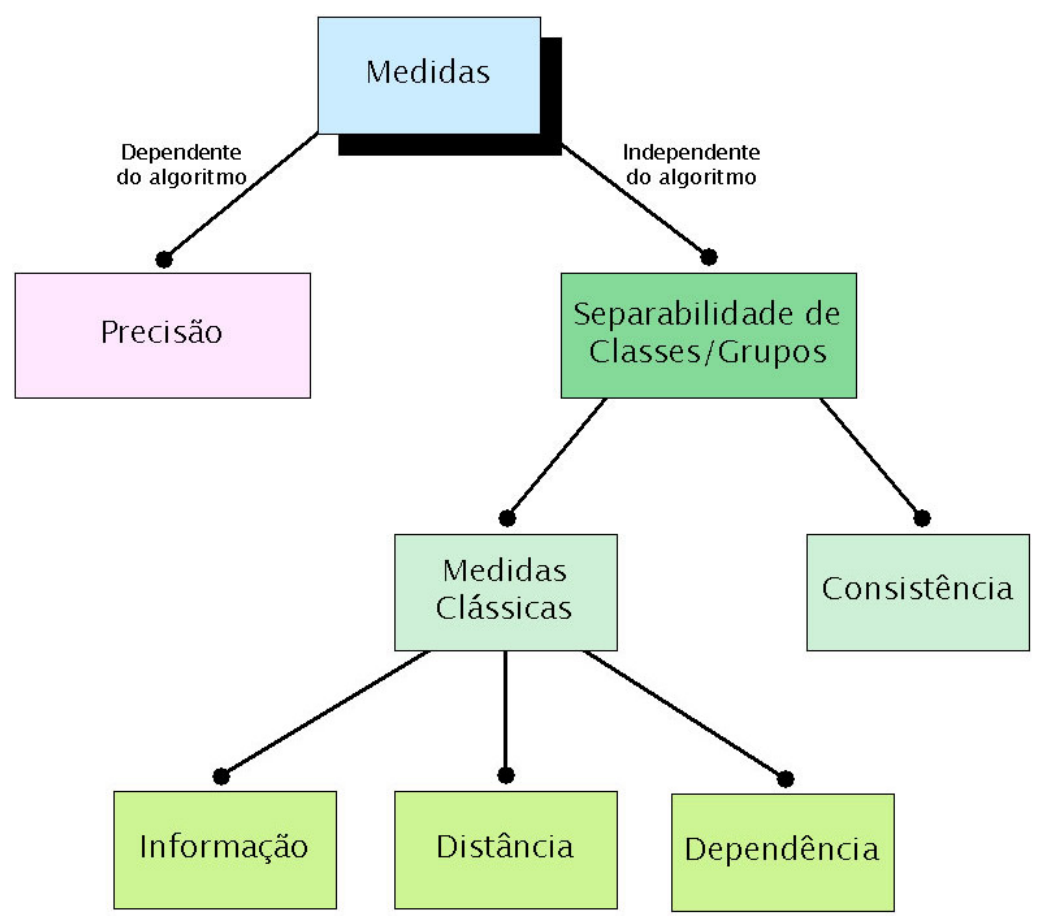

Figura 3.6: Taxonomia de tipos de medidas de importância de atributos (Liu e Motoda, 1998)

tória. Muitas medidas que tratam desse tipo de importância exigem uma discretização prévia de dados numéricos.

Medidas de Dependência. A importância de atributos baseada em dependência, correlação ou associação permite identificar a habilidade de predizer o valor de um atributo a partir do valor de outro. Uma medida clássica de dependência é o coeficiente de correlação. Medidas de dependência podem ser úteis para reduzir a redundância nos dados.

Medidas de Distância. A importância de atributos baseada em distância, separabilidade ou divergência permite destacar atributos que, por exemplo, não separam exemplos de mesma classe. Medidas clássicas de separabilidade incluem a distância inter-classe e a distância intra-classe.

Medidas de Informação. A importância de atributos baseada em informação possibilita determinar o ganho de informação, i.e., a diferença entre as incertezas a priori e a posteriori a partir de um atributo. Uma medida clássica de informação é a entropia, a qual avalia a incerteza inerente aos dados.

Medidas de Precisão. A importância de atributos baseada em precisão (ou acurácia) contribui na obtenção de classificadores com um melhor desempenho. Usualmente, os atributos são avaliados pelo mesmo algoritmo de aprendizado que gera o classificador utilizando os exemplos do 
conjunto de teste. Assim, as medidas de precisão não estão relacionadas à abordagem filtro, pois necessitam de um algoritmo de aprendizado durante a SA.

\subsection{Trabalhos Relacionados}

Ainda que a pesquisa em seleção de atributos para dados multirrótulo seja relativamente recente, é possível observar na literatura a aplicação de várias medidas de importância, bem como publicações que instanciam cada dimensão de seleção de atributos multirrótulo introduzida na Seção 3.1. Na Tabela A. 1 do Apêndice, página 111, são apresentadas informações básicas das 74 publicações identificadas neste trabalho (Spolaôr et al., 2012b) ${ }^{23}$ por meio do método da revisão sistemática, o qual permite uma revisão ampla e reprodutivel da literatura (Kitchenham e Charters, 2007) e, como mencionado, constitui uma das contribuições deste trabalho. Essas informações são sintetizadas na Tabela 3.1, na qual são contabilizados, em valores absolutos e em porcentagem, quantas publicações utilizam cada abordagem ou categoria em cada dimensão. Note que a quantidade total de trabalhos nessa tabela, incluindo os que não tiveram abordagens reconhecidas, é diferente de 74, pois alguns trabalhos representam mais de uma abordagem em uma mesma dimensão.

Tabela 3.1: Quantidade de trabalhos instanciando cada dimensão da SA

\begin{tabular}{ll|l}
\hline Dimensão & Abordagem & Quantidade de trabalhos (\%) \\
\hline \hline \multirow{4}{*}{ interação com classificador } & embutida & $12(16,2 \%)$ \\
& filtro & $50(67,6 \%)$ \\
& wrapper & $7(9,5 \%)$ \\
& não reconhecido & $5(6,8 \%)$ \\
\hline \multirow{3}{*}{ escopo do multirrótulo } & híbrido & $17(23,0 \%)$ \\
& monorrótulo & $33(44,6 \%)$ \\
& multirrótulo & $23(31,1 \%)$ \\
& não reconhecido & $5(6,8 \%)$ \\
\hline \multirow{3}{*}{ dependência de rótulos } & alta ordem & $12(16,2 \%)$ \\
& primeira ordem & $46(62,2 \%)$ \\
& segunda ordem & $11(14,9 \%)$ \\
& não reconhecido & $7(9,5 \%)$ \\
\hline \hline
\end{tabular}

Com relação às dimensões de interação com o classificador e dependência de rótulos, as abordagens filtro e primeira ordem são predominantes $(67,6 \%$ e $62,2 \%$ dos trabalhos, respectivamente). Esse comportamento é explicado, em parte, porque ambas abordagens têm custo computacional relativamente menor que as outras abordagens. Além disso, a abordagem filtro é independente do algoritmo de aprendizado multirrótulo. Com relação à dimensão de escopo do multirrótulo, há uma distribuição mais equitativa entre as abordagens híbrida, monorrótulo e multirrótulo. A quantidade de trabalhos com abordagem

\footnotetext{
${ }^{23}$ Essa revisão foi atualizada recentemente (junho de 2014).
} 
não reconhecida é relativamente baixa, mas indica a necessidade da adoção de uma taxonomia específica para a seleção de atributos multirrótulo, a exemplo do que já existe na SA monorrótulo - Figura 3.6.

Convém ressaltar que algumas informações da Tabela 3.1 serviram como base para decisões tomadas no desenvolvimento deste trabalho. Em particular, optamos pela usual abordagem filtro devido às vantagens dessa abordagem mencionadas anteriormente. Por outro lado, priorizamos as incipientes abordagens de segunda e de alta ordem nas propostas deste trabalho devido a indícios da literatura de que a consideração da dependência de rótulos deve levar a bons resultados.

Na Figura 3.7 é apresentada a quantidade de trabalhos encontrados pela revisão sistemática publicados a partir de 1997 (total: 74 trabalhos). Note que, como a última atualização da revisão foi realizada em junho de 2014, o número de publicações em 2014 ainda está reduzido.

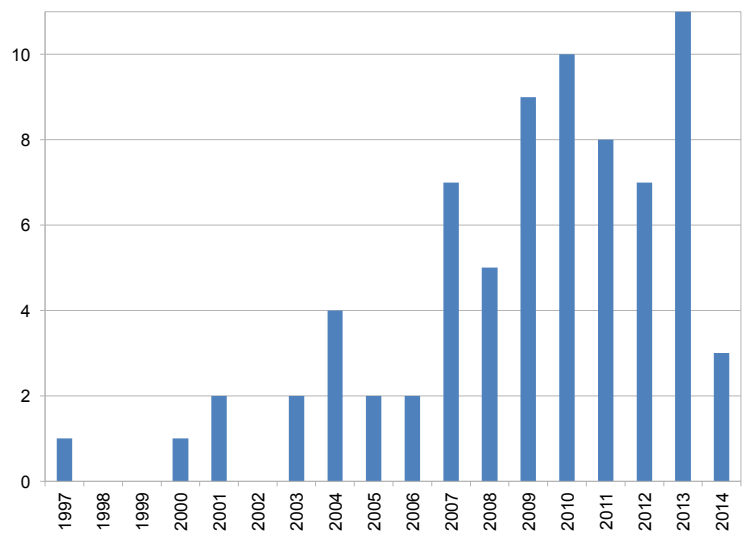

Figura 3.7: Número de trabalhos relacionados por ano de publicação, de acordo com a última atualização da revisão sistemática (junho de 2014)

No restante desta seção, alguns estudos relacionados a este trabalho, identificados por meio do processo de revisão sistemática, são brevemente apresentados em dois grupos:

1. Extensões do algoritmo ReliefF (Kononenko, 1994), relacionadas a uma das propostas deste trabalho - Capítulo 5 -; e

2. Outros métodos para SA multirrótulo propostos na literatura.

Uma característica comum entre esses trabalhos consiste na consideração da dependência de rótulos durante a seleção de atributos. Adicionalmente, a maioria dos trabalhos que usam ReliefF consideram a abordagem filtro.

\subsection{Extensões do Algoritmo ReliefF}

O algoritmo ReliefF monorrótulo é descrito em maiores detalhes no Capítulo 5. De modo geral, a ideia do ReliefF original e de suas extensões para o 
problema multirrótulo é destacar atributos potencialmente úteis na diferenciação de exemplos com rotulações distintas. Para tanto, uma busca pelos $k$ vizinhos mais próximos é realizada, o que envolve por exemplo o uso de uma medida de dissimilaridade entre exemplos no espaço de atributos e, eventualmente, no espaço de rótulos (multirrótulos).

De acordo com a revisão sistemática, os primeiros estudos utilizando ReliefF para SA multirrótulo correspondem ao estágio inicial do desenvolvimento deste trabalho. Nesses trabalhos, uma abordagem de transformação do problema - Seção 2.2.1, página 15 - é aplicada para permitir o uso do algoritmo ReliefF original (monorrótulo).

A primeira adaptação do algoritmo ReliefF para SA multirrótulo identificada na literatura consiste no trabalho de Kong et al. (2012). Nesse caso, o algoritmo é estendido para abordar pares de rótulos sem nenhuma transformação do problema, de modo que o trabalho é categorizado respectivamente como uma estratégia de segunda ordem e de escopo multirrótulo. Embora os subconjuntos de atributos selecionados tenham contribuído na construção de classificadores com melhor desempenho em relação a classificadores gerados ao combinar ReliefF com abordagens de transformação do problema, o algoritmo proposto apresenta uma limitação por ignorar exemplos nos quais ambos rótulos coocorrem.

Posteriormente, três extensões distintas foram propostas quase simultaneamente (Pupo et al., 2013; Slavkov et al., 2013; Spolaôr et al., 2013c), as quais são respectivamente denominadas ReliefF-ML, HMC-ReliefF e RF-ML. Embora todas elas sejam estratégias de alta ordem e de escopo multirrótulo, há características que as diferenciam, como abordado a seguir.

Motivado por propriedades do Relieff original, como consideração de interação entre atributos e aplicabilidade para dados numéricos e categóricos, Pupo et al. (2013) estendem esse algoritmo para tratar dados multirrótulo diretamente. Três diferenciais importantes do algoritmo ReliefF-ML são:

- Aplicação da abordagem embutida de interação com o classificador, pois os valores de importância dos atributos calculados pela extensão são utilizados para ponderar atributos na medida de dissimilaridade de algoritmos de aprendizado lazy;

- Extensão do ReliefF, originalmente proposto para problemas de classificação monorrótulo, ao invés da sua variação para regressão monorrótulo RReliefF (Robnik-Sikonja e Kononenko, 1997); e

- A estratégia adotada possui simultaneamente características de primeira e de alta ordem, pois necessita buscar por vizinhos mais próximos no 
contexto de cada rótulo enquanto utiliza a medida distância Hamming normalizada entre multirrótulos.

Partindo de características positivas do ReliefF, como exploração de suas propriedades teóricas e relativa facilidade para extensão, Slavkov et al. (2013) estendem o algoritmo RReliefF (Robnik-Sikonja e Kononenko, 1997), projetado originalmente para problemas de regressão monorrótulo. A escolha realizada, em detrimento de ReliefF, é justificada pela dispensa da busca por vizinhos próximos de mesma classe e de classe distinta em um problema multirrótulo. O principal diferencial do HMC-ReliefF é que o problema de aprendizado tratado é a classificação hierárquica multirrótulo, de modo que uma medida de dissimilaridade que considera a estrutura hierárquica substitui a medida original do RReliefF e caracteriza o algoritmo como estratégia de alta ordem.

Embora a extensão $R F-M L$ proposta neste trabalho (Spolaôr et al., 2013c) possua características em comum com as duas outras extensões, seu principal diferencial consiste justamente na combinação única entre essas características na seleção de atributos com a abordagem filtro para apoiar aprendizado multirrótulo não hierárquico ( "flat"). Em particular, medidas de dissimilaridade como distância Hamming normalizada - Seção 5.2.1, página 85, Equação 5.9 - e Jaccard, aplicáveis para multirrótulos de exemplos, podem ser utilizadas como parte da busca por vizinhos mais próximos. Além disso, o algoritmo RReliefF é usado como base para a extensão descrita no Capítulo 5 conforme as abordagens filtro e alta ordem.

Convém ressaltar que a complexidade dos algoritmos HMC-ReliefF e $R F$ $M L$ é similar, sendo dominada pela busca pelos $k$ vizinhos mais próximos $N_{t r}$ vezes, i.e., $O\left(N_{t r}^{2} \times M\right)$, em que $N_{t r}$ e $M$ são, respectivamente, o número de exemplos de treinamento e de atributos (Robnik-Sikonja e Kononenko, 2003). Por outro lado, o custo de ReliefF-ML é maior, pois a busca por vizinhos mais próximos é realizada para cada um dos $q$ rótulos, i.e., $O\left(N_{t r}^{2} \times M \times q\right)$.

\subsubsection{Outros Métodos para Seleção de Atributos Multirrótulo Pro- postos na Literatura}

Os trabalhos relacionados tratados nesta seção consideram relações entre rótulos durante a seleção de atributos usando uma medida de importância alternativa ao ReliefF. A maioria deles segue a abordagem filtro, a mais usual na literatura.

Em (Trohidis et al., 2008), a dependência de rótulos é considerada por meio do uso da abordagem de transformação do problema LP. A medida de importância qui-quadrado é aplicada conforme a abordagem filtro nos dados monorrótulo resultantes dessa transformação para dados multiclasse. Assim, 
o método é categorizado como estratégia de alta ordem e escopo híbrido, pois a relação entre vários rótulos é obtida por meio de uma transformação do problema. O método proposto apresenta em geral um melhor desempenho que o uso da abordagem de transformação $B R$ com o usual escopo monorrótulo. No entanto, vale ressaltar que $L P$ apresenta desvantagens, como a geração de um conjunto de dados multiclasse usualmente desbalanceado e a incapacidade de predizer multirrótulos que não participam do conjunto de treinamento.

Em (Lastra et al., 2011) é proposta a adaptação da incerteza simétrica, uma medida de importância de atributos relativamente usual na seleção de atributos monorrótulo conforme a abordagem filtro. Essa adaptação inclui a construção de uma matriz com os valores da medida entre todos os pares de atributos e rótulos, o que implica na consideração da dependência de rótulos durante a seleção de atributos. Posteriormente, é construída uma árvore geradora que possibilita representar graficamente a relevância dos atributos conforme a distância para os rótulos. A incerteza simétrica adaptada é categorizada como estratégia de segunda ordem e escopo multirrótulo, pois pares de rótulos são utilizados durante a avaliação dos atributos e nenhum método de transformação do problema é necessário.

A medida informação mútua, utilizada por Doquire e Verleysen (2011) e avaliada mais extensivamente em (Doquire e Verleysen, 2013), é aplicada junto à abordagem de transformação do problema $L P$ e analisa a importância de atributos baseada em dependência. A informação mútua se destaca em relação a coeficientes de correlação por possibilitar a identificação de relações não-lineares entre atributos e a avaliação de subconjuntos de atributos. Uma das contribuições desse método de seleção de atributos baseado na abordagem filtro consiste no uso de um estimador potencialmente menos sensível ao número de atributos presentes nos dados. Adicionalmente, a dependência de rótulos é considerada por meio da aplicação de uma abordagem de transformação do problema que apresenta funcionalidades como a poda, a qual simplifica o problema e garante que todos os multirrótulos (classes) sejam representados por um determinado número de exemplos (Read, 2008). Como o método de transformação do problema resulta em um conjunto de dados multiclasse que representa múltiplos rótulos, o algoritmo de SA é categorizado como estratégia de alta ordem e escopo híbrido.

Outro trabalho recente baseado na informação mútua é realizado por Lee e Kim (2013). A principal diferença em relação ao trabalho anterior consiste na dispensa de uma abordagem de transformação do problema. Contudo, como esse método considera relações entre cada atributo e cada rótulo, bem como entre cada atributo e cada par de rótulos, a sua complexidade é exponencial. O método proposto é categorizado como estratégia de segunda ordem, pois 
inclui até dois rótulos em cada avaliação, escopo multirrótulo e abordagem filtro.

O último representante da abordagem filtro tratado nesta seção avalia e busca por subconjuntos de atributos por meio da adaptação do Correlationbased Feature Selection (CFS) (Hall, 1999), um algoritmo bem conhecido de seleção de atributos monorrótulo (Jungjit et al., 2012). A extensão original tem como intuito encontrar um subconjunto de atributos pouco correlacionados entre si e muito correlacionados com cada um dos rótulos simples, dispensando qualquer transformação do problema. Assim, é considerada uma estratégia de primeira ordem com escopo multirrótulo. Recentemente, o método se tornou mais sofisticado ao usar o valor absoluto da correlação no CFS e ao ponderar cada correlação entre atributo e rótulo simples com a informação mútua média entre esse rótulo e os demais (Jungjit et al., 2013). Nesse caso, a estratégia passa a ser categorizada como de segunda ordem.

Um método para construir redes Bayesianas proposto em (Gasse et al., 2014) transforma o problema multirrótulo para multiclasse antes de realizar a seleção de atributos utilizando a abordagem embutida. Em particular, a importância de subconjuntos de atributos é estimada por meio de conceitos como Markov blankets e limites de Markov. Um Markov blanket de uma variável randômica ${ }^{24}$ é qualquer subconjunto de variáveis tal que essa variável seja condicionalmente independente das demais variáveis dado esse blanket. Um limite de Markov de uma variável randômica, por sua vez, consiste em qualquer Markov blanket tal que nenhum de seus subconjuntos seja um Markov blanket da variável randômica. Devido à transformação do problema multirrótulo para multiclasse, o método é categorizado como estratégia de alta ordem e escopo híbrido. A dependência de rótulos é considerada, pois redes bayesianas com Markov blanket possibilitam a modelagem de interdependências entre rótulos.

A medida de importância Average Precision (Tsoumakas et al., 2010) é otimizada como função de aptidão por uma meta-heurística ${ }^{25}$ por Shao et al. (2011). Essa medida aborda a relação do ranking entre dois rótulos, sendo portanto categorizada como estratégia de segunda ordem. O método proposto considera a dependência de rótulos na seleção de atributos utilizando a abordagem wrapper por meio de algoritmos de aprendizado multirrótulo do grupo de adaptação do algoritmo, caracterizando a SA com escopo multirrótulo.

A partir da adaptação de um algoritmo para ranking de rótulos baseado em Support Vector Machines, Gu et al. (2011) propõem um algoritmo de aprendizado multirrótulo que considera a correlação de rótulos durante a seleção de

\footnotetext{
${ }^{24}$ Essa variável poderia modelar, por exemplo, um atributo do conjunto de dados.

${ }^{25}$ Estratégia de alto nível para exploração de um espaço de busca (Coello, 2011), como o espaço relacionado à seleção de atributos.
} 
atributos utilizando a abordagem embutida. Esse algoritmo busca encontrar um subconjunto de atributos que minimiza uma medida de importância relacionada a uma função de perda. Adicionalmente, o algoritmo realiza seleção de atributos por meio de uma estratégia de segunda ordem, devido ao estudo da correlação entre rótulos, e do escopo multirrótulo.

Em (Zhang et al., 2009), a medida Hamming Loss é otimizada simultaneamente ao Ranking Loss (Tsoumakas et al., 2010) para estimar o desempenho de classificadores multirrótulo Naive Bayes sem transformação do problema, o que caracteriza escopo multirrótulo. A busca pelo subconjunto de atributos que otimiza um compromisso entre essas medidas é realizado por meio de uma meta-heurística, denominada algoritmo genético multiobjetivo ${ }^{26}$, conforme a abordagem wrapper. O método contempla tanto uma estratégia de alta ordem quanto uma de segunda ordem, respectivamente, por meio de Hamming Loss e de Ranking Loss.

\subsection{Considerações Finais}

Uma breve fundamentação sobre conceitos de seleção de atributos foi apresentada neste capítulo. Um dos pontos mais relevantes consiste na proposta de uma taxonomia com dimensões para categorizar métodos de SA multirrótulo, tais como: interação da seleção de atributos com o algoritmo de aprendizado; escopo do multirrótulo tratado; intensidade de exploração da dependência de rótulos; e tipo de medida de importância de atributos. A taxonomia proposta é flexivel, pois as dimensões atuais podem ser expandidas e há suporte para inclusão de novas dimensões.

Outro ponto relevante neste trabalho, e no desenvolvimento de algoritmos de seleção de atributos multirrótulo em geral, é a aplicação do método de revisão sistemática da literatura. Ao todo, 74 publicações relacionadas foram identificadas e serviram como base para sintetizar informações que propiciam um panorama atualizado da área de pesquisa em seleção de atributos no aprendizado multirrótulo.

Nos Capítulos 4 e 5 são propostos e avaliados, respectivamente, o método LCFS e as extensões RF-ML e RFM-ML para seleção de atributos multirrótulo.

\footnotetext{
${ }^{26}$ Meta-heurística para otimização simultânea de mais de uma função de aptidão, a qual pode modelar uma medida de avaliação multirrótulo, por exemplo.
} 


\section{$-4$ \\ Seleção de Atributos baseada em Transformação do Problema}

A abordagem padrão para seleção de atributos multirrótulo, a qual transforma o conjunto de dados multirrótulo em um ou mais conjuntos monorrótulo antes do uso de algoritmos tradicionais de SA, pode ser implementada por meio da abordagem Binary Relevance. No entanto, essa abordagem conta com uma desvantagem que pode prejudicar posteriormente o aprendizado: a dependência de rótulos é frequentemente ignorada.

Uma alternativa para reduzir esse problema seria construir rótulos a partir de relações entre os rótulos originais e incluir os novos rótulos durante a tarefa de SA. Assim, mesmo se todos rótulos continuarem sendo tratados independentemente por $B R$, alguns deles estariam representando indiretamente associações entre rótulos.

A ideia principal da construção de variáveis (rótulos ou atributos) é obter informação sobre relações entre as variáveis originais a partir dos dados e inferir variáveis adicionais (Motoda e Liu, 2002). Embora a construção de atributos seja menos usual que os métodos de seleção de atributos (Lillywhite et al., 2013), ela já foi usada para auxiliar tanto o aprendizado monorrótulo (García et al., 2014; Piramuthu e Sikora, 2009; Lee et al., 2000) quanto multirrótulo (Prati e Olivetti de Franca, 2013; Duivesteijn et al., 2012; Yang e Gopal, 2012). No entanto, não é de nosso conhecimento a existência de pesquisas sobre construção de rótulos para dados multirrótulo, tampouco o uso dessa ideia para auxiliar a abordagem $B R$ para seleção de atributos.

Neste capítulo é proposto o método Label Construction for Feature Selection (LCFS), o qual constroi variáveis binárias (novos rótulos) a partir de relações 
inerentes a dois ou mais rótulos (Spolaôr et al., 2014b). Essas variáveis são então incluídas como novos rótulos no conjunto de dados original a ser submetido à abordagem padrão para SA multirrótulo. Posteriormente, o conjunto de dados descrito pelos atributos selecionados e pelos rótulos originais pode ser submetido a qualquer algoritmo de aprendizado multirrótulo. As configurações do LCFS atualmente implementadas consideram relações entre dois rótulos, caracterizando o método como uma estratégia de segunda ordem (Zhang e Zhou, 2013), e almejam reduzir a desvantagem do $B R$ mencionada anteriormente.

Neste capítulo, primeiramente são apresentadas abordagens tradicionais de transformação do problema para seleção de atributos, bem como o método proposto LCFS. Também são abordadas as medidas de importância utilizadas e outras informações relacionadas à configuração experimental. Após, a avaliação experimental comparando abordagens tradicionais e configurações do método LCFS em 10 conjuntos de dados benchmark multirrótulo é descrita e discutida.

\subsection{Seleção de Atributos via Abordagens Tradicionais de Transformação do Problema}

Como mencionado, duas abordagens usuais para a realização da seleção de atributos multirrótulo consistem em Binary Relevance e Label Powerset Capítulo 2. A seguir, cada uma dessas abordagens é descrita no contexto de seleção de atributos.

\subsubsection{Abordagem Binary Relevance}

A abordagem $B R$ para seleção de atributos é representada na Figura 4.1. A partir de um conjunto de treinamento $D_{t r}$ e um de teste $D_{t e}$, são realizados os seguintes quatro procedimentos principais para encontrar o subconjunto de atributos selecionados $X^{\prime}$ :

1. Transformação dos dados (T): decompõe $D_{t r}$ em $q$ problemas binários;

2. Seleção de Atributos (SA): aplica uma medida de importância de SA monorrótulo em um determinado problema binário para avaliar atributos individualmente ou em subconjuntos;

3. Combinação dos resultados (C): agrega resultados dos problemas binários de acordo com uma determinada estratégia, de modo que cada atributo ou cada subconjunto de atributos é associado a um valor de impor- 
tância para todos os rótulos. Se a avaliação dos atributos é individual, um limiar ou quantidade de atributos é estabelecido para encontrar $X^{\prime}$;

4. Redução (R): remove de $D_{t r}$ e $D_{t e}$ os atributos não pertencentes a $X^{\prime}$.

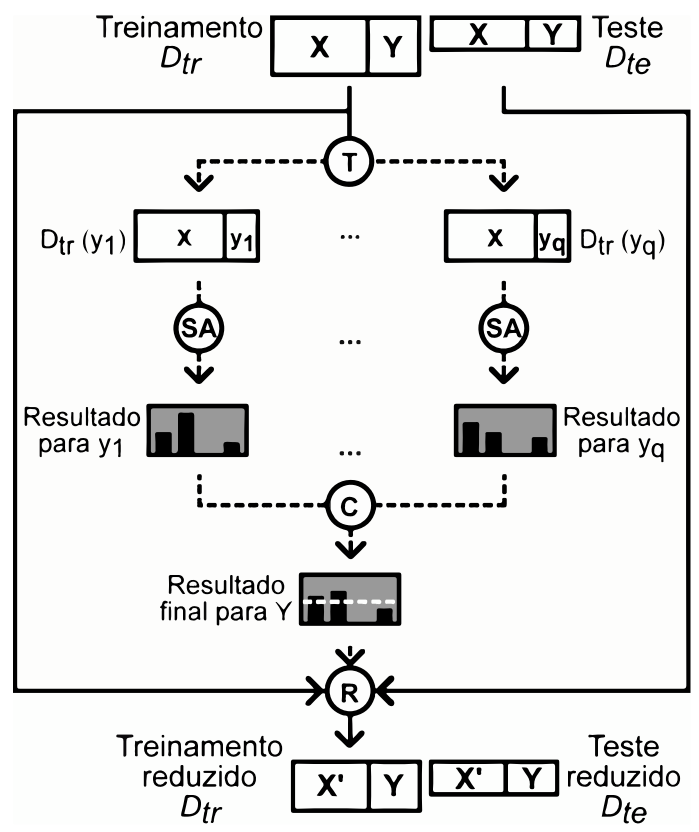

Figura 4.1: Abordagem Binary Relevance para seleção de atributos

$B R$ apresenta complexidade linear em termos do número de rótulos simples $q=|L|$. Assim, essa abordagem é apropriada para um número $q$ não elevado. Porém, uma desvantagem inerente consiste na desconsideração de relações entre rótulos durante a seleção de atributos. Além disso, o algoritmo de SA necessita ser aplicado $q$ vezes, uma por problema monorrótulo.

Diferentes estratégias para combinar resultados de SA monorrótulo têm sido propostas. Como parte deste trabalho, quatro estratégias que combinam o valor de importância de cada atributo estimado em relação a cada rótulo foram avaliadas em 20 conjuntos de dados textuais multirrótulo (Spolaôr e Tsoumakas, 2013).

Seja $v_{j l}$ o valor de importância do atributo $X_{j}$ estimado por um algoritmo de SA monorrótulo para os dados correspondentes ao rótulo simples $y_{l}, l=1 . . q$. As estratégias avaliadas experimentalmente utilizam os valores $v_{j l}$ de cada atributo $X_{j}$ dos conjuntos de dados binários (monorrótulo) correspondentes para estimar o valor de importância final $v_{j}$ do atributo $X_{j}$ no conjunto de dados multirrótulo - Tabela 4.1. As quatro estratégias avaliadas são:

1. Média: o valor de importância final $v_{j}$ de cada atributo $X_{j}$ é dado pela média dos $q$ valores de importância estimados para $X_{j}$ nos $q$ problemas 
Tabela 4.1: Combinação de resultados da avaliação individual de atributos realizada por um algoritmo de SA monorrótulo para estimar os valores de importância final relacionados a um conjunto de dados multirrótulo

\begin{tabular}{r|rrrr|r}
\hline \hline & \multicolumn{5}{|c}{ Valores de importância } \\
\hline Atributos & $y_{1}$ & $y_{2}$ & $\ldots$ & $y_{q}$ & Final \\
\hline$X_{1}$ & $v_{11}$ & $v_{12}$ & $\ldots$ & $v_{1 q}$ & $v_{1}$ \\
$X_{2}$ & $v_{21}$ & $v_{22}$ & $\ldots$ & $v_{2 q}$ & $v_{2}$ \\
$\vdots$ & $\vdots$ & $\vdots$ & $\vdots$ & $\vdots$ & $\vdots$ \\
$X_{M}$ & $v_{M 1}$ & $v_{M 2}$ & $\ldots$ & $v_{M q}$ & $v_{q}$ \\
\hline \hline
\end{tabular}

monorrótulo, como definido pela Equação 4.1;

$$
v_{j}=\bar{v}_{j}=\frac{1}{q} \sum_{l=1}^{q} v_{j l} .
$$

2. Máximo: o valor de importância final $v_{j}$ de cada atributo $X_{j}$ é dado pelo maior $^{27}$ valor de importância estimado para $X_{j}$ dentre os $q$ problemas monorrótulo, como definido pela Equação 4.2;

$$
v_{j}=\hat{v}_{j}=\max _{1 \leq l \leq q} v_{j l}
$$

3. Round-robin (Forman, 2004): inicialmente, a estratégia ordena os valores de importância $v_{j l}$ relacionados a cada rótulo $y_{l}, l=1 . . q$, e inicia um subconjunto de atributos $X^{\prime \prime}-X^{\prime \prime}=\emptyset$. Após, é iniciado um procedimento iterativo, a partir do rótulo $y_{1}$, com a finalidade de inserir em $X^{\prime \prime}$ o atributo $X_{j}$ correspondente ao melhor valor $v_{j l}$ para o rótulo $y_{l}$, bem como atribuir $v_{j l}$ como o valor final $v_{j}$ de $X_{j}$. Se $v_{j l}$ é o melhor valor para $y_{l}$ e $X_{j} \notin X^{\prime \prime}, v_{j l}$ recebe um valor inválido, $X_{j}$ é inserido em $X^{\prime \prime}$ e o procedimento avança para avaliar os valores vinculados ao rótulo seguinte $-y_{l+1}$. Após investigar os valores até $y_{q}$, o procedimento segue para a próxima iteração, partindo novamente de $y_{1}$. A estratégia é repetida sucessivamente até que $X^{\prime \prime}$ contenha os $M$ atributos e os valores finais de importância correspondentes tenham sido registrados;

4. Rand-robin (Forman, 2004): difere de Round-robin ao estabelecer randomicamente o rótulo $y_{l}$ cujos valores de importância $v_{j l}$ serão investigados em um determinado momento. Em outras palavras, ao invés de percorrer sequencialmente os rótulos $y_{l}, l=1 . . q$, Rand-robin escolhe randomicamente um rótulo por vez para avaliação dos valores correspondentes. A probabilidade de escolha de um rótulo $y_{l}$ é inversamente proporcional à frequência de $y_{l}$, de modo a incentivar a seleção de mais atributos para

\footnotetext{
${ }^{27}$ Se a medida de importância inerente deve ser minimizada, a estratégia Máximo é substituída por Mínimo, a qual atribui a $v_{j}$ o menor valor $v_{j l}$ estimado para $X_{j}$.
} 
rótulos raros. Após investigar os valores do rótulo atual, o procedimento realiza novo sorteio e avalia os valores correspondentes do rótulo recém escolhido. Ao final, $X^{\prime \prime}$ contém $M$ atributos e os valores finais correspondentes são registrados.

De modo geral, os valores finais de importância estabelecidos pelas estratégias tradicionais Média e Máximo contribuíram na construção dos melhores classificadores (Spolaôr e Tsoumakas, 2013). Em particular, Média se destacou quando foram utilizados os menores subconjuntos de atributos para gerar os classificadores, o que é considerado um bom resultado para a tarefa de seleção de atributos.

Devido aos bons resultados obtidos pelas estratégias Média e Máximo, foi considerada a ideia de explorar melhor ambas estratégias levando em conta a distribuição dos valores $v_{j l}, l=1 . . q$, de cada atributo $X_{j}$. Assim, foi proposta uma estratégia adicional para combinar os resultados da SA monorrótulo, denominada agregação parcial. Na agregação parcial, é calculado inicialmente o valor médio $\bar{v}_{j}$ para o atributo $X_{j}$ - Equação $4.1-$, bem como o desvio padrão $\sigma_{j}$ correspondente. Essas informações são utilizadas para separar os valores $v_{j l}$ nas seguintes três regiões:

1. Região $r_{1}$ : contém valores $v_{j l}<\left(\bar{v}_{j}-\sigma_{j}\right)$;

2. Região $r_{2}$ : contém valores $v_{j l}$, tal que $\left(\bar{v}_{j}-\sigma_{j}\right) \leq v_{j l} \leq\left(\bar{v}_{j}+\sigma_{j}\right)$;

3. Região $r_{3}$ : contém valores $v_{j l}>\left(\bar{v}_{j}+\sigma_{j}\right)$.

Adicionalmente, a quantidade de valores nessas regiões, $n_{1}, n_{2}$ e $n_{3}$, respectivamente, $\operatorname{com} n_{1}+n_{2}+n_{3}=q$, é contabilizada.

Após, as técnicas Média e Máximo são aplicadas em conjunto com a estratégia agregação parcial, de modo que o valor de importância $v_{j}$ do atributo $X_{j}$ é dado, respectivamente, pela média ou pelo máximo dos valores na região selecionada de acordo com as seguintes regras:

- Se $n_{1}>\frac{q}{2}$, então $v_{j}$ é a média (ou o máximo) dos $n_{1}$ valores de importância em $r_{1}$;

- Caso contrário, se $n_{2}>\frac{q}{2}$, então $v_{j}$ é a média (ou o máximo) dos $n_{2}$ valores de importância em $r_{2}$;

- Caso contrário, se $n_{3}>\frac{q}{2}$, então $v_{j}$ é a média (ou o máximo) dos $n_{3}$ valores de importância em $r_{3}$;

- Caso contrário, usar o caso default, no qual o valor de importância $v_{j}$ é dado pela média (ou o máximo) dos $q$ valores de importância $v_{j l}$ estimados para o atributo $X_{j}$ nos problemas monorrótulo. Em outras palavras, esse caso usa a estratégia Média ou Máximo original. 
Foram realizados experimentos preliminares para avaliar a estratégia de agregação parcial proposta. Foi observado, por exemplo, que houve vários casos em que a opção default não aconteceu. Porém, os resultados obtidos não foram superiores aos atingidos pelas estratégias originais Média e Máximo. Embora diferentes variáveis influenciem nessa avaliação, acredita-se que um dos motivos pelo qual a agregação parcial não foi bem-sucedida seria a menor representatividade dos valores $v_{j}$, os quais são estimados usando menos de $q$ valores $v_{j l}$. Além disso, em alguns casos, agregação parcial pode ignorar todos os valores de importância $v_{j l}$ calculados para determinados rótulos.

Assim, considerando que Média obteve melhores resultados com a abordagem Binary Relevance (Spolaôr e Tsoumakas, 2013), foi decidido utilizar essa estratégia no desenvolvimento deste trabalho.

\subsubsection{Abordagem Label Powerset}

Como mencionado na Seção 2.2.1, página 15, essa abordagem transforma o problema de aprendizado multirrótulo em um problema multiclasse. Dado um conjunto de treinamento $D_{t r}$ multirrótulo com $p$ multirrótulos diferentes, cada exemplo $E_{i}=\left(\mathbf{x}_{i}, Y_{i}\right), i=1 . . N_{t r}$, é transformado em $E_{i}=\left(\mathbf{x}_{i}, l_{i}\right)$, com $l_{i} \in\left\{l_{1}, l_{2}, \ldots l_{p}\right\}$ o monorrótulo que representa o multirrótulo $Y_{i}$. Após, um algoritmo de seleção de atributos monorrótulo é aplicado uma única vez nos dados transformados.

Diferentemente de $B R, L P$ considera alguma relação entre rótulos. Entretanto, como o número $p$ de rótulos no conjunto de dados multiclasse é dado pelo número de multirrótulos distintos em $D_{t r}$, uma desvantagem de $L P$ é que alguns desses rótulos no conjunto de dados multiclasse podem ser associados a uma quantidade muito pequena de exemplos, o que caracteriza dados desbalanceados. Adicionalmente, os $p$ multirrótulos existentes no conjunto de treinamento $D_{t r}$ não necessariamente refletem todos os multirrótulos possíveis no domínio considerado. Assim, a qualidade dos subconjuntos de atributos selecionados e da predição posterior em aplicações práticas pode ser prejudicada.

\subsection{Método Label Construction for Feature Selection}

Dado um conjunto de treinamento multirrótulo $D_{t r}$ com exemplos rotulados pelo conjunto de rótulos simples $L=\left\{y_{1}, y_{2}, y_{3}, \ldots, y_{q}\right\}$, a ideia principal do LCFS, proposto neste trabalho, é construir $q^{\prime}$ novos rótulos simples ao combinar os rótulos originais em pares $\left(y_{i}, y_{j}\right), i \neq j, y_{i} \in L$ e $y_{j} \in L$. Em cada iteração, LCFS seleciona um par de rótulos $\left(y_{i}, y_{j}\right)$ de $L$ e combina os rótulos nesse par para gerar um novo rótulo $y_{i j}$. Após repetir esse procedimento $q^{\prime}$ 
vezes, os $q^{\prime}$ novos rótulos são incluídos no conjunto de rótulos $L$, tal que informações sobre relações entre rótulos podem ser usadas pela abordagem Binary Relevance para seleção de atributos.

O método LCFS consiste de duas etapas, cada uma direcionada à resolução de uma questão diferente:

1. Seleção: quais pares de rótulos $\left(y_{i}, y_{j}\right)$ deveriam ser escolhidos?

2. Geração: como combinar esses rótulos para gerar os novos rótulos $y_{i j}$ ?

Essas etapas são ilustradas na Figura $4.2 \operatorname{com} q^{\prime}=1$.

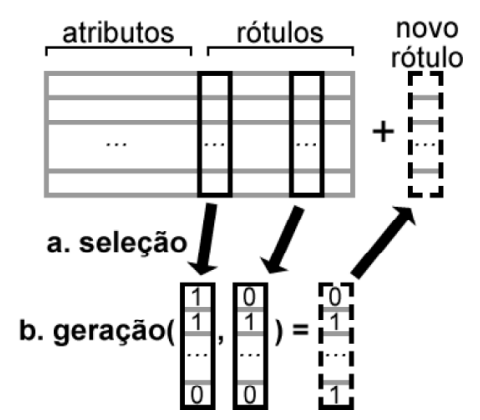

Figura 4.2: Ilustração da aplicação das duas etapas de LCFS para construir $q^{\prime}=1$ novos rótulos

Assim, uma configuração do LCFS é dada pela escolha de uma estratégia para selecionar pares de rótulos e de uma estratégia para combinar os rótulos em cada par selecionado. Um parâmetro adicional consiste no número de novos rótulos $q^{\prime}$ que serão construídos. A seguir, são descritas as duas etapas do método LCFS.

\subsubsection{Etapa 1: Seleção}

Dado o conjunto de rótulos $L=\left\{y_{1}, y_{2}, y_{3}, \ldots, y_{q}\right\}$ do conjunto de treinamento $D_{t r}$, são escolhidos $q^{\prime}$ pares diferentes ${ }^{28}$ de rótulos $\left(y_{i}, y_{j}\right), i \neq j$, de acordo com uma estratégia de seleção. A ideia é que esses pares capturem alguma relação entre os rótulos simples de $D_{t r}$.

LCFS suporta estratégias simples de seleção, tais como a Seleção Randômica $(S R)$, bem como heurísticas baseadas na frequência de rótulos, i.e., no número de exemplos associados a cada rótulo simples original. Duas estratégias que especificamente consideram a frequência de rótulos são a Seleção baseada em Coocorrência $(S C)$ e a Seleção baseada em rótulos relacionados $(S L)$. No $S C$, os pares de rótulos são organizados em ordem decrescente de coocorrência $c_{c}$,

\footnotetext{
${ }^{28}$ Neste trabalho, dois pares de rótulos são considerados diferentes se eles não possuem rótulos em comum. Por exemplo, $\left(y_{1}, y_{2}\right)$ e $\left(y_{1}, y_{3}\right)$ não são considerados diferentes pois compartilham o rótulo $y_{1}$.
} 
i.e., o número de exemplos rotulados por ambos rótulos, permitindo a seleção dos $q^{\prime}$ primeiros pares diferentes. Por outro lado, no SL são contabilizados:

1. O número de exemplos nos quais os rótulos em um par concordam, i.e., são iguais, $c_{e}$; e

2. O número de exemplos nos quais os rótulos em um par discordam, $c_{d}$.

Os pares são então organizados em duas listas, as quais apresentam os pares em ordem decrescente de valores $c_{e}$ e $c_{d}$. Após, é iniciado um procedimento para selecionar o par com o maior valor $-c_{e}$ ou $c_{d}-$, o qual é removido da lista correspondente. Esse procedimento é repetido até que sejam selecionados $q^{\prime}$ pares diferentes por $S L$.

Para ilustrar as estratégias de seleção $S R, S C$ e $S L$, considere o conjunto de dados multirrótulo na Tabela 4.2, com $L=\left\{y_{1}, y_{2}, y_{3}, y_{4}\right\}$, e o número de novos rótulos a serem construídos $q^{\prime}=2$.

Tabela 4.2: Conjunto de dados ilustrativo para o método LCFS

\begin{tabular}{c|cccc}
\hline \hline & $y_{1}$ & $y_{2}$ & $y_{3}$ & $y_{4}$ \\
\hline$E_{1}$ & 1 & 0 & 1 & 0 \\
$E_{2}$ & 1 & 1 & 0 & 0 \\
$E_{3}$ & 0 & 0 & 0 & 1 \\
$E_{4}$ & 1 & 1 & 1 & 0 \\
$E_{5}$ & 1 & 0 & 0 & 1 \\
$E_{6}$ & 1 & 1 & 0 & 1 \\
$E_{7}$ & 0 & 1 & 0 & 1 \\
\hline \hline
\end{tabular}

$S R$ randomicamente seleciona $q^{\prime}=2$ pares diferentes de rótulos. Por outro lado, $S C$ e $S L$ ordenam os pares de rótulos $\left(y_{i}, y_{j}\right)$ em ordem decrescente de acordo com o número de exemplos que verificam uma determinada condição relacionada aos rótulos $y_{i}$ e $y_{j}$.

Dado um par $\left(y_{i}, y_{j}\right), i \neq j$, seja:

- $c_{c}\left(y_{i}, y_{j}\right)$ o número de exemplos rotulados por ambos rótulos, i.e., $(1,1)$;

- $c_{e}\left(y_{i}, y_{j}\right)$ o número de exemplos nos quais os rótulos são iguais, i.e., $(1,1)$ ou $(0,0)$;

- $c_{d}\left(y_{i}, y_{j}\right)$ o número de exemplos nos quais os rótulos são diferentes, i.e., $(1,0)$ ou $(0,1)$.

Os $q^{\prime}=2$ pares de rótulos selecionados e as listas de pares de rótulos ordenadas por SC e $S L$ nas quais os pares selecionados estão em negrito, são descritos a seguir.

SC pares de rótulos selecionados: $\left(\boldsymbol{y}_{1}, \boldsymbol{y}_{2}\right)$ e $\left(\boldsymbol{y}_{3}, \boldsymbol{y}_{4}\right)$

$$
c_{c}\left(\boldsymbol{y}_{\mathbf{1}}, \boldsymbol{y}_{\mathbf{2}}\right)=3, c_{c}\left(y_{1}, y_{3}\right)=2, c_{c}\left(y_{1}, y_{4}\right)=2, c_{c}\left(y_{2}, y_{4}\right)=2, c_{c}\left(y_{2}, y_{3}\right)=1, c_{c}\left(\boldsymbol{y}_{\mathbf{3}}, \boldsymbol{y}_{\mathbf{4}}\right)=0
$$


O par $\left(y_{1}, y_{2}\right)$ é selecionado inicialmente por $S C$, pois possui a coocorrência mais alta. Então essa estratégia procura pelo próximo par na lista ordenada. Como $\left(y_{1}, y_{3}\right)$ não é um par diferente de $\left(y_{1}, y_{2}\right)$ devido ao rótulo comum $y_{1}$, SC procura o próximo elemento da lista, $\left(y_{1}, y_{4}\right)$, e assim sucessivamente até encontrar $\left(y_{3}, y_{4}\right)$, o qual é um par diferente do par $\left(y_{1}, y_{2}\right)$ selecionado previamente. Como $q^{\prime}=2$ pares de rótulos foram selecionados, a execução da estratégia de seleção $S C$ é finalizada.

SL pares de rótulos selecionados: $\left(\boldsymbol{y}_{3}, \boldsymbol{y}_{4}\right)$ e $\left(\boldsymbol{y}_{1}, \boldsymbol{y}_{2}\right)$

$$
\begin{aligned}
& c_{e}\left(\boldsymbol{y}_{1}, \boldsymbol{y}_{2}\right)=4, c_{e}\left(y_{1}, y_{3}\right)=4, c_{e}\left(y_{2}, y_{3}\right)=3, c_{e}\left(y_{2}, y_{4}\right)=3, c_{e}\left(y_{1}, y_{4}\right)=2, c_{e}\left(y_{3}, y_{4}\right)=1 \\
& c_{d}\left(\boldsymbol{y}_{\mathbf{3}}, \boldsymbol{y}_{\mathbf{4}}\right)=6, c_{d}\left(y_{1}, y_{4}\right)=5, c_{d}\left(y_{2}, y_{3}\right)=4, c_{d}\left(y_{2}, y_{4}\right)=4, c_{d}\left(y_{1}, y_{2}\right)=3, c_{d}\left(y_{1}, y_{3}\right)=3
\end{aligned}
$$

Neste caso, as frequências (números de exemplos rotulados) $c_{e}$ e $c_{d}$ relacionadas ao primeiro par de rótulos em cada lista ordenada, $\left(y_{1}, y_{2}\right)$ e $\left(y_{3}, y_{4}\right)$, são comparadas. Como $c_{d}\left(y_{3}, y_{4}\right)>c_{e}\left(y_{1}, y_{2}\right)$, o par $\left(y_{3}, y_{4}\right)$ é selecionado e removido da lista relacionada a $c_{d}$. A estratégia $S L$ procura então o primeiro par de rótulos na lista reordenada de acordo $\operatorname{com} c_{d}$. Entretanto, como o primeiro par nessa lista, $\left(y_{1}, y_{4}\right)$, não é diferente do par selecionado previamente devido ao rótulo comum $y_{4}$, o procedimento avança para o próximo elemento na lista. Uma vez mais, o primeiro elemento dessa lista, $\left(y_{2}, y_{3}\right)$, não é diferente de $\left(y_{3}, y_{4}\right)$. No entanto, como $c_{e}\left(y_{1}, y_{2}\right)=c_{d}\left(y_{2}, y_{3}\right)$, o primeiro elemento da lista ordenada de acordo com $c_{e}$ pode ser considerado. Ao verificar que $\left(y_{1}, y_{2}\right)$ é um par diferente de $\left(y_{3}, y_{4}\right),\left(y_{1}, y_{2}\right)$ é selecionado e removido da lista ordenada por $c_{e}$, resultando no término da estratégia $S L$ com $q^{\prime}=2$ pares de rótulos escolhidos.

Neste exemplo, e com o objetivo de simplificar as explicações na próxima seção, ambas estratégias selecionam os mesmos pares.

\subsubsection{Etapa 2: Geração}

Nesta etapa, ambos rótulos em cada par $\left(y_{i}, y_{j}\right), i \neq j$, selecionado por LCFS são combinados para construir os novos rótulos $y_{i j}$. A ideia é que os valores de $y_{i j}$ representem uma relação entre $y_{i}$ e $y_{j}$. No final da etapa, todos os exemplos em $D_{t r}$ são rotulados pelos $q$ rótulos simples originais e pelos $q^{\prime}$ novos rótulos. Diferentes estratégias de combinação entre variáveis binárias (rótulos) são suportadas por LCFS. Neste trabalho, são utilizados três operadores lógicos simples para gerar os valores dos novos rótulos de cada exemplo em $D_{t r}$. Os operadores lógicos utilizados são:

AND : $y_{i j}=1$ sss $y_{i}=y_{j}=1 ; y_{i j}=0$ caso contrário;

XOR $: y_{i j}=1$ sss $y_{i} \neq y_{j} ; y_{i j}=0$ caso contrário; 
XNOR : $y_{i j}=1$ sss $y_{i}=y_{j} ; y_{i j}=0$ caso contrário.

Para exemplificar, considere os $q^{\prime}=2$ pares de rótulos selecionados na Etapa 1, $\left(y_{1}, y_{2}\right)$ e $\left(y_{3}, y_{4}\right)$, e o conjunto de dados original da Tabela 4.2. A partir desses pares, os rótulos $y_{12}$ e $y_{34}$ são gerados por uma das estratégias e agregados ao conjunto de dados original, como exemplificado na Tabela 4.3.

Tabela 4.3: Conjunto de dados ilustrativo para a Etapa 2 do método LCFS

\begin{tabular}{c|cccc||cc|cc|cc}
\cline { 2 - 11 } & \multicolumn{1}{|c|}{ Rótulos originais } & \multicolumn{2}{c|}{ AND } & \multicolumn{2}{c|}{ XOR } & \multicolumn{2}{c}{ XNOR } \\
& $y_{1}$ & $y_{2}$ & $y_{3}$ & $y_{4}$ & $y_{12}$ & $y_{34}$ & $y_{12}$ & $y_{34}$ & $y_{12}$ & $y_{34}$ \\
\hline$E_{1}$ & 1 & 0 & 1 & 0 & 0 & 0 & 1 & 1 & 0 & 0 \\
$E_{2}$ & 1 & 1 & 0 & 0 & 1 & 0 & 0 & 0 & 1 & 1 \\
$E_{3}$ & 0 & 0 & 0 & 1 & 0 & 0 & 0 & 1 & 1 & 0 \\
$E_{4}$ & 1 & 1 & 1 & 0 & 1 & 0 & 0 & 1 & 1 & 0 \\
$E_{5}$ & 1 & 0 & 0 & 1 & 0 & 0 & 1 & 1 & 0 & 0 \\
$E_{6}$ & 1 & 1 & 0 & 1 & 1 & 0 & 0 & 1 & 1 & 0 \\
$E_{7}$ & 0 & 1 & 0 & 1 & 0 & 0 & 1 & 1 & 0 & 0 \\
\hline \hline
\end{tabular}

Note que cada rótulo $y_{i j}$ gerado por meio do operador AND possui valor 1 nos exemplos em que há coocorrência de rótulos no par $\left(y_{i}, y_{j}\right)$, i.e., quando ambos são 1 , de modo que há uma correspondência entre AND e a estratégia $S C$, bem como um destaque para rótulos coocorrentes. Há também correspondência entre os operadores XOR/XNOR e a estratégia $S L$. De fato, por meio de XNOR, também conhecido como função coincidência, o valor 1 é atribuído para $y_{i j}$ sss os rótulos $y_{i}$ e $y_{j}$ concordam, enquanto o oposto ocorre com XOR. Essas correspondências são exploradas na Seção 4.4 para definir configurações do método LCFS utilizadas nos experimentos realizados neste capítulo.

Após gerar os $q^{\prime}$ novos rótulos, a abordagem tradicional para seleção de atributos $B R$ pode ser aplicada para um conjunto de dados rotulado por $q+q^{\prime}$ rótulos. Note que, ao combinar BR com LCFS, qualquer algoritmo monorrótulo de SA pode ser aplicado ao conjunto de dados expandido com informação de segunda ordem de rótulos (Zhang e Zhou, 2013).

Convém ressaltar que a complexidade do método LCFS é similar à de $B R \mathrm{e}$ depende da configuração adotada. Configurações baseadas na estratégia $S R$, por exemplo, possuem um custo mais baixo para a etapa de seleção que as configurações baseadas na seleção informada de pares de rótulos. Além disso, os operadores mencionados anteriormente para a etapa de geração são pouco custosos, apresentando complexidade linear em termos do número de novos rótulos $q^{\prime}$.

\subsection{Medidas de Importância de Atributos Utilizadas}

Os métodos de SA considerados nos experimentos estimam a importância dos atributos por meio de um dos seguintes algoritmos de ranking de atributos 
monorrótulo, i.e., de avaliação individual de atributos monorrótulo:

- Ganho de informação - IG;

- ReliefF.

De acordo com a revisão sistemática da literatura realizada neste trabalho - Seção 3.3, página 44 -, a medida de importância ganho de informação é uma das mais utilizadas nas publicações relacionadas. Um dos motivos é que essa medida também tem mostrado bom desempenho na seleção de atributos monorrótulo. A medida IG é estritamente univariada, avaliando isoladamente cada atributo $X_{j}, j=1 \ldots M$, de acordo com a dependência entre ele e um rótulo, como definido pela Equação 4.3. Para tanto, é calculada a diferença entre a entropia ${ }^{29}$ do conjunto de treinamento $D_{t r}$ e a soma ponderada da entropia de cada subconjunto $D_{t r_{v}} \subseteq D_{t r}$, em que $D_{t r_{v}}$ consiste no subconjunto de exemplos nos quais $X_{j}$ possui o valor $v$. Assim, por exemplo, se $X_{j}$ possuir $v=10$ valores distintos em $D_{t r}$, a soma é aplicada a 10 subconjuntos $D_{t r_{v}}$.

$$
I G\left(D_{t r}, X_{j}\right)=\operatorname{entropia}\left(D_{t r}\right)-\sum_{v} \frac{\left|D_{t r_{v}}\right| \text { entropia }\left(D_{t r_{v}}\right)}{\left|D_{t r}\right|} .
$$

Quanto maior o valor calculado para um atributo $X_{j}$, mais forte é a dependência entre $X_{j}$ e o rótulo. Para lidar com atributos contínuos, a medida IG necessita uma técnica de discretização. Neste trabalho, é utilizada a opção default do framework Weka ${ }^{30}$ (Witten e Frank, 2011) para SA monorrótulo com essa medida: discretização minimum description length (Fayyad e Irani, 1993).

Por outro lado, a ideia geral da medida de importância $R F$, inerente ao algoritmo ReliefF e a outros de sua família, é recompensar um atributo que possui valores diferentes em exemplos (vizinhos) próximos de classes distintas, bem como penalizar um atributo que possui valores diferentes em vizinhos próximos de mesma classe (Demšar, 2010). No caso binário e com $k=1$ vizinho mais próximo, essa ideia pode ser expressa da seguinte maneira. Sejam $A$ e $S$, respectivamente, os vizinhos mais próximos de mesma classe e de classe distinta a um exemplo sob investigação $E$. Seja $O_{d X}$ o evento relacionado à ocorrência de um valor para o atributo $X_{j}$ que seja diferente para $E$ e um vizinho próximo. A importância do atributo $X_{j}$ nesse cenário pode ser aproximada pela diferença entre as probabilidades condicionais definidas pela Equação 4.4 .

\footnotetext{
${ }^{29}$ Incerteza inerente aos dados.

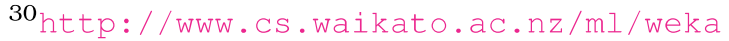




$$
R F\left(X_{j}\right)=P\left(O_{d X} \mid S\right)-P\left(O_{d X} \mid A\right) .
$$

Assim, a probabilidade $P\left(O_{d X} \mid S\right)$ deveria ser maximizada para melhorar a separabilidade entre rótulos, enquanto a probabilidade $P\left(O_{d X} \mid A\right)$ deveria ser minimizada para que exemplos com o mesmo rótulo estejam próximos. Quanto maior o valor de RF, maior a importância do atributo $X_{j}$ para o algoritmo. Os algoritmos da família Relief, incluindo ReliefF, são descritos no Capítulo 5.

Duas vantagens de ReliefF em relação a IG consistem na consideração da interação entre atributos, pois todos os atributos são utilizados para buscas pelos vizinhos mais próximos (Moore e White, 2007), bem como na dispensa de discretização dos dados. Em particular, o fato de ReliefF trabalhar diretamente com atributos categóricos e contínuos é uma vantagem importante, pois a necessidade de discretizar os dados - caso de IG - inclui uma nova variável que é o método de discretização adotado. Além disso, a mesma discretização necessita ser realizada no conjunto de teste, o que pode gerar problemas adicionais em aplicações práticas.

\subsection{Configuração Experimental}

A avaliação experimental realizada consiste na comparação de métodos para seleção de atributos multirrótulo baseada em transformação do problema organizados em dois grupos:

1. Algoritmos de SA baseados nas abordagens tradicionais $B R$ e $L P$;

2. Configurações do método LCFS, proposto neste trabalho.

A partir dos resultados obtidos, foi realizada uma comparação adicional entre o melhor representante de cada grupo.

Os métodos de SA considerados estimam a importância dos atributos por meio de uma das duas medidas monorrótulo IG e $R F$. A partir do ranking final de atributos, os subconjuntos dos melhores atributos $X^{\prime} \subset X,\left|X^{\prime}\right|=$ $10 \% M, 20 \% M, \ldots, 90 \% M$ foram determinados. Esses nove subconjuntos foram usados para descrever versões reduzidas do conjunto de dados multirrótulo original, as quais foram submetidas a um algoritmo de aprendizado multirrótulo para avaliar a qualidade dos atributos selecionados em cada subconjunto em termos de desempenho de classificação.

O algoritmo de aprendizado escolhido é o BRkNN-b, descrito na Seção 2.2.2, página 19. Como algoritmos lazy são suscetíveis a atributos irrelevantes, 
$B R k N N$-b é uma boa alternativa para a avaliação de métodos de seleção de atributos.

O principal parâmetro relacionado aos algoritmos $B R k N N-b$ e ReliefF é o número de vizinhos próximos. Neste trabalho, optou-se por fixar $k=10$ para ambos, seguindo o valor adotado por default no framework Weka para ReliefF. Convém ressaltar, entretanto, que outros valores de $k$ têm sido utilizados como parte deste trabalho (Spolaôr e Monard, 2014; Spolaôr et al., 2014b). Algumas configurações específicas de parâmetros são relatadas nas seções seguintes, enquanto que os demais parâmetros são usados com valores default de Weka (Witten e Frank, 2011) e Mulan (Tsoumakas et al., 2011). Esses frameworks implementam BR, LP, BRkNN-b, IG e ReliefF. O método LCFS proposto neste trabalho é baseado em ambos frameworks e está disponível à comunidade em http://www. labic.icmc.usp.br/pub/mcmonard/ Implementations/Multilabel/lcfs.zip.

Os 10 conjuntos de dados benchmark caracterizados na Seção 2.5, página 32, são considerados nos experimentos descritos neste capítulo. Em todos os experimentos, o desempenho dos classificadores foi estimado por meio das medidas de avaliação F-Measure, Hamming Loss, Accuracy e microaveraged $F$-measure $\left(F_{b}\right)$, descritas na Seção 2.3, página 21. Esse desempenho é estimado por meio da estratégia de validação cruzada de 10 folds com folds pareados.

\subsubsection{Configurações Específicas das Abordagens Tradicionais}

Inicialmente, um ranking de atributos é gerado no procedimento correspondente à seleção de atributos para cada um dos $q$ problemas binários decompostos por BR - Seção 4.1.1. No procedimento de seleção de atributos, a ordenação dos atributos em cada ranking é realizada de acordo com o valor de importância estimado por uma das medidas consideradas - IG ou $R F$. Em seguida, no procedimento de combinação, os $q$ resultados gerados por $B R$ são agregados em um único ranking por meio da estratégia Média, a qual apresentou os melhores resultados em experimentos anteriores (Spolaôr e Tsoumakas, 2013). Adicionalmente, são definidas nove quantidades de atributos para estabelecer um subconjunto $X^{\prime} \subset X:\left|X^{\prime}\right|=10 \% M, 20 \% M, \ldots, 90 \% M$. Cada quantidade de atributos escolhida origina um conjunto de treinamento e de teste reduzido e distinto, os quais são submetidos ao algoritmo de aprendizado $B R k N N-b$ com o objetivo de avaliar a qualidade dos atributos selecionados.

A abordagem $L P$ foi aplicada como mencionado na Seção 4.1.2, de modo que o ranking de atributos gerado a partir dos dados multiclasse usando ganho de informação ou ReliefF serve como base para a definição dos nove subconjuntos dos melhores atributos e a consequente redução dos conjuntos de 
dados multirrótulo originais.

A combinação entre as abordagens $B R$ e $L P$ e as medidas de importância IG e $R F$ resultam em quatro métodos de seleção de atributos:

1. Ganho de informação baseado na abordagem $L P(I G-L P)$;

2. ReliefF baseado na abordagem $L P(R F-L P)$;

3. Ganho de informação baseado na abordagem $B R(I G-B R)$;

4. ReliefF baseado na abordagem $B R(R F-B R)$.

A Seleção de Atributos Randômica (SAR), incluída como referência experimental, não necessita abordagem de transformação do problema multirrótulo. Devido a sua natureza estocástica, são realizadas três execuções da SAR. Em cada execução, cada uma das nove quantidades de atributos é utilizada para definir um conjuntos de dados reduzido, o qual é submetido ao $B R k N N-b$ para classificação. Os resultados finais do SAR, em termos de desempenho preditivo, consistem na média do desempenho dos classificadores gerados nas três execuções.

\subsubsection{Configurações Específicas do Label Construction for Fea- ture Selection}

LCFS é aplicado antes da abordagem BR. As configurações relacionadas a $B R$ são as mesmas descritas na Seção 4.4.1. Com relação a LCFS, quatro configurações combinando diferentes estratégias de Seleção (S) e de Geração (G) são determinadas na Tabela 4.4 .

Tabela 4.4: Configurações do método Label Construction for Feature Selection avaliadas neste trabalho

\begin{tabular}{r|l|l}
\hline \hline Configuração & S & G \\
\hline Seleção baseada em rótulos relacionados e operadores XNOR e XOR (SL-X) & SL & XOR ou XNOR é escolhido a partir de listas \\
Seleção baseada em Coocorrencia e operador AND (SC-A) & SC & AND \\
Seleção Randômica e operador AND (SR-A) & SR & AND \\
Seleção Randômica e operadores XOR e XNOR (SR-X) & SR & XOR ou XNOR é randomicamente escolhido \\
\hline \hline
\end{tabular}

Convém ressaltar que a estratégia $S L$ ordena os pares de rótulos a partir dos valores de $c_{e}$ e $c_{d}$ - Seção 4.2.1. Para um determinado par de rótulos $\left(y_{i}, y_{j}\right)$, a configuração $S L-X$ aplica o operador XNOR para gerar o novo rótulo simples $y_{i j}$ se o par foi selecionado da lista ordenada por $c_{e}$ - número de exemplos nos quais os rótulos em um par concordam. Caso contrário, o operador XOR é aplicado, pois a lista foi ordenada de acordo com o número de exemplos em que os rótulos discordam $\left(c_{d}\right)$. Além de $S L-X, S C-A$ também explora uma correspondência entre estratégias de seleção e de geração, pois tanto SC quanto o operador AND valorizam a coocorrência de rótulos em um par $\left(y_{i}, y_{j}\right)$. 
Por fim, SR-X elege randomicamente XOR ou XNOR para determinar cada rótulo na etapa de geração.

O parâmetro $q^{\prime}$, responsável por estabelecer a quantidade de novos rótulos que serão construídos, é definido como $q^{\prime}=\left\lfloor\frac{q}{2}\right\rfloor$, i.e., todo rótulo simples é considerado uma vez se $q$ é par, ou um rótulo simples é ignorado se $q$ é ímpar.

Devido à estocasticidade da estratégia $S R$, são realizadas três execuções de $S R-A$ e $S R-X$. Do mesmo modo que SAR, os resultados correspondentes a $S R-A$ e $S R-X$, em termos de desempenho preditivo, consistem na média do desempenho dos classificadores $B R k N N-b$ gerados nas três execuções.

A taxonomia para SA multirrótulo descrita na Seção 3.1, página 38, permite classificar os métodos informados que compõem a avaliação experimental como representantes da abordagem filtro para interação com o classificador. As demais dimensões dessa taxonomia são apresentadas na Tabela 4.5 especificamente para cada método.

Tabela 4.5: Categorização dos métodos de seleção de atributos multirrótulo baseados em transformação do problema avaliados neste trabalho de acordo com a taxonomia proposta neste trabalho - Seção 3.1

\begin{tabular}{r|rrr}
\hline \hline & escopo do multirrótulo & dependência de rótulos & medida de importância \\
\hline$I G-L P$ & híbrido & alta ordem & ganho de informação \\
$R F-L P$ & híbrido & alta ordem & $R F$ \\
$I G-B R$ & monorrótulo & primeira ordem & ganho de informação \\
$R F-B R$ & monorrótulo & primeira ordem & $R F$ \\
$L C F S$ & monorrótulo & segunda ordem & ganho de informação \\
\hline \hline
\end{tabular}

\subsection{Resultados e Discussão}

Como mencionado, dois grupos de métodos para seleção de atributos são avaliados neste capítulo. Inicialmente, são comparados os classificadores $B R k N N-b$ construídos com os atributos identificados por cinco algoritmos de seleção de atributos multirrótulo: $I G-L P, R F-L P, I G-B R, R F-B R$ e SAR. Após, são comparados os classificadores $B R k N N-b$ resultantes de quatro configurações do método LCFS: $S L-X, S C-A, S R-A$ e $S R-X$. Ao final, é realizada uma comparação adicional entre o melhor representante dos dois grupos.

\subsubsection{Abordagens Tradicionais de Transformação do Problema}

Nas Tabelas A.4 a A.7 do Apêndice, páginas 113 a 118, são descritos os resultados experimentais em termos de cada medida de avaliação considerada. Em cada célula dessas tabelas, são apresentados três valores relacionados a um classificador no formato:

desempenho médio (desvio padrão) [ranking do classificador] 
Adicionalmente, é destacado em negrito o melhor desempenho por conjunto de dados. Células contendo resultados piores que os de Geral $_{B}$ - Tabela A.2 do Apêndice -, são coloridas em cinza. Na Figura 4.3 são apresentados graficamente os valores de $F$-Measure ${ }^{31}$ obtidos para cada quantidade de atributos $\left|X^{\prime}\right|$. Em particular, cada eixo corresponde a um conjunto de dados, enquanto cada linha colorida consiste no desempenho dos classificadores construídos usando atributos escolhidos por um algoritmo de seleção de atributos. Como F-Measure deve ser maximizada, os melhores resultados são os situados mais longe do centro e da linha tracejada (método SAR). Note que os resultados do conjunto de dados 6-Genbase* não são incluídos nos gráficos, pois apresentam pouca variação em geral. Uma possível explicação consiste na baixa dissimilaridade entre exemplos desse conjunto de dados, o que pode ser ilustrado por meio do dendrograma ${ }^{32}$ da Figura A.4 do Apêndice, página 131.

Em geral, a diferença de desempenho dos classificadores gerados para cada quantidade de atributos é pequena. Algumas das exceções mais notáveis ocorrem no conjunto de dados 8-Magtag5k. Na Figura 4.4 são mostrados os desempenhos dos classificadores (eixo $y$ ) gerados para cada quantidade de atributos (eixo $x$ ) nesse conjunto de dados. No gráfico correspondente à medida Hamming Loss, é adicionada uma linha em ciano para representar os resultados do $\mathrm{Geral}_{B}$ - Tabela A.2 do Apêndice, página 112 -, pois essa é a única medida de avaliação com resultados piores que o baseline para 8-Magtag5k. Além disso, essa medida tem a menor variação de valores - precisão de três casas decimais. Note que os resultados relacionados à SAR estão marcados com cor magenta e tipo de linha distinto.

$O$ algoritmo IG-LP atinge alguns dos melhores resultados nesse conjunto. Em geral, à medida que o número de atributos aumenta, há uma melhoria e um distanciamento maior do baseline $\operatorname{Geral}_{B}$ até um determinado ponto, a partir do qual aspectos como a inclusão de atributos ruins podem levar a uma degradação de desempenho. De fato, a tarefa de seleção de atributos é frequentemente relacionada ao compromisso (tradeoff) entre desempenho de aprendizado e número de atributos.

A dificuldade em selecionar atributos para construir classificadores BRkNN$b$ melhores que Geral $_{B}$ em termos da medida Hamming Loss vai além do conjunto de dados 8-Magtag5k. É possível observar na Tabela A.5 do Apêndice, página 115, mais cinco conjuntos nessa situação: 1-Cal500, 2-Corel5k, 3Corel16k001, 5-Fapesp e 7-Llog-f*. Convém ressaltar em favor dos algoritmos de SA que esses cinco conjuntos de dados são relativamente difíceis de apren-

\footnotetext{
${ }^{31}$ Os gráficos das outras medidas de avaliação consideradas são apresentados nas Figuras A. 1 a A.3 do Apêndice, páginas 128 a 130.

${ }^{32}$ Representação gráfica do agrupamento hierárquico de exemplos baseada na dissimilaridade no espaço de atributos.
} 

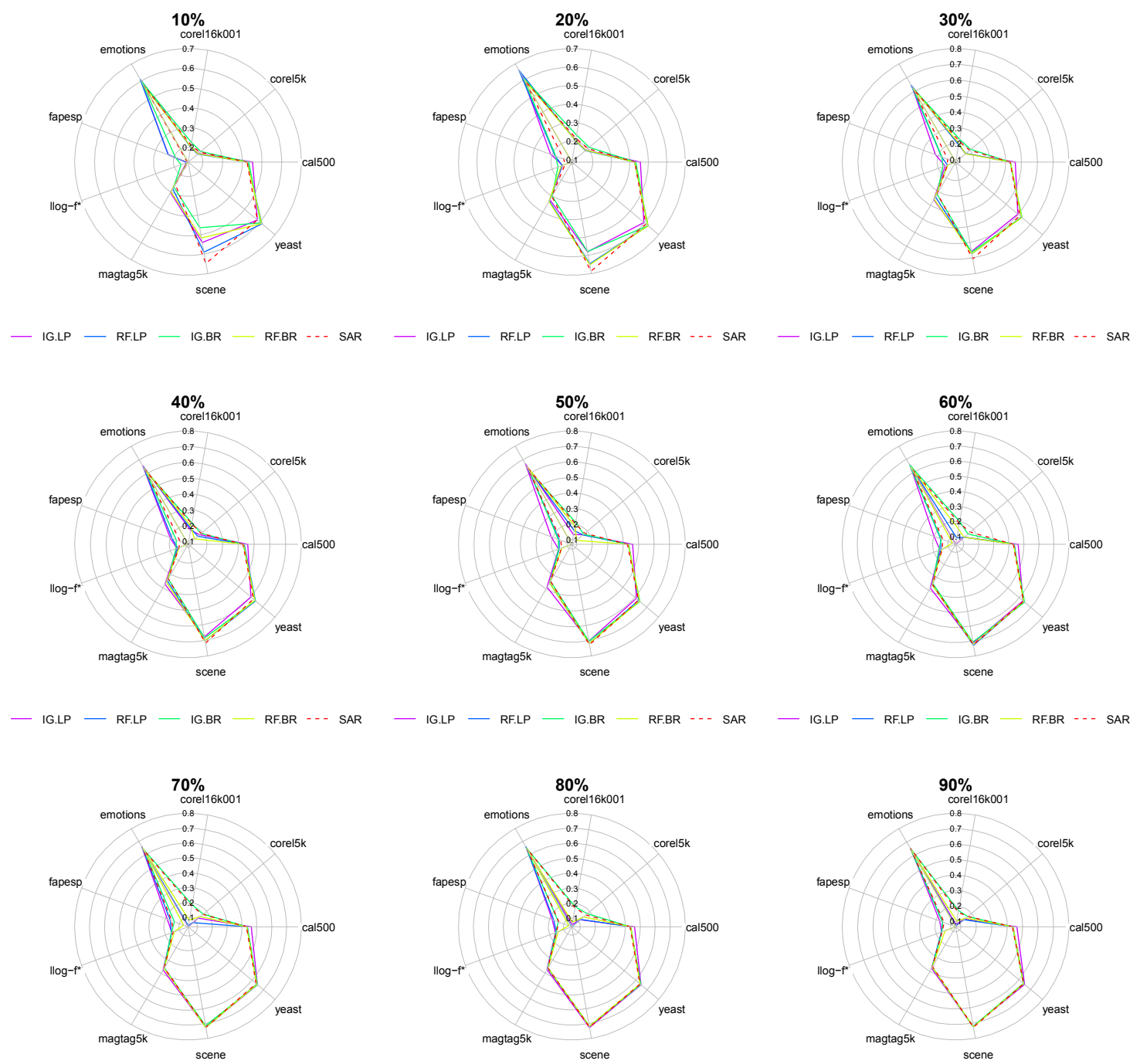

- IG.LP - RF.LP — IG.BR - RF.BR -.. SAR

- IG.LP - RF.LP - IG.BR - RF.BR - - SAR

- IG.LP - RF.LP - IG.BR - RF.BR - . SAR

Figura 4.3: Desempenho de algoritmos de SA em termos dos valores de FMeasure obtidos pelo algoritmo de classificação $B R k N N-b$ 


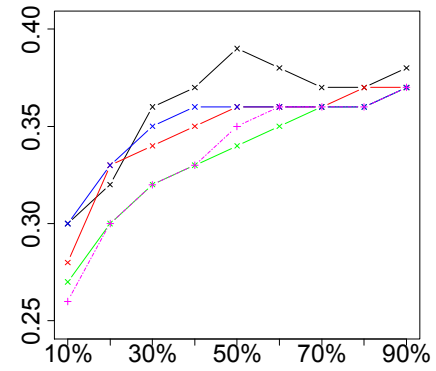

\#1 F-Measure

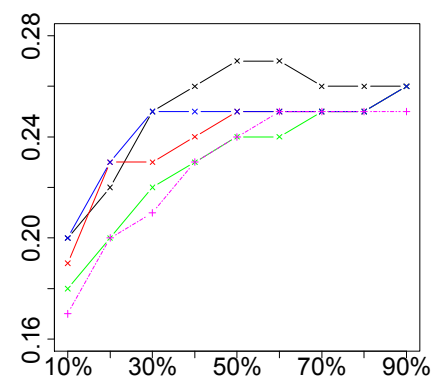

\#3 Accuracy

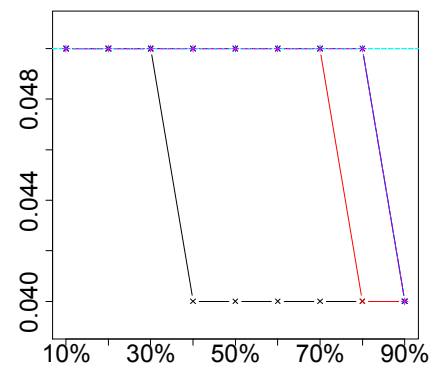

\#2 Hamming Loss

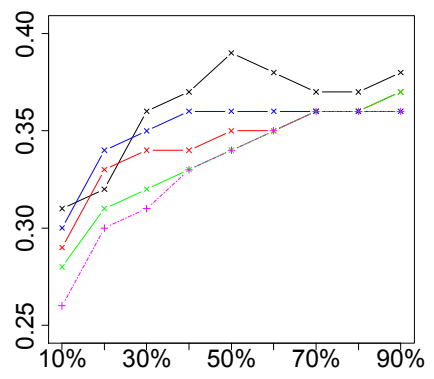

\#4 $F_{b}$

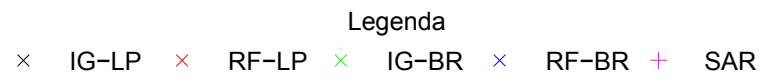

Figura 4.4: Desempenho de algoritmos de SA em termos dos valores das quatro medidas de avaliação obtidos pelo algoritmo de classificação BRkNN-b no conjunto de dados 8-Magtag5k 
der com o algoritmo $B R k N N-b$. De fato, nem mesmo o uso de todos os atributos para construir os classificadores $B R k N N$-b é suficiente para obter valor de Hamming Loss melhor que o de $\mathrm{Geral}_{B}$, como descrito nas células coloridas em cinza na Tabela A.3 do Apêndice, página 113.

Como mencionado, a diferença de desempenho dos classificadores gerados para cada quantidade de atributos é pequena. De fato, ao aplicar o teste estatístico não paramétrico de Friedman (Demšar, 2006; Friedman, 1940), com a hipótese nula de que o desempenho dos classificadores construídos usando os atributos escolhidos pelos cinco algoritmos de SA é equivalente, não foi encontrada diferença significativa, i.e., não foi possível rejeitar a hipótese com nivel de significância $\alpha=0,05$.

Embora não tenha sido encontrada diferença significativa nesse teste, os rankings médios dos classificadores calculados no teste possibilitam identificar qual foi o melhor algoritmo de SA dentre os 10 conjuntos de dados considerados, como representado na Tabela 4.6 para cada quantidade de atributos $\left|X^{\prime}\right|$ e cada medida de avaliação. Note que em algumas células da tabela há mais de um algoritmo de SA, pois os rankings médios atingidos pelos classificadores correspondentes são iguais.

Tabela 4.6: Melhores rankings médios atingidos pelo algoritmo de classificação $B R k N N-b$ para cada quantidade de atributos $\left|X^{\prime}\right|$ selecionada pelos algoritmos de SA e para cada medida de avaliação

\begin{tabular}{|c|c|c|c|c|c|c|c|c|c|c|c|c|c|c|}
\hline & $10 \% M$ & $20 \% M$ & $30 \% M$ & $40 \% M$ & $50 \% M$ & $60 \% M$ & $70 \% M$ & $80 \% M$ & $90 \% M$ & IG-LP & RF-LP & IG-BR & RF-BR & SAR \\
\hline FM & $\begin{array}{l}R F-L P \\
I G-B R\end{array}$ & $I G-B R$ & $I G-L P$ & $I G-L P$ & $I G-L P$ & $I G-B R$ & $I G-L P$ & $I G-L P$ & $I G-L P$ & 6 & 1 & 3 & 0 & 0 \\
\hline$H L$ & $R F-L P$ & $R F-L P$ & $I G-B R$ & $R F-L P$ & $I G-L P$ & $\begin{array}{l}R F-L P \\
I G-B R\end{array}$ & $I G-B R$ & $I G-B R$ & $I G-B R$ & 1 & 4 & 5 & 0 & 0 \\
\hline$A C$ & $I G-L P$ & $I G-B R$ & $I G-L P$ & $R F-L P$ & $I G-L P$ & $\begin{array}{l}R F-L P \\
I G-B R\end{array}$ & $I G-L P$ & $I G-L P$ & $I G-L P$ & 6 & 2 & 2 & 0 & 0 \\
\hline$F_{b}$ & $I G-L P$ & $I G-B R$ & IG-BR & $I G-L P$ & $I G-L P$ & $I G-L P$ & $I G-L P$ & $I G-L P$ & $I G-L P$ & 7 & 0 & 2 & 0 & 0 \\
\hline$\overline{I I G-L P}$ & $\overline{2}$ & $\overline{0}$ & $\overline{2}$ & 2 & $\overline{4}$ & $\overline{1}$ & 3 & 3 & 3 & 20 & & & & \\
\hline$R F-L P$ & 2 & 1 & 0 & 2 & 0 & 2 & 0 & 0 & 0 & & 7 & & & \\
\hline$I G-B R$ & 1 & 3 & 2 & 0 & 0 & 3 & 1 & 1 & 1 & & & 12 & & \\
\hline$R F-B R$ & 0 & 0 & 0 & 0 & 0 & 0 & 0 & 0 & 0 & & & & 0 & \\
\hline SAR & 0 & 0 & 0 & 0 & 0 & 0 & 0 & 0 & 0 & & & & & 0 \\
\hline
\end{tabular}

Com relação aos melhores rankings médios, o algoritmo IG-LP é visivelmente melhor na maioria dos casos, com destaque para os resultados correspondentes às medidas F-Measure (6 casos), Accuracy (6 casos) e microaveraged F-measure ( 7 casos). Convém ressaltar também que IG-LP é o melhor nas quatro medidas de avaliação quando a metade dos atributos $-\left|X^{\prime}\right|=$ $50 \% M$ - é considerada, além de se destacar para $\left|X^{\prime}\right|=70 \% M, 80 \% M, 90 \% M$. Adicionalmente, é possível observar na Tabela A.5 do Apêndice, página 115, que esse algoritmo contribui para obter a menor quantidade de classificadores $B R k N N-b$ piores que Geral $_{B}$ em termos da medida Hamming Loss.

$O$ algoritmo IG-BR também obtém algum destaque na Tabela 4.6, especialmente na medida Hamming Loss para $\left|X^{\prime}\right|=20 \% M$ e $60 \% M$. Ao considerar 
os resultados relacionados a $\mathrm{Geral}_{B}$, é possível notar que IG-BR contribui para obter a menor quantidade de classificadores $B R k N N-b$ piores que o baseline em termos das medidas F-Measure, Accuracy e $F_{b}$.

É importante notar que nenhum classificador construído usando os atributos identificados pela seleção randômica (SAR) atinge o melhor ranking médio. Adicionalmente, vários classificadores relacionados a SAR apresentam desempenho ruim. Entretanto, esse método obteve destaque no conjunto de dados 9-Scene, independentemente da medida de avaliação. Isso pode ser um ponto de partida para estudo sobre propriedades desse conjunto de dados com alguma influência na qualidade da seleção de atributos informada.

Ainda que não há diferença significativa entre todos os algoritmos de SA considerados, IG-LP e IG-BR tiveram algum destaque, o que leva à seguinte questão:

\section{Qual é o melhor algoritmo: IG-LP ou IG-BR?}

Com o intuito de responder essa questão, foi aplicado o teste estatístico não paramétrico de Wilcoxon signed-ranks (Demšar, 2006; Wilcoxon, 1945) especificamente para a comparação entre os dois algoritmos. No entanto, não foi encontrada diferença significativa com $\alpha=0,05$. Logo, a melhor resposta para a questão, baseada nos resultados apresentados, é dada pela análise dos melhores rankings médios calculados no teste de Friedman e apresentados na Tabela 4.6, na qual IG-LP sobressai na maioria dos casos.

\subsubsection{Label Construction for Feature Selection}

Os resultados apresentados na seção anterior são úteis como uma base para a discussão dos experimentos abordados a seguir. Em particular, o método LCFS é investigado em relação à sua capacidade de incrementar a competitividade de $I G-B R$ em relação a $I G-L P$.

Além das quatro configurações do método LCFS, o algoritmo de SA IG-BR é incluído na comparação apresentada a seguir. Convém lembrar que, após a execução de cada configuração, o algoritmo IG-BR é executado nos conjuntos de dados expandidos com $q+q^{\prime}$ rótulos.

Nas Tabelas A.8 a A.11 do Apêndice, páginas 120 a 126, são descritos os resultados experimentais em termos de cada medida de avaliação considerada. Em cada célula dessas tabelas, são apresentados três valores relacionados a um classificador no formato:

desempenho médio (desvio padrão) [ranking do classificador]

Adicionalmente, é destacado em negrito o melhor desempenho por conjunto de dados. Células contendo resultados piores que os de Geral $_{B}$ - Tabela A.2 
do Apêndice - , são coloridas em cinza. Para ilustrar graficamente esses resultados, na Figura 4.5 são apresentados os valores de $F$-Measure ${ }^{33}$ obtidos para cada quantidade de atributos $\left|X^{\prime}\right|$. Cada linha colorida consiste no desempenho dos classificadores construídos usando os atributos escolhidos por uma configuração do LCFS. Como F-Measure deve ser maximizada, os melhores resultados são os situados mais longe do centro e da linha tracejada - algoritmo $I G-B R$. Os resultados do conjunto de dados 6-Genbase* não são incluídos nos gráficos, pois também para LCFS apresentam pouca variação em geral - Figura A.4 do Apêndice, página 131.
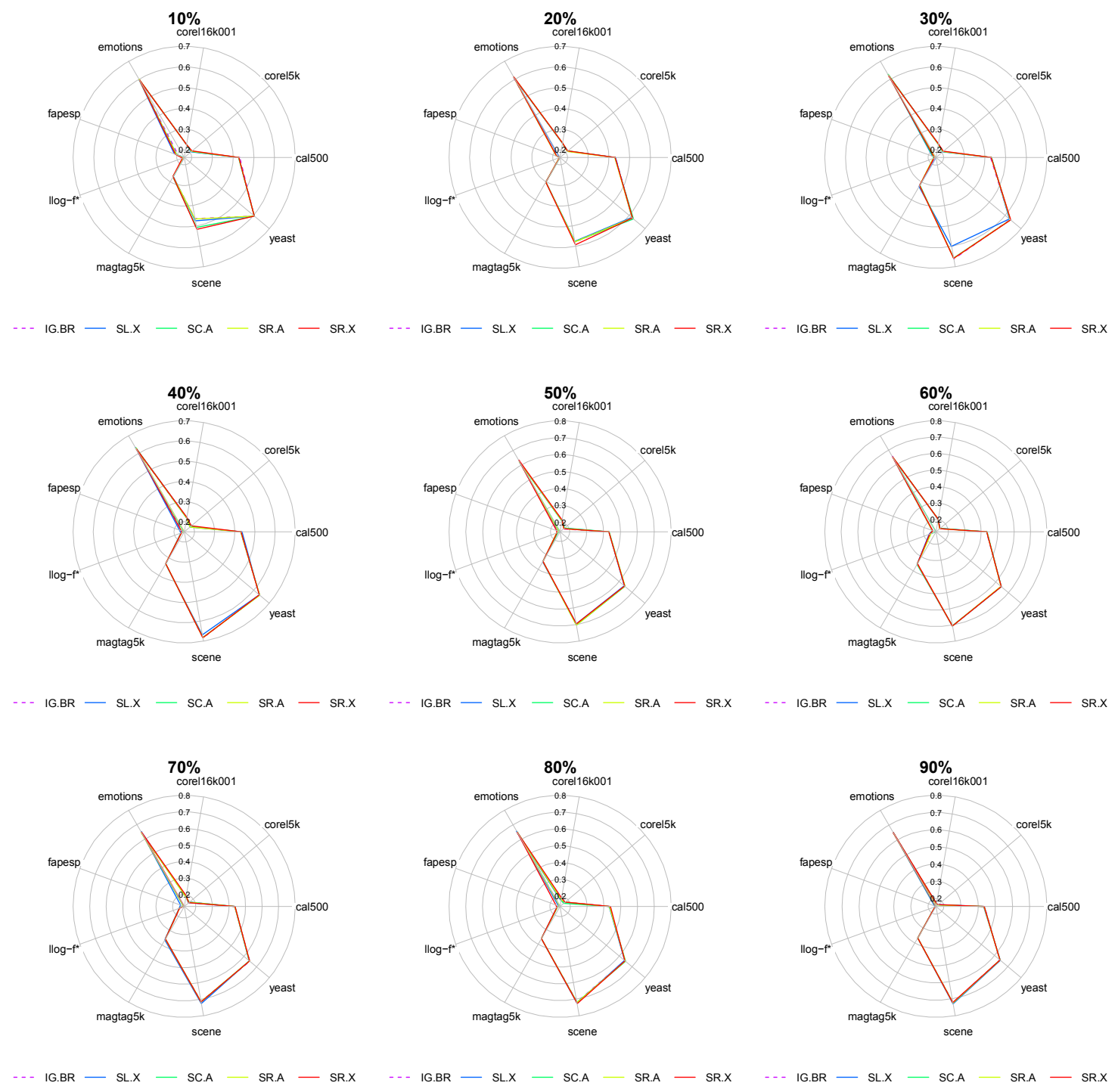

Figura 4.5: Desempenho de configurações do LCFS em termos dos valores de $F-$ Measure obtidos pelo algoritmo de classificação $B R k N N-b$

Assim como observado na Seção 4.5.1, as diferenças no desempenho dos

\footnotetext{
${ }^{33}$ Os gráficos das outras medidas de avaliação consideradas são apresentados nas Figuras A.5 a A.7 do Apêndice, páginas 132 a 134.
} 
classificadores gerados para cada quantidade de atributos é pequena. De fato, ao aplicar o teste de Friedman, não foi encontrada diferença estatística com $\alpha=0,05$.

Ao realizar uma comparação do desempenho dos classificadores resultantes das configurações de LCFS com os resultados do Geral $_{B}$, é possível observar nas Tabelas A.10 e A.11 do Apêndice, páginas 124 e 126, um destaque da configuração $S R-X$ em conjuntos de dados específicos. A partir do subconjunto $\left|X^{\prime}\right|=40 \% M$ selecionado pelo algoritmo IG-BR para o conjunto 2-Corel5k, é gerado um classificador $B R k N N-b$ que é pior que $G_{e r a l}$ nas medidas Accuracy e $F_{b}$. Contudo, ao utilizar o subconjunto selecionado com auxílio de $S R-X$ com a mesma quantidade de atributos, é construído um classificador melhor que o $\mathrm{Geral}_{B}$. Isso também ocorre no conjunto de dados 3-Corel16k001 com $\left|X^{\prime}\right|=90 \% M$. Convém ressaltar que essa é a única configuração do LCFS que leva a alguma melhoria, enquanto as demais atingem resultados similares a IG-BR.

Como realizado na Seção 4.5.1, os rankings médios dos classificadores calculados no teste de Friedman são representados na Tabela 4.7 para cada quantidade de atributos $\left|X^{\prime}\right|$.

Tabela 4.7: Melhores rankings médios atingidos pelo algoritmo de classificação $B R k N N-b$ para cada quantidade de atributos $\left|X^{\prime}\right|$ selecionada pelas configurações do método LCFS e para cada medida de avaliação

\begin{tabular}{|c|c|c|c|c|c|c|c|c|c|c|c|c|c|c|}
\hline & $10 \% M$ & $20 \% M$ & $30 \% M$ & $40 \% M$ & $50 \% M$ & $60 \% M$ & $70 \% M$ & $80 \% M$ & $90 \% M$ & $I G-B R$ & SL-X & $\overline{S C-A}$ & SR-A & SR-X \\
\hline FM & $S L-X$ & $S R-X$ & $S C-A$ & $\begin{array}{l}S L-X \\
S R-X\end{array}$ & $S L-X$ & $I G-B R$ & $S L-X$ & $S R-X$ & $\begin{array}{l}I G-B R \\
S L-X \\
S C-A \\
S R-X\end{array}$ & 2 & 5 & 2 & 0 & 4 \\
\hline$H L$ & $\begin{array}{l}\text { SC-A } \\
\text { SR-X }\end{array}$ & $S R-X$ & $\begin{array}{l}I G-B R \\
S C-A \\
S R-A\end{array}$ & $\begin{array}{l}I G-B R \\
S L-X \\
S C-A \\
S R-A \\
S R-X\end{array}$ & $\begin{array}{l}I G-B R \\
S C-A \\
\text { SR-A }\end{array}$ & $\begin{array}{l}I G-B R \\
S C-A\end{array}$ & $\begin{array}{l}I G-B R \\
S L-X \\
S C-A \\
S R-X\end{array}$ & $\begin{array}{l}I G-B R \\
S L-X \\
S C-A \\
S R-X\end{array}$ & $\begin{array}{l}I G-B R \\
S L-X \\
S C-A\end{array}$ & 7 & 4 & 8 & 3 & 5 \\
\hline$A C$ & $S R-X$ & $S R-X$ & $I G-B R$ & $S L-X$ & $S R-A$ & $S L-X$ & SL-X & $S R-X$ & $\begin{array}{l}I G-B R \\
S R-X\end{array}$ & 2 & 3 & 0 & 1 & 4 \\
\hline$F_{b}$ & $S R-X$ & $\begin{array}{l}S C-A \\
S R-X\end{array}$ & $S C-A$ & $S L-X$ & $\begin{array}{l}\text { SL-X } \\
\text { SR-A } \\
\text { SR-X }\end{array}$ & $\begin{array}{l}I G-B R \\
S L-X\end{array}$ & $S L-X$ & $S R-X$ & $\begin{array}{l}S C-A \\
S R-X\end{array}$ & 1 & 4 & 3 & 1 & 5 \\
\hline$I G-B R$ & 0 & $\overline{0}$ & 2 & 1 & $\overline{1}$ & 3 & $\overline{1}$ & $\overline{1}$ & 3 & 12 & & & & \\
\hline$S L-X$ & 1 & 0 & 0 & 4 & 2 & 2 & 4 & 1 & 2 & & 16 & & & \\
\hline$S C-A$ & 1 & 1 & 3 & 1 & 1 & 1 & 1 & 1 & 3 & & & 13 & & \\
\hline$S R-A$ & 0 & 0 & 1 & 1 & 3 & 0 & 0 & 0 & 0 & & & & 5 & \\
\hline$S R-X$ & 3 & 4 & 0 & 2 & 1 & 0 & 1 & 4 & 3 & & & & & 18 \\
\hline
\end{tabular}

Na Tabela 4.7 é possivel observar que determinadas configurações LCFS levam a classificadores relativamente melhores que os classificadores gerados originalmente por $I G-B R$. É verificado também um destaque similar para as configurações $S L-X$ e $S R-X$ em relação ao número de casos com melhores rankings médios - 16 e 18, respectivamente. Em favor de $S R-X$, há uma quantidade maior de casos destacados, muitos dos quais relacionados às menores quantidades de atributos investigadas: $\left|X^{\prime}\right|=10 \% M$ e $20 \% M$. Em particular, 
com $\left|X^{\prime}\right|=20 \% M$, essa configuração obteve o melhor ranking médio nas quatro medidas de avaliação. Assim, SR-X é considerada a melhor configuração LCFS avaliada neste trabalho.

Ao focar a comparação especificamente nos algoritmos IG-BR e SR-X, foi aplicado o teste de Wilcoxon signed-ranks, revelando a ocorrência de duas diferenças estatísticas com $\alpha=0,05$. Em particular, foi verificada a superioridade significativa de $S R-X$ em relação a $I G-B R$ com $\left|X^{\prime}\right|=80 \% M$ nas medidas de avaliação F-Measure e Accuracy.

Convém ressaltar que $S R-X$ também atinge superioridade significativa em outros experimentos realizados como parte deste trabalho (Spolaôr et al., 2014b). Nesses experimentos, o número de vizinhos próximos $k$ inerente ao algoritmo $B R k N N-b$ foi definido a partir de um estudo preliminar, de modo que o parâmetro $k$ foi estabelecido com um valor específico por conjunto de dados. Os resultados indicam que os classificadores construídos usando atributos selecionados por $S R-X$ são estatisticamente superiores $(\alpha=0,05)$ aos construídos por IG-BR quando a quantidade de atributos é menor $-\left|X^{\prime}\right|=10 \% M \mathrm{e}$ $20 \% M$ - , o que é considerado um bom resultado para a tarefa de seleção de atributos.

Em relação à complexidade, o custo computacional adicional que $S R-X$ exige antes da execução de IG-BR se limita basicamente ao custo de aplicar o operador XOR ou XNOR para criar os $q^{\prime}$ novos rótulos na etapa de geração, pois a seleção dos pares de rótulos é realizada de modo randômico.

A partir dos resultados discutidos nessa seção, opta-se pela inclusão da configuração $S R-X$ em um estudo comparativo com o melhor algoritmo baseado em abordagens tradicionais de transformação do problema: $I G-L P-$ Seção 4.5.1.

IG-LP vs SR-X

Os resultados experimentais de $I G-L P$ e $S R-X$ já foram apresentados na Seção A.2 do Apêndice, página 112. Para investigar a capacidade da configuração $S R-X$ incrementar a competitividade de $I G-B R$ em relação a IG-LP, a seguir são apresentados dois grupos de rankings médios de classificadores:

1. Rankings médios calculados especificamente entre $I G-L P$ e IG-BR;

2. Rankings médios calculados especificamente entre $I G-L P$ e $S R-X$.

Na Tabela 4.8 são apresentados os valores dos rankings médios, calculados dentre os 10 conjuntos de dados considerados, para os classificadores gerados a partir dos atributos selecionados por IG-LP e IG-BR. Os melhores valores para cada quantidade de atributos $\left|X^{\prime}\right|$ e medida de avaliação são destacados 
em negrito. Note que, por ser a única medida de avaliação que deve ser minimizada, Hamming Loss é a única medida na qual quanto maior o ranking médio, melhor é o classificador. De acordo com o teste de Wilcoxon, não foi encontrada diferença significativa entre os dois métodos de seleção de atributos $\operatorname{com} \alpha=0,05$.

Tabela 4.8: Valores dos rankings médios atingidos por classificadores $B R k N N$ $b$ gerados a partir de IG-LP e IG-BR para cada quantidade de atributos selecionados $\left|X^{\prime}\right|$

\begin{tabular}{l|ll|ll|ll|ll}
\hline \hline & \multicolumn{2}{|c|}{ F-Measure } & \multicolumn{2}{|c|}{ Hamming Loss } & \multicolumn{2}{c|}{ Accuracy } & \multicolumn{2}{c}{$F_{b}$} \\
& $I G-L P$ & $I G-B R$ & $I G-L P$ & $I G-B R$ & $I G-L P$ & $I G-B R$ & $I G-L P$ & $I G-B R$ \\
\hline $10 \% M$ & $\mathbf{1 , 4 5}$ & 1,55 & $\mathbf{1 , 5 0}$ & $\mathbf{1 , 5 0}$ & $\mathbf{1 , 4 0}$ & 1,60 & $\mathbf{1 , 4 0}$ & 1,60 \\
$20 \% M$ & $\mathbf{1 , 4 5}$ & 1,55 & 1,40 & $\mathbf{1 , 6 0}$ & $\mathbf{1 , 4 0}$ & 1,60 & $\mathbf{1 , 4 0}$ & 1,60 \\
$30 \% M$ & $\mathbf{1 , 4 5}$ & 1,55 & $\mathbf{1 , 5 0}$ & $\mathbf{1 , 5 0}$ & $\mathbf{1 , 5 0}$ & $\mathbf{1 , 5 0}$ & $\mathbf{1 , 5 0}$ & $\mathbf{1 , 5 0}$ \\
$40 \% M$ & $\mathbf{1 , 4 0}$ & 1,60 & $\mathbf{1 , 5 5}$ & 1,45 & $\mathbf{1 , 5 0}$ & $\mathbf{1 , 5 0}$ & $\mathbf{1 , 5 0}$ & $\mathbf{1 , 5 0}$ \\
$50 \% M$ & $\mathbf{1 , 4 0}$ & 1,60 & $\mathbf{1 , 6 0}$ & 1,40 & $\mathbf{1 , 3 0}$ & 1,70 & $\mathbf{1 , 5 0}$ & $\mathbf{1 , 5 0}$ \\
$60 \% M$ & 1,55 & $\mathbf{1 , 4 5}$ & 1,45 & $\mathbf{1 , 5 5}$ & $\mathbf{1 , 5 0}$ & $\mathbf{1 , 5 0}$ & $\mathbf{1 , 5 0}$ & $\mathbf{1 , 5 0}$ \\
$70 \% M$ & $\mathbf{1 , 4 0}$ & 1,60 & 1,45 & $\mathbf{1 , 5 5}$ & $\mathbf{1 , 4 0}$ & 1,60 & $\mathbf{1 , 4 0}$ & 1,60 \\
$80 \% M$ & $\mathbf{1 , 3 5}$ & 1,65 & 1,35 & $\mathbf{1 , 6 5}$ & $\mathbf{1 , 3 0}$ & 1,70 & $\mathbf{1 , 3 0}$ & 1,70 \\
$90 \% M$ & $\mathbf{1 , 3 5}$ & 1,65 & 1,35 & $\mathbf{1 , 6 5}$ & $\mathbf{1 , 3 0}$ & 1,70 & $\mathbf{1 , 4 0}$ & 1,60 \\
\hline \hline
\end{tabular}

Na Tabela 4.9 são apresentados, com formatação similar à Tabela 4.8 , os valores de rankings médios dos classificadores relacionados a IG- $L P$ e $S R-X$. De acordo com o teste de Wilcoxon, não foi encontrada diferença significativa entre esses métodos $\operatorname{com} \alpha=0,05$.

Tabela 4.9: Valores dos rankings médios atingidos por classificadores BRkNN$b$ gerados a partir de $I G-L P$ e $S R-X$ para cada quantidade de atributos selecionados $\left|X^{\prime}\right|$

\begin{tabular}{c|cc|ll|ll|ll}
\hline \hline & \multicolumn{2}{|c|}{ F-Measure } & \multicolumn{2}{|c|}{ Hamming Loss } & \multicolumn{2}{c}{ Accuracy } & \multicolumn{2}{c}{$F_{b}$} \\
& $I G-L P$ & $S R-X$ & $I G-L P$ & $S R-X$ & $I G-L P$ & $S R-X$ & $I G-L P$ & $S R-X$ \\
\hline $10 \% M$ & $\mathbf{1 , 4 0}$ & 1,60 & $\mathbf{1 , 5 0}$ & $\mathbf{1 , 5 0}$ & $\mathbf{1 , 4 0}$ & 1,60 & $\mathbf{1 , 4 0}$ & 1,60 \\
$20 \% M$ & $\mathbf{1 , 5 0}$ & $\mathbf{1 , 5 0}$ & 1,35 & $\mathbf{1 , 6 5}$ & $\mathbf{1 , 5 0}$ & $\mathbf{1 , 5 0}$ & $\mathbf{1 , 5 0}$ & $\mathbf{1 , 5 0}$ \\
$30 \% M$ & $\mathbf{1 , 4 5}$ & 1,55 & $\mathbf{1 , 5 5}$ & 1,45 & $\mathbf{1 , 4 5}$ & 1,55 & $\mathbf{1 , 5 0}$ & $\mathbf{1 , 5 0}$ \\
$40 \% M$ & $\mathbf{1 , 4 0}$ & 1,60 & $\mathbf{1 , 5 5}$ & 1,45 & $\mathbf{1 , 4 5}$ & 1,55 & $\mathbf{1 , 5 0}$ & $\mathbf{1 , 5 0}$ \\
$50 \% M$ & $\mathbf{1 , 4 0}$ & 1,60 & $\mathbf{1 , 6 5}$ & 1,35 & $\mathbf{1 , 3 5}$ & 1,65 & $\mathbf{1 , 4 0}$ & 1,60 \\
$60 \% M$ & $\mathbf{1 , 4 5}$ & 1,55 & $\mathbf{1 , 5 0}$ & $\mathbf{1 , 5 0}$ & $\mathbf{1 , 5 0}$ & $\mathbf{1 , 5 0}$ & $\mathbf{1 , 4 0}$ & 1,60 \\
$70 \% M$ & $\mathbf{1 , 4 0}$ & 1,60 & 1,45 & $\mathbf{1 , 5 5}$ & $\mathbf{1 , 3 5}$ & 1,65 & $\mathbf{1 , 4 0}$ & 1,60 \\
$80 \% M$ & $\mathbf{1 , 4 0}$ & 1,60 & 1,35 & $\mathbf{1 , 6 5}$ & 1,55 & $\mathbf{1 , 4 5}$ & $\mathbf{1 , 4 0}$ & 1,60 \\
$90 \% M$ & $\mathbf{1 , 3 5}$ & 1,65 & 1,40 & $\mathbf{1 , 6 0}$ & $\mathbf{1 , 4 5}$ & 1,55 & $\mathbf{1 , 4 0}$ & 1,60 \\
\hline \hline
\end{tabular}

Um aspecto no qual a configuração $S R-X$ incrementa a competitividade de $I G-B R$ em relação a $I G-L P$ consiste na melhoria dos valores de ranking médio quando a quantidade de atributos $\left|X^{\prime}\right|$ é menor. SR-X obtém classificadores destacados para quantidades de atributos menores que as quantidades consideradas por IG-BR em geral. A melhoria mais notável envolve a quantidade de atributos $\left|X^{\prime}\right|=20 \% M$, em que $S R$ - $X$ contribui na construção de classificadores destacados nas quatro medidas de avaliação - Tabela 4.9. IG-BR atinge resultado parecido com uma quantidade três vezes maior de atributos: $\left|X^{\prime}\right|=60 \% M$ - Tabela 4.8 .

Convém ressaltar que o uso de LCFS previamente a IG-BR considera alguma relação entre rótulos, como IG-LP faz, mas evita desvantagens da abor- 
dagem LP utilizada pelo último algoritmo, como a limitação dos multirrótulos suportados aqueles que estão presentes no conjunto de treinamento $D_{t r}$. Adicionalmente, $S R-X$ herda determinadas características positivas de $I G-B R$, como a facilidade para dividir o problema em partes menores e independentes e, consequentemente, usar paralelização em computadores com múltiplos processadores (Kononenko e Robnik-Šikonja, 2007).

A superioridade apresentada por $I G-L P$ e $S R-X$ em relação ao $I G-B R$ original sugere que a consideração de relação entre rótulos é importante para a seleção de atributos multirrótulo.

\subsection{Considerações Finais}

Neste capítulo foram apresentados métodos e experimentos relacionados à seleção de atributos multirrótulo baseada em transformação do problema. Em particular, foram considerados quatro algoritmos de seleção de atributos - IG$L P, R F-L P, I G-B R$ e $R F-B R$ - , bem como quatro configurações do método Label Construction for Feature Selection - SL-X, SC-A, SR-A e SR-X - e a seleção de atributos randômica. LCFS foi proposto neste trabalho como uma alternativa para adicionar a consideração de relações entre rótulos à abordagem $B R$.

De modo geral, o algoritmo IG-LP obteve maior destaque dentre os algoritmos de SA. Com o intuito de incrementar a competitividade de $I G-B R$ em relação a $I G-L P$, foram investigadas as configurações do LCFS proposto neste trabalho. Uma delas, $S R-X$, se destacou por ser a única que levou à melhora de alguns classificadores originalmente piores que o baseline Geral $_{B}$. Além disso, ao comparar especificamente os algoritmos $I G-B R$ e $S R-X$, foi verificada a superioridade significativa do último.

Os bons resultados credenciaram a inclusão de $S R-X$ em um estudo comparativo com IG-LP. Embora nenhuma diferença significativa foi encontrada entre ambos, alguns resultados indicam, por exemplo, que $S R-X$ obteve destaque quando a quantidade de atributos usados para construir o classificador foi menor, o que é considerado um bom resultado para a tarefa de SA. Convém ressaltar que o uso de $L C F S$ previamente a $I G-B R$ considera alguma relação entre rótulos, como IG-LP faz, mas evita desvantagens da abordagem $L P$.

Assim, é possível concluir que LCFS, por meio da configuração $S R-X$, incrementou a competitividade de $I G-B R$ em relação a $I G-L P$. Além disso, a superioridade relativa apresentada por $I G-L P$ e $S R-X$ consiste em uma evidência de que a consideração de relação entre rótulos é importante para a seleção de atributos multirrótulo baseada em abordagens de transformação do problema.

No Capítulo 5 são propostas e avaliadas as extensões $R F-M L$ e $R F M-M L$ para SA multirrótulo sem abordagens de transformação do problema. 


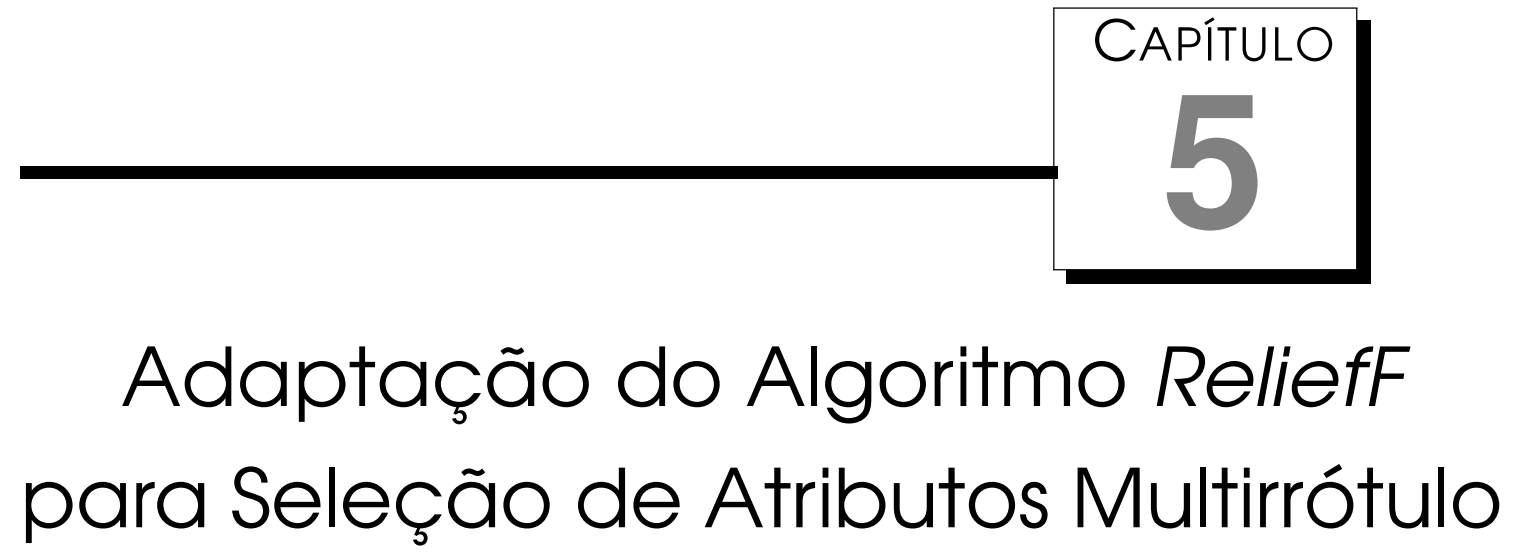

A tradicional família de algoritmos Relief para seleção de atributos monorrótulo se destaca em relação a medidas de importância de atributos estritamente univariadas, como ganho de informação, devido à consideração de interações entre atributos (Moore e White, 2007; Robnik-Sikonja e Kononenko, 2003). O algoritmo pioneiro, denominado Relief (Kira e Rendell, 1992), foi proposto no início dos anos 1990 e era somente capaz de avaliar a qualidade de atributos em dados binários, sem valores faltantes e com pouco ou nenhum ruído. Uma de suas primeiras extensões, denominada ReliefF (Kononenko, 1994) reduzia as limitações mencionadas e suportava dados multiclasse. Sua adaptação para dados com rótulos numéricos, denominada RReliefF (RobnikSikonja e Kononenko, 1997), foi proposta alguns anos mais tarde.

A maneira tradicional para aplicação de algoritmos Relief nos dados multirrótulo ainda é por meio de abordagens de transformação do problema, como exemplificado em trabalhos preliminares que integram este trabalho (Spolaôr et al., 2013b, 2011a). Entretanto, recente e simultaneamente foram publicadas extensões de ReliefF e RReliefF que são capazes de realizar seleção de atributos multirrótulo diretamente, sem nenhuma transformação prévia (Pupo et al., 2013; Slavkov et al., 2013; Spolaôr et al., 2013c). Duas dessas extensões são propostas neste trabalho: ReliefF Multirrótulo e ReliefF Mútuo Multirrótulo. Ambas extensões mantêm propriedades de ReliefF e RReliefF, como a consideração da interação entre atributos, além de combinarem de modo único características das duas outras extensões, permitindo usar a SA filtro no suporte ao aprendizado multirrótulo não hierárquico. 
Neste capítulo são apresentados primeiramente os três representantes da família Relief mencionados anteriormente e as extensões propostas neste trabalho para SA multirrótulo. Após, essas extensões são avaliadas experimentalmente e comparadas com alguns algoritmos, usados no Capítulo 4, que aplicam ReliefF e ganho de informação de acordo com as tradicionais abordagens de transformação do problema Binary Relevance e Label Powerset.

\section{1 Familia de Algoritmos Relief}

As duas extensões propostas neste trabalho são baseadas em ReliefF e RReliefF, descritos brevemente a seguir. O algoritmo Relief, base da família, também é abordado. Uma descrição mais abrangente da família, incluindo pseudocódigos, ilustrações e extensões para seleção de atributos monorrótulo, é apresentada em (Spolaôr et al., 2014a).

\subsubsection{Relief}

O mais antigo algoritmo da família, denominado Relief, foi proposto para dados binários monorrótulo (Kira e Rendell, 1992). Sua ideia principal é destacar atributos que possuem valores diferentes para vizinhos (exemplos) mais próximos de rótulos diferentes, bem como penalizar atributos que possuem valores diferentes para vizinhos mais próximos de rótulos iguais (Demšar, 2010). Para tanto, Relief busca dois vizinhos próximos para cada exemplo $E_{i}$ randomicamente amostrado: um de mesmo rótulo - nearest hit - , e o outro de rótulo distinto - nearest miss. Desse modo, o algoritmo considera $k=1$ vizinhos mais próximos de cada rótulo do problema binário.

Relief usa a medida diff - Equação 5.1 - para calcular a diferença entre os valores de um atributo $X_{j}$ nos exemplos $E_{i}$ e $E_{k}$, denotados por $x_{i j}$ e $x_{k j}$ respectivamente. No Relief, diff é constituída pelas medidas overlap, definida pela Equação 5.2 para atributos categóricos, e Range Normalized Difference (RND), definida pela Equação 5.3 para atributos numéricos.

$$
\begin{gathered}
\text { diff } f\left(X_{j}, E_{i}, E_{k}\right)=\left\{\begin{array}{l}
\text { overlap }\left(X_{j}, E_{i}, E_{k}\right) \text { se } X_{j} \text { écategórico } \\
R N D\left(X_{j}, E_{i}, E_{k}\right) \text { se } X_{j} \text { énumérico. }
\end{array}\right. \\
\text { overlap }\left(X_{j}, E_{i}, E_{k}\right)=\left\{\begin{array}{l}
0 \text { se } x_{i j}=x_{k j} \\
1 \text { se } x_{i j} \neq x_{k j} .
\end{array}\right.
\end{gathered}
$$




$$
R N D\left(X_{j}, E_{i}, E_{k}\right)=\frac{\left|x_{i j}-x_{k j}\right|}{\max \left(X_{j}\right)-\min \left(X_{j}\right)}
$$

No Algoritmo 5.1 é apresentado o pseudocódigo do Relief (Kira e Rendell, 1992). O parâmetro definido pelo usuário $c$ corresponde ao número de iterações, i.e., a quantidade de exemplos considerada para estimar a importância dos atributos.

Entrada: Conjunto de treinamento $D_{t r}$ Número de iterações $c$

Resultado: Vetor de valores de importância de atributos $W$

$1 W \leftarrow \emptyset$

2 for $i=1 \rightarrow c$ do

$3 \quad E_{i} \leftarrow$ randomInstance $\left(D_{t r}\right)$

$A \leftarrow$ nearestHit $\left(E_{i}, D_{t r}\right)$

$S \leftarrow$ nearestMiss $\left(E_{i}, D_{t r}\right)$

for $j=1 \rightarrow M$ do

$W\left(X_{j}\right) \leftarrow W\left(X_{j}\right)+\frac{\operatorname{diff}\left(X_{j}, E_{i}, S\right)-\operatorname{diff}\left(X_{j}, E_{i}, A\right)}{c}$

end

Algoritmo 5.1: Relief

A complexidade do Relief é $O\left(c \times N_{t r} \times M\right)$, onde $c$ é um parâmetro definido pelo usuário, $N_{t r}$ é o número de exemplos de treinamento e $M$ é o número de atributos.

\subsubsection{ReliefF}

O algoritmo ReliefF, por sua vez, estende Relief para tratar dados com mais de dois rótulos (multiclasse), com valores faltantes e/ou ruidosos (Kononenko, 1994). Essas três melhorias são obtidas da seguinte maneira:

- Uso de $k>1$ vizinhos mais próximos para cada rótulo;

- Tratamento probabilístico de valores faltantes durante o cálculo de dissimilaridade entre exemplos no espaço de atributos.

De modo similar ao algoritmo de classificação kNN (Aha e Kibler, 1991), a busca por $k>1$ vizinhos mais próximos garante maior robustez em dados com ruído. Desse modo, para cada exemplo $E_{i}$ com rótulo $y_{i}$, ReliefF trabalha com os $k$ nearest hits $A K\left(E_{i}\right)$ e $k$ nearest misses $S K\left(E_{i}, y_{l}\right)$ para cada rótulo $y_{l}$, $y_{l} \neq y_{i}$.

ReliefF pondera as contribuições dos nearest misses pela frequência relativa de cada rótulo $y_{l}$ no conjunto de treinamento. Para normalizar os pesos, 
o algoritmo divide cada probabilidade previamente calculada pela soma das probabilidades envolvendo misses dos rótulos $y_{l}, y_{l} \neq y_{i}$, i.e., $1-P\left(y_{i}\right)$, onde $y_{i}$ é o rótulo de $E_{i}$. De fato, a classe dos hits $\left(y_{i}\right)$ não participa dos misses, sendo portanto desconsiderada na soma de probabilidades.

Para tratar atributos com valores faltantes, ReliefF modifica a medida diff para avaliar a probabilidade de que dois exemplos tenham valores diferentes em um determinado atributo. Nesse sentido, há duas possiveis situações:

(i) Um dos exemplos possui um valor desconhecido no atributo $X_{j}$. Se o exemplo desconhecido é $E_{i}$, diff é definida pela Equação 5.4. Por outro lado, se o exemplo é $E_{k}$, diff é definida pela Equação 5.5;

$$
\begin{gathered}
\operatorname{diff}\left(X_{j}, E_{i}, E_{k}\right)=1-P\left(x_{k j} \mid y=y_{i}\right) . \\
\operatorname{diff}\left(X_{j}, E_{i}, E_{k}\right)=1-P\left(x_{i j} \mid y=y_{i}\right) .
\end{gathered}
$$

(ii) Se ambos exemplos $E_{i}$ e $E_{k}$ possuem valor desconhecido no atributo $X_{j}$, diff é definida pela Equação 5.6. Seja $\sharp$ valores $\left(X_{j}\right)$ o número de valores de $X_{j}$.

$$
\operatorname{diff}\left(X_{j}, E_{i}, E_{k}\right)=1-\sum_{V}^{\sharp \operatorname{valores}\left(X_{j}\right)}\left(P\left(V \mid y=y_{i}\right) \times P\left(V \mid y=y_{k}\right)\right)
$$

No Algoritmo 5.2, o pseudocódigo de ReliefF é apresentado (Kononenko, 1994). Essa extensão possui dois parâmetros principais definidos pelo usuário: $c$ e $k$.

ReliefF atingiu relativa popularidade para SA filtro, tendo sido implementado em frameworks como Weka (Witten e Frank, 2011) e Orange (Demšar et al., 2013).

\subsubsection{RReliefF}

Além da robustez a valores faltantes e ruidosos, o algoritmo RReliefF é capaz de ranquear atributos em dados com rótulos contínuos - problemas de regressão monorrótulo (Robnik-Sikonja e Kononenko, 1997). Para evitar a busca por exemplos de rótulos iguais (nearest hits), RReliefF modela a probabilidade de que os rótulos contínuos de dois exemplos sejam diferentes como uma função de dissimilaridade entre esses rótulos. A partir dessa probabilidade e do Teorema de Bayes, RReliefF aproxima a estimativa da importância de atributos realizada por ReliefF enquanto dispensa a ideia de nearest hits (Kononenko e Robnik-Šikonja, 2007). 


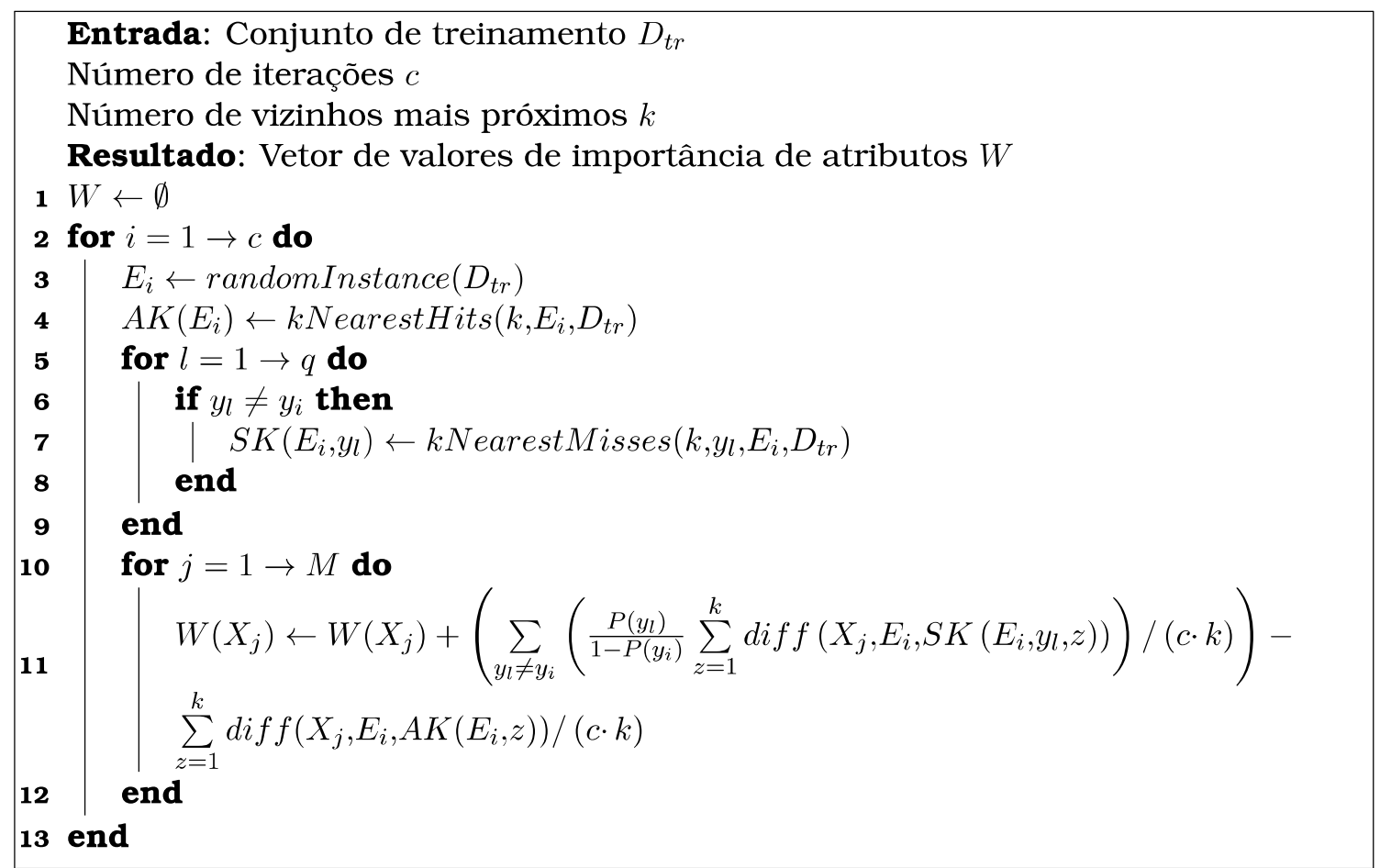

\section{Algoritmo 5.2: ReliefF}

Para melhor entender RReliefF, convém relembrar que a estimativa obtida por Relief pode ser aproximada pela diferença entre probabilidades relacionadas a exemplos - Equação 4.4, página 62. Essa aproximação é reescrita na Equação 5.7 em termos de notações diferentes. Sejam $O_{d X}$ o evento no qual dois exemplos possuem valores diferentes para um determinado atributo, $O_{d Y}$ o evento no qual dois exemplos possuem rótulos diferentes e $O_{d \bar{Y}}=1-O_{d Y}$ o evento no qual dois exemplos possuem o mesmo rótulo.

$$
\text { Relief }\left(X_{j}\right)=P\left(O_{d X} \mid O_{d Y}\right)-P\left(O_{d X} \mid O_{d \bar{Y}}\right)
$$

Ao modificar as equações, por exemplo, para omitir a condição inerente às probabilidades condicionais na Equação 5.7, é possível evitar a noção de exemplos com os mesmos valores de classe em problemas de regressão (Kononenko e Robnik-Šikonja, 2007). A partir da regra de Bayes, os seguintes cálculos reescrevem a probabilidade $P\left(O_{d X} \mid O_{d Y}\right)$ na Equação 5.7. A reformulação da probabilidade $P\left(O_{d X} \mid O_{d \bar{Y}}\right)$ pode ser obtida de modo similar.

$$
\begin{aligned}
P\left(O_{d X} \mid O_{d Y}\right) & =\frac{P\left(O_{d Y} \mid O_{d X}\right) P\left(O_{d X}\right)}{P\left(O_{d Y}\right)} \\
& =\frac{P\left(O_{d Y} \cap O_{d X}\right)}{P\left(O_{d Y}\right)} .
\end{aligned}
$$


Finalmente, ao substituir os termos antigos na Equação 5.7 pelos novos, RReliefF é resumido como definido pela Equação 5.8. Seja $P\left(O_{d Y} \cap O_{d X}\right)$ a probabilidade em que dois exemplos mais próximos possuam diferentes valores de classe e do atributo $X_{j}$. Assim como ocorre com os rótulos, essa probabilidade é modelada por meio de uma função de dissimilaridade. Para tanto, é utilizada a função diff mencionada anteriormente.

$$
\text { RRelief } F\left(X_{j}\right)=\frac{P\left(O_{d Y} \cap O_{d X}\right)}{P\left(O_{d Y}\right)}-\frac{P\left(O_{d X}\right)-P\left(O_{d Y} \cap O_{d X}\right)}{1-P\left(O_{d Y}\right)}
$$

Com essa reformulação, a estimativa de importância pode ser aplicada em dados com rótulos numéricos. No pseudocódigo de RReliefF - Algoritmo 5.3 (Robnik-Sikonja e Kononenko, 1997) -, os pesos específicos são denotados por:

- $W_{d Y}$ para rótulos diferentes;

- $W_{d X}$ para valores de atributos diferentes;

- $W_{d Y X}$ para rótulos e valores de atributos diferentes.

Entrada: Conjunto de treinamento $D_{t r}$

Número de iterações $c$

Número de vizinhos mais próximos $k$

Resultado: Vetor de valores de importância de atributos $W$

$$
\begin{aligned}
& 1 W_{d Y} \leftarrow \emptyset \\
& 2 W_{d X} \leftarrow \emptyset \\
& \text { 3 } W_{d Y X} \leftarrow \emptyset \\
& 4 W \leftarrow \emptyset \\
& 5 \text { for } i=1 \rightarrow c \text { do } \\
& 6 \quad E_{i} \leftarrow \text { randomInstance }\left(D_{t r}\right) \\
& 7 \quad E K \leftarrow k N \text { earestNeighbors }\left(k, E_{i}, D_{t r}\right) \\
& 8 \text { for } z=1 \rightarrow k \text { do } \\
& \mathbf{9} \quad W_{d Y} \leftarrow W_{d Y}+\operatorname{diff}\left(Y, E_{i}, E K_{z}\right) \cdot d\left(E_{i}, E K_{z}\right) \\
& 10 \quad \text { for } j=1 \rightarrow M \text { do } \\
& 11 \quad W_{d X}\left(X_{j}\right) \leftarrow W_{d X}\left(X_{j}\right)+\operatorname{diff}\left(X_{j}, E_{i}, E K_{z}\right) \cdot d\left(E_{i}, E K_{z}\right) \\
& 12 \quad W_{d Y X}\left(X_{j}\right) \leftarrow W_{d Y X}\left(X_{j}\right)+\operatorname{diff}\left(Y, E_{i}, E K_{z}\right) \cdot \operatorname{diff}\left(X_{j}, E_{i}, E K_{z}\right) \cdot d\left(E_{i}, E K_{z}\right) \\
& 13 \text { end } \\
& 14 \text { end } \\
& 15 \text { end } \\
& 16 \text { for } j=1 \rightarrow M \text { do } \\
& 17 \mid W\left(X_{j}\right) \leftarrow W\left(X_{j}\right)+\frac{W_{d Y X}\left(X_{j}\right)}{W_{d Y}}-\frac{W_{d X}\left(X_{j}\right)-W_{d Y X}\left(X_{j}\right)}{c-W_{d Y}} \\
& 18 \text { end }
\end{aligned}
$$

Algoritmo 5.3: RReliefF 
É possível notar que cada um desses pesos possuem relações com as probabilidades na reformulação - Equação 5.8. Em particular, os pesos $W_{d Y}$, $W_{d X}$ e $W_{d Y Y}$ estão relacionados, respectivamente, às probabilidades $P\left(O_{d Y}\right)$, $P\left(O_{d X}\right)$ e $P\left(O_{d Y} \cap O_{d X}\right)$.

O termo $d\left(E_{i}, E K_{z}\right)$ no Algoritmo 5.3 pondera cada vizinho mais próximo $E K_{z}$ de acordo com a dissimilaridade entre esse vizinho e o exemplo randomicamente selecionado $E_{i}$ - distance weighting (Robnik-Sikonja e Kononenko, 2003). Quanto mais próximos os exemplos são, maior é a influência do vizinho. Neste trabalho, essa ponderação não é considerada, de modo que $d\left(E_{i}, E K_{z}\right)=1 / k$, i.e., todos os vizinhos apresentam a mesma contribuição.

\subsection{Extensões Propostas para Seleção de Atributos Multirrótulo via ReliefF}

Ainda que várias extensões da família Relief tenham sido introduzidas para seleção de atributos monorrótulo, somente recentemente foram propostas alternativas para tratar dados multirrótulo diretamente (Pupo et al., 2013; Slavkov et al., 2013; Spolaôr et al., 2013c; Kong et al., 2012; Spolaôr et al., 2011a). A seguir, são descritas duas extensões para SA multirrótulo propostas neste trabalho: ReliefF Multirrótulo (RF-ML) e ReliefF Mútuo Multirrótulo (RFM-ML).

\subsubsection{ReliefF Multirrótulo}

Embora RF-ML seja similar a RReliefF (Robnik-Sikonja e Kononenko, 1997), a extensão proposta neste trabalho se diferencia por introduzir uma função de dissimilaridade $m l d(.,$.$) que considera múltiplos rótulos simultaneamente,$ bem como por buscar $k$ vizinhos multirrótulo. Desse modo, RF-ML dispensa a transformação do problema multirrótulo para monorrótulo e, consequentemente, tem menor custo computacional que a aplicação do ReliefF monorrótulo conforme as tradicionais abordagens Binary Relevance e Label Powerset. É necessário destacar que $R F-M L$ também mantém propriedades como a consideração de interações entre atributos ao analisar a dissimilaridade entre exemplos no espaço de atributos, a qual independe da quantidade e do tratamento dos rótulos dos dados.

O pseudocódigo da extensão RF-ML é apresentado no Algoritmo 5.4. O loop principal, iniciado na Linha 5, percorre cada um dos $E_{i}$ exemplos obtidos por amostragem randômica com reposição ${ }^{34}$ do conjunto $D_{t r}$. Na $i$-ésima iteração, os $k$ vizinhos mais próximos no espaço de atributos de um exemplo $E_{i}$ são identificados (Linha 7). Na $z$-ésima iteração do loop iniciado na Linha 8, a

\footnotetext{
${ }^{34}$ Quando $c=N_{t r}$, a amostra é igual ao conjunto de treinamento $D_{t r}$, dispensando a amostragem randômica.
} 
dissimilaridade $m l d\left(E_{i}, E K_{z}\right)$ entre os multirrótulos de $E_{i}$ e de seu vizinho $E K_{z}$ é acumulada em $W_{d Y}$ (Linha 9). Ao longo do algoritmo, o termo $d\left(E_{i}, E K_{z}\right)$ oferece suporte para o uso de distance weighting, o qual pondera a contribuição de cada vizinho $E K_{z}$ de acordo com sua dissimilaridade no espaço de atributos para $E_{i}$. O loop mais aninhado, iniciado na Linha 10 , acumula em $W_{d X}$ e $W_{d Y X}$ as demais dissimilaridades considerando em cada iteração a diferença diff $\left(X_{j}, E_{i}, E K_{z}\right)$ entre os valores do atributo $X_{j}$ para os dois exemplos. Note que as dissimilaridades nos espaços de atributos e de rótulos para a $j$-ésima iteração são multiplicadas na Linha 12, assim como realizado por RReliefF. O último loop, iniciado na Linha 16, estima a importância de cada atributo usando a mesma formulação derivada do Teorema de Bayes por RReliefF.

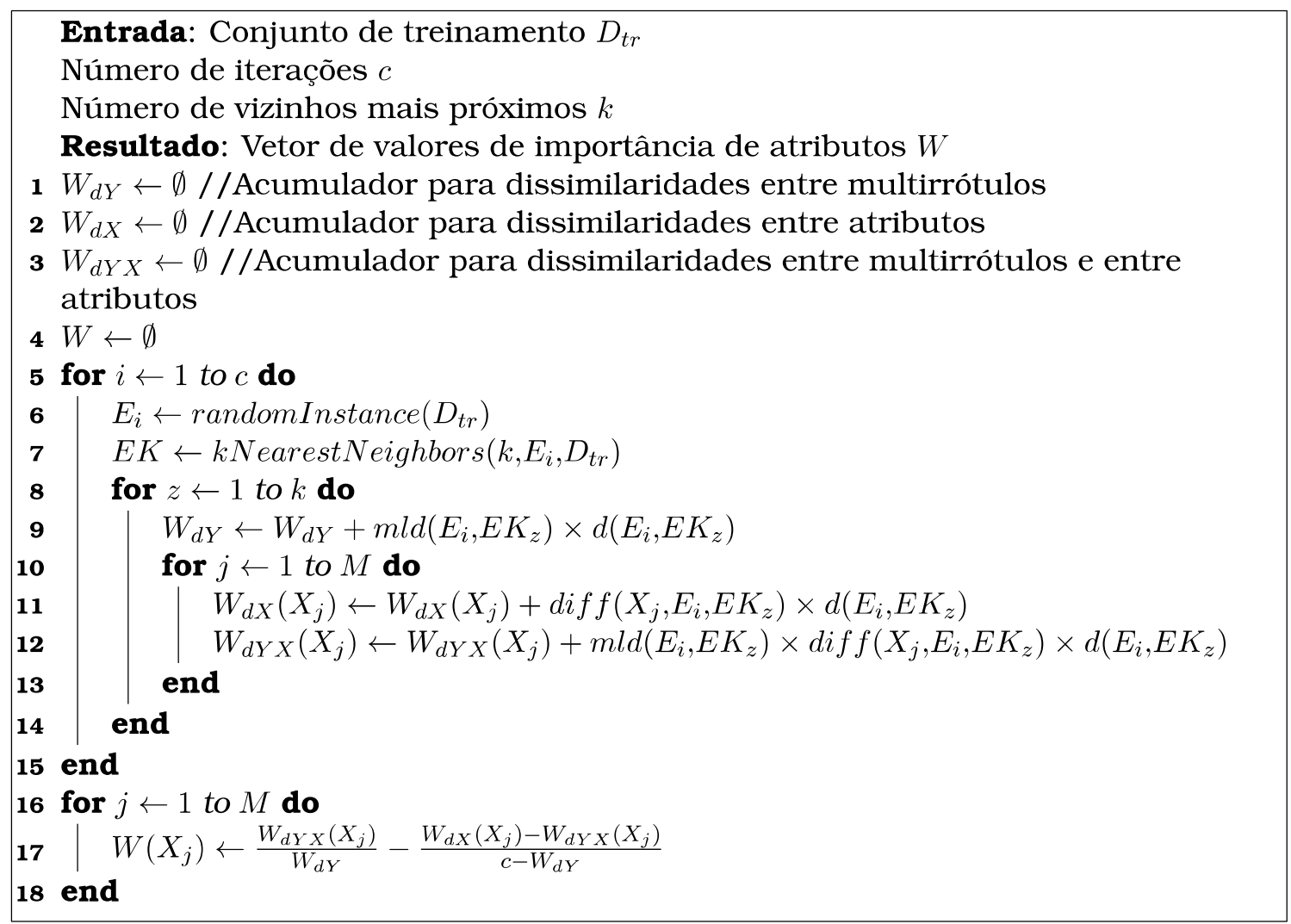

Algoritmo 5.4: ReliefF Multirrótulo

Como mencionado, a introdução da função de dissimilaridade entre multirrótulos $m l d(.,$.$) diferencia R F-M L$ de seu antecessor RReliefF. Qualquer medida de dissimilaridade aplicável a dois conjuntos pode implementar $m l d(.,$.$) . Neste$ trabalho, duas medidas têm sido utilizadas: dissimilaridade Hamming normalizada e dissimilaridade Jaccard.

A dissimilaridade Hamming entre dois multirrótulos $Y_{i}$ e $Y_{j}$ é definida por $\left|Y_{i} \cup Y_{j}\right|-\left|Y_{i} \cap Y_{j}\right|$, o que é equivalente à quantidade de rótulos simples distintos entre os dois multirrótulos (Hamming, 1950). Nessa medida, a ausência e a presença de rótulos são tratados igualmente. Note, por exem- 
plo, que tanto a dissimilaridade Hamming de $\left(\left\{y_{3}, y_{6}\right\},\left\{y_{1}, y_{4}\right\}\right)$ quanto a de $\left(\left\{y_{2}, y_{3}, y_{5}, y_{6}\right\},\left\{y_{1}, y_{2}, y_{4}, y_{5}\right\}\right)$ é igual a 4 . A dissimilaridade Hamming normalizada é definida pela Equação 5.9 para os multirrótulos $Y_{i}$ e $Y_{j}$ presentes em um conjunto de dados com $q$ rótulos.

$$
H D\left(Y_{i}, Y_{j}\right)=\frac{\left|Y_{i} \cup Y_{j}\right|-\left|Y_{i} \cap Y_{j}\right|}{q} .
$$

Por outro lado, a dissimilaridade Jaccard $J D\left(Y_{i}, Y_{j}\right)$ é útil, por exemplo, para casos nos quais $Y_{i}$ e $Y_{j}$ são constituídos por poucos rótulos simples, i.e., quando $\left|Y_{i} \cup Y_{j}\right|<<q$. A ideia é que a medida de dissimilaridade desconsidere os rótulos ausentes em ambos multirrótulos, ou seja, somente rótulos presentes em pelo menos um dos multirrótulos são incluídos no cálculo (Tan et al., 2013). Para tanto, a dissimilaridade Jaccard é definida pela Equação 5.10 a partir dos valores $f_{11}=\left|Y_{i} \cap Y_{j}\right|, f_{10}=\left|Y_{i}-Y_{j}\right|$ e $f_{01}=\left|Y_{j}-Y_{i}\right|$. Note que, assim como a dissimilaridade Hamming normalizada, os valores de $J D$ variam entre $[0,1]$. Além disso, diferentemente de $H D$, é observado que $J D\left(\left\{y_{3}, y_{6}\right\},\left\{y_{1}, y_{4}\right\}\right) \neq$ $J D\left(\left\{y_{2}, y_{3}, y_{5}, y_{6}\right\},\left\{y_{1}, y_{2}, y_{4}, y_{5}\right\}\right)$.

$$
J D\left(Y_{i}, Y_{j}\right)=\frac{f_{10}+f_{01}}{f_{10}+f_{01}+f_{11}} .
$$

A complexidade dos algoritmos Relieff, RReliefF e $R F-M L$ é dominada pela realização da busca pelos $k$ vizinhos mais próximos $c$ vezes, $\operatorname{com} c \leq N_{t r}$, i.e., $O\left(c \times N_{t r} \times M\right)$, em que $N_{t r}$ e $M$ são, respectivamente, o número de exemplos de treinamento e de atributos (Robnik-Sikonja e Kononenko, 2003). Quando $c=$ $N_{t r}$, estimativas mais confiáveis são obtidas, mas a complexidade aumenta $O\left(N_{t r}^{2} \times M\right)$. Especificamente para ReliefF, a busca pelos vizinhos deve ser realizada para cada um dos rótulos do conjunto de dados.

\subsubsection{ReliefF Mútuo Multirrótulo}

A principal diferença entre $R F-M L$ e $R F M-M L$ é que o último estabelece uma vizinhança entre os exemplos $E_{i}$ e $E_{j}$ se e somente se $E_{j}$ é vizinho mais próximo de $E_{i}$ e vice-versa. Apesar de ser mais restrita no sentido que o número de vizinhos mútuos mais próximos $k^{\prime} \leq k$, a vizinhança mútua tem contribuído para obter bons resultados em classificação monorrótulo (Liu et al., 2010) e multirrótulo (Cherman et al., 2013). Além disso, foi verificado teoricamente que a taxa de convergência do $k$ Nearest Neighbor mútuo para problemas de regressão é ótima quando o número de atributos $M \geq 2$ (Guyader e Hengartner, 2013). 
O pseudocódigo da extensão RFM-ML é apresentado no Algoritmo 5.5. Note que o procedimento updatekNearestNeighbors $\left(E K, E_{i}, D_{t r}\right)$ é adicionado na Linha 9 para manter em $E K^{\prime}$ somente os vizinhos mútuos de $E_{i}$, i.e., $E K^{\prime} \subseteq E K$. Em cada iteração desse procedimento, os $k$ vizinhos do exemplo $E K_{z}, z=1 . . k$, são identificados dentre os $c$ exemplos presentes na amostra do conjunto de treinamento $D_{t r}$. Se $E_{i}$ é um desses $k$ vizinhos, $E_{i}$ e $E K_{z}$ são confirmados como vizinhos mútuos, resultando na inclusão de $E K_{z}$ no conjunto $E K^{\prime}$ associado a $E_{i}$.

Entrada: Conjunto de treinamento $D_{t r}$

Número de iterações $c$

Número de vizinhos mais próximos $k$

Resultado: Vetor de valores de importância de atributos $W$

$1 W_{d Y} \leftarrow \emptyset / /$ Acumulador para dissimilaridades entre multirrótulos

$2 W_{d X} \leftarrow \emptyset / /$ Acumulador para dissimilaridades entre atributos

$3 W_{d Y X} \leftarrow \emptyset / /$ Acumulador para dissimilaridades entre multirrótulos e entre atributos

$4 W \leftarrow \emptyset$

$5 N I \leftarrow 0 / /$ Acumulador para número de exemplos ignorados

6 for $i \leftarrow 1$ to $c$ do

$7 \quad E_{i} \leftarrow$ randomInstance $\left(D_{t r}\right)$

$8 \quad E K \leftarrow k N$ earest Neighbors $\left(k, E_{i}, D_{t r}\right)$

$9 \quad E K^{\prime} \leftarrow$ updatekNearest Neighbors $\left(E K, E_{i}, D_{t r}\right)$

$10 \quad k^{\prime} \leftarrow\left|E K^{\prime}\right|$

11 if $k^{\prime}>0$ then

$25 c \leftarrow c-N I / /$ Tamanho da amostra é reduzido ao ignorar NI exemplos

26 for $j \leftarrow 1$ to $M$ do

$27 \mid W\left(X_{j}\right) \leftarrow \frac{W_{d Y X}\left(X_{j}\right)}{W_{d Y}}-\frac{W_{d X}\left(X_{j}\right)-W_{d Y X}\left(X_{j}\right)}{c-W_{d Y}}$

28 end

$/ / E_{i}$ possui vizinho mútuo

for $z \leftarrow 1$ to $k^{\prime}$ do

$W_{d Y} \leftarrow W_{d Y}+\operatorname{mld}\left(E_{i}, E K_{z}^{\prime}\right) \times d\left(E_{i}, E K_{z}^{\prime}\right)$

for $j \leftarrow 1$ to $M$ do

$W_{d X}\left(X_{j}\right) \leftarrow W_{d X}\left(X_{j}\right)+\operatorname{diff}\left(X_{j}, E_{i}, E K_{z}^{\prime}\right) \times d\left(E_{i}, E K_{z}^{\prime}\right)$ $W_{d Y X}\left(X_{j}\right) \leftarrow W_{d Y X}\left(X_{j}\right)+\operatorname{mld}\left(E_{i}, E K_{z}^{\prime}\right) \times \operatorname{diff}\left(X_{j}, E_{i}, E K_{z}^{\prime}\right) \times d\left(E_{i}, E K_{z}^{\prime}\right)$ end

end

end

else

$N I \leftarrow N I+1 / / \mathrm{O}$ descarte de $E_{i}$ é contabilizado end

Algoritmo 5.5: ReliefF Mútuo Multirrótulo

O número de vizinhos mútuos $k^{\prime}$ pode ser igual a zero, como ilustrado na Figura 5.1 pela vizinhança do exemplo $E_{4}$. Note que os $k=3$ vizinhos mais próximos de $E_{4}$ são $E_{2}, E_{3}$ e $E_{6}$, mas nenhum desses três exemplos possui $E_{4}$ como um de seus vizinhos mais próximos. No controle realizado na $\mathrm{Li}$ nha 11 do Algoritmo 5.5, exemplos como $E_{4}$ são ignorados e não influenciam 
na estimativa da importância de atributos. Além disso, como apresentado na Linha 25, o tamanho da amostra $c$ é reduzido antes da estimativa do vetor $W$ ao subtrair o número de exemplos ignorados $N I$.

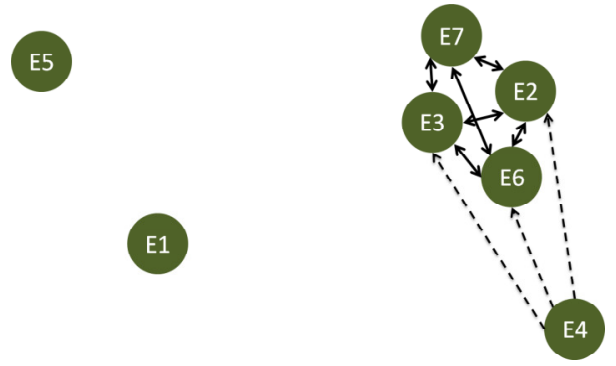

Figura 5.1: Conjunto de dados no qual o exemplo $E_{4}$ possui $k=3$ vizinhos mais próximos, mas nenhum vizinho mútuo $\left(k^{\prime}=0\right)$

Diferentemente do $R F-M L$, a complexidade do algoritmo $R F M-M L$ é dominada pela atualização da vizinhança mútua realizada pelo procedimento update$k N$ earest $N$ eighbors $\left(E K, E_{i}, D_{t r}\right)$ (Linha 9). Em particular, a complexidade $O(k \times$ $\left.N_{t r}^{2} \times M\right)$ de $R F M-M L$ é dominada pela realização de busca por vizinhos mais próximos por cada um dos $k$ vizinhos de $E_{i}, i=1 . . c$ e $c \leq N_{t r}$. Embora relativamente elevada, essa complexidade pode ser reduzida, por exemplo, por meio de estruturas de dados especiais (Liu et al., 2010) ou do cálculo prévio das dissimilaridades entre todos os pares possíveis de exemplos da amostra (Guyader e Hengartner, 2013).

\subsection{Configuração Experimental}

Na avaliação experimental realizada são utilizados os 10 conjuntos de dados benchmark descritos na Seção 2.5, página 32, bem como 45 conjuntos de dados artificiais gerados por meio de uma ferramenta publicamente disponível $^{35}$ (Tomás et al., 2014). A motivação para utilizar os mesmos conjuntos de dados benchmark abordados no Capítulo 4 é incluir no presente estudo comparativo métodos de seleção de atributos multirrótulo previamente avaliados neste trabalho. Por outro lado, conjuntos de dados artificiais proporcionam um ambiente controlado com propriedades conhecidas (Bolón-Canedo et al., 2013), como a solução verdadeira (Muñoz e González-Navarro, 2011).

Como ilustrado na Figura 5.2, as avaliações experimentais realizadas podem ser organizadas em dois grupos principais, os quais abordam:

- Configurações dos algoritmos RF-ML e RFM-ML baseadas nas medidas de dissimilaridade Hamming e Jaccard;

- Algoritmos baseados em ReliefF e algoritmo IG-LP.

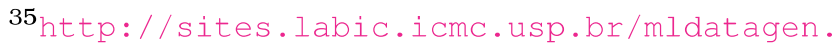




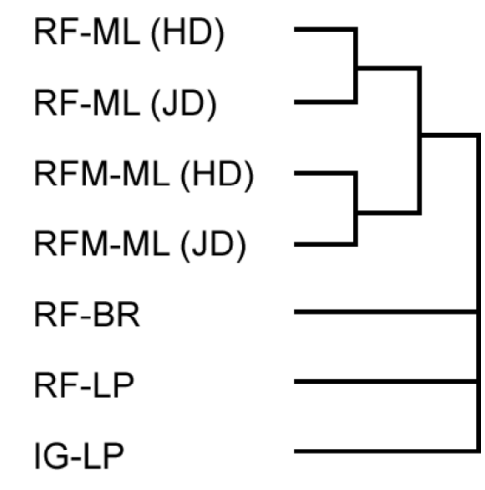

Figura 5.2: Ilustração da configuração experimental

Com relação ao primeiro grupo, duas avaliações específicas são abordadas:

- $R F-M L$ usando a medida de dissimilaridade Hamming versus RF-ML usando a dissimilaridade Jaccard, i.e., RF-ML (HD) vs RF-ML (JD);

- RFM-ML usando a medida Hamming versus RFM-ML usando Jaccard, i.e., RFM-ML (HD) vs RFM-ML (JD).

Após, a melhor configuração é eleita para inclusão no segundo grupo, o qual também contém IG-LP e dois algoritmos baseados em ReliefF - RF-BR e $R F-L P$ - descritos na Seção 4.4, página 63. Em particular, IG-LP, baseado na medida de importância ganho de informação, é escolhido devido ao seu destaque na avaliação experimental envolvendo seleção de atributos baseada em transformação do problema - Seção 4.5.1, página 65.

A taxonomia para SA multirrótulo descrita na Seção 3.1, página 37, permite classificar todos esses algoritmos de SA como representantes da abordagem filtro. As demais dimensões dessa taxonomia são apresentadas especificamente para cada algoritmo na Tabela 5.1.

Tabela 5.1: Categorização dos algoritmos de seleção de atributos multirrótulo baseados em ReliefF e de IG-LP avaliados neste trabalho de acordo com a taxonomia proposta neste trabalho - Seção 3.1

\begin{tabular}{r|rrr}
\hline \hline & escopo do multirrótulo & dependência de rótulos & medida de importância \\
\hline$R F-M L$ & multirrótulo & alta ordem & $R F$ multirrótulo \\
$R F-B R$ & monorrótulo & primeira ordem & $R F$ \\
$R F-L P$ & híbrido & alta ordem & $R F$ \\
$I G-L P$ & híbrido & alta ordem & ganho de informação \\
\hline \hline
\end{tabular}

Assim como realizado no Capítulo 4, cada método de seleção de atributos multirrótulo utilizado gera um ranking de atributos. A partir desse ranking, os subconjuntos dos melhores atributos $X^{\prime} \subset X,\left|X^{\prime}\right|=10 \% M, 20 \% M, \ldots, 90 \% M$ foram determinados para descrever versões reduzidas do conjunto de dados multirrótulo original, as quais foram submetidas ao algoritmo de aprendizado 
multirrótulo lazy $B R k N N-b$. A ideia foi avaliar a qualidade dos atributos selecionados em cada subconjunto em termos do desempenho de classificação do BRkNN-b.

Além das medidas de avaliação de aprendizado multirrótulo descritas na Seção 2.3 , página 21 , são adicionadas na presente avaliação experimental duas medidas específicas para seleção de atributos, as quais são apresentadas na Seção 5.3.2.

Assim como realizado para $R F-B R, R F-L P$ e $B R k N N-b$, optou-se neste trabalho por fixar $k=10$ para $R F-M L$ e $R F M-M L$. Convém ressaltar, entretanto, que outros valores de $k$ foram utilizados como parte deste trabalho (Spolaôr e Monard, 2014; Spolaôr et al., 2014b). Os demais algoritmos são configurados como descrito na Seção 4.4, página 62. Como mencionado, os algoritmos de classificação e de SA estão implementados no framework Mulan (Tsoumakas et al., 2011). Adicionalmente, as extensões propostas RF-ML e RFM-ML estão disponíveis à comunidade em http://www.labic.icmc.usp.br/pub/ momonard/Implementations/Multilabel/rf-ml.zip.

\subsubsection{Conjuntos de Dados Artificiais}

Os 45 conjuntos de dados artificiais foram gerados no formato compatível com o framework Mulan a partir da estratégia HyperCubes, a qual é baseada na estratégia HyperSpheres proposta por Zhang et al. (2009). Tanto HyperCubes quanto HyperSpheres estão implementados em uma ferramenta web, desenvolvida em colaboração (Tomás et al., 2014), denominada Mldatagen.

A principal motivação para HyperCubes é que hipercubos poderiam ser apropriados para avaliar algoritmos de aprendizado multirrótulo, como árvores de decisão (Clare e King, 2001), que classificam exemplos por meio da divisão do espaço usando hiperplanos. Além disso, diferentemente do que ocorre para uma hiperesfera ${ }^{36}$, o volume de um hipercubo sempre cresce à medida que o número de dimensões (atributos) aumenta.

De modo geral, há quatro etapas principais para gerar, por meio de $\mathrm{Hy}$ perCubes, conjuntos de dados artificiais com $M_{r e l}$ atributos relevantes, $M_{i r r}$ atributos irrelevantes e nível de ruído nos multirrótulos $\mu$.

1. Gerar o hipercubo principal, com meia-aresta de tamanho 1 no espaço $\mathbb{R}^{M_{\text {rel }} \text {; }}$

2. Gerar $q$ pequenos hipercubos dentro do hipercubo principal, um por rótulo simples;

3. Gerar $N$ pontos (exemplos) no espaço $\mathbb{R}^{M}$ dentro do hipercubo principal;

36http://divisbyzero.com/2010/05/09/volumes-of-n-dimensional-balls. 
4. Gerar multirrótulos dos exemplos a partir dos pequenos hipercubos em que os pontos correspondentes estão situados e alterar cada rótulo $y_{l}$, $l=1 . . q$, de cada exemplo com probabilidade ${ }^{37} \mu$.

Após configurar apropriadamente os parâmetros de HyperCubes na ferramenta Mldatagen, foram gerados 45 conjuntos de dados artificiais -9 configurações $\times 5$ níveis de ruído $-\operatorname{com} M=20$ atributos. As nove configurações adotadas variam as quantidades de atributos $M_{r e l}$ e $M_{i r r}$, a quantidade de rótulos $q$ e a quantidade de exemplos $N$, como descrito na Tabela 5.2 para cada um dos níveis de ruído considerados: $\mu=0 \%, 5 \%, 10 \%, 20 \%, 40 \%$. Note que para cada configuração é atribuído um nome: dataset $_{1}$, dataset $_{2}, \ldots$, dataset $_{9}$.

Tabela 5.2: Nove configurações para conjuntos de dados artificiais com o mesmo nível de ruído

\begin{tabular}{l|c|c|c}
\hline \hline & $\begin{array}{c}N=10000 \\
q=100\end{array}$ & $\begin{array}{c}N=5000 \\
q=30\end{array}$ & $\begin{array}{c}N=2000 \\
q=10\end{array}$ \\
\hline \hline$M_{\text {rel }}=10, M_{\text {irr }}=10$ & dataset $_{1}$ & dataset $_{4}$ & dataset $_{7}$ \\
$M_{\text {rel }}=5, M_{\text {irr }}=15$ & dataset $_{2}$ & dataset $_{5}$ & dataset $_{8}$ \\
$M_{\text {rel }}=1, M_{\text {irr }}=19$ & dataset $_{3}$ & dataset $_{6}$ & dataset $_{9}$ \\
\hline \hline
\end{tabular}

Ao inserir ruído em um conjunto de dados multirrótulo, novos multirrótulos podem surgir. Quanto maior o nivel de ruído, maior a chance de que o número de multirrótulos distintos do conjunto de dados artificial seja aumentado.

\subsubsection{Medidas de Avaliação Específicas para Seleção de Atribu- tos}

Sejam $M_{r e l}$ e $M_{i r r}$ o tamanho dos eixos $y$ e $x$ respectivamente. Dado um ranking de $M_{r e l}$ atributos relevantes e $M_{i r r}$ irrelevantes, cada atributo $X_{j}, j=1 . . M$, do ranking é considerado em ordem decrescente de valores de importância para plotar uma curva fundamental para a medida Area Under Curve for $\mathrm{Fe}$ ature Ranking evaluation (FR-AUC), proposta neste trabalho (Spolaôr et al., 2013c). Se $X_{j}$ for relevante $\left(X_{R}\right)$, a curva cresce uma unidade no eixo $y$; caso contrário, $X_{j}$ é irrelevante $\left(X_{I}\right)$ e a curva cresce uma unidade no eixo $x$. A partir disso, FR-AUC é calculada como a área sob essa curva, normalizada pela área máxima $-M_{r e l} \times M_{i r r}$. Assim, o valor da medida varia entre 0 e 1 . Quanto maior o valor, melhor o ranking de atributos avaliado.

Para ilustrar, considere um conjunto de dados com $M_{r e l}=5, M_{i r r}=15 \mathrm{e}$ o ranking de atributos $\left(X_{R}, X_{R}, X_{I}, X_{R}, X_{R}, X_{I}, X_{I}, X_{R}, X_{I}, \ldots, X_{I}\right)$. A curva e a área sob a curva, correspondente a FR-AUC, são mostradas na Figura 5.3. Em particular, o valor da medida no exemplo é dado por:

\footnotetext{
${ }^{37}$ Se $y_{l}$ está no multirrótulo $Y$, então $y_{l}$ é removido de $Y$ com probabilidade $\mu$; caso contrário, $y_{l}$ é inserido em $Y$ com a mesma probabilidade.
} 


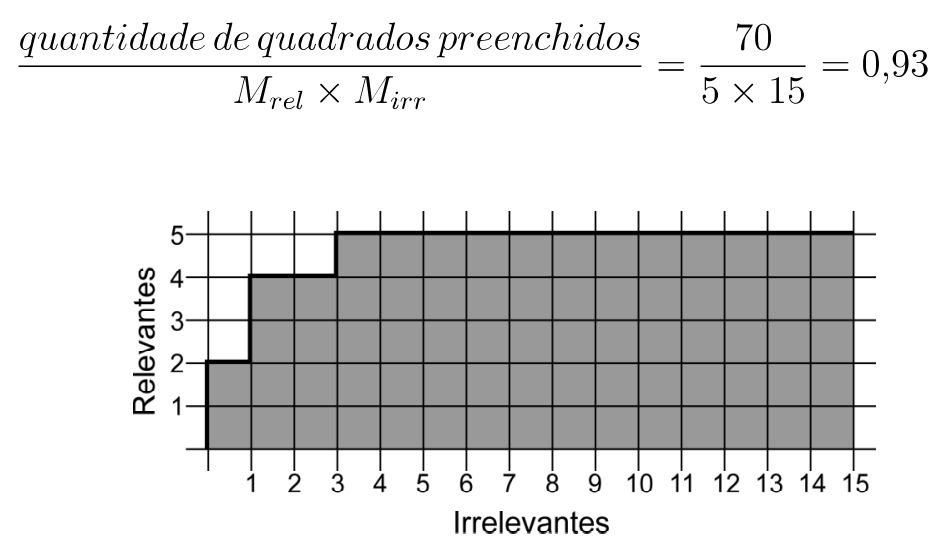

Figura 5.3: Ilustração da medida Area Under Curve for Feature Ranking evaluation

Motivado pela necessidade de medir a estabilidade de subconjuntos de atributos retornados por múltiplas execuções de um algoritmo ${ }^{38}$, Kuncheva (2007) propôs um índice de similaridade que satisfaz três propriedades para os subconjuntos $S_{1}$ e $S_{2}$, os quais possuem o mesmo número de elementos, i.e., $\left|S_{1}\right|=\left|S_{2}\right|=g, 0<g<M$. Seja $r=\left|S_{1} \cap S_{2}\right|$.

1. Monotonicidade: quando maior a intersecção entre $S_{1}$ e $S_{2}$, maior o valor de similaridade;

2. Limites: o índice deveria ser delimitado por constantes independentes de $M$ ou $g$. O valor máximo deveria ser atingido quanto os dois subconjuntos são iguais, i.e., para $r=g$;

3. Correção para o acaso: o índice deveria ter um valor constante para subconjuntos de atributos independentemente gerados de mesmo tamanho, $g$.

Para avaliar a cardinalidade esperada da intersecção entre subconjuntos, $r$ é considerada uma variável randômica obtida de subconjuntos $S_{1}$ e $S_{2}$ randomicamente amostrados de $X$ sem reposição. A partir disso, é formulado o indice de similaridade definido pela Equação 5.11, o qual varia entre 1 (similaridade máxima) e -1 .

$$
\begin{array}{r}
\operatorname{similaridade}\left(S_{1}, S_{2}\right)=\frac{r \times M-g^{2}}{g \times(M-g)}, \\
\text { onde } g=\left|S_{1}\right|=\left|S_{2}\right|
\end{array}
$$

\footnotetext{
${ }^{38}$ Um algoritmo de seleção de atributos estável identificaria subconjuntos de atributos similares se executado múltiplas vezes com a mesma configuração.
} 


\subsection{Resultados e Discussão}

Como mencionado, as avaliações experimentais iniciais comparam $R F-M L$ (HD) vs RF-ML (JD) e RFM-ML (HD) vs RFM-ML (JD). Após, a melhor dessas configurações é eleita para inclusão em uma avaliação experimental que inclui os algoritmos $R F-L P$ e $R F-B R$, baseados em ReliefF, e o algoritmo IG-LP - Figura 5.2, página 88. Medidas de avaliação descritas nas Seções 2.3 e 5.3.2 são adotadas para estimar a qualidade dos algoritmos de SA considerados. Exceto para FR-AUC, os valores apresentados são obtidos por meio da estratégia de validação cruzada de 10 folds com folds pareados.

\subsubsection{Comparação entre Configurações dos Algoritmos RF-ML e RFM-ML}

Estudos preliminares mostraram que o desempenho das duas configurações de $R F-M L$ consideradas, $R F-M L$ com dissimilaridade Hamming e dissimilaridade Jaccard, foi similar nos conjuntos de dados artificiais. Assim, nesta seção é dado enfoque aos experimentos realizados nos 10 conjuntos de dados benchmark descritos na Seção 2.5, página 32.

Nas Tabelas A.12 a A.15 do Apêndice, páginas 135 a 140, são descritos os resultados experimentais em termos de cada medida de avaliação de aprendizado multirrótulo considerada. Em cada célula dessas tabelas, são apresentados três valores relacionados a um classificador no formato:

\section{desempenho médio (desvio padrão) [ranking do classificador]}

Adicionalmente, é destacado em negrito o melhor desempenho por conjunto de dados. Células contendo resultados piores que os de Geral $_{B}$ - Tabela A.2 do Apêndice -, são coloridas em cinza.

Ao considerar o desempenho dos classificadores $B R k N N-b$ gerados a partir dos atributos selecionados pelas configurações $R F-M L$ em relação a Geral $_{B}$, é possivel observar resultados piores, em termos da medida Hamming Loss, em seis conjuntos de dados: 1-Cal500, 2-Corel5k, 3-Corel16k001, 5-Fapesp, 7-Llog-f* e 8-Magtag5k. Esses conjuntos também estão associados a resultados adversos de algoritmos de SA baseados em transformação do problema, como relatado nas Seções 4.5.1 e 4.5.2, páginas 65 e 70. Contudo, assim como ocorre com os métodos LCFS - configuração $S R-X$ - e IG-LP, a partir do subconjunto $\left|X^{\prime}\right|=40 \% M, R F-M L(H D)$ e $R F-M L$ (JD) são capazes de contribuir para gerar classificadores melhores que Geral $_{B}$ no conjunto de dados 8-Magtag5k. Em particular, $R F-M L(J D)$ já atinge esse resultado positivo a partir de um subconjunto menor $-\left|X^{\prime}\right|=30 \% M$. 
Convém ressaltar novamente, em favor dos algoritmos de SA, que nem mesmo o uso de todos os atributos para construir os classificadores BRkNN-b é suficiente para obter valor de Hamming Loss melhor que o de Geral $_{B}$ em cinco conjuntos de dados mencionados anteriormente, como descrito na Tabela A.3 do Apêndice, página 113.

Embora a diferença absoluta no desempenho dos classificadores gerados para cada quantidade de atributos seja pequena em geral, são encontradas diferenças significativas de acordo com o teste estatístico não paramétrico de Wilcoxon signed-ranks (Demšar, 2006; Wilcoxon, 1945) com $\alpha=0,05$. Para cada diferença, a configuração de $R F-M L$ estatisticamente superior é descrita na Tabela 5.3.

Tabela 5.3: Configuração $R F-M L$ significativamente melhor com nível de significância $\alpha=0,05$

\begin{tabular}{l|l|l|l|c}
\hline \hline$\left|X^{\prime}\right|$ & F-Measure & Hamming Loss & Accuracy & $F_{b}$ \\
\hline $20 \%$ & & $R F-M L(H D)$ & & \\
$50 \%$ & $R F-M L(J D)$ & & $R F-M L(J D)$ & $R F-M L(J D)$ \\
$60 \%$ & $R F-M L(J D)$ & & $R F-M L(J D)$ & $R F-M L(J D)$ \\
$80 \%$ & & $R F-M L(H D)$ & & \\
\hline \hline
\end{tabular}

Há um relativo equilíbrio entre as duas configurações, com duas vitórias para RF-ML com dissimilaridade Hamming na medida Hamming Loss e duas vitórias para $R F-M L$ com dissimilaridade Jaccard em cada uma das demais medidas de avaliação. Para complementar a comparação, os rankings médios dos classificadores $B R k N N-b$ calculados dentre os 10 conjuntos de dados benchmark são considerados. Na Tabela 5.4 são apresentados os rankings obtidos para cada quantidade de atributos $\left|X^{\prime}\right|$ e cada medida de avaliação. Note que em algumas células da tabela há mais de uma configuração, pois os rankings médios atingidos pelos classificadores correspondentes são iguais.

Tabela 5.4: Melhores rankings médios atingidos pelo algoritmo de classificação $B R k N N-b$ para cada quantidade de atributos $\left|X^{\prime}\right|$ selecionada pelo algoritmo $R F-M L$ com medidas de dissimilaridade Jaccard e Hamming e para cada medida de avaliação

\begin{tabular}{|c|c|c|c|c|c|c|c|c|c|c|c|}
\hline $\mathrm{FM}$ & $\begin{array}{c}10 \% M \\
R F-M L(J D)\end{array}$ & $\begin{array}{c}20 \% M \\
R F-M L(J D)\end{array}$ & $\begin{array}{c}30 \% M \\
R F-M L(J D)\end{array}$ & $\begin{array}{c}40 \% M \\
R F-M L(J D)\end{array}$ & $\begin{array}{c}50 \% M \\
R F-M L(J D)\end{array}$ & $\begin{array}{c}60 \% M \\
R F-M L(J D)\end{array}$ & $\begin{array}{c}70 \% M \\
R F-M L(J D)\end{array}$ & $\begin{array}{c}\frac{80 \% M}{R-M L(J D)} \\
R-1\end{array}$ & $\begin{array}{c}90 \% M \\
R F-M L(J D)\end{array}$ & \begin{tabular}{|c|}
$R F-M L(J D)$ \\
9
\end{tabular} & $\begin{array}{c}R F-M L(H D) \\
0\end{array}$ \\
\hline$H L$ & $R F-M L(H D)$ & $R F-M L(H D)$ & $R F-M L(H D)$ & RF-ML (JD) & $R F-M L(J D)$ & $R F-M L(J D)$ & $R F-M L(H D)$ & $R F-M L(H D)$ & $R F-M L(J D)$ & 4 & 7 \\
\hline Accuracy & RF-ML (JD) & $R F-M L(H D)$ & $R F-M L(J D)$ & $\frac{R F-M L(I D D)}{R F-M L(J D)}$ & $R F-M L(J D)$ & $R F-M L(J D)$ & $R F-M L(J D)$ & $R F-M L(J D)$ & $R F-M L(J D)$ & 8 & 1 \\
\hline$F_{b}$ & $R F-M L(H D)$ & $\begin{array}{l}R F-M L(J D) \\
R F-M L(H D)\end{array}$ & $R F-M L(J D)$ & $R F-M L(J D)$ & $R F-M L(J D)$ & $R F-M L(J D)$ & $R F-M L(J D)$ & $R F-M L(J D)$ & $R F-M L(J D)$ & 8 & 2 \\
\hline $\begin{array}{l}R F-M L(J D) \\
R F-M L(H D)\end{array}$ & $\begin{array}{l}2 \\
2\end{array}$ & $\begin{array}{l}2 \\
3\end{array}$ & $\begin{array}{l}3 \\
1\end{array}$ & $\begin{array}{l}4 \\
1\end{array}$ & $\begin{array}{c}4 \\
0\end{array}$ & $\begin{array}{l}4 \\
1\end{array}$ & $\begin{array}{l}3 \\
1\end{array}$ & $\begin{array}{l}3 \\
1\end{array}$ & $\begin{array}{l}4 \\
0 \\
0\end{array}$ & 29 & 10 \\
\hline
\end{tabular}

Assim como verificado na Tabela 5.3, há um destaque para $R F-M L$ com dissimilaridade Hamming na medida Hamming Loss e para RF-ML com dissimilaridade Jaccard nas demais medidas de avaliação. Se o critério de escolha da melhor configuração fosse baseada no número de casos com melhores rankings médios, RF-ML com dissimilaridade Jaccard seria eleito. Contudo, ao 
considerar os valores de ranking médio apresentados nas Tabelas A.12 a A.15 do Apêndice, páginas 135 a 140, é verificada a ocorrência de empates, i.e., existência de pelo menos dois classificadores com valores iguais para uma medida de avaliação. Como resumido na Tabela 5.5, a medida Hamming Loss é a que apresenta a maior quantidade de empates nos 90 casos avaliados - 10 conjuntos de dados $\times 9$ tamanhos de subconjuntos de atributos.

Tabela 5.5: Porcentagem de empates nos valores de cada medida de avaliação obtidos pelos classificadores $B R k N N-b$ gerados a partir do algoritmo $R F-M L$ com medidas de dissimilaridade Jaccard e Hamming

\begin{tabular}{rrrr}
\hline \hline F-Measure & Hamming Loss & Accuracy & $F_{b}$ \\
\hline $39 \%$ & $\mathbf{7 3} \%$ & $43 \%$ & $48 \%$ \\
\hline \hline
\end{tabular}

Como os classificadores $B R k N N-b$ apresentam maior dificuldade em termos de Hamming Loss - Tabela A.3 - , uma das mais utilizadas na literatura (Metz et al., 2014), a configuração $R F-M L$ com dissimilaridade Hamming, destacada nessa medida de avaliação mesmo com o relativamente alto número de empates, é eleita para os experimentos comparativos envolvendo algoritmos baseados em ReliefF da Seção 5.4.2. Entretanto, quando pertinente, resultados relacionados à configuração $R F-M L(J D)$ também são comentados.

Antes da comparação direta entre $R F-M L$ e $R F M-M L$, convém considerar a qualidade do algoritmo ReliefF Mútuo Multirrótulo usando as medidas de dissimilaridade Jaccard e Hamming. De modo geral, os resultados relativos são similares ao que foi verificado para $R F-M L$. Assim, ReliefF Mútuo Multirrótulo usando dissimilaridade Hamming se destacou na medida de avaliação Hamming Loss, enquanto que ReliefF Mútuo Multirrótulo com dissimilaridade Jaccard sobressai nas demais medidas.

RF-ML vs RFM-ML

A partir dos resultados obtidos anteriormente, foram realizadas as seguintes quatro comparações, uma por medida de avaliação.

1. F-Measure: RF-ML (JD) vs RFM-ML (JD);

2. Hamming Loss: RF-ML (HD) vs RFM-ML (HD);

3. Accuracy: RF-ML (JD) vs RFM-ML (JD);

4. $F_{b}: R F-M L(J D)$ vs RFM-ML (JD).

Embora a aplicação do teste de Wilcoxon signed-ranks com $\alpha=0,05$ não permitiu encontrar diferença significativa, os rankings médios dos classificadores apresentados na Tabela 5.6 destacam $R F-M L$ em relação a $R F M-M L$, independentemente da medida de avaliação. 
Tabela 5.6: Melhores rankings médios atingidos pelo algoritmo de classificação $B R k N N-b$ para cada quantidade de atributos $\left|X^{\prime}\right|$ selecionada pelos algoritmos $R F-M L$ e RFM-ML e para cada medida de avaliação

\begin{tabular}{|c|c|c|c|c|c|c|c|c|c|c|c|}
\hline & $10 \% M$ & $20 \% M$ & $30 \% M$ & $\overline{440 \% M}$ & $50 \% M$ & $60 \% M$ & $70 \% M$ & $80 \% M$ & $90 \% M$ & RF-ML & RFM-ML \\
\hline FM & $R F-M L$ & $\begin{array}{l}R F-M L \\
R F M-M L\end{array}$ & $\begin{array}{l}R F-M L \\
R F M-M L\end{array}$ & $\begin{array}{l}R F-M L \\
R F M-M L\end{array}$ & $R F-M L$ & $R F-M L$ & $\begin{array}{l}R F-M L \\
R F M-M L\end{array}$ & $\begin{array}{l}R F-M L \\
R F M-M L\end{array}$ & $R F-M L$ & 9 & 5 \\
\hline$H L$ & $R F-M L$ & $R F-M L$ & $R F-M L$ & $R F-M L$ & $\begin{array}{l}R F-M L \\
R F M-M L\end{array}$ & $R F-M L$ & $R F-M L$ & $R F-M L$ & $R F-M L$ & 9 & 1 \\
\hline Accuracy & $R F-M L$ & $\begin{array}{l}R F-M L \\
R F M-M L\end{array}$ & $\begin{array}{l}R F-M L \\
R F M-M L\end{array}$ & $\begin{array}{l}R F-M L \\
R F M-M L\end{array}$ & $\begin{array}{l}R F-M L \\
R F M-M L\end{array}$ & $R F-M L$ & $\begin{array}{l}R F-M L \\
R F M-M L\end{array}$ & $R F-M L$ & $\begin{array}{l}R F-M L \\
R F M-M L\end{array}$ & 9 & 6 \\
\hline$F_{b}$ & $R F-M L$ & $\begin{array}{l}R F-M L \\
R F M-M L\end{array}$ & $\begin{array}{l}R F-M L \\
R F M-M L\end{array}$ & $\begin{array}{l}R F-M L \\
R F M-M L\end{array}$ & $\begin{array}{l}\text { RF-ML } \\
R F M-M L\end{array}$ & $R F-M L$ & $\begin{array}{l}R F-M L \\
R F M-M L\end{array}$ & $R F-M L$ & $R F-M L$ & 9 & 5 \\
\hline$R F-M L$ & 4 & 4 & 4 & 4 & 4 & 4 & 4 & 4 & 4 & 36 & \\
\hline$R F M-M L$ & 0 & 3 & 3 & 3 & 3 & 0 & 3 & 1 & 1 & & 17 \\
\hline
\end{tabular}

Como RF-ML atinge o melhor ranking para todos os classificadores, esse algoritmo é eleito para o estudo comparativo com outros algoritmos baseados em ReliefF.

\subsubsection{Algoritmos baseados em ReliefF e IG-LP}

Nas Tabelas A.16 a A.35 do Apêndice, páginas 142 a 151, são descritos os rankings de atributos e valores de importância estimados por $R F-M L, R F-L P$, $R F-B R$ e IG-LP nos conjuntos de dados artificiais para cada nível de ruído. Convém ressaltar que $I G-L P$ atribui valor de importância mínimo (0) para vários atributos. Para destacar essa desvantagem, os atributos envolvidos foram ordenados em ordem decrescente de índice. Consequentemente, a medida FR-AUC - Seção 5.3.2 - se torna capaz de refletir o mau desempenho do algoritmo.

Na Tabela A.36 do Apêndice, página 151, são apresentados os valores de FR-AUC para os quatro algoritmos de seleção de atributos avaliados nesta seção. Em cada célula dessas tabelas, são apresentados dois valores relacionados a um conjunto de dados no formato:

\section{valor FR-AUC [ranking do algoritmo]}

Adicionalmente, é destacado em negrito o melhor desempenho em cada conjunto. Esses resultados são resumidos na Tabela 5.7, de modo que cada célula contém três valores relacionados a um algoritmo de SA no formato:

valor FR-AUC médio (desvio padrão) [ranking médio do algoritmo]

em que a média é calculada dentre os valores FR-AUC correspondentes a nove conjuntos de dados com um determinado nivel de ruído.

$R F-M L$ se sobressai independentemente do nível de ruído $\mu$. De fato, ao aplicar o teste estatístico não paramétrico de Friedman (Demšar, 2006; Friedman, 1940), com a hipótese nula de que o valor de FR-AUC atingido pelos 
Tabela 5.7: Resumo dos resultados experimentais nos conjuntos de dados artificiais de acordo com FR-AUC: algoritmos de SA baseados em ReliefF e $I G-L P$

\begin{tabular}{l|cccc}
\hline \hline & $R F-M L$ & $R F-L P$ & $R F-B R$ & $I G-L P$ \\
\hline \hline$\mu=0 \%$ & $\mathbf{1 , 0 0}(\mathbf{0 , 0 0})[\mathbf{2 , 1}]$ & $0,78(0,44)[2,6]$ & $0,98(0,07)[2,3]$ & $0,56(0,53)[3,1]$ \\
$\mu=5 \%$ & $\mathbf{1 , 0 0}(\mathbf{0 , 0 0 )}[\mathbf{1 , 7 ]}$ & $0,47(0,51)[2,8]$ & $0,98(0,07)[1,8]$ & $0,14(0,33)[3,7]$ \\
$\mu=10 \%$ & $\mathbf{1 , 0 0}(\mathbf{0 , 0 0}[\mathbf{1 , 7 ]}$ & $0,33(0,50)[3,1]$ & $0,98(0,07)[1,8]$ & $0,11(0,33)[3,5]$ \\
$\mu=20 \%$ & $\mathbf{1 , 0 0}(\mathbf{0 , 0 0 )}[\mathbf{1 , 5 ]}$ & $0,33(0,50)[3,1]$ & $0,97(0,07)[1,9]$ & $0,11(0,33)[3,5]$ \\
$\mu=40 \%$ & $\mathbf{0 , 8 9}(\mathbf{0 , 1 3}[\mathbf{1 , 4 ]}$ & $0,14(0,33)[3,2]$ & $0,88(0,13)[1,7]$ & $0,00(0,00)[3,7]$ \\
\hline todos & $\mathbf{0 , 9 8 ( 0 , 0 7 ) [ \mathbf { 1 , 7 } ]}$ & $0,41(0,49)[2,9]$ & $0,96(0,09)[1,9]$ & $0,18(0,38)[3,5]$ \\
\hline \hline
\end{tabular}

quatro algoritmos de SA é equivalente, foi constatada a rejeição da hipótese com $\alpha=0,05$ para $\mu=5 \%, 10 \%, \ldots, 40 \%$. A partir de cada rejeição, foi utilizado o pós-teste de Nemenyi, o qual afirma que uma diferença entre dois algoritmos é significativa se os rankings médios correspondentes diferem no mínimo pela Diferença Crítica (DC). Quando múltiplos algoritmos são comparados, os resultados do pós-teste podem ser representados graficamente em um diagrama simples, no qual algoritmos que não são significativamente diferentes são conectados por uma linha horizontal. Nas Figuras 5.4 e 5.5 são apresentados os diagramas correspondentes aos resultados do pós-teste para $\mu=10 \% \mathrm{e}$ $\mu=40 \%$, os quais são similares, respectivamente, aos diagramas para $\mu=5 \%$ e $\mu=20 \%$.

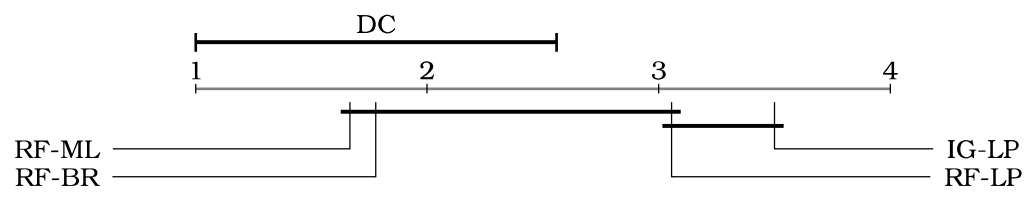

Figura 5.4: Diagrama do pós-teste de Nemenyi para algoritmos baseados em ReliefF e IG-LP nos conjuntos de dados artificiais com $\mu=10 \%$

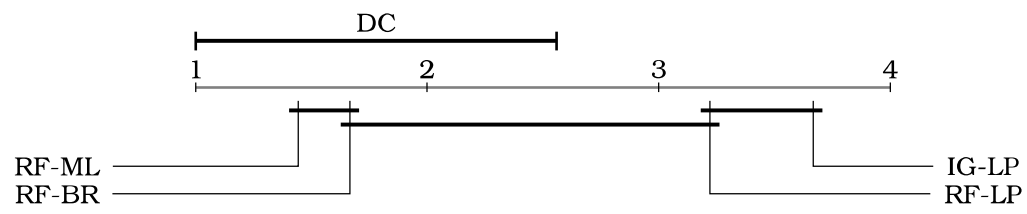

Figura 5.5: Diagrama do pós-teste de Nemenyi para algoritmos baseados em ReliefF e IG-LP nos conjuntos de dados artificiais com $\mu=40 \%$

Nos diagramas fica ainda mais evidente a superioridade de $R F-M L$ em relação a IG-LP. Além disso, nos níveis mais altos de ruído $-\mu=20 \%$ e $40 \%-$, $R F-M L$ é também superior a $R F-L P$. É possivel observar ainda que $R F-M L$ sempre atinge o menor (melhor) ranking médio, como descrito na Tabela 5.7, embora não tenha sido estatisticamente superior a $R F-B R$ em nenhum dos casos avaliados. $R F-B R$, por sua vez, é superior a $I G-L P$ já com $\mu=10 \%$.

Outro aspecto positivo obtido por RF-ML consiste no número de casos nos quais FR-AUC não atinge seu valor máximo, i.e., no número de vezes em que 
há pelo menos um atributo irrelevante ranqueado melhor que um ou mais atributos relevantes de um determinado conjunto de dados. Esse aspecto é retratado na Tabela 5.8 para cada nível de ruído e para todos os 45 conjuntos de dados artificiais.

Tabela 5.8: Número de casos nos quais $F R-A U C$ não atingiu seu valor máximo

\begin{tabular}{l|llll}
\hline \hline & $R F-M L$ & $R F-L P$ & $R F-B R$ & $I G-L P$ \\
\hline \hline$\mu=0 \%$ & $\mathbf{0}$ & 2 & 1 & 4 \\
$\mu=5 \%$ & $\mathbf{0}$ & 5 & 1 & 8 \\
$\mu=10 \%$ & $\mathbf{0}$ & 6 & 1 & 8 \\
$\mu=20 \%$ & $\mathbf{0}$ & 7 & 3 & 8 \\
$\mu=40 \%$ & $\mathbf{5}$ & 8 & 6 & 9 \\
\hline total & $\mathbf{5}$ & 28 & 12 & 37 \\
porcentagem & $\mathbf{1 1 \%}$ & $62 \%$ & $27 \%$ & $82 \%$ \\
\hline \hline
\end{tabular}

Como pode ser observado, $R F-M L$ atinge os melhores resultados para cada nível de ruído, falhando na obtenção do melhor valor de FR-AUC em somente $11 \%$ dos casos, todos eles relacionados ao maior nível de ruído - 40\%. Ainda assim, RF-ML deixa de obter o melhor valor de FR-AUC um número menor de vezes que os outros algoritmos considerados. Esse resultado sugere uma robustez a ruído. $R F-B R$ também obtém algum destaque, embora tenha falhado quase três vezes mais - 12 contra 5 - que $R F-M L$ em atingir o valor máximo FR-AUC. Por fim, os dois algoritmos de seleção de atributos baseados na abordagem $L P$ apresentam os piores resultados, inclusive para o nível de ruído $\mu=0 \%$.

Como mencionado na Seção 5.3.1, podem surgir novos multirrótulos quando ruído é inserido nos conjuntos de dados gerados pela estratégia HyperCubes. A partir disso, é possível supor que o conjunto de dados resultante de $L P$ seja muito desbalanceado, como ocorre frequentemente nessa abordagem, levando à seguinte questão:

À medida que aumenta o nivel de ruído $\mu$, os conjuntos de dados artificiais transformados por LP se tornam mais desbalanceados?

Uma maneira de responder essa questão é por meio da investigação da evolução da quantidade de multirrótulos distintos $D_{d} R$ gerados para cada uma das nove configurações de conjuntos de dados artificiais em função de $\mu$. Essa evolução é representada na Figura 5.6, na qual cada eixo corresponde à uma configuração e cada linha colorida consiste nos valores de $D_{d} R$ relacionados a um determinado nível de ruído.

Como os valores de $D_{d} R$ visivelmente aumentam junto com $\mu$, há um indício de que a abordagem $L P$ gera conjuntos de dados monorrótulo desbalanceados. Esse indício é reforçado quando se observa alguns dos valores máximos atingidos por $D_{d} R$ na Figura 5.6: 10000 e 5000. Como esses valores correspondem às quantidades de exemplos das configurações dataset dataset $_{2}$, dataset $_{3} \mathrm{e}$ 


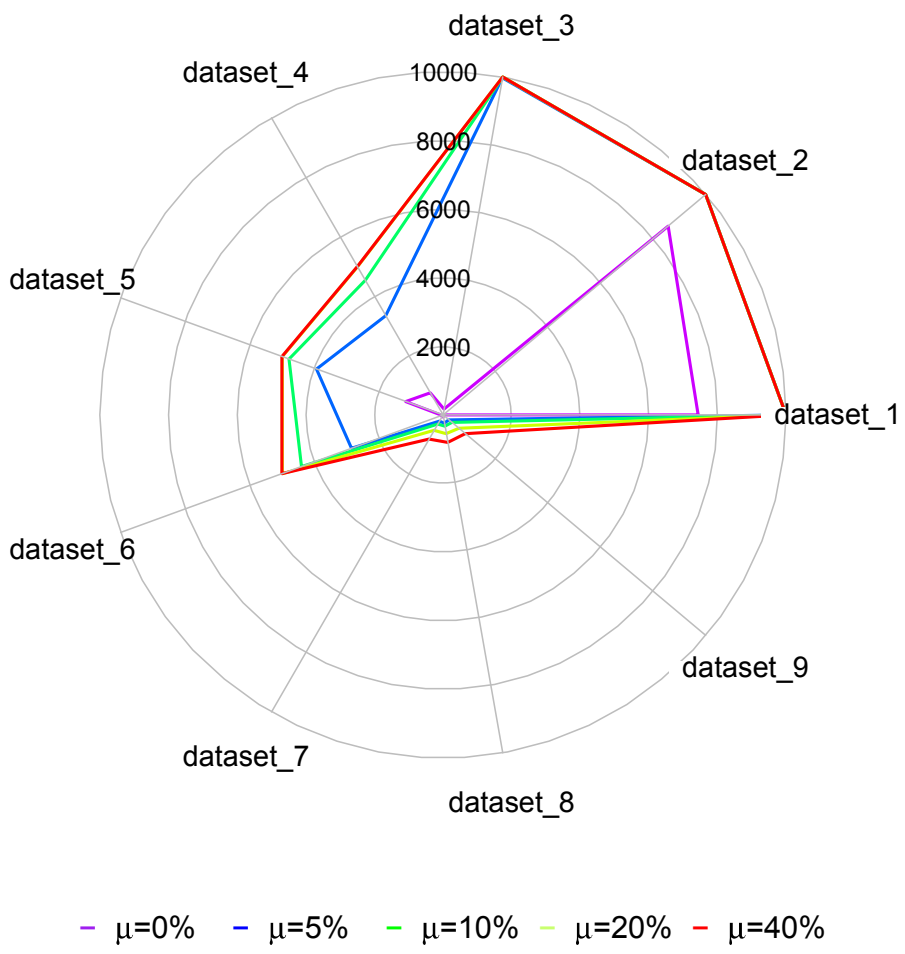

Figura 5.6: Evolução da quantidade de multirrótulos distintos obtidos utilizando a abordagem $L P$ em função do nível de ruído dos 45 conjuntos de dados artificiais

dataset $_{4}$, dataset $_{5}$, dataset $_{6}$, respectivamente, é possivel afirmar que, nos conjuntos resultantes de $L P$, há somente um exemplo por multirrótulo. Portanto, a resposta para a questão anterior é afirmativa.

Embora a relação entre nível de ruído e desbalanceamento para Label Powerset tenha sido encontrada nos conjuntos de dados artificiais, convém ressaltar que os algoritmos de seleção de atributos baseados nessa abordagem exibem resultados ruins mesmo quando $\mu=0 \%$. Isso fortalece o destaque do algoritmo $R F-M L$ proposto neste trabalho.

Convém ressaltar que experimentos usando dissimilaridade Jaccard para $R F-M L$ também foram realizados nos 45 conjuntos de dados artificiais. O desempenho atingido foi semelhante ao observado para a configuração utilizada nos demais experimentos, a qual usa dissimilaridade Hamming.

Experimentos em Conjuntos de Dados Benchmark

Nas Tabelas A.37 a A.40 do Apêndice, páginas 152 a 157, são descritos os resultados experimentais dos algoritmos $R F-M L, R F-B R, R F-L P$ e IG-LP em termos de cada medida de avaliação considerada. Em cada célula dessas 
tabelas, são apresentados três valores relacionados a um classificador $B R k N N$ $b$ no formato:

desempenho médio (desvio padrão) [ranking do classificador]

Adicionalmente, é destacado em negrito o melhor desempenho por conjunto de dados. Células contendo resultados piores que os de Geral $_{B}$ - Tabela A.2 do Apêndice -, são coloridas em cinza.

Embora não foi encontrada diferença significativa entre os quatro algoritmos de acordo com o teste de Friedman, os rankings médios dos classificadores calculados no teste possibilitam identificar qual foi o melhor algoritmo dentre os 10 conjuntos de dados considerados, como mostrado na Tabela 5.9 para cada quantidade de atributos $\left|X^{\prime}\right|$ e cada medida de avaliação. Note que em algumas células da tabela há mais de um algoritmo de SA, pois os rankings médios atingidos pelos classificadores correspondentes são iguais.

Tabela 5.9: Melhores rankings médios atingidos pelo algoritmo de classificação $B R k N N-b$ para cada quantidade de atributos $\left|X^{\prime}\right|$ selecionada pelos algoritmos baseados em ReliefF e IG-LP e para cada medida de avaliação

\begin{tabular}{|c|c|c|c|c|c|c|c|c|c|c|c|c|c|}
\hline & $10 \% M$ & $20 \% M$ & $30 \% M$ & $40 \% M$ & $50 \% M$ & $60 \% M$ & $70 \% M$ & $80 \% M$ & $90 \% M$ & $R F-M L$ & $R F-L P$ & $R F-B R$ & $I G-L P$ \\
\hline FM & $R F-L P$ & $I G-L P$ & $I G-L P$ & $I G-L P$ & $I G-L P$ & $I G-L P$ & $I G-L P$ & $I G-L P$ & $I G-L P$ & 0 & 1 & 0 & 8 \\
\hline$H L$ & $R F-L P$ & $R F-L P$ & $R F-L P$ & $R F-L P$ & $I G-L P$ & $R F-L P$ & $\begin{array}{l}R F-M L \\
I G-L P\end{array}$ & $R F-M L$ & $\begin{array}{l}R F-M L \\
R F-L P \\
R F-B R\end{array}$ & 3 & 6 & 1 & 2 \\
\hline$A C$ & $I G-L P$ & $\begin{array}{l}R F-M L \\
R F-L P\end{array}$ & $I G-L P$ & $I G-L P$ & $I G-L P$ & $I G-L P$ & $I G-L P$ & $I G-L P$ & $I G-L P$ & 1 & 1 & 0 & 8 \\
\hline$F_{b}$ & $I G-L P$ & $R F-M L$ & $I G-L P$ & $I G-L P$ & $I G-L P$ & $I G-L P$ & $I G-L P$ & $I G-L P$ & $I G-L P$ & 1 & 0 & 0 & 8 \\
\hline$R F-M L$ & 0 & 2 & 0 & $\overline{0}$ & 0 & 0 & $\overline{1}$ & $\overline{1}$ & 1 & 5 & & & \\
\hline$R F-L P$ & 2 & 2 & 1 & 1 & 0 & 1 & 0 & 0 & 1 & & 8 & & \\
\hline$R F-B R$ & 0 & 0 & 0 & 0 & 0 & 0 & 0 & 0 & 1 & & & 1 & \\
\hline$I G-L P$ & 2 & 1 & 3 & 3 & 4 & 3 & 4 & 3 & 3 & & & & 26 \\
\hline
\end{tabular}

Com relação aos melhores rankings médios, o algoritmo IG-LP é visivelmente melhor na maioria dos casos - 26 de 36 - seguido por $R F-L P$ e $R F$ $M L$, os quais obtêm destaque em 8 e 5 casos respectivamente. Contudo, ao se considerar os melhores rankings em termos da quantidade de atributos selecionados $\left|X^{\prime}\right|$, é verificado um equilíbrio maior entre esses três algoritmos para as duas menores quantidades $-\left|X^{\prime}\right|=10 \% M$ e $20 \% M$. Nesse caso, RF-LP, $I G-L P$ e $R F-M L$ estão relacionados, respectivamente, a 4,3 e 2 dos melhores rankings calculados.

Embora as medidas de avaliação utilizadas em cada caso sejam diferentes, convém ressaltar que o destaque de IG-LP e $R F-L P$ nos conjuntos de dados benchmark contrasta com o destaque de $R F-M L$ e $R F-B R$ nos conjuntos de dados artificiais. Isso motiva a realização de estudos complementares com o intuito de melhor avaliar esses algoritmos de seleção de atributos.

Note que, na Tabela 5.9, Hamming Loss é a única medida de avaliação em que IG-LP não sobressai nos conjuntos benchmark. Um aspecto que pode ser 
observado na Tabela A.38 do Apêndice, página 154, é que essa medida está relacionada a uma quantidade elevada de empates, i.e., uma alta ocorrência de pelo menos dois classificadores com o mesmo desempenho por conjunto de dados. De fato, como descrito na Tabela 5.10, Hamming Loss atinge a maior quantidade de empates - 99\%. Note que algo similar foi observado para duas configurações do RF-ML - Tabela 5.5.

Tabela 5.10: Porcentagem de empates nos valores de cada medida de avaliação obtidos por classificadores $B R k N N$ - $b$ gerados a partir de algoritmos de SA baseados em ReliefF e IG-LP

\begin{tabular}{rrrc}
\hline \hline F-Measure & Hamming Loss & Accuracy & $F_{b}$ \\
\hline $81 \%$ & $\mathbf{9 9} \%$ & $86 \%$ & $77 \%$ \\
\hline \hline
\end{tabular}

Deve ser observado que, quando se dobra o número de casas decimais para os valores da medida Hamming Loss, o número de empates é reduzido para somente $2 \%$ dos casos. Além disso, há uma mudança na distribuição dos melhores rankings médios, destacando $R F-M L$ com o melhor resultado em 7 das 9 quantidades de atributos, em especial para as menores quantidades $-\left|X^{\prime}\right|=10 \% M, \ldots, 40 \% M$. Nesse novo cenário, IG-LP obtém os melhores resultados somente em quantidades maiores, iniciando em $\left|X^{\prime}\right|=40 \% M$.

A partir disso, é possivel fazer duas considerações:

1. Nos experimentos realizados, a medida Hamming Loss foi a que apresentou a menor variação nos seus valores;

2. Ao aumentar a precisão dos valores de Hamming Loss, foi verificado um destaque do RF-ML, proposto neste trabalho, usando dissimilaridade Hamming para os menores subconjuntos de atributos, o que é considerado um bom resultado para a tarefa de seleção de atributos.

No que se refere à segunda consideração, convém destacar que parece existir uma relação entre a medida de avaliação de aprendizado multirrótulo Hamming Loss e a medida de dissimilaridade Hamming. Esse indício foi reforçado por meio de experimentos usando a medida de dissimilaridade Jaccard para $R F-M L$ nos 10 conjuntos de dados benchmark, os quais indicam que, nesse caso, $R F-M L$ obtém em geral os piores rankings médios em relação a $R F-L P$, $R F-B R$ e $I G-L P$.

Outra maneira de avaliar a qualidade de um algoritmo de seleção de atributos envolve verificar sua capacidade em reduzir a dimensionalidade de um conjunto de dados, ao mesmo tempo que contribui para a geração de um classificador com desempenho superior ou semelhante ao classificador construído usando todos os atributos. Nesse aspecto, de acordo com o teste de Wilcoxon, 
nenhuma diferença significativa foi encontrada nos conjuntos de dados benchmark entre os classificadores $B R k N N$ - $b$ resultantes de IG-LP e os que foram gerados usando todos os atributos. Um resultado similar foi observado entre classificadores resultantes de $R F-M L$ e os classificadores construídos usando todos os atributos, exceto para $\left|X^{\prime}\right|=60 \% M$, onde $R F-M L$ foi significativamente inferior nas medidas de avaliação Accuracy, F-Measure e $F_{b}$.

Desse modo, tanto IG-LP quanto $R F-M L$ se destacam na capacidade de reduzir em 10\%,20\%, . ., 50\%, 70\%,80\%, 90\% a dimensionalidade dos conjuntos de dados benchmark sem perda significativa do desempenho preditivo dos classificadores $B R k N N-b$. Além disso, IG-LP também se destaca para $\left|X^{\prime}\right|=60 \% M$.

Um estudo adicional para complementar a presente avaliação experimental consiste no uso de algoritmos de classificação alternativos a BRkNN- $b$. Como parte deste trabalho, além de BRkNN-b, foram considerados em (Spolaôr e Monard, 2014) dois métodos de aprendizado multirrótulo que usam o algoritmo monorrótulo não lazy Naive Bayes como base: BR e BR+ (Cherman et al., 2012). Nesse contexto, quatro algoritmos de SA foram avaliados nos mesmos conjuntos de dados benchmark adotados neste capítulo:

\section{IG-BR;}

2. RF-BR com $k$ específico para cada conjunto de dados;

3. RF-LP com $k$ específico para cada conjunto de dados;

4. RF-ML com dissimilaridade Hamming e $k$ específico para cada conjunto de dados.

Ao aplicar o teste de Friedman com pós-teste de Nemenyi para 108 experimentos -4 algoritmos de $\mathrm{SA} \times 9$ quantidades de atributos $\times 3$ métodos de aprendizado - foram observadas 14 diferenças significativas $-13 \%$ do total - com $\alpha=0,05$. Das 14 diferenças, a maioria (12) está relacionada ao mesmo algoritmo adotado neste capítulo - BRkNN-b - e indicam inferioridade de $R F-M L$ em relação a $I G-B R$. Contudo, convém ressaltar que $I G-B R$ tem algumas desvantagens, tais como uma complexidade relativamente alta $O\left(N_{t r} \times M \times q\right)$ - e o custo adicional relacionado à discretização de dados após a transformação do problema via $B R$.

Como mencionado na presente avaliação experimental, IG-LP se destaca nos conjuntos de dados benchmark, enquanto que $R F-M L$ com dissimilaridade Hamming sobressai nos conjuntos de dados artificiais. No primeiro contexto, foram consideradas medidas de avaliação de aprendizado, enquanto que no segundo foi utilizada uma medida independente de classificador - FR-AUC. Outra medida de avaliação para SA independente de classificador consiste no 
índice de similaridade entre subconjuntos de atributos — Seção 5.3.2. Na Tabela 5.11 são apresentados os valores médios desse índice, calculados dentre os 10 conjuntos de dados benchmark para $\left|X^{\prime}\right|=10 \% M^{39}$. Nessas tabelas, as menores similaridades médias são destacadas em negrito.

Tabela 5.11: Similaridade média dos subconjuntos de atributos de tamanho $\left|X^{\prime}\right|=10 \% M$ encontrados pelos algoritmos de SA baseados em ReliefF e IG-LP

\begin{tabular}{rrrrr}
\hline \hline & $I G-L P$ & $R F-B R$ & $R F-L P$ & $R F-M L$ \\
\hline$I G-L P$ & & 0,40 & 0,51 & $\mathbf{0 , 2 2}$ \\
$R F-B R$ & 0,40 & & 0,69 & $\mathbf{0 , 2 8}$ \\
$R F-L P$ & 0,51 & 0,69 & & $\mathbf{0 , 3 0}$ \\
$R F-M L$ & $\mathbf{0 , 2 2}$ & $\mathbf{0 , 2 8}$ & $\mathbf{0 , 3 0}$ & \\
\hline \hline
\end{tabular}

Independentemente da quantidade de atributos, $R F-M L$, proposto neste trabalho, apresenta similaridade média menor que a dos demais algoritmos nos conjuntos de dados benchmark. Isso sugere que $R F-M L$ pode ser uma boa opção para ser combinado com outros algoritmos de seleção de atributos por meio de métodos como ensemble (Cannas et al., 2013).

Desse modo, é possível observar que $R F-M L$ se destaca em mais uma medida independente de classificador, enquanto IG-LP segue se sobressaindo em medidas de avaliação de aprendizado. Contudo, convém destacar um aspecto negativo tanto para $R F-L P$ quanto para IG-LP: a dependência da abordagem de transformação do problema Label Powerset. Como mencionado, embora $L P$ considere alguma relação entre rótulos, a conversão de dados multirrótulo para monorrótulo inerente à abordagem LP pode levar a dados desbalanceados. Além disso, há uma limitação nos multirrótulos considerados, pois os multirrótulos estão restritos àqueles presentes no conjunto de treinamento $D_{t r}$, o que pode prejudicar a qualidade dos atributos selecionados e do consequente aprendizado em aplicações práticas.

Por outro lado, $R F-M L$ independe de qualquer abordagem de transformação do problema enquanto mantém propriedades do algoritmo ReliefF, como a consideração de interações entre atributos e a dispensa da discretização de dados. Nesse sentido, se um conjunto de dados possuir fortes interações entre atributos, é esperado que $R F-M L$ obtenha um bom resultado. Adicionalmente, o algoritmo apresenta complexidade menor que a de $R F-B R$.

\subsection{Considerações Finais}

Neste capítulo foram descritas e avaliadas duas extensões propostas neste trabalho para seleção de atributos multirrótulo via ReliefF, $R F-M L$ e $R F M-M L$, as quais são independentes de abordagem de transformação do problema. Foi

\footnotetext{
${ }^{39}$ Nas Tabelas A.41 a A.48 do Apêndice, páginas 159 a 160, são descritos os resultados para as demais quantidades de atributos selecionados.
} 
realizada uma avaliação experimental em 45 conjuntos de dados artificiais e em 10 conjuntos de dados benchmark. Essa avaliação contempla a comparação de configurações das extensões propostas, dentre as quais uma foi eleita - RF-ML com medida de dissimilaridade Hamming - para comparação com os algoritmos baseados em transformação do problema $R F-B R, R F-L P$ e $I G-L P$. Enquanto $R F-B R$ e $R F-L P$ são baseados no algoritmo tradicional ReliefF, $I G-L P$ foi destacado em relação a outros algoritmos baseados em transformação do problema.

Nos conjuntos de dados artificiais, o algoritmo $R F-M L$ proposto neste trabalho obtém, em vários casos, superioridade significativa em relação a $I G-L P$, além de apresentar uma possível robustez a ruído. Embora não foi encontrada diferença significativa entre os quatro algoritmos nos conjuntos de dados benchmark, IG-LP é geralmente melhor quando os rankings médios dos classificadores são considerados.

Embora as medidas de avaliação utilizadas em cada caso sejam diferentes, convém ressaltar que o destaque de $I G-L P$ e $R F-L P$ nos conjuntos de dados benchmark contrasta com o destaque de $R F-M L$ e $R F-B R$ nos conjuntos de dados artificiais. Assim, estudos complementares foram realizados com o intuito de melhor avaliar esses algoritmos.

Um aumento na precisão dos valores da medida Hamming Loss, a qual apresenta a menor variação nos seus valores, leva ao destaque do algoritmo proposto $R F-M L$ para os menores subconjuntos de atributos avaliados. Isso sugere, por exemplo, uma possível relação entre Hamming Loss e a medida de dissimilaridade Hamming usada por RF-ML. Adicionalmente, em um estudo complementar com uma medida de avaliação independente de classificador, $R F-M L$ apresenta similaridade média menor que a dos demais algoritmos nos conjuntos de dados benchmark.

Como mencionado, é importante destacar que $I G-L P$ e $R F-L P$ apresentam desvantagens inerentes à abordagem Label Powerset que podem, por exemplo, prejudicar a qualidade dos atributos selecionados e do consequente aprendizado em aplicações práticas. Por outro lado, $R F-M L$ evita essas desvantagens, é mais veloz que $R F-L P$ e $R F-B R$ e dispensa discretização de dados, além de considerar relações entre rótulos - o algoritmo trata diretamente o problema multirrótulo sem a necessidade de transformá-lo.

No próximo capítulo são apresentadas as conclusões deste trabalho, incluindo limitações e trabalhos futuros. 


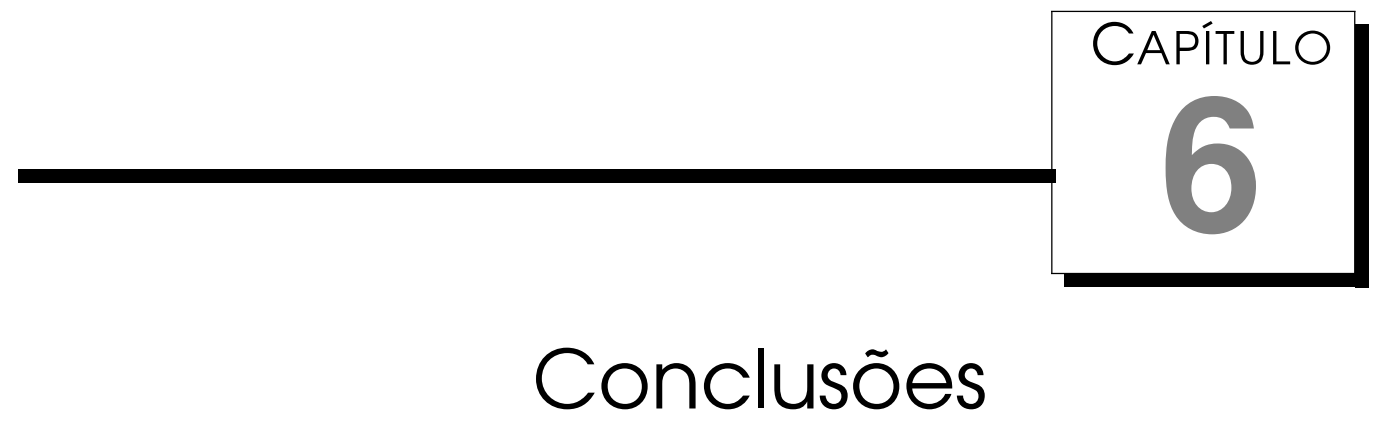

Neste capítulo são apresentadas as conclusões decorrentes desta tese de doutorado. O objetivo do trabalho, bem como cada contribuição principal apresentada na tese, limitações e trabalhos futuros correspondentes, são considerados brevemente.

O objetivo do trabalho consistiu no projeto e na implementação de algoritmos filtro de seleção de atributos multirrótulo que consideram relações entre rótulos, visando verificar a hipótese de que algoritmos de seleção de atributos em dados multirrótulo que consideram a dependência de rótulos teriam um melhor desempenho que aqueles que ignoram essa informação.

As seguintes contribuições estão diretamente relacionadas ao objetivo e foram apresentadas em capítulos específicos desta tese:

Capítulo 3. Revisão Sistemática sobre Seleção de Atributos Multirrótulo;

Capítulo 4. Seleção de Atributos de Segunda Ordem via Construção de Rótulos;

Capítulo 5. Extensão do Algoritmo Relieff para Seleção de Atributos Multirrótulo.

\subsection{Revisão Sistemática sobre Seleção de Atributos Multirrótulo}

A revisão sistemática realizada neste trabalho permitiu explorar, de modo amplo, rigoroso e reprodutível, a literatura de seleção de atributos multirrótulo. Um dos aspectos positivos provenientes da aplicação desse método foi 
a sintese de 74 publicações relacionadas, a qual estabelece um panorama contemporâneo da área de pesquisa de interesse. Com relação ao objetivo, a revisão sistemática evidencia que a consideração da dependência de rótulos na seleção de atributos é incipiente. De fato, vários dos trabalhos categorizados em termos de intensidade de exploração da dependência de rótulos como estratégias de segunda ordem e alta ordem foram publicados somente a partir de 2011.

Uma limitação do processo de revisão sistemática, também compartilhada por outros tipos de revisão bibliográfica, é que ela deve ser reaplicada para atualizar o panorama da área de pesquisa considerada. Embora o processo de revisão sistemática facilite a reaplicação, devido ao estabelecimento prévio de um protocolo reprodutivel, o uso de ferramentas computacionais de apoio, como o StArt ${ }^{40}$ (Hernandes et al., 2012), se faz importante no gerenciamento a longo prazo. Nesse sentido, um trabalho futuro consiste na comparação das ferramentas computacionais disponíveis para suporte à revisão sistemática. Esse estudo poderia ainda fomentar o desenvolvimento de novos frameworks para atender lacunas eventualmente encontradas.

\subsection{Seleção de Atributos de Segunda Ordem via Construção de Rótulos}

Uma maneira inovadora por meio da qual este trabalho atendeu ao objetivo consiste na proposta do método Label Construction for Feature Selection como uma alternativa para adicionar a consideração de relações entre rótulos à abordagem Binary Relevance de transformação do problema multirrótulo para monorrótulo. Esse método pode ser aplicado quando há interesse na consideração de relações de segunda ordem para a seleção de atributos, independentemente da medida de importância utilizada.

A avaliação experimental realizada em 10 conjuntos de dados benchmark aponta que a configuração $S R-X$ se destacou como a única configuração LCFS que levou à melhora de alguns classificadores originalmente piores que o baseline $\mathrm{Geral}_{B}$, além de obter a maior quantidade de classificadores BRkNN-b com ranking médio máximo. Adicionalmente, um estudo específico para comparação entre essa configuração e IG-BR indica, em alguns casos, que o primeiro foi significativamente superior em termos de desempenho preditivo (teste de Wilcoxon, $\alpha=0,05)$. O método LCFS, por meio da configuração $S R-X$, também incrementou a competitividade de $I G-B R$ em relação ao melhor método de seleção de atributos baseado em transformação do problema - IG-LP. No entanto, não foi encontrada diferença significativa entre SR-X e IG-LP.

\footnotetext{
40 http://lapes.dc.ufscar.br/tools/start_tool
} 
Com relação ao objetivo, essa avaliação experimental contribuiu ao obter uma evidência de que a consideração de relação entre rótulos é importante para a seleção de atributos multirrótulo baseada em abordagens de transformação do problema. Em particular, isso pode ser verificado pela superioridade relativa exibida por $I G-L P$ e $S R-X$ em relação a métodos baseados especificamente na abordagem $B R$, a qual frequentemente ignora a dependência de rótulos. Convém ressaltar que $S R-X$ evita desvantagens da abordagem $L P$, como o usual desbalanceamento dos dados multiclasse resultantes e a incapacidade de predizer multirrótulos que não participam do conjunto de treinamento.

Uma limitação do método LCFS consiste no aumento temporário da quantidade de rótulos simples de um conjunto de dados multirrótulo durante a tarefa de seleção de atributos. Essa limitação tem implicação na complexidade, em termos do número de rótulos, inerente aos algoritmos de SA baseados na abordagem $B R$. Alternativas que poderiam ser investigadas em trabalho futuro incluem a realização de seleção ou redução de rótulos (label selection ou label extraction) (Pacharawongsakda e Theeramunkong, 2013; Charte et al., 2012) para eliminar rótulos pouco significativos gerados pelo LCFS. É possível que, além da diminuição no custo computacional, outros benefícios sejam verificados com essas alternativas, a exemplo do que pode ser verificado com a seleção de atributos. Adicionalmente, a avaliação do parâmetro $q^{\prime}$, responsável por estabelecer a quantidade de novos rótulos, pode ser explorada.

Outro trabalho futuro consiste na proposta e avaliação de configurações mais sofisticadas para o método LCFS. Algumas delas poderiam ser baseadas, por exemplo, na ponderação dos pares de rótulos de acordo com a intensidade da relação entre os rótulos envolvidos, de modo similar ao que foi realizado em um método de SA multirrótulo independente de transformação do problema (Jungjit et al., 2013).

\subsection{Extensão do Algoritmo Relieff para Seleção de Atributos Multirrótulo}

Outra maneira pela qual este trabalho perseguiu seu objetivo foi por meio da proposta das adaptações $R F-M L$ e $R F M-M L$, baseadas em ReliefF e RReliefF, para realizar SA diretamente, sem nenhuma transformação do problema. Desse modo, os multirrótulos presentes no conjunto de dados, bem como as relações entre os rótulos simples, são preservados. Diferentemente do método LCFS, essa alternativa é especifica para a medida de importância inerente a algoritmos da família Relief, podendo ser apropriada quando há interesse em um método de SA multirrótulo que dispensa discretização de dados numéricos e considera interações entre atributos. A principal modificação realizada por 
ambas extensões consiste no uso da dissimilaridade entre conjuntos de rótulos (multirrótulos), ao invés da dissimilaridade entre valores de classe monorrótulo inerente a RReliefF, para modelar a probabilidade de que dois exemplos tenham rotulação diferente.

A avaliação experimental foi realizada em 45 conjuntos de dados artificiais, gerados com um framework desenvolvido em colaboração (Tomás et al., 2014) e em 10 conjuntos de dados benchmark. Inicialmente, foi verificado o destaque de $R F-M L$ em relação a $R F M-M L$, o que motivou a escolha do primeiro para comparação com métodos de seleção de atributos baseados em transformação do problema que combinam as abordagens $B R$ e $L P$ com o ReliefF original.

Nos conjuntos de dados artificiais, em alguns casos, $R F-M L$ foi significativamente superior (teste de Friedman, $\alpha=0,05$ ) a IG-LP e $R F-L P$ em termos da medida $F R-A U C$, a qual é independente da tarefa de classificação. Adicionalmente, os únicos casos em que RF-ML não encontrou subconjuntos de atributos esperados ocorreram no mais alto nivel de ruído avaliado $-\mu=40 \%-$, o que sugere uma robustez a ruído. Por outro lado, nos conjuntos de dados benchmark, embora não houve diferença significativa, IG-LP levou em geral a classificadores $B R k N N$ - $b$ com melhor ranking médio do que os classificadores correspondentes a $R F-M L$.

A divergência verificada entre os resultados provenientes de conjuntos de dados artificiais e benchmark motivou a realização de estudos complementares envolvendo $R F-M L$ e IG-LP. Um dos aspectos identificados nesses estudos indica que o algoritmo $R F-M L$ se destacou em conjuntos de dados benchmark quando houve um aumento na precisão dos resultados da medida de avaliação com menor variação de seus valores - Hamming Loss. Consequentemente, parece haver uma relação entre Hamming Loss e a medida de dissimilaridade Hamming utilizada por $R F-M L$. Outro aspecto é que tanto IG-LP quanto $R F-M L$ se destacaram na capacidade de reduzir a dimensionalidade dos conjuntos de dados benchmark sem perda significativa de desempenho preditivo. Ainda nos conjuntos benchmark, RF-ML selecionou em geral subconjuntos de atributos com a menor similaridade média em relação aos subconjuntos identificados pelos demais métodos. Isso pode motivar sua inclusão em ensembles de algoritmos de seleção de atributos multirrótulo. Ensembles de métodos de SA já têm sido estudados em dados monorrótulo (Prati, 2012).

Embora $R F-M L$ evite desvantagens da abordagem $L P$, como a limitação dos multirrótulos considerados àqueles que estão presentes no conjunto de treinamento, isso não se refletiu em superioridade significativa, em termos de desempenho preditivo, nos conjuntos de dados benchmark multirrótulo. Alternativas que poderiam ser estudadas em trabalhos futuros incluem a investigação da relação entre medidas de dissimilaridade para $R F-M L$ e medidas de 
avaliação multirrótulo, bem como a avaliação empírica de outros parâmetros do algoritmo, como exemplificado em (Slavkov et al., 2013) para SA multirrótulo hierárquica. Adicionalmente, um estudo mais aprofundado de características dos conjuntos de dados com auxílio, por exemplo, de frameworks para análise visual (Carvalho et al., 2014), é importante para melhor entendimento dos resultados que foram obtidos.

Uma limitação inerente às avaliações experimentais apresentadas consiste na dificuldade em avaliar classificadores multirrótulo. Diferentemente do que ocorre no aprendizado monorrótulo, também é necessário levar em conta predições parcialmente corretas. Múltiplas medidas de avaliação deveriam ser utilizadas, a exemplo do que foi realizado neste trabalho, pois cada medida considera explicita ou implicitamente diferentes aspectos do classificador. Assim, mecanismos que, como o baseline $\operatorname{Geral}_{B}$, auxiliam na análise de múltiplas medidas de avaliação multirrótulo deveriam ser investigados no futuro.

Aplicações dos métodos propostos em conjuntos de dados reais, como o conjunto 5-Fapesp construído por membros de nosso laboratório, também são de interesse no futuro. Além disso, a proposta de algoritmos multirrótulo como o apresentado em (Jungjit et al., 2013), os quais avaliam subconjuntos de atributos ao invés de avaliar atributos individualmente, atenderia outra limitação deste trabalho.

Convém ressaltar que as implementações desenvolvidas neste trabalho estão publicamente disponíveis em http://www.labic.icmc. usp.br/pub/mcmonard/Implementations/Multilabel/lcfs.zip e em http: //www.labic.icmc.usp.br/pub/mcmonard/Implementations/ 


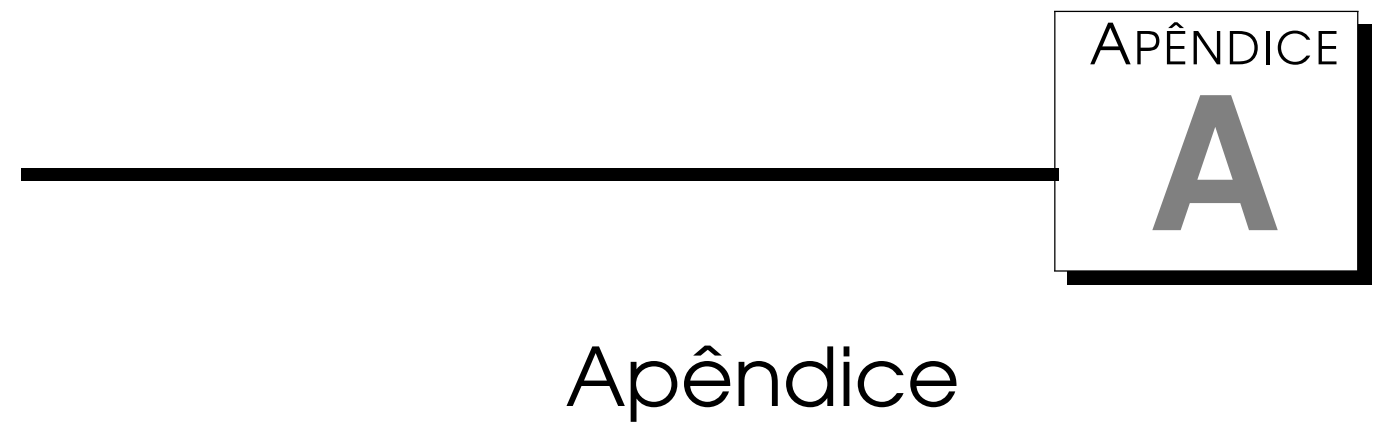

\section{A. 1 Resultados Complementares Relacionados à Re- visão Sistemática}

Nesta seção são apresentadas informações básicas dos 74 trabalhos identificados pelo processo de revisão sistemática aplicado neste trabalho. $\mathrm{Na}$ Tabela A.1 são apresentados o ID e a referência à publicação, bem como as abordagens que instanciam as três dimensões propostas para a seleção de atributos multirrótulo descritas na Seção 3.1, Página 38.

Tabela A. 1: Informações dos 74 trabalhos encontrados na revisão sistemática

\begin{tabular}{|c|c|c|c|c|}
\hline ID & referência & $\begin{array}{l}\text { interação com o } \\
\text { algoritmo de indução }\end{array}$ & $\begin{array}{l}\text { escopo do } \\
\text { multirrótulo }\end{array}$ & $\begin{array}{l}\text { dependência } \\
\text { de rótulos }\end{array}$ \\
\hline 1 & (Oliveira et al., 2010) & wrapper & monorrótulo & primeira ordem \\
\hline 2 & (Saleh e El-Sonbaty, 2007) & filtro & multirrótulo & primeira ordem \\
\hline 3 & (Luo et al., 2011) & filtro & monorrótulo & primeira ordem \\
\hline 4 & (Mittal e Cheong, 2004) & filtro & híbrido & primeira ordem \\
\hline 5 & (Wandekokem et al., 2010) & wrapper & monorrótulo & primeira ordem \\
\hline 6 & (Noh et al., 2004) & embutida & multirrótulo & segunda ordem \\
\hline 7 & (Esuli et al., 2008) & embutida & híbrido & primeira ordem \\
\hline 8 & (Olsson e Oard, 2006) & filtro & monorrótulo & primeira ordem \\
\hline 9 & (Novovičová et al., 2007) & filtro & multirrótulo & primeira ordem \\
\hline 10 & (Skarmeta et al., 2000) & filtro & monorrótulo & primeira ordem \\
\hline 11 & (Wandekokem et al., 2011) & wrapper & monorrótulo & primeira ordem \\
\hline 12 & (Nardiello et al., 2003) & filtro & monorrótulo & primeira ordem \\
\hline 13 & (Chen et al., 2007) & filtro & híbrido & alta ordem \\
\hline 14 & (Wang et al., 2009) & filtro & não reconhecido & não reconhecido \\
\hline 15 & (Yang et al., 2007) & filtro & monorrótulo & primeira ordem \\
\hline 16 & (Shimosaka et al., 2009) & não reconhecido & não reconhecido & não reconhecido \\
\hline 17 & (Doquire e Verleysen, 2011) & filtro & híbrido & alta ordem \\
\hline 18 & (Zhang et al., 2009) & wrapper & multirrótulo & alta ordem e segunda ordem \\
\hline 19 & (Li et al., 2010a) & embutida & multirrótulo & segunda ordem \\
\hline 20 & (Cong e Tong, 2008) & filtro & monorrótulo & primeira ordem \\
\hline 21 & (Islam e Sluzek, 2010) & embutida & híbrido & alta ordem \\
\hline 22 & (Mayne e Perry, 2009) & filtro & monorrótulo & primeira ordem \\
\hline 23 & (Tan, 2007) & filtro & multirrótulo & primeira ordem \\
\hline 24 & (Gonçalves e Quaresma, 2005) & filtro & multirrótulo & primeira ordem \\
\hline 25 & (Naula et al., 2011) & wrapper & multirrótulo e monorrótulo & primeira ordem \\
\hline 26 & (Chou e Hsu, 2005) & embutida & multirrótulo & alta ordem \\
\hline 27 & (Kong e Yu, 2010) & não reconhecido & multirrótulo & não reconhecido \\
\hline
\end{tabular}


Tabela A.1: Informações dos 74 trabalhos encontrados na RS (continuação)

\begin{tabular}{|c|c|c|c|c|}
\hline ID & referência & $\begin{array}{l}\text { interação com o } \\
\text { algoritmo de indução }\end{array}$ & $\begin{array}{l}\text { escopo do } \\
\text { multirrótulo }\end{array}$ & $\begin{array}{l}\text { dependência } \\
\text { de rótulos }\end{array}$ \\
\hline 28 & (Li et al., 2008) & não reconhecido & não reconhecido & não reconhecido \\
\hline 29 & (Chang et al., 2008) & filtro & monorrótulo & primeira ordem \\
\hline 30 & (Bhowmick et al., 2009) & filtro & monorrótulo & primeira ordem \\
\hline 31 & (Wang e Jiang, 2009) & não reconhecido & não reconhecido & não reconhecido \\
\hline 32 & (Bekkerman et al., 2001) & filtro & monorrótulo & primeira ordem \\
\hline 33 & (Mengle e Goharian, 2009) & filtro & híbrido & primeira ordem \\
\hline 34 & (Tsoumakas e Vlahavas, 2007) & filtro & monorrótulo & primeira ordem \\
\hline 35 & (Lewis et al., 2004) & filtro & monorrótulo & primeira ordem \\
\hline 36 & (Zhang e Yang, 2003) & filtro & monorrótulo & primeira ordem \\
\hline 37 & (Wei et al., 2009) & filtro & hibrido & segunda ordem \\
\hline 38 & (Jiang et al., 2009) & não reconhecido & não reconhecido & não reconhecido \\
\hline 39 & (Pfister e Robinson, 2010) & filtro & hibrido & segunda ordem \\
\hline 40 & (Li et al., 2010b) & wrapper & híbrido & segunda ordem \\
\hline 41 & (Toutanova et al., 2001) & filtro & multirrótulo & primeira ordem \\
\hline 42 & (Esuli ct al., 2006) & cmbutida & híbrido & primeira ordem \\
\hline 43 & (Park et al., 2011) & filtro & monorrótulo & primeira ordem \\
\hline 44 & (Largeron et al., 2010) & filtro & hibrido & primeira ordem \\
\hline 45 & (Dendamrongvit e Kubat, 2010) & embutida & monorrótulo & primeira ordem \\
\hline 46 & (Pons-Porrata et al., 2007) & filtro & híbrido & primeira ordem \\
\hline 47 & (Wei et al., 2010) & filtro & multirrótulo & alta ordem \\
\hline 48 & (Trohidis et al., 2008) & filtro & híbrido e monorrótulo & alta ordem e primeira ordem \\
\hline 49 & (Zheng et al., 2004) & filtro & híbrido & primeira ordem \\
\hline 50 & (Liu et al., 2012) & filtro & monorrótulo & primeira ordem \\
\hline 51 & (Gu et al., 2011) & embutida & multirrótulo & segunda ordem \\
\hline 52 & (Lastra et al., 2011) & filtro & multirrótulo & segunda ordem \\
\hline 53 & (Dendamrongvit et al., 2011) & filtro & monorrótulo & primeira ordem \\
\hline 54 & (Shao et al., 201 1) & wrapper & multirrótulo & segunda ordem \\
\hline 55 & (Lee et al., 2012) & filtro & multirrótulo & segunda ordem \\
\hline 56 & (Ye et al., 2012) & filtro & monorrótulo & primeira ordem \\
\hline 57 & (Lee e Kim, 2013) & filtro & multirrótulo & segunda ordem \\
\hline 58 & (Kong et al., 2012) & filtro & multirrótulo & segunda ordem \\
\hline 59 & (Spolaôr et al., 2012a) & filtro & multirrótulo e monorrótulo & primeira ordem \\
\hline 60 & (Jungjit et al., 2012) & filtro & multirrótulo & primeira ordem \\
\hline 61 & (Spolaôr et al., 2013b) & filtro & hibrido e monorrótulo & primeira ordem \\
\hline 62 & (Yang e Pedersen, 1997) & filtro & monorrótulo & primeira ordem \\
\hline 63 & (Li e Zhang, 2012) & filtro & monorrótulo & primeira ordem \\
\hline 64 & (Spolaôr e Tsoumakas, 2013) & filtro & monorrótulo & primeira ordem \\
\hline 65 & (Slavkov et al., 2013) & filtro & multirrótulo & alta ordem \\
\hline 66 & (Karabulut, 2013) & filtro & monorrótulo & primeira ordem \\
\hline 67 & (Naula et al., 2014) & embutida & monorrótulo & primeira ordem \\
\hline 68 & (Doquire e Verleysen, 2013) & filtro & hibrido & alta ordem \\
\hline 69 & (Spolaôr et al., 2013c) & filtro & multirrótulo & alta ordem \\
\hline 70 & (Pupo et al., 2013) & filtro & multirrótulo & não reconhecido \\
\hline 71 & (Rios e Kavuluru, 2013) & filtro & monorrótulo & primeira ordem \\
\hline 72 & (Jungjit et al., 2013) & filtro & multirrótulo & segunda ordem \\
\hline 73 & (Gasse et al., 2014) & embutida & híbrido & alta ordem \\
\hline 74 & (Liu et al., 2014) & embutida & monorrótulo & primeira ordem \\
\hline
\end{tabular}

\section{A.2 Resultados Experimentais Complementares ao Ca- pítulo 4}

Tabela A.2: Resultados do classificador baseline Geral $_{B}$ nos 10 conjuntos de dados considerados

\begin{tabular}{l|llll}
\hline \hline & F-Measure & Hamming Loss & Accuracy & $F_{b}$ \\
\hline \hline 1-Cal50O & 0,46 & 0,16 & 0,30 & 0,46 \\
2-Corel5k & 0,18 & 0,01 & 0,12 & 0,19 \\
3-Corel16k001 & 0,16 & 0,03 & 0,11 & 0,17 \\
4-Emotions & 0,30 & 0,33 & 0,23 & 0,31 \\
5-Fapesp & 0,07 & 0,04 & 0,06 & 0,08 \\
6-Genbase* & 0,26 & 0,06 & 0,26 & 0,23 \\
7-Llog-f* & 0,12 & 0,03 & 0,11 & 0,11 \\
8-Magtag5k & 0,19 & 0,05 & 0,12 & 0,19 \\
9-Scene & 0,20 & 0,27 & 0,19 & 0,21 \\
10-Yeast & 0,55 & 0,26 & 0,42 & 0,57 \\
\hline \hline
\end{tabular}


Tabela A.3: Desempenho dos classificadores $B R k N N-b$ construídos usando todos os atributos dos 10 conjuntos de dados considerados

\begin{tabular}{l|llll}
\hline \hline & F-Measure & Hamming Loss & Accuracy & $F_{b}$ \\
\hline \hline 1-Cal500 & 0,44 & 0,16 & 0,29 & 0,44 \\
2-Corel5k & 0,16 & 0,01 & 0,11 & 0,16 \\
3-Corel16k001 & 0,15 & 0,03 & 0,10 & 0,16 \\
4-Emotions & 0,64 & 0,21 & 0,55 & 0,66 \\
5-Fapesp & 0,14 & 0,05 & 0,12 & 0,14 \\
6-Genbase* & 0,96 & 0,00 & 0,95 & 0,95 \\
7-Llog-f* & 0,16 & 0,03 & 0,14 & 0,16 \\
8-Magtag5k & 0,37 & 0,04 & 0,26 & 0,37 \\
9-Scene & 0,73 & 0,10 & 0,72 & 0,72 \\
10-Yeast & 0,64 & 0,21 & 0,53 & 0,66 \\
\hline \hline
\end{tabular}

\section{A.2. 1 Abordagens Tradicionais de Transformação do Problema}

Tabela A.4: Resultados experimentais de acordo com F-measure baseada em exemplos: cinco métodos de SA baseados em abordagens tradicionais de transformação do problema

\begin{tabular}{|c|c|c|c|c|c|}
\hline & $I G-L P$ & $R F-L P$ & $I G-B R$ & $R F-B R$ & SAR \\
\hline & \multicolumn{5}{|c|}{$\left|X^{\prime}\right|=10 \% M$} \\
\hline 1-Cal500 & $0,45(0,02)[1,0]$ & $0,43(0,01)[2,5]$ & $0,43(0,02)[2,5]$ & $0,42(0,02)[4,5]$ & $0,42(0,01)[4,5]$ \\
\hline 2-Corel5k & $0,19(0,01)[4,0]$ & $0,19(0,01)[4,0]$ & $0,21(0,01)[1,0]$ & $0,19(0,01)[4,0]$ & $0,20(0,01)[2,0]$ \\
\hline 3-Corel16k001 & $0,20(0,01)[4,0]$ & $0,20(0,01)[4,0]$ & $0,23(0,00)[1,0]$ & $0,20(0,01)[4,0]$ & $0,21(0,00)[2,0]$ \\
\hline 4-Emotions & $0,60(0,04)[2,5]$ & $0,61(0,04)[1,0]$ & $0,60(0,03)[2,5]$ & $0,57(0,05)[4,5]$ & $0,57(0,05)[4,5]$ \\
\hline 5-Fapesp & $0,23(0,07)[1,5]$ & $0,23(0,05)[1,5]$ & $0,19(0,05)[3,0]$ & $0,13(0,05)[4,5]$ & $0,13(0,05)[4,5]$ \\
\hline 6-Genbase* & $0,99(0,01)[1,0]$ & $0,96(0,02)[3,0]$ & $0,96(0,01)[3,0]$ & $0,96(0,02)[3,0]$ & $0,38(0,08)[5,0]$ \\
\hline 7-Llog-f* & $0,13(0,02)[3,5]$ & $0,13(0,03)[3,5]$ & $0,16(0,03)[1,0]$ & $0,13(0,01)[3,5]$ & $0,13(0,03)[3,5]$ \\
\hline 8-Magtag5k & $0,30(0,01)[1,5]$ & $0,28(0,02)[3,0]$ & $0,27(0,02)[4,0]$ & $0,30(0,02)[1,5]$ & $0,26(0,02)[5,0]$ \\
\hline 9-Scene & $0,54(0,03)[3,0]$ & $0,59(0,04)[2,0]$ & $0,46(0,03)[5,0]$ & $0,52(0,03)[4,0]$ & $0,65(0,03)[1,0]$ \\
\hline \multirow[t]{2}{*}{ 10-Yeast } & $0,58(0,02)[4,5]$ & $0,61(0,02)[1,5]$ & $0,60(0,02)[3,0]$ & $0,61(0,02)[1,5]$ & $0,58(0,02)[4,5]$ \\
\hline & \multicolumn{5}{|c|}{$\left|X^{\prime}\right|=20 \% M$} \\
\hline 1-Cal500 & $0,46(0,01)[1,0]$ & $0,43(0,02)[3,5]$ & $0,43(0,01)[3,5]$ & $0,43(0,02)[3,5]$ & $0,43(0,01)[3,5]$ \\
\hline 2-Corel5k & $0,19(0,01)[3,0]$ & $0,18(0,01)[4,5]$ & $0,21(0,01)[1,0]$ & $0,18(0,01)[4,5]$ & $0,20(0,01)[2,0]$ \\
\hline 3-Corel16k001 & $0,21(0,01)[3,0]$ & $0,20(0,01)[4,5]$ & $0,23(0,00)[1,0]$ & $0,20(0,01)[4,5]$ & $0,22(0,01)[2,0]$ \\
\hline 4-Emotions & $0,62(0,03)[2,0]$ & $0,66(0,06)[1,0]$ & $0,61(0,03)[3,0]$ & $0,60(0,03)[4,0]$ & $0,59(0,04)[5,0]$ \\
\hline 5-Fapesp & $0,21(0,05)[1,0]$ & $0,18(0,05)[2,5]$ & $0,18(0,07)[2,5]$ & $0,09(0,04)[5,0]$ & $0,13(0,04)[4,0]$ \\
\hline 6-Genbase* & $0,99(0,01)[1,0]$ & $0,96(0,02)[3,0]$ & $0,96(0,01)[3,0]$ & $0,96(0,02)[3,0]$ & $0,48(0,12)[5,0]$ \\
\hline 7-Llog-f* & $0,14(0,02)[3,5]$ & $0,14(0,02)[3,5]$ & $0,17(0,02)[1,0]$ & $0,15(0,01)[2,0]$ & $0,13(0,03)[5,0]$ \\
\hline 8-Magtag $5 k$ & $0,32(0,01)[3,0]$ & $0,33(0,01)[1,5]$ & $0,30(0,01)[4,5]$ & $0,33(0,02)[1,5]$ & $0,30(0,02)[4,5]$ \\
\hline 9-Scene & $0,58(0,03)[4,5]$ & $0,65(0,04)[2,5]$ & $0,58(0,02)[4,5]$ & $0,65(0,03)[2,5]$ & $0,69(0,03)[1,0]$ \\
\hline \multirow[t]{2}{*}{ 10-Yeast } & $0,60(0,02)[5,0]$ & $0,63(0,02)[1,0]$ & $0,62(0,02)[2,5]$ & $0,62(0,02)[2,5]$ & $0,61(0,02)[4,0]$ \\
\hline & \multicolumn{5}{|c|}{$\left|X^{\prime}\right|=30 \% M$} \\
\hline 1-Cal500 & $0,46(0,01)[1,0]$ & $0,43(0,02)[3,5]$ & $0,43(0,01)[3,5]$ & $0,43(0,02)[3,5]$ & $0,43(0,01)[3,5]$ \\
\hline 2-Corel5k & $0,16(0,01)[4,5]$ & $0,17(0,01)[3,0]$ & $0,21(0,01)[1,0]$ & $0,16(0,01)[4,5]$ & $0,20(0,01)[2,0]$ \\
\hline 3-Corel16k001 & $0,20(0,01)[3,5]$ & $0,19(0,01)[5,0]$ & $0,23(0,00)[1,0]$ & $0,20(0,01)[3,5]$ & $0,22(0,01)[2,0]$ \\
\hline 4-Emotions & $0,63(0,04)[2,0]$ & $0,64(0,04)[1,0]$ & $0,62(0,04)[3,5]$ & $0,62(0,04)[3,5]$ & $0,61(0,05)[5,0]$ \\
\hline 5-Fapesp & $0,22(0,05)[1,0]$ & $0,18(0,07)[2,5]$ & $0,18(0,05)[2,5]$ & $0,08(0,04)[5,0]$ & $0,14(0,04)[4,0]$ \\
\hline 6-Genbase* & $0,99(0,01)[1,0]$ & $0,96(0,02)[3,0]$ & $0,96(0,01)[3,0]$ & $0,96(0,02)[3,0]$ & $0,62(0,13)[5,0]$ \\
\hline 7-Llog-f* & $0,16(0,02)[2,0]$ & $0,14(0,02)[3,5]$ & $0,17(0,04)[1,0]$ & $0,13(0,03)[5,0]$ & $0,14(0,03)[3,5]$ \\
\hline 8-Magtag5k & $0,36(0,01)[1,0]$ & $0,34(0,01)[3,0]$ & $0,32(0,02)[4,5]$ & $0,35(0,01)[2,0]$ & $0,32(0,01)[4,5]$ \\
\hline 9-Scene & $0,66(0,03)[4,5]$ & $0,67(0,03)[2,5]$ & $0,66(0,02)[4,5]$ & $0,67(0,03)[2,5]$ & $0,71(0,02)[1,0]$ \\
\hline \multirow[t]{2}{*}{ 10-Yeast } & $0,60(0,02)[5,0]$ & $0,63(0,02)[2,0]$ & $0,63(0,02)[2,0]$ & $0,63(0,02)[2,0]$ & $0,61(0,02)[4,0]$ \\
\hline & \multicolumn{5}{|c|}{$\left|X^{\prime}\right|=40 \% M$} \\
\hline 1-Cal500 & $0,46(0,01)[1,0]$ & $0,44(0,02)[2,0]$ & $0,43(0,02)[4,0]$ & $0,43(0,02)[4,0]$ & $0,43(0,02)[4,0]$ \\
\hline 2-Corel5k & $0,18(0,01)[3,0]$ & $0,16(0,01)[4,0]$ & $0,19(0,01)[1,5]$ & $0,13(0,01)[5,0]$ & $0,19(0,01)[1,5]$ \\
\hline 3-Corel16k001 & $0,17(0,01)[5,0]$ & $0,18(0,01)[4,0]$ & $0,22(0,01)[1,0]$ & $0,20(0,01)[3,0]$ & $0,21(0,01)[2,0]$ \\
\hline
\end{tabular}


Tabela A.4: Resultados para F-measure baseada em exemplos (continuação)

\begin{tabular}{|c|c|c|c|c|c|}
\hline & $I G-L P$ & $R F-L P$ & $I G-B R$ & $R F-B R$ & SAR \\
\hline 4-Emotions & $0,65(0,03)[1,5]$ & $0,65(0,05)[1,5]$ & $0,62(0,03)[4,5]$ & $0,63(0,04)[3,0]$ & $0,62(0,04)[4,5]$ \\
\hline 5-Fapesp & $0,20(0,05)[1,0]$ & $0,19(0,05)[2,0]$ & $0,16(0,05)[3,0]$ & $0,08(0,03)[5,0]$ & $0,13(0,05)[4,0]$ \\
\hline 6-Genbase* & $0,99(0,01)[1,0]$ & $0,96(0,02)[3,0]$ & $0,96(0,01)[3,0]$ & $0,96(0,02)[3,0]$ & $0,69(0,13)[5,0]$ \\
\hline $7-L \log -f^{*}$ & $0,16(0,02)[1,5]$ & $0,15(0,03)[3,0]$ & $0,16(0,03)[1,5]$ & $0,14(0,03)[4,5]$ & $0,14(0,03)[4,5]$ \\
\hline 8-Magtag $5 k$ & $0,37(0,01)[1,0]$ & $0,35(0,01)[3,0]$ & $0,33(0,01)[4,5]$ & $0,36(0,01)[2,0]$ & $0,33(0,02)[4,5]$ \\
\hline 9-Scene & $0,68(0,02)[4,5]$ & $0,70(0,03)[2,5]$ & $0,68(0,02)[4,5]$ & $0,70(0,02)[2,5]$ & $0,72(0,02)[1,0]$ \\
\hline \multirow[t]{2}{*}{ 10-Yeast } & $0,60(0,02)[5,0]$ & $0,64(0,02)[1,5]$ & $0,64(0,02)[1,5]$ & $0,63(0,02)[3,0]$ & $0,62(0,02)[4,0]$ \\
\hline & & & $\left|X^{\prime}\right|=50 \% M$ & & \\
\hline 1-Cal500 & $0,46(0,01)[1,0]$ & $0,44(0,02)[2,0]$ & $0,43(0,02)[4,0]$ & $0,43(0,01)[4,0]$ & $0,43(0,01)[4,0]$ \\
\hline 2-Corel5k & $0,16(0,01)[3,5]$ & $0,16(0,01)[3,5]$ & $0,17(0,01)[2,0]$ & $0,11(0,01)[5,0]$ & $0,18(0,02)[1,0]$ \\
\hline 3-Corel16k001 & $0,13(0,02)[5,0]$ & $0,16(0,01)[4,0]$ & $0,21(0,01)[1,0]$ & $0,19(0,01)[3,0]$ & $0,20(0,01)[2,0]$ \\
\hline 4-Emotions & $0,67(0,04)[1,0]$ & $0,64(0,04)[3,0]$ & $0,62(0,05)[5,0]$ & $0,64(0,04)[3,0]$ & $0,64(0,06)[3,0]$ \\
\hline 5-Fapesp & $0,21(0,06)[1,0]$ & $0,15(0,07)[3,0]$ & $0,16(0,05)[2,0]$ & $0,07(0,04)[5,0]$ & $0,14(0,05)[4,0]$ \\
\hline 6-Genbase* & $0,99(0,01)[1,0]$ & $0,96(0,02)[3,0]$ & $0,96(0,01)[3,0]$ & $0,96(0,02)[3,0]$ & $0,75(0,11)[5,0]$ \\
\hline 7-Llog-f* & $0,16(0,03)[1,5]$ & $0,15(0,04)[3,0]$ & $0,16(0,03)[1,5]$ & $0,14(0,03)[4,5]$ & $0,14(0,03)[4,5]$ \\
\hline 8-Magtag5k & $0,39(0,01)[1,0]$ & $0,36(0,01)[2,5]$ & $0,34(0,01)[5,0]$ & $0,36(0,01)[2,5]$ & $0,35(0,01)[4,0]$ \\
\hline 9-Scene & $0,70(0,03)[4,5]$ & $0,72(0,04)[2,5]$ & $0,70(0,03)[4,5]$ & $0,72(0,03)[2,5]$ & $0,73(0,03)[1,0]$ \\
\hline \multirow[t]{2}{*}{ 10-Yeast } & $0,61(0,0$ & $0,63(0,02)[3,5]$ & $0,64(0,02)[1,5]$ & $0,64(0,02)[1,5]$ & $0,63(0,02)[3,5]$ \\
\hline & & & $\left|X^{\prime}\right|=60 \% M$ & & \\
\hline $1-\mathrm{Cc}$ & $0,46(0, C$ & $0,44(0,0$ & $0,43(0,02)[4,0]$ & $43(0,02$ & 0,4310 \\
\hline $2-\mathrm{Cc}$ & $0,12(0,0$ & $0,12(0,01)[4,0]$ & $0,15(0,01)[2,0]$ & $0,12(0,01)[$ & $0,17(0$, \\
\hline 3-Corel16k001 & $0,05(0,01)[5,0]$ & $0,09(0,01)[4,0]$ & $0,20(0,01)$ & $0,15(0,02)[3,0]$ & $0,19(0,01)[2,0]$ \\
\hline 4-Emotions & $0,65(0,05)[2,5]$ & $0,65(0,03)[2,5]$ & $0,66(0,05)[1,0]$ & $0,63(0,04)[4,0]$ & $0,62(0$ \\
\hline 5-Fapesp & $0,20(0,06)[1,0]$ & $0,15(0,06)[2,5]$ & $0,15(0,05)[2,5]$ & $0,07(0,04)[5,0]$ & $0,14(0,05)[4,0]$ \\
\hline 6-Genbase* & $0,99(0,01)[1,0]$ & $0,96(0,02)[3,0]$ & $0,96(0,01)[3,0]$ & $0,96(0,02)[3,0]$ & $0,78(0,11)[5,0]$ \\
\hline $7-L \log -f^{*}$ & $0,16(0,03)[2,0]$ & $0,15(0,03)[3,0]$ & $0,17(0,04)[1,0]$ & $0,14(0,03)[4,5]$ & $0,14(0, \mathrm{C}$ \\
\hline 8-Magtag5k & $0,38(0,0$ & $0,36(0,01)[3,0]$ & $0,35(0,01$ & $0,36(0,01)$ & $0,36(0,01)[3,0]$ \\
\hline 9-Scene & $0,71(0,0$ & $0,73(0,03)[1,0]$ & $0,71(0,03)[4,5]$ & $0,72(0,03)[2,5]$ & $0,72(0,03)[2,5]$ \\
\hline \multirow[t]{2}{*}{ 10-Yeast } & $0,63(0,0$ & $0,64(0,02)[2,5]$ & $0,64(0,02)[2,5]$ & $0,64(0,02)[2,5]$ & $0,64(0$, \\
\hline & & & $\left|X^{\prime}\right|=7$ & & \\
\hline $1-C$ & $46(0$, &, $44(0$, & $0,43(0, \mathrm{C}$ & & 0,4310 \\
\hline $2-\mathrm{Co}$ & $0,13(0,0$ & $0,08(0,01)[5,0]$ & $0,17(0,01)[1,5]$ & $0,14(0,02)[3,0]$ & $0,17(0,02)[1,5]$ \\
\hline 3-Corel16k001 & $0,04(0,01)[5,0]$ & $0,05(0,01)[4,0]$ & $0,19(0,01)[1$ & $0,08(0,01)[3,0]$ & $0,18(0$, \\
\hline 4-Emotions & $0,66(0,05)[1,0]$ & $0,65(0,04)[2,5]$ & $0,64(0,05$ & $0,65(0,04)[2,5]$ & $0,63(0$ \\
\hline 5-Fapesp & $0,19(0,06)[1,0]$ & $0,16(0,05)[2,0]$ & $0,14(0,04)[4,0]$ & $0,07(0,03)[5,0]$ & $0,15(0, \mathrm{C}$ \\
\hline 6-Genbase* & $0,99(0,01)[1,0]$ & $0,96(0,02)[3,0]$ & $0,96(0,01)[3,0]$ & $0,96(0,02)[3,0]$ & $0,86(0,09)[5,0]$ \\
\hline 7-Llog-f* & $0,15(0,04)[3,0]$ & $0,16(0,03)[1,5]$ & $0,16(0,02)[1,5]$ & $0,14(0,03)[4,5]$ & $0,14(0,03)[4,5]$ \\
\hline 8-Magtag5k & $0,37(0,01)[1,0]$ & $0,36(0,01)[3,5]$ & $0,36(0,0$ & $0,36(0,01)[3,5]$ & $0,36(0,01)[3,5]$ \\
\hline 9-Scene & $0,72(0,0$ & $0,72(0,03)[3,5]$ & $0,71(0,03)[5,0]$ & $0,73(0,03)[1,5]$ & $0,73(0,03)[1,5]$ \\
\hline \multirow[t]{2}{*}{ 10-Yeast } & $0,64(0,02)[3,5]$ & $0,64(0,02)[3,5]$ & $0,65(0,02)[1,0]$ & $0,64(0,02)[3,5]$ & $0,64(0,02)[3,5]$ \\
\hline & & & $\left|X^{\prime}\right|=80 \% M$ & & \\
\hline $1-C$ & 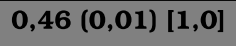 & 0,10, & $0,44(0, \mathrm{C}$ & & $0,43(0$ \\
\hline 2-Corel5k & $0,12(0,02)[4,5]$ & $0,12(0,02)[4,5]$ & $0,18(0,01)[1,0]$ & $0,14(0,02)[3,0]$ & $0,17(0,02)[2,0]$ \\
\hline 3-Corel16kOO & $0,06(0,02)[4,0]$ & $0,04(0,01)[5,0]$ & $0,18(0,01)[1,0]$ & $0,07(0,02)[3,0]$ & $0,17(0,01)[2,0]$ \\
\hline 4-Emotions & $0,64(0,03)[4,0]$ & $0,66(0,03)[1,0]$ & $0,65(0,03)[2,0]$ & $0,64(0,05)[4,0]$ & $0,64(0,04)[4,0]$ \\
\hline 5-Fapesp & $0,18(0,06)[1,0]$ & $0,17(0,05)[2,0]$ & $0,14(0,05)[3,5]$ & $0,06(0,03)[5,0]$ & $0,14(0,05)[3,5]$ \\
\hline 6-Genbase* & $0,99(0,01)[1,0]$ & $0,96(0,02)[3,0]$ & $0,96(0,01)[3,0]$ & $0,96(0,02)[3,0]$ & $0,92(0,06)[5,0]$ \\
\hline 7-Llog-f* & $0,16(0,03)[1,5]$ & $0,15(0,02)[3,5]$ & $0,16(0,03)[1,5]$ & $0,14(0,03)[5,0]$ & $0,15(0,03)[3,5]$ \\
\hline 8-Magtag5k & $0,37(0,01)[1,5]$ & $0,37(0,01)[1,5]$ & $0,36(0,01)[4,0]$ & $0,36(0,01)[4,0]$ & $0,36(0,01)[4,0]$ \\
\hline 9-Scene & $0,73(0,02)[1,0]$ & $0,72(0,03)[3,5]$ & $0,72(0,03)[3,5]$ & $0,72(0,03)[3,5]$ & $0,72(0,02)[3,5]$ \\
\hline \multirow[t]{2}{*}{ 10-Yeast } & $0,65(0,02)[1,0]$ & $0,64(0,02)[3,5]$ & $0,64(0,02)[3,5]$ & $0,64(0,02)[3,5]$ & $0,64(0,02)[3,5]$ \\
\hline & & & $\left|X^{\prime}\right|=90 \% M$ & & \\
\hline 1-Cal50O & $0,46(0,01)[1,0]$ & $0,4+(0,0)[0,0]$ & $0,44(0,01)[3,0]$ & & $0,43(0,02)[5,0]$ \\
\hline 2-Corel5k & $0,14(0,01)[4,5]$ & $0,14(0,01)[4,5]$ & $0,17(0,01)[1,5]$ & $0,15(0,02)[3,0]$ & $0,17(0,01)[1,5]$ \\
\hline 3-Corel16kOO1 & $0,08(0,00)[4,0]$ & $0,07(0,00)[5,0]$ & $0,17(0,01)$ & $0,10(0,01)[3,0]$ & $0,16(0,01)[2,0]$ \\
\hline 4-Emotions & $0,65(0,04)[2,5]$ & $0,65(0,04)[2,5]$ & $0,65(0,04)[2,5]$ & $0,64(0,05)[5,0]$ & $0,65(0,05)[2,5]$ \\
\hline $5-F a$ & $0,18(0,07)[1,0]$ & $0,16(0,05)[2,5]$ & $0,16(0,05)[2,5]$ & $0,07(0,03)[5,0]$ & $0,15(0,05)[4,0]$ \\
\hline 6-Genbase* & $0,99(0,01)[1,0]$ & $0,96(0,02)[3,0]$ & $0,96(0,01)[3,0]$ & $0,96(0,02)[3,0]$ & $0,94(0,04)[5,0]$ \\
\hline
\end{tabular}


Tabela A.4: Resultados para F-measure baseada em exemplos (continuação)

\begin{tabular}{l|lllll}
\hline & $I G-L P$ & $R F-L P$ & $I G-B R$ & $R F-B R$ & SAR \\
\hline 7-Llog-f* & $0,16(0,03)[2,5]$ & $0,16(0,02)[2,5]$ & $0,16(0,03)[2,5]$ & $0,14(0,03)[5,0]$ & $0,16(0,03)[2,5]$ \\
8-Magtag5k & $\mathbf{0 , 3 8 ( 0 , 0 1 ) [ 1 , 0 ]}$ & $0,37(0,01)[3,5]$ & $0,37(0,01)[3,5]$ & $0,37(0,01)[3,5]$ & $0,37(0,01)[3,5]$ \\
9-Scene & $0,72(0,02)[3,5]$ & $0,72(0,03)[3,5]$ & $0,72(0,02)[3,5]$ & $0,72(0,03)[3,5]$ & $\mathbf{0 , 7 3}(\mathbf{0 , 0 3}[\mathbf{1 , 0}]$ \\
10-Yeast & $\mathbf{0 , 6 5}(\mathbf{0 , 0 2})[\mathbf{1 , 0}]$ & $0,64(0,02)[3,5]$ & $0,64(0,02)[3,5]$ & $0,64(0,02)[3,5]$ & $0,64(0,02)[3,5]$ \\
\hline
\end{tabular}

Tabela A.5: Resultados experimentais de acordo com Hamming Loss: cinco métodos de SA baseados em abordagens tradicionais de transformação do problema

\begin{tabular}{|c|c|c|c|c|c|}
\hline & $I G-L P$ & $R F-L P$ & $I G-B R$ & $R F-B R$ & SAR \\
\hline & \multicolumn{5}{|c|}{$\left|X^{\prime}\right|=10 \% M$} \\
\hline 1-Cal500 & $0,16(0,01)[5,0]$ & $0,17(0,00)[2,5]$ & $0,17(0,00)[2,5]$ & $0,17(0,01)[2,5]$ & $0,17(0,00)[2,5]$ \\
\hline 2-Corel5k & $0,02(0,00)[2,5]$ & $0,02(0,00)[2,5]$ & $0,01(0,00)[5,0]$ & $0,02(0,00)[2,5]$ & $0,02(0,00)[2,5]$ \\
\hline 3-Corel16k001 & $0,03(0,00)[3,0]$ & $0,03(0,00)[3,0]$ & $0,03(0,00)[3,0]$ & $0,03(0,00)[3,0]$ & $0,03(0,00)[3,0]$ \\
\hline 4-Emotions & $0,24(0,03)[3,5]$ & $0,23(0,03)[5,0]$ & $0,24(0,02)[3,5]$ & $0,25(0,02)[2,0]$ & $0,26(0,03)[1,0]$ \\
\hline 5-Fapesp & $0,04(0,00)[4,0]$ & $0,04(0,00)[4,0]$ & $0,04(0,00)[4,0]$ & $0,05(0,01)[1,5]$ & $0,05(0,00)[1,5]$ \\
\hline 6-Genbase* & $0,00(0,00)[3,5]$ & $0,00(0,00)[3,5]$ & $0,00(0,00)[3,5]$ & $0,00(0,00)[3,5]$ & $0,05(0,01)[1,0]$ \\
\hline 7-Llog-f* & $0,03(0,00)[3,0]$ & $0,03(0,00)[3,0]$ & $0,03(0,00)[3,0]$ & $0,03(0,00)[3,0]$ & $0,03(0,00)[3,0]$ \\
\hline 8-Magtag5k & $0,05(0,00)[3,0]$ & $0,05(0,00)[3,0]$ & $0,05(0,00)[3,0]$ & $0,05(0,00)[3,0]$ & $0,05(0,00)[3,0]$ \\
\hline 9-Scene & $0,16(0,01)[3,0]$ & $0,14(0,01)[4,0]$ & $0,19(0,01)[1,0]$ & $0,17(0,01)[2,0]$ & $0,12(0,01)[5,0]$ \\
\hline \multirow[t]{2}{*}{ 10-Yeast } & $0,24(0,01)[1,5]$ & $0,23(0,01)[4,0]$ & $0,23(0,01)[4,0]$ & $0,23(0,01)[4,0]$ & $0,24(0,01)[1,5]$ \\
\hline & \multicolumn{5}{|c|}{$\left|X^{\prime}\right|=20 \% M$} \\
\hline 1-Cal500 & $0,16(0,00)[5,0]$ & $0,17(0,01)[2,5]$ & $0,17(0,01)[2,5]$ & $0,17(0,01)[2,5]$ & $0,17(0,00)[2,5]$ \\
\hline 2-Corel5k & $0,02(0,00)[2,5]$ & $0,02(0,00)[2,5]$ & $0,01(0,00)[5,0]$ & $0,02(0,00)[2,5]$ & $0,02(0,00)[2,5]$ \\
\hline 3-Corel16k001 & $0,03(0,00)[3,0]$ & $0,03(0,00)[3,0]$ & $0,03(0,00)[3,0]$ & $0,03(0,00)[3,0]$ & $0,03(0,00)[3,0]$ \\
\hline 4-Emotions & $0,23(0,02)[3,5]$ & $0,20(0,03)[5,0]$ & $0,23(0,02)[3,5]$ & $0,24(0,01)[1,5]$ & $0,24(0,02)[1,5]$ \\
\hline 5-Fapesp & $0,05(0,00)[2,0]$ & $0,04(0,00)[4,5]$ & $0,04(0,00)[4,5]$ & $0,05(0,01)[2,0]$ & $0,05(0,00)[2,0]$ \\
\hline 6-Genbase* & $0,00(0,00)[3,5]$ & $0,00(0,00)[3,5]$ & $0,00(0,00)[3,5]$ & $0,00(0,00)[3,5]$ & $0,05(0,01)[1,0]$ \\
\hline 7-Llog-f* & $0,03(0,00)[3,0]$ & $0,03(0,00)[3,0]$ & $0,03(0,00)[3,0]$ & $0,03(0,00)[3,0]$ & $0,03(0,00)[3,0]$ \\
\hline 8-Magtag5k & $0,05(0,00)[3,0]$ & $0,05(0,00)[3,0]$ & $0,05(0,00)[3,0]$ & $0,05(0,00)[3,0]$ & $0,05(0,00)[3,0]$ \\
\hline 9-Scene & $0,15(0,01)[1,5]$ & $0,12(0,01)[3,5]$ & $0,15(0,01)[1,5]$ & $0,12(0,01)[3,5]$ & $0,11(0,01)[5,0]$ \\
\hline \multirow[t]{2}{*}{ 10-Yeast } & $0,23(0,01)[1,5]$ & $0,22(0,01)[4,0]$ & $0,22(0,01)[4,0]$ & $0,22(0,01)[4,0]$ & $0,23(0,01)[1,5]$ \\
\hline & \multicolumn{5}{|c|}{$\left|X^{\prime}\right|=30 \% M$} \\
\hline 1-Cal500 & $0,16(0,00)[5,0]$ & $0,17(0,01)[2,5]$ & $0,17(0,00)[2,5]$ & $0,17(0,01)[2,5]$ & $0,17(0,01)[2,5]$ \\
\hline 2-Corel5k & $0,01(0,00)[4,0]$ & $0,02(0,00)[1,5]$ & $0,01(0,00)[4,0]$ & $0,02(0,00)[1,5]$ & $0,01(0,00)[4,0]$ \\
\hline 3-Corel16k001 & $0,03(0,00)[3,0]$ & $0,03(0,00)[3,0]$ & $0,03(0,00)[3,0]$ & $0,03(0,00)[3,0]$ & $0,03(0,00)[3,0]$ \\
\hline 4-Emotions & $0,22(0,02)[3,5]$ & $0,21(0,02)[5,0]$ & $0,22(0,02)[3,5]$ & $0,23(0,02)[1,5]$ & $0,23(0,03)[1,5]$ \\
\hline 5-Fapesp & $0,04(0,00)[4,0]$ & $0,04(0,00)[4,0]$ & $0,04(0,00)[4,0]$ & $0,05(0,01)[1,5]$ & $0,05(0,00)[1,5]$ \\
\hline 6-Genbase* & $0,00(0,00)[3,5]$ & $0,00(0,00)[3,5]$ & $0,00(0,00)[3,5]$ & $0,00(0,00)[3,5]$ & $0,03(0,01)[1,0]$ \\
\hline 7-Llog-f* & $0,03(0,00)[3,0]$ & $0,03(0,00)[3,0]$ & $0,03(0,00)[3,0]$ & $0,03(0,00)[3,0]$ & $0,03(0,00)[3,0]$ \\
\hline 8-Magtag5k & $0,05(0,00)[3,0]$ & $0,05(0,00)[3,0]$ & $0,05(0,00)[3,0]$ & $0,05(0,00)[3,0]$ & $0,05(0,00)[3,0]$ \\
\hline 9-Scene & $0,12(0,01)[1,5]$ & $0,11(0,01)[3,5]$ & $0,12(0,01)[1,5]$ & $0,11(0,01)[3,5]$ & $0,10(0,01)[5,0]$ \\
\hline \multirow[t]{2}{*}{ 10-Yeast } & $0,23(0,01)[1,0]$ & $0,22(0,01)[3,0]$ & $0,21(0,01)[5,0]$ & $0,22(0,01)[3,0]$ & $0,22(0,01)[3,0]$ \\
\hline & \multicolumn{5}{|c|}{$\left|X^{\prime}\right|=40 \% M$} \\
\hline 1-Cal500 & $0,16(0,00)[4,5]$ & $0,16(0,00)[4,5]$ & $0,17(0,01)[2,0]$ & $0,17(0,01)[2,0]$ & $0,17(0,0$ \\
\hline 2-Corel5k & $0,01(0,00)[4,0]$ & $0,02(0,00)[1,5]$ & $0,01(0,00)[4,0]$ & $0,02(0,00)[1,5]$ & $0,01(0,00)[4,0]$ \\
\hline 3-Corel16k001 & $0,03(0,00)[3,0]$ & $0,03(0,00)[3,0]$ & $0,03(0,00)[3,0]$ & $0,03(0,00)[3,0]$ & $0,03(0,00)[3,0]$ \\
\hline 4-Emotions & $0,21(0,02)[4,5]$ & $0,21(0,03)[4,5]$ & $0,22(0,02)[2,0]$ & $0,22(0,02)[2,0]$ & $0,22(0,02)[2,0]$ \\
\hline 5-Fapesp & $0,05(0,00)[2,0]$ & $0,04(0,00)[4,5]$ & $0,04(0,00)[4,5]$ & $0,05(0,01)[2,0]$ & $0,05(0,00)[2,0]$ \\
\hline 6-Genbase ${ }^{*}$ & $0,00(0,00)[3,5]$ & $0,00(0,00)[3,5]$ & $0,00(0,00)[3,5]$ & $0,00(0,00)[3,5]$ & $0,03(0,01)[1,0]$ \\
\hline 7-Llog-f* & $0,03(0,00)[3,0]$ & $0,03(0,00)[3,0]$ & $0,03(0,00)[3,0]$ & $0,03(0,00)[3,0]$ & $0,03(0,00)[3,0]$ \\
\hline 8-Magtag5k & $0,04(0,00)[5,0]$ & $0,05(0,00)[2,5]$ & $0,05(0,00)[2,5]$ & $0,05(0,00)[2,5]$ & $0,05(0,00)[2,5]$ \\
\hline 9-Scene & $0,11(0,01)[1,5]$ & $0,10(0,01)[4,0]$ & $0,11(0,01)[1,5]$ & $0,10(0,01)[4,0]$ & $0,10(0,01)[4,0]$ \\
\hline \multirow[t]{2}{*}{ 10-Yeast } & $0,23(0,01)[1,0]$ & $0,21(0,01)[4,0]$ & $0,21(0,01)[4,0]$ & $0,21(0,01)[4,0]$ & $0,22(0,01)[2,0]$ \\
\hline & \multicolumn{5}{|c|}{$\left|X^{\prime}\right|=50 \% M$} \\
\hline 1-Cal500 & $0,16(0,00)[4,5]$ & $0,16(0,01)[4,5]$ & $0,17(0,01)[2,0]$ & $0,17(0,00)[2,0]$ & $0,17(0,00)[2,0]$ \\
\hline 2-Corel5k & $0,01(0,00)[3,5]$ & $0,01(0,00)[3,5]$ & $0,01(0,00)[3,5]$ & $0,02(0,00)[1,0]$ & $0,01(0,00)[3,5]$ \\
\hline
\end{tabular}


Tabela A.5: Resultados para Hamming Loss (continuação)

\begin{tabular}{|c|c|c|c|c|c|}
\hline & $I G-L P$ & $R F-L P$ & $I G-B R$ & $R F-B R$ & SAR \\
\hline 3-Corel16k001 & $0,03(0,00)[3,0]$ & $0,03(0,00)[3,0]$ & $0,03(0,00)[3,0]$ & $0,03(0,00)[3,0]$ & $0,03(0,00)[3,0]$ \\
\hline 4-Emotions & $0,20(0,02)[5,0]$ & $0,21(0,03)[3,5]$ & $0,22(0,03)[1,5]$ & $0,21(0,02)[3,5]$ & $0,22(0,03)[1,5]$ \\
\hline 5-Fapesp & $0,04(0,00)[4,0]$ & $0,04(0,00)[4,0]$ & $0,04(0,00)[4,0]$ & $0,05(0,01)[1,5]$ & $0,05(0,01)[1,5]$ \\
\hline 6-Genbase* & $0,00(0,00)[3,5]$ & $0,00(0,00)[3,5]$ & $0,00(0,00)[3,5]$ & $0,00(0,00)[3,5]$ & $0,02(0,01)[1,0]$ \\
\hline 7-Llog-f* & $0,03(0,00)[3,0]$ & $0,03(0,00)[3,0]$ & $0,03(0,00)[3,0]$ & $0,03(0,00)[3,0]$ & $0,03(0,00)[3,0]$ \\
\hline 8-Magtag5k & $0,04(0,00)[5,0]$ & $0,05(0,00)[2,5]$ & $0,05(0,00)[2,5]$ & $0,05(0,00)[2,5]$ & $0,05(0,00)[2,5]$ \\
\hline 9-Scene & $0,10(0,01)[3,0]$ & $0,10(0,01)[3,0]$ & $0,10(0,01)[3,0]$ & $0,10(0,01)[3,0]$ & $0,10(0,01)[3,0]$ \\
\hline \multirow[t]{2}{*}{ 10-Yeast } & $0,22(0,01)[1,0]$ & $0,21(0,01)[3,5]$ & $0,21(0,01)[3,5]$ & $0,21(0,01)[3,5]$ & $0,21(0,01)[3,5]$ \\
\hline & & & $\left|X^{\prime}\right|=60 \% M$ & & \\
\hline 1-Cal50O & $0,10(0,00)[4,0]$ & $0,16(0,01)[4,5]$ & $0,17(0,01)[2,0]$ & $0,17(0,0$ & $0,17(0,00)[2,0]$ \\
\hline 2-Corel5k & $0,02(0,00)[2,5]$ & $0,02(0,00)[2,5]$ & $0,01(0,00)[5,0]$ & $0,02(0,00)[2,5]$ & $0,02(0,00)[2,5]$ \\
\hline 3-Corel16k001 & $0,04(0,00)[1,0]$ & $0,03(0,00)[3,5]$ & $0,03(0,00)[3,5]$ & $0,03(0,00)[3,5]$ & $0,03(0,00)[3,5]$ \\
\hline 4-Emotions & $0,21(0,03)[2,5]$ & $0,20(0,02)[4,5]$ & $0,20(0,03)[4,5]$ & $0,21(0,02)[2,5]$ & $0,22(0,02)[1,0]$ \\
\hline 5-Fapesp & $0,04(0,00)[4,0]$ & $0,04(0,00)[4,0]$ & $0,04(0,00)[4,0]$ & $0,05(0,01)[1,5]$ & $0,05(0,00)[1,5]$ \\
\hline 6-Genbase* & $0,00(0,00)[3,5]$ & $0,00(0,00)[3,5]$ & $0,00(0,00)[3,5]$ & $0,00(0,00)[3,5]$ & $0,02(0,01)[1,0]$ \\
\hline 7-Llog-f* & $0,03(0,00)[3,0]$ & $0,03(0,00)[3,0]$ & $0,03(0,00)[3,0]$ & $0,03(0,00)[3,0]$ & $0,03(0,00)[3,0]$ \\
\hline 8-Magtag $5 k$ & $0,04(0,00)[5,0]$ & $0,05(0,00)[2,5]$ & $0,05(0,00)[2,5]$ & $0,05(0,00)[2,5]$ & $0,05(0,00)[2,5]$ \\
\hline 9-Scene & $0,10(0,01)[3,0]$ & $0,10(0,01)[3,0]$ & $0,10(0,01)[3,0]$ & $0,10(0,01)[3,0]$ & $0,10(0,01)[3,0]$ \\
\hline \multirow[t]{2}{*}{ 10-Yeast } & $0,21(0,01)[3,0]$ & $0,21(0,01)[3,0]$ & $0,21(0,01)[3,0]$ & $0,21(0,01)[3,0]$ & $0,21(0,01)[3,0]$ \\
\hline & & & $\left|X^{\prime}\right|=70 \% M$ & & \\
\hline 1-Cal500 & $16(0$, & {$[2,5]$} & $0,17(0,1$ & $0,17(0$ & 0,17 (C \\
\hline 2-Corel5k & $0,02(0,00)[2,0]$ & $0,02(0,00)[2,0]$ & $0,01(0, C$ & $0,02(0,00)[2,0]$ & $0,01(0$ \\
\hline 3-Corel16k001 & $0,04(0,00)[2,0]$ & $0,04(0,00)[2,0]$ & $0,03(0,00)[4,5]$ & $0,04(0,00)[2,0]$ & $0,03(0$ \\
\hline 4-Emotions & $0,21(0,0$ & $0,20(0,02)[5,0]$ & $0,21(0,02)[3,0]$ & $0,21(0,02)[3,0]$ & $0,22(0$, \\
\hline 5-Fapesp & $0,04(0,01)[4,0]$ & $0,04(0,00)[4,0]$ & $0,04(0,00)[4,0]$ & $0,05(0,01)[1,5]$ & $0,05(0,00)[1,5]$ \\
\hline 6-Genbase* & $0,00(0,00)[3,5]$ & $0,00(0,00)[3,5]$ & $0,00(0,00)[3,5]$ & $0,00(0,00)[3,5]$ & $0,01(0$, \\
\hline $7-L \log -f^{*}$ & $0,03(0,00)[3,0]$ & $0,03(0,00)[3,0]$ & $0,03(0,00)[3,0]$ & $0,03(0,00)[3,0]$ & $0,03(0$ \\
\hline 8-Magtag $5 k$ & $0,04(0,00)[5,0]$ & $0,05(0,00)[2,5]$ & $0,05(0,00)[2,5]$ & $0,05(0,00)[2,5]$ & $0,05(0,00)[2,5]$ \\
\hline 9-Scene & $0,10(0,01)[3,0]$ & $0,10(0,01)[3,0]$ & $0,10(0,01)[3,0]$ & $0,10(0,01)[3,0]$ & $0,10(0,01)[3,0]$ \\
\hline \multirow[t]{2}{*}{ 10-Yeast } & $0,21(0,01)[2,5]$ & $0,21(0,01)[2,5]$ & $0,20(0,01)[5,0]$ & $0,21(0,01)[2,5]$ & $0,21(0$, \\
\hline & & & $\left|X^{\prime}\right|=8$ & & \\
\hline 1-Cal50O & - & {$[\mathbf{3 , 5}]$} & $0,16(0$ & & $0,17(0$ \\
\hline 2-Corel5k & $0,02(0,00)[2,0]$ & $0,02(0,00)[2,0]$ & $0,01(0$ & $0,02(0,00)[2,0]$ & $0,01(0$ \\
\hline 3-Corel16kO & $0,04(0,00)[2,0]$ & $0,04(0,00)[2,0]$ & $0,03(0,00)[4,5]$ & $0,04(0,00)[2,0]$ & $0,03(0$ \\
\hline 4-Emotions & $0,22(0,02)[1,0]$ & $0,20(0,01)[5,0]$ & $0,21(0,01)[3,0]$ & $0,21(0,02)[3,0]$ & $0,21(0,02)[3,0]$ \\
\hline 5-Fapesp & $0,05(0,00)[2,0]$ & $0,04(0,00)[4,5]$ & $0,04(0,00)[4,5]$ & $0,05(0,01)[2,0]$ & $0,05(0,00)[2,0]$ \\
\hline 6-Genbase* & $0,00(0,00)[3,5]$ & $0,00(0,00)[3,5]$ & $0,00(0,00)[3,5]$ & $0,00(0,00)[3,5]$ & $0,01(0,00)[1,0]$ \\
\hline 7-Llog-f* & $0,03(0,00)[3,0]$ & $0,03(0,00)[3,0]$ & $0,03(0,00)[3,0]$ & $0,03(0,00)[3,0]$ & $0,03(0,00)[3,0]$ \\
\hline 8-Magtag5k & $0,04(0,00)[4,5]$ & $0,04(0,00)[4,5]$ & $0,05(0,00)[2,0]$ & $0,05(0,00)[2,0]$ & $0,05(0,00)[2,0]$ \\
\hline 9-Scene & $0,10(0,01)[3,0]$ & $0,10(0,01)[3,0]$ & $0,10(0,01)[3,0]$ & $0,10(0,01)[3,0]$ & $0,10(0,01)[3,0]$ \\
\hline \multirow[t]{2}{*}{ 10-Yeast } & $0,21(0,01)[3,0]$ & $0,21(0,01)[3,0]$ & $0,21(0,01)[3,0]$ & $0,21(0,01)[3,0]$ & $0,21(0,01)[3,0]$ \\
\hline & & & $\left|X^{\prime}\right|=90 \% M$ & & \\
\hline 1-Cal500 & $0,20,0$ & {$[\mathbf{0}, \mathbf{0}]$} & $0,16(0$, & {$[3,5]$} & $0,17(0$ \\
\hline 2-Corel5k & $0,02(0,00)[2,0]$ & $0,02(0,00)[2,0]$ & $0,01(0,00)[4,5]$ & $0,02(0,00)[2,0]$ & $0,01(0,00)[4,5]$ \\
\hline 3-Corel16k001 & $0,04(0,00)[1,5]$ & $0,04(0,00)[1,5]$ & $0,03(0,00)[4,0]$ & $0,03(0,00)[4,0]$ & $0,03(0,00)[4,0]$ \\
\hline 4-Emotions & $0,21(0,02)[3,0]$ & $0,21(0,02)[3,0]$ & $0,21(0,02)[3,0]$ & $0,21(0,02)[3,0]$ & $0,21(0,02)[3,0]$ \\
\hline 5-Fapesp & $0,05(0,00)[2,0]$ & $0,04(0,01)[4,5]$ & $0,04(0,00)[4,5]$ & $0,05(0,01)[2,0]$ & $0,05(0,00)[2,0]$ \\
\hline 6-Genbase* & $0,00(0,00)[3,5]$ & $0,00(0,00)[3,5]$ & $0,00(0,00)[3,5]$ & $0,00(0,00)[3,5]$ & $0,01(0,00)[1,0]$ \\
\hline 7-Llog-f* & $0,03(0,00)[3,0]$ & $0,03(0,00)[3,0]$ & $0,03(0,00)[3,0]$ & $0,03(0,00)[3,0]$ & $0,03(0,00)[3,0]$ \\
\hline 8-Magtag5k & $0,04(0,00)[3,0]$ & $0,04(0,00)[3,0]$ & $0,04(0,00)[3,0]$ & $0,04(0,00)[3,0]$ & $0,04(0,00)[3,0]$ \\
\hline 9-Scene & $0,10(0,01)[3,0]$ & $0,10(0,01)[3,0]$ & $0,10(0,01)[3,0]$ & $0,10(0,01)[3,0]$ & $0,10(0,01)[3,0]$ \\
\hline 10-Yeast & $0,21(0,01)[3,0]$ & $0,21(0,01)[3,0]$ & $0,21(0,01)[3,0]$ & $0,21(0,01)[3,0]$ & $0,21(0,01)[3,0]$ \\
\hline
\end{tabular}


Tabela A.6: Resultados experimentais de acordo com Accuracy: cinco métodos de SA baseados em abordagens tradicionais de transformação do problema

\begin{tabular}{|c|c|c|c|c|c|}
\hline & $I G-L P$ & $R F-L P$ & $I G-B R$ & $R F-B R$ & SAR \\
\hline & \multicolumn{5}{|c|}{$\left|X^{\prime}\right|=10 \% M$} \\
\hline 1-Cal500 & $0,30(0,01)[1,0]$ & $0,28(0,01)[3,5]$ & $0,28(0,01)[3,5]$ & $0,28(0,02)[3,5]$ & $0,28(0,01)[3,5]$ \\
\hline 2-Corel5k & $0,12(0,00)[4,0]$ & $0,12(0,00)[4,0]$ & $0,14(0,01)[1,0]$ & $0,12(0,00)[4,0]$ & $0,13(0,01)[2,0]$ \\
\hline 3-Corel16k001 & $0,13(0,00)[4,0]$ & $0,13(0,00)[4,0]$ & $0,15(0,00)[1,0]$ & $0,13(0,00)[4,0]$ & $0,14(0,00)[2,0]$ \\
\hline 4-Emotions & $0,51(0,04)[1,5]$ & $0,51(0,04)[1,5]$ & $0,50(0,03)[3,0]$ & $0,48(0,05)[4,5]$ & $0,48(0,05)[4,5]$ \\
\hline 5-Fapesp & $0,18(0,06)[2,0]$ & $0,19(0,05)[1,0]$ & $0,16(0,04)[3,0]$ & $0,10(0,05)[4,5]$ & $0,10(0,04)[4,5]$ \\
\hline 6-Genbase ${ }^{*}$ & $0,99(0,01)[1,0]$ & $0,95(0,02)[3,5]$ & $0,95(0,02)[3,5]$ & $0,96(0,02)[2,0]$ & $0,38(0,08)[5,0]$ \\
\hline 7-Llog-f* & $0,12(0,02)[3,0]$ & $0,12(0,02)[3,0]$ & $0,15(0,02)[1,0]$ & $0,12(0,01)[3,0]$ & $0,11(0,02)[5,0]$ \\
\hline 8-Magtag5k & $0,20(0,00)[1,5]$ & $0,19(0,01)[3,0]$ & $0,18(0,01)[4,0]$ & $0,20(0,01)[1,5]$ & $0,17(0,02)[5,0]$ \\
\hline 9-Scene & $0,53(0,03)[3,0]$ & $0,58(0,04)[2,0]$ & $0,46(0,03)[5,0]$ & $0,51(0,03)[4,0]$ & $0,64(0,03)[1,0]$ \\
\hline \multirow[t]{2}{*}{ 10-Yeast } & $0,47(0,02)[4,5]$ & $0,50(0,02)[1,5]$ & $0,49(0,02)[3,0]$ & $0,50(0,02)[1,5]$ & $0,47(0,02)[4,5]$ \\
\hline & \multicolumn{5}{|c|}{$\left|X^{\prime}\right|=20 \% M$} \\
\hline 1-Cal500 & $0,30(0,01)[1,0]$ & $0,28(0,01)[3,5]$ & $0,28(0,01)[3,5]$ & $0,28(0,01)[3,5]$ & $0,28(0,01)[3,5]$ \\
\hline 2-Corel5k & $0,12(0,01)[4,0]$ & $0,12(0,01)[4,0]$ & $0,14(0,00)[1,0]$ & $0,12(0,01)[4,0]$ & $0,13(0,01)[2,0]$ \\
\hline 3-Corel16k001 & $0,13(0,00)[4,0]$ & $0,13(0,00)[4,0]$ & $0,16(0,00)[1,0]$ & $0,13(0,00)[4,0]$ & $0,14(0,01)[2,0]$ \\
\hline 4-Emotions & $0,53(0,04)[2,0]$ & $0,57(0,05)[1,0]$ & $0,52(0,03)[3,0]$ & $0,51(0,03)[4,0]$ & $0,50(0,04)[5,0]$ \\
\hline 5-Fapesp & $0,16(0,05)[1,0]$ & $0,15(0,05)[2,5]$ & $0,15(0,07)[2,5]$ & $0,07(0,03)[5,0]$ & $0,10(0,03)[4,0]$ \\
\hline 6-Genbase $e^{*}$ & $0,99(0,01)[1,0]$ & $0,95(0,02)[3,5]$ & $0,95(0,02)[3,5]$ & $0,96(0,02)[2,0]$ & $0,47(0,12)[5,0]$ \\
\hline $7-L \log -f^{*}$ & $0,13(0,02)[3,5]$ & $0,13(0,02)[3,5]$ & $0,15(0,02)[1,0]$ & $0,14(0,01)[2,0]$ & $0,11(0,02)[5,0]$ \\
\hline 8-Magtag5k & $0,22(0,01)[3,0]$ & $0,23(0,01)[1,5]$ & $0,20(0,01)[4,5]$ & $0,23(0,01)[1,5]$ & $0,20(0,01)[4,5]$ \\
\hline 9-Scene & $0,57(0,03)[4,5]$ & $0,64(0,04)[2,5]$ & $0,57(0,02)[4,5]$ & $0,64(0,03)[2,5]$ & $0,68(0,03)[1,0]$ \\
\hline \multirow[t]{2}{*}{ 10-Yeast } & $0,48(0,02)[5,0]$ & $0,51(0,02)[2,0]$ & $0,51(0,02)[2,0]$ & $0,51(0,02)[2,0]$ & $0,50(0,02)[4,0]$ \\
\hline & \multicolumn{5}{|c|}{$\left|X^{\prime}\right|=30 \% M$} \\
\hline 1-Cal500 & $0,30(0,01)[1,0]$ & $0,28(0,01)[3,5]$ & $0,28(0,01)[3,5]$ & $0,28(0,01)[3,5]$ & $0,28(0,01)[3,5]$ \\
\hline 2-Corel5k & $0,11(0,01)[4,0]$ & $0,11(0,01)[4,0]$ & $0,14(0,01)[1,0]$ & $0,11(0,01)[4,0]$ & $0,13(0,01)[2,0]$ \\
\hline 3-Corel16k001 & $0,13(0,01)[4,0]$ & $0,13(0,00)[4,0]$ & $0,15(0,00)[1,0]$ & $0,13(0,00)[4,0]$ & $0,14(0,00)[2,0]$ \\
\hline 4-Emotions & $0,54(0,04)[2,0]$ & $0,55(0,03)[1,0]$ & $0,53(0,04)[3,5]$ & $0,53(0,04)[3,5]$ & $0,52(0,05)[5,0]$ \\
\hline 5-Fapesp & $0,17(0,05)[1,0]$ & $0,15(0,06)[2,5]$ & $0,15(0,05)[2,5]$ & $0,06(0,03)[5,0]$ & $0,11(0,03)[4,0]$ \\
\hline 6-Genbase* & $0,99(0,01)[1,0]$ & $0,95(0,02)[3,5]$ & $0,95(0,02)[3,5]$ & $0,96(0,02)[2,0]$ & $0,60(0,13)[5,0]$ \\
\hline 7-Llog-f* & $0,14(0,01)[2,0]$ & $0,13(0,02)[3,5]$ & $0,15(0,03)[1,0]$ & $0,12(0,03)[5,0]$ & $0,13(0,03)[3,5]$ \\
\hline 8-Magtag5k & $0,25(0,01)[1,5]$ & $0,23(0,01)[3,0]$ & $0,22(0,01)[4,0]$ & $0,25(0,01)[1,5]$ & $0,21(0,01)[5,0]$ \\
\hline 9-Scene & $0,65(0,03)[4,5]$ & $0,66(0,03)[2,5]$ & $0,65(0,02)[4,5]$ & $0,66(0,03)[2,5]$ & $0,69(0,02)[1,0]$ \\
\hline \multirow[t]{2}{*}{ 10-Yeast } & $0,49(0,02)[5,0]$ & $0,52(0,02)[2,0]$ & $0,52(0,02)[2,0]$ & $0,52(0,02)[2,0]$ & $0,50(0,02)[4,0]$ \\
\hline & \multicolumn{5}{|c|}{$\left|X^{\prime}\right|=40 \% M$} \\
\hline 1-Cal500 & $0,30(0,01)[1,0]$ & $0,29(0,01)[2,0]$ & $0,28(0,01)[4,0]$ & $0,28(0,02)[4,0]$ & $0,28(0,01)[4,0]$ \\
\hline 2-Corel5k & $0,11(0,01)[3,0]$ & $0,10(0,01)[4,0]$ & $0,12(0,00)[1,5]$ & $0,09(0,01)[5,0]$ & $0,12(0,01)[1,5]$ \\
\hline 3-Corel16k001 & $0,11(0,01)[5,0]$ & $0,12(0,00)[4,0]$ & $0,15(0,00)[1,0]$ & $0,13(0,00)[3,0]$ & $0,14(0,00)[2,0]$ \\
\hline 4-Emotions & $0,56(0,03)[1,5]$ & $0,56(0,05)[1,5]$ & $0,53(0,04)[4,5]$ & $0,54(0,04)[3,0]$ & $0,53(0,05)[4,5]$ \\
\hline 5-Fapesp & $0,15(0,04)[2,0]$ & $0,16(0,05)[1,0]$ & $0,14(0,05)[3,0]$ & $0,06(0,02)[5,0]$ & $0,11(0,04)[4,0]$ \\
\hline 6-Genbase* & $0,99(0,01)[1,0]$ & $0,95(0,02)[3,5]$ & $0,95(0,02)[3,5]$ & $0,96(0,02)[2,0]$ & $0,68(0,13)[5,0]$ \\
\hline 7-Llog-f* & $0,14(0,02)[2,5]$ & $0,14(0,03)[2,5]$ & $0,15(0,03)[1,0]$ & $0,13(0,03)[4,5]$ & $0,13(0,03)[4,5]$ \\
\hline 8-Magtag5k & $0,26(0,01)[1,0]$ & $0,24(0,01)[3,0]$ & $0,23(0,01)[4,5]$ & $0,25(0,01)[2,0]$ & $0,23(0,02)[4,5]$ \\
\hline 9-Scene & $0,67(0,02)[4,5]$ & $0,69(0,03)[2,5]$ & $0,67(0,02)[4,5]$ & $0,69(0,02)[2,5]$ & $0,71(0,02)[1,0]$ \\
\hline \multirow[t]{2}{*}{ 10-Yeast } & $0,49(0,02)[5,0]$ & $0,53(0,02)[2,0]$ & $0,53(0,02)[2,0]$ & $0,53(0,02)[2,0]$ & $0,51(0,02)[4,0]$ \\
\hline & \multicolumn{5}{|c|}{$\left|X^{\prime}\right|=50 \% M$} \\
\hline 1-Cal500 & $0,31(0,01)[1,0]$ & $0,29(0,01)[2,0]$ & $0,28(0,01)[4,0]$ & $0,28(0,01)[4,0]$ & $0,28(0,01)[4,0]$ \\
\hline 2-Corel5k & $0,10(0,01)[4,0]$ & $0,11(0,01)[2,5]$ & $0,11(0,01)[2,5]$ & $0,07(0,01)[5,0]$ & $0,12(0,01)[1,0]$ \\
\hline 3-Corel16k001 & $0,08(0,01)[5,0]$ & $0,10(0,01)[4,0]$ & $0,14(0,01)[1,0]$ & $0,12(0,00)[3,0]$ & $0,13(0,01)[2,0]$ \\
\hline 4-Emotions & $0,58(0,04)[1,0]$ & $0,55(0,05)[2,5]$ & $0,53(0,04)[5,0]$ & $0,55(0,04)[2,5]$ & $0,54(0,06)[4,0]$ \\
\hline 5-Fapesp & $0,16(0,05)[1,0]$ & $0,13(0,06)[3,0]$ & $0,14(0,04)[2,0]$ & $0,05(0,03)[5,0]$ & $0,12(0,05)[4,0]$ \\
\hline 6-Genbase* & $0,99(0,01)[1,0]$ & $0,95(0,02)[3,5]$ & $0,95(0,02)[3,5]$ & $0,96(0,02)[2,0]$ & $0,74(0,11)[5,0]$ \\
\hline $7-L \log -f^{*}$ & $0,15(0,03)[1,0]$ & $0,14(0,04)[2,5]$ & $0,14(0,03)[2,5]$ & $0,13(0,03)[4,5]$ & $0,13(0,03)[4,5]$ \\
\hline 8-Magtag5k & $0,27(0,01)[1,0]$ & $0,25(0,01)[2,5]$ & $0,24(0,01)[4,5]$ & $0,25(0,01)[2,5]$ & $0,24(0,01)[4,5]$ \\
\hline 9-Scene & $0,69(0,03)[4,5]$ & $0,71(0,04)[2,5]$ & $0,69(0,03)[4,5]$ & $0,71(0,03)[2,5]$ & $0,72(0,03)[1,0]$ \\
\hline \multirow[t]{2}{*}{ 10-Yeast } & $0,50(0,02)[5,0]$ & $0,52(0,02)[3,5]$ & $0,54(0,02)[1,0]$ & $0,53(0,02)[2,0]$ & $0,52(0,02)[3,5]$ \\
\hline & \multicolumn{5}{|c|}{$\left|X^{\prime}\right|=60 \% M$} \\
\hline
\end{tabular}


Tabela A.6: Resultados para Accuracy (continuação)

\begin{tabular}{|c|c|c|c|c|c|}
\hline & $I G-L P$ & $R F-L P$ & $I G-B R$ & $R F-B R$ & SAR \\
\hline 1-Cal500 & $0,31(0,01)[1,0]$ & $0,29(0,01)[2,0]$ & $0,28(0,01)[4,0]$ & $0,28(0,02)[4,0]$ & $0,28(0,01)[4,0]$ \\
\hline 2-Corel5k & $0,08(0,01)[4,0]$ & $0,08(0,01)[4,0]$ & $0,10(0,01)[2,0]$ & $0,08(0,01)[4,0]$ & $0,11(0,02)[1,0]$ \\
\hline 3-Corell6k001 & $0,03(0,01)[5,0]$ & $0,06(0,00)[4,0]$ & $0,13(0,00)[1,0]$ & $0,10(0,01)[3,0]$ & $0,12(0,01)[2,0]$ \\
\hline 4-Emotions & $0,56(0,06)[3,0]$ & $0,57(0,03)[1,5]$ & $0,57(0,05)[1,5]$ & $0,55(0,04)[4,0]$ & $0,53(0,05)[5,0]$ \\
\hline 5-Fapesp & $0,16(0,05)[1,0]$ & $0,14(0,06)[2,0]$ & $0,13(0,05)[3,0]$ & $0,05(0,03)[5,0]$ & $0,11(0,04)[4,0]$ \\
\hline 6-Genbase $e^{*}$ & $0,99(0,01)[1,0]$ & $0,95(0,02)[3,5]$ & $0,95(0,02)[3,5]$ & $0,96(0,02)[2,0]$ & $0,77(0,11)[5,0]$ \\
\hline 7-Llog-f* & $0,14(0,03)[2,5]$ & $0,14(0,03)[2,5]$ & $0,15(0,04)[1,0]$ & $0,13(0,03)[4,5]$ & $0,13(0,03)[4,5]$ \\
\hline 8-Magtag5k & $0,27(0,01)[1,0]$ & $0,25(0,01)[3,0]$ & $0,24(0,01)[5,0]$ & $0,25(0,01)[3,0]$ & $0,25(0,01)[3,0]$ \\
\hline 9-Scene & $0,70(0,03)[4,5]$ & $0,72(0,03)[1,0]$ & $0,70(0,03)[4,5]$ & $0,71(0,03)[2,5]$ & $0,71(0,03)[2,5]$ \\
\hline \multirow[t]{2}{*}{ 10-Yeast } & $0,52(0,02)[5,0]$ & $0,53(0,02)[3,0]$ & $0,54(0,02)[1,0]$ & $0,53(0,02)[3,0]$ & $0,53(0,02)[3,0]$ \\
\hline & & & $\left|X^{\prime}\right|=70 \% M$ & & \\
\hline 1-Cal500 & $0,31(0,01)[1,0]$ & $0,29(0,01)$ & $0,28(0,01)[4,0]$ & $0,28(0,01)[4,0]$ & $0,28(0,01)[4,0]$ \\
\hline 2-Corel5k & $0,08(0,01)[4,0]$ & $0,05(0,01)[5,0]$ & $0,11(0,01)[1,5]$ & $0,09(0,01)[3,0]$ & $0,11(0,01)[1,5]$ \\
\hline 3-Corel16k001 & $0,03(0,00)[4,5]$ & $0,03(0,00)[4,5]$ & $0,13(0,01)[1,0]$ & $0,05(0,01)[3,0]$ & $0,12(0,01)[2,0]$ \\
\hline 4-Emotions & $0,56(0,05)[2,5]$ & $0,57(0,04)[1,0]$ & $0,55(0,05)[4,0]$ & $0,56(0,04)[2,5]$ & $0,54(0,05)[5,0]$ \\
\hline 5-Fapesp & $0,15(0,05)[1,0]$ & $0,14(0,05)[2,0]$ & $0,12(0,04)[3,5]$ & $0,05(0,02)[5,0]$ & $0,12(0,04)[3,5]$ \\
\hline 6-Genbase $e^{*}$ & $0,99(0,01)[1,0]$ & $0,95(0,02)[3,5]$ & $0,95(0,02)[3,5]$ & $0,96(0,02)[2,0]$ & $0,85(0,09)[5,0]$ \\
\hline 7-Llog-f* & $0,14(0,03)[2,0]$ & $0,14(0,03)[2,0]$ & $0,14(0,02)[2,0]$ & $0,12(0,03)[5,0]$ & $0,13(0,03)[4,0]$ \\
\hline 8-Magtag5k & $0,26(0,01)[1,0]$ & $0,25(0,01)[3,5]$ & $0,25(0,01)[3,5]$ & $0,25(0,01)[3,5]$ & $0,25(0,01)[3,5]$ \\
\hline 9-Scene & $0,71(0,02)[2,5]$ & $0,71(0,03)[2,5]$ & $0,70(0,03)[5,0]$ & $0,71(0,03)[2,5]$ & $0,71(0,03)[2,5]$ \\
\hline \multirow[t]{2}{*}{ 10-Yeast } & $0,54(0,02)[1,5]$ & $0,53(0,02)[4,0]$ & $0,54(0,02)[1,5]$ & $0,53(0,02)[4,0]$ & $0,53(0,02)[4,0]$ \\
\hline & & & $\left|X^{\prime}\right|=80 \% M$ & & \\
\hline 1-Cal500 & $0,31(0,01)[1,0]$ & $0,29(0,0$ & $0,29(0,01)[3,0]$ & 1) $[3,0]$ & $0,28(0$ \\
\hline 2-Corel5k & $0,08(0,02)[4,5]$ & $0,08(0,01)[4,5]$ & $0,12(0,01)[1,0]$ & $0,09(0,01)[3,0]$ & $0,11(0,01)[2,0]$ \\
\hline 3-Corel16k001 & $0,04(0,01)[4,0]$ & $0,03(0,01)[5,0]$ & $0,12(0,01)[1,0]$ & $0,05(0,01)[3,0]$ & $0,11(0,01)[2,0]$ \\
\hline 4-Emotions & $0,55(0,03)[4,0]$ & $0,57(0,03)[1,0]$ & $0,56(0,04)[2,0]$ & $0,55(0,05)[4,0]$ & $0,55(0$, \\
\hline 5-Fapesp & $0,14(0,05)[2,0]$ & $0,15(0,05)[1,0]$ & $0,12(0,06)[3,0]$ & $0,05(0,03)[5,0]$ & $0,11(0,05)[4,0]$ \\
\hline 6-Genbase $e^{*}$ & $0,99(0,01)[1,0]$ & $0,95(0,02)[3,5]$ & $0,95(0,02)[3,5]$ & $0,96(0,02)[2,0]$ & $0,91(0,06)[5,0]$ \\
\hline 7-Llog-f* & $0,14(0,03)[2,5]$ & $0,14(0,02)[2,5]$ & $0,14(0,03)[2,5]$ & $0,13(0,03)[5,0]$ & $0,14(0,03)[2,5]$ \\
\hline 8-Magtag5k & $0,26(0,01)[1,0]$ & $0,25(0,01)[3,5]$ & $0,25(0,01)[3,5]$ & $0,25(0,01)[3,5]$ & $0,25(0,01)[3,5]$ \\
\hline 9-Scene & $0,71(0,02)[3,0]$ & $0,71(0,03)[3,0]$ & $0,71(0,03)[3,0]$ & $0,71(0,02)[3,0]$ & $0,71(0,02)[3,0]$ \\
\hline \multirow[t]{2}{*}{ 10-Yeast } & $0,54(0,02)[1,0]$ & $0,53(0,02)[3,5]$ & $0,53(0,02)[3,5]$ & $0,53(0,02)[3,5]$ & $0,53(0,02)[3,5]$ \\
\hline & & & $\left|X^{\prime}\right|=90 \% M$ & & \\
\hline 1-Cal500 & $0,31(0,01)[1,0]$ & $0,29(0,01)[3,0]$ & $0,29(0,01)[3,0]$ & $0,29(0,01)[3,0]$ & $0,28(0,01)[5,0]$ \\
\hline 2-Corel5k & $0,09(0,01)[4,5]$ & $0,09(0,01)[4,5]$ & $0,11(0,01)[1,5]$ & $0,10(0,01)[3,0]$ & $0,11(0,01)[1,5]$ \\
\hline 3-Corel16k001 & $0,05(0,00)[4,5]$ & $0,05(0,00)[4,5]$ & $0,12(0,00)[1,0]$ & $0,07(0,01)[3,0]$ & $0,11(0,01)[2,0]$ \\
\hline 4-Emotions & $0,56(0,05)[2,5]$ & $0,56(0,04)[2,5]$ & $0,56(0,04)[2,5]$ & $0,55(0,05)[5,0]$ & $0,56(0,06)[2,5]$ \\
\hline 5-Fapesp & $0,13(0,06)[2,0]$ & $0,13(0,05)[2,0]$ & $0,13(0,05)[2,0]$ & $0,05(0,02)[5,0]$ & $0,12(0,04)[4,0]$ \\
\hline 6-Genbase $e^{*}$ & $0,99(0,01)[1,0]$ & $0,95(0,02)[3,5]$ & $0,95(0,02)[3,5]$ & $0,96(0,02)[2,0]$ & $0,93(0,04)[5,0]$ \\
\hline 7-Llog-f* & $0,15(0,03)[2,0]$ & $0,15(0,02)[2,0]$ & $0,15(0,03)[2,0]$ & $0,13(0,03)[5,0]$ & $0,14(0,03)[4,0]$ \\
\hline 8-Magtag $5 k$ & $0,26(0,01)[2,5]$ & $0,26(0,01)[2,5]$ & $0,26(0,01)[2,5]$ & $0,26(0,01)[2,5]$ & $0,25(0,01)[5,0]$ \\
\hline 9-Scene & $0,71(0,02)[3,5]$ & $0,71(0,02)[3,5]$ & $0,71(0,02)[3,5]$ & $0,71(0,02)[3,5]$ & $0,72(0,03)[1,0]$ \\
\hline 10-Yeast & $0,54(0,02)[1,0]$ & $0,53(0,02)[3,5]$ & $0,53(0,02)[3,5]$ & $0,53(0,02)[3,5]$ & $0,53(0,02)[3,5]$ \\
\hline
\end{tabular}

Tabela A.7: Resultados experimentais de acordo com $F_{b}$ : cinco métodos de SA baseados em abordagens tradicionais de transformação do problema

\begin{tabular}{|c|c|c|c|c|c|}
\hline & $I G-L P$ & $R F-L P$ & $I G-B R$ & $R F-B R$ & SAR \\
\hline & \multicolumn{5}{|c|}{$\left|X^{\prime}\right|=10 \% M$} \\
\hline 1-Cal500 & $0,45(0,01)[1,0]$ & $0,43(0,01)[3,5]$ & $0,43(0,02)[3,5]$ & $0,43(0,02)[3,5]$ & $0,43(0,01)[3,5]$ \\
\hline 2-Corel5k & $0,20(0,01)[3,0]$ & $0,19(0,01)[4,5]$ & $0,21(0,01)[1,5]$ & $0,19(0,01)[4,5]$ & $0,21(0,01)[1,5]$ \\
\hline 3-Corel16k001 & $0,21(0,01)[4,0]$ & $0,21(0,01)[4,0]$ & $0,23(0,00)[1,0]$ & $0,21(0,01)[4,0]$ & $0,22(0,00)[2,0]$ \\
\hline 4-Emotions & $0,62(0,05)[2,5]$ & $0,63(0,04)[1,0]$ & $0,62(0,03)[2,5]$ & $0,59(0,04)[4,5]$ & $0,59(0,05)[4,5]$ \\
\hline 5-Fapesp & $0,23(0,07)[1,0]$ & $0,22(0,05)[2,0]$ & $0,19(0,05)[3,0]$ & $0,12(0,05)[5,0]$ & $0,13(0,05)[4,0]$ \\
\hline 6-Genbase* & $0,99(0,01)[1,0]$ & $0,95(0,02)[3,0]$ & $0,95(0,01)[3,0]$ & $0,95(0,02)[3,0]$ & $0,37(0,09)[5,0]$ \\
\hline 7-Llog-f* & $0,13(0,02)[3,0]$ & $0,13(0,03)[3,0]$ & $0,17(0,02)[1,0]$ & $0,13(0,02)[3,0]$ & $0,12(0,03)[5,0]$ \\
\hline 8-Magtag $5 k$ & $0,31(0,00)[1,0]$ & $0,29(0,01)[3,0]$ & $0,28(0,01)[4,0]$ & $0,30(0,01)[2,0]$ & $0,26(0,02)[5,0]$ \\
\hline 9-Scene & $0,54(0,03)[3,0]$ & $0,59(0,04)[2,0]$ & $0,46(0,03)[5,0]$ & $0,51(0,02)[4,0]$ & $0,65(0,03)[1,0]$ \\
\hline
\end{tabular}


Tabela A.7: Resultados para $F_{b}$ (continuação)

\begin{tabular}{|c|c|c|c|c|c|}
\hline & $I G-L P$ & $R F-L P$ & $I G-B R$ & $R F-B R$ & SAR \\
\hline \multirow[t]{2}{*}{ 10-Yeast } & $0,60(0,02$ & $0,63(0,01$ & $0,62(0,02)[3,0]$ & $0,63(0,02$ & $0,60(0$ \\
\hline & \multicolumn{5}{|c|}{$\left|X^{\prime}\right|=20 \% M$} \\
\hline 1-Cal500 & $0,46(0,01)[1,0]$ & $0,43(0,02)[3,5]$ & $0,43(0,01)[3,5]$ & $0,43(0,02)[3,5]$ & $0,43(0,02)[3,5]$ \\
\hline 2-Corel5k & $0,19(0,01)[3,5]$ & $0,18(0,01)[5,0]$ & $0,21(0,01)[1,0]$ & $0,19(0,01)[3,5]$ & $0,20(0,01)[2,0]$ \\
\hline 3-Corel16k001 & $0,21(0,01)[4,0]$ & $0,21(0,00)[4,0]$ & $0,24(0,00)[1,0]$ & $0,21(0,01)[4,0]$ & $0,22(0,01)[2,0]$ \\
\hline 4-Emotions & $0,64(0,03)[2,0]$ & $0,67(0,05)[1,0]$ & $0,63(0,03)[3,0]$ & $0,61(0,03)[4,5]$ & $0,61(0,03)[4,5]$ \\
\hline 5-Fapesp & $0,21(0,05)[1,0]$ & $0,18(0,05)[2,0]$ & $0,17(0,07)[3,0]$ & $0,08(0,04)[5,0]$ & $0,13(0,05)[4,0]$ \\
\hline 6-Genbase $e^{*}$ & $0,99(0,01)[1,0]$ & $0,95(0,02)[3,0]$ & $0,95(0,01)[3,0]$ & $0,95(0,02)[3,0]$ & $0,48(0,11)[5,0]$ \\
\hline 7-Llog-f* & $0,14(0,02)[3,5]$ & $0,14(0,02)[3,5]$ & $0,16(0,02)[1,0]$ & $0,15(0,01)[2,0]$ & $0,12(0,03)[5,0]$ \\
\hline 8-Magtag5k & $0,32(0,01)[3,0]$ & $0,33(0,01)[2,0]$ & $0,31(0,01)[4,0]$ & $0,34(0,02)[1,0]$ & $0,30(0,02)[5,0]$ \\
\hline 9-Scene & $0,58(0,03)[4,5]$ & $0,64(0,04)[3,0]$ & $0,58(0,02)[4,5]$ & $0,65(0,03)[2,0]$ & $0,68(0,03)[1,0]$ \\
\hline \multirow[t]{2}{*}{ 10-Yeast } & $0,61(0,02)[5,0]$ & $0,64(0,01)[2,0]$ & $0,64(0,02)[2,0]$ & $0,64(0,02)[2,0]$ & $0,62(0,02)[4,0]$ \\
\hline & \multicolumn{5}{|c|}{$\left|X^{\prime}\right|=30 \% M$} \\
\hline $1-\mathrm{Ca}$ & $0,46(0,01)[1,0]$ & $0,43(0,02)$ & $0,43(0,01)[3,5]$ & $0,43(0,02)[3,5]$ & $0,43(0,02)[3,5]$ \\
\hline 2-Corel5k & $0,17(0,01)[4,0]$ & $0,17(0,01)[4,0]$ & $0,21(0,01)[1,0]$ & $0,17(0,01)[4,0]$ & $0,20(0$, \\
\hline 3-Corel16k001 & $0,20(0,01)[4,5]$ & $0,20(0,01)[4,5]$ & $0,23(0,00)[1,0]$ & $0,21(0,00)[3,0]$ & $0,22(0$ \\
\hline 4-Emotions & $0,65(0,03)[2,0]$ & $0,66(0,03)[1,0]$ & $0,64(0,04)[3,0]$ & $0,63(0,04)[4,5]$ & $0,63(0$, \\
\hline 5-Fapesp & $0,22(0,05)[1,0]$ & $0,17(0,06)[2,5]$ & $0,17(0,05)[2,5]$ & $0,08(0,04)[5,0]$ & $0,14(0,04)[4,0]$ \\
\hline 6-Genbase* & $0,99(0,01)[1,0]$ & $0,95(0,02)[3,0]$ & $0,95(0,01)[3,0]$ & $0,95(0,02)[3,0]$ & $0,62(0$ \\
\hline 7-Llog-f* & $0,16(0,02)[2,0]$ & $0,14(0,02)[3,5]$ & $0,17(0,04)[1,0]$ & $0,13(0,03)[5,0]$ & $0,14(0,03)[3,5]$ \\
\hline 8-Magtag5k & $0,36(0,01)[1,0]$ & $0,34(0,01)[3,0]$ & $0,32(0,02)[4,0]$ & $0,35(0,01)[2,0]$ & $0,31(0,01)[5,0]$ \\
\hline 9-Scene & $0,65(0,03)[5,0]$ & $0,67(0,03)[2,5]$ & $0,66(0,02)[4,0]$ & $0,67(0,03)[2,5]$ & $0,70(0,02)[1,0]$ \\
\hline \multirow[t]{2}{*}{ 10-Yeast } & $0,61(0,02)[5,0]$ & $0,64(0,02)[2,5]$ & $0,65(0,02)[1,0]$ & $0,64(0,02)[2,5]$ & $0,63(0$ \\
\hline & \multicolumn{5}{|c|}{$\left|X^{\prime}\right|=40 \% M$} \\
\hline $1-C$ & $0,46(0,01)[1,0]$ & $0,44(0,02)$ & $0,43(0,02)[4,5]$ & $0,44(0,02)[2,5]$ & ,5] \\
\hline $2-C$ & $0,18(0,01)[3,0]$ & $0,16(0,01)[4,0]$ & $0,19(0,01)[1,5]$ & $0,14(0,01)[5,0]$ & 0,19 (0 \\
\hline 3-Corel16kO & $0,18(0,01)[5,0]$ & $0,19(0,01)$ & $0,22(0,1$ & $0,20(0,00)[3,0]$ & 0,22 \\
\hline 4-Emotions & $0,67(0,03)[1,5]$ & $0,67(0,05)[1,5]$ & $0,64(0,03)[4,5]$ & $0,65(0,03)[3,0]$ & $0,64(0$ \\
\hline 5-Fapesp & $0,20(0,05)[1,0]$ & $0,18(0,05)[2,0]$ & $0,15(0,04)[3,0]$ & $0,08(0,03)[5,0]$ & $0,13(0$ \\
\hline 6-Genbase* & $0,99(0,01)[1,0]$ & $0,95(0,02)[3,0]$ & $0,95(0,01)[3,0]$ & $0,95(0,02)[3,0]$ & $0,68(0$ \\
\hline $7-L \log -f^{*}$ & $0,16(0,02)[1,5]$ & $0,15(0,03)[3,0]$ & $0,16(0,02)[1,5]$ & $0,14(0,03)[4,5]$ & $0,14(0$, \\
\hline 8-Magtag5k & $0,37(0,01)[1,0]$ & $0,34(0,01)$ & $0,33(0,01)[4,5]$ & $0,36(0,01)[2,0]$ & $0,33(0$ \\
\hline 9-Scene & $0,67(0,02)[5,0]$ & $0,70(0,03)[2,5]$ & $0,68(0,02)[4,0]$ & $0,70(0,02)[2,5]$ & $0,71(0,02)[1,0]$ \\
\hline \multirow[t]{2}{*}{ 10-Yeast } & $0,62(0,02)[5,0]$ & $0,65(0,02)[2,0]$ & $0,65(0,02)[2,0]$ & $0,65(0,02)[2,0]$ & $0,64(0$, \\
\hline & \multicolumn{5}{|c|}{$\left|X^{\prime}\right|=50 \% M$} \\
\hline $1-\mathrm{Co}$ & $0,46(0,0)$ & $0,44(0,02)$ & $0,43(0,02)[4,5]$ & $0,44(0,02)[2,5]$ & $4,5]$ \\
\hline 2-Corel5k & $0,17(0,01)[2,5]$ & $0,16(0,01)[4,0]$ & $0,17(0,01)[2,5]$ & $0,11(0,01)[5,0]$ & 0,18 (C \\
\hline 3-Corel16kO & $0,14(0,02)[5,0]$ & $0,16(0,01)[4,0]$ & $0,21(0,0$ & $0,19(0,01)[3,0]$ & 0,21 \\
\hline 4-Emotions & $0,69(0,03)[1,0]$ & $0,66(0,04)[2,5]$ & $0,64(0,04)[5,0]$ & $0,66(0,04)[2,5]$ & $0,65(0$ \\
\hline 5-Fapesp & $0,21(0,06)[1,0]$ & $0,15(0,06)[2,5]$ & $0,15(0,04)[2,5]$ & $0,07(0,03)[5,0]$ & $0,14(0$, \\
\hline 6-Genbase ${ }^{*}$ & $0,99(0,01)[1,0]$ & $0,95(0,02)[3,0]$ & $0,95(0,01)[3,0]$ & $0,95(0,02)[3,0]$ & $0,75(0$, \\
\hline 7-Llog-f* & $0,16(0,03)[1,5]$ & $0,15(0,03)[3,0]$ & $0,16(0,02)[1,5]$ & $0,14(0,03)[4,5]$ & $0,14(0$, \\
\hline 8-Magtag5k & $0,39(0,01)[1,0]$ & $0,35(0,01)[3,0]$ & $0,34(0,01)[4,5]$ & $0,36(0,01)[2,0]$ & $0,34(0$, \\
\hline 9-Scene & $0,70(0,03)[4,5]$ & $0,71(0,03)[2,5]$ & $0,70(0,02)[4,5]$ & $0,71(0,03)[2,5]$ & $0,72(0,03)[1,0]$ \\
\hline \multirow[t]{2}{*}{ 10-Yeast } & $0,63(0,01)[5,0]$ & $0,65(0,01)[3,5]$ & $0,66(0,02)[1,5]$ & $0,66(0,02)[1,5]$ & $0,65(0,02)[3,5]$ \\
\hline & \multicolumn{5}{|c|}{$\left|X^{\prime}\right|=60 \% M$} \\
\hline $1-\mathrm{Ca}$ & 4610 & $0,44(0,02)$ & $0,43(0,02)[4,0]$ & 0,4310 & $0,43(C$ \\
\hline 2-Corel5k & $0,12(0,02)[4,0]$ & $0,12(0,02)[4,0]$ & $0,16(0,01)[2,0]$ & $0,12(0,01)[4,0]$ & $0,17(0,03)[1,0]$ \\
\hline 3-Corel16kO & $0,05(0,01)[5,0]$ & $0,09(0,01)[4,0]$ & $0,20(0,01)[1,0]$ & $0,15(0,02)[3,0]$ & $0,19(0$ \\
\hline 4-Emotions & $0,67(0,05)[2,0]$ & $0,67(0,03)[2,0]$ & $0,67(0,05)[2,0]$ & $0,65(0,04)[4,0]$ & $0,64(0,05)[5,0]$ \\
\hline 5-Fapesp & $0,19(0,05)[1,0]$ & $0,14(0,06)[2,5]$ & $0,14(0,05)[2,5]$ & $0,07(0,04)[5,0]$ & $0,13(0,05)[4,0]$ \\
\hline 6-Genbase* & $0,99(0,01)[1,0]$ & $0,95(0,02)[3,0]$ & $0,95(0,01)[3,0]$ & $0,95(0,02)[3,0]$ & $0,78(0,11)[5,0]$ \\
\hline 7-Llog-f* & $0,16(0,02)[2,0]$ & $0,15(0,03)[3,0]$ & $0,17(0,04)[1,0]$ & $0,14(0,03)[4,5]$ & $0,14(0,03)[4,5]$ \\
\hline 8-Magtag5k & $0,38(0,01)[1,0]$ & $0,35(0,01)[4,0]$ & $0,35(0,01)[4,0]$ & $0,36(0,01)[2,0]$ & $0,35(0,01)[4,0]$ \\
\hline 9-Scene & $0,71(0,03)[4,0]$ & $0,72(0,03)[2,0]$ & $0,70(0,03)[5,0]$ & $0,72(0,03)[2,0]$ & $0,72(0,03)[2,0]$ \\
\hline \multirow[t]{2}{*}{ 10-Yeast } & $0,65(0,01)[3,5]$ & $0,65(0,02)[3,5]$ & $0,66(0,02)[1,0]$ & $0,65(0,02)[3,5]$ & $0,65(0,02)[3,5]$ \\
\hline & \multicolumn{5}{|c|}{$\left|X^{\prime}\right|=70 \% M$} \\
\hline 1-Cal500 & $0,46(0,01)[1,0]$ & $0,44(0,02)[2,5]$ & $0,44(0,02)[2,5]$ & ,02) $[4,5]$ & $43(0,01)[4,5]$ \\
\hline
\end{tabular}


Tabela A.7: Resultados para $F_{b}$ (continuação)

\begin{tabular}{|c|c|c|c|c|c|}
\hline & $I G-L P$ & $R F-L P$ & $I G-B R$ & $R F-B R$ & SAR \\
\hline 2-Corel5k & $0,13(0,02)[4,0]$ & $0,08(0,01)[5,0]$ & $0,17(0,01)[1,5]$ & $0,14(0,02)[3,0]$ & $0,17(0,02)[1,5]$ \\
\hline 3-Corel16k001 & $0,04(0,01)[5,0]$ & $0,05(0,01)[4,0]$ & $0,19(0,01)[1,0]$ & $0,08(0,01)[3,0]$ & $0,18(0,02)[2,0]$ \\
\hline 4-Emotions & $0,67(0,04)[2,0]$ & $0,67(0,03)[2,0]$ & $0,66(0,04)[4,0]$ & $0,67(0,04)[2,0]$ & $0,65(0,05)[5,0]$ \\
\hline 5-Fapesp & $0,19(0,06)[1,0]$ & $0,15(0,05)[2,5]$ & $0,12(0,04)[4,0]$ & $0,07(0,03)[5,0]$ & $0,15(0,04)[2,5]$ \\
\hline 6-Genbase $e^{*}$ & $0,99(0,01)[1,0]$ & $0,95(0,02)[3,0]$ & $0,95(0,01)[3,0]$ & $0,95(0,02)[3,0]$ & $0,86(0,08)[5,0]$ \\
\hline 7-Llog-f* & $0,15(0,03)[3,5]$ & $0,16(0,03)[1,5]$ & $0,16(0,02)[1,5]$ & $0,14(0,04)[5,0]$ & $0,15(0,03)[3,5]$ \\
\hline 8-Magtag $5 k$ & $0,37(0,01)[1,0]$ & $0,36(0,01)[3,5]$ & $0,36(0,01)[3,5]$ & $0,36(0,01)[3,5]$ & $0,36(0,01)[3,5]$ \\
\hline 9-Scene & $0,72(0,02)[2,5]$ & $0,72(0,03)[2,5]$ & $0,71(0,03)[5,0]$ & $0,72(0,03)[2,5]$ & $0,72(0,03)[2,5]$ \\
\hline \multirow[t]{2}{*}{ 10-Yeast } & $0,66(0,01)[2,0]$ & $0,65(0,02)[4,5]$ & $0,66(0,02)[2,0]$ & $0,66(0,02)[2,0]$ & $0,65(0,02)[4,5]$ \\
\hline & & & $\left|X^{\prime}\right|=80 \% M$ & & \\
\hline 1-Cal500 & $0,46(0,01)[1,0]$ & $0,44(0,02)[3,5]$ & $0,44(0,02)[3,5]$ & $0,44(0,02)[3,5]$ & $0,44(0,02)[3,5]$ \\
\hline 2-Corel5k & $0,12(0,02)[4,5]$ & $0,12(0,02)[4,5]$ & $0,18(0,01)[1,0]$ & $0,15(0,02)[3,0]$ & $0,17(0,02)[2,0]$ \\
\hline 3-Corel16k001 & $0,06(0,02)[4,0]$ & $0,05(0,01)[5,0]$ & $0,18(0,01)[1,0]$ & $0,08(0,02)[3,0]$ & $0,17(0,01)[2,0]$ \\
\hline 4-Emotions & $0,65(0,03)[5,0]$ & $0,67(0,03)[1,5]$ & $0,67(0,03)[1,5]$ & $0,66(0,05)[3,5]$ & $0,66(0,04)[3,5]$ \\
\hline 5-Fapesp & $0,18(0,06)[1,0]$ & $0,16(0,03)[2,0]$ & $0,13(0,04)[3,5]$ & $0,06(0,03)[5,0]$ & $0,13(0,05)[3,5]$ \\
\hline 6-Genbase & $0,99(0,01)[1,0]$ & $0,95(0,02)[3,0]$ & $0,95(0,01)[3,0]$ & $0,95(0,02)[3,0]$ & $0,91(0,06)[5,0]$ \\
\hline 7-Llog-f* & $0,16(0,03)[1,5]$ & $0,15(0,02)[3,5]$ & $0,16(0,03)[1,5]$ & $0,14(0,02)[5,0]$ & $0,15(0,03)[3,5]$ \\
\hline 8-Magtag5k & $0,37(0,01)[1,0]$ & $0,36(0,01)[3,5]$ & $0,36(0,01)[3,5]$ & $0,36(0,01)[3,5]$ & $0,36(0,01)[3,5]$ \\
\hline 9-Scene & $0,72(0,02)[1,5]$ & $0,71(0,03)[4,0]$ & $0,71(0,03)[4,0]$ & $0,71(0,03)[4,0]$ & $0,72(0,02)[1,5]$ \\
\hline \multirow[t]{2}{*}{ 10-Yeast } & $0,66(0,01)[2,0]$ & $0,66(0,02)[2,0]$ & $0,66(0,02)[2,0]$ & $0,65(0,02)[4,5]$ & $0,65(0,02)[4,5]$ \\
\hline & & & $\left|X^{\prime}\right|=90 \% M$ & & \\
\hline 1-Cal500 & $0,47(0,01)[1,0]$ & $0,44(0,02)[3,5]$ & $0,44(0,01)[3,5]$ & $0,44(0,02)[3,5]$ & $0,44(0,02)[3,5]$ \\
\hline 2-Corel5k & $0,14(0,01)[4,5]$ & $0,14(0,01)[4,5]$ & $0,17(0,01)[1,5]$ & $0,15(0,02)[3,0]$ & $0,17(0,01)[1,5]$ \\
\hline 3-Corel16k001 & $0,09(0,01)[4,0]$ & $0,07(0,00)[5,0]$ & $0,18(0,01)[1,0]$ & $0,11(0,01)[3,0]$ & $0,17(0,01)[2,0]$ \\
\hline 4-Emotions & $0,66(0,04)[3,5]$ & $0,66(0,04)[3,5]$ & $0,67(0,03)[1,0]$ & $0,66(0,04)[3,5]$ & $0,66(0,05)[3,5]$ \\
\hline 5-Fapesp & $0,17(0,07)[1,0]$ & $0,15(0,05)[2,5]$ & $0,15(0,05)[2,5]$ & $0,07(0,02)[5,0]$ & $0,14(0,04)[4,0]$ \\
\hline 6-Genbase $e^{*}$ & $0,99(0,01)[1,0]$ & $0,95(0,02)[3,0]$ & $0,95(0,01)[3,0]$ & $0,95(0,02)[3,0]$ & $0,93(0,03)[5,0]$ \\
\hline 7-Llog-f* & $0,16(0,03)[2,5]$ & $0,16(0,02)[2,5]$ & $0,16(0,02)[2,5]$ & $0,14(0,03)[5,0]$ & $0,16(0,03)[2,5]$ \\
\hline 8-Magtag5k & $0,38(0,01)[1,0]$ & $0,37(0,01)[2,5]$ & $0,37(0,01)[2,5]$ & $0,36(0,01)[4,5]$ & $0,36(0,01)[4,5]$ \\
\hline 9-Scene & $0,72(0,02)[3,0]$ & $0,72(0,03)[3,0]$ & $0,72(0,02)[3,0]$ & $0,72(0,03)[3,0]$ & $0,72(0,03)[3,0]$ \\
\hline 10-Yeast & $0,66(0,02)[2,5]$ & $0,66(0,02)[2,5]$ & $0,65(0,02)[5,0]$ & $0,66(0,02)[2,5]$ & $0,66(0,02)[2,5]$ \\
\hline
\end{tabular}

\section{A.2.2 Label Construction for Feature Selection}

Tabela A.8: Resultados experimentais de acordo com F-measure baseada em exemplos: quatro configurações do LCFS e algoritmo $I G-B R$

\begin{tabular}{|c|c|c|c|c|c|}
\hline & $I G-B R$ & SL-X & $S C-A$ & SR-A & SR-X \\
\hline & \multicolumn{5}{|c|}{$\left|X^{\prime}\right|=10 \% M$} \\
\hline 1-Cal500 & $0,43(0,02)[3,0]$ & $0,43(0,01)[3,0]$ & $0,43(0,02)[3,0]$ & $0,43(0,02)[3,0]$ & $0,43(0,02)[3,0]$ \\
\hline 2-Corel5k & $0,21(0,01)[3,0]$ & $0,21(0,01)[3,0]$ & $0,21(0,01)[3,0]$ & $0,21(0,01)[3,0]$ & $0,21(0,01)[3,0]$ \\
\hline 3-Corell6k001 & $0,23(0,00)[3,0]$ & $0,23(0,00)[3,0]$ & $0,23(0,00)[3,0]$ & $0,23(0,00)[3,0]$ & $0,23(0,00)[3,0]$ \\
\hline 4-Emotions & $0,60(0,03)[2,0]$ & $0,60(0,03)[2,0]$ & $0,59(0,03)[4,5]$ & $0,60(0,03)[2,0]$ & $0,59(0,03)[4,5]$ \\
\hline 5-Fapesp & $0,19(0,05)[5,0]$ & $0,21(0,07)[1,5]$ & $0,20(0,04)[3,5]$ & $0,20(0,06)[3,5]$ & $0,21(0,06)[1,5]$ \\
\hline 6-Genbase* & $0,96(0,01)[3,0]$ & $0,96(0,01)[3,0]$ & $0,96(0,01)[3,0]$ & $0,96(0,01)[3,0]$ & $0,96(0,01)[3,0]$ \\
\hline $7-\operatorname{Llog}-f^{*}$ & $0,16(0,03)[4,5]$ & $0,17(0,03)[2,0]$ & $0,16(0,03)[4,5]$ & $0,17(0,03)[2,0]$ & $0,17(0,03)[2,0]$ \\
\hline 8-Magtag5k & $0,27(0,02)[3,0]$ & $0,27(0,01)[3,0]$ & $0,27(0,01)[3,0]$ & $0,27(0,02)[3,0]$ & $0,27(0,02)[3,0]$ \\
\hline 9-Scene & $0,46(0,03)[5,0]$ & $0,47(0,04)[3,5]$ & $0,51(0,06)[2,0]$ & $0,47(0,02)[3,5]$ & $0,52(0,04)[1,0]$ \\
\hline \multirow[t]{2}{*}{ 10-Yeast } & $0,60(0,02)[4,0]$ & $0,61(0,02)[1,5]$ & $0,60(0,01)[4,0]$ & $0,61(0,02)[1,5]$ & $0,60(0,02)[4,0]$ \\
\hline & \multicolumn{5}{|c|}{$\left|X^{\prime}\right|=20 \% M$} \\
\hline 1-Cal500 & $0,43(0,01)[3,0]$ & $0,43(0,01)[3,0]$ & $0,43(0,01)[3,0]$ & $0,43(0,01)[3,0]$ & $0,43(0,01)[3,0]$ \\
\hline 2-Corel5k & $0,21(0,01)[3,0]$ & $0,21(0,01)[3,0]$ & $0,21(0,01)[3,0]$ & $0,21(0,01)[3,0]$ & $0,21(0,01)[3,0]$ \\
\hline 3-Corel16k001 & $0,23(0,00)[3,0]$ & $0,23(0,00)[3,0]$ & $0,23(0,00)[3,0]$ & $0,23(0,00)[3,0]$ & $0,23(0,00)[3,0]$ \\
\hline 4-Emotions & $0,61(0,03)[3,0]$ & $0,61(0,04)[3,0]$ & $0,61(0,03)[3,0]$ & $0,61(0,03)[3,0]$ & $0,61(0,03)[3,0]$ \\
\hline 5-Fapesp & $0,18(0,07)[2,5]$ & $0,17(0,05)[5,0]$ & $0,18(0,05)[2,5]$ & $0,18(0,07)[2,5]$ & $0,18(0,06)[2,5]$ \\
\hline 6-Genbase* & $0,96(0,01)[3,0]$ & $0,96(0,01)[3,0]$ & $0,96(0,01)[3,0]$ & $0,96(0,01)[3,0]$ & $0,96(0,01)[3,0]$ \\
\hline
\end{tabular}


Tabela A.8: Resultados para F-measure baseada em exemplos (continuação)

\begin{tabular}{|c|c|c|c|c|c|}
\hline & $I G-B R$ & $S L-X$ & $S C-A$ & SR-A & SR-X \\
\hline 7-Llog-f* & $17(0,02)[2,5]$ & $0,17(0,02)[2,5]$ & $0,16(0,03)[5,0]$ & $0,17(0,03)[2,5]$ & $0,17(0,03)[2,5]$ \\
\hline 8-Magtag5k & $0,30(0,01)[3,0]$ & $0,30(0,02)[3,0]$ & $0,30(0,01)[3,0]$ & $0,30(0,02)[3,0]$ & $0,30(0,02)[3,0]$ \\
\hline 9-Scene & $58(0,02)[3,5]$ & $0,58(0,03)[3,5]$ & $0,58(0,02)[3,5]$ & $0,58(0,02)[3,5]$ & $0,59(0,04)[1,0]$ \\
\hline \multirow[t]{2}{*}{ 10-Yeast } & $62(0,02)[3,5]$ & $0,62(0,02)[$ & $0,63(0,02)[1,0]$ & $0,62(0,02)[3,5]$ & $0,62(0,02)[3,5]$ \\
\hline & & & $\left|X^{\prime}\right|=30 \% M$ & & \\
\hline 1-Cal50O & $0,43(0,01)[3,0]$ & $0,43(0,01)[3,0]$ & $0,43(0,02)[3,0]$ & $0,43(0,02)[3,0]$ & $0,43(0,01)[3,0]$ \\
\hline 2-Corel5k & $0,21(0,01)[2,5]$ & $0,20(0,01)[5,0]$ & $0,21(0,01)[2,5]$ & $0,21(0,01)[2,5]$ & $0,21(0,01)[2,5]$ \\
\hline 3-Corel16k001 & $0,23(0,00)[3,0]$ & $0,23(0,01)[3,0]$ & $0,23(0,00)[3,0]$ & $0,23(0,01)[3,0]$ & $0,23(0,00)[3,0]$ \\
\hline 4-Emotions & $0,62(0,04)[3,0]$ & $0,61(0,03)[5,0]$ & $0,63(0,04)[1,0]$ & $0,62(0,04)[3,0]$ & $0,62(0,05)[3,0]$ \\
\hline 5-Fapesp & $0,18(0,05)[2,0]$ & $0,18(0,07)[2,0]$ & $0,18(0,05)[2,0]$ & $0,17(0,06)[4,5]$ & $0,17(0,05)[4,5]$ \\
\hline 6-Genbase* & $0,96(0,01)[3,0]$ & $0,96(0,01)[3,0]$ & $0,96(0,01)[3,0]$ & $0,96(0,01)[3,0]$ & $0,96(0,01)[3,0]$ \\
\hline 7-Llog-f* & $0,17(0,04)[3,5]$ & $0,16(0,03)[5,0]$ & $0,18(0,04)[1,5]$ & $0,18(0,03)[1,5]$ & $0,17(0,03)[3,5]$ \\
\hline 8-Magtag5k & $0,32(0,02)[3,5]$ & $0,33(0,01)[1,0]$ & $0,32(0,02)[3,5]$ & $0,32(0,02)[3,5]$ & $0,32(0,02)[3,5]$ \\
\hline 9-Scene & $0,66(0,02)[2,5]$ & $0,60(0,03)[5,0]$ & $0,66(0,03)[2,5]$ & $0,66(0,02)[2,5]$ & $0,66(0,03)[2,5]$ \\
\hline \multirow[t]{2}{*}{ 10-Yeast } & $0,63(0,02)[3,0]$ & $0,63(0,02)[3,0]$ & $0,63(0,02)[3,0]$ & $0,63(0,02)[3,0]$ & $0,63(0,02)[3,0]$ \\
\hline & & & $\left|X^{\prime}\right|=40 \% M$ & & \\
\hline $1-C$ & $0,43(0,02$ & $0,44(0$, & $0,43(0,02)[3,5]$ & $0,43(0, \mathrm{C}$ & $0,43(0$, \\
\hline 2-Corel5k & $0,19(0,01)$ & $0,19(0,0$ & $0,19(0, c$ & $0,19(0,0$ & $0,20(0,01)[1,0]$ \\
\hline 3-Corel16k001 & $0,22(0,01)[3,0]$ & $0,22(0,01)[3,0]$ & $0,22(0$, & $0,22(0,01)$ & $0,22(0$, \\
\hline tions & $0,62(0,03)$ & 0,6310 & $0,63(0$, & $0,63(0, \mathrm{C}$ & $0,62(0$, \\
\hline & $0,16(0,05)[3$ & $0,17(0,0$ & $0,15(0, c$ & $0,15(0,05)$ & $0,17(0,06)$ \\
\hline 6-Genbase* & $0,96(0,01)[3,0]$ & $0,96(0,01)[3,0]$ & $0,96(0,01)[3,0]$ & $0,96(0,01)[3,0]$ & $0,96(0,01)[3,0]$ \\
\hline 7-Llog-f* & $0,16(0,03)[4,0]$ & $0,17(0,0$ & $0,16(0$ & $0,17(0,0$ & $0,16(0,03)$ \\
\hline 8-Magtag5k & $0,33(0,01)[3,0]$ & $0,33(0,01)[3,0]$ & $0,33(0,01)[3,0]$ & $0,33(0,01)[3,0]$ & $0,33(0,01)[3,0]$ \\
\hline 9-Scene & $0,68(0,02)[2,5]$ & $0,67(0,03)[5,0]$ & $0,68(0,03)[2,5]$ & $0,68(0,02)[2,5]$ & $0,68(0,04)[2,5]$ \\
\hline \multirow[t]{2}{*}{ 10-Yeast } & $0,64(0,02)[2,5]$ & $0,63(0,0$ & $0,64(0,02)[2,5]$ & $0,64(0,02)[2,5]$ & $0,64(0,02)[2,5]$ \\
\hline & & & $\left|X^{\prime}\right|=$ & & \\
\hline $1-C$ & 4310,1 & 0,4310 & $0,43(0$, & 0,43 & $0,43(0$ \\
\hline 2-Corel5k & $0,17(0,01)[4,0]$ & $0,18(0,0$ & $0,18(0$, & $0,17(0,0$ & $0,17(0,01)[4,0]$ \\
\hline 3-Corel16k001 & $0,21(0,01)[3,0]$ & $0,21(0, C$ & $0,21(0$, & $0,21(0$, & $0,21(0,01)[3,0]$ \\
\hline 4-Emotions & $0,62(0,05$ & $0,62(0$, & $0,62(0$ & $0,62(0$ & 0,6310 \\
\hline & $0,16(0,05)[1,5]$ & $0,15(0,0$ & $0,14(0$, & $0,15(0$, & $0,16(0$, \\
\hline 6-Genbase* & $0,96(0,01)[3,0]$ & $0,96(0,01)[3,0]$ & $0,96(0,01)[3,0]$ & $0,96(0,01)[3,0]$ & $0,96(0,01)[3,0]$ \\
\hline 7-Llog-f* & $0,16(0,03)[3,5]$ & $0,16(0, c$ & $0,16(0$ & $0,17(0,03)[1,0]$ & $0,16(0,03)[3,5]$ \\
\hline 8-Magtag $5 k$ & $0,34(0,01)[3,5]$ & 0,3510, & $0,34(0$ & $0,34(0$, & $0,34(0,01)$ \\
\hline 9-Scene & $0,70(0,03)[3,0]$ & $0,70(0,03)[3,0]$ & 0,7010 & 0,7010 & $0,70(0,02)[3,0]$ \\
\hline \multirow[t]{2}{*}{ 10-Yeast } & $0,64(0,02)[3,0]$ & $0,64(0,0$ & $0,64(0,02)[3,0]$ & $0,64(0, C$ & $0,64(0,02)[3,0]$ \\
\hline & & & $\left|X^{\prime}\right|=$ & & \\
\hline $1-\mathrm{Ca}$ & 43( & 0,43 & $0,44(0$ & 0.43 & $0,4 i$ \\
\hline 2-Corel5k & $0,15(0,01)[4,0]$ & $0,16(0,02)[1,5]$ & $0,16(0,01)[1,5]$ & $0,15(0,01)[4,0]$ & $0,15(0,01)[4,0]$ \\
\hline 3-Corel16kOc & $0,20(0,01)[3,0]$ & $0,20(0,01)[3,0]$ & $0,20(0$, & $0,20(0,01)[3,0]$ & $0,20(0,00)[3,0]$ \\
\hline 4-Emotions & $0,66(0,05)[1,0]$ & $0,64(0,05)[4,5]$ & $0,64(0$, & $0,65(0$ & $0,65(0$ \\
\hline & $0,15(0,05)[2,0]$ & $0,15(0$ & & 0,15 & $0,14(0$ \\
\hline 6-Genbase & $0,96(0,01)[3,0]$ & $0,96(0,01)[3,0]$ & $0,96(0,01)[3,0]$ & $0,96(0,01)[3,0]$ & $0,96(0,01)[3,0]$ \\
\hline 7-Llog-f* & $0,17(0,04)[1,5]$ & $0,17(0,03)[1,5]$ & $0,16(0,03)[4,0]$ & $0,16(0,04)[4,0]$ & $0,16(0,04)[4,0]$ \\
\hline 8-Magtag5k & $0,35(0,01)[3,0]$ & $0,35(0,02)[3,0]$ & $0,35(0,01)[3,0]$ & $0,35(0,01)[3,0]$ & $0,35(0,01)[3,0]$ \\
\hline 9-Scene & $0,71(0,03)[3,0]$ & $0,71(0,02)[3,0]$ & $0,71(0,02)[3,0]$ & $0,71(0,03)[3,0]$ & $0,71(0,03)[3,0]$ \\
\hline \multirow[t]{2}{*}{ 10-Yeast } & $0,64(0,02)[3,0]$ & $0,64(0,02)[3,0]$ & $0,64(0,02)[3,0]$ & $0,64(0,02)[3,0]$ & $0,64(0,02)[3,0]$ \\
\hline & & & $\left|X^{\prime}\right|=$ & & \\
\hline $1-\mathrm{Ca}$ & , & $0,4=10$ & $0,44(0$, & 0,40 & $0,44(0$ \\
\hline 2-Corel5k & $0,17(0,01)[2,5]$ & $0,16(0,01)[5,0]$ & $0,17(0,01)[2,5]$ & $0,17(0,01)[2,5]$ & $0,17(0,01)[2,5]$ \\
\hline 3-Corel16koc & $0,19(0,01)[3,0]$ & $0,19(0,01)[3,0]$ & $0,19(0,01)[3,0]$ & $0,19(0,01)[3,0]$ & $0,19(0,01)[3,0]$ \\
\hline 4-Emotions & $0,64(0,0$ & 0,6510 & $0,64(0$, & $0,65(0$ & $0,65(0$ \\
\hline 5-Fapesp & $0,14(0,04)[2,0]$ & $0,15(0,05)[1,0]$ & $0,13(0,04)[4,0]$ & $0,13(0,04)[4,0]$ & $0,13(0,05)[4,0]$ \\
\hline 6-Genbase $e^{*}$ & $0,96(0,01)[3,0]$ & $0,96(0,01)[3,0]$ & $0,96(0,01)[3,0]$ & $0,96(0,01)[3,0]$ & $0,96(0,01)[3,0]$ \\
\hline 7-Llog-f* & $0,16(0,02)[3,0]$ & $0,16(0,03)[3,0]$ & $0,16(0,03)[3,0]$ & $0,16(0,03)[3,0]$ & $0,16(0,03)[3,0]$ \\
\hline 8-Magtag5k & $0,36(0,01)[3,0]$ & $0,36(0,01)[3,0]$ & $0,36(0,01)[3,0]$ & $0,36(0,01)[3,0]$ & $0,36(0,01)[3,0]$ \\
\hline 9-Scene & $0,71(0,03)[4,5]$ & $0,73(0,02)[1,0]$ & $0,72(0,03)[2,5]$ & $0,71(0,03)[4,5]$ & $0,72(0,02)[2,5]$ \\
\hline
\end{tabular}


Tabela A.8: Resultados para F-measure baseada em exemplos (continuação)

\begin{tabular}{|c|c|c|c|c|c|}
\hline & $I G-B R$ & $S L-X$ & $S C-A$ & $S R-A$ & SR-X \\
\hline 10-Yeast & $0,65(0,02)[1,0]$ & $0,64(0,02)[3,5]$ & $0,64(0,02)[3,5]$ & $0,64(0,02)[3,5]$ & $0,64(0,02)[3,5]$ \\
\hline & \multicolumn{5}{|c|}{$\left|X^{\prime}\right|=80 \% M$} \\
\hline 1-Cal500 & $0,44(0,02)[1,5]$ & $0,43(0,02)[4,0]$ & $0,43(0,02)[4,0]$ & $0,43(0,02)[4,0]$ & $0,44(0,01)[1,5]$ \\
\hline 2-Corel5k & $0,18(0,01)[2,0]$ & $0,17(0,01)[4,5]$ & $0,17(0,01)[4,5]$ & $0,18(0,01)[2,0]$ & $0,18(0,01)[2,0]$ \\
\hline 3-Corel16k001 & $0,18(0,01)[3,0]$ & $0,17(0,01)[5,0]$ & $0,18(0,01)[3,0]$ & $0,18(0,01)[3,0]$ & $0,19(0,01)[1,0]$ \\
\hline 4-Emotions & $0,65(0,03)[3,0]$ & $0,66(0,04)[1,0]$ & $0,65(0,05)[3,0]$ & $0,64(0,03)[5,0]$ & $0,65(0,04)[3,0]$ \\
\hline 5-Fapesp & $0,14(0,05)[4,0]$ & $0,15(0,04)[2,0]$ & $0,14(0,05)[4,0]$ & $0,14(0,05)[4,0]$ & $0,17(0,07)[1,0]$ \\
\hline 6-Genbase* & $0,96(0,01)[3,0]$ & $0,96(0,01)[3,0]$ & $0,96(0,01)[3,0]$ & $0,96(0,01)[3,0]$ & $0,96(0,01)[3,0]$ \\
\hline $7-L \log -f^{*}$ & $0,16(0,03)[3,0]$ & $0,16(0,03)[3,0]$ & $0,16(0,02)[3,0]$ & $0,16(0,03)[3,0]$ & $0,16(0,03)[3,0]$ \\
\hline 8-Magtag5k & $0,36(0,01)[3,0]$ & $0,36(0,01)[3,0]$ & $0,36(0,01)[3,0]$ & $0,36(0,01)[3,0]$ & $0,36(0,01)[3,0]$ \\
\hline 9-Scene & $0,72(0,03)[4,0]$ & $0,73(0,02)[1,5]$ & $0,72(0,03)[4,0]$ & $0,72(0,03)[4,0]$ & $0,73(0,02)[1,5]$ \\
\hline \multirow[t]{2}{*}{ 10-Yeast } & $0,64(0,02)[3,5]$ & $0,64(0,02)[3,5]$ & $0,65(0,02)[1,0]$ & $0,64(0,02)[3,5]$ & $0,64(0,02)[3,5]$ \\
\hline & \multicolumn{5}{|c|}{$\left|X^{\prime}\right|=90 \% M$} \\
\hline 1-Cal500 & $0,44(0,01)[2,0]$ & $0,43(0,02)[4,5]$ & $0,44(0,01)[2,0]$ & $0,43(0,02)[4,5]$ & $0,44(0,01)[2,0]$ \\
\hline 2-Corel5k & $0,17(0,01)[3,0]$ & $0,17(0,01)[3,0]$ & $0,17(0,01)[3,0]$ & $0,17(0,01)[3,0]$ & $0,17(0,01)[3,0]$ \\
\hline 3-Corel16k001 & $0,17(0,01)[3,0]$ & $0,17(0,01)[3,0]$ & $0,17(0,01)[3,0]$ & $0,17(0,01)[3,0]$ & $0,17(0,01)[3,0]$ \\
\hline 4-Emotions & $0,65(0,04)[3,0]$ & $0,65(0,04)[3,0]$ & $0,65(0,04)[3,0]$ & $0,65(0,04)[3,0]$ & $0,65(0,04)[3,0]$ \\
\hline 5-Fapesp & $0,16(0,05)[2,5]$ & $0,16(0,05)[2,5]$ & $0,16(0,05)[2,5]$ & $0,15(0,05)[5,0]$ & $0,16(0,05)[2,5]$ \\
\hline 6-Genbase* & $0,96(0,01)[3,0]$ & $0,96(0,01)[3,0]$ & $0,96(0,01)[3,0]$ & $0,96(0,01)[3,0]$ & $0,96(0,01)[3,0]$ \\
\hline $7-L \log -f^{*}$ & $0,16(0,03)[3,0]$ & $0,16(0,03)[3,0]$ & $0,16(0,03)[3,0]$ & $0,16(0,03)[3,0]$ & $0,16(0,03)[3,0]$ \\
\hline 8-Magtag5k & $0,37(0,01)[3,0]$ & $0,37(0,01)[3,0]$ & $0,37(0,01)[3,0]$ & $0,37(0,01)[3,0]$ & $0,37(0,01)[3,0]$ \\
\hline 9-Scene & $0,72(0,02)[3,5]$ & $0,73(0,02)[1,0]$ & $0,72(0,02)[3,5]$ & $0,72(0,02)[3,5]$ & $0,72(0,02)[3,5]$ \\
\hline 10-Yeast & $0,64(0,02)[3,0]$ & $0,64(0,02)[3,0]$ & $0,64(0,02)[3,0]$ & $0,64(0,02)[3,0]$ & $0,64(0,02)[3,0]$ \\
\hline
\end{tabular}

Tabela A.9: Resultados experimentais de acordo com Hamming Loss: quatro configurações do LCFS e algoritmo IG-BR

\begin{tabular}{|c|c|c|c|c|c|}
\hline & $I G-B R$ & SL-X & $S C-A$ & $S R-A$ & SR-X \\
\hline & \multicolumn{5}{|c|}{$\left|X^{\prime}\right|=10 \% M$} \\
\hline 1-Cal500 &, $17(0,00)[3,0]$ & $0,17(0,00)[3,0]$ & $0,17(0,01)[3,0]$ & $0,17(0,01)[3,0]$ & $0,17(0,01)[3,0]$ \\
\hline 2-Corel5k & $0,01(0,00)[3,0]$ & $0,01(0,00)[3,0]$ & $0,01(0,00)[3,0]$ & $0,01(0,00)[3,0]$ & $0,01(0,00)[3,0]$ \\
\hline 3-Corel16k001 & $0,03(0,00)[3,0]$ & $0,03(0,00)[3,0]$ & $0,03(0,00)[3,0]$ & $0,03(0,00)[3,0]$ & $0,03(0,00)[3,0]$ \\
\hline 4-Emotions & $0,24(0,02)[3,0]$ & $0,24(0,01)[3,0]$ & $0,24(0,02)[3,0]$ & $0,24(0,01)[3,0]$ & $0,24(0,02)[3,0]$ \\
\hline 5-Fapesp & $0,04(0,00)[3,0]$ & $0,04(0,00)[3,0]$ & $0,04(0,00)[3,0]$ & $0,04(0,00)[3,0]$ & $0,04(0,00)[3,0]$ \\
\hline 6-Genbase* & $0,00(0,00)[3,0]$ & $0,00(0,00)[3,0]$ & $0,00(0,00)[3,0]$ & $0,00(0,00)[3,0]$ & $0,00(0,00)[3,0]$ \\
\hline $7-L \log -f^{*}$ & $0,03(0,00)[3,0]$ & $0,03(0,00)[3,0]$ & $0,03(0,00)[3,0]$ & $0,03(0,00)[3,0]$ & $0,03(0,00)[3,0]$ \\
\hline 8-Magtag5k & $0,05(0,00)[3,0]$ & $0,05(0,00)[3,0]$ & $0,05(0,00)[3,0]$ & $0,05(0,00)[3,0]$ & $0,05(0,00)[3,0]$ \\
\hline 9-Scene & $0,19(0,01)[1,5]$ & $0,18(0,02)[3,0]$ & $0,17(0,02)[4,5]$ & $0,19(0,01)[1,5]$ & $0,17(0,01)[4,5]$ \\
\hline \multirow[t]{2}{*}{ 10-Yeast } & $0,23(0,01)[3,0]$ & $0,23(0,0$ & $0,23(0,01)[3,0]$ & 0,23 ( & 0,23 \\
\hline & \multicolumn{5}{|c|}{$\left|X^{\prime}\right|=20 \% M$} \\
\hline 1-Cal & $0,17(0,01)[3,0]$ & $0,17(0, \mathrm{C}$ & $0,17(0,00)[3,0]$ & $0,17(0$ & $0,17(0$, \\
\hline 2-Corel5k & $0,01(0,00)[3,0]$ & $0,01(0,00)[3,0]$ & $0,01(0,00)[3,0]$ & $0,01(0,00)[3,0]$ & $0,01(0,00)[3,0]$ \\
\hline 3-Corel16k001 & $0,03(0,00)[3,0]$ & $0,03(0,00)[3,0]$ & $0,03(0,00)[3,0]$ & $0,03(0,00)[3,0]$ & $0,03(0,00)[3,0]$ \\
\hline 4-Emotions & $0,23(0,02)[3,5]$ & $0,23(0,02)[3,5]$ & $0,24(0,02)[1,0]$ & $0,23(0,02)[3,5]$ & $0,23(0,02)[3,5]$ \\
\hline 5-Fapesp & $0,04(0,00)[3,0]$ & $0,04(0,00)[3,0]$ & $0,04(0,00)[3,0]$ & $0,04(0,00)[3,0]$ & $0,04(0,00)[3,0]$ \\
\hline 6-Genbase* & $0,00(0,00)[3,0]$ & $0,00(0,00)[3,0]$ & $0,00(0,00)[3,0]$ & $0,00(0,00)[3,0]$ & $0,00(0,00)[3,0]$ \\
\hline 7-Llog-f* & $0,03(0,00)[3,0]$ & $0,03(0,00)[3,0]$ & $0,03(0,00)[3,0]$ & $0,03(0,00)[3,0]$ & $0,03(0,00)[3,0]$ \\
\hline 8-Magtag5k & $0,05(0,00)[3,0]$ & $0,05(0,00)[3,0]$ & $0,05(0,00)[3,0]$ & $0,05(0,00)[3,0]$ & $0,05(0,00)[3,0]$ \\
\hline 9-Scene & $0,15(0,01)[2,5]$ & $0,15(0,01)[2,5]$ & $0,15(0,01)[2,5]$ & $0,15(0,01)[2,5]$ & $0,14(0,01)[5,0]$ \\
\hline \multirow[t]{2}{*}{ 10-Yeast } & $0,22(0,01)[3,0]$ & $0,22(0,01)[3,0]$ & $0,22(0,01)[3,0]$ & $0,22(0,01)[3,0]$ & $0,22(0,01)[3,0]$ \\
\hline & \multicolumn{5}{|c|}{$\left|X^{\prime}\right|=30 \% M$} \\
\hline 1-Cal50O & $0,17(0,00)[3,0]$ & $0,17(0,00)[3,0]$ & $0,17(0,01)[3,0]$ & $0,17(0$, & $0,17(0, \mathrm{C}$ \\
\hline 2-Corel5k & $0,01(0,00)[3,0]$ & $0,01(0,00)[3,0]$ & $0,01(0,00)[3,0]$ & $0,01(0,00)[3,0]$ & $0,01(0,00)[3,0]$ \\
\hline 3-Corel16k001 & $0,03(0,00)[3,0]$ & $0,03(0,00)[3,0]$ & $0,03(0,00)[3,0]$ & $0,03(0,00)[3,0]$ & $0,03(0,00)[3,0]$ \\
\hline 4-Emotions & $0,22(0,02)[4,0]$ & $0,23(0,02)[1,5]$ & $0,22(0,03)[4,0]$ & $0,22(0,02)[4,0]$ & $0,23(0,02)[1,5]$ \\
\hline 5-Fapesp & $0,04(0,00)[3,0]$ & $0,04(0,01)[3,0]$ & $0,04(0,00)[3,0]$ & $0,04(0,00)[3,0]$ & $0,04(0,00)[3,0]$ \\
\hline 6-Genbase* & $0,00(0,00)[3,0]$ & $0,00(0,00)[3,0]$ & $0,00(0,00)[3,0]$ & $0,00(0,00)[3,0]$ & $0,00(0,00)[3,0]$ \\
\hline 7-Llog-f* & $0,03(0,00)[3,0]$ & $0,03(0,00)[3,0]$ & $0,03(0,00)[3,0]$ & $0,03(0,00)[3,0]$ & $0,03(0,00)[3,0]$ \\
\hline
\end{tabular}

continua na próxima página 
Tabela A.9: Resultados para Hamming Loss (continuação)

\begin{tabular}{|c|c|c|c|c|c|}
\hline & $I G-B R$ & SL-X & $S C-A$ & SR-A & SR-X \\
\hline 8-Magtag5k & $0,05(0,00)[3,0]$ & $0,05(0,00)[3,0]$ & $0,05(0,00)[3,0]$ & $0,05(0,00)[3,0]$ & $0,05(0,00)[3,0]$ \\
\hline 9-Scene & $0,12(0,01)[3,5]$ & $0,14(0,01)[1,0]$ & $0,12(0,01)[3,5]$ & $0,12(0,01)[3,5]$ & $0,12(0,01)[3,5]$ \\
\hline \multirow[t]{2}{*}{ 10-Yeast } & $0,21(0,01)[3,5]$ & $0,22(0,01)[1,0]$ & $0,21(0,01)[3,5]$ & $0,21(0,01)[3,5]$ & $0,21(0,01)[3,5]$ \\
\hline & & & $\left|X^{\prime}\right|=40 \% M$ & & \\
\hline 1-Cal50O &, $17(0,01)[2,5]$ & $0,16(0,01)[5,0]$ & $0,17(0,01)[2,5]$ & $0,17(0,01)[2,5]$ & $0,17(0,01)[2,5]$ \\
\hline 2-Corel5k & $0,01(0,00)[3,0]$ & $0,01(0,00)[3,0]$ & $0,01(0,00)[3,0]$ & $0,01(0,00)[3,0]$ & $0,01(0,00)[3,0]$ \\
\hline 3-Corel16k001 & $0,03(0,00)[3,0]$ & $0,03(0,00)[3,0]$ & $0,03(0,00)[3,0]$ & $0,03(0,00)[3,0]$ & $0,03(0,00)[3,0]$ \\
\hline 4-Emotions & $0,22(0,02)[3,0]$ & $0,22(0,03)[3,0]$ & $0,22(0,02)[3,0]$ & $0,22(0,03)[3,0]$ & $0,22(0,02)[3,0]$ \\
\hline 5-Fapesp & $0,04(0,00)[3,0]$ & $0,04(0,00)[3,0]$ & $0,04(0,00)[3,0]$ & $0,04(0,00)[3,0]$ & $0,04(0,00)[3,0]$ \\
\hline 6-Genbase* & $0,00(0,00)[3,0]$ & $0,00(0,00)[3,0]$ & $0,00(0,00)[3,0]$ & $0,00(0,00)[3,0]$ & $0,00(0,00)[3,0]$ \\
\hline $7-L \log -f^{*}$ & $0,03(0,00)[3,0]$ & $0,03(0,00)[3,0]$ & $0,03(0,00)[3,0]$ & $0,03(0,00)[3,0]$ & $0,03(0,00)[3,0]$ \\
\hline 8-Magtag $5 k$ & $0,05(0,00)[3,0]$ & $0,05(0,00)[3,0]$ & $0,05(0,00)[3,0]$ & $0,05(0,00)[3,0]$ & $0,05(0,00)[3,0]$ \\
\hline 9-Scene & $0,11(0,01)[3,5]$ & $0,12(0,01)[1,0]$ & $0,11(0,01)[3,5]$ & $0,11(0,01)[3,5]$ & $0,11(0,01)[3,5]$ \\
\hline \multirow[t]{2}{*}{ 10-Yeast } & $0,21(0,01)[3,0]$ & $0,21(0,01)[3,0]$ & $0,21(0,01)[3,0]$ & $0,21(0,01)[3,0]$ & $0,21(0,01)[3,0]$ \\
\hline & & & $\left|X^{\prime}\right|=50 \% M$ & & \\
\hline 1-Cal500 & 17 & 0,17 & $0,17(0,0$ & 0,17 & $0,17(0$ \\
\hline 2-Corel5k & $0,01(0,00)[3,0]$ & $0,01(0,0$ & $0,01(0$, & $0,01(0$ & $0,01(0$ \\
\hline 3-Corel16k001 & $0,03(0,00)[3,0]$ & $0,03(0,00)[3,0]$ & $0,03(0,00)[3,0]$ & $0,03(0,00)[3,0]$ & $0,03(0,00)[3,0]$ \\
\hline 4-Emotions & $0,22(0,03)[3,5]$ & $0,23(0,03)[1,0]$ & $0,22(0,0$ & $0,22(0, C$ & $0,22(0, \mathrm{C}$ \\
\hline 5-Fapesp & $0,04(0,00)[3,0]$ & $0,04(0,00)[3,0]$ & $0,04(0,00)[3,0]$ & $0,04(0,00)[3,0]$ & $0,04(0,00)[3,0]$ \\
\hline 6-Genbase* & $0,00(0,00)[3,0]$ & $0,00(0,00)[3,0]$ & $0,00(0,00)[3,0]$ & $0,00(0$, & $0,00(0$, \\
\hline $7-L \log -f^{*}$ & $0,03(0,00)[3,0]$ & $0,03(0,00)[3,0]$ & $0,03(0, \mathrm{C}$ & $0,03(0$ & $0,03(0$ \\
\hline 8-Magtag $5 k$ & $0,05(0,00)[3,0]$ & $0,05(0, \mathrm{C}$ & $0,05(0$, & 0,0510 & 0,0510 \\
\hline 9-Scene & $0,10(0,01)[4,0]$ & $0,11(0$, & $0,10(0$ & 0,1010 & $0,11(0$, \\
\hline \multirow[t]{2}{*}{ 10-Yeast } & $0,21(0$, & $0,21(0$, & $0,21(0,01)[3,0]$ & $0,21(0$ & $0,21(0$, \\
\hline & & & $\left|X^{\prime}\right|=$ & & \\
\hline $1-\mathrm{Co}$ & $2,5]$ & 0,17 & $0,16(0$ & 0,17 & 0,17 \\
\hline $2-C$ & $0,01(0,00)[3,0]$ & $0,01(0$ & $0,01(0$ & $0,01(C$ & 0,0110 \\
\hline 3-Corel16k001 & $0,03(0,00)[3,0]$ & $0,03(0, C$ & $0,03(0$, & $0,03(0$ & 0,0310 \\
\hline 4-Emotions & 03) $[5,0]$ & $0,21(0,0$ & $0,21(0,0$ & $0,21(0$ & 0,21 \\
\hline 5-Fapesp & $0,04(0,00)[3,0]$ & $0,04(0, \mathrm{C}$ & $0,04(0, c$ & $0,04(0$ & $0,04(0$, \\
\hline 6-Genbase* & $0,00(0,00)[3,0]$ & $0,00(0,00)[3,0]$ & $0,00(0,00)[3,0]$ & $0,00(0$, & $0,00(0$, \\
\hline $7-L \log -f^{*}$ & $0,03(0,00)[3,0]$ & $0,03(0,00)[3,0]$ & $0,03(0, \mathrm{C}$ & $0,03(0$ & $0,03(0$ \\
\hline 8-Magtag $5 k$ & $0,05(0,00)[3,0]$ & $0,05(0,00)[3,0]$ & $0,05(0,1$ & 0,0510 & $0,05(0,00)[3,0]$ \\
\hline 9-Scene & $0,10(0,01)[3,0]$ & $0,10(0, c$ & $0,10(0, c$ & 0,1010 & $0,10(0,01)[3,0]$ \\
\hline \multirow[t]{2}{*}{ 10-Yeast } & $0,21(0,01)[3,0]$ & $0,21(0,0$ & $0,21(0,01)[3,0]$ & $0,21(0, \mathrm{C}$ & $0,21(0, \mathrm{C}$ \\
\hline & & & $\left|X^{\prime}\right|=70 \% M$ & & \\
\hline $1-C$ & & & $0,16(0$ & & כ] \\
\hline 2-Corel5k & {$[3,0]$} & 0,01 & 0,0110 & 0,01 & $0,01(C$ \\
\hline 3-Corel16kO & $0,03(0,00)[3,0]$ & $0,03(0,00)[3,0]$ & $0,03(0,0$ & $0,03(0$ & $0,03(0$ \\
\hline 4-Emotions & $0,02)[3,0]$ & $0,21(0,02)[3,0]$ & $0,21(0,02)[3,0]$ & $0,21(0$, & $0,21(0,02)[3,0]$ \\
\hline 5-Fapesp & $0,04(0,00)[3,0]$ & $0,04(0,00)[3,0]$ & $0,04(0,00)[3,0]$ & $0,04(0$, & $0,04(0,00)[3,0]$ \\
\hline 6-Genbase* & $0,00(0,00)[3,0]$ & $0,00(0,00)[3,0]$ & $0,00(0,00)[3,0]$ & $0,00(0, C$ & $0,00(0, c$ \\
\hline $7-L \log -f^{*}$ & $0,03(0,00)[3,0]$ & $0,03(0,00)[3,0]$ & $0,03(0,00)[3,0]$ & 0,0310, & 0,0310 \\
\hline 8-Magtag5k & $0,05(0,00)[3,0]$ & $0,05(0,00)[3,0]$ & $0,05(0,00)[3,0]$ & $0,05(0,00)[3,0]$ & $0,05(0,00)[3,0]$ \\
\hline 9-Scene & $0,10(0,01)[3,0]$ & $0,10(0,01)[3,0]$ & $0,10(0,01)[3,0]$ & $0,10(0,01)[3,0]$ & $0,10(0,01)[3,0]$ \\
\hline \multirow[t]{2}{*}{ 10-Yeast } & $0,20(0,01)[5,0]$ & $0,21(0,01)[2,5]$ & $0,21(0,01)[2,5]$ & $0,21(0$ & $0,21(0$ \\
\hline & & & $\left|X^{\prime}\right|=80 \% M$ & & \\
\hline 1-Cal500 & & 0,1 & $0,17(0$ & & 0,1 \\
\hline 2-Corel5k & $0,01(0,00)[3,0]$ & $0,01(0,00)[3,0]$ & $0,01(0$, & $0,01(C$ & $0,01(0$ \\
\hline 3-Corel16k001 & $0,03(0,00)[3,0]$ & $0,03(0,1$ & $0,03(0$, & 0,0310 & $0,03(0,00)[3,0]$ \\
\hline 4-Emotions & $0,21(0,01)[2,5]$ & $0,20(0,02)[5,0]$ & $0,21(0,03)[2,5]$ & $0,21(0,02)[2,5]$ & $0,21(0,02)[2,5]$ \\
\hline 5-Fapesp & $0,04(0,00)[3,0]$ & $0,04(0,00)[3,0]$ & $0,04(0,00)[3,0]$ & $0,04(0,00)[3,0]$ & $0,04(0,01)[3,0]$ \\
\hline 6-Genbase $e^{*}$ & $0,00(0,00)[3,0]$ & $0,00(0,00)[3,0]$ & $0,00(0,00)[3,0]$ & $0,00(0,00)[3,0]$ & $0,00(0,00)[3,0]$ \\
\hline 7-Llog-f* & $0,03(0,00)[3,0]$ & $0,03(0,00)[3,0]$ & $0,03(0,00)[3,0]$ & $0,03(0,00)[3,0]$ & $0,03(0,00)[3,0]$ \\
\hline 8-Magtag $5 k$ & $0,05(0,00)[3,0]$ & $0,05(0,00)[3,0]$ & $0,05(0,00)[3,0]$ & $0,05(0,00)[3,0]$ & $0,05(0,00)[3,0]$ \\
\hline 9-Scene & $0,10(0,01)[3,0]$ & $0,10(0,01)[3,0]$ & $0,10(0,01)[3,0]$ & $0,10(0,01)[3,0]$ & $0,10(0,01)[3,0]$ \\
\hline 10-Yeast & $0,21(0,01)[2,5]$ & $0,21(0,01)[2,5]$ & $0,20(0,01)[5,0]$ & $0,21(0,01)[2,5]$ & $0,21(0,01)[2,5]$ \\
\hline
\end{tabular}


Tabela A.9: Resultados para Hamming Loss (continuação)

\begin{tabular}{|c|c|c|c|c|c|}
\hline & $I G-B R$ & $S L-X$ & $S C-A$ & SR-A & SR-X \\
\hline & \multicolumn{5}{|c|}{$\left|X^{\prime}\right|=90 \% M$} \\
\hline 1-Cal500 & $0,16(0,00)[5,0]$ & $0,17(0,01)[2,5]$ & $0,17(0,00)[2,5]$ & $0,17(0,00)[2,5]$ & $0,17(0,00)[2,5]$ \\
\hline 2-Corel5k & $0,01(0,00)[3,0]$ & $0,01(0,00)[3,0]$ & $0,01(0,00)[3,0]$ & $0,01(0,00)[3,0]$ & $0,01(0,00)[3,0]$ \\
\hline 3-Corel16k001 & $0,03(0,00)[3,0]$ & $0,03(0,00)[3,0]$ & $0,03(0,00)[3,0]$ & $0,03(0,00)[3,0]$ & $0,03(0,00)[3,0]$ \\
\hline 4-Emotions & $0,21(0,02)[2,5]$ & $0,21(0,02)[2,5]$ & $0,20(0,02)[5,0]$ & $0,21(0,02)[2,5]$ & $0,21(0,02)[2,5]$ \\
\hline 5-Fapesp & $0,04(0,00)[3,0]$ & $0,04(0,00)[3,0]$ & $0,04(0,00)[3,0]$ & $0,04(0,00)[3,0]$ & $0,04(0,00)[3,0]$ \\
\hline 6-Genbase ${ }^{*}$ & $0,00(0,00)[3,0]$ & $0,00(0,00)[3,0]$ & $0,00(0,00)[3,0]$ & $0,00(0,00)[3,0]$ & $0,00(0,00)[3,0]$ \\
\hline 7-Llog-f* & $0,03(0,00)[3,0]$ & $0,03(0,00)[3,0]$ & $0,03(0,00)[3,0]$ & $0,03(0,00)[3,0]$ & $0,03(0,00)[3,0]$ \\
\hline 8-Magtag5k & $0,04(0,00)[3,0]$ & $0,04(0,00)[3,0]$ & $0,04(0,00)[3,0]$ & $0,04(0,00)[3,0]$ & $0,04(0,00)[3,0]$ \\
\hline 9-Scene & $0,10(0,01)[2,5]$ & $0,09(0,01)[5,0]$ & $0,10(0,01)[2,5]$ & $0,10(0,01)[2,5]$ & $0,10(0,01)[2,5]$ \\
\hline 10-Yeast & $0,21(0,01)[3,0]$ & $0,21(0,01)[3,0]$ & $0,21(0,01)[3,0]$ & $0,21(0,01)[3,0]$ & $0,21(0,01)[3,0]$ \\
\hline
\end{tabular}

Tabela A.10: Resultados experimentais de acordo com Accuracy: quatro configurações do LCFS e algoritmo IG-BR

\begin{tabular}{|c|c|c|c|c|c|}
\hline & $I G-B R$ & $S L-X$ & $S C-A$ & $S R-A$ & SR-X \\
\hline & \multicolumn{5}{|c|}{$\left|X^{\prime}\right|=10 \% M$} \\
\hline 1-Cal500 & $0,28(0,01)[3,0]$ & $0,28(0,01)[3,0]$ & $0,28(0,02)[3,0]$ & $0,28(0,01)[3,0]$ & $0,28(0,01)[3,0]$ \\
\hline 2-Corel5k & $0,14(0,01)[2,5]$ & $0,13(0,01)[5,0]$ & $0,14(0,01)[2,5]$ & $0,14(0,01)[2,5]$ & $0,14(0,01)[2,5]$ \\
\hline 3-Corel16k001 & $0,15(0,00)[3,0]$ & $0,15(0,00)[3,0]$ & $0,15(0,00)[3,0]$ & $0,15(0,00)[3,0]$ & $0,15(0,00)[3,0]$ \\
\hline 4-Emotions & $0,50(0,03)[4,0]$ & $0,51(0,02)[1,5]$ & $0,50(0,03)[4,0]$ & $0,51(0,02)[1,5]$ & $0,50(0,03)[4,0]$ \\
\hline 5-Fapesp & $0,16(0,04)[5,0]$ & $0,18(0,06)[1,0]$ & $0,17(0,04)[3,0]$ & $0,17(0,06)[3,0]$ & $0,17(0,06)[3,0]$ \\
\hline 6-Genbase* & $0,95(0,02)[3,0]$ & $0,95(0,02)[3,0]$ & $0,95(0,02)[3,0]$ & $0,95(0,02)[3,0]$ & $0,95(0,02)[3,0]$ \\
\hline 7-Llog-f* & $0,15(0,02)[3,5]$ & $0,15(0,02)[3,5]$ & $0,15(0,03)[3,5]$ & $0,15(0,03)[3,5]$ & $0,16(0,03)[1,0]$ \\
\hline 8-Magtag $5 k$ & $0,18(0,01)[3,0]$ & $0,18(0,01)[3,0]$ & $0,18(0,01)[3,0]$ & $0,18(0,01)[3,0]$ & $0,18(0,01)[3,0]$ \\
\hline 9-Scene & $0,46(0,03)[4,5]$ & $0,47(0,04)[3,0]$ & $0,50(0,06)[2,0]$ & $0,46(0,02)[4,5]$ & $0,51(0,04)[1,0]$ \\
\hline \multirow[t]{2}{*}{ 10-Yeast } & $0,49(0,02)[3,0]$ & $0,49(0,02)[3,0]$ & $0,49(0,01)[3,0]$ & $0,49(0,02)[3,0]$ & $0,49(0,02)[3,0]$ \\
\hline & \multicolumn{5}{|c|}{$\left|X^{\prime}\right|=20 \% M$} \\
\hline 1-Cal500 & $0,28(0,01)[3,0]$ & $0,28(0,01)[3,0]$ & $0,28(0,01)[3,0]$ & $0,28(0,01)[3,0]$ & $0,28(0,01)[3,0]$ \\
\hline 2-Corel5k & $0,14(0,00)[3,0]$ & $0,14(0,01)[3,0]$ & $0,14(0,01)[3,0]$ & $0,14(0,00)[3,0]$ & $0,14(0,00)[3,0]$ \\
\hline 3-Corel16kOO1 & $0,16(0,00)[2,5]$ & $0,15(0,00)[5,0]$ & $0,16(0,00)[2,5]$ & $0,16(0,00)[2,5]$ & $0,16(0,00)[2,5]$ \\
\hline 4-Emotions & $0,52(0,03)[3,0]$ & $0,52(0,04)[3,0]$ & $0,52(0,04)[3,0]$ & $0,52(0,03)[3,0]$ & $0,52(0,04)[3,0]$ \\
\hline 5-Fapesp & $0,15(0,07)[2,5]$ & $0,14(0,05)[5,0]$ & $0,15(0,04)[2,5]$ & $0,15(0,06)[2,5]$ & $0,15(0,05)[2,5]$ \\
\hline 6-Genbase* & $0,95(0,02)[3,0]$ & $0,95(0,02)[3,0]$ & $0,95(0,02)[3,0]$ & $0,95(0,02)[3,0]$ & $0,95(0,02)[3,0]$ \\
\hline 7-Llog-f* & $0,15(0,02)[3,0]$ & $0,15(0,02)[3,0]$ & $0,15(0,03)[3,0]$ & $0,15(0,03)[3,0]$ & $0,15(0,03)[3,0]$ \\
\hline 8-Magtag $5 k$ & $0,20(0,01)[3,0]$ & $0,20(0,01)[3,0]$ & $0,20(0,01)[3,0]$ & $0,20(0,01)[3,0]$ & $0,20(0,01)[3,0]$ \\
\hline 9-Scene & $0,57(0,02)[3,5]$ & $0,57(0,03)[3,5]$ & $0,57(0,02)[3,5]$ & $0,57(0,02)[3,5]$ & $0,58(0,04)[1,0]$ \\
\hline \multirow[t]{2}{*}{ 10-Yeast } & $0,51(0,02)[2,5]$ & $0,50(0,02)[5,0]$ & $0,51(0,02)[2,5]$ & $0,51(0,02)[2,5]$ & $0,51(0,02)[2,5]$ \\
\hline & \multicolumn{5}{|c|}{$\left|X^{\prime}\right|=30 \% M$} \\
\hline 1-Cal500 & $0,28(0,01)[3,0]$ & $0,28(0,01)[3,0]$ & $0,28(0,02)[3,0]$ & $0,28(0,01)[3,0]$ & $0,28(0,01)[3,0]$ \\
\hline 2-Corel5k & $0,14(0,01)[1,5]$ & $0,13(0,01)[4,0]$ & $0,13(0,01)[4,0]$ & $0,13(0,01)[4,0]$ & $0,14(0,01)[1,5]$ \\
\hline 3-Corel16k001 & $0,15(0,00)[3,0]$ & $0,15(0,00)[3,0]$ & $0,15(0,00)[3,0]$ & $0,15(0,00)[3,0]$ & $0,15(0,00)[3,0]$ \\
\hline 4-Emotions & $0,53(0,04)[3,0]$ & $0,52(0,03)[5,0]$ & $0,54(0,05)[1,0]$ & $0,53(0,04)[3,0]$ & $0,53(0,05)[3,0]$ \\
\hline 5-Fapesp & $0,15(0,05)[2,0]$ & $0,15(0,07)[2,0]$ & $0,15(0,04)[2,0]$ & $0,14(0,06)[4,5]$ & $0,14(0,05)[4,5]$ \\
\hline 6-Genbase* & $0,95(0,02)[3,0]$ & $0,95(0,02)[3,0]$ & $0,95(0,02)[3,0]$ & $0,95(0,02)[3,0]$ & $0,95(0,02)[3,0]$ \\
\hline 7-Llog-f* & $0,15(0,03)[4,0]$ & $0,15(0,03)[4,0]$ & $0,16(0,04)[1,5]$ & $0,16(0,03)[1,5]$ & $0,15(0,03)[4,0]$ \\
\hline 8-Magtag $5 k$ & $0,22(0,01)[2,5]$ & $0,22(0,01)[2,5]$ & $0,21(0,01)[5,0]$ & $0,22(0,01)[2,5]$ & $0,22(0,01)[2,5]$ \\
\hline 9-Scene & $0,65(0,02)[2,5]$ & $0,59(0,03)[5,0]$ & $0,65(0,03)[2,5]$ & $0,65(0,02)[2,5]$ & $0,65(0,03)[2,5]$ \\
\hline \multirow[t]{2}{*}{ 10-Yeast } & $0,52(0,02)[3,0]$ & $0,52(0,02)[3,0]$ & $0,52(0,02)[3,0]$ & $0,52(0,02)[3,0]$ & $0,52(0,02)[3,0]$ \\
\hline & \multicolumn{5}{|c|}{$\left|X^{\prime}\right|=40 \% M$} \\
\hline 1-Cal500 & $0,28(0,01)[3,5]$ & $0,29(0,02)[1,0]$ & $0,28(0,02)[3,5]$ & $0,28(0,02)[3,5]$ & $0,28(0,01)[3,5]$ \\
\hline 2-Corel5k & $0,12(0,00)[3,5]$ & $0,12(0,01)[3,5]$ & $0,12(0,01)[3,5]$ & $0,12(0,01)[3,5]$ & $0,13(0,01)[1,0]$ \\
\hline 3-Corel16k001 & $0,15(0,00)[3,0]$ & $0,15(0,01)[3,0]$ & $0,15(0,01)[3,0]$ & $0,15(0,00)[3,0]$ & $0,15(0,00)[3,0]$ \\
\hline 4-Emotions & $0,53(0,04)[4,5]$ & $0,54(0,05)[2,0]$ & $0,54(0,03)[2,0]$ & $0,54(0,04)[2,0]$ & $0,53(0,04)[4,5]$ \\
\hline 5-Fapesp & $0,14(0,05)[2,5]$ & $0,15(0,06)[1,0]$ & $0,13(0,03)[4,5]$ & $0,13(0,04)[4,5]$ & $0,14(0,05)[2,5]$ \\
\hline 6-Genbase* & $0,95(0,02)[3,0]$ & $0,95(0,02)[3,0]$ & $0,95(0,02)[3,0]$ & $0,95(0,02)[3,0]$ & $0,95(0,02)[3,0]$ \\
\hline 7-Llog-f* & $0,15(0,03)[3,0]$ & $0,15(0,03)[3,0]$ & $0,15(0,03)[3,0]$ & $0,15(0,03)[3,0]$ & $0,15(0,03)[3,0]$ \\
\hline 8-Magtag $5 k$ & $0,23(0,01)[2,5]$ & $0,23(0,01)[2,5]$ & $0,23(0,01)[2,5]$ & $0,23(0,01)[2,5]$ & $0,22(0,01)[5,0]$ \\
\hline
\end{tabular}


Tabela A.10: Resultados para Accuracy (continuação)

\begin{tabular}{|c|c|c|c|c|c|}
\hline & $I G-B R$ & $S L-X$ & $S C-A$ & SR-A & SR-X \\
\hline 9-Scene & $67(0,02)[2,5]$ & $0,66(0,03)[5,0]$ & $0,67(0,03)[2,5]$ & $0,67(0,02)[2,5]$ & $0,67(0,04)[2,5]$ \\
\hline \multirow[t]{2}{*}{ 10-Yeast } & $53(0,02)[3,0]$ & $0,53(0,02)[3,0]$ & $0,53(0,02)[3,0]$ & $0,53(0,02)[3,0]$ & $0,53(0,02)[3,0]$ \\
\hline & & & $\left|X^{\prime}\right|=50 \% M$ & & \\
\hline 1-Cal500 & $28(0,01)[3,0]$ & $0,28(0,01)[3,0]$ & $0,28(0,01)[3,0]$ & $0,28(0, C$ & $0,28(0,0$ \\
\hline 2-Corel5k & $0,11(0,01)[3,5]$ & $0,11(0,01)[3,5]$ & $0,12(0,01)[1,0]$ & $0,11(0,01)[3,5]$ & $0,11(0,01)[3,5]$ \\
\hline 3-Corel16k001 & $0,14(0,01)[3,0]$ & $0,14(0,00)[3,0]$ & $0,14(0,00)[3,0]$ & $0,14(0,00)[3,0]$ & $0,14(0,01)[3,0]$ \\
\hline 4-Emotions & $0,53(0,04)[3,5]$ & $0,53(0,04)[3,5]$ & $0,53(0,05)[3,5]$ & $0,53(0,04)[3,5]$ & $0,54(0,04)[1,0]$ \\
\hline 5-Fapesp & $0,14(0,04)[1,5]$ & $0,13(0,04)[3,5]$ & $0,12(0,05)[5,0]$ & $0,13(0,04)[3,5]$ & $0,14(0,05)[1,5]$ \\
\hline 6-Genbase* & $0,95(0,02)[3,0]$ & $0,95(0,02)[3,0]$ & $0,95(0,02)[3,0]$ & $0,95(0,02)[3,0]$ & $0,95(0,02)[3,0]$ \\
\hline 7-Llog-f* & $0,14(0,03)[4,5]$ & $0,15(0,03)[2,0]$ & $0,14(0,03)[4,5]$ & $0,15(0,03)[2,0]$ & $0,15(0,03)[2,0]$ \\
\hline 8-Magtag5k & $0,24(0,01)[3,0]$ & $0,24(0,01)[3,0]$ & $0,24(0,01)[3,0]$ & $0,24(0,01)[3,0]$ & $0,24(0,01)[3,0]$ \\
\hline 9-Scene & $0,69(0,03)[2,0]$ & $0,68(0,03)[4,5]$ & $0,69(0,03)[2,0]$ & $0,69(0,03)[2,0]$ & $0,68(0,02)[4,5]$ \\
\hline \multirow[t]{2}{*}{ 10-Yeast } & $0,54(0,02)[2,0]$ & $0,53(0,02)[4,5]$ & $0,54(0,02)[2,0]$ & $0,54(0,02)[2,0]$ & $0,53(0,0$ \\
\hline & & & $\left|X^{\prime}\right|=60 \% M$ & & \\
\hline $1-C$ & $28(0,0$ & 0,2810 & $0,28(0,01)[3,0]$ & 0,2810 & $0,2810,1$ \\
\hline $2-\mathrm{Cc}$ & $0,10(0,01)[3,5]$ & $0,11(0,0$ & $0,10(0,0)$ & $0,10(0,01)[3,5]$ & $0,10(0,01)[3,5]$ \\
\hline $3-\mathrm{Co}$ & $0,13(0,00)[3,0]$ & $0,13(0,00)[3,0]$ & $0,13(0,01)[3,0]$ & $0,13(0, C$ & $0,13(0,0$ \\
\hline 4-Emotions & $0,57(0,05)[1,0]$ & $0,56(0,0$ & $0,55(0,0$ & $0,56(0,0$ & $0,56(0,05)[3,0]$ \\
\hline $5-F C$ & $0,13(0,05)[2,5]$ & $0,13(0,05)[2,5]$ & $0,11(0,0$ & $0,13(0, C$ & $0,13(0,0$ \\
\hline 6-Genbase* & $0,95(0,02)[3,0]$ & $0,95(0,02)[3,0]$ & $0,95(0,02)[3,0]$ & $0,95(0,02)[3,0]$ & $0,95(0,02)[3,0]$ \\
\hline & $0,15(0,0$ & $0,15(0,0$ & $0,14(0$, & $0,14(0$, & $0,15(0$, \\
\hline 8-Magtag & $0,24(0,01)[3,0]$ & $0,24(0,01)[3,0]$ & $0,24(0,01)[3,0]$ & $0,24(0,01)[3,0]$ & $0,24(0,01)[3,0]$ \\
\hline & $0,70(0,0$ & $0,70(0,0$ & $0,69(0,0$ & 0,7010 & 0,7010 \\
\hline \multirow[t]{2}{*}{ 10-Yeast } & $54(0,0$ & $0,54(0, c$ & $0,54(0,02)[2,0]$ & $0,53(0$, & $0,53(0$ \\
\hline & & & $\left|X^{\prime}\right|=$ & & \\
\hline $1-C$ & $\overline{28}$ & $0,28(0$ & $0,29(0,1$ & 0,28 & 0,2910 \\
\hline $2-C$ & $0,11(0,0$ & $0,11(0$, & $0,11(0$, & $0,11(0$ & $0,11(0$ \\
\hline $3-C$ & $0,13(0,0$ & $0,13(0$, & $0,13(0$, & $0,12(0$, & 0,1310 \\
\hline $4-E r$ & $0,55(0,0$ & $0,56(0$, & $0,55(0$ & 0,5610 & 0,5610 \\
\hline & $0,12(0,0$ & $0,14(0$, & $0,12(0,0$ & $0,12(0$, & $0,12(0$ \\
\hline 6-Genbase & $0,95(0,0$ & $0,95(0,0$ & $0,95(0$, & 0,9510 & $0,95(0$ \\
\hline & & $0,15(0,03)[1,0]$ & $0,14(0,03)$ & $0,14(0,03)$ & $0,14(0,03)[3,5]$ \\
\hline 8-Magtag & $0,25(0,01)[3,0]$ & $0,25(0,01)[3,0]$ & $0,25(0,01)[3,0]$ & $0,25(0,01)[3,0]$ & $0,25(0,01)[3,0]$ \\
\hline & $0,70(0,03$ & $0,71(0,0$ & $0,70(0, c$ & $0,70(0$, & $0,70(0,02)[3,5]$ \\
\hline \multirow[t]{2}{*}{ 10-Yeast } & $54(0,0$ & $0,54(0, c$ & $0,54(0,02)[3,0]$ & $0,54(0$, & $0,54(0,02)[3,0]$ \\
\hline & & & $\left|X^{\prime}\right|=$ & & \\
\hline $1-\mathrm{Co}$ & 29 & $28(0$ & $0,28(0, r$ & 0,28 & 0,29 (C \\
\hline $2-C$ & $0,12(0,0$ & $0,11(0, \mathrm{C}$ & $0,11(0, \mathrm{c}$ & $0,12(0$ & $0,12(0$ \\
\hline $3-C$ & $0,12(0,0$ & $0,12(0, c$ & $0,12(0$, & $0,12(0$, & $0,13(0$ \\
\hline 4-Emotions & $0,56(0,0$ & $0,57(0$, & $0,56(0$, & 0,55 & $0,56(0$, \\
\hline & 0,12 & $0,12(0$, & $0,12(0$, & 0,12 & $0,14(0$ \\
\hline 6-Genbase & $0,95(0,02)[3,0]$ & $0,95(0,02)[3,0]$ & $0,95(0,02)[3,0]$ & $0,95(0,02)[3,0]$ & $0,95(0,02)[3,0]$ \\
\hline 7-Llog-f* & 0,14 & $0,15(0$, & $0,14(0,0$ & 0,14 & $0,14(0,03)$ \\
\hline 8-Magtag & $0,25(0,01)[3,0]$ & $0,25(0,01)[3,0]$ & $0,25(0,01)[3,0]$ & $0,25(0,01)[3,0]$ & $0,25(0,01)[3,0]$ \\
\hline 9-Scene & $0,71(0,0$ & $0,71(0$, & $0,71(0, C$ & $0,71(0$, & $0,72(0,02)[1,0]$ \\
\hline \multirow[t]{2}{*}{ 10-Yeast } & $0,53(0,02)[4,5]$ & $0,53(0,02)[4,5]$ & $0,54(0,02)[2,0]$ & $0,54(0,02)[2,0]$ & $0,54(0,02)[2,0]$ \\
\hline & & & $\left|X^{\prime}\right|=90 \% M$ & & \\
\hline & 0 & 0,2010 & $0,28(0,0$ & 0,2010 & 0,2910 \\
\hline $2-\mathrm{Co}$ & $0,11(0,01)[3,0]$ & $0,11(0$ & $0,11(0$ & $0,11(C$ & $0,11(0,01)[3,0]$ \\
\hline 3-Corel16kOc & $0,12(0,00)[1,5]$ & $0,11(0,01)[4,0]$ & $0,11(0,01)[4,0]$ & $0,11(0,01)[4,0]$ & $0,12(0,00)[1,5]$ \\
\hline 4-Emotions & $0,56(0,04)[2,5]$ & $0,56(0,04)[2,5]$ & $0,56(0,03)[2,5]$ & $0,55(0,04)[5,0]$ & $0,56(0,03)[2,5]$ \\
\hline 5-Fapesp & $0,13(0,05)[2,5]$ & $0,13(0,05)[2,5]$ & $0,13(0,05)[2,5]$ & $0,12(0,05)[5,0]$ & $0,13(0,05)[2,5]$ \\
\hline 6-Genbase $e^{*}$ & $0,95(0,02)[3,0]$ & $0,95(0,02)[3,0]$ & $0,95(0,02)[3,0]$ & $0,95(0,02)[3,0]$ & $0,95(0,02)[3,0]$ \\
\hline 7-Llog-f* & $0,15(0,03)[3,0]$ & $0,15(0,03)[3,0]$ & $0,15(0,03)[3,0]$ & $0,15(0,03)[3,0]$ & $0,15(0,03)[3,0]$ \\
\hline 8-Magtag5 & $0,26(0,01)[3,0]$ & $0,26(0,01)[3,0]$ & $0,26(0,01)[3,0]$ & $0,26(0,01)[3,0]$ & $0,26(0,01)[3,0]$ \\
\hline & $0,71(0,02)[3,5]$ & $0,72(0,02)[1,0]$ & $0,71(0,02)[3,5]$ & $0,71(0,02)[3,5]$ & $0,71(0,02)[3,5]$ \\
\hline 10-Yeast & $0,53(0,02)[3,0]$ & $0,53(0,02)[3,0]$ & $0,53(0,02)[3,0]$ & $0,53(0,02)[3,0]$ & $0,53(0,02)[3,0]$ \\
\hline
\end{tabular}


Tabela A.11: Resultados experimentais de acordo com $F_{b}$ : quatro configurações do LCFS e algoritmo IG-BR

\begin{tabular}{|c|c|c|c|c|c|}
\hline & $I G-B R$ & $S L-X$ & $S C-A$ & SR-A & SR-X \\
\hline & \multicolumn{5}{|c|}{$\left|X^{\prime}\right|=10 \% M$} \\
\hline 1-Cal500 & $0,43(0,02)[3,0]$ & $0,43(0,01)[3,0]$ & $0,43(0,02)[3,0]$ & $0,43(0,02)[3,0]$ & $0,43(0,02)[3,0]$ \\
\hline 2-Corel5k & $0,21(0,01)[3,5]$ & $0,21(0,01)[3,5]$ & $0,21(0,01)[3,5]$ & $0,21(0,01)[3,5]$ & $0,22(0,01)[1,0]$ \\
\hline 3-Corel16k001 & $0,23(0,00)[3,0]$ & $0,23(0,00)[3,0]$ & $0,23(0,00)[3,0]$ & $0,23(0,00)[3,0]$ & $0,23(0,00)[3,0]$ \\
\hline 4-Emotions & $0,62(0,03)[2,5]$ & $0,62(0,02)[2,5]$ & $0,62(0,03)[2,5]$ & $0,62(0,02)[2,5]$ & $0,61(0,03)[5,0]$ \\
\hline 5-Fapesp & $0,19(0,05)[5,0]$ & $0,21(0,06)[1,0]$ & $0,20(0,04)[3,0]$ & $0,20(0,06)[3,0]$ & $0,20(0,06)[3,0]$ \\
\hline 6-Genbase $e^{*}$ & $0,95(0,01)[3,0]$ & $0,95(0,01)[3,0]$ & $0,95(0,01)[3,0]$ & $0,95(0,01)[3,0]$ & $0,95(0,01)[3,0]$ \\
\hline 7-Llog-f* & $0,17(0,02)[2,5]$ & $0,17(0,02)[2,5]$ & $0,16(0,03)[5,0]$ & $0,17(0,03)[2,5]$ & $0,17(0,03)[2,5]$ \\
\hline 8-Magtag5k & $0,28(0,01)[2,5]$ & $0,27(0,01)[5,0]$ & $0,28(0,01)[2,5]$ & $0,28(0,01)[2,5]$ & $0,28(0,01)[2,5]$ \\
\hline 9-Scene & $0,46(0,03)[4,5]$ & $0,47(0,04)[3,0]$ & $0,51(0,06)[2,0]$ & $0,46(0,02)[4,5]$ & $0,52(0,04)[1,0]$ \\
\hline \multirow[t]{2}{*}{ 10-Yeast } & $0,62(0,02)[3,0]$ & $0,62(0,02)[3,0]$ & $0,62(0,01)[3,0]$ & $0,62(0,02)[3,0]$ & $0,62(0,02)[3,0]$ \\
\hline & \multicolumn{5}{|c|}{$\left|X^{\prime}\right|=20 \% M$} \\
\hline 1-Cal500 & $0,43(0,01)[3,0]$ & $0,43(0,01)[3,0]$ & $0,43(0,01)[3,0]$ & $0,43(0,02)[3,0]$ & $0,43(0,01)[3,0]$ \\
\hline 2-Corel5k & $0,21(0,01)[4,0]$ & $0,21(0,01)[4,0]$ & $0,22(0,01)[1,5]$ & $0,21(0,01)[4,0]$ & $0,22(0,01)[1,5]$ \\
\hline 3-Corel16k001 & $0,24(0,00)[3,0]$ & $0,24(0,00)[3,0]$ & $0,24(0,00)[3,0]$ & $0,24(0,00)[3,0]$ & $0,24(0,00)[3,0]$ \\
\hline 4-Emotions & $0,63(0,03)[2,5]$ & $0,63(0,03)[2,5]$ & $0,62(0,03)[5,0]$ & $0,63(0,03)[2,5]$ & $0,63(0,03)[2,5]$ \\
\hline 5-Fapesp & $0,17(0,07)[3,0]$ & $0,16(0,05)[5,0]$ & $0,18(0,04)[1,0]$ & $0,17(0,06)[3,0]$ & $0,17(0,05)[3,0]$ \\
\hline 6-Genbase* & $0,95(0,01)[3,0]$ & $0,95(0,01)[3,0]$ & $0,95(0,01)[3,0]$ & $0,95(0,01)[3,0]$ & $0,95(0,01)[3,0]$ \\
\hline 7-Llog-f* & $0,16(0,02)[4,0]$ & $0,16(0,02)[4,0]$ & $0,17(0,03)[1,5]$ & $0,17(0,03)[1,5]$ & $0,16(0,03)[4,0]$ \\
\hline 8-Magtag5k & $0,31(0,01)[3,0]$ & $0,31(0,02)[3,0]$ & $0,31(0,01)[3,0]$ & $0,31(0,01)[3,0]$ & $0,31(0,01)[3,0]$ \\
\hline 9-Scene & $0,58(0,02)[3,0]$ & $0,57(0,03)[5,0]$ & $0,58(0,02)[3,0]$ & $0,58(0,02)[3,0]$ & $0,59(0,04)[1,0]$ \\
\hline \multirow[t]{2}{*}{ 10-Yeast } & $0,64(0,02)[2,0]$ & $0,63(0,02)[4,5]$ & $0,64(0,02)[2,0]$ & $0,63(0,02)[4,5]$ & $0,64(0,02)[2,0]$ \\
\hline & \multicolumn{5}{|c|}{$\left|X^{\prime}\right|=30 \% M$} \\
\hline 1-Cal50O & $0,43(0,01)[3,0]$ & $0,43(0,01)[3,0]$ & $0,43(0,02)[3,0]$ & $0,43(0,02)[3,0]$ & $0,43(0,02)[3,0]$ \\
\hline 2-Corel5k & $0,21(0,01)[3,0]$ & $0,21(0,01)[3,0]$ & $0,21(0,01)[3,0]$ & $0,21(0,01)[3,0]$ & $0,21(0,01)[3,0]$ \\
\hline 3-Corel16k001 & $0,23(0,00)[3,0]$ & $0,23(0,01)[3,0]$ & $0,23(0,00)[3,0]$ & $0,23(0,00)[3,0]$ & $0,23(0,00)[3,0]$ \\
\hline 4-Emotions & $0,64(0,04)[3,0]$ & $0,63(0,03)[5,0]$ & $0,65(0,04)[1,0]$ & $0,64(0,04)[3,0]$ & $0,64(0,04)[3,0]$ \\
\hline 5-Fapesp & $0,17(0,05)[2,0]$ & $0,17(0,07)[2,0]$ & $0,17(0,05)[2,0]$ & $0,16(0,06)[4,5]$ & $0,16(0,06)[4,5]$ \\
\hline 6-Genbase* & $0,95(0,01)[3,0]$ & $0,95(0,01)[3,0]$ & $0,95(0,01)[3,0]$ & $0,95(0,01)[3,0]$ & $0,95(0,01)[3,0]$ \\
\hline 7-Llog-f* & $0,17(0,04)[3,0]$ & $0,16(0,03)[5,0]$ & $0,18(0,04)[1,0]$ & $0,17(0,03)[3,0]$ & $0,17(0,04)[3,0]$ \\
\hline 8-Magtag5k & $0,32(0,02)[3,5]$ & $0,33(0,01)[1,0]$ & $0,32(0,02)[3,5]$ & $0,32(0,02)[3,5]$ & $0,32(0,02)[3,5]$ \\
\hline 9-Scene & $0,66(0,02)[2,0]$ & $0,60(0,03)[5,0]$ & $0,65(0,03)[4,0]$ & $0,66(0,02)[2,0]$ & $0,66(0,03)[2,0]$ \\
\hline \multirow[t]{2}{*}{ 10-Yeast } & $0,65(0,02)[2,5]$ & $0,64(0,01)[5,0]$ & $0,65(0,02)[2,5]$ & $0,65(0,01)[2,5]$ & $0,65(0,02)[2,5]$ \\
\hline & \multicolumn{5}{|c|}{$\left|X^{\prime}\right|=40 \% M$} \\
\hline 1-Cal500 & $0,43(0,02) \mid$ & $0,44(0$ & $0,43(0,02)[3,5]$ & $0,43(0$ & $0,43(0,02)[3,5]$ \\
\hline 2-Corel5k & $0,19(0,01)[3,5]$ & $0,19(0,01)[3,5]$ & $0,19(0,01)[3,5]$ & $0,19(0,01)[3,5]$ & $0,20(0,01)[1,0]$ \\
\hline 3-Corel16k001 & $0,22(0,01)[3,5]$ & $0,22(0,01)[3,5]$ & $0,22(0,01)[3,5]$ & $0,22(0,01)[3,5]$ & $0,23(0,01)[1,0]$ \\
\hline 4-Emotions & $0,64(0,03)[4,5]$ & $0,65(0,04)[2,0]$ & $0,65(0,02)[2,0]$ & $0,65(0,04)[2,0]$ & $0,64(0,03)[4,5]$ \\
\hline 5-Fapesp & $0,15(0,04)[3,5]$ & $0,17(0,05)[1,0]$ & $0,14(0,04)[5,0]$ & $0,15(0,04)[3,5]$ & $0,16(0,05)[2,0]$ \\
\hline 6-Genbase* & $0,95(0,01)[3,0]$ & $0,95(0,01)[3,0]$ & $0,95(0,01)[3,0]$ & $0,95(0,01)[3,0]$ & $0,95(0,01)[3,0]$ \\
\hline 7-Llog-f* & $0,16(0,02)[4,0]$ & $0,17(0,04)[1,5]$ & $0,16(0,03)[4,0]$ & $0,17(0,03)[1,5]$ & $0,16(0,03)[4,0]$ \\
\hline 8-Magtag5k & $0,33(0,01)[3,0]$ & $0,33(0,01)[3,0]$ & $0,33(0,01)[3,0]$ & $0,33(0,01)[3,0]$ & $0,33(0,01)[3,0]$ \\
\hline 9-Scene & $0,68(0,02)[2,5]$ & $0,67(0,03)[5,0]$ & $0,68(0,03)[2,5]$ & $0,68(0,02)[2,5]$ & $0,68(0,03)[2,5]$ \\
\hline \multirow[t]{2}{*}{ 10-Yeast } & $0,65(0,02)[3,0]$ & $0,65(0,02)[3,0]$ & $0,65(0,02)[3,0]$ & $0,65(0,02)[3,0]$ & $0,65(0,02)[3,0]$ \\
\hline & \multicolumn{5}{|c|}{$\left|X^{\prime}\right|=50 \% M$} \\
\hline 1-Cal500 & $0,43(0,02)[4,0]$ & $0,43(0,0$ & $0,44(0,02)[1,5]$ & $0,44(0$ & $0,43(0,02)[4,0]$ \\
\hline 2-Corel5k & $0,17(0,01)[4,0]$ & $0,18(0,02)[1,5]$ & $0,18(0,01)[1,5]$ & $0,17(0,01)[4,0]$ & $0,17(0,01)[4,0]$ \\
\hline 3-Corell6kOc & $0,21(0,01)[3,5]$ & $0,21(0,01)[3,5]$ & $0,21(0,01)[3,5]$ & $0,21(0,00)[3,5]$ & $0,22(0,01)[1,0]$ \\
\hline 4-Emotions & $0,64(0,04)[3,5]$ & $0,64(0,04)[3,5]$ & $0,64(0,05)[3,5]$ & $0,64(0,05)[3,5]$ & $0,65(0,04)[1,0]$ \\
\hline 5-Fapesp & $0,15(0,04)[2,0]$ & $0,14(0,04)[3,5]$ & $0,13(0,06)[5,0]$ & $0,14(0,04)[3,5]$ & $0,16(0,04)[1,0]$ \\
\hline 6-Genbase* & $0,95(0,01)[3,0]$ & $0,95(0,01)[3,0]$ & $0,95(0,01)[3,0]$ & $0,95(0,01)[3,0]$ & $0,95(0,01)[3,0]$ \\
\hline 7-Llog-f* & $0,16(0,02)[4,0]$ & $0,17(0,03)[1,5]$ & $0,16(0,03)[4,0]$ & $0,17(0,03)[1,5]$ & $0,16(0,03)[4,0]$ \\
\hline 8-Magtag5k & $0,34(0,01)[3,5]$ & $0,35(0,01)[1,0]$ & $0,34(0,01)[3,5]$ & $0,34(0,01)[3,5]$ & $0,34(0,01)[3,5]$ \\
\hline 9-Scene & $0,70(0,02)[2,0]$ & $0,69(0,03)[4,5]$ & $0,70(0,03)[2,0]$ & $0,70(0,03)[2,0]$ & $0,69(0,02)[4,5]$ \\
\hline \multirow[t]{2}{*}{ 10-Yeast } & $0,66(0,02)[3,0]$ & $0,66(0,01)[3,0]$ & $0,66(0,02)[3,0]$ & $0,66(0,02)[3,0]$ & $0,66(0,02)[3,0]$ \\
\hline & \multicolumn{5}{|c|}{$\left|X^{\prime}\right|=60 \% M$} \\
\hline
\end{tabular}


Tabela A. 11: Resultados para $F_{b}$ (continuação)

\begin{tabular}{|c|c|c|c|c|c|}
\hline & $I G-B R$ & $S L-X$ & $S C-A$ & SR-A & SR-X \\
\hline 1-Cal500 & $43(0,02)[5,0]$ & $0,44(0,01)[2,5]$ & $0,44(0,02)[2,5]$ & $0,44(0,02)[2,5]$ & $0,44(0,02)[2,5]$ \\
\hline 2-Corel5k & $16(0,01)[2,0]$ & $0,16(0,02)[2,0]$ & $0,16(0,01)[2,0]$ &, $15(0,01)[4,5]$ & $0,15(0,01)[4,5]$ \\
\hline 3-Corel16k001 & $0,20(0,01)[4,0]$ & $0,20(0,01)[4,0]$ & $0,21(0,01)[1,5]$ & $0,21(0,01)[1,5]$ & $0,20(0,00)[4,0]$ \\
\hline 4-Emotions & $0,67(0,05)[1,5]$ & $0,66(0,05)[4,0]$ & $0,66(0,04)[4,0]$ & $0,66(0,04)[4,0]$ & $0,67(0,05)[1,5]$ \\
\hline 5-Fapesp & $0,14(0,05)[2,0]$ & $0,14(0,05)[2,0]$ & $0,12(0,05)[5,0]$ & $0,14(0,05)[2,0]$ & $0,13(0,04)[4,0]$ \\
\hline 6-Genbase* & $0,95(0,01)[3,0]$ & $0,95(0,01)[3,0]$ & $0,95(0,01)[3,0]$ & $0,95(0,01)[3,0]$ & $0,95(0,01)[3,0]$ \\
\hline 7-Llog-f* & $0,17(0,04)[1,5]$ & $0,17(0,02)[1,5]$ & $0,16(0,02)[4,0]$ & $0,16(0,03)[4,0]$ & $0,16(0,03)[4,0]$ \\
\hline 8-Magtag5k & $0,35(0,01)[3,0]$ & $0,35(0,02)[3,0]$ & $0,35(0,01)[3,0]$ & $0,35(0,01)[3,0]$ & $0,35(0,01)[3,0]$ \\
\hline 9-Scene & $0,70(0,03)[3,0]$ & $0,70(0,02)[3,0]$ & $0,70(0,02)[3,0]$ & $0,70(0,03)[3,0]$ & $0,70(0,03)[3,0]$ \\
\hline \multirow[t]{2}{*}{ 10-Yeast } & $0,66(0,02)[3,0]$ & $0,66(0,01)[3,0]$ & $0,66(0,02)[3,0]$ & $0,66(0,02)[3,0]$ & $0,66(0,02)[3,0]$ \\
\hline & & & $\left|X^{\prime}\right|=70 \% M$ & & \\
\hline $1-\mathrm{Ca}$ & & 0,44 & $0,44(0,0)$ & 0,44 & $0,44(0$ \\
\hline 2-Corel5k & $0,17(0,01)[2,5]$ & $0,16(0,01)[5,0]$ & $0,17(0,0$ & $0,17(0,01)[2,5]$ & $0,17(0,01)[2,5]$ \\
\hline 3-Corel16k001 & $0,19(0,01)[3,5]$ & $0,19(0,01)[3,5]$ & $0,19(0,0$ & $0,19(0,01)[3,5]$ & $0,20(0,01)[1,0]$ \\
\hline 4-Emotions & $0,66(0,04)[3,5]$ & $0,67(0,03)[1,0]$ & $0,66(0,0$ & $0,66(0$, & $0,66(0$, \\
\hline 5-Fapesp & $0,12(0,04)[3,0]$ & $0,14(0,04)[1,0]$ & $0,12(0,0$ & $0,12(0$, & $0,11(0,0$ \\
\hline 6-Genbase* & $0,95(0,01)[3,0]$ & $0,95(0,01)[3,0]$ & $0,95(0,01)]$ & $0,95(0,01)[3,0]$ & $0,95(0,01)[3,0]$ \\
\hline 7-Llog-f* & $0,16(0,02)[3,0]$ & $0,16(0,03)[3,0]$ & $0,16(0,03)[3,0]$ & $0,16(0,03)[3,0]$ & $0,16(0,03)[3,0]$ \\
\hline 8-Magtag5k & $0,36(0,01)[3,0]$ & $0,36(0,01)[3,0]$ & $0,36(0,01)[3,0]$ & $0,36(0,01)[3,0]$ & $0,36(0,01)[3,0]$ \\
\hline $9-\mathrm{Sc}$ & $0,71(0,03)[3,5]$ & $0,72(0$, & $0,71(0, c$ & $0,71(0$, & $0,71(0,02)[3,5]$ \\
\hline \multirow[t]{2}{*}{ 10-Yeast } & $0,66(0,02)[3,0]$ & $0,66(0$, & $0,66(0,02)[3,0]$ & 0,6610 & $0,66(0,02)[3,0]$ \\
\hline & & & $\left|X^{\prime}\right|=$ & & \\
\hline 1-Cal500 & - & 0,4 & $0,44(0$ & ]] & 0,4 \\
\hline $2-C$ & $0,18(0,01)[2,0]$ & $0,17(0, \mathrm{C}$ & $0,17($ & $0,18(0$ & $0,18(0$, \\
\hline 3-Corel16k001 & $0,18(0,01)[3,5]$ & $0,18(0$, & $0,18($ & $0,18(0$ & $0,19(0$, \\
\hline 4-Emotions & $0,67(0,03)[2,5]$ & $0,67(0$, & $0,67(0$, & $0,66(0$ & $0,67(0$, \\
\hline 5-Fapesp & $0,13(0,04)[4,0]$ & $0,14(0,0$ & $0,13(0,04)[4,0]$ & $0,13(0$, & $0,15(0,06)[1,0]$ \\
\hline 6-Genbase* & $0,95(0,01)[3,0]$ & $0,95(0,01)[3,0]$ & $0,95(0,01)[3,0]$ & $0,95(0,01)[3,0]$ & $0,95(0, c$ \\
\hline 7-Llog-f* & $(0,03)[2,5]$ & $0,16(0,02)[2,5]$ & $0,15(0,02)[5,0]$ & $0,16(0,02)[2,5]$ & $0,16(0,02)[2,5]$ \\
\hline 8-Magtag & $0,36(0,01)[3,0]$ & $0,36(0,01)[3,0]$ & $0,36(0,01)[3,0]$ & $0,36(0,01)[3,0]$ & $0,36(0,01)[3,0]$ \\
\hline 9-Scene & $0,71(0,03)[4,5]$ & $0,72(0$ & $0,71(0,03)[4,5]$ & $0,72(0$ & $0,72(0,02)[2,0]$ \\
\hline \multirow[t]{2}{*}{ 10-Yeast } & $0,66(0,02)[3,0]$ & $0,66(0,02)[3,0]$ & $0,66(0,02)[3,0]$ & $0,66(0,02)[3,0]$ & $0,66(0,02)[3,0]$ \\
\hline & & & $\left|X^{\prime}\right|=$ & & \\
\hline 1-Cal500 & $0,4+(0,01)[2,0]$ & $0,43(0$, & $0,44(0, C$ & 0,4470 & $0,44(0$ \\
\hline 2-Corel5k & $0,17(0,01)[3,0]$ & $0,17(0,01)[3,0]$ & $0,17(0,01)[3,0]$ & $0,17(0,01)[3,0]$ & $0,17(0,01)[3,0]$ \\
\hline 3-Corel16koc & $0,18(0,01)[1,5]$ & $0,17(0,01)[4,0]$ & $0,17(0,01)[4,0]$ & $0,17(0,01)[4,0]$ & $0,18(0,01)[1,5]$ \\
\hline 4-Emotions & $0,67(0,03)[2,5]$ & $0,67(0, C$ & $0,67(0,03)[2,5]$ & $0,66(0,0$ & $0,67(0$, \\
\hline 5-Fapesp & $0,15(0,05)[3,5]$ & $0,15(0,04)[3,5]$ & $0,16(0,05)[1,0]$ & $0,15(0,05)[3,5]$ & $0,15(0,05)[3,5]$ \\
\hline 6-Genbase* & $0,95(0,01)[3,0]$ & $0,95(0,01)[3,0]$ & $0,95(0,01)[3,0]$ & $0,95(0,01)[3,0]$ & $0,95(0,01)[3,0]$ \\
\hline 7-Llog-f* & $0,16(0,02)[3,0]$ & $0,16(0,02)[3,0]$ & $0,16(0,02)[3,0]$ & $0,16(0,02)[3,0]$ & $0,16(0,02)[3,0]$ \\
\hline 8-Magtag5k & $0,37(0,01)[3,0]$ & $0,37(0,01)[3,0]$ & $0,37(0,01)[3,0]$ & $0,37(0,01)[3,0]$ & $0,37(0,01)[3,0]$ \\
\hline 9-Scene & $0,72(0,02)[3,5]$ & $0,73(0,02)[1,0]$ & $0,72(0,02)[3,5]$ & $0,72(0,02)[3,5]$ & $0,72(0,02)[3,5]$ \\
\hline 10-Yeast & $0,65(0,02)[4,5]$ & $0,66(0,02)[2,0]$ & $0,66(0,02)[2,0]$ & $0,65(0,02)[4,5]$ & $0,66(0,02)[2,0]$ \\
\hline
\end{tabular}



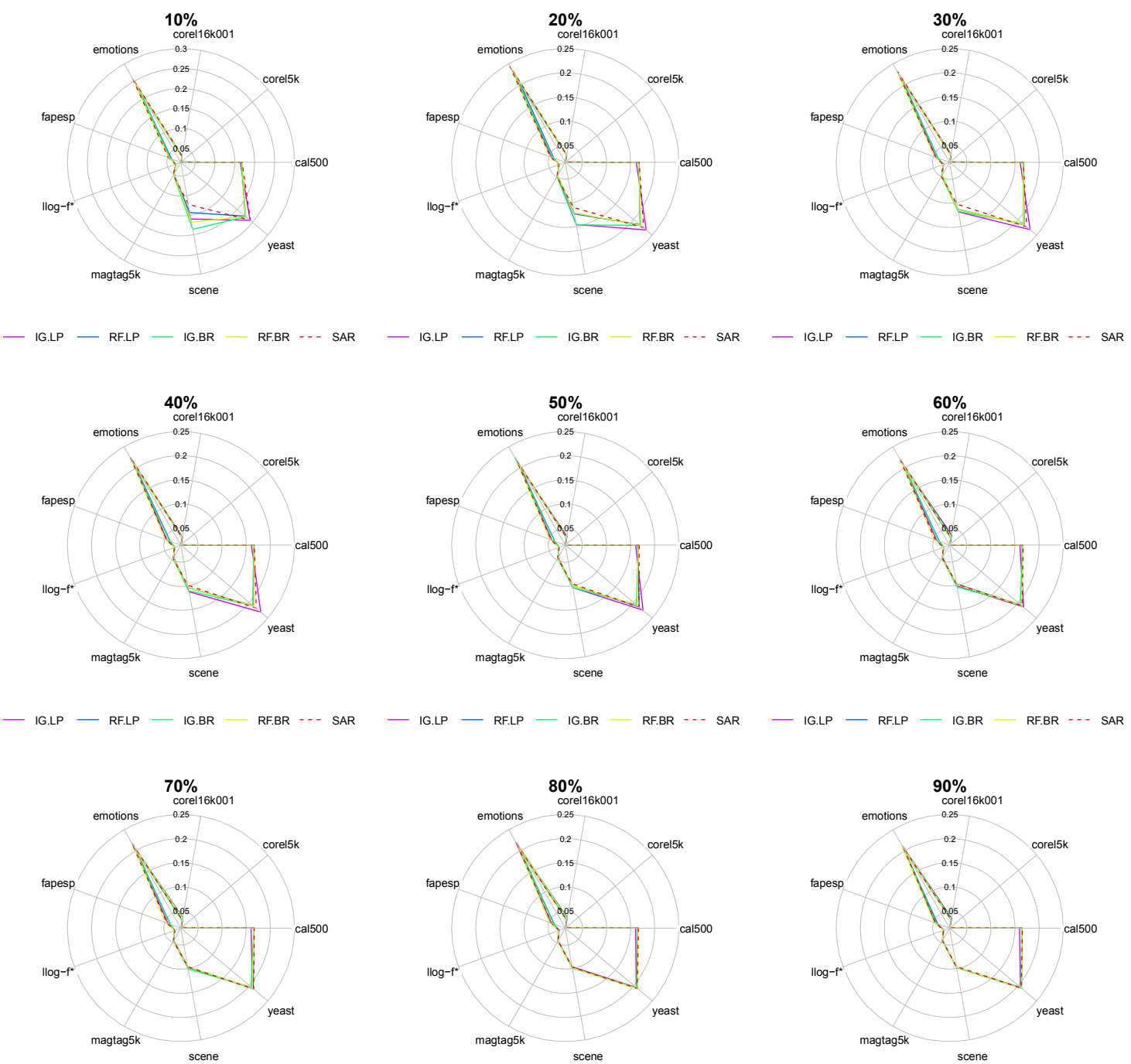

- IG.LP - RF.LP - IG.BR - RF.BR -.. SAR

- IG.LP - RF.LP - IG.BR - RF.BR -.. SAR

- IG.LP - RF.LP - IG.BR - RF.BR -.. SAR

Figura A. 1: Desempenho de algoritmos de SA em termos dos valores de Hamming Loss obtidos pelo algoritmo de classificação $B R k N N-b$ 

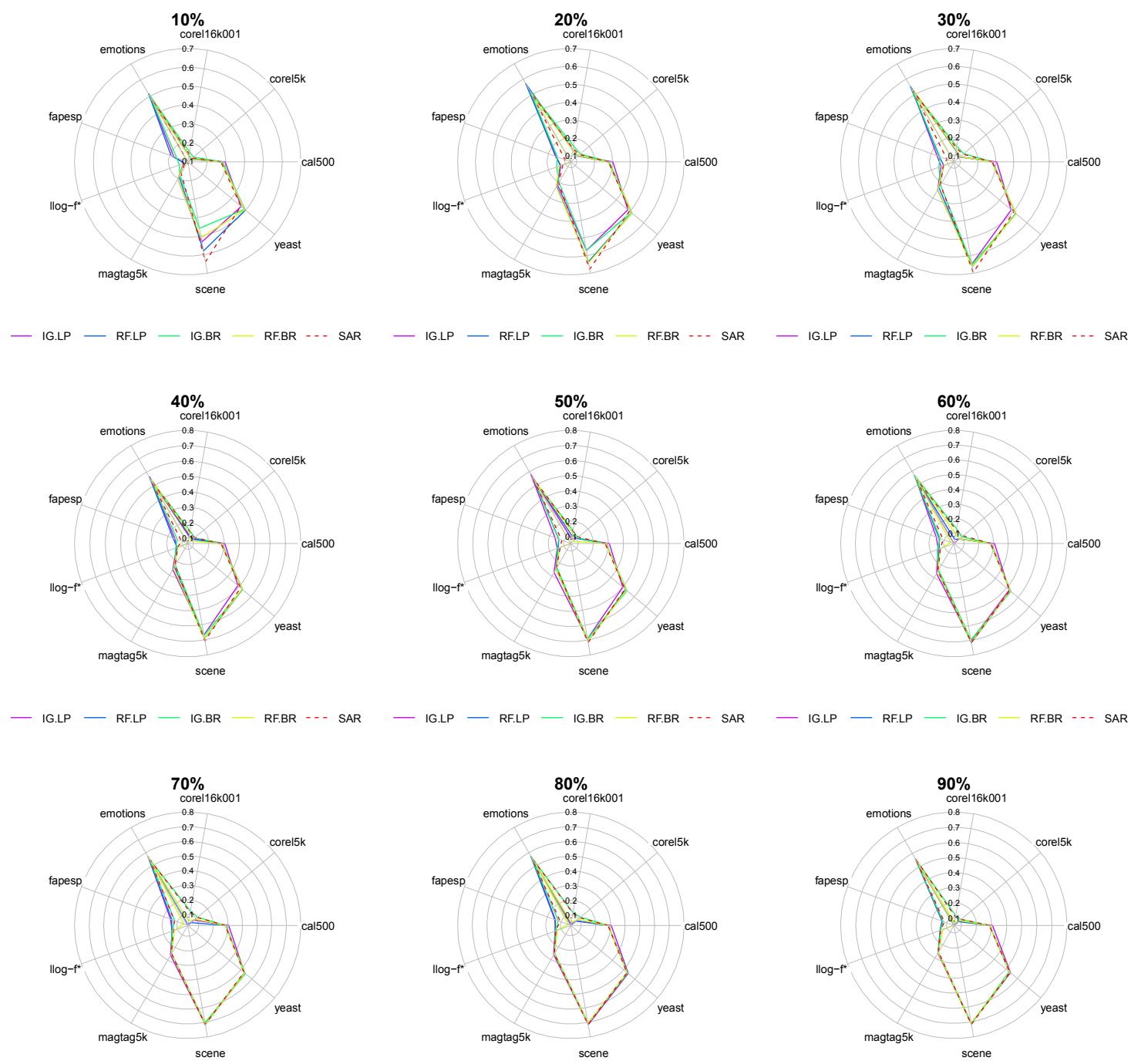

- IG.LP - RF.LP — IG.BR - RF.BR -.. SAR

- IG.LP - RF.LP — IG.BR - RF.BR - . SAR

- IG.LP - RF.LP - IG.BR - RF.BR - - SAR

Figura A.2: Desempenho de algoritmos de SA em termos dos valores de Accuracy obtidos pelo algoritmo de classificação $B R k N N-b$ 

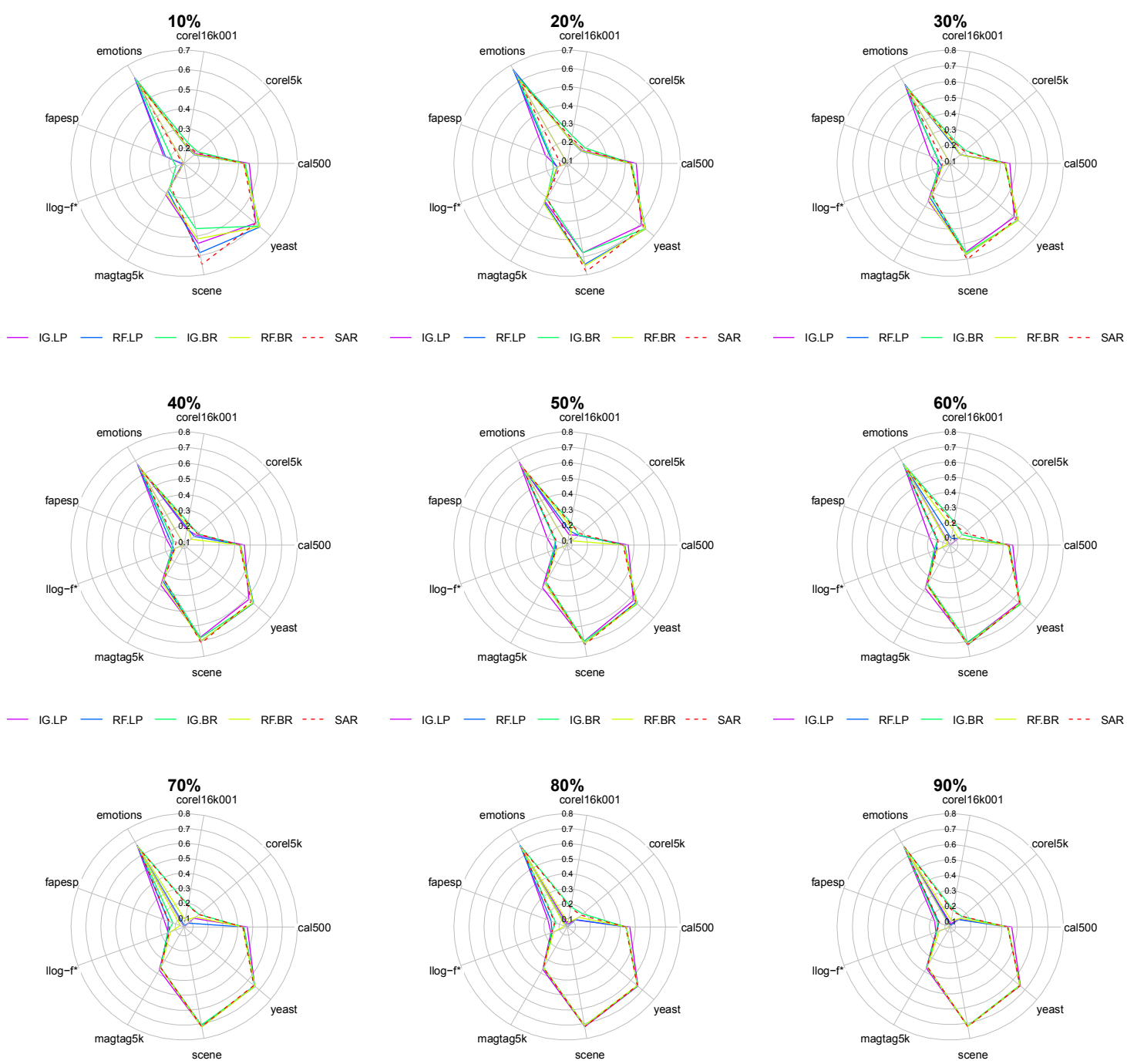

- IG.LP — RF.LP — IG.BR - RF.BR -.. SAR

- IG.LP — RF.LP - IG.BR - RF.BR -.. SAR

- IG.LP - RF.LP - IG.BR - RF.BR -.. SAR

Figura A.3: Desempenho de algoritmos de SA em termos dos valores de $F_{b}$ obtidos pelo algoritmo de classificação $B R k N N-b$ 


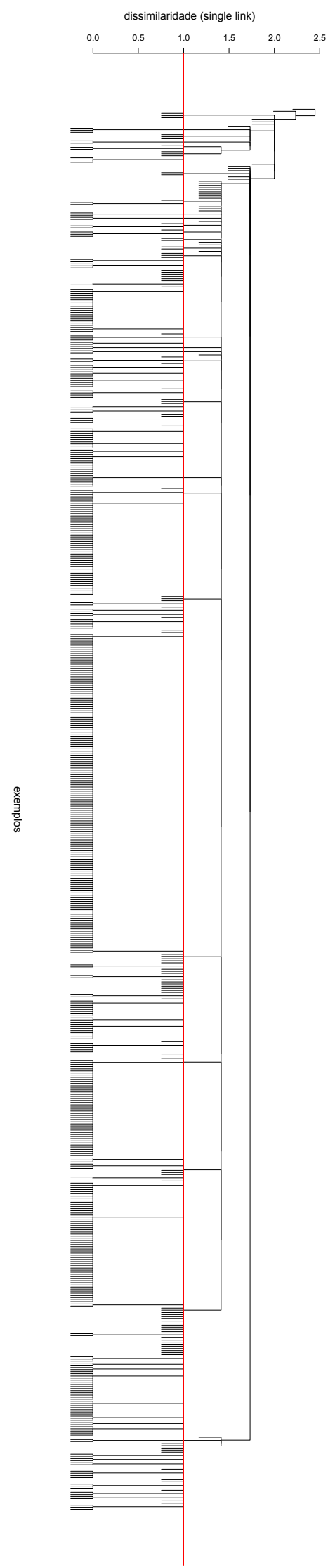

Figura A.4: Agrupamento hierárquico dos exemplos do conjunto de dados 6Genbase* por meio do método single link (Jain e Dubes, 1988) 

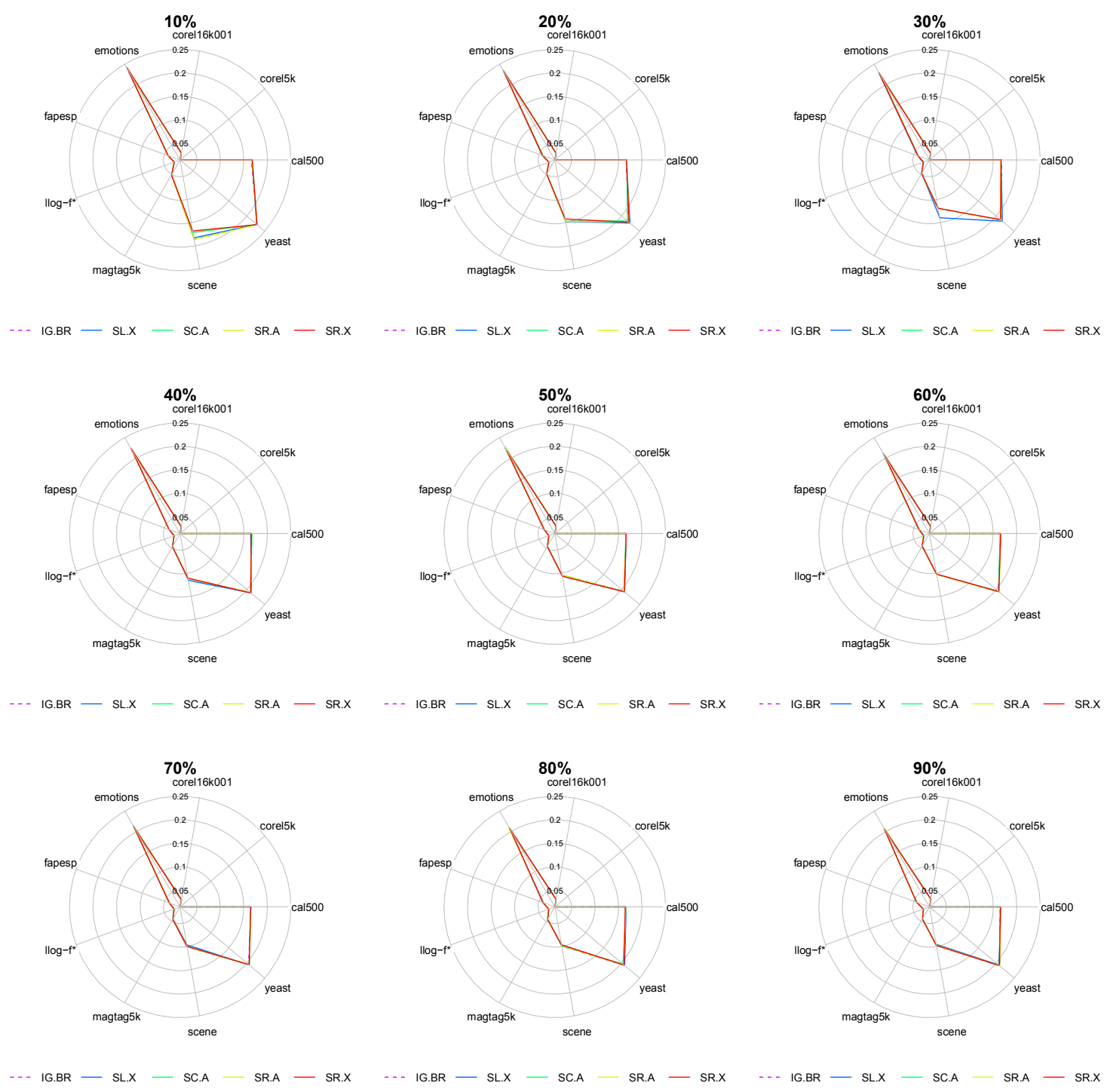

-- IG.BR - SL.X - SC.A SR.A - SR.X

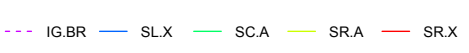

IG.BR - SL.X - SC.A SR.A - SR.X

Figura A.5: Desempenho de configurações do LCFS em termos dos valores de Hamming Loss obtidos pelo algoritmo de classificação BRkNN-b 

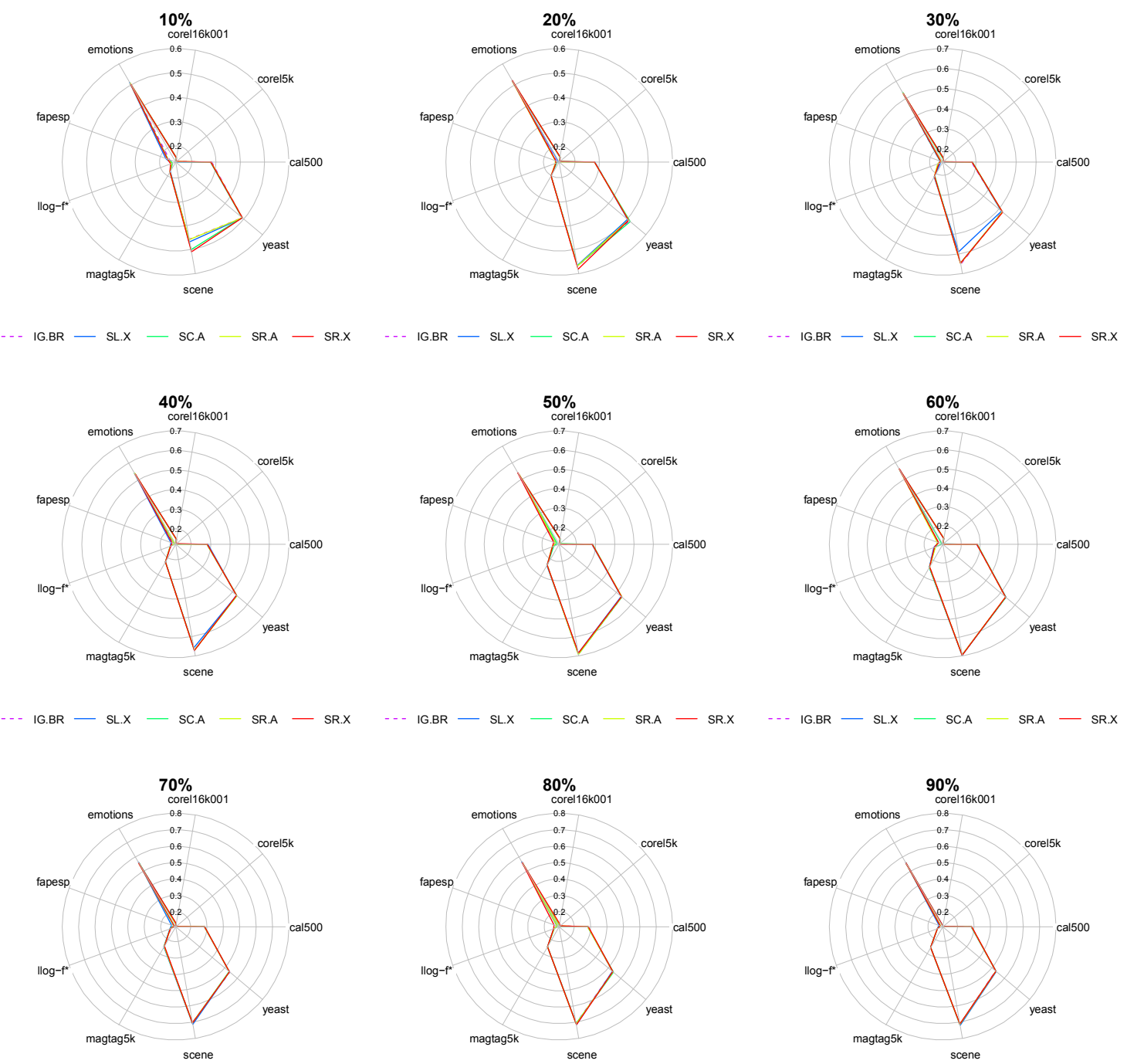

-. IG.BR - SL.X - SC.A - SR.A - SR.X

- - IG.BR - SL.X - SC.A - SR.A - SR.X

-. IG.BR - SL.X - SC.A - SR.A - SR.X

Figura A.6: Desempenho de configurações do LCFS em termos dos valores de Accuracy obtidos pelo algoritmo de classificação $B R k N N-b$ 

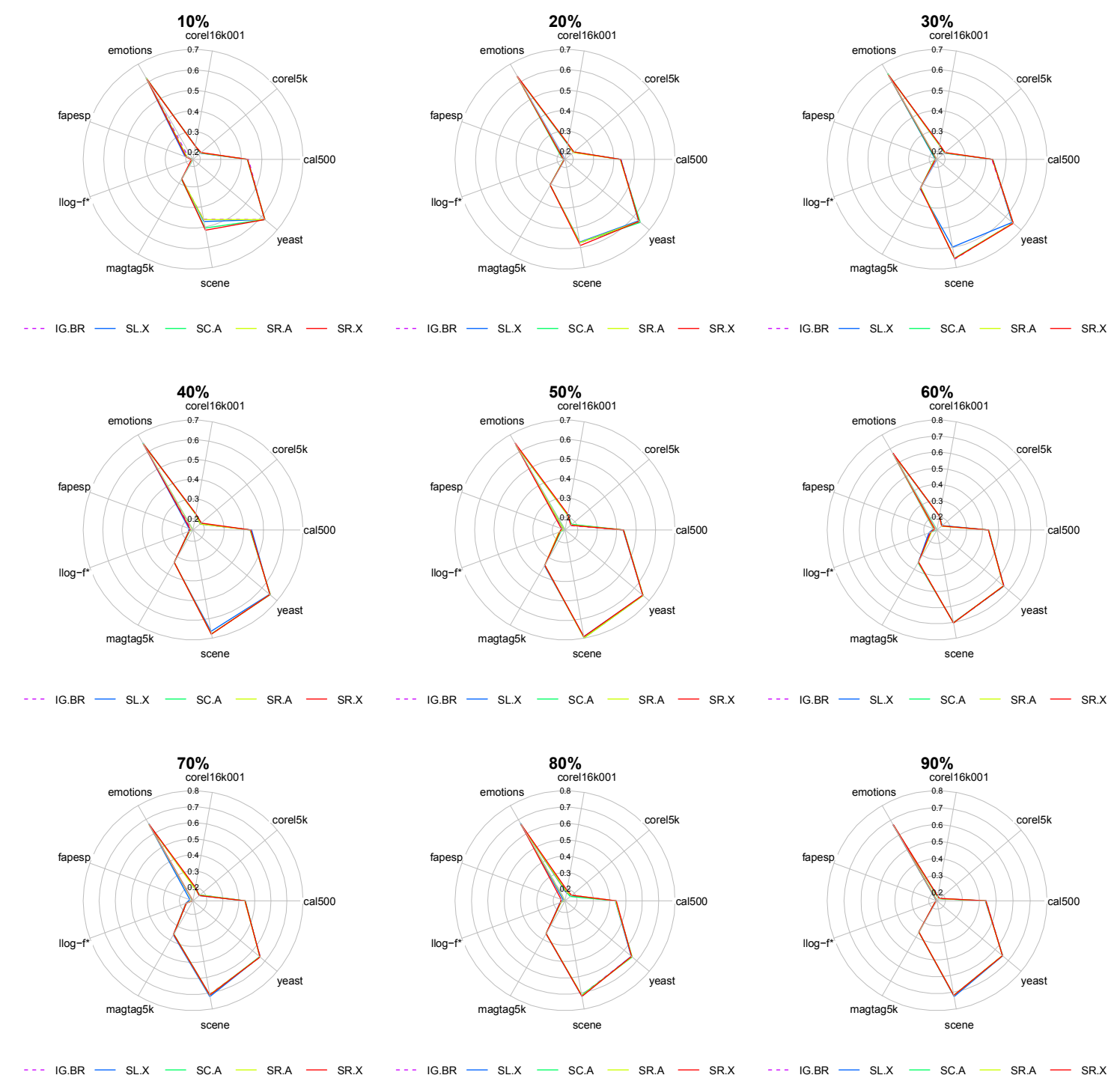

Figura A.7: Desempenho de configurações do LCFS em termos dos valores de $F_{b}$ obtidos pelo algoritmo de classificação $B R k N N-b$ 


\section{A.3 Resultados Experimentais Complementares ao Ca- pítulo 5}

\section{A.3. 1 Configurações dos Algoritmos RF-ML e RFM-ML}

Tabela A.12: Resultados experimentais de acordo com F-measure baseada em exemplos: algoritmo de SA RF-ML com medidas de dissimilaridade Jaccard e Hamming

\begin{tabular}{|c|c|c|}
\hline & $R F-M L$ Jaccard & $R F-M L$ Hamming \\
\hline & & $\left|X^{\prime}\right|=10 \% M$ \\
\hline 1-Cal500 & $0,43(0,02)[1,0]$ & $0,42(0,01)[2,0]$ \\
\hline 2-Corel5k & $0,19(0,01)[1,5]$ & $0,19(0,01)[1,5]$ \\
\hline 3-Corel16k001 & $0,21(0,01)[1,5]$ & $0,21(0,01)[1,5]$ \\
\hline 4-Emotions & $0,59(0,04)[2,0]$ & $0,60(0,04)[1,0]$ \\
\hline 5-Fapesp & $0,15(0,07)[2,0]$ & $0,17(0,05)[1,0]$ \\
\hline 6-Genbase* & $0,96(0,02)[1,5]$ & $0,96(0,02)[1,5]$ \\
\hline $7-L \log -f^{*}$ & $0,14(0,02)[1,0]$ & $0,12(0,03)[2,0]$ \\
\hline 8-Magtag5k & $0,34(0,01)[1,0]$ & $0,33(0,01)[2,0]$ \\
\hline 9-Scene & $0,49(0,06)[2,0]$ & $0,50(0,07)[1,0]$ \\
\hline \multirow[t]{2}{*}{ 10-Yeast } & $0,61(0,02)[1,0]$ & $0,60(0,02)[2,0]$ \\
\hline & & $\left|X^{\prime}\right|=20 \% M$ \\
\hline 1-Cal500 & $0,44(0,02)[1,0]$ & $0,43(0,02)[2,0]$ \\
\hline 2-Corel5k & $0,18(0,01)[1,5]$ & $0,18(0,01)[1,5]$ \\
\hline 3-Corel16k001 & $0,20(0,01)[2,0]$ & $0,21(0,01)[1,0]$ \\
\hline 4-Emotions & $0,60(0,06)[2,0]$ & $0,63(0,04)[1,0]$ \\
\hline 5-Fapesp & $0,13(0,08)[1,0]$ & $0,12(0,06)[2,0]$ \\
\hline 6-Genbase* & $0,96(0,02)[1,5]$ & $0,96(0,02)[1,5]$ \\
\hline $7-L \log -f^{*}$ & $0,16(0,03)[1,0]$ & $0,14(0,02)[2,0]$ \\
\hline 8-Magtag $5 k$ & $0,37(0,01)[1,0]$ & $0,35(0,02)[2,0]$ \\
\hline 9-Scene & $0,60(0,03)[1,5]$ & $0,60(0,03)[1,5]$ \\
\hline \multirow[t]{2}{*}{ 10-Yeast } & $0,62(0,02)[2,0]$ & $0,63(0,02)[1,0]$ \\
\hline & & $\left|X^{\prime}\right|=30 \% M$ \\
\hline 1-Cal500 & $0,44(0,02)[1,0]$ & $0,43(0,01)[2,0]$ \\
\hline 2-Corel5k & $0,17(0,01)[1,5]$ & $0,17(0,01)[1,5]$ \\
\hline 3-Corel16k001 & $0,20(0,01)[2,0]$ & $0,21(0,00)[1,0]$ \\
\hline 4-Emotions & $0,63(0,04)[1,0]$ & $0,62(0,05)[2,0]$ \\
\hline 5-Fapesp & $0,13(0,08)[1,0]$ & $0,10(0,05)[2,0]$ \\
\hline 6-Genbase* & $0,96(0,02)[1,5]$ & $0,96(0,02)[1,5]$ \\
\hline $7-L \log -f^{*}$ & $0,17(0,03)[1,0]$ & $0,14(0,03)[2,0]$ \\
\hline 8-Magtag5k & $0,37(0,01)[1,0]$ & $0,35(0,01)[2,0]$ \\
\hline 9-Scene & $0,62(0,02)[2,0]$ & $0,63(0,03)[1,0]$ \\
\hline \multirow[t]{2}{*}{ 10-Yeast } & $0,63(0,02)[2,0]$ & $0,64(0,02)[1,0]$ \\
\hline & & $\left|X^{\prime}\right|=40 \% M$ \\
\hline 1-Cal500 & $0,44(0,02)[1,0]$ & $0,43(0,01)[2,0]$ \\
\hline 2-Corel5k & $0,15(0,01)[1,5]$ & $0,15(0,02)[1,5]$ \\
\hline 3-Corel16k001 & $0,19(0,01)[2,0]$ & $0,20(0,01)[1,0]$ \\
\hline 4-Emotions & $0,62(0,04)[2,0]$ & $0,64(0,05)[1,0]$ \\
\hline 5-Fapesp & $0,13(0,08)[1,0]$ & $0,09(0,05)[2,0]$ \\
\hline 6-Genbase* & $0,96(0,02)[1,5]$ & $0,96(0,02)[1,5]$ \\
\hline 7-Llog-f* & $0,16(0,03)[1,0]$ & $0,15(0,03)[2,0]$ \\
\hline 8-Magtag $5 k$ & $0,38(0,01)[1,0]$ & $0,37(0,01)[2,0]$ \\
\hline 9-Scene & $0,66(0,03)[1,0]$ & $0,65(0,03)[2,0]$ \\
\hline \multirow[t]{2}{*}{ 10-Yeast } & $0,64(0,02)[1,5]$ & $0,64(0,02)[1,5]$ \\
\hline & & $\left|X^{\prime}\right|=50 \% M$ \\
\hline 1-Cal500 & $0,44(0,02)[1,0]$ & $0,43(0,01)[2,0]$ \\
\hline
\end{tabular}


Tabela A.12: F-measure baseada em exemplos (continuação)

\begin{tabular}{|c|c|c|}
\hline & RF-ML Jaccard & $R F-M L$ Hamming \\
\hline 2-Corel5k & $0,13(0,02)[1,0]$ & $0,12(0,02)[2,0]$ \\
\hline 3-Corel16k001 & $0,18(0,01)[1,0]$ & $0,17(0,01)[2,0]$ \\
\hline 4-Emotions & $0,64(0,03)[1,0]$ & $0,63(0,05)[2,0]$ \\
\hline 5-Fapesp & $0,13(0,09)[1,0]$ & $0,08(0,04)[2,0]$ \\
\hline 6-Genbase* & $0,96(0,02)[1,5]$ & $0,96(0,02)[1,5]$ \\
\hline 7-Llog-f* & $0,15(0,03)[1,5]$ & $0,15(0,03)[1,5]$ \\
\hline 8-Magtag5k & $0,37(0,01)[1,5]$ & $0,37(0,01)[1,5]$ \\
\hline 9-Scene & $0,67(0,03)[1,5]$ & $0,67(0,03)[1,5]$ \\
\hline \multirow[t]{2}{*}{ 10-Yeast } & $0,64(0,02)[1,5]$ & $0,64(0,02)[1,5]$ \\
\hline & & $\left|X^{\prime}\right|=60 \% M$ \\
\hline 1-Cal500 & $0,44(0,02)[1,0]$ & $0,43(0,01)[2,0]$ \\
\hline 2-Corel5k & $0,11(0,01)[1,5]$ & $0,11(0,02)[1,5]$ \\
\hline 3-Corel16k001 & $0,16(0,01)[1,0]$ & $0,15(0,01)[2,0]$ \\
\hline 4-Emotions & $0,65(0,05)[1,0]$ & $0,63(0,04)[2,0]$ \\
\hline 5-Fapesp & $0,13(0,09)[1,0]$ & $0,06(0,05)[2,0]$ \\
\hline 6-Genbase* & $0,96(0,02)[1,5]$ & $0,96(0,02)[1,5]$ \\
\hline $7-L \log -f^{*}$ & $0,15(0,03)[1,0]$ & $0,14(0,03)[2,0]$ \\
\hline 8-Magtag5k & $0,37(0,01)[1,5]$ & $0,37(0,01)[1,5]$ \\
\hline 9-Scene & $0,70(0,03)[1,5]$ & $0,70(0,03)[1,5]$ \\
\hline \multirow[t]{2}{*}{ 10-Yeast } & $0,64(0,02)[1,5]$ & $0,64(0,02)[1,5]$ \\
\hline & & $\left|X^{\prime}\right|=70 \% M$ \\
\hline 1-Cal500 & $0,43(0,02)[1,5]$ & $0,43(0,01)[1,5]$ \\
\hline 2-Corel5k & $0,11(0,02)[1,5]$ & $0,11(0,02)[1,5]$ \\
\hline 3-Corel16k001 & $0,16(0,01)[1,0]$ & $0,13(0,01)[2,0]$ \\
\hline 4-Emotions & $0,65(0,05)[1,0]$ & $0,64(0,04)[2,0]$ \\
\hline 5-Fapesp & $0,12(0,08)[1,0]$ & $0,05(0,04)[2,0]$ \\
\hline 6-Genbase* & $0,96(0,02)[1,5]$ & $0,96(0,02)[1,5]$ \\
\hline 7-Llog-f* & $0,15(0,03)[1,0]$ & $0,14(0,03)[2,0]$ \\
\hline 8-Magtag $5 k$ & $0,37(0,01)[1,5]$ & $0,37(0,01)[1,5]$ \\
\hline 9-Scene & $0,72(0,03)[1,5]$ & $0,72(0,03)[1,5]$ \\
\hline \multirow[t]{2}{*}{ 10-Yeast } & $0,64(0,02)[1,5]$ & $0,64(0,02)[1,5]$ \\
\hline & & $\left|X^{\prime}\right|=80 \% M$ \\
\hline 1-Cal500 & $0,43(0,02)[2,0]$ & $0,44(0,01)[1,0]$ \\
\hline 2-Corel5k & $0,10(0,01)[1,5]$ & $0,10(0,01)[1,5]$ \\
\hline 3-Corel16k001 & $0,15(0,01)[1,0]$ & $0,11(0,01)[2,0]$ \\
\hline 4-Emotions & $0,65(0,04)[2,0]$ & $0,66(0,04)[1,0]$ \\
\hline 5-Fapesp & $0,12(0,08)[1,0]$ & $0,04(0,04)[2,0]$ \\
\hline 6-Genbase* & $0,96(0,02)[1,5]$ & $0,96(0,02)[1,5]$ \\
\hline 7-Llog-f* & $0,16(0,03)[1,0]$ & $0,14(0,03)[2,0]$ \\
\hline 8-Magtag5k & $0,37(0,01)[1,5]$ & $0,37(0,01)[1,5]$ \\
\hline 9-Scene & $0,73(0,03)[1,5]$ & $0,73(0,03)[1,5]$ \\
\hline \multirow[t]{2}{*}{ 10-Yeast } & $0,64(0,02)[1,5]$ & $0,64(0,02)[1,5]$ \\
\hline & & $\left|X^{\prime}\right|=90 \% M$ \\
\hline 1-Cal500 & $0,43(0,02)[2,0]$ & $0,44(0,02)[1,0]$ \\
\hline 2-Corel5k & $0,10(0,02)[2,0]$ & $0,12(0,01)[1,0]$ \\
\hline 3-Corel16k001 & $0,10(0,01)[2,0]$ & $0,11(0,01)[1,0]$ \\
\hline 4-Emotions & $0,66(0,03)[1,0]$ & $0,64(0,05)[2,0]$ \\
\hline 5-Fapesp & $0,10(0,07)[1,0]$ & $0,04(0,04)[2,0]$ \\
\hline 6-Genbase* & $0,96(0,02)[1,5]$ & $0,96(0,02)[1,5]$ \\
\hline 7-Llog-f* & $0,15(0,03)[1,0]$ & $0,14(0,04)[2,0]$ \\
\hline 8-Magtag5k & $0,37(0,01)[1,5]$ & $0,37(0,01)[1,5]$ \\
\hline 9-Scene & $0,73(0,03)[1,5]$ & $0,73(0,03)[1,5]$ \\
\hline 10-Yeast & $0,65(0,02)[1,0]$ & $0,64(0,02)[2,0]$ \\
\hline
\end{tabular}


Tabela A.13: Resultados experimentais de acordo com Hamming Loss: algoritmo de SA RF-ML com medidas de dissimilaridade Jaccard e Hamming

\begin{tabular}{|c|c|c|}
\hline & $R F-M L$ Jaccard & RF-ML Hamming \\
\hline & \multicolumn{2}{|c|}{$\left|X^{\prime}\right|=10 \% M$} \\
\hline 1-Cal500 & $0,17(0,01)[1,5]$ & $0,17(0,00)[1,5]$ \\
\hline 2-Corel5k & $0,02(0,00)[1,0]$ & $0,01(0,00)[2,0]$ \\
\hline 3-Corel16k001 & $0,03(0,00)[1,5]$ & $0,03(0,00)[1,5]$ \\
\hline 4-Emotions & $0,24(0,02)[1,5]$ & $0,24(0,02)[1,5]$ \\
\hline 5-Fapesp & $0,05(0,00)[1,0]$ & $0,04(0,00)[2,0]$ \\
\hline 6-Genbase* & $0,00(0,00)[1,5]$ & $0,00(0,00)[1,5]$ \\
\hline 7-Llog-f* & $0,03(0,00)[1,5]$ & $0,03(0,00)[1,5]$ \\
\hline 8-Magtag $5 k$ & $0,05(0,00)[1,5]$ & $0,05(0,00)[1,5]$ \\
\hline 9-Scene & $0,18(0,02)[1,0]$ & $0,17(0,02)[2,0]$ \\
\hline \multirow[t]{2}{*}{ 10-Yeast } & $0,23(0,01)[1,5]$ & $0,23(0,01)[1,5]$ \\
\hline & \multicolumn{2}{|c|}{$\left|X^{\prime}\right|=20 \% M$} \\
\hline 1-Cal500 & $0,17(0,00)[1,5]$ & $0,17(0,01)[1,5]$ \\
\hline 2-Corel5k & $0,02(0,00)[1,0]$ & $0,01(0,00)[2,0]$ \\
\hline 3-Corel16k001 & $0,03(0,00)[1,5]$ & $0,03(0,00)[1,5]$ \\
\hline 4-Emotions & $0,24(0,03)[1,0]$ & $0,22(0,02)[2,0]$ \\
\hline 5-Fapesp & $0,05(0,01)[1,0]$ & $0,04(0,00)[2,0]$ \\
\hline 6-Genbase* & $0,00(0,00)[1,5]$ & $0,00(0,00)[1,5]$ \\
\hline $7-L \log -f^{*}$ & $0,03(0,00)[1,5]$ & $0,03(0,00)[1,5]$ \\
\hline 8-Magtag5k & $0,05(0,00)[1,5]$ & $0,05(0,00)[1,5]$ \\
\hline 9-Scene & $0,14(0,01)[1,5]$ & $0,14(0,01)[1,5]$ \\
\hline \multirow[t]{2}{*}{ 10-Yeast } & $0,22(0,01)[1,5]$ & $0,22(0,01)[1,5]$ \\
\hline & \multicolumn{2}{|c|}{$\left|X^{\prime}\right|=30 \% M$} \\
\hline 1-Cal500 & $0,17(0,01)[1,5]$ & $0,17(0,00)[1,5]$ \\
\hline 2-Corel5k & $0,02(0,00)[1,0]$ & $0,01(0,00)[2,0]$ \\
\hline 3-Corel16k001 & $0,03(0,00)[1,5]$ & $0,03(0,00)[1,5]$ \\
\hline 4-Emotions & $0,22(0,02)[1,5]$ & $0,22(0,03)[1,5]$ \\
\hline 5-Fapesp & $0,05(0,01)[1,0]$ & $0,04(0,00)[2,0]$ \\
\hline 6-Genbase* & $0,00(0,00)[1,5]$ & $0,00(0,00)[1,5]$ \\
\hline $7-L \log -f^{*}$ & $0,03(0,00)[1,5]$ & $0,03(0,00)[1,5]$ \\
\hline 8-Magtag $5 k$ & $0,04(0,00)[2,0]$ & $0,05(0,00)[1,0]$ \\
\hline 9-Scene & $0,13(0,01)[1,5]$ & $0,13(0,01)[1,5]$ \\
\hline \multirow[t]{2}{*}{ 10-Yeast } & $0,21(0,01)[1,5]$ & $0,21(0,01)[1,5]$ \\
\hline & \multicolumn{2}{|c|}{$\left|X^{\prime}\right|=40 \% M$} \\
\hline 1-Cal500 & $0,16(0,01)[2,0]$ & $0,17(0,00)[1,0]$ \\
\hline 2-Corel5k & $0,02(0,00)[1,5]$ & $0,02(0,00)[1,5]$ \\
\hline 3-Corel16k001 & $0,03(0,00)[1,5]$ & $0,03(0,00)[1,5]$ \\
\hline 4-Emotions & $0,22(0,02)[1,5]$ & $0,22(0,02)[1,5]$ \\
\hline 5-Fapesp & $0,05(0,01)[1,0]$ & $0,04(0,00)[2,0]$ \\
\hline 6-Genbase* & $0,00(0,00)[1,5]$ & $0,00(0,00)[1,5]$ \\
\hline $7-L \log -f^{*}$ & $0,03(0,00)[1,5]$ & $0,03(0,00)[1,5]$ \\
\hline 8-Magtag5k & $0,04(0,00)[1,5]$ & $0,04(0,00)[1,5]$ \\
\hline 9-Scene & $0,12(0,01)[1,5]$ & $0,12(0,01)[1,5]$ \\
\hline \multirow[t]{2}{*}{ 10-Yeast } & $0,21(0,01)[1,5]$ & $0,21(0,01)[1,5]$ \\
\hline & \multicolumn{2}{|c|}{$\left|X^{\prime}\right|=50 \% M$} \\
\hline 1-Cal500 & $0,16(0,01)[2,0]$ & $0,17(0,00)[1,0]$ \\
\hline 2-Corel5k & $0,02(0,00)[1,5]$ & $0,02(0,00)[1,5]$ \\
\hline 3-Corel16k001 & $0,03(0,00)[1,5]$ & $0,03(0,00)[1,5]$ \\
\hline 4-Emotions & $0,21(0,02)[2,0]$ & $0,22(0,02)[1,0]$ \\
\hline 5-Fapesp & $0,05(0,01)[1,0]$ & $0,04(0,00)[2,0]$ \\
\hline 6-Genbase* & $0,00(0,00)[1,5]$ & $0,00(0,00)[1,5]$ \\
\hline $7-L \log -f^{*}$ & $0,03(0,00)[1,5]$ & $0,03(0,00)[1,5]$ \\
\hline 8-Magtag5k & $0,04(0,00)[1,5]$ & $0,04(0,00)[1,5]$ \\
\hline 9-Scene & $0,12(0,01)[1,5]$ & $0,12(0,01)[1,5]$ \\
\hline \multirow[t]{2}{*}{ 10-Yeast } & $0,21(0,01)[1,5]$ & $0,21(0,01)[1,5]$ \\
\hline & \multicolumn{2}{|c|}{$\left|X^{\prime}\right|=60 \% M$} \\
\hline
\end{tabular}


Tabela A.13: Hamming Loss (continuação)

\begin{tabular}{|c|c|c|}
\hline & $R F-M L$ Jaccard & $R F-M L$ Hamming \\
\hline 1-Cal500 & $0,17(0,00)[1,5]$ & $0,17(0,00)[1,5]$ \\
\hline 2-Corel5k & $0,02(0,00)[1,5]$ & $0,02(0,00)[1,5]$ \\
\hline 3-Corel16k001 & $0,03(0,00)[1,5]$ & $0,03(0,00)[1,5]$ \\
\hline 4-Emotions & $0,21(0,02)[2,0]$ & $0,22(0,01)[1,0]$ \\
\hline 5-Fapesp & $0,05(0,01)[1,0]$ & $0,04(0,00)[2,0]$ \\
\hline 6-Genbase* & $0,00(0,00)[1,5]$ & $0,00(0,00)[1,5]$ \\
\hline 7-Llog-f* & $0,03(0,00)[1,5]$ & $0,03(0,00)[1,5]$ \\
\hline 8-Magtag5k & $0,04(0,00)[1,5]$ & $0,04(0,00)[1,5]$ \\
\hline 9-Scene & $0,11(0,01)[1,5]$ & $0,11(0,01)[1,5]$ \\
\hline 10-Yeast & $0,21(0,01)[1,5]$ & $0,21(0,01)[1,5]$ \\
\hline & \multicolumn{2}{|c|}{$\left|X^{\prime}\right|=70 \% M$} \\
\hline 1-Cal500 & $0,17(0,01)[1,5]$ & $0,17(0,00)[1,5]$ \\
\hline 2-Corel5k & $0,02(0,00)[1,5]$ & $0,02(0,00)[1,5]$ \\
\hline 3-Corel16k001 & $0,03(0,00)[1,5]$ & $0,03(0,00)[1,5]$ \\
\hline 4-Emotions & $0,21(0,02)[1,5]$ & $0,21(0,02)[1,5]$ \\
\hline 5-Fapesp & $0,05(0,01)[1,0]$ & $0,04(0,00)[2,0]$ \\
\hline 6-Genbase ${ }^{*}$ & $0,00(0,00)[1,5]$ & $0,00(0,00)[1,5]$ \\
\hline 7-Llog-f* & $0,03(0,00)[1,5]$ & $0,03(0,00)[1,5]$ \\
\hline 8-Magtag5k & $0,04(0,00)[1,5]$ & $0,04(0,00)[1,5]$ \\
\hline 9-Scene & $0,10(0,01)[1,5]$ & $0,10(0,01)[1,5]$ \\
\hline \multirow[t]{2}{*}{ 10-Yeast } & $0,21(0,01)[1,5]$ & $0,21(0,01)[1,5]$ \\
\hline & \multicolumn{2}{|c|}{$\left|X^{\prime}\right|=80 \% M$} \\
\hline 1-Cal500 & $0,17(0,01)[1,0]$ & $0,16(0,00)[2,0]$ \\
\hline 2-Corel5k & $0,02(0,00)[1,5]$ & $0,02(0,00)[1,5]$ \\
\hline 3-Corel16k001 & $0,03(0,00)[1,5]$ & $0,03(0,00)[1,5]$ \\
\hline 4-Emotions & $0,21(0,02)[1,0]$ & $0,20(0,02)[2,0]$ \\
\hline 5-Fapesp & $0,05(0,01)[1,0]$ & $0,04(0,00)[2,0]$ \\
\hline 6-Genbase ${ }^{*}$ & $0,00(0,00)[1,5]$ & $0,00(0,00)[1,5]$ \\
\hline 7-Llog-f* & $0,03(0,00)[1,5]$ & $0,03(0,00)[1,5]$ \\
\hline 8-Magtag5k & $0,04(0,00)[1,5]$ & $0,04(0,00)[1,5]$ \\
\hline 9-Scene & $0,10(0,01)[1,5]$ & $0,10(0,01)[1,5]$ \\
\hline \multirow[t]{2}{*}{ 10-Yeast } & $0,21(0,01)[1,5]$ & $0,21(0,01)[1,5]$ \\
\hline & \multicolumn{2}{|c|}{$\left|X^{\prime}\right|=90 \% M$} \\
\hline 1-Cal500 & $0,17(0,01)[1,5]$ & $0,17(0,01)[1,5]$ \\
\hline 2-Corel5k & $0,02(0,00)[1,5]$ & $0,02(0,00)[1,5]$ \\
\hline 3-Corel16k001 & $0,03(0,00)[1,5]$ & $0,03(0,00)[1,5]$ \\
\hline 4-Emotions & $0,20(0,02)[2,0]$ & $0,21(0,02)[1,0]$ \\
\hline 5-Fapesp & $0,05(0,01)[1,0]$ & $0,04(0,00)[2,0]$ \\
\hline 6-Genbase* & $0,00(0,00)[1,5]$ & $0,00(0,00)[1,5]$ \\
\hline 7-Llog-f* & $0,03(0,00)[1,5]$ & $0,03(0,00)[1,5]$ \\
\hline 8-Magtag5k & $0,04(0,00)[1,5]$ & $0,04(0,00)[1,5]$ \\
\hline 9-Scene & $0,09(0,01)[2,0]$ & $0,10(0,01)[1,0]$ \\
\hline 10-Yeast & $0,20(0,01)[2,0]$ & $0,21(0,01)[1,0]$ \\
\hline
\end{tabular}

Tabela A. 14: Resultados experimentais de acordo com Accuracy: algoritmo de SA RF-ML com medidas de dissimilaridade Jaccard e Hamming

\begin{tabular}{|c|c|c|}
\hline & $R F-M L$ Jaccard & $R F-M L$ Hamming \\
\hline & \multicolumn{2}{|c|}{$\left|X^{\prime}\right|=10 \% M$} \\
\hline 1-Cal500 & $0,28(0,01)[1,0]$ & $0,27(0,01)[2,0]$ \\
\hline 2-Corel5k & $0,12(0,01)[1,5]$ & $0,12(0,00)[1,5]$ \\
\hline 3-Corel16k001 & $0,14(0,00)[1,5]$ & $0,14(0,00)[1,5]$ \\
\hline 4-Emotions & $0,50(0,04)[1,5]$ & $0,50(0,04)[1,5]$ \\
\hline 5-Fapesp & $0,12(0,06)[2,0]$ & $0,14(0,05)[1,0]$ \\
\hline 6-Genbase* & $0,95(0,02)[1,5]$ & $0,95(0,02)[1,5]$ \\
\hline $7-L \log -f^{*}$ & $0,12(0,02)[1,0]$ & $0,11(0,03)[2,0]$ \\
\hline 8-Magtag5k & $0,24(0,01)[1,0]$ & $0,23(0,01)[2,0]$ \\
\hline 9-Scene & $0,48(0,06)[2,0]$ & $0,49(0,07)[1,0]$ \\
\hline
\end{tabular}


Tabela A. 14: Accuracy (continuação)

\begin{tabular}{|c|c|c|}
\hline & $R F-M L$ Jaccard & $R F-M L$ Hamming \\
\hline 10-Yeast & $0,49(0,02)[1,5]$ & $0,49(0,02)[1,5]$ \\
\hline & \multicolumn{2}{|c|}{$\left|X^{\prime}\right|=20 \% M$} \\
\hline 1-Cal50O & $0,29(0,01)[1,0]$ & $0,28(0,01)[2,0]$ \\
\hline 2-Corel5k & $0,11(0,00)[2,0]$ & $0,12(0,00)[1,0]$ \\
\hline 3-Corel16k001 & $0,13(0,00)[2,0]$ & $0,14(0,00)[1,0]$ \\
\hline 4-Emotions & $0,51(0,06)[2,0]$ & $0,54(0,04)[1,0]$ \\
\hline 5-Fapesp & $0,10(0,07)[2,0]$ & $0,11(0,06)[1,0]$ \\
\hline 6-Genbase* & $0,95(0,02)[1,5]$ & $0,95(0,02)[1,5]$ \\
\hline $7-\log -f^{*}$ & $0,15(0,02)[1,0]$ & $0,13(0,02)[2,0]$ \\
\hline 8-Magtag5k & $0,26(0,01)[1,0]$ & $0,25(0,01)[2,0]$ \\
\hline 9-Scene & $0,59(0,03)[2,0]$ & $0,60(0,03)[1,0]$ \\
\hline \multirow[t]{2}{*}{ 10-Yeast } & $0,51(0,02)[1,5]$ & $0,51(0,02)[1,5]$ \\
\hline & \multicolumn{2}{|c|}{$\left|X^{\prime}\right|=30 \% M$} \\
\hline 1-Cal50O & $0,29(0,02)[1,0]$ & $0,28(0,01)[2,0]$ \\
\hline 2-Corel5k & $0,11(0,01)[1,5]$ & $0,11(0,00)[1,5]$ \\
\hline 3-Corel16k001 & $0,13(0,00)[2,0]$ & $0,14(0,00)[1,0]$ \\
\hline 4-Emotions & $0,54(0,04)[1,0]$ & $0,53(0,05)[2,0]$ \\
\hline 5-Fapesp & $0,10(0,07)[1,0]$ & $0,09(0,04)[2,0]$ \\
\hline 6-Genbase* & $0,95(0,02)[1,5]$ & $0,95(0,02)[1,5]$ \\
\hline 7-Llog-f* & $0,16(0,02)[1,0]$ & $0,13(0,03)[2,0]$ \\
\hline 8-Magtag5k & $0,26(0,01)[1,0]$ & $0,25(0,01)[2,0]$ \\
\hline 9-Scene & $0,61(0,02)[2,0]$ & $0,62(0,03)[1,0]$ \\
\hline \multirow[t]{2}{*}{ 10-Yeast } & $0,52(0,02)[2,0]$ & $0,53(0,02)[1,0]$ \\
\hline & \multicolumn{2}{|c|}{$\left|X^{\prime}\right|=40 \% M$} \\
\hline 1-Cal500 & $0,29(0,01)[1,0]$ & $0,28(0,01)[2,0]$ \\
\hline 2-Corel5k & $0,10(0,01)[1,5]$ & $0,10(0,01)[1,5]$ \\
\hline 3-Corel16k001 & $0,12(0,00)[2,0]$ & $0,13(0,00)[1,0]$ \\
\hline 4-Emotions & $0,54(0,04)[1,5]$ & $0,54(0,05)[1,5]$ \\
\hline 5-Fapesp & $0,10(0,07)[1,0]$ & $0,09(0,05)[2,0]$ \\
\hline 6-Genbase* & $0,95(0,02)[1,5]$ & $0,95(0,02)[1,5]$ \\
\hline $7-L \log -f^{*}$ & $0,15(0,03)[1,0]$ & $0,14(0,03)[2,0]$ \\
\hline 8-Magtag5k & $0,26(0,01)[1,5]$ & $0,26(0,01)[1,5]$ \\
\hline 9-Scene & $0,65(0,03)[1,0]$ & $0,64(0,03)[2,0]$ \\
\hline \multirow[t]{2}{*}{ 10-Yeast } & $0,53(0,02)[2,0]$ & $0,54(0,02)[1,0]$ \\
\hline & \multicolumn{2}{|c|}{$\left|X^{\prime}\right|=50 \% M$} \\
\hline 1-Cal50O & $0,29(0,01)[1,0]$ & $0,28(0,01)[2,0]$ \\
\hline 2-Corel5k & $0,09(0,01)[1,0]$ & $0,08(0,01)[2,0]$ \\
\hline 3-Corel16k001 & $0,12(0,00)[1,5]$ & $0,12(0,01)[1,5]$ \\
\hline 4-Emotions & $0,55(0,03)[1,0]$ & $0,54(0,05)[2,0]$ \\
\hline 5-Fapesp & $0,10(0,07)[1,0]$ & $0,07(0,04)[2,0]$ \\
\hline 6-Genbase* & $0,95(0,02)[1,5]$ & $0,95(0,02)[1,5]$ \\
\hline $7-L \log -f^{*}$ & $0,14(0,03)[1,5]$ & $0,14(0,03)[1,5]$ \\
\hline 8-Magtag5k & $0,26(0,01)[1,5]$ & $0,26(0,01)[1,5]$ \\
\hline 9-Scene & $0,66(0,03)[1,5]$ & $0,66(0,02)[1,5]$ \\
\hline \multirow[t]{2}{*}{ 10-Yeast } & $0,53(0,02)[1,5]$ & $0,53(0,02)[1,5]$ \\
\hline & \multicolumn{2}{|c|}{$\left|X^{\prime}\right|=60 \% M$} \\
\hline 1-Cal50O & $0,29(0,01)[1,0]$ & $0,28(0,01)[2,0]$ \\
\hline 2-Corel5k & $0,07(0,01)[2,0]$ & $0,08(0,01)[1,0]$ \\
\hline 3-Corel16k001 & $0,11(0,00)[1,0]$ & $0,10(0,01)[2,0]$ \\
\hline 4-Emotions & $0,56(0,05)[1,0]$ & $0,54(0,03)[2,0]$ \\
\hline 5-Fapesp & $0,10(0,08)[1,0]$ & $0,05(0,04)[2,0]$ \\
\hline 6-Genbase* & $0,95(0,02)[1,5]$ & $0,95(0,02)[1,5]$ \\
\hline $7-L \log -f^{*}$ & $0,13(0,03)[1,5]$ & $0,13(0,03)[1,5]$ \\
\hline 8-Magtag5k & $0,26(0,01)[1,5]$ & $0,26(0,01)[1,5]$ \\
\hline 9-Scene & $0,69(0,03)[1,5]$ & $0,69(0,03)[1,5]$ \\
\hline \multirow[t]{2}{*}{ 10-Yeast } & $0,54(0,02)[1,5]$ & $0,54(0,02)[1,5]$ \\
\hline & \multicolumn{2}{|c|}{$\left|X^{\prime}\right|=70 \% M$} \\
\hline 1-Cal500 & $0,28(0,02)[2,0]$ & $0,29(0,01)[1,0]$ \\
\hline
\end{tabular}


Tabela A. 14: Accuracy (continuação)

\begin{tabular}{|c|c|c|}
\hline & $R F-M L$ Jaccard & $R F-M L$ Hamming \\
\hline 2-Corel5k & $0,07(0,01)[1,5]$ & $0,07(0,01)[1,5]$ \\
\hline 3-Corel16k001 & $0,10(0,00)[1,0]$ & $0,08(0,01)[2,0]$ \\
\hline 4-Emotions & $0,56(0,05)[1,0]$ & $0,55(0,04)[2,0]$ \\
\hline 5-Fapesp & $0,10(0,07)[1,0]$ & $0,05(0,04)[2,0]$ \\
\hline 6-Genbase* & $0,95(0,02)[1,5]$ & $0,95(0,02)[1,5]$ \\
\hline 7-Llog-f* & $0,13(0,03)[1,5]$ & $0,13(0,03)[1,5]$ \\
\hline 8-Magtag5k & $0,26(0,00)[1,5]$ & $0,26(0,01)[1,5]$ \\
\hline 9-Scene & $0,71(0,02)[1,5]$ & $0,71(0,03)[1,5]$ \\
\hline 10-Yeast & $0,54(0,02)[1,5]$ & $0,54(0,02)[1,5]$ \\
\hline & \multicolumn{2}{|c|}{$\left|X^{\prime}\right|=80 \% M$} \\
\hline 1-Cal500 & $0,28(0,02)[2,0]$ & $0,29(0,01)[1,0]$ \\
\hline 2-Corel5k & $0,07(0,01)[1,5]$ & $0,07(0,01)[1,5]$ \\
\hline 3-Corel16k001 & $0,09(0,01)[1,0]$ & $0,07(0,01)[2,0]$ \\
\hline 4-Emotions & $0,56(0,04)[2,0]$ & $0,57(0,04)[1,0]$ \\
\hline 5-Fapesp & $0,09(0,07)[1,0]$ & $0,04(0,04)[2,0]$ \\
\hline 6-Genbase* & $0,95(0,02)[1,5]$ & $0,95(0,02)[1,5]$ \\
\hline 7-Llog-f* & $0,14(0,03)[1,0]$ & $0,13(0,03)[2,0]$ \\
\hline 8-Magtag5k & $0,26(0,01)[1,5]$ & $0,26(0,01)[1,5]$ \\
\hline 9-Scene & $0,72(0,03)[1,5]$ & $0,72(0,03)[1,5]$ \\
\hline \multirow[t]{2}{*}{ 10-Yeast } & $0,53(0,02)[1,5]$ & $0,53(0,02)[1,5]$ \\
\hline & \multicolumn{2}{|c|}{$\left|X^{\prime}\right|=90 \% M$} \\
\hline 1-Cal500 & $0,28(0,01)[2,0]$ & $0,29(0,01)[1,0]$ \\
\hline 2-Corel5k & $0,07(0,01)[2,0]$ & $0,08(0,01)[1,0]$ \\
\hline 3-Corel16k001 & $0,07(0,01)[1,5]$ & $0,07(0,01)[1,5]$ \\
\hline 4-Emotions & $0,57(0,03)[1,0]$ & $0,56(0,04)[2,0]$ \\
\hline 5-Fapesp & $0,08(0,06)[1,0]$ & $0,04(0,04)[2,0]$ \\
\hline 6-Genbase* & $0,95(0,02)[1,5]$ & $0,95(0,02)[1,5]$ \\
\hline 7-Llog-f* & $0,14(0,03)[1,0]$ & $0,13(0,04)[2,0]$ \\
\hline 8-Magtag5k & $0,26(0,01)[1,5]$ & $0,26(0,01)[1,5]$ \\
\hline 9-Scene & $0,72(0,03)[1,5]$ & $0,72(0,03)[1,5]$ \\
\hline 10-Yeast & $0,54(0,03)[1,0]$ & $0,53(0,02)[2,0]$ \\
\hline
\end{tabular}

Tabela A.15: Resultados experimentais de acordo com $F_{b}$ : algoritmo de SA $R F-M L$ com medidas de dissimilaridade Jaccard e Hamming

\begin{tabular}{|c|c|c|}
\hline & $R F-M L$ Jaccard & RF-ML Hamming \\
\hline & \multicolumn{2}{|c|}{$\left|X^{\prime}\right|=10 \% M$} \\
\hline 1-Cal500 & $0,43(0,02)[1,5]$ & $0,43(0,01)[1,5]$ \\
\hline 2-Corel5k & $0,19(0,01)[1,5]$ & $0,19(0,01)[1,5]$ \\
\hline 3-Corel16k001 & $0,21(0,01)[2,0]$ & $0,22(0,01)[1,0]$ \\
\hline 4-Emotions & $0,61(0,04)[1,5]$ & $0,61(0,03)[1,5]$ \\
\hline 5-Fapesp & $0,15(0,07)[1,5]$ & $0,15(0,04)[1,5]$ \\
\hline 6-Genbase* & $0,95(0,02)[1,5]$ & $0,95(0,02)[1,5]$ \\
\hline 7-Llog-f* & $0,14(0,02)[1,0]$ & $0,12(0,03)[2,0]$ \\
\hline 8-Magtag $5 k$ & $0,34(0,01)[1,5]$ & $0,34(0,01)[1,5]$ \\
\hline 9-Scene & $0,49(0,06)[2,0]$ & $0,50(0,07)[1,0]$ \\
\hline \multirow[t]{2}{*}{ 10-Yeast } & $0,62(0,02)[1,5]$ & $0,62(0,02)[1,5]$ \\
\hline & \multicolumn{2}{|c|}{$\left|X^{\prime}\right|=20 \% M$} \\
\hline 1-Cal500 & $0,44(0,02)[1,0]$ & $0,43(0,02)[2,0]$ \\
\hline 2-Corel5k & $0,18(0,01)[2,0]$ & $0,19(0,01)[1,0]$ \\
\hline 3-Corel16koO1 & $0,21(0,01)[2,0]$ & $0,22(0,01)[1,0]$ \\
\hline 4-Emotions & $0,62(0,05)[2,0]$ & $0,65(0,05)[1,0]$ \\
\hline 5-Fapesp & $0,14(0,08)[1,0]$ & $0,11(0,05)[2,0]$ \\
\hline 6-Genbase* & $0,95(0,02)[1,5]$ & $0,95(0,02)[1,5]$ \\
\hline 7-Llog-f* & $0,16(0,03)[1,0]$ & $0,14(0,02)[2,0]$ \\
\hline 8-Magtag $5 k$ & $0,37(0,01)[1,0]$ & $0,36(0,01)[2,0]$ \\
\hline 9-Scene & $0,59(0,03)[2,0]$ & $0,60(0,03)[1,0]$ \\
\hline
\end{tabular}


Tabela A. 15: $F_{b}$ (continuação)

\begin{tabular}{|c|c|c|}
\hline & $R F-M L$ Jaccard & $R F-M L$ Hamming \\
\hline \multirow[t]{2}{*}{ 10-Yeast } & $0,64(0,02)[1,5]$ & $0,64(0,01)[1,5]$ \\
\hline & \multicolumn{2}{|c|}{$\left|X^{\prime}\right|=30 \% M$} \\
\hline 1-Cal500 & $0,44(0,02)[1,0]$ & $0,43(0,01)[2,0]$ \\
\hline 2-Corel5k & $0,17(0,01)[1,5]$ & $0,17(0,01)[1,5]$ \\
\hline 3-Corel16k001 & $0,20(0,01)[2,0]$ & $0,21(0,00)[1,0]$ \\
\hline 4-Emotions & $0,65(0,03)[1,0]$ & $0,63(0,05)[2,0]$ \\
\hline 5-Fapesp & $0,14(0,08)[1,0]$ & $0,09(0,04)[2,0]$ \\
\hline 6-Genbase* & $0,95(0,02)[1,5]$ & $0,95(0,02)[1,5]$ \\
\hline 7-Llog-f* & $0,17(0,03)[1,0]$ & $0,14(0,02)[2,0]$ \\
\hline 8-Magtag5k & $0,37(0,01)[1,0]$ & $0,35(0,01)[2,0]$ \\
\hline 9-Scene & $0,62(0,02)[2,0]$ & $0,63(0,03)[1,0]$ \\
\hline \multirow[t]{2}{*}{ 10-Yeast } & $0,65(0,02)[1,5]$ & $0,65(0,01)[1,5]$ \\
\hline & \multicolumn{2}{|c|}{$\left|X^{\prime}\right|=40 \% M$} \\
\hline 1-Cal500 & $0,44(0,02)[1,0]$ & $0,43(0,01)[2,0]$ \\
\hline 2-Corel5k & $0,16(0,01)[1,0]$ & $0,15(0,02)[2,0]$ \\
\hline 3-Corel16k001 & $0,19(0,01)[2,0]$ & $0,20(0,01)[1,0]$ \\
\hline 4-Emotions & $0,64(0,03)[2,0]$ & $0,65(0,04)[1,0]$ \\
\hline 5-Fapesp & $0,13(0,08)[1,0]$ & $0,08(0,05)[2,0]$ \\
\hline 6-Genbase* & $0,95(0,02)[1,5]$ & $0,95(0,02)[1,5]$ \\
\hline $7-L \log -f^{*}$ & $0,17(0,02)[1,0]$ & $0,15(0,03)[2,0]$ \\
\hline 8-Magtag5k & $0,37(0,01)[1,5]$ & $0,37(0,01)[1,5]$ \\
\hline 9-Scene & $0,66(0,03)[1,0]$ & $0,65(0,03)[2,0]$ \\
\hline \multirow[t]{2}{*}{ 10-Yeast } & $0,65(0,02)[2,0]$ & $0,66(0,01)[1,0]$ \\
\hline & \multicolumn{2}{|c|}{$\left|X^{\prime}\right|=50 \% M$} \\
\hline 1-Cal500 & $0,44(0,02)[1,0]$ & $0,43(0,01)[2,0]$ \\
\hline 2-Corel5k & $0,13(0,02)[1,0]$ & $0,12(0,02)[2,0]$ \\
\hline 3-Corel16k001 & $0,18(0,01)[1,5]$ & $0,18(0,01)[1,5]$ \\
\hline 4-Emotions & $0,65(0,03)[1,0]$ & $0,64(0,04)[2,0]$ \\
\hline 5-Fapesp & $0,13(0,09)[1,0]$ & $0,07(0,04)[2,0]$ \\
\hline 6-Genbase* & $0,95(0,02)[1,5]$ & $0,95(0,02)[1,5]$ \\
\hline $7-L \log -f^{*}$ & $0,15(0,02)[1,5]$ & $0,15(0,03)[1,5]$ \\
\hline 8-Magtag5k & $0,37(0,01)[1,5]$ & $0,37(0,01)[1,5]$ \\
\hline 9-Scene & $0,66(0,03)[1,5]$ & $0,66(0,03)[1,5]$ \\
\hline \multirow[t]{2}{*}{ 10-Yeast } & $0,65(0,02)[1,5]$ & $0,65(0,02)[1,5]$ \\
\hline & \multicolumn{2}{|c|}{$\left|X^{\prime}\right|=60 \% M$} \\
\hline 1-Cal500 & $0,44(0,02)[1,5]$ & $0,44(0,01)[1,5]$ \\
\hline 2-Corel5k & $0,11(0,02)[1,5]$ & $0,11(0,01)[1,5]$ \\
\hline 3-Corel16k001 & $0,17(0,01)[1,0]$ & $0,15(0,01)[2,0]$ \\
\hline 4-Emotions & $0,67(0,04)[1,0]$ & $0,65(0,03)[2,0]$ \\
\hline 5-Fapesp & $0,13(0,09)[1,0]$ & $0,05(0,04)[2,0]$ \\
\hline 6-Genbase* & $0,95(0,02)[1,5]$ & $0,95(0,02)[1,5]$ \\
\hline 7-Llog-f* & $0,15(0,03)[1,0]$ & $0,14(0,03)[2,0]$ \\
\hline 8-Magtag5k & $0,37(0,01)[1,5]$ & $0,37(0,01)[1,5]$ \\
\hline 9-Scene & $0,69(0,03)[1,5]$ & $0,69(0,03)[1,5]$ \\
\hline \multirow[t]{2}{*}{ 10-Yeast } & $0,66(0,02)[1,5]$ & $0,66(0,02)[1,5]$ \\
\hline & \multicolumn{2}{|c|}{$\left|X^{\prime}\right|=70 \% M$} \\
\hline 1-Cal500 & $0,44(0,02)[1,5]$ & $0,44(0,01)[1,5]$ \\
\hline 2-Corel5k & $0,11(0,02)[1,5]$ & $0,11(0,02)[1,5]$ \\
\hline 3-Corel16k001 & $0,16(0,01)[1,0]$ & $0,13(0,01)[2,0]$ \\
\hline 4-Emotions & $0,67(0,04)[1,0]$ & $0,65(0,03)[2,0]$ \\
\hline 5-Fapesp & $0,12(0,08)[1,0]$ & $0,04(0,03)[2,0]$ \\
\hline 6-Genbase* & $0,95(0,02)[1,5]$ & $0,95(0,02)[1,5]$ \\
\hline $7-L \log -f^{*}$ & $0,15(0,03)[1,0]$ & $0,14(0,03)[2,0]$ \\
\hline 8-Magtag5k & $0,37(0,01)[1,5]$ & $0,37(0,01)[1,5]$ \\
\hline 9-Scene & $0,72(0,02)[1,5]$ & $0,72(0,03)[1,5]$ \\
\hline \multirow[t]{2}{*}{ 10-Yeast } & $0,66(0,02)[1,5]$ & $0,66(0,02)[1,5]$ \\
\hline & \multicolumn{2}{|c|}{$\left|X^{\prime}\right|=80 \% M$} \\
\hline 1-Cal500 & $0,44(0,02)[1,5]$ & $0,44(0,01)[1,5]$ \\
\hline
\end{tabular}


Tabela A. 15: $F_{b}$ (continuação)

\begin{tabular}{|c|c|c|}
\hline & $R F-M L$ Jaccard & $R F-M L$ Hamming \\
\hline 2-Corel5k & $0,10(0,01)[1,5]$ & $0,10(0,01)[1,5]$ \\
\hline 3-Corel16k001 & $0,15(0,01)[1,0]$ & $0,12(0,01)[2,0]$ \\
\hline 4-Emotions & $0,67(0,04)[1,5]$ & $0,67(0,04)[1,5]$ \\
\hline 5-Fapesp & $0,11(0,08)[1,0]$ & $0,03(0,03)[2,0]$ \\
\hline 6-Genbase* & $0,95(0,02)[1,5]$ & $0,95(0,02)[1,5]$ \\
\hline 7-Llog-f* & $0,16(0,03)[1,0]$ & $0,14(0,03)[2,0]$ \\
\hline 8-Magtag5k & $0,37(0,01)[1,5]$ & $0,37(0,01)[1,5]$ \\
\hline 9-Scene & $0,72(0,03)[1,5]$ & $0,72(0,03)[1,5]$ \\
\hline \multirow[t]{2}{*}{ 10-Yeast } & $0,66(0,02)[1,5]$ & $0,66(0,02)[1,5]$ \\
\hline & \multicolumn{2}{|c|}{$\left|X^{\prime}\right|=90 \% M$} \\
\hline 1-Cal500 & $0,44(0,02)[1,5]$ & $0,44(0,02)[1,5]$ \\
\hline 2-Corel5k & $0,10(0,02)[2,0]$ & $0,12(0,01)[1,0]$ \\
\hline 3-Corel16k001 & $0,10(0,01)[2,0]$ & $0,11(0,01)[1,0]$ \\
\hline 4-Emotions & $0,67(0,03)[1,0]$ & $0,66(0,04)[2,0]$ \\
\hline 5-Fapesp & $0,10(0,07)[1,0]$ & $0,03(0,03)[2,0]$ \\
\hline 6-Genbase* & $0,95(0,02)[1,5]$ & $0,95(0,02)[1,5]$ \\
\hline 7-Llog-f* & $0,15(0,03)[1,0]$ & $0,14(0,04)[2,0]$ \\
\hline 8-Magtag5k & $0,37(0,01)[1,5]$ & $0,37(0,01)[1,5]$ \\
\hline 9-Scene & $0,73(0,03)[1,0]$ & $0,72(0,03)[2,0]$ \\
\hline 10-Yeast & $0,66(0,02)[1,5]$ & $0,66(0,02)[1,5]$ \\
\hline
\end{tabular}

\section{A.3.2 Algoritmos baseados em Relieff e IG-LP}

\section{Conjuntos de Dados Artificiais}

Tabela A.16: Rankings de atributos e valores de importância estimados por $R F-M L$ nos conjuntos de dados artificiais com $\mu=0 \%$

\begin{tabular}{|c|c|c|c|c|c|c|c|c|c|c|c|c|c|c|c|c|c|c|}
\hline & \multicolumn{2}{|c|}{ dataset $_{1}$} & \multicolumn{2}{|c|}{$\overline{\text { dataset }_{2}}$} & \multicolumn{2}{|c|}{ dataset $_{3}$} & \multicolumn{2}{|c|}{ dataset $_{4}$} & \multicolumn{2}{|c|}{ dataset $_{5}$} & \multicolumn{2}{|c|}{$\overline{\text { dataset }_{6}}$} & \multicolumn{2}{|c|}{ dataset $_{7}$} & \multicolumn{2}{|c|}{$\overline{\text { dataset }_{8}}$} & \multicolumn{2}{|c|}{ dataset $_{9}$} \\
\hline 10 & $\overline{\mathbf{X 3}}$ & 0,01 & $\overline{\mathrm{X} 4}$ & $\overline{0,01}$ & $\overline{\mathbf{X 1}}$ & 0,12 & $\overline{\mathrm{X1}}$ & 0,02 & $\overline{X 5}$ & 0,02 & $\overline{\mathbf{X 1}}$ & 0,14 & $\mathbf{X 1}$ & $\overline{0,02}$ & $\mathbf{X 2}$ & 0,03 & $\overline{\mathbf{X 1}}$ & 0,14 \\
\hline $3^{\mathbf{o}}$ & X9 & 0,01 & $\mathbf{X 3}$ & 0,01 & X18 & 0,00 & X10 & 0,01 & $\mathbf{X 2}$ & 0,02 & X8 & 0,00 & $\mathbf{x 1 0}$ & 0,02 & X3 & 0,02 & X19 & 0,00 \\
\hline $6^{\circ}$ & $\mathrm{X5}$ & 0,01 & X15 & 0,00 & $\mathrm{X} 3$ & 0,00 & $\mathbf{X 9}$ & 0,01 & $\mathrm{X} 12$ & 0,00 & $\mathrm{X} 3$ & 0,00 & $\mathbf{X 3}$ & 0,01 & $\mathrm{X} 18$ & 0,00 & $\mathrm{X} 16$ & 0,00 \\
\hline $7^{\mathrm{o}}$ & $\mathbf{x 1}$ & 0,01 & $\mathrm{X} 8$ & 0,00 & X17 & 0,00 & $\mathbf{X 4}$ & 0,01 & X9 & 0,00 & $\mathrm{X} 13$ & 0,00 & $\mathbf{x 8}$ & 0,01 & X20 & 0,00 & X10 & 0,00 \\
\hline 80 & X4 & 0,01 & $\mathrm{X} 16$ & 0,00 & $\mathrm{X} 11$ & 0,00 & X8 & 0,01 & $\mathrm{X} 20$ & 0,00 & $\mathrm{X} 11$ & 0,00 & X9 & 0,01 & $\mathrm{X} 13$ & 0,00 & $\mathrm{X} 6$ & 0,00 \\
\hline 110 & $\mathrm{X} 18$ & $-0,01$ & X9 & 0,00 & X7 & 0,00 & X17 & $-0,01$ & X16 & 0,00 & $\mathrm{X} 2$ & $-0,01$ & X20 & $-0,01$ & X7 & 0,00 & X7 & $-0,01$ \\
\hline $12^{\circ}$ & X16 & $-0,01$ & $\mathrm{X} 18$ & 0,0 & X5 & & X16 & $-0,01$ & $\mathrm{X} 18$ & 0,00 & $\mathrm{X}^{7}$ & $-0,01$ & $\mathrm{X}$ & $-0,01$ & X11 & 0,00 & X15 & $-0,01$ \\
\hline 130 & X20 & $-0,0$ & $\mathrm{X} 17$ & 0,00 & X16 & 0 , & X14 & -0 , & $\mathrm{XlC}$ & 0,00 & $\mathrm{X}$ & -0 , & X18 & -0 , & X9 & 0,00 & X18 & $-0,01$ \\
\hline $14^{\circ}$ & X14 & $-0,01$ & X13 & 0,00 & X19 & 0,00 & X19 & $-0,01$ & X17 & 0,00 & X19 & $-0,01$ & X19 & $-0,01$ & X10 & $-0,01$ & $\mathrm{X} 14$ & $-0,01$ \\
\hline $15^{\circ}$ & X19 & $-0,01$ & X19 & 0,00 & X4 & 0,00 & $\mathrm{X} 12$ & $-0,01$ & X7 & 0,00 & $\mathrm{X} 6$ & $-0,01$ & $\mathrm{X} 16$ & $-0,01$ & X16 & $-0,01$ & X20 & $-0,01$ \\
\hline $16^{\circ}$ & $\mathrm{X} 13$ & $-0,01$ & $\mathrm{X} 11$ & 0,00 & X6 & $-0,01$ & X20 & $-0,01$ & $\mathrm{X} 11$ & 0,00 & X15 & $-0,01$ & $\mathrm{X} 12$ & $-0,01$ & $\mathrm{X} 12$ & $-0,01$ & $\mathrm{X} 17$ & $-0,01$ \\
\hline $17^{\circ}$ & X11 & $-0,0$ & $\mathrm{X} 7$ & 0,0 & $\mathrm{X} 2$ & -0 , & $\mathrm{X}$ & -0 , & $\mathrm{X}$ & 0 , & $\mathrm{X}$ & & $\mathrm{X} 17$ & & X6 & & $\mathrm{X}$ & $-0,01$ \\
\hline
\end{tabular}


Tabela A.17: Rankings de atributos e valores de importância estimados por $R F-M L$ nos conjuntos de dados artificiais $\operatorname{com} \mu=5 \%$

\begin{tabular}{|c|c|c|c|c|c|c|c|c|c|c|c|c|c|c|c|c|c|c|}
\hline & \multicolumn{2}{|c|}{ dataset $_{1}$} & \multicolumn{2}{|c|}{ dataset $_{2}$} & \multicolumn{2}{|c|}{ dataset $_{3}$} & \multicolumn{2}{|c|}{ dataset $_{4}$} & \multicolumn{2}{|c|}{ dataset $_{5}$} & \multicolumn{2}{|c|}{ dataset $_{6}$} & \multicolumn{2}{|c|}{ dataset $_{7}$} & \multicolumn{2}{|c|}{ dataset $_{8}$} & \multicolumn{2}{|c|}{ dataset $_{9}$} \\
\hline 10 & X3 & 0,00 & $\mathbf{X 4}$ & 0,01 & $\mathrm{X1}$ & 0,09 & $\mathrm{X1}$ & 0,01 & $\mathbf{X 5}$ & 0,01 & $\mathrm{X} 1$ & 0,10 & $\mathbf{X 1}$ & 0,01 & $\mathrm{X2}$ & 0,02 & $\mathbf{X 1}$ & 0,11 \\
\hline $2^{\mathrm{O}}$ & X9 & 0,00 & X5 & 0,01 & $\mathrm{X} 12$ & 0,00 & X3 & 0,01 & $\mathbf{x 1}$ & 0,01 & X14 & 0,00 & $\mathbf{x 1 0}$ & 0,01 & X5 & 0,02 & $\mathrm{X} 12$ & 0,00 \\
\hline 30 & X8 & 0,00 & X3 & 0,01 & $\mathrm{X} 18$ & 0,00 & $\mathbf{X 1 0}$ & 0,01 & $\mathbf{X 2}$ & 0,01 & $\mathrm{X} 4$ & 0,00 & $\mathbf{X 4}$ & 0,01 & X3 & 0,01 & X19 & 0,00 \\
\hline 40 & $\mathbf{X} 2$ & 0,00 & $\mathbf{X 1}$ & 0,01 & $\mathrm{X} 10$ & 0,00 & X6 & 0,01 & $\mathbf{X 4}$ & 0,01 & X3 & 0,00 & X6 & 0,01 & $\mathbf{X 1}$ & 0,01 & $\mathrm{X} 16$ & 0,00 \\
\hline $5^{0}$ & X4 & 0,00 & X2 & 0,01 & $\mathrm{X} 2$ & 0,00 & X5 & 0,01 & X3 & 0,00 & X8 & 0,00 & $\mathbf{X} 2$ & 0,01 & X4 & 0,01 & $\mathrm{X} 14$ & 0,00 \\
\hline 60 & X6 & 0,00 & X15 & 0,00 & $\mathrm{X} 17$ & 0,00 & X2 & 0,01 & $\mathrm{X} 12$ & 0,00 & $\mathrm{X} 20$ & 0,00 & X8 & 0,01 & X20 & 0,00 & X5 & 0,00 \\
\hline $7^{\circ} \stackrel{0}{ }$ & $\mathbf{x 1}$ & 0,00 & X20 & 0,00 & X9 & 0,00 & X8 & 0,01 & $\mathrm{X} 10$ & 0,00 & $\mathrm{X} 7$ & 0,00 & X9 & 0,01 & $\mathrm{X} 18$ & 0,00 & X6 & 0,00 \\
\hline 80 & X5 & 0,00 & $\mathrm{X} 8$ & 0,00 & $\mathrm{X} 3$ & 0,00 & X9 & 0,01 & X9 & 0,00 & $\mathrm{X} 13$ & 0,00 & X3 & 0,01 & X19 & 0,00 & $\mathrm{X} 11$ & 0,00 \\
\hline 9 음 & X7 & 0,00 & X18 & 0,00 & X11 & 0,00 & X4 & 0,01 & $\mathrm{X} 11$ & 0,00 & $\mathrm{X} 11$ & 0,00 & X5 & 0,01 & X14 & 0,00 & X7 & 0,00 \\
\hline $10^{\circ}$ & X10 & 0,00 & $\mathrm{X} 12$ & 0,00 & $\mathrm{X} 7$ & 0,00 & X7 & 0,01 & $\mathrm{X} 16$ & 0,00 & $\mathrm{X} 2$ & 0,00 & X7 & 0,01 & X10 & 0,00 & $\mathrm{X} 10$ & 0,00 \\
\hline 110 & X18 & 0,00 & $\mathrm{X} 11$ & 0,00 & X19 & 0,00 & X17 & 0,00 & $\mathrm{X} 17$ & 0,00 & $\mathrm{X} 10$ & 0,00 & X16 & $-0,01$ & X11 & 0,00 & $\mathrm{X} 4$ & 0,00 \\
\hline $12^{\circ}$ 은 & X14 & 0,00 & X16 & 0,00 & X16 & 0,00 & X18 & $-0,01$ & $\mathrm{X} 18$ & 0,00 & X9 & 0,00 & X20 & $-0,01$ & X9 & 0,00 & $\mathrm{X} 18$ & 0,00 \\
\hline $13^{\circ}$ & X19 & 0,00 & X19 & 0,00 & X5 & 0,00 & X15 & $-0,01$ & $\mathrm{X} 13$ & 0,00 & $\mathrm{X} 18$ & 0,00 & $\mathrm{X} 17$ & $-0,01$ & X13 & 0,00 & $\mathrm{X} 13$ & 0,00 \\
\hline $14^{\circ}$ & $\mathrm{X} 16$ & 0,00 & X9 & 0,00 & $\mathrm{X} 4$ & 0,00 & $\mathrm{X} 12$ & $-0,01$ & X7 & 0,00 & X19 & 0,00 & X19 & $-0,01$ & X7 & 0,00 & $\mathrm{X} 17$ & 0,00 \\
\hline 150 & $\mathrm{X} 20$ & 0,00 & $\mathrm{X} 6$ & 0,00 & $\mathrm{X} 8$ & 0,00 & X16 & $-0,01$ & $\mathrm{X} 14$ & 0,00 & X5 & 0,00 & X14 & $-0,01$ & $\mathrm{X} 17$ & 0,00 & X15 & 0,00 \\
\hline $16^{\circ}$ & X11 & 0,00 & $\mathrm{X} 17$ & 0,00 & X6 & 0,00 & X19 & $-0,01$ & $\mathrm{X} 20$ & 0,00 & X6 & 0,00 & $\mathrm{X} 18$ & $-0,01$ & X16 & 0,00 & $\mathrm{X} 20$ & 0,00 \\
\hline $17^{\circ}$ & $\mathrm{X} 13$ & 0,00 & $\mathrm{X} 13$ & 0,00 & $\mathrm{X} 20$ & 0,00 & X11 & $-0,01$ & X15 & 0,00 & X15 & 0,00 & $\mathrm{X} 11$ & $-0,01$ & $\mathrm{X} 12$ & 0,00 & $\mathrm{X} 8$ & $-0,01$ \\
\hline $18^{\circ}$ & X12 & 0,00 & X14 & 0,00 & X15 & 0,00 & X14 & $-0,01$ & X19 & 0,00 & X16 & $-0,01$ & X12 & $-0,01$ & $\mathrm{X} 6$ & 0,00 & X9 & $-0,01$ \\
\hline $19^{\circ}$ 의 & X15 & 0,00 & $\mathrm{X} 7$ & 0,00 & $\mathrm{X} 13$ & 0,00 & X20 & $-0,01$ & $\mathrm{X} 6$ & 0,00 & $\mathrm{X} 12$ & $-0,01$ & $\mathrm{X} 13$ & $-0,01$ & $\mathrm{X} 8$ & $-0,01$ & $\mathrm{X} 2$ & $-0,01$ \\
\hline $20^{\circ}$ & X17 & 0,00 & $\mathrm{X} 10$ & 0,00 & X14 & 0,00 & X13 & $-0,01$ & X8 & 0,00 & $\mathrm{X} 17$ & $-0,01$ & X15 & $-0,01$ & X15 & $-0,01$ & X3 & $-0,01$ \\
\hline
\end{tabular}

Tabela A.18: Rankings de atributos e valores de importância estimados por $R F-M L$ nos conjuntos de dados artificiais com $\mu=10 \%$

\begin{tabular}{|c|c|c|c|c|c|c|c|c|c|c|c|c|c|c|c|c|c|c|}
\hline & \multicolumn{2}{|c|}{ dataset $_{1}$} & \multicolumn{2}{|c|}{ dataset $_{2}$} & \multicolumn{2}{|c|}{ dataset $_{3}$} & \multicolumn{2}{|c|}{ dataset $_{4}$} & \multicolumn{2}{|c|}{ dataset $_{5}$} & \multicolumn{2}{|c|}{ dataset $_{6}$} & \multicolumn{2}{|c|}{ dataset $_{7}$} & \multicolumn{2}{|c|}{ dataset $_{8}$} & \multicolumn{2}{|c|}{ dataset $_{9}$} \\
\hline 10 & $\mathbf{X 3}$ & 0,00 & $\mathrm{X4}$ & 0,01 & $\mathbf{X 1}$ & 0,07 & $\mathbf{X 1}$ & 0,01 & $\mathbf{X 1}$ & 0,01 & $\mathrm{X1}$ & 0,08 & $\mathbf{x 1}$ & 0,01 & $\mathbf{X 2}$ & 0,02 & $\mathbf{x 1}$ & 0,08 \\
\hline 20 & X9 & 0,00 & X5 & 0,01 & $\mathrm{X} 12$ & 0,00 & X3 & 0,01 & $\mathbf{X 5}$ & 0,01 & $\mathrm{X} 14$ & 0,00 & X2 & 0,01 & $\mathbf{X 5}$ & 0,01 & X11 & 0,00 \\
\hline 30 & X8 & 0,00 & X3 & 0,01 & X11 & 0,00 & $\mathbf{x 1 0}$ & 0,00 & X2 & 0,01 & $\mathrm{X} 20$ & 0,00 & X6 & 0,01 & X3 & 0,01 & $\mathrm{X} 12$ & 0,00 \\
\hline $4 \underline{0}$ & X6 & 0,00 & $\mathbf{x 1}$ & 0,00 & $\mathrm{X} 18$ & 0,00 & X7 & 0,00 & $\mathbf{X 4}$ & 0,01 & X3 & 0,00 & $\mathbf{x 1 0}$ & 0,01 & $\mathbf{x 1}$ & 0,01 & X19 & 0,00 \\
\hline 5 음 & X2 & 0,00 & X2 & 0,00 & $\mathrm{X} 3$ & 0,00 & X5 & 0,00 & X3 & 0,00 & X4 & 0,00 & $\mathbf{X 4}$ & 0,01 & X4 & 0,01 & $\mathrm{X} 18$ & 0,00 \\
\hline $6^{\circ}$ & X5 & 0,00 & $\mathrm{X} 8$ & 0,00 & X17 & 0,00 & X6 & 0,00 & X18 & 0,00 & X11 & 0,00 & X8 & 0,01 & $\mathrm{X} 18$ & 0,00 & $\mathrm{X} 16$ & 0,00 \\
\hline $7 \underline{0}$ & X4 & 0,00 & X15 & 0,00 & $\mathrm{X} 2$ & 0,00 & X9 & 0,00 & X11 & 0,00 & X9 & 0,00 & X9 & 0,01 & $\mathrm{X} 12$ & 0,00 & $\times 10$ & 0,00 \\
\hline $8^{\circ}$ & X7 & 0,00 & X20 & 0,00 & X10 & 0,00 & $\mathbf{X 2}$ & 0,00 & $\mathrm{X} 12$ & 0,00 & $\mathrm{X} 2$ & 0,00 & $\mathbf{x 3}$ & 0,01 & X19 & 0,00 & $\mathrm{X} 13$ & 0,00 \\
\hline 90 & $\mathbf{x 1}$ & 0,00 & $\mathrm{X} 12$ & 0,00 & X5 & 0,00 & X8 & 0,00 & $\mathrm{X} 16$ & 0,00 & $\mathrm{X} 19$ & 0,00 & X7 & 0,01 & $\mathrm{X} 16$ & 0,00 & $\mathrm{X} 4$ & 0,00 \\
\hline $10^{0}$ & X10 & 0,00 & X16 & 0,00 & X7 & 0,00 & $\times 4$ & 0,00 & $\mathrm{X} 10$ & 0,00 & $\mathrm{X} 13$ & 0,00 & X5 & 0,00 & X6 & 0,00 & X8 & 0,00 \\
\hline 110 & X14 & 0,00 & X13 & 0,00 & X9 & 0,00 & X19 & 0,00 & $\mathrm{X} 7$ & 0,00 & $\mathrm{X} 8$ & 0,00 & $\mathrm{X} 18$ & 0,00 & $\mathrm{X} 10$ & 0,00 & $\mathrm{X} 6$ & 0,00 \\
\hline 120 & X20 & 0,00 & $\mathrm{X} 18$ & 0,00 & X8 & 0,00 & X20 & 0,00 & $\mathrm{X} 17$ & 0,00 & X7 & 0,00 & X20 & 0,00 & X7 & 0,00 & $\mathrm{X} 14$ & 0,00 \\
\hline 130 & X18 & 0,00 & X19 & 0,00 & X16 & 0,00 & $\mathrm{X} 17$ & 0,00 & X8 & 0,00 & X6 & 0,00 & X19 & 0,00 & $\mathrm{X} 20$ & 0,00 & X15 & 0,00 \\
\hline $14^{\circ}$ & $\mathrm{X} 12$ & 0,00 & $\mathrm{X} 17$ & 0,00 & X20 & 0,00 & $\mathrm{X} 12$ & 0,00 & $\mathrm{X} 20$ & 0,00 & X15 & 0,00 & X16 & 0,00 & X15 & 0,00 & $\mathrm{X} 17$ & 0,00 \\
\hline $15^{\circ}$ & X15 & 0,00 & $\mathrm{X} 10$ & 0,00 & X19 & 0,00 & X11 & 0,00 & X14 & 0,00 & $\mathrm{X} 18$ & 0,00 & X13 & $-0,01$ & $\mathrm{X} 13$ & 0,00 & X5 & 0,00 \\
\hline $16^{0}$ & X16 & 0,00 & $\mathrm{X} 6$ & 0,00 & $\mathrm{X} 4$ & 0,00 & X16 & 0,00 & X9 & 0,00 & $\mathrm{X} 12$ & 0,00 & $\mathrm{X} 12$ & $-0,01$ & $\mathrm{X} 14$ & 0,00 & X7 & 0,00 \\
\hline $17^{\circ}$ & X19 & 0,00 & $\mathrm{X} 11$ & 0,00 & X13 & 0,00 & X18 & 0,00 & $\mathrm{X} 13$ & 0,00 & X5 & 0,00 & X17 & $-0,01$ & X8 & 0,00 & X20 & 0,00 \\
\hline $18^{\circ}$ & X13 & 0,00 & X9 & 0,00 & X6 & 0,00 & X14 & 0,00 & X6 & 0,00 & $\mathrm{X} 10$ & 0,00 & X14 & $-0,01$ & $\mathrm{X} 17$ & 0,00 & $\mathrm{X} 2$ & $-0,01$ \\
\hline 190 & X11 & 0,00 & $\mathrm{X} 14$ & 0,00 & X14 & 0,00 & X15 & 0,00 & X19 & 0,00 & $\mathrm{X} 16$ & 0,00 & X15 & $-0,01$ & X9 & 0,00 & X3 & $-0,01$ \\
\hline $20 \underline{0}$ & $\mathrm{X} 17$ & 0,00 & $\mathrm{X} 7$ & 0,00 & X15 & 0,00 & X13 & 0,00 & X15 & 0,00 & X17 & 0,00 & X11 & $-0,01$ & $\mathrm{X} 11$ & 0,00 & X9 & $-0,01$ \\
\hline
\end{tabular}

Tabela A.19: Rankings de atributos e valores de importância estimados por $R F-M L$ nos conjuntos de dados artificiais com $\mu=20 \%$

\begin{tabular}{|c|c|c|c|c|c|c|c|c|c|c|c|c|c|c|c|c|c|c|}
\hline & \multicolumn{2}{|c|}{ dataset $_{1}$} & \multicolumn{2}{|c|}{ dataset $_{2}$} & \multicolumn{2}{|c|}{ dataset $_{3}$} & \multicolumn{2}{|c|}{ dataset $_{4}$} & \multicolumn{2}{|c|}{ dataset $_{5}$} & \multicolumn{2}{|c|}{ dataset $_{6}$} & \multicolumn{2}{|c|}{ dataset $_{7}$} & \multicolumn{2}{|c|}{ dataset $_{8}$} & \multicolumn{2}{|c|}{ dataset $_{9}$} \\
\hline 10 & $\mathrm{X8}$ & 0,00 & $\mathrm{X4}$ & 0,00 & $\mathrm{X1}$ & 0,03 & $\mathbf{X 1}$ & 0,00 & $\mathbf{x 5}$ & 0,00 & $\mathbf{X 1}$ & 0,04 & X10 & 0,01 & $\mathbf{X 2}$ & 0,01 & $\overline{x 1}$ & 0,05 \\
\hline 20 & X4 & 0,00 & X5 & 0,00 & X3 & 0,00 & X4 & 0,00 & $\mathbf{x 1}$ & 0,00 & $\mathrm{X} 13$ & 0,00 & X6 & 0,01 & $\mathbf{X 5}$ & 0,01 & $\mathrm{X} 11$ & 0,00 \\
\hline $3^{0}$ & X9 & 0,00 & $\mathbf{X 1}$ & 0,00 & X9 & 0,00 & X3 & 0,00 & X2 & 0,00 & X3 & 0,00 & X4 & 0,01 & $\mathbf{X 4}$ & 0,00 & $\mathrm{X} 12$ & 0,00 \\
\hline $4^{0}$ & X5 & 0,00 & $\mathbf{X 3}$ & 0,00 & X7 & 0,00 & $\mathbf{X 8}$ & 0,00 & X4 & 0,00 & $\mathrm{X} 20$ & 0,00 & $\mathbf{x} 1$ & 0,00 & $\mathbf{X 1}$ & 0,00 & X16 & 0,00 \\
\hline $5^{\circ}$ - & X6 & 0,00 & X2 & 0,00 & $\mathrm{X} 2$ & 0,00 & X6 & 0,00 & X3 & 0,00 & $\mathrm{X} 10$ & 0,00 & $\mathbf{X 3}$ & 0,00 & X3 & 0,00 & $\mathrm{X} 17$ & 0,00 \\
\hline $6^{\circ}-$ & X3 & 0,00 & X15 & 0,00 & $\mathrm{X} 11$ & 0,00 & X10 & 0,00 & X12 & 0,00 & X11 & 0,00 & $\mathbf{X 2}$ & 0,00 & X11 & 0,00 & X18 & 0,00 \\
\hline $7^{\circ} \underline{0}$ & $\mathbf{X} 2$ & 0,00 & X9 & 0,00 & $\mathrm{X} 12$ & 0,00 & X9 & 0,00 & X14 & 0,00 & X4 & 0,00 & X9 & 0,00 & X9 & 0,00 & $\mathrm{X} 4$ & 0,00 \\
\hline $8^{\circ}$ & $\mathbf{x} 1$ & 0,00 & $\mathrm{X} 12$ & 0,00 & $\mathrm{X} 10$ & 0,00 & X5 & 0,00 & $\mathrm{X} 13$ & 0,00 & X6 & 0,00 & $\mathbf{X 8}$ & 0,00 & $\mathrm{X} 13$ & 0,00 & X8 & 0,00 \\
\hline 90 & X7 & 0,00 & X14 & 0,00 & $\mathrm{X} 8$ & 0,00 & $\mathbf{X 2}$ & 0,00 & $\mathrm{X} 10$ & 0,00 & $\mathrm{X} 14$ & 0,00 & X7 & 0,00 & $\mathrm{X} 12$ & 0,00 & $\mathrm{X} 6$ & 0,00 \\
\hline $10^{\circ}$ & $\mathbf{x} 10$ & 0,00 & X19 & 0,00 & $\mathrm{X} 4$ & 0,00 & X7 & 0,00 & X9 & 0,00 & X19 & 0,00 & X5 & 0,00 & X6 & 0,00 & X19 & 0,00 \\
\hline 110 & X12 & 0,00 & X16 & 0,00 & X18 & 0,00 & X17 & 0,00 & X16 & 0,00 & X8 & 0,00 & X18 & 0,00 & $\mathrm{X} 20$ & 0,00 & $\mathrm{X} 2$ & 0,00 \\
\hline $12^{\circ}$ & X16 & 0,00 & X13 & 0,00 & X17 & 0,00 & X20 & 0,00 & $\mathrm{X} 17$ & 0,00 & X7 & 0,00 & X15 & 0,00 & $\mathrm{X} 10$ & 0,00 & X5 & 0,00 \\
\hline $13^{0}$ & $\mathrm{X} 17$ & 0,00 & X18 & 0,00 & X16 & 0,00 & X19 & 0,00 & X6 & 0,00 & $\mathrm{X} 18$ & 0,00 & $\mathrm{X} 11$ & 0,00 & $\mathrm{X} 14$ & 0,00 & $\mathrm{X} 10$ & 0,00 \\
\hline 140 & $\mathrm{X} 18$ & 0,00 & X17 & 0,00 & X5 & 0,00 & X15 & 0,00 & X18 & 0,00 & $\mathrm{X} 12$ & 0,00 & X14 & 0,00 & X19 & 0,00 & $\mathrm{X} 13$ & 0,00 \\
\hline 150 & X19 & 0,00 & X7 & 0,00 & X13 & 0,00 & X16 & 0,00 & $\mathrm{X} 20$ & 0,00 & X9 & 0,00 & X16 & 0,00 & $\mathrm{X} 8$ & 0,00 & X14 & 0,00 \\
\hline $16^{0}$ & $\mathrm{X} 13$ & 0,00 & X20 & 0,00 & $\mathrm{X} 20$ & 0,00 & X14 & 0,00 & X15 & 0,00 & $\mathrm{X} 2$ & 0,00 & X20 & 0,00 & X7 & 0,00 & $\mathrm{X} 7$ & 0,00 \\
\hline $17^{\mathrm{o}}$ & X20 & 0,00 & $\mathrm{X} 10$ & 0,00 & X14 & 0,00 & $\mathrm{X} 18$ & 0,00 & X7 & 0,00 & X15 & 0,00 & X19 & 0,00 & $\mathrm{X} 18$ & 0,00 & $\mathrm{X} 20$ & 0,00 \\
\hline $18^{\circ}$ & $\mathrm{X} 14$ & 0,00 & $\mathrm{X} 8$ & 0,00 & $\mathrm{X} 6$ & 0,00 & $\mathrm{X} 12$ & 0,00 & X11 & 0,00 & X5 & 0,00 & X13 & 0,00 & $\mathrm{X} 17$ & 0,00 & X3 & 0,00 \\
\hline 190 & X15 & 0,00 & X11 & 0,00 & X19 & 0,00 & X11 & 0,00 & X19 & 0,00 & $\mathrm{X} 17$ & 0,00 & $\mathrm{X} 12$ & 0,00 & $\mathrm{X} 16$ & 0,00 & X9 & 0,00 \\
\hline 20 응 & $\mathrm{X} 11$ & 0,00 & X6 & 0,00 & X15 & 0,00 & X13 & 0,00 & X8 & 0,00 & X16 & 0,00 & $\mathrm{X} 17$ & 0,00 & X15 & 0,00 & X15 & 0,00 \\
\hline
\end{tabular}


Tabela A.20: Rankings de atributos e valores de importância estimados por $R F-M L$ nos conjuntos de dados artificiais $\operatorname{com} \mu=40 \%$

\begin{tabular}{|c|c|c|c|c|c|c|c|c|c|c|c|c|c|c|c|c|c|c|}
\hline & \multicolumn{2}{|c|}{ dataset $_{1}$} & \multicolumn{2}{|c|}{ dataset $_{2}$} & \multicolumn{2}{|c|}{ dataset $_{3}$} & \multicolumn{2}{|c|}{ dataset $_{4}$} & \multicolumn{2}{|c|}{ dataset $_{5}$} & \multicolumn{2}{|c|}{ dataset $_{6}$} & \multicolumn{2}{|c|}{ dataset $_{7}$} & \multicolumn{2}{|c|}{ dataset $_{8}$} & \multicolumn{2}{|c|}{ dataset $_{9}$} \\
\hline 10 & X3 & 0,00 & $\mathbf{X 5}$ & 0,00 & $\mathbf{x 1}$ & 0,00 & $\mathbf{x 1}$ & 0,00 & $\mathrm{X} 17$ & 0,00 & $\mathbf{X 1}$ & 0,00 & $\mathbf{X 1}$ & 0,00 & $\mathbf{X 2}$ & 0,00 & $\mathbf{X 1}$ & 0,00 \\
\hline 30 & X9 & 0,00 & $\mathbf{X 1}$ & 0,00 & $\mathrm{X} 2$ & 0,00 & $\times 10$ & 0,00 & X7 & 0,00 & X16 & 0,00 & X4 & 0,00 & X6 & 0,00 & $\mathrm{X} 2$ & 0,00 \\
\hline 50 & X4 & 0,00 & $\mathbf{X 2}$ & 0,00 & $\mathrm{X} 10$ & 0,00 & X8 & 0,00 & X15 & 0,00 & X19 & 0,00 & $\mathbf{X 3}$ & 0,00 & X13 & 0,00 & X13 & 0,00 \\
\hline $6=$ & X6 & 0,00 & X15 & 0,00 & X15 & 0,00 & X14 & 0,00 & $\mathrm{X} 12$ & 0,00 & X18 & 0,00 & X14 & 0,00 & $\mathbf{x} 3$ & 0,00 & X17 & 0,00 \\
\hline $7 \stackrel{0}{-}$ & X20 & 0,00 & X16 & 0,00 & X14 & 0,00 & $\mathbf{X 5}$ & 0,00 & $\mathbf{X 2}$ & 0,00 & $\mathrm{X} 4$ & 0,00 & X8 & 0,00 & $\mathbf{X 5}$ & 0,00 & X15 & 0,00 \\
\hline $8^{0}$ & $\mathbf{X 1}$ & 0,00 & X17 & 0,00 & X18 & 0,00 & X12 & 0,00 & X11 & 0,00 & X8 & 0,00 & X7 & 0,00 & $\mathbf{X 1}$ & 0,00 & X5 & 0,00 \\
\hline 110 & X12 & 0,00 & X9 & 0,00 & X17 & 0,00 & X18 & 0,00 & $\mathbf{X} 4$ & 0,00 & X20 & 0,00 & X20 & 0,00 & X9 & 0,00 & X7 & 0,00 \\
\hline $12^{\circ}$ & X19 & 0,00 & X20 & 0,00 & $\mathrm{X} 4$ & 0,00 & X3 & 0,00 & X14 & 0,00 & X3 & 0,00 & X6 & 0,00 & X4 & 0,00 & $\mathrm{X} 10$ & 0,00 \\
\hline $13^{0}$ & X13 & 0,00 & X18 & 0,00 & X8 & 0,00 & X2 & 0,00 & $\mathrm{X} 18$ & 0,00 & X9 & 0,00 & X19 & 0,00 & X11 & 0,00 & X9 & 0,00 \\
\hline $14^{\mathrm{O}}$ & X14 & 0,00 & X13 & 0,00 & X3 & 0,00 & X13 & 0,00 & $\mathrm{X} 16$ & 0,00 & $\mathrm{X} 12$ & 0,00 & X11 & 0,00 & X7 & 0,00 & X3 & 0,00 \\
\hline $15^{0}$ & $\mathbf{X} 2$ & 0,00 & X14 & 0,00 & X9 & 0,00 & X20 & 0,00 & X10 & 0,00 & $\mathrm{X} 2$ & 0,00 & $\mathbf{X} 2$ & 0,00 & $\mathrm{X} 12$ & 0,00 & X8 & 0,00 \\
\hline $16^{\circ}$ & X11 & 0,00 & X8 & 0,00 & $\mathrm{X} 12$ & 0,00 & X19 & 0,00 & X5 & 0,00 & $\mathrm{X} 13$ & 0,00 & X13 & 0,00 & X19 & 0,00 & X6 & 0,00 \\
\hline $17^{\circ}$ & X16 & 0,00 & $\mathrm{X} 12$ & 0,00 & X13 & 0,00 & X11 & 0,00 & $\mathrm{X} 20$ & 0,00 & $\mathrm{X} 14$ & 0,00 & X18 & 0,00 & $\mathrm{X} 17$ & 0,00 & X18 & 0,00 \\
\hline
\end{tabular}

Tabela A.21: Rankings de atributos e valores de importância estimados por $R F-L P$ nos conjuntos de dados artificiais com $\mu=0 \%$

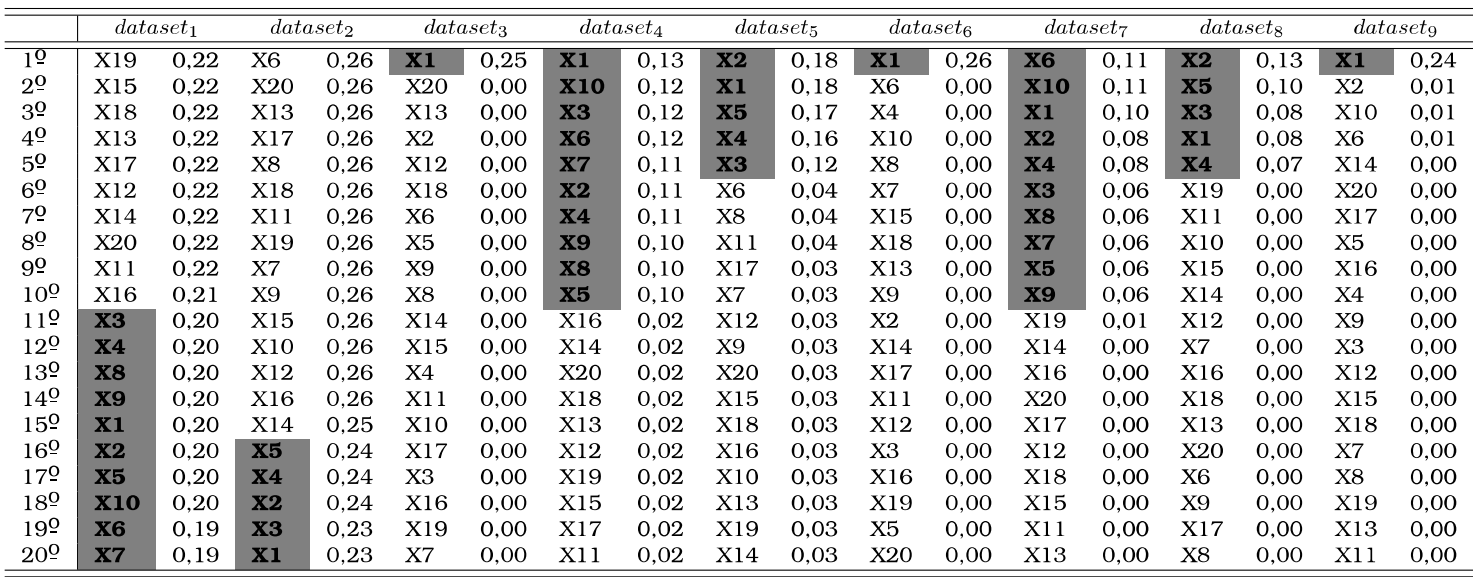


Tabela A.22: Rankings de atributos e valores de importância estimados por $R F-L P$ nos conjuntos de dados artificiais com $\mu=5 \%$

\begin{tabular}{|c|c|c|c|c|c|c|c|c|c|c|c|c|c|c|c|c|c|c|}
\hline & \multicolumn{2}{|c|}{ dataset $_{1}$} & \multicolumn{2}{|c|}{ dataset $_{2}$} & \multicolumn{2}{|c|}{ dataset $_{3}$} & \multicolumn{2}{|c|}{ dataset $_{4}$} & \multicolumn{2}{|c|}{ dataset $_{5}$} & \multicolumn{2}{|c|}{ dataset $_{6}$} & \multicolumn{2}{|c|}{ dataset $_{7}$} & \multicolumn{2}{|c|}{ dataset $_{8}$} & \multicolumn{2}{|c|}{ dataset $_{9}$} \\
\hline 10 & X19 & 0,33 & X15 & 0,34 & $\mathrm{X} 4$ & 0,33 & X11 & 0,19 & X19 & 0,23 & $\mathbf{x 1}$ & 0,25 & X6 & 0,09 & $\mathbf{X 2}$ & 0,11 & $\mathbf{X 1}$ & 0,21 \\
\hline 20 & $\mathrm{X} 12$ & 0,33 & X6 & 0,33 & $\mathrm{X} 6$ & 0,33 & X13 & 0,19 & $\mathrm{X} 10$ & 0,23 & X3 & 0,16 & X10 & 0,08 & X5 & 0,08 & $\mathrm{X} 2$ & 0,03 \\
\hline 3 음 & X14 & 0,33 & X13 & 0,33 & X16 & 0,33 & X19 & 0,19 & $\mathrm{X} 12$ & 0,23 & $\mathrm{X} 10$ & 0,16 & $\mathbf{X 1}$ & 0,08 & X3 & 0,07 & $\mathrm{X} 7$ & 0,02 \\
\hline 40 & $\mathrm{X} 17$ & 0,33 & X11 & 0,33 & X15 & 0,33 & X20 & 0,19 & $\mathrm{X} 13$ & 0,23 & X16 & 0,15 & $\mathbf{X 2}$ & 0,07 & $\mathbf{X 1}$ & 0,07 & $\mathrm{X} 17$ & 0,02 \\
\hline 50 & $\mathrm{X} 18$ & 0,33 & X9 & 0,33 & X5 & 0,33 & $\mathrm{X} 17$ & 0,18 & $\mathrm{X} 17$ & 0,23 & X7 & 0,15 & $\mathbf{X 4}$ & 0,06 & X4 & 0,06 & $\mathrm{X} 20$ & 0,02 \\
\hline $6^{\circ}$ & X16 & 0,33 & $\mathrm{X} 10$ & 0,33 & X3 & 0,33 & X16 & 0,18 & X9 & 0,23 & X5 & 0,15 & X3 & 0,06 & X18 & 0,02 & X14 & 0,02 \\
\hline $7^{\circ}$ & X15 & 0,33 & X8 & 0,33 & $\mathrm{X} 8$ & 0,33 & $\mathbf{X} 1$ & 0,18 & $\mathbf{X 1}$ & 0,23 & $\mathrm{X} 11$ & 0,15 & X5 & 0,05 & $\mathrm{X} 10$ & 0,02 & X15 & 0,02 \\
\hline $8^{\circ}$ & X20 & 0,33 & $\mathrm{X} 20$ & 0,33 & X11 & 0,33 & X14 & 0,18 & X11 & 0,23 & X6 & 0,15 & X8 & 0,05 & $\mathrm{X} 12$ & 0,02 & X19 & 0,02 \\
\hline 90 & X13 & 0,33 & X17 & 0,33 & $\mathrm{X} 12$ & 0,33 & X15 & 0,18 & X16 & 0,23 & X19 & 0,15 & X7 & 0,05 & $\mathrm{X} 14$ & 0,02 & $\mathrm{X} 4$ & 0,02 \\
\hline $10^{\circ}$ & X11 & 0,33 & X7 & 0,33 & X7 & 0,33 & $\mathrm{X} 18$ & 0,18 & X6 & 0,23 & $\mathrm{X} 4$ & 0,15 & X9 & 0,05 & X19 & 0,02 & $\mathrm{X} 10$ & 0,02 \\
\hline $11 \stackrel{0}{0}$ & $\mathrm{X} 4$ & 0,26 & X16 & 0,33 & X17 & 0,33 & $\mathrm{X} 12$ & 0,18 & X15 & 0,23 & X9 & 0,15 & X19 & 0,02 & $\mathrm{X} 11$ & 0,02 & X5 & 0,02 \\
\hline $12^{\circ}$ & X3 & 0,26 & X19 & 0,33 & X14 & 0,33 & X10 & 0,17 & $\mathrm{X} 20$ & 0,23 & X8 & 0,15 & X15 & 0,02 & X7 & 0,02 & X9 & 0,02 \\
\hline $13^{0}$ & X9 & 0,26 & X18 & 0,33 & X20 & 0,33 & X6 & 0,17 & X8 & 0,23 & $\mathrm{X} 13$ & 0,15 & X16 & 0,02 & $\mathrm{X} 20$ & 0,02 & X8 & 0,02 \\
\hline 140 & X8 & 0,26 & X14 & 0,33 & X18 & 0,33 & X7 & 0,17 & X14 & 0,23 & X18 & 0,15 & X17 & 0,01 & $\mathrm{X} 17$ & 0,02 & X6 & 0,02 \\
\hline $15^{\circ}$ & $\mathbf{X 1}$ & 0,25 & $\mathrm{X} 12$ & 0,33 & $\mathrm{X} 2$ & 0,33 & X2 & 0,17 & $\mathbf{X} 2$ & 0,23 & $\mathrm{X} 12$ & 0,15 & $\mathrm{X} 18$ & 0,01 & $\mathrm{X} 16$ & 0,02 & $\mathrm{X} 16$ & 0,02 \\
\hline 160 & X5 & 0,25 & $\mathbf{X 2}$ & 0,26 & X9 & 0,33 & X3 & 0,17 & X7 & 0,23 & $\mathrm{X} 14$ & 0,15 & X13 & 0,01 & $\mathrm{X} 13$ & 0,02 & $\mathrm{X} 11$ & 0,02 \\
\hline $17^{\circ} \underline{0}$ & X2 & 0,25 & $\mathbf{X 4}$ & 0,26 & X19 & 0,33 & X4 & 0,17 & $\mathrm{X} 18$ & 0,23 & X15 & 0,15 & X11 & 0,01 & $\mathrm{X} 8$ & 0,01 & X3 & 0,02 \\
\hline $18^{\circ}$ & $\mathbf{X 1 0}$ & 0,25 & $\mathbf{X 5}$ & 0,26 & $\mathrm{X} 10$ & 0,33 & X9 & 0,16 & X5 & 0,22 & $\mathrm{X} 20$ & 0,15 & $\mathrm{X} 14$ & 0,01 & X6 & 0,01 & $\mathrm{X} 12$ & 0,02 \\
\hline 190 & X6 & 0,25 & X3 & 0,25 & X13 & 0,33 & X8 & 0,16 & X4 & 0,22 & $\mathrm{X} 17$ & 0,15 & X20 & 0,01 & X9 & 0,01 & $\mathrm{X} 18$ & 0,02 \\
\hline 20 응 & X7 & 0,25 & $\mathbf{X 1}$ & 0,25 & X1 & 0,25 & X5 & 0,15 & X3 & 0,18 & $\mathrm{X} 2$ & 0,15 & $\mathrm{X} 12$ & 0,01 & X15 & 0,01 & X13 & 0,01 \\
\hline
\end{tabular}

Tabela A.23: Rankings de atributos e valores de importância estimados por $R F-L P$ nos conjuntos de dados artificiais $\operatorname{com} \mu=10 \%$

\begin{tabular}{|c|c|c|c|c|c|c|c|c|c|c|c|c|c|c|c|c|c|c|}
\hline \multirow[b]{2}{*}{10} & \multicolumn{2}{|c|}{ dataset $_{1}$} & \multicolumn{2}{|c|}{ dataset $_{2}$} & \multicolumn{2}{|c|}{ dataset $_{3}$} & \multicolumn{2}{|c|}{ dataset $_{4}$} & \multicolumn{2}{|c|}{ dataset $_{5}$} & \multicolumn{2}{|c|}{ dataset $_{6}$} & \multicolumn{2}{|c|}{ dataset $_{7}$} & \multicolumn{2}{|c|}{ dataset $_{8}$} & \multicolumn{2}{|c|}{ dataset $_{9}$} \\
\hline & X19 & 0,33 & X15 & 0,34 & $\mathrm{X} 4$ & 0,34 & $\mathrm{X} 13$ & 0,29 & $\mathrm{X} 8$ & 0,31 & $\mathrm{X} 12$ & 0,28 & $\mathrm{X6}$ & 0,08 & $\mathbf{X 2}$ & 0,09 & $\mathrm{X} 1$ & 0,19 \\
\hline $2 \underline{O}$ & $\mathrm{X} 12$ & 0,33 & $\mathrm{X} 6$ & 0,33 & $\mathrm{X} 6$ & 0,33 & X15 & 0,29 & X9 & 0,31 & X13 & 0,28 & $\mathbf{x 1 0}$ & 0,07 & $\mathbf{X 5}$ & 0,06 & $\mathrm{X} 12$ & 0,03 \\
\hline $3^{0}$ & $\mathrm{X} 14$ & 0,33 & $\mathrm{X} 13$ & 0,33 & X15 & 0,33 & X20 & 0,29 & $\mathrm{X} 12$ & 0,31 & X7 & 0,28 & $\mathbf{X 1}$ & 0,07 & X3 & 0,06 & $\mathrm{X} 5$ & 0,03 \\
\hline $4 \stackrel{0}{ }$ & $\mathrm{X} 18$ & 0,33 & $\mathrm{X} 11$ & 0,33 & $\mathrm{X} 16$ & 0,33 & $\mathrm{X} 16$ & 0,29 & X15 & 0,31 & $\mathrm{x} 8$ & 0,28 & $\mathbf{x 4}$ & 0,06 & $\mathbf{x 1}$ & 0,05 & $\mathrm{X} 18$ & 0,03 \\
\hline $5^{0}$ & $\mathrm{X} 17$ & 0,33 & $\mathrm{X} 10$ & 0,33 & X3 & 0,33 & X11 & 0,29 & X14 & 0,31 & $\mathrm{X} 4$ & 0,28 & X2 & 0,06 & X4 & 0,05 & X7 & 0,03 \\
\hline $6^{\circ}$ & X16 & 0,33 & X9 & 0,33 & X12 & 0,33 & $\mathrm{X} 17$ & 0,29 & X19 & 0,31 & X11 & 0,28 & X3 & 0,05 & X15 & 0,03 & X6 & 0,03 \\
\hline $7^{\mathrm{O}}$ & X15 & 0,33 & X8 & 0,33 & $\mathrm{X} 8$ & 0,33 & X18 & 0,28 & X10 & 0,31 & X16 & 0,27 & X7 & 0,05 & X11 & 0,02 & X10 & 0,03 \\
\hline $8^{\circ}$ & $\mathrm{X} 13$ & 0,33 & $\mathrm{X} 20$ & 0,33 & X17 & 0,33 & X12 & 0,28 & $\mathrm{X} 13$ & 0,31 & X3 & 0,27 & X8 & 0,04 & $\mathrm{X} 10$ & 0,02 & $\mathrm{X} 13$ & 0,03 \\
\hline 90 & $\mathrm{X} 20$ & 0,33 & $\mathrm{X7}$ & 0,33 & $\mathrm{X} 11$ & 0,33 & X19 & 0,28 & $\mathrm{X} 20$ & 0,31 & X17 & 0,27 & X9 & 0,04 & $\mathrm{X} 7$ & 0,02 & X16 & 0,03 \\
\hline $10 \underline{0}$ & X11 & 0,33 & X19 & 0,33 & X5 & 0,33 & X14 & 0,28 & X6 & 0,31 & X20 & 0,27 & X5 & 0,04 & $\mathrm{X} 12$ & 0,02 & X14 & 0,03 \\
\hline $11 \stackrel{0}{0}$ & X4 & 0,26 & $\mathrm{X} 17$ & 0,33 & $\mathrm{X} 7$ & 0,33 & $\mathbf{X 1}$ & 0,24 & $\mathrm{X} 7$ & 0,31 & X18 & 0,27 & X16 & 0,02 & $\mathrm{X} 14$ & 0,02 & $\mathrm{X} 4$ & 0,03 \\
\hline 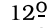 & $\mathbf{X 3}$ & 0,26 & X16 & 0,33 & X14 & 0,33 & X7 & 0,24 & $\mathrm{X} 11$ & 0,31 & X5 & 0,27 & X14 & 0,02 & $\mathrm{X} 13$ & 0,02 & X15 & 0,03 \\
\hline 13 음 & $\mathbf{X 9}$ & 0,26 & $\mathrm{X} 18$ & 0,33 & $\mathrm{X} 20$ & 0,33 & $\mathbf{X 6}$ & 0,23 & $\mathrm{X} 17$ & 0,31 & $\mathrm{X} 10$ & 0,27 & $\mathrm{X} 18$ & 0,02 & X9 & 0,02 & $\mathrm{X} 3$ & 0,03 \\
\hline $14 \stackrel{0}{0}$ & X8 & 0,26 & X14 & 0,33 & $\mathrm{X} 2$ & 0,33 & $\mathbf{X} 4$ & 0,23 & X18 & 0,31 & $\mathrm{X} 2$ & 0,27 & X19 & 0,02 & $\mathrm{X} 18$ & 0,02 & X17 & 0,03 \\
\hline $15 \underline{0}$ & $\mathbf{X 1}$ & 0,25 & $\mathrm{X} 12$ & 0,33 & X18 & 0,33 & $\mathbf{X 3}$ & 0,23 & X16 & 0,31 & X9 & 0,27 & X15 & 0,02 & X16 & 0,02 & X20 & 0,03 \\
\hline $16^{\circ}$ & X5 & 0,25 & $\mathbf{x} 2$ & 0,26 & X9 & 0,33 & $\mathbf{X} 10$ & 0,23 & $\mathbf{X} 1$ & 0,26 & X14 & 0,27 & $\mathrm{X} 12$ & 0,02 & $\mathrm{X} 20$ & 0,02 & $\mathrm{X} 2$ & 0,03 \\
\hline $17^{\mathrm{o}}$ & $\mathbf{X} 2$ & 0,25 & $\mathbf{X 4}$ & 0,26 & X19 & 0,33 & $\mathbf{X 2}$ & 0,23 & X2 & 0,26 & X15 & 0,27 & $\mathrm{X} 17$ & 0,02 & $\mathrm{X} 8$ & 0,02 & X9 & 0,03 \\
\hline $18^{\circ}$ & $\mathbf{x 1 0}$ & 0,25 & X5 & 0,26 & X13 & 0,33 & X9 & 0,22 & X5 & 0,25 & $\mathrm{X} 6$ & 0,27 & X13 & 0,02 & X19 & 0,02 & X11 & 0,02 \\
\hline 19 응 & X6 & 0,25 & X3 & 0,25 & $\mathrm{X} 10$ & 0,33 & X8 & 0,21 & $\mathbf{x 4}$ & 0,25 & X19 & 0,27 & X20 & 0,02 & X6 & 0,02 & X19 & 0,02 \\
\hline $20^{\circ}$ & $\mathbf{x 7}$ & 0,25 & $\mathbf{x} 1$ & 0,25 & $\mathbf{X 1}$ & 0,25 & X5 & 0,21 & $\mathbf{x} 3$ & 0,21 & $\mathbf{X} 1$ & 0,26 & $\mathrm{X} 11$ & 0,02 & $\mathrm{X} 17$ & 0,02 & $\mathrm{X} 8$ & 0,02 \\
\hline
\end{tabular}

Tabela A.24: Rankings de atributos e valores de importância estimados por $R F-L P$ nos conjuntos de dados artificiais com $\mu=20 \%$

\begin{tabular}{|c|c|c|c|c|c|c|c|c|c|c|c|c|c|c|c|c|c|c|}
\hline & \multicolumn{2}{|c|}{ dataset $_{1}$} & \multicolumn{2}{|c|}{ dataset $_{2}$} & \multicolumn{2}{|c|}{ dataset $_{3}$} & \multicolumn{2}{|c|}{ dataset $_{4}$} & \multicolumn{2}{|c|}{ dataset $_{5}$} & \multicolumn{2}{|c|}{ dataset $_{6}$} & \multicolumn{2}{|c|}{ dataset $_{7}$} & \multicolumn{2}{|c|}{ dataset $_{8}$} & \multicolumn{2}{|c|}{ dataset $_{9}$} \\
\hline 10 & X19 & 0,33 & X15 & 0,34 & $\mathrm{X} 4$ & 0,34 & X11 & 0,33 & X19 & 0,34 & X16 & 0,34 & X6 & 0,06 & $\mathbf{X 2}$ & 0,06 & $\mathbf{X 1}$ & 0,16 \\
\hline 20 & X12 & 0,33 & $\mathrm{X} 6$ & 0,33 & X6 & 0,33 & $\mathrm{X} 16$ & 0,33 & $\mathrm{X} 12$ & 0,33 & X8 & 0,34 & $\mathbf{X 1 0}$ & 0,06 & X3 & 0,05 & X13 & 0,05 \\
\hline $3^{0}$ & X14 & 0,33 & X13 & 0,33 & X15 & 0,33 & X15 & 0,33 & X15 & 0,33 & X7 & 0,34 & $\mathbf{X 1}$ & 0,05 & X5 & 0,05 & X10 & 0,05 \\
\hline $4 \stackrel{0}{-0}$ & $\mathrm{X} 18$ & 0,33 & $\mathrm{X} 11$ & 0,33 & X16 & 0,33 & X13 & 0,33 & $\mathrm{X} 10$ & 0,33 & X3 & 0,34 & X4 & 0,05 & $\mathbf{X} 1$ & 0,05 & X7 & 0,05 \\
\hline $5^{0}$ & X17 & 0,33 & $\mathrm{X} 10$ & 0,33 & X3 & 0,33 & X19 & 0,33 & X9 & 0,33 & $\mathrm{X} 4$ & 0,34 & X7 & 0,05 & X6 & 0,04 & X14 & 0,05 \\
\hline $6^{\circ}$ & X16 & 0,33 & X9 & 0,33 & $\mathrm{X} 12$ & 0,33 & $\mathrm{X} 17$ & 0,33 & X13 & 0,33 & $\mathrm{X} 12$ & 0,34 & $\mathbf{X 2}$ & 0,05 & $\mathrm{X} 11$ & 0,04 & X8 & 0,05 \\
\hline $7^{\mathrm{O}}$ & X15 & 0,33 & $\mathrm{X} 8$ & 0,33 & $\mathrm{X} 8$ & 0,33 & X14 & 0,33 & $\mathrm{X} 8$ & 0,33 & $\mathrm{X} 20$ & 0,33 & $\mathbf{X 5}$ & 0,05 & $\mathrm{X} 4$ & 0,04 & $\mathrm{X} 2$ & 0,05 \\
\hline $8^{\circ}$ & $\mathrm{X} 13$ & 0,33 & X20 & 0,33 & X17 & 0,33 & X20 & 0,33 & X14 & 0,33 & X5 & 0,33 & X9 & 0,04 & X12 & 0,04 & X17 & 0,05 \\
\hline $9 \stackrel{0}{0}$ & X20 & 0,33 & X7 & 0,33 & $\mathrm{X} 11$ & 0,33 & X18 & 0,33 & $\mathrm{X} 11$ & 0,33 & $\mathrm{X} 6$ & 0,33 & X8 & 0,04 & X20 & 0,04 & X20 & 0,05 \\
\hline $10^{0}$ & X11 & 0,33 & X19 & 0,33 & X5 & 0,33 & $\mathrm{X} 12$ & 0,33 & X7 & 0,33 & $\mathrm{X} 11$ & 0,33 & X3 & 0,04 & X8 & 0,04 & $\mathrm{X} 4$ & 0,05 \\
\hline $11 \stackrel{0}{0}$ & X4 & 0,26 & X17 & 0,33 & $\mathrm{X} 7$ & 0,33 & $\mathbf{X 1}$ & 0,27 & X6 & 0,33 & $\mathrm{X} 2$ & 0,33 & $\mathrm{X} 12$ & 0,04 & X9 & 0,04 & X11 & 0,05 \\
\hline $12^{\circ}$ & $\mathbf{X 3}$ & 0,26 & X16 & 0,33 & X14 & 0,33 & X7 & 0,27 & X20 & 0,33 & X13 & 0,33 & X13 & 0,04 & X19 & 0,04 & X9 & 0,05 \\
\hline $13^{0}$ & X9 & 0,26 & X18 & 0,33 & X20 & 0,33 & $\mathbf{X 4}$ & 0,26 & X17 & 0,33 & $\mathrm{X} 17$ & 0,33 & X11 & 0,04 & X13 & 0,04 & X19 & 0,04 \\
\hline $14^{\mathrm{O}}$ & X8 & 0,26 & X14 & 0,33 & $\mathrm{X} 2$ & 0,33 & X6 & 0,26 & X16 & 0,33 & X14 & 0,33 & X16 & 0,04 & $\mathrm{X} 18$ & 0,04 & X18 & 0,04 \\
\hline $15^{\circ}$ & $\mathbf{X 1}$ & 0,25 & X12 & 0,33 & X18 & 0,33 & X3 & 0,26 & X18 & 0,33 & X18 & 0,33 & $\mathrm{X} 17$ & 0,04 & X15 & 0,04 & X3 & 0,04 \\
\hline 160 & $\mathbf{X 5}$ & 0,25 & $\mathbf{X} 2$ & 0,26 & X9 & 0,33 & $\mathbf{X 1 0}$ & 0,26 & $\mathbf{X} 1$ & 0,28 & X19 & 0,33 & X15 & 0,04 & $\mathrm{X} 10$ & 0,04 & $\mathrm{X} 6$ & 0,04 \\
\hline $17^{0} \stackrel{0}{ }$ & X2 & 0,25 & X4 & 0,26 & X19 & 0,33 & X2 & 0,26 & X2 & 0,27 & X15 & 0,33 & X18 & 0,03 & X16 & 0,04 & X12 & 0,04 \\
\hline 180 & $\mathbf{x 1 0}$ & 0,25 & $\mathbf{X 5}$ & 0,26 & X13 & 0,33 & X9 & 0,25 & $\mathbf{X 5}$ & 0,27 & $\mathrm{X} 10$ & 0,33 & X14 & 0,03 & $\mathrm{X} 17$ & 0,03 & X15 & 0,04 \\
\hline 190 & X6 & 0,25 & X3 & 0,25 & $\mathrm{X} 10$ & 0,33 & X8 & 0,25 & X4 & 0,27 & X9 & 0,33 & X19 & 0,03 & X14 & 0,03 & X16 & 0,04 \\
\hline 200 & $\mathbf{X 7}$ & 0,25 & $\mathbf{X} 1$ & 0,25 & $\mathbf{X 1}$ & 0,25 & $\mathbf{X 5}$ & 0,24 & x3 & 0,23 & $\mathbf{X 1}$ & 0,26 & $\mathrm{X} 20$ & 0,03 & $\mathrm{X} 7$ & 0,03 & $\mathrm{X} 5$ & 0,04 \\
\hline
\end{tabular}


Tabela A.25: Rankings de atributos e valores de importância estimados por $R F-L P$ nos conjuntos de dados artificiais com $\mu=40 \%$

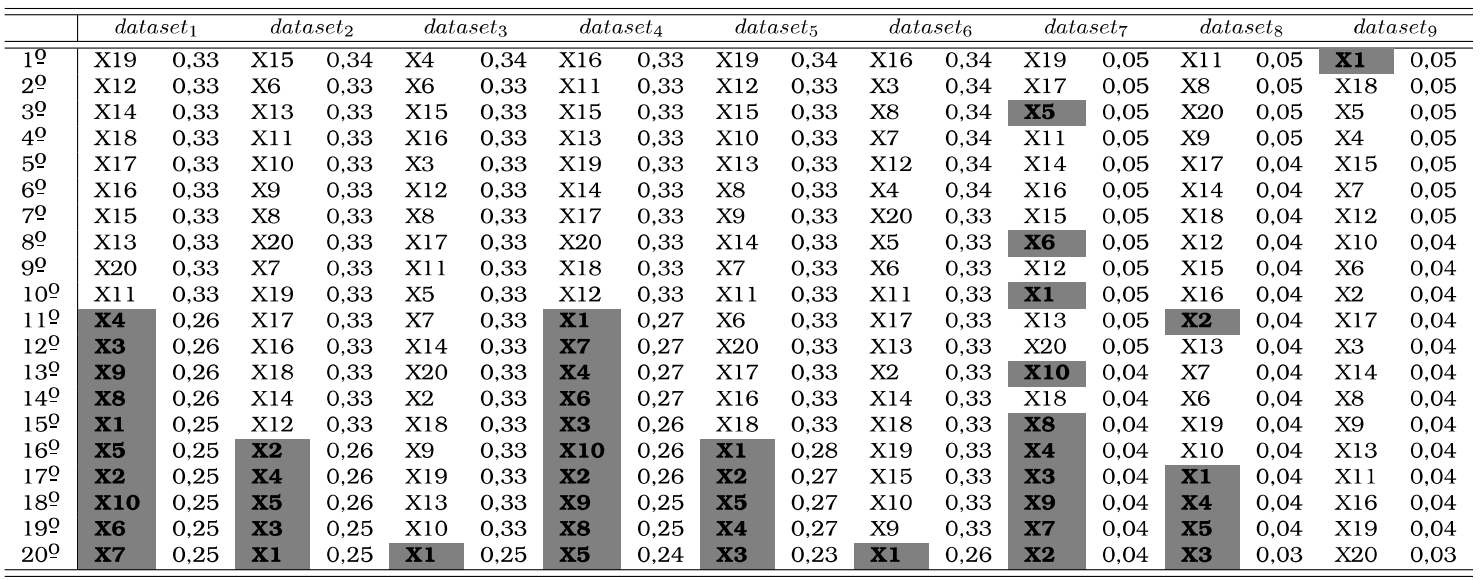

Tabela A.26: Rankings de atributos e valores de importância estimados por $R F-B R$ nos conjuntos de dados artificiais com $\mu=0 \%$

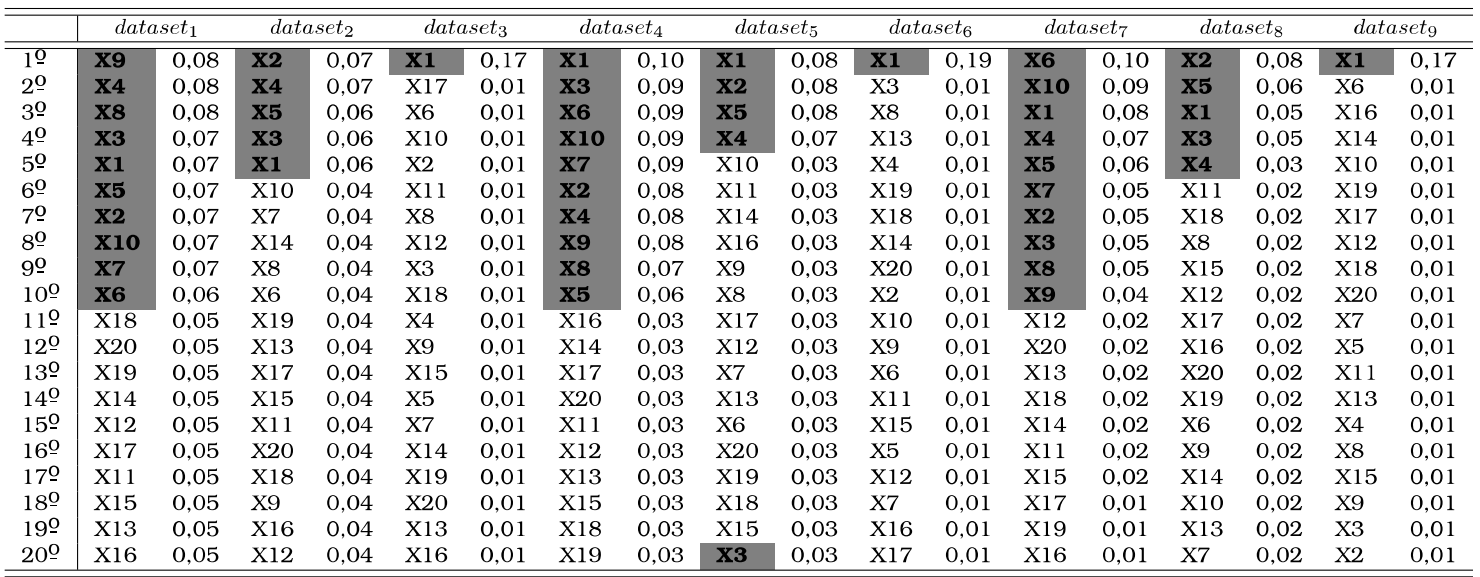


Tabela A.27: Rankings de atributos e valores de importância estimados por $R F-B R$ nos conjuntos de dados artificiais $\operatorname{com} \mu=5 \%$

\begin{tabular}{|c|c|c|c|c|c|c|c|c|c|c|c|c|c|c|c|c|c|c|}
\hline & \multicolumn{2}{|c|}{ dataset $_{1}$} & \multicolumn{2}{|c|}{ dataset $_{2}$} & \multicolumn{2}{|c|}{ dataset $_{3}$} & \multicolumn{2}{|c|}{ dataset $_{4}$} & \multicolumn{2}{|c|}{ dataset $_{5}$} & \multicolumn{2}{|c|}{ dataset $_{6}$} & \multicolumn{2}{|c|}{ dataset $_{7}$} & \multicolumn{2}{|c|}{ dataset $_{8}$} & \multicolumn{2}{|c|}{ dataset $_{9}$} \\
\hline 10 & X9 & 0,03 & $\mathrm{X4}$ & 0,03 & $\mathbf{X 1}$ & 0,11 & $\mathbf{X 1}$ & 0,04 & $\mathbf{X 1}$ & 0,03 & $\mathbf{X 1}$ & 0,13 & X6 & 0,04 & $\mathbf{X 2}$ & 0,05 & $\mathbf{x 1}$ & 0,12 \\
\hline $2^{\circ}$ & $\mathbf{X 4}$ & 0,03 & X5 & 0,02 & $\mathrm{X} 17$ & 0,01 & $\mathbf{X 3}$ & 0,03 & X5 & 0,03 & X3 & 0,01 & $\mathbf{x 1}$ & 0,04 & X5 & 0,03 & X14 & 0,01 \\
\hline 30 & X8 & 0,03 & $\mathbf{X 2}$ & 0,02 & X6 & 0,01 & X6 & 0,03 & $\mathbf{X 2}$ & 0,03 & $\mathrm{X} 4$ & 0,01 & X10 & 0,04 & X3 & 0,03 & X16 & 0,01 \\
\hline 40 & X3 & 0,03 & $\mathbf{X 3}$ & 0,02 & X10 & 0,01 & X4 & 0,03 & $\mathbf{X 4}$ & 0,03 & X13 & 0,01 & X4 & 0,03 & $\mathbf{X 1}$ & 0,03 & X7 & 0,01 \\
\hline $5^{\underline{0}}$ & X5 & 0,03 & $\mathbf{X} 1$ & 0,02 & $\mathrm{X} 2$ & 0,01 & $\mathbf{X 1 0}$ & 0,03 & $\mathrm{X} 10$ & 0,02 & X8 & 0,01 & $\mathbf{X 2}$ & 0,03 & X4 & 0,02 & X18 & 0,01 \\
\hline $6^{\circ}$ & $\mathbf{x 1}$ & 0,03 & X14 & 0,02 & $\mathrm{X} 11$ & 0,01 & X7 & 0,03 & $\mathrm{X} 11$ & 0,02 & X20 & 0,01 & X7 & 0,03 & X11 & 0,02 & X19 & 0,01 \\
\hline $7 \stackrel{0}{-}$ & $\mathbf{X 2}$ & 0,03 & X7 & 0,02 & $\mathrm{X} 12$ & 0,01 & X9 & 0,03 & $\mathrm{X} 16$ & 0,02 & $\mathrm{X} 14$ & 0,01 & X5 & 0,03 & X18 & 0,01 & $\mathrm{X} 12$ & 0,01 \\
\hline $8^{0}$ & X6 & 0,03 & X20 & 0,02 & X8 & 0,01 & $\mathbf{X 2}$ & 0,03 & $\mathrm{X} 17$ & 0,02 & $\mathrm{X} 2$ & 0,01 & X3 & 0,03 & X20 & 0,01 & X6 & 0,01 \\
\hline 90 & $\mathbf{X 1 0}$ & 0,03 & X17 & 0,02 & $\mathrm{X} 4$ & 0,01 & X8 & 0,03 & $\mathrm{X} 7$ & 0,02 & X19 & 0,01 & X8 & 0,03 & $\mathrm{X} 17$ & 0,01 & X17 & 0,01 \\
\hline $10^{\circ}$ & X7 & 0,02 & X11 & 0,02 & X3 & 0,01 & X5 & 0,03 & $\mathrm{X} 14$ & 0,02 & $\mathrm{X} 18$ & 0,01 & X9 & 0,03 & $\mathrm{X} 12$ & 0,01 & X20 & 0,01 \\
\hline $11^{\circ}$ & X18 & 0,02 & X10 & 0,02 & X18 & 0,01 & X16 & 0,02 & X8 & 0,02 & X10 & 0,01 & X12 & 0,02 & $\mathrm{X} 8$ & 0,01 & X10 & 0,01 \\
\hline $12^{\mathrm{O}}$ & X14 & 0,02 & X13 & 0,02 & X7 & 0,01 & X14 & 0,02 & X9 & 0,02 & X6 & 0,01 & X13 & 0,02 & X15 & 0,01 & X15 & 0,01 \\
\hline $13^{\circ}$ & $\mathrm{X} 20$ & 0,02 & X8 & 0,02 & X19 & 0,01 & $\mathrm{X} 17$ & 0,02 & $\mathrm{X} 20$ & 0,02 & X15 & 0,01 & X17 & 0,02 & X13 & 0,01 & X5 & 0,01 \\
\hline $14^{\mathrm{O}}$ & X19 & 0,02 & $\mathrm{X} 18$ & 0,02 & $\mathrm{X} 14$ & 0,01 & X11 & 0,02 & $\mathrm{X} 6$ & 0,02 & X9 & 0,01 & X15 & 0,02 & X10 & 0,01 & X11 & 0,01 \\
\hline $15^{\mathrm{o}}$ & $\mathrm{X} 12$ & 0,02 & X19 & 0,02 & X5 & 0,01 & $\mathrm{X} 20$ & 0,02 & $\mathrm{X} 12$ & 0,02 & X11 & 0,01 & X19 & 0,02 & X16 & 0,01 & X13 & 0,01 \\
\hline $16^{\mathrm{O}}$ & X17 & 0,02 & X6 & 0,02 & X9 & 0,01 & X15 & 0,02 & $\mathrm{X} 13$ & 0,02 & X5 & 0,01 & $\mathrm{X} 11$ & 0,02 & X9 & 0,01 & X8 & 0,01 \\
\hline $17^{\circ}$ & X11 & 0,02 & X16 & 0,02 & X15 & 0,01 & $\mathrm{X} 12$ & 0,02 & X19 & 0,02 & X7 & 0,01 & X20 & 0,02 & $\mathrm{X} 14$ & 0,01 & $\mathrm{X} 4$ & 0,00 \\
\hline $18^{\circ}$ & X15 & 0,02 & X9 & 0,02 & X16 & 0,01 & X18 & 0,02 & X15 & 0,02 & $\mathrm{X} 17$ & 0,01 & X18 & 0,02 & X6 & 0,01 & X3 & 0,00 \\
\hline $19^{\circ}$ & X13 & 0,02 & X15 & 0,02 & $\mathrm{X} 13$ & 0,01 & X13 & 0,02 & $\mathrm{X} 18$ & 0,02 & $\mathrm{X} 12$ & 0,01 & X16 & 0,02 & X19 & 0,01 & X9 & 0,00 \\
\hline $20^{0}$ & X16 & 0,02 & $\mathrm{X} 12$ & 0,02 & X20 & 0,01 & X19 & 0,02 & X3 & 0,02 & X16 & 0,01 & X14 & 0,02 & X7 & 0,01 & $\mathrm{X} 2$ & 0,00 \\
\hline
\end{tabular}

Tabela A.28: Rankings de atributos e valores de importância estimados por $R F-B R$ nos conjuntos de dados artificiais com $\mu=10 \%$

\begin{tabular}{|c|c|c|c|c|c|c|c|c|c|c|c|c|c|c|c|c|c|c|}
\hline & \multicolumn{2}{|c|}{ dataset $_{1}$} & \multicolumn{2}{|c|}{ dataset $_{2}$} & \multicolumn{2}{|c|}{ dataset $_{3}$} & \multicolumn{2}{|c|}{ dataset $_{4}$} & \multicolumn{2}{|c|}{ dataset $_{5}$} & \multicolumn{2}{|c|}{ dataset $_{6}$} & \multicolumn{2}{|c|}{ dataset $_{7}$} & \multicolumn{2}{|c|}{ dataset $_{8}$} & \multicolumn{2}{|c|}{ dataset $_{9}$} \\
\hline 10 & X9 & 0,02 & $\mathrm{X4}$ & 0,02 & $\mathbf{X 1}$ & 0,08 & $\overline{X 1}$ & 0,02 & $\mathbf{X 1}$ & 0,02 & $\mathbf{X 1}$ & 0,09 & $\mathrm{X} 6$ & 0,03 & $\mathbf{X 2}$ & 0,03 & $\overline{X 1}$ & 0,09 \\
\hline $2^{\circ}$ & $\mathbf{x 8}$ & 0,02 & X5 & 0,02 & X17 & 0,01 & $\mathbf{X 1 0}$ & 0,02 & X5 & 0,02 & $\mathrm{X} 4$ & 0,01 & $\mathbf{x 1}$ & 0,03 & $\mathbf{X 5}$ & 0,02 & X18 & 0,01 \\
\hline 30 & X4 & 0,02 & X3 & 0,02 & $\mathrm{X} 6$ & 0,01 & $\mathbf{X 3}$ & 0,02 & $\mathbf{X} 2$ & 0,02 & X3 & 0,01 & $\mathbf{X 1 0}$ & 0,02 & $\mathbf{x 3}$ & 0,02 & X6 & 0,01 \\
\hline $4^{\circ}$ & X3 & 0,02 & $\mathbf{X 1}$ & 0,02 & X10 & 0,01 & X6 & 0,02 & X4 & 0,02 & X20 & 0,01 & X4 & 0,02 & $\mathbf{x 1}$ & 0,02 & X14 & 0,01 \\
\hline $5^{\circ}$ & X5 & 0,02 & X2 & 0,02 & X11 & 0,01 & X9 & 0,02 & X16 & 0,01 & X19 & 0,01 & $\mathbf{X 2}$ & 0,02 & X4 & 0,02 & X11 & 0,01 \\
\hline $6^{\circ}$ & X6 & 0,02 & X17 & 0,01 & $\mathrm{X} 8$ & 0,01 & X7 & 0,02 & $\mathrm{X} 10$ & 0,01 & $\mathrm{X} 2$ & 0,01 & X7 & 0,02 & X12 & 0,01 & X12 & 0,01 \\
\hline $7^{\circ}=$ & $\mathbf{X 1}$ & 0,02 & X7 & 0,01 & $\mathrm{X} 2$ & 0,01 & X4 & 0,02 & X11 & 0,01 & $\mathrm{X} 8$ & 0,01 & X8 & 0,02 & $\mathrm{X} 18$ & 0,01 & X10 & 0,01 \\
\hline 80 & $\mathbf{X} 2$ & 0,02 & X14 & 0,01 & $\mathrm{X} 12$ & 0,01 & X2 & 0,02 & X17 & 0,01 & $\mathrm{X} 13$ & 0,01 & X5 & 0,02 & X15 & 0,01 & X16 & 0,01 \\
\hline $9^{\circ}$ & $\mathbf{X 1 0}$ & 0,02 & X10 & 0,01 & X19 & 0,01 & X5 & 0,02 & X14 & 0,01 & X9 & 0,01 & X9 & 0,02 & X6 & 0,01 & X17 & 0,00 \\
\hline $10^{0} \underline{0}$ & X7 & 0,02 & X11 & 0,01 & X3 & 0,01 & X8 & 0,02 & X20 & 0,01 & $\mathrm{X} 14$ & 0,01 & X3 & 0,02 & X8 & 0,01 & X13 & 0,00 \\
\hline 110 & $\mathrm{X} 20$ & 0,02 & $\mathrm{X} 8$ & 0,01 & $\mathrm{X} 7$ & 0,01 & X16 & 0,02 & X7 & 0,01 & X15 & 0,00 & $\mathrm{X} 12$ & 0,01 & $\mathrm{X} 17$ & 0,01 & X8 & 0,00 \\
\hline $12^{\mathrm{O}}$ & X12 & 0,02 & X20 & 0,01 & X14 & 0,01 & X14 & 0,02 & X9 & 0,01 & X5 & 0,00 & X13 & 0,01 & X16 & 0,01 & X19 & 0,00 \\
\hline $13^{\circ} \underline{0}$ & $\mathrm{X} 18$ & 0,02 & X13 & 0,01 & $\mathrm{X} 4$ & 0,01 & $\mathrm{X} 20$ & 0,02 & $\mathrm{X} 8$ & 0,01 & $\mathrm{X} 18$ & 0,00 & $\mathrm{X} 20$ & 0,01 & X11 & 0,01 & $\mathrm{X} 20$ & 0,00 \\
\hline $14^{\mathrm{O}}$ & X14 & 0,02 & X19 & 0,01 & $\times 5$ & 0,01 & X17 & 0,02 & $\mathrm{X} 6$ & 0,01 & $\mathrm{X} 6$ & 0,00 & X18 & 0,01 & X10 & 0,01 & $\mathrm{X} 4$ & 0,00 \\
\hline 150 & X17 & 0,02 & X12 & 0,01 & X18 & 0,01 & X11 & 0,02 & X18 & 0,01 & $\mathrm{X} 10$ & 0,00 & X19 & 0,01 & $\mathrm{X} 20$ & 0,01 & X15 & 0,00 \\
\hline $16^{\circ}$ & X19 & 0,02 & $\mathrm{x} 6$ & 0,01 & $\mathrm{X} 20$ & 0,00 & X18 & 0,02 & X19 & 0,01 & X11 & 0,00 & X17 & 0,01 & X19 & 0,01 & $\mathrm{X} 7$ & 0,00 \\
\hline $17^{\circ} \stackrel{0}{\circ}$ & X15 & 0,02 & X16 & 0,01 & X16 & 0,00 & X12 & 0,02 & X12 & 0,01 & $\mathrm{X} 7$ & 0,00 & X15 & 0,01 & X14 & 0,01 & X5 & 0,00 \\
\hline $18^{\circ}$ & X11 & 0,02 & X18 & 0,01 & X15 & 0,00 & X19 & 0,02 & $\mathrm{X} 13$ & 0,01 & $\mathrm{X} 12$ & 0,00 & X14 & 0,01 & X9 & 0,01 & $\mathrm{X} 3$ & 0,00 \\
\hline 190 & X13 & 0,01 & X15 & 0,01 & X9 & 0,00 & X15 & 0,02 & X15 & 0,01 & X17 & 0,00 & X16 & 0,01 & X7 & 0,01 & $\mathrm{X} 2$ & 0,00 \\
\hline 20 - & X16 & 0,01 & X9 & 0,01 & $\mathrm{X} 13$ & 0,00 & $\mathrm{X} 13$ & 0,02 & X3 & 0,01 & X16 & 0,00 & X11 & 0,01 & X13 & 0,01 & X9 & 0,00 \\
\hline
\end{tabular}


Tabela A.29: Rankings de atributos e valores de importância estimados por $R F-B R$ nos conjuntos de dados artificiais com $\mu=20 \%$

\begin{tabular}{|c|c|c|c|c|c|c|c|c|c|c|c|c|c|c|c|c|c|c|}
\hline & \multicolumn{2}{|c|}{ dataset $_{1}$} & \multicolumn{2}{|c|}{ dataset $_{2}$} & \multicolumn{2}{|c|}{ dataset $_{3}$} & \multicolumn{2}{|c|}{ dataset $_{4}$} & \multicolumn{2}{|c|}{ dataset $_{5}$} & \multicolumn{2}{|c|}{ dataset $_{6}$} & \multicolumn{2}{|c|}{ dataset $_{7}$} & \multicolumn{2}{|c|}{ dataset $_{8}$} & \multicolumn{2}{|c|}{ dataset $_{9}$} \\
\hline 10 & X9 & 0,01 & $\mathrm{X} 4$ & 0,01 & $\mathbf{x 1}$ & 0,04 & $\mathbf{X 1}$ & 0,01 & $\mathbf{X 1}$ & 0,01 & $\mathbf{X 1}$ & 0,05 & X6 & 0,01 & $\mathbf{X 2}$ & 0,02 & $\mathbf{X 1}$ & 0,05 \\
\hline 30 & $\mathbf{X 8}$ & 0,01 & X3 & 0,01 & X8 & 0,00 & X6 & 0,01 & $\mathbf{X 2}$ & 0,01 & X13 & 0,00 & $\mathbf{X 1 0}$ & 0,01 & X3 & 0,01 & $\mathrm{X} 17$ & 0,00 \\
\hline $5^{\mathbf{o}}$ & X5 & 0,01 & $\mathbf{X} 2$ & 0,01 & X6 & 0,00 & X10 & 0,01 & $\mathrm{X} 10$ & 0,01 & $\mathrm{X} 20$ & 0,00 & $\mathbf{X 2}$ & 0,01 & X4 & 0,01 & X7 & 0,00 \\
\hline 60 & X3 & 0,01 & X7 & 0,01 & $\mathrm{X} 11$ & 0,00 & $\mathbf{X 3}$ & 0,01 & $\mathrm{X} 14$ & 0,01 & $\mathrm{X} 4$ & 0,00 & X3 & 0,01 & X11 & 0,01 & X6 & 0,00 \\
\hline $7 \stackrel{0}{-}$ & $\mathbf{X 1}$ & 0,01 & X14 & 0,01 & X12 & 0,00 & $\mathbf{X 2}$ & 0,01 & $\mathrm{X} 16$ & 0,01 & X6 & 0,00 & X15 & 0,01 & X8 & 0,01 & $\mathrm{X} 10$ & 0,00 \\
\hline $8-$ & $\mathbf{X} 2$ & 0,01 & $\mathrm{X} 13$ & 0,01 & $\mathrm{X} 10$ & 0,00 & $\mathbf{X 7}$ & 0,01 & $\mathrm{X} 6$ & 0,01 & $\mathrm{X} 8$ & 0,00 & X7 & 0,01 & $\mathrm{X} 10$ & 0,01 & X14 & 0,00 \\
\hline $11^{\mathrm{O}}$ & X12 & 0,01 & $\mathrm{X} 12$ & 0,01 & X7 & 0,00 & X8 & 0,01 & $\mathrm{X} 20$ & 0,01 & $\mathrm{X} 18$ & 0,00 & X11 & 0,01 & X17 & 0,00 & X8 & 0,00 \\
\hline $12^{\circ}$ & X17 & 0,01 & X10 & 0,01 & $\mathrm{X} 4$ & 0,00 & X5 & 0,01 & $\mathrm{X} 17$ & 0,01 & X11 & 0,00 & $\mathrm{X} 12$ & 0,01 & X16 & 0,00 & $\mathrm{X} 11$ & 0,00 \\
\hline $13^{0}$ & X20 & 0,01 & X18 & 0,01 & X9 & 0,00 & X17 & 0,01 & X8 & 0,01 & $\mathrm{X} 2$ & 0,00 & $\mathrm{X} 18$ & 0,01 & X14 & 0,00 & X5 & 0,00 \\
\hline $14^{\mathrm{O}}$ & X18 & 0,01 & X19 & 0,01 & X18 & 0,00 & X15 & 0,01 & X7 & 0,01 & X5 & 0,00 & X9 & 0,01 & X13 & 0,00 & $\mathrm{X} 20$ & 0,00 \\
\hline $15^{0}$ & X14 & 0,01 & $\mathrm{X} 20$ & 0,01 & X13 & 0,00 & X14 & 0,01 & X11 & 0,01 & X9 & 0,00 & X13 & 0,01 & X6 & 0,00 & X4 & 0,00 \\
\hline $16^{\circ}$ & X19 & 0,01 & X15 & 0,01 & X5 & 0,00 & $\mathrm{X} 12$ & 0,01 & X15 & 0,01 & X7 & 0,00 & X20 & 0,01 & X20 & 0,00 & $\mathrm{X} 2$ & 0,00 \\
\hline $17^{\circ}$ & X15 & 0,01 & X8 & 0,01 & X16 & 0,00 & X18 & 0,01 & $\mathrm{X} 18$ & 0,01 & X15 & 0,00 & X19 & 0,01 & X15 & 0,00 & X15 & 0,00 \\
\hline
\end{tabular}

Tabela A.30: Rankings de atributos e valores de importância estimados por $R F-B R$ nos conjuntos de dados artificiais $\operatorname{com} \mu=40 \%$

\begin{tabular}{|c|c|c|c|c|c|c|c|c|c|c|c|c|c|c|c|c|c|c|}
\hline & \multicolumn{2}{|c|}{ dataset $_{1}$} & \multicolumn{2}{|c|}{ dataset $_{2}$} & \multicolumn{2}{|c|}{$\overline{\text { dataset }_{3}}$} & \multicolumn{2}{|c|}{ dataset $_{4}$} & \multicolumn{2}{|c|}{ dataset $_{5}$} & \multicolumn{2}{|c|}{ dataset $_{6}$} & \multicolumn{2}{|c|}{ dataset $_{7}$} & \multicolumn{2}{|c|}{ dataset $_{8}$} & \multicolumn{2}{|c|}{ dataset $_{9}$} \\
\hline 19 & X3 & 0,00 & $\mathrm{X4}$ & 0,00 & $\mathbf{X 1}$ & 0,00 & $\overline{\mathrm{X} 1}$ & 0,00 & $\overline{X 1}$ & 0,00 & $\overline{\mathrm{X1}}$ & 0,00 & $\mathbf{X 1 0}$ & 0,00 & $\mathrm{X2}$ & 0,00 & $\mathbf{X 1}$ & 0,00 \\
\hline 2 & $\mathrm{X4}$ & 0,00 & $\mathrm{X5}$ & 0,00 & X10 & 0,00 & $\mathrm{X4}$ & 0,00 & $\mathrm{X} 17$ & 0,00 & $\mathrm{X} 19$ & 0,00 & $\mathbf{X 1}$ & 0,00 & $\mathrm{X} 20$ & 0,00 & $\mathrm{X} 4$ & 0,00 \\
\hline 30 & X9 & 0,00 & $\mathbf{X 1}$ & 0,00 & X16 & 0,00 & $\mathbf{x 1 0}$ & 0,00 & X7 & 0,00 & X5 & 0,00 & X5 & 0,00 & X13 & 0,00 & $\mathrm{X} 12$ & 0,00 \\
\hline 40 & $\mathrm{x} 6$ & 0,00 & $\mathrm{x} 3$ & 0,00 & X14 & 0,00 & $\mathrm{x} 8$ & 0,00 & $\times 10$ & 0,00 & $\mathrm{X7}$ & 0,00 & $\mathbf{x 3}$ & 0,00 & $\mathrm{X5}$ & 0,00 & $\mathrm{X} 13$ & 0,00 \\
\hline $5^{0}$ & $\mathrm{X} 12$ & 0,00 & $\mathrm{X} 18$ & 0,00 & $\mathrm{X} 2$ & 0,00 & X13 & 0,00 & $\mathrm{X} 12$ & 0,00 & $\mathrm{X} 18$ & 0,00 & $\mathrm{X4}$ & 0,00 & $\mathbf{X 3}$ & 0,00 & X8 & 0,00 \\
\hline 60 & X10 & 0,00 & X2 & 0,00 & $\mathrm{X} 4$ & 0,00 & X7 & 0,00 & $\mathbf{X} 2$ & 0,00 & $\mathrm{X} 4$ & 0,00 & X15 & 0,00 & X11 & 0,00 & $\mathrm{X} 2$ & 0,00 \\
\hline $7^{\circ} \underline{0}$ & X5 & 0,00 & X10 & 0,00 & X13 & 0,00 & X5 & 0,00 & $\mathbf{X 4}$ & 0,00 & X14 & 0,00 & $\mathrm{X} 14$ & 0,00 & X6 & 0,00 & X10 & 0,00 \\
\hline $8^{0}$ & X19 & 0,00 & X15 & 0,00 & X11 & 0,00 & X6 & 0,00 & X5 & 0,00 & X9 & 0,00 & X20 & 0,00 & X17 & 0,00 & X5 & 0,00 \\
\hline $9^{0}$ & $\mathrm{X} 20$ & 0,00 & $\mathrm{X} 17$ & 0,00 & X3 & 0,00 & X3 & 0,00 & $\mathrm{X} 13$ & 0,00 & X16 & 0,00 & $\mathrm{X} 13$ & 0,00 & $\mathrm{X} 8$ & 0,00 & $\mathrm{X} 17$ & 0,00 \\
\hline $10^{0}$ & $\mathbf{X 1}$ & 0,00 & X6 & 0,00 & $\mathrm{X} 12$ & 0,00 & X16 & 0,00 & $\mathrm{X} 14$ & 0,00 & $\mathrm{X} 20$ & 0,00 & X6 & 0,00 & $\mathbf{X 1}$ & 0,00 & $\mathrm{X} 7$ & 0,00 \\
\hline 11 은 & X8 & 0,00 & X9 & 0,00 & X15 & 0,00 & X12 & 0,00 & X3 & 0,00 & X6 & 0,00 & X7 & 0,00 & X16 & 0,00 & X15 & 0,00 \\
\hline $12^{\mathrm{O}}$ & X7 & 0,00 & X16 & 0,00 & X9 & 0,00 & X14 & 0,00 & X18 & 0,00 & X15 & 0,00 & X18 & 0,00 & $\mathrm{X} 12$ & 0,00 & X9 & 0,00 \\
\hline 13 은 & $\mathrm{X} 2$ & 0,00 & $\mathrm{X} 13$ & 0,00 & $\mathrm{X} 5$ & 0,00 & $\mathbf{x} 2$ & 0,00 & X9 & 0,00 & $\mathrm{X} 17$ & 0,00 & X11 & 0,00 & $\mathrm{X} 7$ & 0,00 & X19 & 0,00 \\
\hline $14^{\mathrm{O}}$ & $\mathrm{X} 13$ & 0,00 & X20 & 0,00 & X7 & 0,00 & X9 & 0,00 & X15 & 0,00 & $\mathrm{X} 10$ & 0,00 & X19 & 0,00 & X19 & 0,00 & $\mathrm{X} 11$ & 0,00 \\
\hline 150 & X14 & 0,00 & $\mathrm{X} 12$ & 0,00 & X17 & 0,00 & X15 & 0,00 & $\mathrm{X} 16$ & 0,00 & X2 & 0,00 & X8 & 0,00 & X18 & 0,00 & X3 & 0,00 \\
\hline 160 & X15 & 0,00 & X7 & 0,00 & X6 & 0,00 & $\times 20$ & 0,00 & $\mathrm{X} 11$ & 0,00 & X8 & 0,00 & X9 & 0,00 & X15 & 0,00 & X16 & 0,00 \\
\hline $17^{\circ}$ & $\mathrm{X} 11$ & 0,00 & $\mathrm{X} 8$ & 0,00 & X8 & 0,00 & $\mathrm{X} 11$ & 0,00 & X8 & 0,00 & $\mathrm{X} 13$ & 0,00 & X2 & 0,00 & X9 & 0,00 & X14 & 0,00 \\
\hline $18^{\circ}$ & $\mathrm{X} 18$ & 0,00 & X11 & 0,00 & X18 & 0,00 & $\mathrm{X} 17$ & 0,00 & $\mathrm{x} 19$ & 0,00 & $\mathrm{X} 12$ & 0,00 & X16 & 0,00 & X4 & 0,00 & X20 & 0,00 \\
\hline 19 은 & $\mathrm{X} 16$ & 0,00 & X14 & 0,00 & X19 & 0,00 & X18 & 0,00 & X6 & 0,00 & $\mathrm{X} 3$ & 0,00 & $\mathrm{X} 12$ & 0,00 & X14 & 0,00 & X18 & 0,00 \\
\hline $20^{\circ}$ & $\mathrm{X} 17$ & 0,00 & X19 & 0,00 & X20 & 0,00 & X19 & 0,00 & $\mathrm{X} 20$ & 0,00 & $\mathrm{X} 11$ & 0,00 & X17 & 0,00 & X10 & 0,00 & X6 & 0,00 \\
\hline
\end{tabular}


Tabela A.31: Rankings de atributos e valores de importância estimados por $I G-L P$ nos conjuntos de dados artificiais com $\mu=0 \%$

\begin{tabular}{|c|c|c|c|c|c|c|c|c|c|c|c|c|c|c|c|c|c|c|}
\hline & \multicolumn{2}{|c|}{ dataset $_{1}$} & \multicolumn{2}{|c|}{ dataset $_{2}$} & \multicolumn{2}{|c|}{ dataset $_{3}$} & \multicolumn{2}{|c|}{ dataset $_{4}$} & \multicolumn{2}{|c|}{ dataset $_{5}$} & \multicolumn{2}{|c|}{ dataset $_{6}$} & \multicolumn{2}{|c|}{ dataset $_{7}$} & \multicolumn{2}{|c|}{ dataset $_{8}$} & \multicolumn{2}{|c|}{ dataset $_{9}$} \\
\hline 10 & X20 & 0,00 & $\mathrm{X} 20$ & 0,00 & $\mathbf{X 1}$ & 6,87 & $\mathrm{X} 20$ & 0,00 & $\mathrm{X} 20$ & 0,00 & $\mathbf{X 1}$ & 5,12 & $\mathbf{X 1}$ & 0,87 & $\mathbf{X 2}$ & 1,38 & $\mathbf{x 1}$ & 3,57 \\
\hline 20 & X19 & 0,00 & X19 & 0,00 & X8 & 0,00 & X19 & 0,00 & X19 & 0,00 & X8 & 0,00 & $\mathbf{x 6}$ & 0,81 & X5 & 1,03 & X8 & 0,00 \\
\hline 30 & X18 & 0,00 & X18 & 0,00 & X7 & 0,00 & X18 & 0,00 & $\mathrm{X} 18$ & 0,00 & X7 & 0,00 & X10 & 0,77 & X3 & 0,79 & X7 & 0,00 \\
\hline $4 \underline{0}$ & X17 & 0,00 & $\mathrm{X} 17$ & 0,00 & X10 & 0,00 & $\mathrm{X} 17$ & 0,00 & $\mathrm{X} 17$ & 0,00 & $\mathrm{X} 10$ & 0,00 & $\mathbf{X 2}$ & 0,76 & X4 & 0,78 & X10 & 0,00 \\
\hline $5^{\underline{0}}$ & $\mathrm{X} 16$ & 0,00 & $\mathrm{X} 16$ & 0,00 & X9 & 0,00 & $\mathrm{X} 16$ & 0,00 & $\mathrm{X} 16$ & 0,00 & X9 & 0,00 & $\mathbf{X 3}$ & 0,63 & X1 & 0,75 & X9 & 0,00 \\
\hline $6^{\circ}$ & X15 & 0,00 & X15 & 0,00 & X3 & 0,00 & X15 & 0,00 & X15 & 0,00 & X3 & 0,00 & $\mathbf{X 4}$ & 0,60 & X9 & 0,00 & X3 & 0,00 \\
\hline $7^{\circ}$ & X14 & 0,00 & $\mathrm{X} 14$ & 0,00 & $\mathrm{X} 2$ & 0,00 & $\mathrm{X} 14$ & 0,00 & $\mathrm{X} 14$ & 0,00 & $\mathrm{X} 2$ & 0,00 & X9 & 0,55 & X10 & 0,00 & $\mathrm{X} 2$ & 0,00 \\
\hline $8^{\mathrm{O}}$ & X13 & 0,00 & $\mathrm{X} 13$ & 0,00 & X6 & 0,00 & $\mathrm{X} 13$ & 0,00 & $\mathrm{X} 13$ & 0,00 & X6 & 0,00 & X7 & 0,53 & X6 & 0,00 & X6 & 0,00 \\
\hline $9 \underline{0}$ & $\mathrm{X} 12$ & 0,00 & $\mathrm{X} 12$ & 0,00 & X4 & 0,00 & $\mathrm{X} 12$ & 0,00 & $\mathrm{X} 12$ & 0,00 & $\mathrm{X} 4$ & 0,00 & X5 & 0,37 & X8 & 0,00 & $\mathrm{X} 4$ & 0,00 \\
\hline $10^{\circ}-$ & $\mathrm{X} 11$ & 0,00 & $\mathrm{X} 11$ & 0,00 & X5 & 0,00 & $\mathrm{X} 11$ & 0,00 & $\mathrm{X} 11$ & 0,00 & X5 & 0,00 & X8 & 0,37 & X7 & 0,00 & X5 & 0,00 \\
\hline $11 \stackrel{0}{-}$ & $\mathbf{X} 10$ & 0,00 & X10 & 0,00 & X17 & 0,00 & $\mathbf{X 1 0}$ & 0,00 & $\mathrm{X} 10$ & 0,00 & $\mathrm{X} 17$ & 0,00 & X15 & 0,00 & X17 & 0,00 & X17 & 0,00 \\
\hline $12^{\circ}$ & X9 & 0,00 & X9 & 0,00 & X16 & 0,00 & X9 & 0,00 & X9 & 0,00 & X16 & 0,00 & X20 & 0,00 & X16 & 0,00 & X16 & 0,00 \\
\hline $13^{\circ}$ & $\mathbf{X 8}$ & 0,00 & X8 & 0,00 & $\mathrm{X} 20$ & 0,00 & X8 & 0,00 & X8 & 0,00 & $\mathrm{X} 20$ & 0,00 & X16 & 0,00 & X20 & 0,00 & X20 & 0,00 \\
\hline $14^{\circ}$ & X7 & 0,00 & X7 & 0,00 & X18 & 0,00 & X7 & 0,00 & X7 & 0,00 & $\mathrm{X} 18$ & 0,00 & $\mathrm{X} 17$ & 0,00 & X18 & 0,00 & X18 & 0,00 \\
\hline $15^{\circ}$ & X6 & 0,00 & X6 & 0,00 & X19 & 0,00 & X6 & 0,00 & X6 & 0,00 & X19 & 0,00 & X18 & 0,00 & X19 & 0,00 & X19 & 0,00 \\
\hline $16^{\circ}$ & X5 & 0,00 & X5 & 0,00 & X12 & 0,00 & X5 & 0,00 & $\times 5$ & 0,00 & $\mathrm{X} 12$ & 0,00 & $\mathrm{X} 12$ & 0,00 & $\mathrm{X} 12$ & 0,00 & $\mathrm{X} 12$ & 0,00 \\
\hline $17^{\circ} \stackrel{\mathrm{O}}{ }$ & $\mathbf{X 4}$ & 0,00 & $\mathbf{X 4}$ & 0,00 & X11 & 0,00 & $\mathbf{X 4}$ & 0,00 & $\mathbf{X 4}$ & 0,00 & X11 & 0,00 & X11 & 0,00 & X11 & 0,00 & X11 & 0,00 \\
\hline $18^{\circ}$ & X3 & 0,00 & X3 & 0,00 & X15 & 0,00 & X3 & 0,00 & X3 & 0,00 & X15 & 0,00 & X19 & 0,00 & X15 & 0,00 & X15 & 0,00 \\
\hline $19^{\circ}$ & $\mathbf{X 2}$ & 0,00 & X2 & 0,00 & X13 & 0,00 & X2 & 0,00 & X2 & 0,00 & X13 & 0,00 & X14 & 0,00 & X13 & 0,00 & $\mathrm{X} 13$ & 0,00 \\
\hline $20^{\circ}-$ & $\mathbf{X 1}$ & 0,00 & $\mathbf{X 1}$ & 0,00 & X14 & 0,00 & $\mathbf{X 1}$ & 0,00 & $\mathbf{X 1}$ & 0,00 & X14 & 0,00 & X13 & 0,00 & X14 & 0,00 & X14 & 0,00 \\
\hline
\end{tabular}

Tabela A.32: Rankings de atributos e valores de importância estimados por $I G-L P$ nos conjuntos de dados artificiais com $\mu=5 \%$

\begin{tabular}{|c|c|c|c|c|c|c|c|c|c|c|c|c|c|c|c|c|c|c|}
\hline & \multicolumn{2}{|c|}{ dataset $_{1}$} & \multicolumn{2}{|c|}{ dataset $_{2}$} & \multicolumn{2}{|c|}{ dataset $_{3}$} & \multicolumn{2}{|c|}{ dataset $_{4}$} & \multicolumn{2}{|c|}{ dataset $_{5}$} & \multicolumn{2}{|c|}{ dataset $_{6}$} & \multicolumn{2}{|c|}{ dataset $_{7}$} & \multicolumn{2}{|c|}{ dataset $_{8}$} & \multicolumn{2}{|c|}{ dataset $_{9}$} \\
\hline $1 \stackrel{0}{ }$ & $\mathrm{X} 20$ & 0,00 & $\mathrm{X} 20$ & 0,00 & $\mathrm{X} 20$ & 0,00 & $\mathrm{X} 20$ & 0,00 & $\mathrm{X} 20$ & 0,00 & $\mathrm{X} 20$ & 0,00 & $\mathbf{X 6}$ & 0,35 & $\mathrm{X} 2$ & 0,60 & $\mathrm{X1}$ & 3,12 \\
\hline $2^{\circ}$ & X19 & 0,00 & X19 & 0,00 & X19 & 0,00 & X19 & 0,00 & X19 & 0,00 & X19 & 0,00 & $\mathrm{X} 20$ & 0,00 & X20 & 0,00 & X8 & 0,00 \\
\hline $3^{0}$ & X18 & 0,00 & $\mathrm{X} 18$ & 0,00 & X18 & 0,00 & $\mathrm{X} 18$ & 0,00 & X18 & 0,00 & $\mathrm{X} 18$ & 0,00 & X19 & 0,00 & X19 & 0,00 & X7 & 0,00 \\
\hline $4^{\circ}$ & $\mathrm{X} 17$ & 0,00 & $\mathrm{X} 17$ & 0,00 & $\mathrm{X} 17$ & 0,00 & $\mathrm{X} 17$ & 0,00 & $\mathrm{X} 17$ & 0,00 & $\mathrm{X} 17$ & 0,00 & X18 & 0,00 & X18 & 0,00 & X10 & 0,00 \\
\hline 50 & $\mathrm{X} 16$ & 0,00 & $\mathrm{X} 16$ & 0,00 & $\mathrm{X} 16$ & 0,00 & $\mathrm{X} 16$ & 0,00 & X16 & 0,00 & $\mathrm{X} 16$ & 0,00 & $\mathrm{X} 17$ & 0,00 & X17 & 0,00 & X9 & 0,00 \\
\hline $6^{\circ}=$ & X15 & 0,00 & X15 & 0,00 & X15 & 0,00 & X15 & 0,00 & X15 & 0,00 & X15 & 0,00 & $\mathrm{X} 16$ & 0,00 & X16 & 0,00 & X3 & 0,00 \\
\hline $7^{\circ} \stackrel{0}{-}$ & X14 & 0,00 & X14 & 0,00 & X14 & 0,00 & X14 & 0,00 & X14 & 0,00 & X14 & 0,00 & X15 & 0,00 & X15 & 0,00 & $\mathrm{X} 2$ & 0,00 \\
\hline $8^{\circ} \underline{0}$ & $\mathrm{X} 13$ & 0,00 & X13 & 0,00 & X13 & 0,00 & $\mathrm{X} 13$ & 0,00 & X13 & 0,00 & X13 & 0,00 & $\mathrm{X} 14$ & 0,00 & X14 & 0,00 & $\mathrm{X} 6$ & 0,00 \\
\hline $9 \stackrel{0}{9}$ & $\mathrm{X} 12$ & 0,00 & $\mathrm{X} 12$ & 0,00 & $\mathrm{X} 12$ & 0,00 & $\mathrm{X} 12$ & 0,00 & $\mathrm{X} 12$ & 0,00 & $\mathrm{X} 12$ & 0,00 & $\mathrm{X} 13$ & 0,00 & $\mathrm{X} 13$ & 0,00 & $\mathrm{X} 4$ & 0,00 \\
\hline $10 \underline{0}$ & X11 & 0,00 & X11 & 0,00 & X11 & 0,00 & X11 & 0,00 & X11 & 0,00 & X11 & 0,00 & $\mathrm{X} 12$ & 0,00 & $\mathrm{X} 12$ & 0,00 & X5 & 0,00 \\
\hline $11 \stackrel{0}{0}$ & $\mathbf{X 1 0}$ & 0,00 & X10 & 0,00 & X10 & 0,00 & $\mathbf{X} 10$ & 0,00 & X10 & 0,00 & X10 & 0,00 & X11 & 0,00 & X11 & 0,00 & X17 & 0,00 \\
\hline $12^{\mathrm{O}}$ & $\mathbf{X 9}$ & 0,00 & X9 & 0,00 & X9 & 0,00 & X9 & 0,00 & X9 & 0,00 & X9 & 0,00 & $\mathbf{X 1 0}$ & 0,00 & X10 & 0,00 & X16 & 0,00 \\
\hline $13^{0}$ & X8 & 0,00 & X8 & 0,00 & $\mathrm{X} 8$ & 0,00 & X8 & 0,00 & X8 & 0,00 & X8 & 0,00 & X9 & 0,00 & X9 & 0,00 & X20 & 0,00 \\
\hline 149 & X7 & 0,00 & X7 & 0,00 & $\mathrm{X} 7$ & 0,00 & X7 & 0,00 & X7 & 0,00 & X7 & 0,00 & X8 & 0,00 & X8 & 0,00 & X18 & 0,00 \\
\hline 150 & $\mathbf{X 6}$ & 0,00 & X6 & 0,00 & $\mathrm{X} 6$ & 0,00 & $\mathbf{X 6}$ & 0,00 & X6 & 0,00 & $\mathrm{X} 6$ & 0,00 & X7 & 0,00 & X7 & 0,00 & X19 & 0,00 \\
\hline $16^{\circ}$ & $\mathbf{X 5}$ & 0,00 & X5 & 0,00 & $\mathrm{X} 5$ & 0,00 & X5 & 0,00 & $\mathbf{X 5}$ & 0,00 & $\mathrm{X5}$ & 0,00 & $\mathbf{X 5}$ & 0,00 & X6 & 0,00 & $\mathrm{X} 12$ & 0,00 \\
\hline $17^{\circ} \stackrel{0}{2}$ & X4 & 0,00 & X4 & 0,00 & $\mathrm{X} 4$ & 0,00 & X4 & 0,00 & X4 & 0,00 & $\mathrm{X} 4$ & 0,00 & X4 & 0,00 & $\times 5$ & 0,00 & X11 & 0,00 \\
\hline $18^{\circ}$ & $\mathbf{X 3}$ & 0,00 & $\mathbf{X 3}$ & 0,00 & $\mathrm{X} 3$ & 0,00 & $\mathbf{X 3}$ & 0,00 & X3 & 0,00 & $\mathrm{X} 3$ & 0,00 & $\mathbf{X 3}$ & 0,00 & X4 & 0,00 & X15 & 0,00 \\
\hline $19 \stackrel{0}{0}$ & $\mathbf{X 2}$ & 0,00 & $\mathbf{X 2}$ & 0,00 & $\mathrm{X} 2$ & 0,00 & $\mathbf{X 2}$ & 0,00 & $\mathbf{X 2}$ & 0,00 & $\mathrm{X} 2$ & 0,00 & $\mathbf{X 2}$ & 0,00 & X3 & 0,00 & X13 & 0,00 \\
\hline $20 \stackrel{0}{ }$ & $\mathbf{X} 1$ & 0,00 & X1 & 0,00 & $\mathbf{X 1}$ & 0,00 & $\mathbf{X} 1$ & 0,00 & X1 & 0,00 & $\mathbf{X} 1$ & 0,00 & $\mathbf{X 1}$ & 0,00 & $\mathbf{x} 1$ & 0,00 & X14 & 0,00 \\
\hline
\end{tabular}


Tabela A.33: Rankings de atributos e valores de importância estimados por IG-LP nos conjuntos de dados artificiais com $\mu=10 \%$

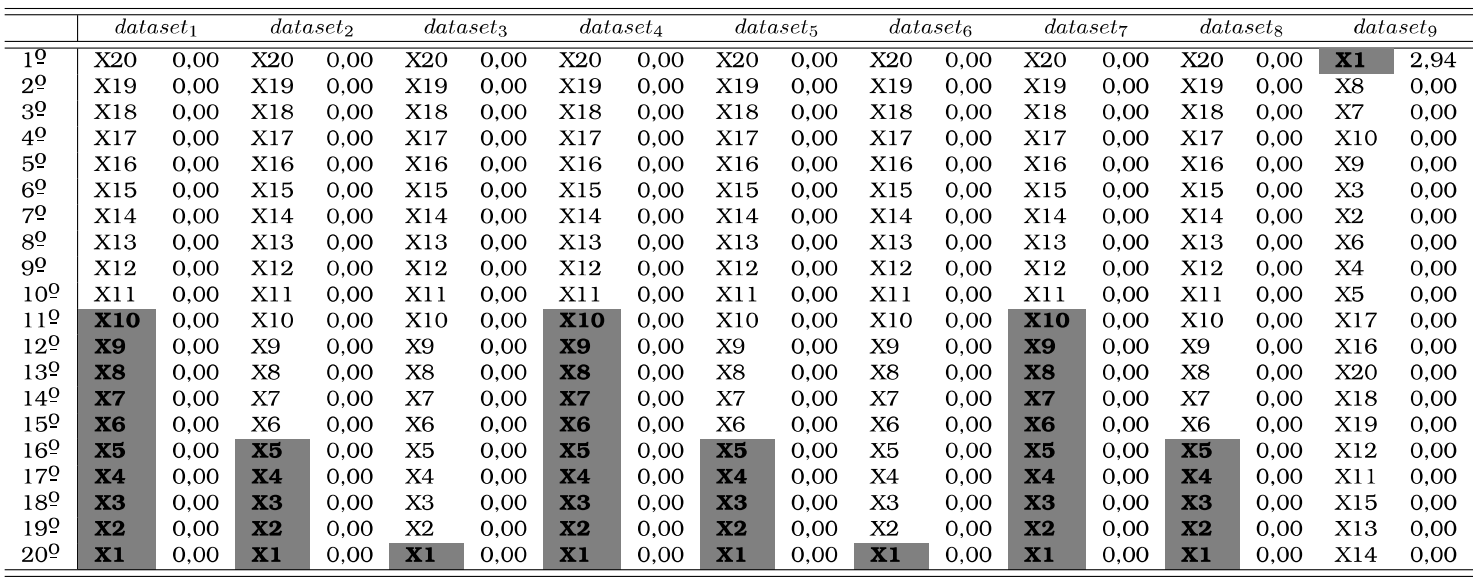

Tabela A.34: Rankings de atributos e valores de importância estimados por IG-LP nos conjuntos de dados artificiais com $\mu=20 \%$

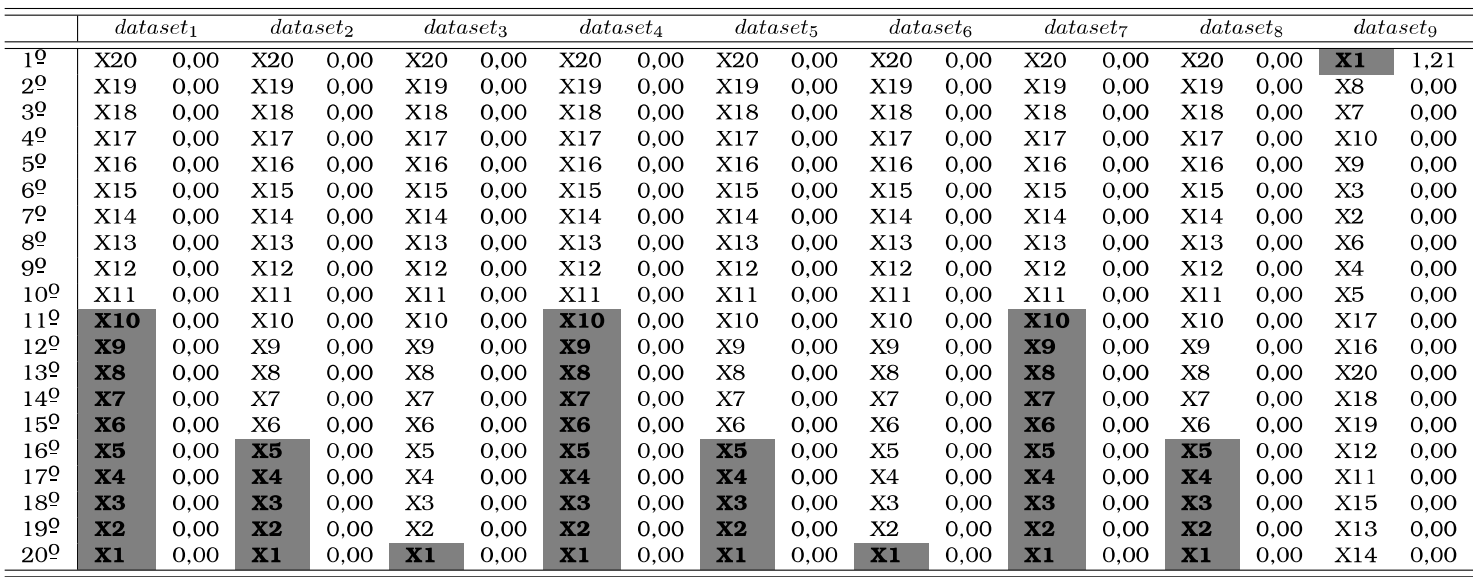


Tabela A.35: Rankings de atributos e valores de importância estimados por IG-LP nos conjuntos de dados artificiais com $\mu=40 \%$

\begin{tabular}{|c|c|c|c|c|c|c|c|c|c|c|c|c|c|c|c|c|c|c|}
\hline & \multicolumn{2}{|c|}{ dataset $_{1}$} & \multicolumn{2}{|c|}{ dataset $_{2}$} & \multicolumn{2}{|c|}{ dataset $_{3}$} & \multicolumn{2}{|c|}{ dataset $_{4}$} & \multicolumn{2}{|c|}{ dataset $_{5}$} & \multicolumn{2}{|c|}{ dataset $_{6}$} & \multicolumn{2}{|c|}{ dataset $_{7}$} & \multicolumn{2}{|c|}{ dataset $_{8}$} & \multicolumn{2}{|c|}{ dataset $_{9}$} \\
\hline 10 & X20 & 0,00 & $\mathrm{X} 20$ & 0,00 & $\mathrm{X} 20$ & 0,00 & $\mathrm{X} 20$ & 0,00 & $\mathrm{X} 20$ & 0,00 & $\mathrm{X} 20$ & 0,00 & $\mathrm{X} 20$ & 0,00 & $\mathrm{X} 20$ & 0,00 & $\mathrm{X} 20$ & 0,00 \\
\hline 20 & X19 & 0,00 & X19 & 0,00 & X19 & 0,00 & X19 & 0,00 & X19 & 0,00 & X19 & 0,00 & X19 & 0,00 & X19 & 0,00 & X19 & 0,00 \\
\hline 30 & X18 & 0,00 & X18 & 0,00 & X18 & 0,00 & X18 & 0,00 & X18 & 0,00 & $\mathrm{X} 18$ & 0,00 & $\mathrm{X} 18$ & 0,00 & X18 & 0,00 & X18 & 0,00 \\
\hline 40 & X17 & 0,00 & $\mathrm{X} 17$ & 0,00 & $\mathrm{X} 17$ & 0,00 & $\mathrm{X} 17$ & 0,00 & X17 & 0,00 & $\mathrm{X} 17$ & 0,00 & $\mathrm{X} 17$ & 0,00 & X17 & 0,00 & X17 & 0,00 \\
\hline $5^{\underline{0}}$ & $\mathrm{X} 16$ & 0,00 & $\mathrm{X} 16$ & 0,00 & $\mathrm{X} 16$ & 0,00 & $\mathrm{X} 16$ & 0,00 & X16 & 0,00 & X16 & 0,00 & $\mathrm{X} 16$ & 0,00 & X16 & 0,00 & X16 & 0,00 \\
\hline $6^{\circ}$ & X15 & 0,00 & X15 & 0,00 & X15 & 0,00 & X15 & 0,00 & X15 & 0,00 & X15 & 0,00 & X15 & 0,00 & X15 & 0,00 & X15 & 0,00 \\
\hline $7 \stackrel{0}{-}$ & X14 & 0,00 & $\mathrm{X} 14$ & 0,00 & $\mathrm{X} 14$ & 0,00 & $\mathrm{X} 14$ & 0,00 & X14 & 0,00 & $\mathrm{X} 14$ & 0,00 & X14 & 0,00 & X14 & 0,00 & X14 & 0,00 \\
\hline $8^{0}$ & X13 & 0,00 & $\mathrm{X} 13$ & 0,00 & $\mathrm{X} 13$ & 0,00 & $\mathrm{X} 13$ & 0,00 & $\mathrm{X} 13$ & 0,00 & $\mathrm{X} 13$ & 0,00 & $\mathrm{X} 13$ & 0,00 & X13 & 0,00 & $\mathrm{X} 13$ & 0,00 \\
\hline $9 \underline{0}$ & $\mathrm{X} 12$ & 0,00 & $\mathrm{X} 12$ & 0,00 & $\mathrm{X} 12$ & 0,00 & $\mathrm{X} 12$ & 0,00 & $\mathrm{X} 12$ & 0,00 & $\mathrm{X} 12$ & 0,00 & $\mathrm{X} 12$ & 0,00 & $\mathrm{X} 12$ & 0,00 & $\mathrm{X} 12$ & 0,00 \\
\hline 10 - & $\mathrm{X} 11$ & 0,00 & $\mathrm{X} 11$ & 0,00 & $\mathrm{X} 11$ & 0,00 & $\mathrm{X} 11$ & 0,00 & X11 & 0,00 & X11 & 0,00 & X11 & 0,00 & X11 & 0,00 & X11 & 0,00 \\
\hline 110 & $\mathbf{X} 10$ & 0,00 & X10 & 0,00 & X10 & 0,00 & $\mathbf{X 1 0}$ & 0,00 & X10 & 0,00 & X10 & 0,00 & $\mathbf{x} 10$ & 0,00 & X10 & 0,00 & X10 & 0,00 \\
\hline $12^{\circ}$ & X9 & 0,00 & X9 & 0,00 & X9 & 0,00 & X9 & 0,00 & X9 & 0,00 & X9 & 0,00 & $\mathbf{X 9}$ & 0,00 & X9 & 0,00 & X9 & 0,00 \\
\hline $13^{\circ}$ & X8 & 0,00 & $\mathrm{X} 8$ & 0,00 & X8 & 0,00 & X8 & 0,00 & X8 & 0,00 & X8 & 0,00 & X8 & 0,00 & X8 & 0,00 & X8 & 0,00 \\
\hline $14^{\mathrm{O}}$ & X7 & 0,00 & X7 & 0,00 & X7 & 0,00 & X7 & 0,00 & X7 & 0,00 & X7 & 0,00 & X7 & 0,00 & X7 & 0,00 & X7 & 0,00 \\
\hline 150 & X6 & 0,00 & X6 & 0,00 & $\mathrm{X} 6$ & 0,00 & X6 & 0,00 & X6 & 0,00 & $\mathrm{X} 6$ & 0,00 & X6 & 0,00 & X6 & 0,00 & X6 & 0,00 \\
\hline 160 & X5 & 0,00 & X5 & 0,00 & X5 & 0,00 & X5 & 0,00 & X5 & 0,00 & X5 & 0,00 & X5 & 0,00 & X5 & 0,00 & X5 & 0,00 \\
\hline $17^{\circ} \underline{0}$ & X4 & 0,00 & X4 & 0,00 & $\mathrm{X} 4$ & 0,00 & X4 & 0,00 & X4 & 0,00 & $\mathrm{X} 4$ & 0,00 & X4 & 0,00 & X4 & 0,00 & $\mathrm{X} 4$ & 0,00 \\
\hline $18^{\circ}$ & $\mathbf{X 3}$ & 0,00 & X3 & 0,00 & X3 & 0,00 & X3 & 0,00 & $\mathbf{X 3}$ & 0,00 & X3 & 0,00 & $\mathbf{X 3}$ & 0,00 & X3 & 0,00 & X3 & 0,00 \\
\hline 19 은 & X2 & 0,00 & X2 & 0,00 & $\mathrm{X} 2$ & 0,00 & X2 & 0,00 & X2 & 0,00 & $\mathrm{X} 2$ & 0,00 & X2 & 0,00 & $\mathrm{X} 2$ & 0,00 & $\mathrm{X} 2$ & 0,00 \\
\hline 20 - & $\mathbf{X 1}$ & 0,00 & $\mathbf{X 1}$ & 0,00 & $\mathbf{X 1}$ & 0,00 & $\mathbf{X 1}$ & 0,00 & $\mathbf{x 1}$ & 0,00 & $\mathbf{X 1}$ & 0,00 & $\mathbf{x 1}$ & 0,00 & $\mathbf{X 1}$ & 0,00 & $\mathbf{X 1}$ & 0,00 \\
\hline
\end{tabular}

Tabela A.36: Resultados experimentais nos conjuntos de dados artificiais de acordo com FR-AUC: algoritmos de SA baseados em ReliefF e IG-LP

\begin{tabular}{|c|c|c|c|c|}
\hline & $R F-M L$ & $R F-L P$ & $R F-B R$ & $I G-L P$ \\
\hline & \multicolumn{4}{|c|}{$\mu=0 \% M$} \\
\hline dataset_1 & $1,00[1,5]$ & $0,00[3,5]$ & $1,00[1,5]$ & $0,00[3,5]$ \\
\hline dataset_2 & $1,00[1,5]$ & $0,00[3,5]$ & $1,00[1,5]$ & $0,00[3,5]$ \\
\hline dataset_3 & $1,00[2,5]$ & $1,00[2,5]$ & $1,00[2,5]$ & $1,00[2,5]$ \\
\hline dataset_4 & $1,00[2,0]$ & $1,00[2,0]$ & $1,00[2,0]$ & $0,00[4,0]$ \\
\hline dataset_5 & $1,00[1,5]$ & $1,00[1,5]$ & $0,80[3,0]$ & $0,00[4,0]$ \\
\hline dataset_6 & $1,00[2,5]$ & $1,00[2,5]$ & $1,00[2,5]$ & $1,00[2,5]$ \\
\hline dataset_7 & $1,00[2,5]$ & $1,00[2,5]$ & $1,00[2,5]$ & $1,00[2,5]$ \\
\hline dataset_8 & $1,00[2,5]$ & $1,00[2,5]$ & $1,00[2,5]$ & $1,00[2,5]$ \\
\hline \multirow[t]{2}{*}{ dataset_9 } & $1,00[2,5]$ & $1,00[2,5]$ & $1,00[2,5]$ & $1,00[2,5]$ \\
\hline & \multicolumn{4}{|c|}{$\mu=5 \% M$} \\
\hline dataset_1 & $1,00[1,5]$ & $0,00[3,5]$ & $1,00[1,5]$ & $0,00[3,5]$ \\
\hline dataset_2 & $1,00[1,5]$ & $0,00[3,5]$ & $1,00[1,5]$ & $0,00[3,5]$ \\
\hline dataset_3 & $1,00[1,5]$ & $0,00[3,5]$ & $1,00[1,5]$ & $0,00[3,5]$ \\
\hline dataset_4 & $1,00[1,5]$ & $0,04[3,0]$ & $1,00[1,5]$ & $0,00[4,0]$ \\
\hline dataset_5 & $1,00[1,0]$ & $0,15[3,0]$ & $0,80[2,0]$ & $0,00[4,0]$ \\
\hline dataset_6 & $1,00[2,0]$ & $1,00[2,0]$ & $1,00[2,0]$ & $0,00[4,0]$ \\
\hline dataset_7 & $1,00[2,0]$ & $1,00[2,0]$ & $1,00[2,0]$ & $0,10[4,0]$ \\
\hline dataset_8 & $1,00[2,0]$ & $1,00[2,0]$ & $1,00[2,0]$ & $0,20[4,0]$ \\
\hline \multirow[t]{2}{*}{ dataset_9 } & $1,00[2,5]$ & $1,00[2,5]$ & $1,00[2,5]$ & $1,00[2,5]$ \\
\hline & \multicolumn{4}{|c|}{$\mu=10 \% M$} \\
\hline dataset_1 & $1,00[1,5]$ & $0,00[3,5]$ & $1,00[1,5]$ & $0,00[3,5]$ \\
\hline data & $1,00[1,5]$ & $0,00[3,5]$ & $1,00[1,5]$ & $0,00[3,5]$ \\
\hline dataset_3 & $1,00[1,5]$ & $0,00[3,5]$ & $1,00[1,5]$ & $0,00[3,5]$ \\
\hline dataset_4 & $1,00[1,5]$ & $0,00[3,5]$ & $1,00[1,5]$ & $0,00[3,5]$ \\
\hline dataset_5 & $1,00[1,0]$ & $0,00[3,5]$ & $0,80[2,0]$ & $0,00[3,5]$ \\
\hline dataset_6 & $1,00[1,5]$ & $0,00[3,5]$ & $1,00[1,5]$ & $0,00[3,5]$ \\
\hline dataset_7 & $1,00[2,0]$ & $1,00[2,0]$ & $1,00[2,0]$ & $0,00[4,0]$ \\
\hline dataset_8 & $1,00[2,0]$ & $1,00[2,0]$ & $1,00[2,0]$ & $0,00[4,0]$ \\
\hline \multirow[t]{2}{*}{ dataset_9 } & $1,00[2,5]$ & $1,00[2,5]$ & $1,00[2,5]$ & $1,00[2,5]$ \\
\hline & \multicolumn{4}{|c|}{$\mu=20 \% M$} \\
\hline dataset_1 & $1,00[1,5]$ & $0,00[3,5]$ & $1,00[1,5]$ & $0,00[3,5]$ \\
\hline dataset_2 & $1,00[1,5]$ & $0,00[3,5]$ & $1,00[1,5]$ & $0,00[3,5]$ \\
\hline dataset_3 & $1,00[1,5]$ & $0,00[3,5]$ & $1,00[1,5]$ & $0,00[3,5]$ \\
\hline dataset_4 & $1,00[1,0]$ & $0,00[3,5]$ & $0,96[2,0]$ & $0,00[3,5]$ \\
\hline dataset_5 & $1,00[1,0]$ & $0,00[3,5]$ & $0,80[2,0]$ & $0,00[3,5]$ \\
\hline dataset_6 & $1,00[1,5]$ & $0,00[3,5]$ & $1,00[1,5]$ & $0,00[3,5]$ \\
\hline
\end{tabular}


Tabela A.36: FR-AUC (continuação)

\begin{tabular}{l|llll}
\hline & $R F-M L$ & $R F-L P$ & $R F-B R$ & $I G-L P$ \\
\hline dataset_7 & $\mathbf{1 , 0 0}[\mathbf{1 , 5}]$ & $\mathbf{1 , 0 0}[\mathbf{1 , 5}]$ & $0,93[3,0]$ & $0,00[4,0]$ \\
dataset_8 & $\mathbf{1 , 0 0}[\mathbf{1 , 5}]$ & $0,97[3,0]$ & $\mathbf{1 , 0 0}[\mathbf{1 , 5}]$ & $0,00[4,0]$ \\
dataset_9 & $\mathbf{1 , 0 0}[\mathbf{2 , 5}]$ & $\mathbf{1 , 0 0}[\mathbf{2 , 5}]$ & $\mathbf{1 , 0 0}[\mathbf{2 , 5}]$ & $\mathbf{1 , 0 0}[\mathbf{2 , 5}]$ \\
\hline & \multicolumn{4}{|c}{$\mu=40 \% M$} \\
\hline dataset_1 & $\mathbf{0 , 9 2}[\mathbf{1 , 0 ]}$ & $0,00[3,5]$ & $0,86[2,0]$ & $0,00[3,5]$ \\
dataset_2 & $\mathbf{1 , 0 0}[\mathbf{1 , 0}]$ & $0,00[3,5]$ & $0,99[2,0]$ & $0,00[3,5]$ \\
dataset_3 & $\mathbf{1 , 0 0}[\mathbf{1 , 5}]$ & $0,00[3,5]$ & $\mathbf{1 , 0 0}[\mathbf{1 , 5}]$ & $0,00[3,5]$ \\
dataset_4 & $0,79[2,0]$ & $0,00[3,5]$ & $\mathbf{0 , 8 8}[\mathbf{1 , 0}]$ & $0,00[3,5]$ \\
dataset_5 & $0,67[2,0]$ & $0,00[3,5]$ & $\mathbf{0 , 7 6}[\mathbf{1 , 0}]$ & $0,00[3,5]$ \\
dataset_6 & $\mathbf{1 , 0 0}[\mathbf{1 , 5}]$ & $0,00[3,5]$ & $\mathbf{1 , 0 0}[\mathbf{1 , 5}]$ & $0,00[3,5]$ \\
dataset_7 & $\mathbf{0 , 8 9}[\mathbf{1 , 0}]$ & $0,16[3,0]$ & $0,71[2,0]$ & $0,00[4,0]$ \\
dataset_8 & $\mathbf{0 , 7 5}[\mathbf{1 , 0}]$ & $0,07[3,0]$ & $0,69[2,0]$ & $0,00[4,0]$ \\
dataset_9 & $\mathbf{1 , 0 0}[\mathbf{2 , 0}]$ & $\mathbf{1 , 0 0}[\mathbf{2 , 0}]$ & $\mathbf{1 , 0 0}[\mathbf{2 , 0}]$ & $0,00[4,0]$ \\
\hline
\end{tabular}

\section{Conjuntos de dados benchmark}

Tabela A.37: Resultados experimentais de acordo com $F$-measure baseada em exemplos: algoritmos de SA baseados em ReliefF e IG-LP

\begin{tabular}{|c|c|c|c|c|}
\hline & $R F-M L$ & $\overline{R F}-\overline{B R}$ & $R F-L P$ & $I G-L P$ \\
\hline & \multicolumn{4}{|c|}{$\left|X^{\prime}\right|=10 \% M$} \\
\hline 1-Cal500 & $0,42(0,01)[3,5]$ & $0,43(0,01)[2,0]$ & $0,42(0,02)[3,5]$ & $0,45(0,02)[1,0]$ \\
\hline 2-Corel5k & $0,19(0,01)[2,5]$ & $0,19(0,01)[2,5]$ & $0,19(0,01)[2,5]$ & $0,19(0,01)[2,5]$ \\
\hline 3-Corel16k001 & $0,21(0,01)[1,0]$ & $0,20(0,01)[3,0]$ & $0,20(0,01)[3,0]$ & $0,20(0,01)[3,0]$ \\
\hline 4-Emotions & $0,60(0,04)[2,5]$ & $0,61(0,04)[1,0]$ & $0,57(0,05)[4,0]$ & $0,60(0,05)[2,5]$ \\
\hline 5-Fapesp & $0,17(0,05)[3,0]$ & $0,23(0,05)[1,5]$ & $0,13(0,05)[4,0]$ & $0,23(0,06)[1,5]$ \\
\hline 6-Genbase* & $0,96(0,02)[3,0]$ & $0,96(0,02)[3,0]$ & $0,96(0,02)[3,0]$ & $0,99(0,01)[1,0]$ \\
\hline $7-L \log -f^{*}$ & $0,12(0,03)[4,0]$ & $0,13(0,03)[2,0]$ & $0,13(0,01)[2,0]$ & $0,13(0,03)[2,0]$ \\
\hline 8-Magtag5k & $0,33(0,01)[1,0]$ & $0,28(0,02)[4,0]$ & $0,30(0,02)[2,5]$ & $0,30(0,01)[2,5]$ \\
\hline 9-Scene & $0,50(0,07)[4,0]$ & $0,59(0,04)[1,0]$ & $0,52(0,03)[3,0]$ & $0,54(0,04)[2,0]$ \\
\hline \multirow[t]{2}{*}{ 10-Yeast } & $0,60(0,02)[3,0]$ & $0,61(0,02)[1,5]$ & $0,61(0,02)[1,5]$ & $0,58(0,02)[4,0]$ \\
\hline & \multicolumn{4}{|c|}{$\left|X^{\prime}\right|=20 \% M$} \\
\hline 1-Cal500 & $0,43(0,02)[3,0]$ & $0,43(0,02)[3,0]$ & $0,43(0,02)[3,0]$ & $0,46(0,02)[1,0]$ \\
\hline 2-Corel5k & $0,18(0,01)[3,0]$ & $0,18(0,01)[3,0]$ & $0,18(0,01)[3,0]$ & $0,19(0,00)[1,0]$ \\
\hline 3-Corel16k001 & $0,21(0,01)[1,5]$ & $0,20(0,01)[3,5]$ & $0,20(0,01)[3,5]$ & $0,21(0,00)[1,5]$ \\
\hline 4-Emotions & $0,63(0,04)[2,0]$ & $0,66(0,06)[1,0]$ & $0,60(0,03)[4,0]$ & $0,62(0,04)[3,0]$ \\
\hline 5-Fapesp & $0,12(0,06)[3,0]$ & $0,18(0,05)[2,0]$ & $0,09(0,04)[4,0]$ & $0,21(0,06)[1,0]$ \\
\hline 6-Genbase ${ }^{*}$ & $0,96(0,02)[3,0]$ & $0,96(0,02)[3,0]$ & $0,96(0,02)[3,0]$ & $0,99(0,01)[1,0]$ \\
\hline $7-L \log -f^{*}$ & $0,14(0,02)[3,0]$ & $0,14(0,02)[3,0]$ & $0,15(0,01)[1,0]$ & $0,14(0,02)[3,0]$ \\
\hline 8-Magtag5k & $0,35(0,02)[1,0]$ & $0,33(0,01)[2,5]$ & $0,33(0,02)[2,5]$ & $0,32(0,01)[4,0]$ \\
\hline 9-Scene & $0,60(0,03)[3,0]$ & $0,65(0,04)[1,5]$ & $0,65(0,03)[1,5]$ & $0,58(0,02)[4,0]$ \\
\hline \multirow[t]{2}{*}{ 10-Yeast } & $0,63(0,02)[1,5]$ & $0,63(0,02)[1,5]$ & $0,62(0,02)[3,0]$ & $0,60(0,02)[4,0]$ \\
\hline & \multicolumn{4}{|c|}{$\left|X^{\prime}\right|=30 \% M$} \\
\hline 1-Cal500 & $0,43(0,01)[3,0]$ & $0,43(0,02)[3,0]$ & $0,43(0,02)[3,0]$ & $0,46(0,02)[1,0]$ \\
\hline 2-Corel5k & $0,17(0,01)[1,5]$ & $0,17(0,01)[1,5]$ & $0,16(0,01)[3,5]$ & $0,16(0,01)[3,5]$ \\
\hline 3-Corel16k001 & $0,21(0,00)[1,0]$ & $0,19(0,01)[4,0]$ & $0,20(0,01)[2,5]$ & $0,20(0,01)[2,5]$ \\
\hline 4-Emotions & $0,62(0,05)[3,5]$ & $0,64(0,04)[1,0]$ & $0,62(0,04)[3,5]$ & $0,63(0,04)[2,0]$ \\
\hline 5-Fapesp & $0,10(0,05)[3,0]$ & $0,18(0,07)[2,0]$ & $0,08(0,04)[4,0]$ & $0,22(0,06)[1,0]$ \\
\hline 6-Genbase* & $0,96(0,02)[3,0]$ & $0,96(0,02)[3,0]$ & $0,96(0,02)[3,0]$ & $0,99(0,01)[1,0]$ \\
\hline 7-Llog-f* & $0,14(0,03)[2,5]$ & $0,14(0,02)[2,5]$ & $0,13(0,03)[4,0]$ & $0,16(0,01)[1,0]$ \\
\hline 8-Magtag5k & $0,35(0,01)[2,5]$ & $0,34(0,01)[4,0]$ & $0,35(0,01)[2,5]$ & $0,36(0,01)[1,0]$ \\
\hline 9-Scene & $0,63(0,03)[4,0]$ & $0,67(0,03)[1,5]$ & $0,67(0,03)[1,5]$ & $0,66(0,03)[3,0]$ \\
\hline \multirow[t]{2}{*}{ 10-Yeast } & $0,64(0,02)[1,0]$ & $0,63(0,02)[2,5]$ & $0,63(0,02)[2,5]$ & $0,60(0,02)[4,0]$ \\
\hline & \multicolumn{4}{|c|}{$\left|X^{\prime}\right|=40 \% M$} \\
\hline 1-Cal500 & $0,43(0,01)[3,5]$ & $0,44(0,02)[2,0]$ & $0,43(0,02)[3,5]$ & $0,46(0,01)[1,0]$ \\
\hline 2-Corel5k & $0,15(0,02)[3,0]$ & $0,16(0,01)[2,0]$ & $0,13(0,01)[4,0]$ & $0,18(0,01)[1,0]$ \\
\hline
\end{tabular}


Tabela A.37: Resultados para F-measure baseada em exemplos (continuação)

\begin{tabular}{|c|c|c|c|c|}
\hline & $R F-M L$ & $R F-B R$ & $R F-L P$ & $I G-L P$ \\
\hline 3-Corel16k001 & $0,20(0,01)[1,5]$ & $0,18(0,01)[3,0]$ & $0,20(0,01)[1,5]$ & $0,17(0,01)[4,0]$ \\
\hline 4-Emotions & $0,64(0,05)[3,0]$ & $0,65(0,05)[1,5]$ & $0,63(0,04)[4,0]$ & $0,65(0,04)[1,5]$ \\
\hline 5-Fapesp & $0,09(0,05)[3,0]$ & $0,19(0,05)[2,0]$ & $0,08(0,03)[4,0]$ & $0,20(0,04)[1,0]$ \\
\hline 6-Genbase* & $0,96(0,02)[3,0]$ & $0,96(0,02)[3,0]$ & $0,96(0,02)[3,0]$ & $0,99(0,01)[1,0]$ \\
\hline $7-L \log -f^{*}$ & $0,15(0,03)[2,5]$ & $0,15(0,03)[2,5]$ & $0,14(0,03)[4,0]$ & $0,16(0,02)[1,0]$ \\
\hline 8-Magtag5k & $0,37(0,01)[1,5]$ & $0,35(0,01)[4,0]$ & $0,36(0,01)[3,0]$ & $0,37(0,01)[1,5]$ \\
\hline 9-Scene & $0,65(0,03)[4,0]$ & $0,70(0,03)[1,5]$ & $0,70(0,02)[1,5]$ & $0,68(0,02)[3,0]$ \\
\hline \multirow[t]{2}{*}{ 10-Yeast } & $0,64(0,02)[1,5]$ & $0,64(0,02)[1,5]$ & $0,63(0,02)[3,0]$ & $0,60(0,02)[4,0]$ \\
\hline & & & $\left|X^{\prime}\right|=50 \% M$ & \\
\hline 1-Cal500 & $0,43(0,01)[3,5]$ & $0,44(0,02)[2,0]$ & $0,43(0,01)[3,5]$ & $0,46(0,01)[1,0]$ \\
\hline 2-Corel5k & $0,12(0,02)[3,0]$ & $0,16(0,01)[1,5]$ & $0,11(0,01)[4,0]$ & $0,16(0,01)[1,5]$ \\
\hline 3-Corel16k001 & $0,17(0,01)[2,0]$ & $0,16(0,01)[3,0]$ & $0,19(0,01)[1,0]$ & $0,13(0,01)[4,0]$ \\
\hline 4-Emotions & $0,63(0,05)[4,0]$ & $0,64(0,04)[2,5]$ & $0,64(0,04)[2,5]$ & $0,67(0,03)[1,0]$ \\
\hline 5-Fapesp & $0,08(0,04)[3,0]$ & $0,15(0,07)[2,0]$ & $0,07(0,04)[4,0]$ & $0,21(0,04)[1,0]$ \\
\hline 6-Genbase* & $0,96(0,02)[3,0]$ & $0,96(0,02)[3,0]$ & $0,96(0,02)[3,0]$ & $0,99(0,01)[1,0]$ \\
\hline 7-Llog-f* & $0,15(0,03)[2,5]$ & $0,15(0,04)[2,5]$ & $0,14(0,03)[4,0]$ & $0,16(0,03)[1,0]$ \\
\hline 8-Magtag5k & $0,37(0,01)[2,0]$ & $0,36(0,01)[3,5]$ & $0,36(0,01)[3,5]$ & $0,39(0,01)[1,0]$ \\
\hline 9-Scene & $0,67(0,03)[4,0]$ & $0,72(0,04)[1,5]$ & $0,72(0,03)[1,5]$ & $0,70(0,03)[3,0]$ \\
\hline \multirow[t]{2}{*}{ 10-Yeast } & $0,64(0,02)[1,5]$ & $0,63(0,02)[3,0]$ & $0,64(0,02)[1,5]$ & $0,61(0,01)[4,0]$ \\
\hline & & & $\left|X^{\prime}\right|=60 \% M$ & \\
\hline 1-Cal500 & $0,43(0,01)[3,5]$ & $0,44(0,02)[2,0]$ & $0,43(0,02)[3,5]$ & $0,46(0,01)[1,0]$ \\
\hline 2-Corel5k & $0,11(0,02)[4,0]$ & $0,12(0,01)[2,0]$ & $0,12(0,01)[2,0]$ & $0,12(0,02)[2,0]$ \\
\hline 3-Corel16k001 & $0,15(0,01)[1,5]$ & $0,09(0,01)[3,0]$ & $0,15(0,02)[1,5]$ & $0,05(0,01)[4,0]$ \\
\hline 4-Emotions & $0,63(0,04)[3,5]$ & $0,65(0,03)[1,5]$ & $0,63(0,04)[3,5]$ & $0,65(0,04)[1,5]$ \\
\hline 5-Fapesp & $0,06(0,05)[4,0]$ & $0,15(0,06)[2,0]$ & $0,07(0,04)[3,0]$ & $0,20(0,04)[1,0]$ \\
\hline 6-Genbase* & $0,96(0,02)[3,0]$ & $0,96(0,02)[3,0]$ & $0,96(0,02)[3,0]$ & $0,99(0,01)[1,0]$ \\
\hline 7-Llog-f* & $0,14(0,03)[3,5]$ & $0,15(0,03)[2,0]$ & $0,14(0,03)[3,5]$ & $0,16(0,03)[1,0]$ \\
\hline 8-Magtag5k & $0,37(0,01)[2,0]$ & $0,36(0,01)[3,5]$ & $0,36(0,01)[3,5]$ & $0,38(0,01)[1,0]$ \\
\hline 9-Scene & $0,70(0,03)[4,0]$ & $0,73(0,03)[1,0]$ & $0,72(0,03)[2,0]$ & $0,71(0,02)[3,0]$ \\
\hline \multirow[t]{2}{*}{ 10-Yeast } & $0,64(0,02)[2,0]$ & $0,64(0,02)[2,0]$ & $0,64(0,02)[2,0]$ & $0,63(0,02)[4,0]$ \\
\hline & & & $\left|X^{\prime}\right|=70 \% M$ & \\
\hline 1-Cal500 & $0,43(0,01)[3,5]$ & $0,44(0,01)[2,0]$ & $0,43(0,02)[3,5]$ & $0,46(0,01)[1,0]$ \\
\hline 2-Corel5k & $0,11(0,02)[3,0]$ & $0,08(0,01)[4,0]$ & $0,14(0,02)[1,0]$ & $0,13(0,01)[2,0]$ \\
\hline 3-Corel16k001 & $0,13(0,01)[1,0]$ & $0,05(0,01)[3,0]$ & $0,08(0,01)[2,0]$ & $0,04(0,01)[4,0]$ \\
\hline 4-Emotions & $0,64(0,04)[4,0]$ & $0,65(0,04)[2,5]$ & $0,65(0,04)[2,5]$ & $0,66(0,04)[1,0]$ \\
\hline 5-Fapesp & $0,05(0,04)[4,0]$ & $0,16(0,05)[2,0]$ & $0,07(0,03)[3,0]$ & $0,19(0,05)[1,0]$ \\
\hline 6-Genbase* & $0,96(0,02)[3,0]$ & $0,96(0,02)[3,0]$ & $0,96(0,02)[3,0]$ & $0,99(0,01)[1,0]$ \\
\hline 7-Llog-f* & $0,14(0,03)[3,5]$ & $0,16(0,03)[1,0]$ & $0,14(0,03)[3,5]$ & $0,15(0,03)[2,0]$ \\
\hline 8-Magtag5k & $0,37(0,01)[1,5]$ & $0,36(0,01)[3,5]$ & $0,36(0,01)[3,5]$ & $0,37(0,01)[1,5]$ \\
\hline 9-Scene & $0,72(0,03)[3,0]$ & $0,72(0,03)[3,0]$ & $0,73(0,03)[1,0]$ & $0,72(0,02)[3,0]$ \\
\hline \multirow[t]{2}{*}{ 10-Yeast } & $0,64(0,02)[2,5]$ & $0,64(0,02)[2,5]$ & $0,64(0,02)[2,5]$ & $0,64(0,02)[2,5]$ \\
\hline & & & $\left|X^{\prime}\right|=80 \% M$ & \\
\hline 1-Cal500 & $0,44(0,01)[3,0]$ & $0,44(0,01)[3,0]$ & $0,44(0,02)[3,0]$ & $0,46(0,01)[1,0]$ \\
\hline 2-Corel5k & $0,10(0,01)[4,0]$ & $0,12(0,02)[2,5]$ & $0,14(0,02)[1,0]$ & $0,12(0,02)[2,5]$ \\
\hline 3-Corel16k001 & $0,11(0,01)[1,0]$ & $0,04(0,01)[4,0]$ & $0,07(0,02)[2,0]$ & $0,06(0,02)[3,0]$ \\
\hline 4-Emotions & $0,66(0,04)[1,5]$ & $0,66(0,03)[1,5]$ & $0,64(0,05)[3,5]$ & $0,64(0,04)[3,5]$ \\
\hline 5-Fapesp & $0,04(0,04)[4,0]$ & $0,17(0,05)[2,0]$ & $0,06(0,03)[3,0]$ & $0,18(0,04)[1,0]$ \\
\hline 6-Genbase ${ }^{*}$ & $0,96(0,02)[3,0]$ & $0,96(0,02)[3,0]$ & $0,96(0,02)[3,0]$ & $0,99(0,01)[1,0]$ \\
\hline $7-L \log -f^{*}$ & $0,14(0,03)[3,5]$ & $0,15(0,02)[2,0]$ & $0,14(0,03)[3,5]$ & $0,16(0,03)[1,0]$ \\
\hline 8-Magtag5k & $0,37(0,01)[2,0]$ & $0,37(0,01)[2,0]$ & $0,36(0,01)[4,0]$ & $0,37(0,01)[2,0]$ \\
\hline 9-Scene & $0,73(0,03)[1,5]$ & $0,72(0,03)[3,5]$ & $0,72(0,03)[3,5]$ & $0,73(0,03)[1,5]$ \\
\hline \multirow[t]{2}{*}{ 10-Yeast } & $0,64(0,02)[3,0]$ & $0,64(0,02)[3,0]$ & $0,64(0,02)[3,0]$ & $0,65(0,02)[1,0]$ \\
\hline & & & $\left|X^{\prime}\right|=90 \% M$ & \\
\hline 1-Cal500 & $0,44(0,02)[3,0]$ & $0,44(0,02)[3,0]$ & $0,44(0,01)[3,0]$ & $0,46(0,02)[1,0]$ \\
\hline 2-Corel5k & $0,12(0,01)[4,0]$ & $0,14(0,01)[2,5]$ & $0,15(0,02)[1,0]$ & $0,14(0,02)[2,5]$ \\
\hline 3-Corel16k001 & $0,11(0,01)[1,0]$ & $0,07(0,00)[4,0]$ & $0,10(0,01)[2,0]$ & $0,08(0,01)[3,0]$ \\
\hline 4-Emotions & $0,64(0,05)[3,5]$ & $0,65(0,04)[1,5]$ & $0,64(0,05)[3,5]$ & $0,65(0,03)[1,5]$ \\
\hline 5-Fapesp & $0,04(0,04)[4,0]$ & $0,16(0,05)[2,0]$ & $0,07(0,03)[3,0]$ & $0,18(0,04)[1,0]$ \\
\hline
\end{tabular}


Tabela A.37: Resultados para F-measure baseada em exemplos (continuação)

\begin{tabular}{|c|c|c|c|c|}
\hline & $R F-M L$ & $R F-B R$ & $R F-L P$ & $I G-L P$ \\
\hline 6-Genbase & $0,96(0,02)[3,0]$ & $0,96(0,02)[3,0]$ & $0,96(0,02)[3,0]$ & $0,99(0,01)[1,0]$ \\
\hline 7-Llog-f* & $0,14(0,04)[3,5]$ & $0,16(0,02)[1,5]$ & $0,14(0,03)[3,5]$ & $0,16(0,03)[1,5]$ \\
\hline 8-Magtag $5 k$ & $0,37(0,01)[3,0]$ & $0,37(0,01)[3,0]$ & $0,37(0,01)[3,0]$ & $0,38(0,01)[1,0]$ \\
\hline 9-Scene & $0,73(0,03)[1,0]$ & $0,72(0,03)[3,0]$ & $0,72(0,03)[3,0]$ & $0,72(0,02)[3,0]$ \\
\hline 10-Yeast & $0,64(0,02)[3,0]$ & $0,64(0,02)[3,0]$ & $0,64(0,02)[3,0]$ & $0,65(0,02)[1,0]$ \\
\hline
\end{tabular}

Tabela A.38: Resultados experimentais de acordo com Hamming Loss: algoritmos de SA baseados em ReliefF e IG- $L P$

\begin{tabular}{|c|c|c|c|c|}
\hline & $R F-M L$ & $R F-B R$ & $R F-L P$ & $I G-L P$ \\
\hline & \multicolumn{4}{|c|}{$\left|X^{\prime}\right|=10 \% M$} \\
\hline 1-Cal500 & ,00) $[2,0]$ & {$[2,0]$} & $0,17(0,01)[2,0]$ & $0,01)[4,0]$ \\
\hline 2-Corel5k & $0,01(0,00)[4,0]$ & $0,02(0,00)[2,0]$ & $0,02(0,00)[2,0]$ & $0,02(0,00)[2,0]$ \\
\hline 3-Corel16k001 & $0,03(0,00)[2,5]$ & $0,03(0,00)[2,5]$ & $0,03(0,00)[2,5]$ & $0,03(0,00)[2,5]$ \\
\hline 4-Emotions & $0,24(0,02)[2,5]$ & $0,23(0,03)[4,0]$ & $0,25(0,02)[1,0]$ & $0,24(0,03)[2,5]$ \\
\hline 5-Fapesp & $0,04(0,00)[3,0]$ & $0,04(0,00)[3,0]$ & $0,05(0,01)[1,0]$ & $0,04(0,00)[3,0]$ \\
\hline 6-Genbase* & $0,00(0,00)[2,5]$ & $0,00(0,00)[2,5]$ & $0,00(0,00)[2,5]$ & $0,00(0,00)[2,5]$ \\
\hline $7-L \log -f^{*}$ & $0,03(0,00)[2,5]$ & $0,03(0,00)[2,5]$ & $0,03(0,00)[2,5]$ & $0,03(0,00)[2,5]$ \\
\hline 8-Magtag5k & $0,05(0,00)[2,5]$ & $0,05(0,00)[2,5]$ & $0,05(0,00)[2,5]$ & $0,05(0,00)[2,5]$ \\
\hline 9-Scene & $0,17(0,02)[1,5]$ & $0,14(0,01)[4,0]$ & $0,17(0,01)[1,5]$ & $0,16(0,01)[3,0]$ \\
\hline \multirow[t]{2}{*}{ 10-Yeast } & $0,23(0,01)[3,0]$ & $0,23(0,01)[3,0]$ & $0,23(0,01)[3,0]$ & $0,24(0,01)[1,0]$ \\
\hline & \multicolumn{4}{|c|}{$\left|X^{\prime}\right|=20 \% M$} \\
\hline 1-Cal500 & $0,17(0,01)[2,0]$ & $0,17(0,01)[2,0]$ & $0,17(0,01)[2,0]$ & $0,16(0,01)[4,0]$ \\
\hline 2-Corel5k & $0,01(0,00)[4,0]$ & $0,02(0,00)[2,0]$ & $0,02(0,00)[2,0]$ & $0,02(0,00$ \\
\hline 3-Corel16k001 & $0,03(0,00)[2,5]$ & $0,03(0,00)[2,5]$ & $0,03(0,00)[2,5]$ & $0,03(0,00)[2,5]$ \\
\hline 4-Emotions & $0,22(0,02)[3,0]$ & $0,20(0,03)[4,0]$ & $0,24(0,01)[1,0]$ & $0,23(0,02)[2,0]$ \\
\hline 5-Fapesp & $0,04(0,00)[3,5]$ & $0,04(0,00)[3,5]$ & $0,05(0,01)[1,5]$ & $0,05(0,00)[1,5]$ \\
\hline 6-Genbase $e^{*}$ & $0,00(0,00)[2,5]$ & $0,00(0,00)[2,5]$ & $0,00(0,00)[2,5]$ & $0,00(0,00)[2,5]$ \\
\hline $7-L \log -f^{*}$ & $0,03(0,00)[2,5]$ & $0,03(0,00)[2,5]$ & $0,03(0,00)[2,5]$ & $0,03(0,00)[2,5]$ \\
\hline 8-Magtag5k & $0,05(0,00)[2,5]$ & $0,05(0,00)[2,5]$ & $0,05(0,00)[2,5]$ & $0,05(0,00)[2,5]$ \\
\hline 9-Scene & $0,14(0,01)[2,0]$ & $0,12(0,01)[3,5]$ & $0,12(0,01)[3,5]$ & $0,15(0,01)[1,0]$ \\
\hline \multirow[t]{2}{*}{ 10-Yeast } & $0,22(0,01)[3,0]$ & $0,22(0,01)[3,0]$ & $0,22(0,01)[3,0]$ & $0,23(0,0$ \\
\hline & \multicolumn{4}{|c|}{$\left|X^{\prime}\right|=30 \% M$} \\
\hline 1-Cal500 & $0,17(0,00)[2,0]$ & $0,17(0,01)[2,0]$ & $0,17(0, \mathrm{C}$ & 0,1610 \\
\hline 2-Corel5k & $0,01(0,00)[3,5]$ & $0,02(0,00)[1,5]$ & $0,02(0,00)[1,5]$ & $0,01(0,00)[3,5]$ \\
\hline 3-Corel16k001 & $0,03(0,00)[2,5]$ & $0,03(0,00)[2,5]$ & $0,03(0,00)[2,5]$ & $0,03(0,00)[2,5]$ \\
\hline 4-Emotions & $0,22(0,03)[2,5]$ & $0,21(0,02)[4,0]$ & $0,23(0,02)[1,0]$ & $0,22(0,02)[2,5]$ \\
\hline 5-Fapesp & $0,04(0,00)[3,0]$ & $0,04(0,00)[3,0]$ & $0,05(0,01)[1,0]$ & $0,04(0,00)[3,0]$ \\
\hline 6-Genbase $e^{*}$ & $0,00(0,00)[2,5]$ & $0,00(0,00)[2,5]$ & $0,00(0,00)[2,5]$ & $0,00(0,00)[2,5]$ \\
\hline $7-L \log -f^{*}$ & $0,03(0,00)[2,5]$ & $0,03(0,00)[2,5]$ & $0,03(0,00)[2,5]$ & $0,03(0,00)[2,5]$ \\
\hline 8-Magtag5k & $0,05(0,00)[2,5]$ & $0,05(0,00)[2,5]$ & $0,05(0,00)[2,5]$ & $0,05(0,00)[2,5]$ \\
\hline 9-Scene & $0,13(0,01)[1,0]$ & $0,11(0,01)[3,5]$ & $0,11(0,01)[3,5]$ & $0,12(0,01)[2,0]$ \\
\hline \multirow[t]{2}{*}{ 10-Yeast } & $0,21(0,01)[4,0]$ & $0,22(0,01)[2,5]$ & $0,22(0,01)[2,5]$ & $0,23(0,01)[1,0]$ \\
\hline & \multicolumn{4}{|c|}{$\left|X^{\prime}\right|=40 \% M$} \\
\hline 1-Cal500 & $0,17(0,00)[1,5]$ & $0,16(0,00)[3,5]$ & $0,17(0,0$ & $0,16(0,0)$ \\
\hline 2-Corel5k & $0,02(0,00)[2,0]$ & $0,02(0,00)[2,0]$ & $0,02(0,00)[2,0]$ & $0,01(0,00)[4,0]$ \\
\hline 3-Corel16k001 & $0,03(0,00)[2,5]$ & $0,03(0,00)[2,5]$ & $0,03(0,00)[2,5]$ & $0,03(0,00)[2,5]$ \\
\hline 4-Emotions & $0,22(0,02)[1,5]$ & $0,21(0,03)[3,5]$ & $0,22(0,02)[1,5]$ & $0,21(0,02)[3,5]$ \\
\hline 5-Fapesp & $0,04(0,00)[3,5]$ & $0,04(0,00)[3,5]$ & $0,05(0,01)[1,5]$ & $0,05(0,00)[1,5]$ \\
\hline 6-Genbase* & $0,00(0,00)[2,5]$ & $0,00(0,00)[2,5]$ & $0,00(0,00)[2,5]$ & $0,00(0,00)[2,5]$ \\
\hline $7-L \log -f^{*}$ & $0,03(0,00)[2,5]$ & $0,03(0,00)[2,5]$ & $0,03(0,00)[2,5]$ & $0,03(0,00)[2,5]$ \\
\hline 8-Magtag5k & $0,04(0,00)[3,5]$ & $0,05(0,00)[1,5]$ & $0,05(0,00)[1,5]$ & $0,04(0,00)[3,5]$ \\
\hline 9-Scene & $0,12(0,01)[1,0]$ & $0,10(0,01)[3,5]$ & $0,10(0,01)[3,5]$ & $0,11(0,01)[2,0]$ \\
\hline \multirow[t]{2}{*}{ 10-Yeast } & $0,21(0,01)[3,0]$ & $0,21(0,01)[3,0]$ & $0,21(0,01)[3,0]$ & $0,23(0,01)[1,0]$ \\
\hline & \multicolumn{4}{|c|}{$\left|X^{\prime}\right|=50 \% M$} \\
\hline 1-Cal500 & $0,17(0,00)[1,5]$ & $0,16(0,01)[3,5]$ & $0,17(0$ & $0,16(0$ \\
\hline 2-Corel5k & $0,02(0,00)[1,5]$ & $0,01(0,00)[3,5]$ & $0,02(0,00)[1,5]$ & $0,01(0,00)[3,5]$ \\
\hline 3-Corel16k001 & $0,03(0,00)[2,5]$ & $0,03(0,00)[2,5]$ & $0,03(0,00)[2,5]$ & $0,03(0,00)[2,5]$ \\
\hline
\end{tabular}


Tabela A.38: Resultados para Hamming Loss (continuação)

\begin{tabular}{|c|c|c|c|c|}
\hline & $R F-M L$ & $R F-B R$ & $R F-L P$ & $I G-L P$ \\
\hline 4-Emotions & $0,22(0,02)[1,0]$ & $0,21(0,03)[2,5]$ & $0,21(0,02)[2,5]$ & $0,20(0,02)[4,0]$ \\
\hline 5-Fapesp & $0,04(0,00)[3,0]$ & $0,04(0,00)[3,0]$ & $0,05(0,01)[1,0]$ & $0,04(0,00)[3,0]$ \\
\hline 6-Genbase* & $0,00(0,00)[2,5]$ & $0,00(0,00)[2,5]$ & $0,00(0,00)[2,5]$ & $0,00(0,00)[2,5]$ \\
\hline 7-Llog-f* & $0,03(0,00)[2,5]$ & $0,03(0,00)[2,5]$ & $0,03(0,00)[2,5]$ & $0,03(0,00)[2,5]$ \\
\hline 8-Magtag $5 k$ & $0,04(0,00)[3,5]$ & $0,05(0,00)[1,5]$ & $0,05(0,00)[1,5]$ & $0,04(0,00)[3,5]$ \\
\hline 9-Scene & $0,12(0,01)[1,0]$ & $0,10(0,01)[3,0]$ & $0,10(0,01)[3,0]$ & $0,10(0,01)[3,0]$ \\
\hline 10-Yeast & $0,21(0,01)[3,0]$ & $0,21(0,01)[3,0]$ & $0,21(0,01)[3,0]$ & $0,22(0,01)[1,0]$ \\
\hline & \multicolumn{4}{|c|}{$\left|X^{\prime}\right|=60 \% M$} \\
\hline 1-Cal500 & $0,17(0,00)[1,5]$ & $0,16(0,01)[3,5]$ & $0,17(0,01)[1,5]$ & $0,16(0,00)[3,5]$ \\
\hline 2-Corel5k & $0,02(0,00)[2,5]$ & $0,02(0,00)[2,5]$ & $0,02(0,00)[2,5]$ & $0,02(0,00)[2,5]$ \\
\hline 3-Corel16k001 & $0,03(0,00)[3,0]$ & $0,03(0,00)[3,0]$ & $0,03(0,00)[3,0]$ & $0,04(0,00)[1,0]$ \\
\hline 4-Emotions & $0,22(0,01)[1,0]$ & $0,20(0,02)[4,0]$ & $0,21(0,02)[2,5]$ & $0,21(0,02)[2,5]$ \\
\hline 5-Fapesp & $0,04(0,00)[3,0]$ & $0,04(0,00)[3,0]$ & $0,05(0,01)[1,0]$ & $0,04(0,00)[3,0]$ \\
\hline 6-Genbase* & $0,00(0,00)[2,5]$ & $0,00(0,00)[2,5]$ & $0,00(0,00)[2,5]$ & $0,00(0,00)[2,5]$ \\
\hline $7-L \log -f^{*}$ & $0,03(0,00)[2,5]$ & $0,03(0,00)[2,5]$ & $0,03(0,00)[2,5]$ & $0,03(0,00)[2,5]$ \\
\hline 8-Magtag5k & $0,04(0,00)[3,5]$ & $0,05(0,00)[1,5]$ & $0,05(0,00)[1,5]$ & $0,04(0,00)[3,5]$ \\
\hline 9-Scene & $0,11(0,01)[1,0]$ & $0,10(0,01)[3,0]$ & $0,10(0,01)[3,0]$ & $0,10(0,01)[3,0]$ \\
\hline \multirow[t]{2}{*}{ 10-Yeast } & $0,21(0,01)[2,5]$ & $0,21(0,01)[2,5]$ & $0,21(0,01)[2,5]$ & $0,21(0,01)[2,5]$ \\
\hline & \multicolumn{4}{|c|}{$\left|X^{\prime}\right|=70 \% M$} \\
\hline 1-Cal500 & $0,17(0,00)[2,0]$ & $0,17(0,00)[2,0]$ & $0,17(0,00)[2,0]$ & $0,16(0,00)[4,0]$ \\
\hline 2-Corel5k & $0,02(0,00)[2,5]$ & $0,02(0,00)[2,5]$ & $0,02(0,00)[2,5]$ & $0,02(0,00)[2,5]$ \\
\hline 3-Corel16k001 & $0,03(0,00)[4,0]$ & $0,04(0,00)[2,0]$ & $0,04(0,00)[2,0]$ & $0,04(0,00)[2,0]$ \\
\hline 4-Emotions & $0,21(0,02)[2,0]$ & $0,20(0,02)[4,0]$ & $0,21(0,02)[2,0]$ & $0,21(0,02)[2,0]$ \\
\hline 5-Fapesp & $0,04(0,00)[3,0]$ & $0,04(0,00)[3,0]$ & $0,05(0,01)[1,0]$ & $0,04(0,00)[3,0]$ \\
\hline 6-Genbase* & $0,00(0,00)[2,5]$ & $0,00(0,00)[2,5]$ & $0,00(0,00)[2,5]$ & $0,00(0,00)[2,5]$ \\
\hline 7-Llog-f* & $0,03(0,00)[2,5]$ & $0,03(0,00)[2,5]$ & $0,03(0,00)[2,5]$ & $0,03(0,00)[2,5]$ \\
\hline 8-Magtag5k & $0,04(0,00)[3,5]$ & $0,05(0,00)[1,5]$ & $0,05(0,00)[1,5]$ & $0,04(0,00)[3,5]$ \\
\hline 9-Scene & $0,10(0,01)[2,5]$ & $0,10(0,01)[2,5]$ & $0,10(0,01)[2,5]$ & $0,10(0,01)[2,5]$ \\
\hline \multirow[t]{2}{*}{ 10-Yeast } & $0,21(0,01)[2,5]$ & $0,21(0,01)[2,5]$ & $0,21(0,01)[2,5]$ & $0,21(0,01)[2,5]$ \\
\hline & \multicolumn{4}{|c|}{$\left|X^{\prime}\right|=80 \% M$} \\
\hline 1-Cal500 & $0,16(0,00)[2,5]$ & $0,16(0,00)[2,5]$ & $0,16(0,00)[2,5]$ & $0,16(0,00)[2,5]$ \\
\hline 2-Corel5k & $0,02(0,00)[2,5]$ & $0,02(0,00)[2,5]$ & $0,02(0,00)[2,5]$ & $0,02(0,00)[2,5]$ \\
\hline 3-Corel16k001 & $0,03(0,00)[4,0]$ & $0,04(0,00)[2,0]$ & $0,04(0,00)[2,0]$ & $0,04(0,00)[2,0]$ \\
\hline 4-Emotions & $0,20(0,02)[3,5]$ & $0,20(0,01)[3,5]$ & $0,21(0,02)[2,0]$ & $0,22(0,02)[1,0]$ \\
\hline 5-Fapesp & $0,04(0,00)[3,5]$ & $0,04(0,00)[3,5]$ & $0,05(0,01)[1,5]$ & $0,05(0,00)[1,5]$ \\
\hline 6-Genbase* & $0,00(0,00)[2,5]$ & $0,00(0,00)[2,5]$ & $0,00(0,00)[2,5]$ & $0,00(0,00)[2,5]$ \\
\hline 7-Llog-f* & $0,03(0,00)[2,5]$ & $0,03(0,00)[2,5]$ & $0,03(0,00)[2,5]$ & $0,03(0,00)[2,5]$ \\
\hline 8-Magtag5k & $0,04(0,00)[3,0]$ & $0,04(0,00)[3,0]$ & $0,05(0,00)[1,0]$ & $0,04(0,00)[3,0]$ \\
\hline 9-Scene & $0,10(0,01)[2,5]$ & $0,10(0,01)[2,5]$ & $0,10(0,01)[2,5]$ & $0,10(0,01)[2,5]$ \\
\hline \multirow[t]{2}{*}{ 10-Yeast } & $0,21(0,01)[2,5]$ & $0,21(0,01)[2,5]$ & $0,21(0,01)[2,5]$ & $0,21(0,01)[2,5]$ \\
\hline & \multicolumn{4}{|c|}{$\left|X^{\prime}\right|=90 \% M$} \\
\hline 1-Cal500 & $0,17(0,01)[1,0]$ & $0,16(0,01)[3,0]$ & $0,16(0,00)[3,0]$ & $0,16(0,01)[3,0]$ \\
\hline 2-Corel5k & $0,02(0,00)[2,5]$ & $0,02(0,00)[2,5]$ & $0,02(0,00)[2,5]$ & $0,02(0,00)[2,5]$ \\
\hline 3-Corel16k001 & $0,03(0,00)[3,5]$ & $0,04(0,00)[1,5]$ & $0,03(0,00)[3,5]$ & $0,04(0,00)[1,5]$ \\
\hline 4-Emotions & $0,21(0,02)[2,5]$ & $0,21(0,02)[2,5]$ & $0,21(0,02)[2,5]$ & $0,21(0,02)[2,5]$ \\
\hline 5-Fapesp & $0,04(0,00)[3,5]$ & $0,04(0,01)[3,5]$ & $0,05(0,01)[1,5]$ & $0,05(0,00)[1,5]$ \\
\hline 6-Genbase* & $0,00(0,00)[2,5]$ & $0,00(0,00)[2,5]$ & $0,00(0,00)[2,5]$ & $0,00(0,00)[2,5]$ \\
\hline 7-Llog-f* & $0,03(0,00)[2,5]$ & $0,03(0,00)[2,5]$ & $0,03(0,00)[2,5]$ & $0,03(0,00)[2,5]$ \\
\hline 8-Magtag $5 k$ & $0,04(0,00)[2,5]$ & $0,04(0,00)[2,5]$ & $0,04(0,00)[2,5]$ & $0,04(0,00)[2,5]$ \\
\hline 9-Scene & $0,10(0,01)[2,5]$ & $0,10(0,01)[2,5]$ & $0,10(0,01)[2,5]$ & $0,10(0,01)[2,5]$ \\
\hline 10-Yeast & $0,21(0,01)[2,5]$ & $0,21(0,01)[2,5]$ & $0,21(0,01)[2,5]$ & $0,21(0,01)[2,5]$ \\
\hline
\end{tabular}

Tabela A.39: Resultados experimentais de acordo com Accuracy: algoritmos de SA baseados em ReliefF e IG-LP

\begin{tabular}{c|cccc}
\hline & $R F-M L$ & $R F-B R$ & $R F-L P$ & $I G-L P$ \\
\hline & & & $\left|X^{\prime}\right|=10 \% M$ & \\
\hline
\end{tabular}


Tabela A.39: Resultados para Accuracy (continuação)

\begin{tabular}{|c|c|c|c|c|}
\hline & $R F-M L$ & $R F-B R$ & $R F-L P$ & $I G-L P$ \\
\hline 1-Cal500 & $0,27(0,01)[4,0]$ & $0,28(0,01)[2,5]$ & $0,28(0,02)[2,5]$ & $0,30(0,02)[1,0]$ \\
\hline 2-Corel5k & $0,12(0,00)[2,5]$ & $0,12(0,00)[2,5]$ & $0,12(0,00)[2,5]$ & $0,12(0,00)[2,5]$ \\
\hline 3-Corel16k001 & $0,14(0,00)[1,0]$ & $0,13(0,00)[3,0]$ & $0,13(0,00)[3,0]$ & $0,13(0,00)[3,0]$ \\
\hline 4-Emotions & $0,50(0,04)[3,0]$ & $0,51(0,04)[1,5]$ & $0,48(0,05)[4,0]$ & $0,51(0,05)[1,5]$ \\
\hline 5-Fapesp & $0,14(0,05)[3,0]$ & $0,19(0,05)[1,0]$ & $0,10(0,05)[4,0]$ & $0,18(0,06)[2,0]$ \\
\hline 6-Genbase* & $0,95(0,02)[3,5]$ & $0,95(0,02)[3,5]$ & $0,96(0,02)[2,0]$ & $0,99(0,02)[1,0]$ \\
\hline 7-Llog-f* & $0,11(0,03)[4,0]$ & $0,12(0,02)[2,0]$ & $0,12(0,01)[2,0]$ & $0,12(0,02)[2,0]$ \\
\hline 8-Magtag5k & $0,23(0,01)[1,0]$ & $0,19(0,01)[4,0]$ & $0,20(0,01)[2,5]$ & $0,20(0,01)[2,5]$ \\
\hline 9-Scene & $0,49(0,07)[4,0]$ & $0,58(0,04)[1,0]$ & $0,51(0,03)[3,0]$ & $0,53(0,04)[2,0]$ \\
\hline 10-Yeast & $0,49(0,02)[3,0]$ & $0,50(0,02)[1,5]$ & $0,50(0,02)[1,5]$ & $0,47(0,02)[4,0]$ \\
\hline & \multicolumn{4}{|c|}{$\left|X^{\prime}\right|=20 \% M$} \\
\hline 1-Cal500 & $0,28(0,01)[3,0]$ & $0,28(0,01)[3,0]$ & $0,28(0,01)[3,0]$ & $0,30(0,01)[1,0]$ \\
\hline 2-Corel5k & $0,12(0,00)[2,5]$ & $0,12(0,01)[2,5]$ & $0,12(0,01)[2,5]$ & $0,12(0,00)[2,5]$ \\
\hline 3-Corel16k001 & $0,14(0,00)[1,0]$ & $0,13(0,00)[3,0]$ & $0,13(0,00)[3,0]$ & $0,13(0,00)[3,0]$ \\
\hline 4-Emotions & $0,54(0,04)[2,0]$ & $0,57(0,05)[1,0]$ & $0,51(0,03)[4,0]$ & $0,53(0,03)[3,0]$ \\
\hline 5-Fapesp & $0,11(0,06)[3,0]$ & $0,15(0,05)[2,0]$ & $0,07(0,03)[4,0]$ & $0,16(0,05)[1,0]$ \\
\hline 6-Genbase* & $0,95(0,02)[3,5]$ & $0,95(0,02)[3,5]$ & $0,96(0,02)[2,0]$ & $0,99(0,02)[1,0]$ \\
\hline 7-Llog-f* & $0,13(0,02)[3,0]$ & $0,13(0,02)[3,0]$ & $0,14(0,01)[1,0]$ & $0,13(0,02)[3,0]$ \\
\hline 8-Magtag5k & $0,25(0,01)[1,0]$ & $0,23(0,01)[2,5]$ & $0,23(0,01)[2,5]$ & $0,22(0,01)[4,0]$ \\
\hline 9-Scene & $0,60(0,03)[3,0]$ & $0,64(0,04)[1,5]$ & $0,64(0,03)[1,5]$ & $0,57(0,02)[4,0]$ \\
\hline \multirow[t]{2}{*}{ 10-Yeast } & $0,51(0,02)[2,0]$ & $0,51(0,02)[2,0]$ & $0,51(0,02)[2,0]$ & $0,48(0,02)[4,0]$ \\
\hline & \multicolumn{4}{|c|}{$\left|X^{\prime}\right|=30 \% M$} \\
\hline 1-Cal500 & $0,28(0,01)[3,0]$ & $0,28(0,01)[3,0]$ & $0,28(0,01)[3,0]$ & $0,30(0,01)[1,0]$ \\
\hline 2-Corel5k & $0,11(0,00)[2,5]$ & $0,11(0,01)[2,5]$ & $0,11(0,01)[2,5]$ & $0,11(0,01)[2,5]$ \\
\hline 3-Corel16k001 & $0,14(0,00)[1,0]$ & $0,13(0,00)[3,0]$ & $0,13(0,00)[3,0]$ & $0,13(0,0$ \\
\hline 4-Emotions & $0,53(0,05)[3,5]$ & $0,55(0,03)[1,0]$ & $0,53(0,04)[3,5]$ & $0,54(0,04)[2,0]$ \\
\hline 5-Fapesp & $0,09(0,04)[3,0]$ & $0,15(0,06)[2,0]$ & $0,06(0,03)[4,0]$ & $0,17(0,06)[1,0]$ \\
\hline 6-Genbase* & $0,95(0,02)[3,5]$ & $0,95(0,02)[3,5]$ & $0,96(0,02)[2,0]$ & $0,99(0,02)[1,0]$ \\
\hline 7-Llog-f* & $0,13(0,03)[2,5]$ & $0,13(0,02)[2,5]$ & $0,12(0,03)[4,0]$ & $0,14(0,01)[1,0]$ \\
\hline 8-Magtag5k & $0,25(0,01)[2,0]$ & $0,23(0,01)[4,0]$ & $0,25(0,01)[2,0]$ & $0,25(0,01)[2,0]$ \\
\hline 9-Scene & $0,62(0,03)[4,0]$ & $0,66(0,03)[1,5]$ & $0,66(0,03)[1,5]$ & $0,65(0,03)[3,0]$ \\
\hline \multirow[t]{2}{*}{ 10-Yeast } & $0,53(0,02)[1,0]$ & $0,52(0,02)[2,5]$ & $0,52(0,02)[2,5]$ & $0,49(0,02)[4,0]$ \\
\hline & \multicolumn{4}{|c|}{$\left|X^{\prime}\right|=40 \% M$} \\
\hline 1-Cal500 & $0,28(0,01)[3,5]$ & $0,29(0,01)[2,0]$ & $0,28(0,02)[3,5]$ & $0,30(0,01)[1,0]$ \\
\hline 2-Corel5k & $0,10(0,01)[2,5]$ & $0,10(0,01)[2,5]$ & $0,09(0,01)[4,0]$ & $0,11(0,01)[1,0]$ \\
\hline 3-Corel16k001 & $0,13(0,00)[1,5]$ & $0,12(0,00)[3,0]$ & $0,13(0,00)[1,5]$ & $0,11(0,00)[4,0]$ \\
\hline 4-Emotions & $0,54(0,05)[3,5]$ & $0,56(0,05)[1,5]$ & $0,54(0,04)[3,5]$ & $0,56(0,04)[1,5]$ \\
\hline 5-Fapesp & $0,09(0,05)[3,0]$ & $0,16(0,05)[1,0]$ & $0,06(0,02)[4,0]$ & $0,15(0,04)[2,0]$ \\
\hline 6-Genbase* & $0,95(0,02)[3,5]$ & $0,95(0,02)[3,5]$ & $0,96(0,02)[2,0]$ & $0,99(0,02)[1,0]$ \\
\hline $7-L \log -f^{*}$ & $0,14(0,03)[2,0]$ & $0,14(0,03)[2,0]$ & $0,13(0,03)[4,0]$ & $0,14(0,02)[2,0]$ \\
\hline 8-Magtag5k & $0,26(0,01)[1,5]$ & $0,24(0,01)[4,0]$ & $0,25(0,01)[3,0]$ & $0,26(0,01)[1,5]$ \\
\hline 9-Scene & $0,64(0,03)[4,0]$ & $0,69(0,03)[1,5]$ & $0,69(0,02)[1,5]$ & $0,67(0,02)[3,0]$ \\
\hline \multirow[t]{2}{*}{ 10-Yeast } & $0,54(0,02)[1,0]$ & $0,53(0,02)[2,5]$ & $0,53(0,02)[2,5]$ & $0,49(0,02)[4,0]$ \\
\hline & \multicolumn{4}{|c|}{$\left|X^{\prime}\right|=50 \% M$} \\
\hline 1-Cal500 & $0,28(0,01)[3,5]$ & $0,29(0,01)[2,0]$ & $0,28(0,01)[3,5]$ & $0,31(0,01)[1,0]$ \\
\hline 2-Corel5k & $0,08(0,01)[3,0]$ & $0,11(0,01)[1,0]$ & $0,07(0,01)[4,0]$ & $0,10(0,01)[2,0]$ \\
\hline 3-Corel16k001 & $0,12(0,01)[1,5]$ & $0,10(0,01)[3,0]$ & $0,12(0,00)[1,5]$ & $0,08(0,01)[4,0]$ \\
\hline 4-Emotions & $0,54(0,05)[4,0]$ & $0,55(0,05)[2,5]$ & $0,55(0,04)[2,5]$ & $0,58(0,03)[1,0]$ \\
\hline 5-Fapesp & $0,07(0,04)[3,0]$ & $0,13(0,06)[2,0]$ & $0,05(0,03)[4,0]$ & $0,16(0,04)[1,0]$ \\
\hline 6-Genbase* & $0,95(0,02)[3,5]$ & $0,95(0,02)[3,5]$ & $0,96(0,02)[2,0]$ & $0,99(0,02)[1,0]$ \\
\hline 7-Llog-f* & $0,14(0,03)[2,5]$ & $0,14(0,04)[2,5]$ & $0,13(0,03)[4,0]$ & $0,15(0,03)[1,0]$ \\
\hline 8-Magtag5k & $0,26(0,01)[2,0]$ & $0,25(0,01)[3,5]$ & $0,25(0,01)[3,5]$ & $0,27(0,01)[1,0]$ \\
\hline 9-Scene & $0,66(0,02)[4,0]$ & $0,71(0,04)[1,5]$ & $0,71(0,03)[1,5]$ & $0,69(0,03)[3,0]$ \\
\hline \multirow[t]{2}{*}{ 10-Yeast } & $0,53(0,02)[1,5]$ & $0,52(0,02)[3,0]$ & $0,53(0,02)[1,5]$ & $0,50(0,02)[4,0]$ \\
\hline & \multicolumn{4}{|c|}{$\left|X^{\prime}\right|=60 \% M$} \\
\hline 1-Cal500 & $0,28(0,01)[3,5]$ & $0,29(0,01)[2,0]$ & $0,28(0,02)[3,5]$ & $0,31(0,01)[1,0]$ \\
\hline 2-Corel5k & $0,08(0,01)[2,5]$ & $0,08(0,01)[2,5]$ & $0,08(0,01)[2,5]$ & $0,08(0,01)[2,5]$ \\
\hline 3-Corel16k & $0,10(0,01)[1,5]$ & $0,06(0,00)[3,0]$ & $0,10(0,01)[1,5]$ & $0,03(0,01)[4,0]$ \\
\hline
\end{tabular}


Tabela A.39: Resultados para Accuracy (continuação)

\begin{tabular}{|c|c|c|c|c|}
\hline & $R F-M L$ & $R F-B R$ & $R F-L P$ & $I G-L P$ \\
\hline 4-Emotions & $0,54(0,03)[4,0]$ & $0,57(0,03)[1,0]$ & $0,55(0,04)[3,0]$ & $0,56(0,04)[2,0]$ \\
\hline 5-Fapesp & $0,05(0,04)[3,5]$ & $0,14(0,06)[2,0]$ & $0,05(0,03)[3,5]$ & $0,16(0,04)[1,0]$ \\
\hline 6-Genbase* & $0,95(0,02)[3,5]$ & $0,95(0,02)[3,5]$ & $0,96(0,02)[2,0]$ & $0,99(0,02)[1,0]$ \\
\hline 7-Llog-f* & $0,13(0,03)[3,5]$ & $0,14(0,03)[1,5]$ & $0,13(0,03)[3,5]$ & $0,14(0,03)[1,5]$ \\
\hline 8-Magtag $5 k$ & $0,26(0,01)[2,0]$ & $0,25(0,01)[3,5]$ & $0,25(0,01)[3,5]$ & $0,27(0,01)[1,0]$ \\
\hline 9-Scene & $0,69(0,03)[4,0]$ & $0,72(0,03)[1,0]$ & $0,71(0,03)[2,0]$ & $0,70(0,02)[3,0]$ \\
\hline 10-Yeast & $0,54(0,02)[1,0]$ & $0,53(0,02)[2,5]$ & $0,53(0,02)[2,5]$ & $0,52(0,02)[4,0]$ \\
\hline & \multicolumn{4}{|c|}{$\left|X^{\prime}\right|=70 \% M$} \\
\hline 1-Cal500 & $0,29(0,01)[2,5]$ & $0,29(0,01)[2,5]$ & $0,28(0,01)[4,0]$ & $0,31(0,01)[1,0]$ \\
\hline 2-Corel5k & $0,07(0,01)[3,0]$ & $0,05(0,01)[4,0]$ & $0,09(0,01)[1,0]$ & $0,08(0,01)[2,0]$ \\
\hline 3-Corel16k001 & $0,08(0,01)[1,0]$ & $0,03(0,00)[3,5]$ & $0,05(0,01)[2,0]$ & $0,03(0,00)[3,5]$ \\
\hline 4-Emotions & $0,55(0,04)[4,0]$ & $0,57(0,04)[1,0]$ & $0,56(0,04)[2,5]$ & $0,56(0,04)[2,5]$ \\
\hline 5-Fapesp & $0,05(0,04)[3,5]$ & $0,14(0,05)[2,0]$ & $0,05(0,02)[3,5]$ & $0,15(0,05)[1,0]$ \\
\hline 6-Genbase* & $0,95(0,02)[3,5]$ & $0,95(0,02)[3,5]$ & $0,96(0,02)[2,0]$ & $0,99(0,02)[1,0]$ \\
\hline 7-Llog-f* & $0,13(0,03)[3,0]$ & $0,14(0,03)[1,5]$ & $0,12(0,03)[4,0]$ & $0,14(0,03)[1,5]$ \\
\hline 8-Magtag5k & $0,26(0,01)[1,5]$ & $0,25(0,01)[3,5]$ & $0,25(0,01)[3,5]$ & $0,26(0,01)[1,5]$ \\
\hline 9-Scene & $0,71(0,03)[2,5]$ & $0,71(0,03)[2,5]$ & $0,71(0,03)[2,5]$ & $0,71(0,02)[2,5]$ \\
\hline \multirow[t]{2}{*}{ 10-Yeast } & $0,54(0,02)[1,5]$ & $0,53(0,02)[3,5]$ & $0,53(0,02)[3,5]$ & $0,54(0,02)[1,5]$ \\
\hline & \multicolumn{4}{|c|}{$\left|X^{\prime}\right|=80 \% M$} \\
\hline 1-Cal500 & $0,29(0,01)[3,0]$ & $0,29(0,01)[3,0]$ & $0,29(0,01)[3,0]$ & $0,31(0,01)[1,0]$ \\
\hline 2-Corel5k & $0,07(0,01)[4,0]$ & $0,08(0,01)[2,5]$ & $0,09(0,01)[1,0]$ & $0,08(0,01)[2,5]$ \\
\hline 3-Corel16k001 & $0,07(0,01)[1,0]$ & $0,03(0,01)[4,0]$ & $0,05(0,01)[2,0]$ & $0,04(0,01)[3,0]$ \\
\hline 4-Emotions & $0,57(0,04)[1,5]$ & $0,57(0,03)[1,5]$ & $0,55(0,05)[3,5]$ & $0,55(0,04)[3,5]$ \\
\hline 5-Fapesp & $0,04(0,04)[4,0]$ & $0,15(0,05)[1,0]$ & $0,05(0,03)[3,0]$ & $0,14(0,04)[2,0]$ \\
\hline 6-Genbase* & $0,95(0,02)[3,5]$ & $0,95(0,02)[3,5]$ & $0,96(0,02)[2,0]$ & $0,99(0,02)[1,0]$ \\
\hline $7-L \log -f^{*}$ & $0,13(0,03)[3,5]$ & $0,14(0,02)[1,5]$ & $0,13(0,03)[3,5]$ & $0,14(0,03)[1,5]$ \\
\hline 8-Magtag5k & $0,26(0,01)[1,5]$ & $0,25(0,01)[3,5]$ & $0,25(0,01)[3,5]$ & $0,26(0,01)[1,5]$ \\
\hline 9-Scene & $0,72(0,03)[1,0]$ & $0,71(0,03)[3,0]$ & $0,71(0,02)[3,0]$ & $0,71(0,03)[3,0]$ \\
\hline \multirow[t]{2}{*}{ 10-Yeast } & $0,53(0,02)[3,0]$ & $0,53(0,02)[3,0]$ & $0,53(0,02)[3,0]$ & $0,54(0,02)[1,0]$ \\
\hline & \multicolumn{4}{|c|}{$\left|X^{\prime}\right|=90 \% M$} \\
\hline 1-Cal500 & $0,29(0,01)[3,0]$ & $0,29(0,01)[3,0]$ & $0,29(0,01)[3,0]$ & $0,31(0,02)[1,0]$ \\
\hline 2-Corel5k & $0,08(0,01)[4,0]$ & $0,09(0,01)[2,5]$ & $0,10(0,01)[1,0]$ & $0,09(0,01)[2,5]$ \\
\hline 3-Corel16k001 & $0,07(0,01)[1,5]$ & $0,05(0,00)[3,5]$ & $0,07(0,01)[1,5]$ & $0,05(0,00)[3,5]$ \\
\hline 4-Emotions & $0,56(0,04)[2,0]$ & $0,56(0,04)[2,0]$ & $0,55(0,05)[4,0]$ & $0,56(0,03)[2,0]$ \\
\hline 5-Fapesp & $0,04(0,04)[4,0]$ & $0,13(0,05)[1,5]$ & $0,05(0,02)[3,0]$ & $0,13(0,04)[1,5]$ \\
\hline 6-Genbase $e^{*}$ & $0,95(0,02)[3,5]$ & $0,95(0,02)[3,5]$ & $0,96(0,02)[2,0]$ & $0,99(0,02)[1,0]$ \\
\hline 7-Llog-f* & $0,13(0,04)[3,5]$ & $0,15(0,02)[1,5]$ & $0,13(0,03)[3,5]$ & $0,15(0,03)[1,5]$ \\
\hline 8-Magtag5k & $0,26(0,01)[2,5]$ & $0,26(0,01)[2,5]$ & $0,26(0,01)[2,5]$ & $0,26(0,01)[2,5]$ \\
\hline 9-Scene & $0,72(0,03)[1,0]$ & $0,71(0,02)[3,0]$ & $0,71(0,02)[3,0]$ & $0,71(0,02)[3,0]$ \\
\hline 10-Yeast & $0,53(0,02)[3,0]$ & $0,53(0,02)[3,0]$ & $0,53(0,02)[3,0]$ & $0,54(0,02)[1,0]$ \\
\hline
\end{tabular}

Tabela A.40: Resultados experimentais de acordo com $F_{b}$ : algoritmos de SA baseados em ReliefF e IG-LP

\begin{tabular}{|c|c|c|c|c|}
\hline & $R F-M L$ & $\overline{R F-B R}$ & $R F-L P$ & $I G-L P$ \\
\hline & \multicolumn{4}{|c|}{$\left|X^{\prime}\right|=10 \% M$} \\
\hline 1-Cal500 & $0,43(0,01)[3,0]$ & $0,43(0,01)[3,0]$ & $0,43(0,02)[3,0]$ & $0,45(0,02)[1,0]$ \\
\hline 2-Corel5k & $0,19(0,01)[3,0]$ & $0,19(0,01)[3,0]$ & $0,19(0,01)[3,0]$ & $0,20(0,01)[1,0]$ \\
\hline 3-Corel16k001 & $0,22(0,01)[1,0]$ & $0,21(0,01)[3,0]$ & $0,21(0,01)[3,0]$ & $0,21(0,00)[3,0]$ \\
\hline 4-Emotions & $0,61(0,03)[3,0]$ & $0,63(0,04)[1,0]$ & $0,59(0,04)[4,0]$ & $0,62(0,05)[2,0]$ \\
\hline 5-Fapesp & $0,15(0,04)[3,0]$ & $0,22(0,05)[2,0]$ & $0,12(0,05)[4,0]$ & $0,23(0,05)[1,0]$ \\
\hline 6-Genbase* & $0,95(0,02)[3,0]$ & $0,95(0,02)[3,0]$ & $0,95(0,02)[3,0]$ & $0,99(0,01)[1,0]$ \\
\hline $7-L \log -f^{*}$ & $0,12(0,03)[4,0]$ & $0,13(0,03)[2,0]$ & $0,13(0,02)[2,0]$ & $0,13(0,03)[2,0]$ \\
\hline 8-Magtag5k & $0,34(0,01)[1,0]$ & $0,29(0,01)[4,0]$ & $0,30(0,01)[3,0]$ & $0,31(0,01)[2,0]$ \\
\hline 9-Scene & $0,50(0,07)[4,0]$ & $0,59(0,04)[1,0]$ & $0,51(0,02)[3,0]$ & $0,54(0,04)[2,0]$ \\
\hline \multirow[t]{2}{*}{ 10-Yeast } & $0,62(0,02)[3,0]$ & $0,63(0,01)[1,5]$ & $0,63(0,02)[1,5]$ & $0,60(0,02)[4,0]$ \\
\hline & \multicolumn{4}{|c|}{$\left|X^{\prime}\right|=20 \% M$} \\
\hline
\end{tabular}


Tabela A.40: Resultados para $F_{b}$ (continuação)

\begin{tabular}{|c|c|c|c|c|}
\hline & $R F-M L$ & $R F-B R$ & $R F-L P$ & $I G-L P$ \\
\hline 1-Cal500 & $0,43(0,02)[3,0]$ & $0,43(0,02)[3,0]$ & $0,43(0,02)[3,0]$ & $0,46(0,02)[1,0]$ \\
\hline 2-Corel5k & $0,19(0,01)[2,0]$ & $0,18(0,01)[4,0]$ & $0,19(0,01)[2,0]$ & $0,19(0,00)[2,0]$ \\
\hline 3-Corel16k001 & $0,22(0,01)[1,0]$ & $0,21(0,00)[3,0]$ & $0,21(0,01)[3,0]$ & $0,21(0,01)[3,0]$ \\
\hline 4-Emotions & $0,65(0,05)[2,0]$ & $0,67(0,05)[1,0]$ & $0,61(0,03)[4,0]$ & $0,64(0,03)[3,0]$ \\
\hline 5-Fapesp & $0,11(0,05)[3,0]$ & $0,18(0,05)[2,0]$ & $0,08(0,04)[4,0]$ & $0,21(0,06)[1,0]$ \\
\hline 6-Genbase* & $0,95(0,02)[3,0]$ & $0,95(0,02)[3,0]$ & $0,95(0,02)[3,0]$ & $0,99(0,01)[1,0]$ \\
\hline $7-L \log -f^{*}$ & $0,14(0,02)[3,0]$ & $0,14(0,02)[3,0]$ & $0,15(0,01)[1,0]$ & $0,14(0,03)[3,0]$ \\
\hline 8-Magtag $5 k$ & $0,36(0,01)[1,0]$ & $0,33(0,01)[3,0]$ & $0,34(0,02)[2,0]$ & $0,32(0,01)[4,0]$ \\
\hline 9-Scene & $0,60(0,03)[3,0]$ & $0,64(0,04)[2,0]$ & $0,65(0,03)[1,0]$ & $0,58(0,02)[4,0]$ \\
\hline \multirow[t]{2}{*}{ 10-Yeast } & $0,64(0,01)[2,0]$ & $0,64(0,01)[2,0]$ & $0,64(0,02)[2,0]$ & $0,61(0,02)[4,0]$ \\
\hline & \multicolumn{4}{|c|}{$\left|X^{\prime}\right|=30 \% M$} \\
\hline 1-Cal50O & $0,43(0,01)[3,0]$ & $0,43(0,02)[3,0]$ & $0,43(0,02)[3,0]$ & $0,46(0,02)[1,0]$ \\
\hline 2-Corel5k & $0,17(0,01)[2,5]$ & $0,17(0,01)[2,5]$ & $0,17(0,01)[2,5]$ & $0,17(0,01)[2,5]$ \\
\hline 3-Corel16k001 & $0,21(0,00)[1,5]$ & $0,20(0,01)[3,5]$ & $0,21(0,00)[1,5]$ & $0,20(0,01)[3,5]$ \\
\hline 4-Emotions & $0,63(0,05)[3,5]$ & $0,66(0,03)[1,0]$ & $0,63(0,04)[3,5]$ & $0,65(0,04)[2,0]$ \\
\hline 5-Fapesp & $0,09(0,04)[3,0]$ & $0,17(0,06)[2,0]$ & $0,08(0,04)[4,0]$ & $0,22(0,05)[1,0]$ \\
\hline 6-Genbase* & $0,95(0,02)[3,0]$ & $0,95(0,02)[3,0]$ & $0,95(0,02)[3,0]$ & $0,99(0,01)[1,0]$ \\
\hline 7-Llog-f* & $0,14(0,02)[2,5]$ & $0,14(0,02)[2,5]$ & $0,13(0,03)[4,0]$ & $0,16(0,01)[1,0]$ \\
\hline 8-Magtag5k & $0,35(0,01)[2,5]$ & $0,34(0,01)[4,0]$ & $0,35(0,01)[2,5]$ & $0,36(0,01)[1,0]$ \\
\hline 9-Scene & $0,63(0,03)[4,0]$ & $0,67(0,03)[1,5]$ & $0,67(0,03)[1,5]$ & $0,65(0,03)[3,0]$ \\
\hline \multirow[t]{2}{*}{ 10-Yeast } & $0,65(0,01)[1,0]$ & $0,64(0,02)[2,5]$ & $0,64(0,02)[2,5]$ & $0,61(0,02)[4,0]$ \\
\hline & \multicolumn{4}{|c|}{$\left|X^{\prime}\right|=40 \% M$} \\
\hline 1-Cal500 & $0,43(0,01)[4,0]$ & $0,44(0,02)[2,5]$ & $0,44(0,02)[2,5]$ & $0,46(0,01)[1,0]$ \\
\hline 2-Corel5k & $0,15(0,02)[3,0]$ & $0,16(0,01)[2,0]$ & $0,14(0,01)[4,0]$ & $0,18(0,01)[1,0]$ \\
\hline 3-Corel16k001 & $0,20(0,01)[1,5]$ & $0,19(0,01)[3,0]$ & $0,20(0,00)[1,5]$ & $0,18(0,01)[4,0]$ \\
\hline 4-Emotions & $0,65(0,04)[3,5]$ & $0,67(0,05)[1,5]$ & $0,65(0,03)[3,5]$ & $0,67(0,04)[1,5]$ \\
\hline 5-Fapesp & $0,08(0,05)[3,5]$ & $0,18(0,05)[2,0]$ & $0,08(0,03)[3,5]$ & $0,20(0,04)[1,0]$ \\
\hline 6-Genbase* & $0,95(0,02)[3,0]$ & $0,95(0,02)[3,0]$ & $0,95(0,02)[3,0]$ & $0,99(0,01)[1,0]$ \\
\hline $7-L \log -f^{*}$ & $0,15(0,03)[2,5]$ & $0,15(0,03)[2,5]$ & $0,14(0,03)[4,0]$ & $0,16(0,02)[1,0]$ \\
\hline 8-Magtag5k & $0,37(0,01)[1,5]$ & $0,34(0,01)[4,0]$ & $0,36(0,01)[3,0]$ & $0,37(0,01)[1,5]$ \\
\hline 9-Scene & $0,65(0,03)[4,0]$ & $0,70(0,03)[1,5]$ & $0,70(0,02)[1,5]$ & $0,67(0,02)[3,0]$ \\
\hline \multirow[t]{2}{*}{ 10-Yeast } & $0,66(0,01)[1,0]$ & $0,65(0,02)[2,5]$ & $0,65(0,02)[2,5]$ & $0,62(0,02)[4,0]$ \\
\hline & \multicolumn{4}{|c|}{$\left|X^{\prime}\right|=50 \% M$} \\
\hline 1-Cal500 & $0,43(0,01)[4,0]$ & $0,44(0,02)[2,5]$ & $0,44(0,02)[2,5]$ & $0,46(0,01)[1,0]$ \\
\hline 2-Corel5k & $0,12(0,02)[3,0]$ & $0,16(0,01)[2,0]$ & $0,11(0,01)[4,0]$ & $0,17(0,01)[1,0]$ \\
\hline 3-Corel16k001 & $0,18(0,01)[2,0]$ & $0,16(0,01)[3,0]$ & $0,19(0,01)[1,0]$ & $0,14(0,01)[4,0]$ \\
\hline 4-Emotions & $0,64(0,04)[4,0]$ & $0,66(0,04)[2,5]$ & $0,66(0,04)[2,5]$ & $0,69(0,03)[1,0]$ \\
\hline 5-Fapesp & $0,07(0,04)[3,5]$ & $0,15(0,06)[2,0]$ & $0,07(0,03)[3,5]$ & $0,21(0,04)[1,0]$ \\
\hline 6-Genbase* & $0,95(0,02)[3,0]$ & $0,95(0,02)[3,0]$ & $0,95(0,02)[3,0]$ & $0,99(0,01)[1,0]$ \\
\hline $7-L \log -f^{*}$ & $0,15(0,03)[2,5]$ & $0,15(0,03)[2,5]$ & $0,14(0,03)[4,0]$ & $0,16(0,03)[1,0]$ \\
\hline 8-Magtag5k & $0,37(0,01)[2,0]$ & $0,35(0,01)[4,0]$ & $0,36(0,01)[3,0]$ & $0,39(0,01)[1,0]$ \\
\hline 9-Scene & $0,66(0,03)[4,0]$ & $0,71(0,03)[1,5]$ & $0,71(0,03)[1,5]$ & $0,70(0,03)[3,0]$ \\
\hline \multirow[t]{2}{*}{ 10-Yeast } & $0,65(0,02)[2,5]$ & $0,65(0,01)[2,5]$ & $0,66(0,02)[1,0]$ & $0,63(0,01)[4,0]$ \\
\hline & \multicolumn{4}{|c|}{$\left|X^{\prime}\right|=60 \% M$} \\
\hline 1-Cal500 & $0,44(0,01)[2,5]$ & $0,44(0,02)[2,5]$ & $0,43(0,02)[4,0]$ & $0,46(0,01)[1,0]$ \\
\hline 2-Corel5k & $0,11(0,01)[4,0]$ & $0,12(0,02)[2,0]$ & $0,12(0,01)[2,0]$ & $0,12(0,02)[2,0]$ \\
\hline 3-Corel16k001 & $0,15(0,01)[1,5]$ & $0,09(0,01)[3,0]$ & $0,15(0,02)[1,5]$ & $0,05(0,01)[4,0]$ \\
\hline 4-Emotions & $0,65(0,03)[3,5]$ & $0,67(0,03)[1,5]$ & $0,65(0,04)[3,5]$ & $0,67(0,04)[1,5]$ \\
\hline 5-Fapesp & $0,05(0,04)[4,0]$ & $0,14(0,06)[2,0]$ & $0,07(0,04)[3,0]$ & $0,19(0,04)[1,0]$ \\
\hline 6-Genbase* & $0,95(0,02)[3,0]$ & $0,95(0,02)[3,0]$ & $0,95(0,02)[3,0]$ & $0,99(0,01)[1,0]$ \\
\hline $7-L \log -f^{*}$ & $0,14(0,03)[3,5]$ & $0,15(0,03)[2,0]$ & $0,14(0,03)[3,5]$ & $0,16(0,02)[1,0]$ \\
\hline 8-Magtag5k & $0,37(0,01)[2,0]$ & $0,35(0,01)[4,0]$ & $0,36(0,01)[3,0]$ & $0,38(0,01)[1,0]$ \\
\hline 9-Scene & $0,69(0,03)[4,0]$ & $0,72(0,03)[1,5]$ & $0,72(0,03)[1,5]$ & $0,71(0,02)[3,0]$ \\
\hline \multirow[t]{2}{*}{ 10-Yeast } & $0,66(0,02)[1,0]$ & $0,65(0,02)[3,0]$ & $0,65(0,02)[3,0]$ & $0,65(0,02)[3,0]$ \\
\hline & \multicolumn{4}{|c|}{$\left|X^{\prime}\right|=70 \% M$} \\
\hline 1-Cal500 & $0,44(0,01)[2,5]$ & $0,44(0,02)[2,5]$ & $0,43(0,02)[4,0]$ & $0,46(0,01)[1,0]$ \\
\hline 2-Corel5k & $0,11(0,02)[3,0]$ & $0,08(0,01)[4,0]$ & $0,14(0,02)[1,0]$ & $0,13(0,01)[2,0]$ \\
\hline 3-Corel16k001 & $0,13(0,01)[1,0]$ & $0,05(0,01)[3,0]$ & $0,08(0,01)[2,0]$ & $0,04(0,01)[4,0]$ \\
\hline
\end{tabular}


Tabela A.40: Resultados para $F_{b}$ (continuação)

\begin{tabular}{|c|c|c|c|c|}
\hline & $R F-M L$ & $R F-B R$ & $R F-L P$ & $I G-L P$ \\
\hline 4-Emotions & $0,65(0,03)[4,0]$ & $0,67(0,03)[2,0]$ & $0,67(0,04)[2,0]$ & $0,67(0,04)[2,0]$ \\
\hline 5-Fapesp & $0,04(0,03)[4,0]$ & $0,15(0,05)[2,0]$ & $0,07(0,03)[3,0]$ & $0,19(0,04)[1,0]$ \\
\hline 6-Genbase* & $0,95(0,02)[3,0]$ & $0,95(0,02)[3,0]$ & $0,95(0,02)[3,0]$ & $0,99(0,01)[1,0]$ \\
\hline 7-Llog-f* & $0,14(0,03)[3,5]$ & $0,16(0,03)[1,0]$ & $0,14(0,04)[3,5]$ & $0,15(0,03)[2,0]$ \\
\hline 8-Magtag5k & $0,37(0,01)[1,5]$ & $0,36(0,01)[3,5]$ & $0,36(0,01)[3,5]$ & $0,37(0,01)[1,5]$ \\
\hline 9-Scene & $0,72(0,03)[2,5]$ & $0,72(0,03)[2,5]$ & $0,72(0,03)[2,5]$ & $0,72(0,02)[2,5]$ \\
\hline 10-Yeast & $0,66(0,02)[2,0]$ & $0,65(0,02)[4,0]$ & $0,66(0,02)[2,0]$ & $0,66(0,02)[2,0]$ \\
\hline & \multicolumn{4}{|c|}{$\left|X^{\prime}\right|=80 \% M$} \\
\hline 1-Cal500 & $0,44(0,01)[3,0]$ & $0,44(0,02)[3,0]$ & $0,44(0,02)[3,0]$ & $0,46(0,01)[1,0]$ \\
\hline 2-Corel5k & $0,10(0,01)[4,0]$ & $0,12(0,02)[2,5]$ & $0,15(0,02)[1,0]$ & $0,12(0,02)[2,5]$ \\
\hline 3-Corel16k001 & $0,12(0,01)[1,0]$ & $0,05(0,01)[4,0]$ & $0,08(0,02)[2,0]$ & $0,06(0,02)[3,0]$ \\
\hline 4-Emotions & $0,67(0,04)[1,5]$ & $0,67(0,03)[1,5]$ & $0,66(0,05)[3,0]$ & $0,65(0,04)[4,0]$ \\
\hline 5-Fapesp & $0,03(0,03)[4,0]$ & $0,16(0,03)[2,0]$ & $0,06(0,03)[3,0]$ & $0,18(0,04)[1,0]$ \\
\hline 6-Genbase* & $0,95(0,02)[3,0]$ & $0,95(0,02)[3,0]$ & $0,95(0,02)[3,0]$ & $0,99(0,01)[1,0]$ \\
\hline 7-Llog-f* & $0,14(0,03)[3,5]$ & $0,15(0,02)[2,0]$ & $0,14(0,02)[3,5]$ & $0,16(0,02)[1,0]$ \\
\hline 8-Magtag5k & $0,37(0,01)[1,5]$ & $0,36(0,01)[3,5]$ & $0,36(0,01)[3,5]$ & $0,37(0,01)[1,5]$ \\
\hline 9-Scene & $0,72(0,03)[1,5]$ & $0,71(0,03)[3,5]$ & $0,71(0,03)[3,5]$ & $0,72(0,03)[1,5]$ \\
\hline \multirow[t]{2}{*}{ 10-Yeast } & $0,66(0,02)[2,0]$ & $0,66(0,02)[2,0]$ & $0,65(0,02)[4,0]$ & $0,66(0,02)[2,0]$ \\
\hline & \multicolumn{4}{|c|}{$\left|X^{\prime}\right|=90 \% M$} \\
\hline 1-Cal500 & $0,44(0,02)[3,0]$ & $0,44(0,02)[3,0]$ & $0,44(0,02)[3,0]$ & $0,47(0,02)[1,0]$ \\
\hline 2-Corel5k & $0,12(0,01)[4,0]$ & $0,14(0,01)[2,5]$ & $0,15(0,02)[1,0]$ & $0,14(0,02)[2,5]$ \\
\hline 3-Corel16k001 & $0,11(0,01)[1,5]$ & $0,07(0,00)[4,0]$ & $0,11(0,01)[1,5]$ & $0,09(0,01)[3,0]$ \\
\hline 4-Emotions & $0,66(0,04)[2,5]$ & $0,66(0,04)[2,5]$ & $0,66(0,04)[2,5]$ & $0,66(0,03)[2,5]$ \\
\hline 5-Fapesp & $0,03(0,03)[4,0]$ & $0,15(0,05)[2,0]$ & $0,07(0,02)[3,0]$ & $0,17(0,04)[1,0]$ \\
\hline 6-Genbase* & $0,95(0,02)[3,0]$ & $0,95(0,02)[3,0]$ & $0,95(0,02)[3,0]$ & $0,99(0,01)[1,0]$ \\
\hline $7-\log -f^{*}$ & $0,14(0,04)[3,5]$ & $0,16(0,02)[1,5]$ & $0,14(0,03)[3,5]$ & $0,16(0,02)[1,5]$ \\
\hline 8-Magtag5k & $0,37(0,01)[2,5]$ & $0,37(0,01)[2,5]$ & $0,36(0,01)[4,0]$ & $0,38(0,01)[1,0]$ \\
\hline 9-Scene & $0,72(0,03)[2,5]$ & $0,72(0,03)[2,5]$ & $0,72(0,03)[2,5]$ & $0,72(0,02)[2,5]$ \\
\hline 10-Yeast & $0,66(0,02)[2,5]$ & $0,66(0,02)[2,5]$ & $0,66(0,02)[2,5]$ & $0,66(0,02)[2,5]$ \\
\hline
\end{tabular}

Tabela A.41: Similaridade média dos subconjuntos de atributos de tamanho $\left|X^{\prime}\right|=20 \% M$ encontrados por algoritmos de SA baseados em ReliefF e IG-LP

\begin{tabular}{rrrrr}
\hline \hline & $I G-L P$ & $R F-B R$ & $R F-L P$ & $R F-M L$ \\
\hline IG-LP & & 0,46 & 0,54 & $\mathbf{0 , 2 4}$ \\
RF-BR & 0,46 & & 0,64 & $\mathbf{0 , 3 3}$ \\
RF-LP & 0,54 & 0,64 & & $\mathbf{0 , 2 8}$ \\
RF-ML & $\mathbf{0 , 2 4}$ & $\mathbf{0 , 3 3}$ & $\mathbf{0 , 2 8}$ & \\
\hline
\end{tabular}

Tabela A.42: Similaridade média dos subconjuntos de atributos de tamanho $\left|X^{\prime}\right|=30 \% M$ encontrados por algoritmos de SA baseados em ReliefF e IG-LP

\begin{tabular}{rrrrr}
\hline \hline & $I G-L P$ & $R F-B R$ & $R F-L P$ & $R F-M L$ \\
\hline IG-LP & & 0,49 & 0,55 & $\mathbf{0 , 2 5}$ \\
RF-BR & 0,49 & & 0,68 & $\mathbf{0 , 3 2}$ \\
RF-LP & 0,55 & 0,68 & & $\mathbf{0 , 2 9}$ \\
RF-ML & $\mathbf{0 , 2 5}$ & $\mathbf{0 , 3 2}$ & $\mathbf{0 , 2 9}$ & \\
\hline \hline
\end{tabular}


Tabela A.43: Similaridade média dos subconjuntos de atributos de tamanho $\left|X^{\prime}\right|=40 \% M$ encontrados por algoritmos de SA baseados em ReliefF e IG-LP

\begin{tabular}{rrrrr}
\hline \hline & $I G-L P$ & $R F-B R$ & $R F-L P$ & $R F-M L$ \\
\hline IG-LP & & 0,44 & 0,49 & $\mathbf{0 , 2 4}$ \\
RF-BR & 0,44 & & 0,66 & $\mathbf{0 , 3 3}$ \\
RF-LP & 0,49 & 0,66 & & $\mathbf{0 , 2 9}$ \\
RF-ML & $\mathbf{0 , 2 4}$ & $\mathbf{0 , 3 3}$ & $\mathbf{0 , 2 9}$ & \\
\hline \hline
\end{tabular}

Tabela A.44: Similaridade média dos subconjuntos de atributos de tamanho $\left|X^{\prime}\right|=50 \% M$ encontrados por algoritmos de SA baseados em ReliefF e $I G-L P$

\begin{tabular}{rrrrr}
\hline \hline & $I G-L P$ & $R F-B R$ & $R F-L P$ & $R F-M L$ \\
\hline IG-LP & & 0,39 & 0,46 & $\mathbf{0 , 2 3}$ \\
RF-BR & 0,39 & & 0,69 & $\mathbf{0 , 3 2}$ \\
RF-LP & 0,46 & 0,69 & & $\mathbf{0 , 2 8}$ \\
RF-ML & $\mathbf{0 , 2 3}$ & $\mathbf{0 , 3 2}$ & $\mathbf{0 , 2 8}$ & \\
\hline \hline
\end{tabular}

Tabela A.45: Similaridade média dos subconjuntos de atributos de tamanho $\left|X^{\prime}\right|=60 \% M$ encontrados por algoritmos de SA baseados em ReliefF e IG-LP

\begin{tabular}{rrrrr}
\hline \hline & $I G-L P$ & $R F-B R$ & $R F-L P$ & $R F-M L$ \\
\hline IG-LP & & 0,40 & 0,48 & $\mathbf{0 , 2 3}$ \\
RF-BR & 0,40 & & 0,71 & $\mathbf{0 , 2 8}$ \\
RF-LP & 0,48 & 0,71 & & $\mathbf{0 , 2 4}$ \\
RF-ML & $\mathbf{0 , 2 3}$ & $\mathbf{0 , 2 8}$ & $\mathbf{0 , 2 4}$ & \\
\hline \hline
\end{tabular}

Tabela A.46: Similaridade média dos subconjuntos de atributos de tamanho $\left|X^{\prime}\right|=70 \% M$ encontrados por algoritmos de SA baseados em ReliefF e IG-LP

\begin{tabular}{rrrrr}
\hline \hline & $I G-L P$ & $R F-B R$ & $R F-L P$ & $R F-M L$ \\
\hline IG-LP & & 0,36 & 0,46 & $\mathbf{0 , 1 6}$ \\
RF-BR & 0,36 & & 0,69 & $\mathbf{0 , 2 5}$ \\
RF-LP & 0,46 & 0,69 & & $\mathbf{0 , 2 1}$ \\
RF-ML & $\mathbf{0 , 1 6}$ & $\mathbf{0 , 2 5}$ & $\mathbf{0 , 2 1}$ & \\
\hline \hline
\end{tabular}

Tabela A.47: Similaridade média dos subconjuntos de atributos de tamanho $\left|X^{\prime}\right|=80 \% M$ encontrados por algoritmos de SA baseados em ReliefF e IG-LP

\begin{tabular}{rrrrr}
\hline \hline & $I G-L P$ & $R F-B R$ & $R F-L P$ & $R F-M L$ \\
\hline IG-LP & & 0,31 & 0,41 & $\mathbf{0 , 0 8}$ \\
RF-BR & 0,31 & & 0,64 & $\mathbf{0 , 2 2}$ \\
RF-LP & 0,41 & 0,64 & & $\mathbf{0 , 2 0}$ \\
RF-ML & $\mathbf{0 , 0 8}$ & $\mathbf{0 , 2 2}$ & $\mathbf{0 , 2 0}$ & \\
\hline \hline
\end{tabular}

Tabela A.48: Similaridade média dos subconjuntos de atributos de tamanho $\left|X^{\prime}\right|=90 \% M$ encontrados por algoritmos de SA baseados em ReliefF e IG-LP

\begin{tabular}{rrrrr}
\hline \hline & $I G-L P$ & $R F-B R$ & $R F-L P$ & $R F-M L$ \\
\hline IG-LP & & 0,26 & 0,38 & $\mathbf{0 , 0 0}$ \\
RF-BR & 0,26 & & 0,60 & $\mathbf{0 , 1 7}$ \\
RF-LP & 0,38 & 0,60 & & $\mathbf{0 , 0 8}$ \\
RF-ML & $\mathbf{0 , 0 0}$ & $\mathbf{0 , 1 7}$ & $\mathbf{0 , 0 8}$ & \\
\hline \hline
\end{tabular}




\section{Referências Bibliográficas}

Aha, D. e Kibler, D. (1991). Instance-based learning algorithms. Machine Learning, 6:37-66. Citado na página 79.

Baranauskas, J. A. (2001). Extração Automática de Conhecimento por Múltiplos Indutores. Tese de doutorado, ICMC-Universidade de São Paulo - Brasil. Citado na página 39.

Bekkerman, R., El-Yaniv, R., Tishby, N., e Winter, Y. (2001). On feature distributional clustering for text categorization. In International ACM Special Interest Group on Information Retrieval Conference on Research and Development in Information Retrieval, páginas 146-153. Citado na página 112.

Bhowmick, P. K., Basu, A., Mitra, P., e Prasad, A. (2009). Multi-label text classification approach for sentence level news emotion analysis. In International Conference on Pattern Recognition and Machine Intelligence, páginas 261-266. Citado nas páginas 2, 34, e 112.

Bolón-Canedo, V., Sánchez-Maroño, N., e Alonso-Betanzos, A. (2013). A review of feature selection methods on synthetic data. Knowledge and Information Systems, 34(3):483-519. Citado na página 87.

Boutell, M. R., Luo, J., Shen, X., e Brown, C. M. (2004). Learning multi-label scene classification. Pattern Recognition, 37:1757-1771. Citado nas páginas 2 e 34.

Brinker, K. e Hüllermeier, E. (2007). Case-based multilabel ranking. In International Joint Conference on Artifical Intelligence, páginas 702-707. Citado na página 18.

Cannas, L. M., Dessì, N., e Pes, B. (2013). Assessing similarity of feature selection techniques in high-dimensional domains. Pattern Recognition Letters, 34(12):1446-1453. Citado nas páginas 7 e 102. 
Carvalho, A. C. P. L. F. d. e Freitas, A. A. (2009). A tutorial on multi-label classification techniques. In Abraham, A., Hassanien, A.-E., e Snášel, V., editors, Foundations of Computational Intelligence Volume 5, volume 205 of Studies in Computational Intelligence, páginas 177-195. Springer Berlin Heidelberg. Citado na página 14.

Carvalho, V. A. M., Spolaôr, N., Cherman, E. A., e Monard, M. C. (2014). A framework for multi-label exploratory data analysis: ML-EDA. In Conferencia Latinoamericana en Informática, páginas 1-12. Citado nas páginas 7 e 109.

Cerri, R., Carvalho, A. C. P. L. F. d., e Freitas, A. A. (2012). Adapting non-hierarchical multilabel classification methods for hierarchical multilabel classification. Intelligent Data Analysis, 15:861-887. Citado nas páginas 12 e 35.

Chang, Y.-C., Chen, S.-M., e Liau, C.-J. (2008). Multilabel text categorization based on a new linear classifier learning method and a category-sensitive refinement method. Expert Systems with Applications, 34(3):1948-1953. Citado na página 112 .

Charte, F., Rivera, A., del Jesus, M., e Herrera, F. (2012). Improving multilabel classifiers via label reduction with association rules. In Corchado, E., Snášel, V., Abraham, A., Wozniak, M., Graña, M., e Cho, S.-B., editors, Hybrid Artificial Intelligent Systems, volume 7209 of Lecture Notes in Computer Science, páginas 188-199. Springer Berlin Heidelberg. Citado na página 107.

Chekina, L., Gutfreund, D., Kontorovich, A., Rokach, L., e Shapira, B. (2013). Exploiting label dependencies for improved sample complexity. Machine Learning, 91(1):1-42. Citado na página 29.

Chen, W., Yan, J., Zhang, B., Chen, Z., e Yang, Q. (2007). Document transformation for multi-label feature selection in text categorization. In IEEE International Conference on Data Mining, páginas 451-456. Citado nas páginas 34 e 111.

Cherman, E. A., Metz, J., e Monard, M. C. (2012). Incorporating label dependency into the binary relevance framework for multi-label classification. Expert Systems with Applications, 39(2):1647-1655. Citado nas páginas 16, 29, e 101 .

Cherman, E. A., Spolaôr, N., Valverde-Rebaza, J. C., e Monard, M. C. (2013). Algoritmos de aprendizado baseado em grafos para classificação multirrótulo. In Encontro Nacional de Inteligência Artificial e Computacional, páginas 1-12. Citado nas páginas 7,19 , e 85. 
Cherman, E. A., Spolaôr, N., Valverde-Rebaza, J. C., e Monard, M. C. (2014). Lazy multi-label learning algorithms based on mutuality strategies. Journal of Intelligent and Robotic Systems, 0:1-17. Em processo de revisão. Citado na página 7.

Chou, S. e Hsu, C.-L. (2005). Mmdt: a multi-valued and multi-labeled decision tree classifier for data mining. Expert Systems with Applications, 28(4):799812. Citado na página 111 .

Clare, A. e King, R. D. (2001). Knowledge discovery in multi-label phenotype data. In European Conference on Principles of Data Mining and Knowledge Discovery, páginas 42-53. Springer Berlin Heidelberg. Citado nas páginas 18 e 89.

Coello, C. A. C. (2011). Evolutionary multi-objective optimization: Basic concepts and some applications in pattern recognition. In Martínez-Trinidad, J., Carrasco-Ochoa, J., Ben-Youssef Brants, C., e Hancock, E., editors, Pattern Recognition, volume 6718 of Lecture Notes in Computer Science, páginas 22-33. Springer Berlin Heidelberg. Citado na página 49.

Cong, H. e Tong, L. H. (2008). Grouping of triz inventive principles to facilitate automatic patent classification. Expert Systems with Applications, 34(1):788-795. Citado nas páginas 16 e 111.

Covões, T. F. (2010). Seleção de atributos via agrupamento. Dissertação de Mestrado, ICMC-Universidade de São Paulo - Brasil. Citado na página 39.

Dembczyński, K., Waegeman, W., Cheng, W., e Hüllermeier, E. (2010). On label dependence in multi-label classification. In International Workshop on Learning from Multi-Label Data in International Conference of Machine Learning, páginas 5-12. Citado na página 29.

Dembczyński, K., Waegeman, W., Cheng, W., e Hüllermeier, E. (2012). On label dependence and loss minimization in multi-label classification. Machine Learning, 88(1-2):5-45. Citado na página 3.

Demšar, J. (2006). Statistical comparison of classifiers over multiple data sets. Journal of Machine Learning Research, 7(1):1-30. Citado nas páginas 69, 70, 93, e 95.

Demšar, J. (2010). Algorithms for subsetting attribute values with Relief. Machine Learning, 78:421-428. Citado nas páginas 61 e 78.

Demšar, J., Curk, T., Erjavec, A., Črt Gorup, Hočevar, T., Milutinovič, M., Možina, M., Polajnar, M., Toplak, M., Starič, A., Štajdohar, M., Umek, L., 
Žagar, L., Žbontar, J., Žitnik, M., e Zupan, B. (2013). Orange: Data mining toolbox in Python. Journal of Machine Learning Research, 14:2349-2353. Citado na página 80.

Dendamrongvit, S. e Kubat, M. (2010). Undersampling approach for imbalanced training sets and induction from multi-label text-categorization domains. In Pacific-Asia International Conference on Knowledge Discovery and Data Mining: New Frontiers in Applied Data Mining, páginas 40-52. Citado na página 112.

Dendamrongvit, S., Vateekul, P., e Kubat, M. (2011). Irrelevant attributes and imbalanced classes in multi-label text-categorization domains. Intelligent Data Analysis, 15(6):843-859. Citado nas páginas 34 e 112.

Doquire, G. e Verleysen, M. (2011). Feature selection for multi-label classification problems. In Cabestany, J., Rojas, I., e Joya, G., editors, Advances in Computational Intelligence, chapter 2, páginas 9-16. Springer Berlin Heidelberg. Citado nas páginas 48 e 111.

Doquire, G. e Verleysen, M. (2013). Mutual information-based feature selection for multilabel classification. Neurocomputing, 122:148-155. Citado nas páginas 2,48 , e 112 .

Duivesteijn, W., Loza Mencía, E., Fürnkranz, J., e Knobbe, A. (2012). Multilabel LeGo - enhancing multi-label classifiers with local patterns. In Hollmén, J., Klawonn, F., e Tucker, A., editors, Advances in Intelligent Data Analysis XI, volume 7619 of Lecture Notes in Computer Science, páginas 114125. Springer Berlin Heidelberg. Citado na página 51.

Elisseef, A. e Weston, J. (2002). Kernel methods for multi-labelled classification and categorical regression problems. Advances in Neural Information Processing Systems, 14:681-687. Citado na página 34.

Esuli, A., Fagni, T., e Sebastiani, F. (2006). TreeBoost.MH: A boosting algorithm for multi-label hierarchical text categorization. In Crestani, F., Ferragina, P., e Sanderson, M., editors, String Processing and Information Retrieval, páginas 13-24. Springer Berlin Heidelberg. Citado nas páginas 2, 41, e 112 .

Esuli, A., Fagni, T., e Sebastiani, F. (2008). Boosting multi-label hierarchical text categorization. Information Retrieval, 11(4):287-313. Citado nas páginas 2, 34, e 111 . 
Fan, R.-E., Chang, K.-W., Hsieh, C.-J., Wang, X.-R., e Lin, C.-J. (2008). LIBLINEAR: A library for large linear classification. Journal of Machine Learning Research, 9:1871-1874. Citado na página 16.

Fayyad, U. M. e Irani, K. B. (1993). Multi-interval discretization of continuousvalued attributes for classification learning. In International Joint Conference on Artificial Intelligence, páginas 1022-1029. Citado na página 61 .

Flach, P. (2012). Machine Learning: the Art and Science of Algorithms that Make Sense of Data. Cambridge University Press. Citado na página 20.

Forman, G. (2004). A pitfall and solution in multi-class feature selection for text classification. HPL-2004-86. Hewlett Packard. Citado na página 54.

Friedman, M. (1940). A comparison of alternative tests of significance for the problem of $m$ rankings. Mathematical Statistics, 11:86-92. Citado nas páginas 69 e 95.

García, D., González, A., e Pérez, R. (2014). A feature construction approach for genetic iterative rule learning algorithm. Journal of Computer and System Sciences, 80(1):101-117. Citado na página 51.

Gasse, M., Aussem, A., e Elghazel, H. (2014). A hybrid algorithm for Bayesian network structure learning with application to multi-label learning. Expert Systems with Application, 41:6755-6772. Citado nas páginas 49 e 112.

Godbole, S. e Sarawagi, S. (2004). Discriminative methods for multi-labeled classification. In Pacific-Asia Conference on Knowledge Discovery and Data Mining, páginas 22-30. Citado nas páginas 20 e 29.

Gonçalves, T. e Quaresma, P. (2005). Is linguistic information relevant for the classification of legal texts? In International Conference on Artificial Intelligence and Law, páginas 168-176. Citado na página 111.

Gu, Q., Li, Z., e Han, J. (2011). Correlated multi-label feature selection. In ACM International Conference on Information and Knowledge Management, páginas 1087-1096. Citado nas páginas 49 e 112.

Guyader, A. e Hengartner, N. (2013). On the mutual nearest neighbors estimate in regression. Journal of Machine Learning Research, 14:2361-2376. Citado nas páginas 85 e 87.

Hall, M. A. (1999). Correlation-based Feature Selection for Machine Learning. Tese de doutorado, University of Waikato - Nova Zelândia. Citado na página 49. 
Hamming, R. W. (1950). Error detecting and error correcting codes. Bell System Technical, 29(2):147-160. Citado na página 84.

Han, J. e Kamber, M. (2011). Data Mining: Concepts and Techniques. Morgan Kaufmann, $3^{\text {a }}$ edição. Citado na página 1.

Hastie, T., Tibshirani, R., e Friedman, J. (2001). The Elements of Statistical Learning. Springer New York. Citado na página 37.

Heider, D., Senge, R., Cheng, W., e Hüllermeier, E. (2013). Multilabel classification for exploiting cross-resistance information in HIV-1 drug resistance prediction. Bioinformatics, 29(16):1946-1952. Citado nas páginas 2 e 35.

Hernandes, E. C. M., Zamboni, A. B., Thommazo, A. D., e Fabbri, S. C. P. F. (2012). Using gqm and tam to evaluate start - a tool that supports systematic review. CLEI Electronic journal, 15(1):1-13. Citado na página 106.

Islam, M. S. e Sluzek, A. (2010). Hierarchical object categorization with automatic feature selection. In Computer Science and Information Technology (IMCSIT), Proceedings of the 2010 International Multiconference on, páginas 45-51. Citado na página 111.

Jain, A. K. e Dubes, R. C. (1988). Algorithms for Clustering Data. Prentice-Hall, Inc. Citado na página 131.

Jiang, W., Cotton, C., Chang, S.-F., Ellis, D., e Loui, A. (2009). Short-term audio-visual atoms for generic video concept classification. In ACM International Conference on Multimedia, páginas 5-14. Citado na página 112.

John, G. H., Kohavi, R., e Pfleger, K. (1994). Irrelevant features and the subset selection problem. In International Conference on Machine Learning, páginas 121-129. Citado na página 39.

Jungjit, S., Freitas, A. A., Michaelis, M., e Cinatl, J. (2012). A multi-label correlation-based feature selection method for the classification of neuroblastoma microarray data. In Industrial Conference on Data Mining Workshops, páginas 149-157. Citado nas páginas 49 e 112.

Jungit, S., Freitas, A. A., Michaelis, M., e Cinatl, J. (2013). Two extensions to multi-label correlation-based feature selection: A case study in bioinformatics. In IEEE International Conference on Systems, Man, and Cybernetics, páginas 1519-1524. Citado nas páginas 49, 107, 109, e 112.

Karabulut, M. (2013). Fuzzy unordered rule induction algorithm in text categorization on top of geometric particle swarm optimization term selection. Knowledge-Based Systems, 54:288-297. Citado nas páginas 2, 16, e 112. 
Kira, K. e Rendell, L. A. (1992). A practical approach to feature selection. In International Workshop on Machine learning, páginas 249-256. Citado nas páginas 77,78 , e 79 .

Kitchenham, B. A. e Charters, S. (2007). Guidelines for performing systematic literature reviews in software engineering. Relatório técnico, Evidence-based Software Engineering - Reino Unido. Citado nas páginas 4 e 44.

Kohavi, R. e John, G. H. (1997). Wrappers for feature subset selection. Artificial Intelligence, 97(1-2):273-324. Citado na página 38.

Kong, D., Ding, C., Huang, H., e Zhao, H. (2012). Multi-label ReliefF and F-statistic feature selections for image annotation. In IEEE Conference on Computer Vision and Pattern Recognition, páginas 2352-2359. Citado nas páginas $7,46,83$, e 112 .

Kong, X. e Yu, P. (2010). Multi-label feature selection for graph classification. In International Conference on Data Mining, páginas 274-283. Citado na página 111 .

Kononenko, I. (1994). Estimating attributes: Analysis and extensions of Relief. In Bergadano, F. e Raedt, L., editors, Machine Learning - ECML-94, volume 784 of Lecture Notes in Computer Science, páginas 171-182. Springer Berlin Heidelberg. Citado nas páginas 6, 45, 77, 79, e 80.

Kononenko, I. e Kukar, M. (2007). Machine Learning and Data Mining: Introduction to Principles and Algorithms. Horwood Publishing Limited. Citado na página 14 .

Kononenko, I. e Robnik-Šikonja, M. (2007). Weighting and local methods non-myopic feature quality evaluation with (R)ReliefF. In Liu, H. e Motoda, H., editors, Computational Methods of Feature Selection, páginas 169-191. Chapman \& Hall/CRC. Citado nas páginas 75, 80, e 81.

Kuncheva, L. I. (2007). A stability index for feature selection. In IASTED International Multi-Conference: Artificial Intelligence and Applications, páginas 390-395. Citado na página 91.

Largeron, C., Moulin, C., e Géry, M. (2010). UJM at INEX 2009 XML mining track. In Focused Retrieval and Evaluation / International Conference on Initiative for the Evaluation of XML Retrieval, páginas 426-433. Citado na página 112 .

Lastra, G., Luaces, O., Quevedo, J. R., e Bahamonde, A. (2011). Graphical feature selection for multilabel classification tasks. In International Confe- 
rence on Advances in Intelligent Data Analysis, páginas 246-257. Citado nas páginas 2, 48, e 112 .

Lee, H. D. (2005). Seleção de Atributos Importantes para a Extração de Conhecimento de Bases de Dados. Tese de doutorado, ICMC-Universidade de São Paulo - Brasil. Citado na página 40.

Lee, H. D., Monard, M. C., e Baranauskas, J. A. (2000). A practical approach to knowledge-driven constructive induction. In Argentine Symposium on Artificial Intelligence, páginas 71-85. Citado na página 51.

Lee, J. e Kim, D.-W. (2013). Feature selection for multi-label classification using multivariate mutual information. Pattern Recognition Letters, 34(3):349-357. Citado nas páginas 7, 48, e 112.

Lee, j., Lim, H., e Kim, D.-W. (2012). Approximating mutual information for multi-label feature selection. Electronics Letters, 48(15):929-930. Citado na página 112.

Lewis, D. D., Yang, Y., Rose, T. G., e Li, F. (2004). Rcv1: A new benchmark collection for text categorization research. Journal of Machine Learning Research, 5:361-397. Citado nas páginas 24 e 112.

Li, F., Dai, Q., Xu, W., e Er, G. (2008). Multilabel neighborhood propagation for region-based image retrieval. IEEE Transactions on Multimedia, 10(8):15921604. Citado na página 112.

Li, G.-Z., You, M., Ge, L., Yang, J., e Yang., M. (2010a). Feature selection for semi-supervised multi-label learning with application to gene function analysis. In ACM International Conference on Bioinformatics and Computational Biology, páginas 354-357. Citado na página 111.

Li, R., Zhang, Y., Lu, Z., Lu, J., e Tian, Y. (2010b). Technique of image retrieval based on multi-label image annotation. In International Conference on MultiMedia and Information Technology, páginas 10-13. Citado na página 112.

Li, S. e Zhang, Z. (2012). An ensemble multi-label feature selection algorithm based on information entropy. In International Conference on Machine Learning, páginas 1-8. Citado na página 112.

Lillywhite, K., Lee, D.-J., Tippetts, B., e Archibald, J. (2013). A feature construction method for general object recognition. Pattern Recognition, 46(12):3300-3314. Citado na página 51.

Liu, G.-P., Yan, J.-J., Wang, Y.-Q., Fu, J.-J., Xu, Z.-X., Guo, R., e Qian, P. (2012). Application of multilabel learning using the relevant feature for each 
label in chronic syndrome diagnosis. Evidence-Based Complementary and Alternative Medicine, 2012:1-9. Citado na página 112.

Liu, H. e Motoda, H. (1998). Feature Selection for Knowledge Discovery and Data Mining. Kluwer Academic Publishers. Citado nas páginas 38, 42, e 43.

Liu, H. e Motoda, H. (2007). Computational Methods of Feature Selection. Chapman \& Hall/CRC. Citado nas páginas 2 e 39.

Liu, H., Zhang, S., e Wu, X. (2014). Mlslr: Multilabel learning via sparse logistic regression. Information Sciences, 281:310-320. Citado na página 112.

Liu, H., Zhang, S., Zhao, J., Zhao, X., e Mo, Y. (2010). A new classification algorithm using mutual nearest neighbors. In International Conference on Grid and Cooperative Computing, páginas 52-57. Citado nas páginas 85 e 87.

Lorena, A. C., Costa, I. G., Spolaôr, N., e de Souto, M. C. (2012). Analysis of complexity indices for classification problems: Cancer gene expression data. Neurocomputing, 75(1):33-42. Brazilian Symposium on Neural Networks (SBRN 2010) International Conference on Hybrid Artificial Intelligence Systems (HAIS 2010). Citado na página 7.

Luo, Q., Chen, E., e Xiong, H. (2011). A semantic term weighting scheme for text categorization. Expert Systems with Applications, 38(10):12708-12716. Citado na página 111.

Luo, X. e Zincir-Heywood, N. A. (2005). Evaluation of two systems on multiclass multi-label document classification. In International Symposium on Methodologies for Intelligent Systems, páginas 161-169. Citado na página 18.

Marques, G., Domingues, M. A., Langlois, T., e Gouyon, F. (2011). Three current issues in music autotagging. In International Society for Music Information Retrieval Conference, páginas 795-800. Citado na página 32.

Mayne, A. e Perry, R. (2009). Hierarchically classifying documents with multiple labels. In IEEE Symposium on Computational Intelligence and Data Mining, páginas 133-139. Citado na página 111.

Mengle, S. e Goharian, N. (2009). Passage detection using text classification. Journal of the American Society for Information Science and Technology, 60(4):814-825. Citado na página 112. 
Metz, J. (2011). Abordagens para aprendizado semissupervisionado multirrótulo e hierárquico. Tese de doutorado, ICMC-Universidade de São Paulo Brasil. Citado na página 12.

Metz, J., Abreu, L. F., Cherman, E. A., e Monard, M. C. (2012). On the estimation of predictive evaluation measure baselines for multi-label learning. In Pavón, J., Duque-Méndez, N. D., e Fuentes-Fernández, R., editors, Advances in Artificial Intelligence - IBERAMIA 2012, volume 7637 of Lecture Notes in Computer Science, páginas 189-198. Springer Berlin Heidelberg. Citado na página 26.

Metz, J., Spolaôr, N., Cherman, E. A., e Monard, M. C. (2014). The learning power of multi-label algorithms: analyzing literature results in contrast to a simple multi-label baseline classifier. Artificial Intelligence Review, 0:1-25. Em processo de revisão. Citado nas páginas 7, 26, 27, 28, e 94.

Mitchell, T. M. (1997). Machine Learning. McGraw-Hill. Citado na página 2.

Mittal, A. e Cheong, L.-F. (2004). Addressing the problems of bayesian network classification of video using high-dimensional features. IEEE Transactions on Knowledge and Data Engineering, 16(2):230-244. Citado na página 111.

Moore, J. e White, B. (2007). Tuning ReliefF for genome-wide genetic analysis. In Marchiori, E., Moore, J. H., e Rajapakse, J. C., editors, Evolutionary Computation, Machine Learning and Data Mining in Bioinformatics, páginas 166-175. Springer Berlin Heidelberg. Citado nas páginas 62 e 77.

Motoda, H. e Liu, H. (2002). Feature selection, extraction and construction. Communication of Institute of Information and Computing Machinery, 5:6772. Citado na página 51 .

Muñoz, L. A. B. e González-Navarro, F. F. (2011). Review and evaluation of feature selection algorithms in synthetic problems. Relatório Técnico Arxiv: $1101.2320 .13 \mathrm{pg}$. Citado na página 87.

Nardiello, P., Sebastiani, F., e Sperduti, A. (2003). Discretizing continuous attributes in AdaBoost for text categorization. In Sebastiani, F., editor, Advances in Information Retrieval, volume 2633 of Lecture Notes in Computer Science, páginas 320-334. Springer Berlin Heidelberg. Citado na página 111.

Naula, P., Airola, A., Salakoski, T., e Pahikkala, T. (2014). Multi-label learning under feature extraction budgets. Pattern Recognition Letters, 40:56-65. Citado na página 112 . 
Naula, P., Pahikkala, T., Airola, A., e Salakoski, T. (2011). Learning multilabel predictors under sparsity budget. In Kofod-Petersen, A., Heintz, F., e Langseth, H., editors, Scandinavian Conference on Artificial Intelligence, volume 227 of Frontiers in Artificial Intelligence and Applications, páginas 30-39. IOS Press. Citado na página 111.

Noh, H. G., Song, M. S., e Park, S. H. (2004). An unbiased method for constructing multilabel classification trees. Computational Statistics \& Data Analysis, 47(1):149-164. Citado na página 111.

Novovičová, J., Somol, P., Haindl, M., e Pudil, P. (2007). Conditional mutual information based feature selection for classification task. In Rueda, L., Mery, D., e Kittler, J., editors, Progress in Pattern Recognition, Image Analysis and Applications, volume 4756 of Lecture Notes in Computer Science, páginas 417-426. Springer Berlin Heidelberg. Citado na página 111.

Oliveira, M. V., Wandekokem, E. W., Mendel, E., Fabris, F., Varejão, F. M., Rauber, T. W., e Batista, R. J. (2010). A comparison of two feature-based ensemble methods for constructing motor pump fault diagnosis classifiers. In IEEE International Conference on Tools with Artificial Intelligence, páginas 417-420. Citado na página 111.

Olsson, J. O. S. e Oard, D. W. (2006). Combining feature selectors for text classification. In ACM International Conference on Information and Knowledge Management, páginas 798-799. Citado nas páginas 16 e 111.

Pacharawongsakda, E. e Theeramunkong, T. (2013). A two-stage dual space reduction framework for multi-label classification. In Li, J., Cao, L., Wang, C., Tan, K., Liu, B., Pei, J., e Tseng, V., editors, Trends and Applications in Knowledge Discovery and Data Mining, volume 7867 of Lecture Notes in Computer Science, páginas 330-341. Springer Berlin Heidelberg. Citado na página 107.

Park, H., Park, D., e Kwon, H.-C. (2011). Two-phase prediction of protein functions from biological literature based on gini-index. In International Conference on Ubiquitous Information Management and Communication, páginas 61:1-61:10. Citado na página 112.

Pfister, T. e Robinson, P. (2010). Speech emotion classification and public speaking skill assessment. In Salah, A., Gevers, T., Sebe, N., e Vinciarelli, A., editors, Human Behavior Understanding, volume 6219 of Lecture Notes in Computer Science, páginas 151-162. Springer Berlin Heidelberg. Citado na página 112. 
Piramuthu, S. e Sikora, R. (2009). Iterative feature construction for improving inductive learning algorithms. Expert Systems with Applications, 36(2):3401-3406. Citado na página 51.

Pons-Porrata, A., Gil-GarcÃa, R., e Berlanga-Llavori, R. (2007). Using typical testors for feature selection in text categorization. In Rueda, L., Mery, D., e Kittler, J., editors, Progress in Pattern Recognition, Image Analysis and Applications, volume 4756 of Lecture Notes in Computer Science, páginas 643-652. Springer Berlin Heidelberg. Citado na página 112.

Prati, R. C. (2012). Combining feature ranking algorithms through rank aggregation. In International Joint Conference on Neural Networks, páginas 1-8. Citado na página 108.

Prati, R. C. e Olivetti de Franca, F. (2013). Extending features for multilabel classification with swarm biclustering. In IEEE Congress on Evolutionary Computation, páginas 2964-2971. Citado na página 51.

Pupo, O. G. R., Morell, C., e Soto, S. V. (2013). ReliefF-ML: An extension of ReliefF algorithm to multi-label learning. In Ruiz-Shulcloper, J. e Sanniti di Baja, G., editors, Progress in Pattern Recognition, Image Analysis, Computer Vision, and Applications, volume 8259 of Lecture Notes in Computer Science, páginas 528-535. Springer Berlin Heidelberg. Citado nas páginas 7, 46, 77, 83, e 112 .

Pyle, D. (1999). Data Preparation for Data Mining. Morgan Kaufmann. Citado na página 1 .

Quinlan, J. R. (1993). C4.5: Programs for Machine Learning. Morgan Kaufmann. Citado na página 18.

Read, J. (2008). A pruned problem transformation method for multi-label classification. In New Zealand Computer Science Research Student Conference, páginas 143-150. Citado na página 48.

Read, J., Bifet, A., Holmes, G., e Pfahringer, B. (2012). Scalable and efficient multi-label classification for evolving data streams. Machine Learning, 88(12):243-272. Citado na página 18.

Read, J., Pfahringer, B., Holmes, G., e Frank, E. (2009). Classifier chains for multi-label classification. In Buntine, W., Grobelnik, M., Mladenic, D., e Shawe-Taylor, J., editors, Machine Learning and Knowledge Discovery in Databases, volume 5782, páginas 254-269. Springer Berlin Heidelberg. Citado nas páginas 16 e 29. 
Reis, D. M. D., Cherman, E. A., Spolaôr, N., e Monard, M. C. (2012). Extensões do algoritmo de aprendizado de máquina multirrótulo BRkNN. In Encontro Nacional de Inteligência Artificial, páginas 1-12. Citado nas páginas 7 e 19.

Rios, A. e Kavuluru, R. (2013). Supervised extraction of diagnosis codes from emrs: Role of feature selection, data selection, and probabilistic thresholding. In IEEE International Conference on Healthcare Informatics, páginas 66-73. Citado nas páginas 16 e 112.

Robnik-Sikonja, M. e Kononenko, I. (1997). An adaptation of Relief for attribute estimation in regression. In International Conference on Machine Learning, páginas 296-304. Citado nas páginas 46, 47, 77, 80, 82, e 83.

Robnik-Sikonja, M. e Kononenko, I. (2003). Theoretical and empirical analysis of ReliefF and RReliefF. Machine Learning, 53(1-2):23-69. Citado nas páginas $6,47,77,83$, e 85 .

Rossi, R. G. e Rezende, S. O. (2011). Building a topic hierarchy using the bagof-related-words representation. In Symposium on Document Engineering, páginas 195-204. ACM. Citado na página 32.

Saleh, S. N. e El-Sonbaty, Y. (2007). A feature selection algorithm with redundancy reduction for text classification. In International Symposium on Computer and Information Sciences, páginas 1-6. Citado na página 111.

Shao, H., Li, G. Z., Liu, G. P., e Wang, Y. Q. (2011). Symptom selection for multi-label data of inquiry diagnosis in traditional chinese medicine. Science China Information Sciences, páginas 1-13. Citado nas páginas 49 e 112 .

Shimosaka, M., Nejigane, Y., Mori, T., e Sato, T. (2009). Fast online action recognition with efficient structured boosting. In IEEE International Conference on Multimedia and Expo, páginas 706-709. Citado na página 111.

Skarmeta, A. G., Bensaid, A., e Tazi, N. (2000). Data mining for text categorization with semi-supervised agglomerative hierarchical clustering. International Journal of Intelligent Systems, 15(7):633-646. Citado na página 111.

Slavkov, I., Karcheska, J., Kocev, D., Kalajdziski, S., e Džeroski, S. (2013). Extending ReliefF for hierarchical multi-label classification. In Workshop on New Frontiers in Mining Complex Patterns - European Conference on Machine Learning/Principles and Practice of Knowledge Discovery in Databases, páginas 156-167. Citado nas páginas 7, 46, 47, 77, 83, 109, e 112.

Spolaôr, N., Cherman, E. A., Metz, J., e Monard, M. C. (2013a). A systematic review on experimental multi-label learning. Relatório Técnico do ICMCUniversidade de São Paulo № 392. 31 pg. Citado nas páginas 7, 26, e 27. 
Spolaôr, N., Cherman, E. A., e Monard, M. C. (2011a). Uso do ReliefF para seleção de atributos em dados multirrótulo. In Conferencia Latinoamericana en Informática, páginas 960-975. Citado nas páginas 2, 77, e 83.

Spolaôr, N., Cherman, E. A., Monard, M. C., e Lee, H. D. (2012a). Filter approach feature selection methods to support multi-label learning based on ReliefF and information gain. In Barros, L. N., Finger, M., Pozo, A. T., Gimenénez-Lugo, G. A., e Castilho, M., editors, Advances in Artificial Intelligence - SBIA 2012, Lecture Notes in Computer Science, páginas 72-81. Springer Berlin Heidelberg. Citado nas páginas 18 e 112.

Spolaôr, N., Cherman, E. A., Monard, M. C., e Lee, H. D. (2013b). A comparison of multi-label feature selection methods using the problem transformation approach. Electronic Notes in Theoretical Computer Science, 292:135151. Citado nas páginas $2,7,16,17,77$, e 112 .

Spolaôr, N., Cherman, E. A., Monard, M. C., e Lee, H. D. (2013c). ReliefF for multi-label feature selection. In Brazilian Conference on Intelligent Systems, páginas 6-11. IEEE. Citado nas páginas 6, 46, 47, 77, 83, 90, e 112.

Spolaôr, N., Lorena, A. C., e Lee, H. D. (2011b). Multi-objective genetic algorithm evaluation in feature selection. In Takahashi, R., Deb, K., Wanner, E., e Greco, S., editors, Evolutionary Multi-Criterion Optimization, volume 6576 of Lecture Notes in Computer Science, páginas 462-476. Springer Berlin Heidelberg. Citado na página 7.

Spolaôr, N. e Monard, M. C. (2014). Evaluating ReliefF-based multi-label feature selection algorithms. In Ibero-American Conference on Artificial Intelligence, páginas 1-12. Springer Berlin Heidelberg. Em vias de impressão. Citado nas páginas 23, 63, 89, e 101.

Spolaôr, N., Monard, M. C., e Lee, H. D. (2012b). A systematic review to identify feature selection publications in multi-labeled data. Relatório Técnico do ICMC-Universidade de São Paulo № 374.31 pg. Citado nas páginas 2, 5, 30 , e 44 .

Spolaôr, N., Monard, M. C., e Lee, H. D. (2014a). The Relief family algorithms for feature selection in single-label datasets and some recent extensions. Relatório Técnico do ICMC-Universidade de São Paulo. Citado nas páginas 7 e 78.

Spolaôr, N., Monard, M. C., Tsoumakas, G., e Lee, H. D. (2014b). Label construction for multi-label feature selection. In Brazilian Conference on Intelligent Systems, páginas 1-6. IEEE. Em vias de impressão. Citado nas páginas $5,52,63,73$, e 89. 
Spolaôr, N. e Tsoumakas, G. (2013). Evaluating feature selection methods for multi-label text classification. In BioASQ workshop, páginas 1-12. Citado nas páginas $16,53,55,56,63$, e 112 .

Spyromitros, E., Tsoumakas, G., e Vlahavas, I. (2008). An empirical study of lazy multilabel classification algorithms. In Hellenic Conference on Artificial Intelligence, páginas 401-406. Springer Berlin Heidelberg. Citado nas páginas 18 e 19.

Tan, F. (2007). Improving Feature Selection Techniques for Machine Learning. Tese de doutorado, Georgia State University - Estados Unidos. Citado na página 111.

Tan, P.-N., Steinbach, M., e Kumar, V. (2013). Introduction to Data Mining. Addison-Wesley. Citado na página 85.

Tomás, J. T., Spolaôr, N., Cherman, E. A., e Monard, M. C. (2013). A framework to generate synthetic multi-label datasets. In Conferencia Latinoamericana en Informática, páginas 1-23. Citado na página 7.

Tomás, J. T., Spolaôr, N., Cherman, E. A., e Monard, M. C. (2014). A framework to generate synthetic multi-label datasets. Electronic Notes in Theoretical Computer Science, 302:155-176. Citado nas páginas 7, 87, 89, e 108.

Toutanova, K., Chen, F., Popat, K., e Hofmann, T. (2001). Text classification in a hierarchical mixture model for small training sets. In International Conference on Information and Knowledge Management, páginas 105-113. Citado na página 112 .

Trohidis, K., Tsoumakas, G., Kalliris, G., e Vlahavas, I. (2008). Multi-label classification of music into emotions. In International Conference on Music Information Retrieval, páginas 1-6. Citado nas páginas 2, 16, 17, 47, e 112.

Tsoumakas, G. (2013). Personal communication. Citado na página 31.

Tsoumakas, G., Dimou, A., Spyromitros, E., Mezaris, V., Kompatsiaris, I., e Vlahavas, I. (2009). Correlation based pruning of stacked binary relevance models for multi-label learning. In International Workshop on Learning from Multi-Label Data, páginas 101-116. Citado na página 29.

Tsoumakas, G. e Katakis, I. (2007). Multi-label classification: An overview. International Journal of Data Warehousing and Mining, 3:1-13. Citado nas páginas 20 e 30.

Tsoumakas, G., Katakis, I., e Vlahavas, I. (2010). Mining multi-label data. In Maimon, O. e Rokach, L., editors, Data Mining and Knowledge Discovery, 
páginas 1-19. Springer United States. Citado nas páginas 2, 14, 15, 16, $20,24,38,49$, e 50.

Tsoumakas, G., Spyromitros-Xioufis, E., Vilcek, J., e Vlahavas, I. (2011). Mulan: A java library for multi-label learning. Journal of Machine Learning Research, 12:2411-2414. Citado nas páginas 6, 63, e 89.

Tsoumakas, G. e Vlahavas, I. (2007). Random k-labelsets: An ensemble method for multilabel classification. In European Conference on Machine Learning, páginas 406-417. Citado nas páginas 16, 29, e 112.

Wandekokem, E. D., Varejão, F. M., e Rauber, T. W. (2010). An overproduceand-choose strategy to create classifier ensembles with tuned SVM parameters applied to real-world fault diagnosis. In Iberoamerican Congress Conference on Progress in Pattern Recognition, Image Analysis, Computer Vision, and Applications, páginas 500-508. Citado na página 111 .

Wandekokem, E. W., Mendel, E., Fabris, F., Oliveira, M. V. D., Batista, R. J., Varejão, F. M., e Rauber, T. W. (2011). Diagnosing multiple faults in oil rig motor pumps using support vector machine classifier ensembles. Integrated Computer-Aided Engineering, 18:61-74. Citado na página 111.

Wang, B., Jia, Y., Han, Y., e Han, W. (2009). Effective feature selection on data with uncertain labels. In IEEE International Conference on Data Engineering, páginas 1657-1662. Citado na página 111.

Wang, Z. e Jiang, M. (2009). Multi-labeled chinese text categorization based on the boosting algorithms. In International Conference on Natural Computation, páginas 205-208. Citado nas páginas 34 e 112.

Wei, Q., Yang, Z., Junping, Z., e Wang, Y. (2009). Semi-supervised multilabel learning algorithm using dependency among labels. In International Conference on Machine Learning and Computing, páginas 112-116. Citado na página 112 .

Wei, Z., Miao, D., Zhao, R., Xie, C., e Zhang, Z. (2010). Visualizing search results based on multi-label classification. In IEEE International Conference on Progress in Informatics and Computing, volume 1, páginas 203-207. Citado na página 112 .

Wilcoxon, F. (1945). Individual comparisons by ranking methods. Biometrics Bulletin, 1(6):80-83. Citado nas páginas 70 e 93.

Wilson, D. R. e Martinez, T. R. (2000). Reduction Techniques for ExemplarBased Learning Algorithms. Machine learning, 38(3):257-286. Citado na página 19. 
Witten, I. H. e Frank, E. (2011). Data Mining: Practical Machine Learning Tools and Techniques. Morgan Kaufmann, 3ª edição. Citado nas páginas 61, 63, e 80 .

Worring, M., Snoek, C., de Rooij, O., Nguyen, G., e Smeulders, A. (2007). The mediamill semantic video search engine. In IEEE International Conference on Acoustics, Speech and Signal Processing, páginas 1213-1216. Citado na página 34 .

Yang, J., Jiang, Y.-G., Hauptmann, A. G., e Ngo, C.-W. (2007). Evaluating bag-of-visual-words representations in scene classification. In International Workshop on Workshop on Multimedia Information Retrieval, páginas 197206. Citado na página 111.

Yang, Y. e Gopal, S. (2012). Multilabel classification with meta-level features in a learning-to-rank framework. Machine Learning, 88(1-2):47-68. Citado na página 51 .

Yang, Y. e Pedersen, J. O. (1997). A comparative study on feature selection in text categorization. In International Conference on Machine Learning, páginas 412-420. Citado nas páginas 16 e 112.

Ye, L., Xu, R., e Xu, J. (2012). Emotion prediction of news articles from reader's perspective based on multi-label classification. In International Conference on Machine Learning and Cybernetics, páginas 2019-2024. Citado nas páginas 16 e 112 .

Younes, Z., Abdallah, F., Denoeux, T., e Snoussi, H. (2011). A dependent multilabel classification method derived from the $\mathrm{k}$-nearest neighbor rule. EURASIP Journal on Advances in Signal Processing, 2011:1-14. Citado nas páginas 18 e 29.

Zhang, J. e Yang, Y. (2003). Robustness of regularized linear classification methods in text categorization. In International ACM SIGIR Conference on Research and Development in Information Retrieval, páginas 190-197. Citado na página 112 .

Zhang, M.-L., Peña, J. M., e Robles, V. (2009). Feature selection for multi-label naive bayes classification. Information Sciences, 179:3218-3229. Citado nas páginas 20, 50, 89, e 111 .

Zhang, M.-L. e Zhang, K. (2010). Multi-label learning by exploiting label dependency. In ACM SIGKDD International Conference on Knowledge Discovery and Data Mining, páginas 999-1008. Citado na página 41. 
Zhang, M.-L. e Zhou, Z.-H. (2005). A k-nearest neighbor based algorithm for multi-label classification. IEEE International Conference on Granular Computing, 2:718-721. Citado nas páginas 18 e 19.

Zhang, M.-L. e Zhou, Z.-H. (2013). A review on multi-label learning algorithms (in press). IEEE Transactions on Knowledge and Data Engineering, 99:1-59. Citado nas páginas 2, 3, 14, 15, 17, 20, 21, 29, 38, 41, 52, e 60.

Zheng, Z., Wu, X., e Srihari, R. (2004). Feature selection for text categorization on imbalanced data. Special Interest Group on Knowledge Discovery and Data Mining Explorations Newsletter, 6:80-89. Citado na página 112. 